\title{
Gesamtheitliches \\ Performance Measurement \\ Vorgehensmodell und informationstechnische Ausgestaltung
}

\section{Dissertation}

\begin{abstract}
zur Erlangung der Würde
eines Doktors der Wirtschafts- und Sozialwissenschaften, vorgelegt der Wirtschafts- und Sozialwissenschaftlichen Fakultät der Universität Freiburg in der Schweiz
\end{abstract}

Von

\section{Thomas Wettstein}

aus St. Peterzell (SG)

Genehmigt von der Wirtschafts- und Sozialwissenschaftlichen Fakultät am 12. November 2002 auf Antrag von

Herrn Prof. Dr. Marino Widmer (erster Referent) und Frau Prof. Dr. Stephanie Teufel (zweite Referentin)

Freiburg i. Ü. 2002 
Mit der Annahme einer Dissertation beabsichtigt die Wirtschafts- und Sozialwissenschaftliche Fakultät der Universität Freiburg nicht, zu den darin enthaltenen wissenschaftlichen Meinungen des Verfassers Stellung zu nehmen (Fakultätsbeschluss vom 23. Januar 1990). 


\section{Vorwort}

Das KTI-Projekt „PROMOSYS“ des Departements für Informatik an der Universität Fribourg (Schweiz), in welchem ich seit 1999 bei der Firma F. Hoffmann-La Roche AG an einem Teilprojekt mitarbeiten durfte, war die Initialzündung für die vorliegende

Dissertation. Enstatnden ist sie aus einer Problemstellung der Praxis, für welche die Wissenschaft keine befriedigende Lösung anbieten konnte.

Für die spontane Übernahme der Rolle des Erstreferenten, sowie die intensive und professionelle Betreuung der Arbeit, möchte ich mich bei Prof. Dr. Marino Widmer ganz herzlich bedanken. Frau Prof. Dr. Stephanie Teufel hat das Koreferat übernommen. Dafür und für die unkomplizierte und angenehme Zusammenarbeit gebührt Ihr mein Dank.

Das Entstehen dieser Arbeit ist ein Spiegelbild des Lebens; bestehend aus Hochs und Tiefs. Prof. Dr. Jürg Kohlas, Prof. Dr. Jacques Pasquier-Rocha und Prof. Dr. Andreas Meier haben im entscheidenden Moment wesentlich dazu beigetragen, dass die vorliegende Arbeit abgeschlossen werden konnte. Besten Dank! Letzterem gebührt mein zusätzliches Dankeschönn für die Unterstützung in der ersten Phase.

Während meiner ganzen Doktorandenzeit durfte ich von einem intensiven Austausch mit PD Dr. Peter Küng profitieren. Mit seiner mehrjährigen Forschungstätigkeit auf dem Gebiet des Prozess Performance Measurements fand ich in ihm einen kompetenten Experten.

Neben meiner Studien- und Doktorandenzeit war ich immer teilzeitig in der Praxis tätig. Dies verlangte von meinen Vorgesetzten ein hotes Mass an Flexibilität, um mir die strukturellen und zeitlichen Voraussetzungen für meine Zeit an der Universität zu schaffen. Dafür und für die vielen wertvollen persönlichen und fachlichen Erfahrungen möchte ich mich ganz herzlich bei Hansjakob Langenegger und Ueli Gerber bedanken.

Während meiner Zeit am Departement für Informatik teilte ich mit Stefan Hüsemann das Büro und meine Pflichten als Assistent. Die konstruktive Zusammenarbeit hat einiges zum Gelingen dieser Dissertation beigetragen. Hierzu gehört natürlich auch die angenehme Atmosphäre am Department für Informatik. Speziell möchte ich hier Prof. Heinz Gröflin erwähnen. Ich und unser Hund Yonago werden Ihn in guter Erinnerung behalten. 
Der grösste Dank gebührt schliesslich meiner Partnerin Frau Cornelia Rumo für die Unterstützung und das Verständnis, welches Sie mir während dieser Zeit entgegengebracht hat.

Bern, im November 2002

Thomas Wettstein.

Aus Gründen der Lesbarkeit wird in der vorliegenden Dissertation stellvertretend für die weibliche und männliche Form nur Letztere verwendet. Die Arbeit wurde in der neuen deutschen Rechtschreibung verfasst. Bei mehreren zulässigen Varianten wurde die neue Variante resp. die Hauptvariante gewählt. 
Inhaltsübersicht

I. TEIL GRUNDLAGEN ...............................................................................................

1 EINFÜHRUNG ................................................................................................................

2 BEGRIFFE ................................................................................................................... 15

3 BESTEHENDE PERFORMANCE-MEASUREMENT-ANSÄTZE ......................33

II. TEIL BESTIMMUNG DER ANFORDERUNGEN AN EIN

PERFORMANCE-MEASUREMENT-SYSTEM................................................................79

4 ANFORDERUNGEN AN EIN PMS

III. TEIL ORGANISATORISCHE KONZEPTION EINES PMS 109

5 BEZUGSRAHMEN FÜR EIN PERFORMANCE-MEASUREMENT-SYSTEM..

6 PROZESS ZUR ERSTELLUNG DER INDIKATORENHIERARCHIE............127

7 PROZESSE FÜR DEN BETRIEB EINES PERFORMANCEMEASUREMENT-SYSTEMS 181

IV. TEIL TECHNISCHE KONZEPTION EINES PMS 193

8 ARCHITEKTUR DES IT-SYSTEMS FÜR DAS PERFORMANCE MEASUREMENT 
9 PROZESS FÜR DEN ENTWURF EINES MULTIDIMENSIONALEN DATENMODELLS ZUR VERWALTUNG VON PERFORMANCE-DATEN 213

10 PROTOTYP EINES RECHNERGESTÜTZTEN SYSTEMS FÜR DAS PERFORMANCE MEASUREMENT

V. TEIL SCHLUSS. .273

11 ZUSAMMENFASSUNG UND AUSBLICK .275

LITERATURVERZEICHNIS .281

VI. TEIL ANHÄNGE .299

ANHANG A: ERHEBUNG ZUM STAND DES PERFORMANCE MEASUREMENT UND DEREN IT-SYSTEME IN DER PRAXIS .301

ANHANG B: ERGEBNISSE DER ERHEBUNG ZUM STAND DES PERFORMANCE MEASUREMENT UND DEREN IT-SYSTEME IN DER PRAXIS - BERICHT FÜR TEILNEHMENDE UNTERNEHMEN .315 ANHANG C: ÜBERSICHT ÜBER KOMMERZIELLE IT-SYSTEME FÜR DAS PM .333 


\section{Inhaltsverzeichnis}

VORWORT ..................................................................................................................... III

INHALTSÜBERSICHT ........................................................................................................ V

ABBILDUNGSVERZEICHNIS ...................................................................................XVII

TABELLENVERZEICHNIS........................................................................................XXI

ABKÜRZUNGSVERZEICHNIS .............................................................................. XXV

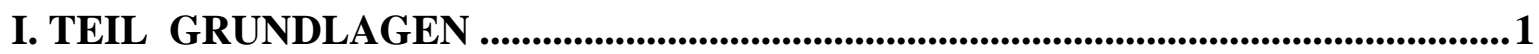

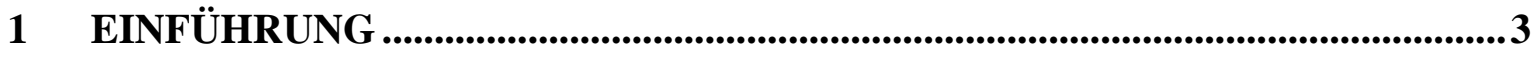

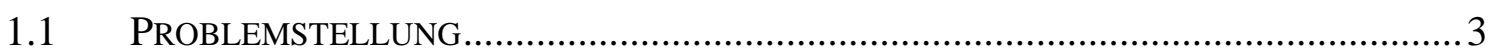

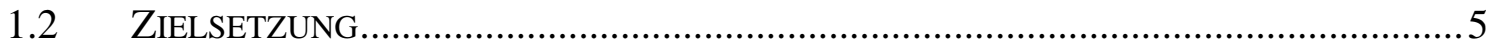

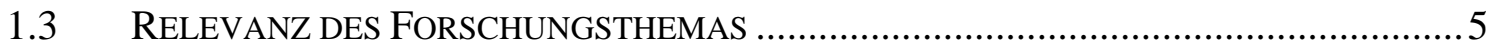

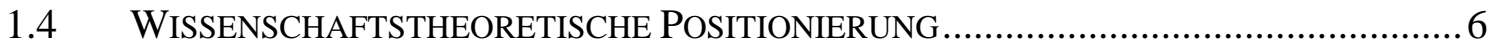

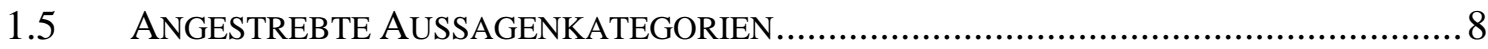

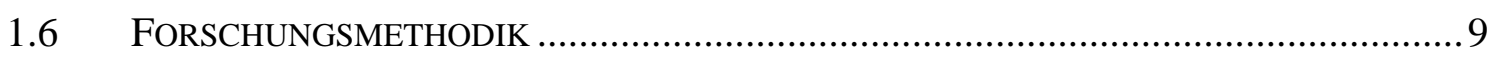

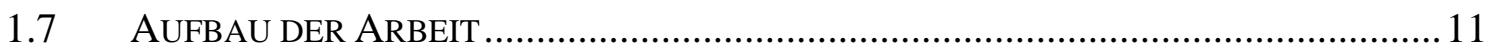

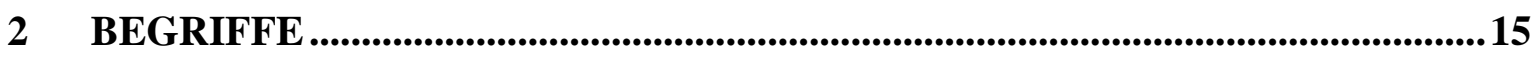

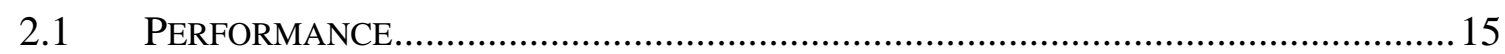

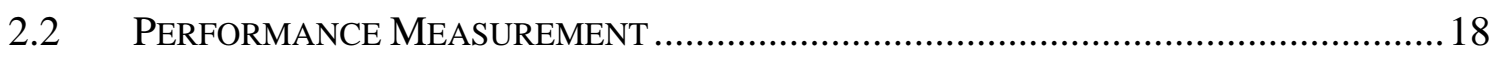

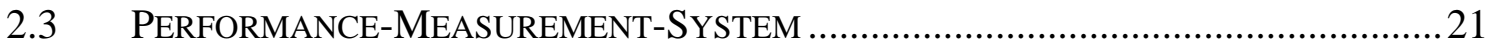

2.4 IT-SYSTEM FÜR DAS PERFORMANCE MEASUREMENT …......................................24

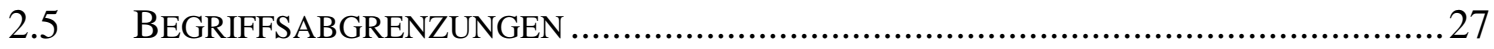

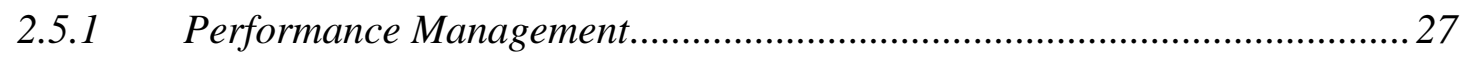

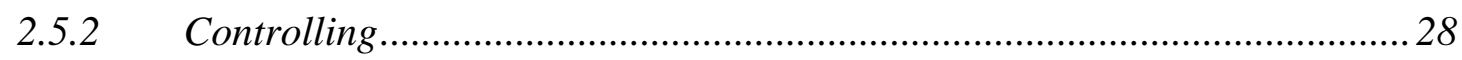

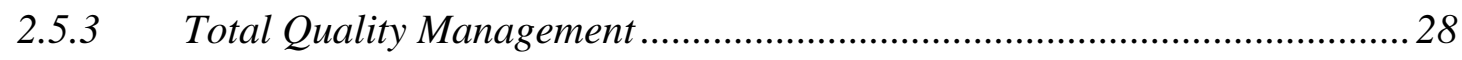

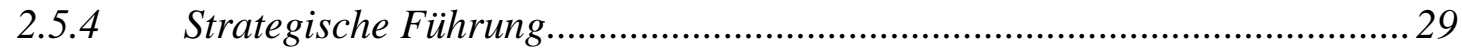

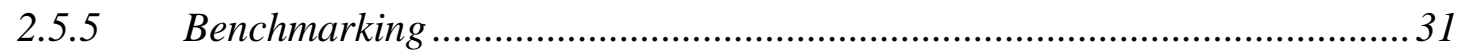


3.1 Historische Entwicklung Des Performance MEASUREMENT.........................33

3.1.1 Ein Ansatz aus dem Finanzbereich ............................................................34

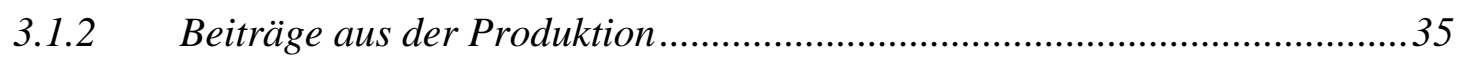

3.1.3 Der Beitrag aus dem Personalbereich.........................................................37

3.1.4 Erste ganzheitliche Systeme ....................................................................... 37

3.1.5 Unterstützung der Ansätze durch Informationstechnologie...........................42

3.2 Vier AKtuelle Ansätze des Performance Measurement ............................44

3.2.1 Der EFQM-Ansatz als Instrument für das Self-Assessment.........................44

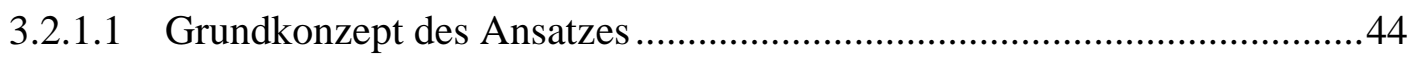

3.2.1.2 Charakterisierung des EFQM-Ansatzes auf Grund von Sekundärliteratur .48

3.2.1.3 Weiterentwicklungen und Varianten des Ansatzes..................................49

3.2.1.4 Berücksichtigung der Informationstechnologie im EFQM-Ansatz ..........50

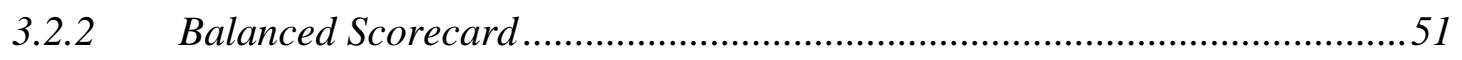

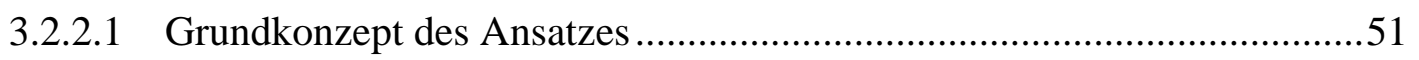

3.2.2.2 Charakterisierung der Balanced Scorecard auf Grund von Sekundärliteratur. .57

3.2.2.3 Weiterentwicklungen und Varianten des Balanced-Scorecard-Ansatzes.58

3.2.2.4 Berücksichtigung der Informationstechnologie im BSC-Ansatz..............58

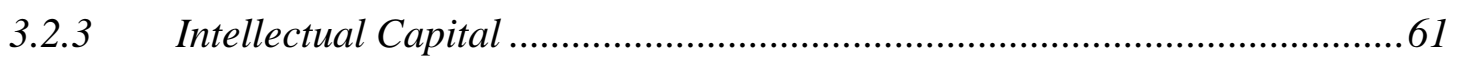

3.2.3.1 Grundkonzept des Ansatzes ...............................................................61

3.2.3.2 Charakterisierung des IC-Ansatzes auf Grund von Sekundärliteratur......65

3.2.3.3 Weiterentwicklungen und Varianten des Ansatzes..................................66

3.2.3.4 Berücksichtigung der Informationstechnologie im Intellectual-Capital-

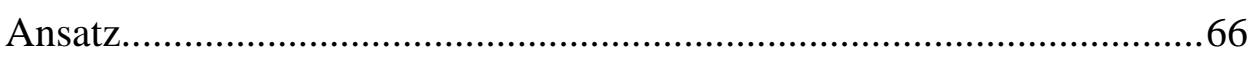

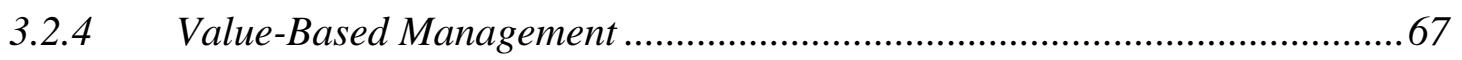

3.2.4.1 Grundkonzept des Ansatzes ..................................................................67

3.2.4.2 Charakterisierung des VBM-Ansatzes auf Grund von Sekundärliteratur.72

3.2.4.3 Weiterentwicklungen und Varianten des Ansatzes...................................72

3.2.4.4 Berücksichtigung der Informationstechnologie im VBM-Ansatz ............73

3.3 VERGLEICHENDE BEURTEILUNG DER PMS-ANSÄTZE ..........................................74

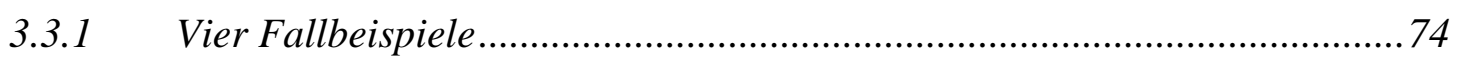

3.3.2 Beurteilung der PMS-Ansätze bezüglich der vier Fallbeispiele ...................76 


\section{TEIL BESTIMMUNG DER ANFORDERUNGEN AN EIN PERFORMANCE-

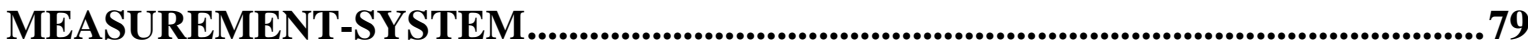

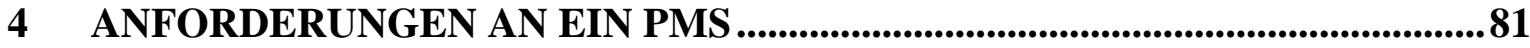

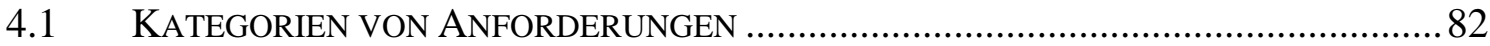

4.2 ANFORDERUNGEN AN PMS AUS DER LITERATUR ................................................ 84

4.3 ERKENNTNISSE AUS EMPIRISCHEN UNTERSUCHUNGEN ZU FRAGEN DES

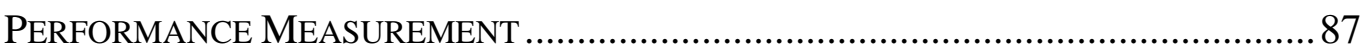

4.3.1 Verbreitung von bestimmten Performance-Indikatoren .............................88

4.3.2 Wirkungszusammenhänge von verschiedenen Indikatoren .........................89

4.3.3 Verbreitung von Performance-Measurement-Konzepten ............................90

4.3.4 Ausgestaltung und Reifegrad von PMS ...................................................92

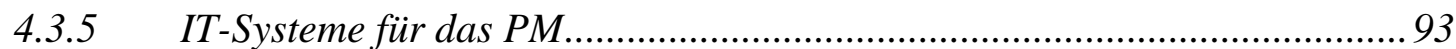

4.4 Empirische Studie Zum Thema „STANd Des Performance Measurement UND DEREN IT-SYSTEME IN DER PRAXIS“ ......................................................... 94

4.4.1 Gegenstandsbereich und Methode der empirischen Studie ..........................99

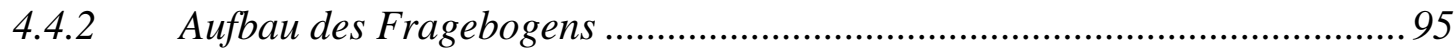

4.4.3 Elektronische Umsetzung des Fragebogens ............................................... 95

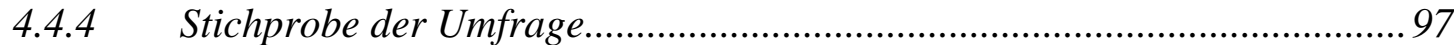

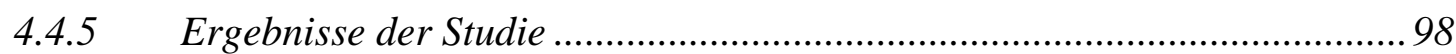

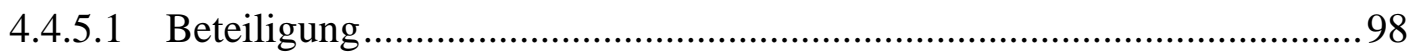

4.4.5.2 Stand der Performance-Measurement-Systeme......................................98

4.4.5.3 Stand der IT-Systeme für das Performance Measurement ...................... 100

4.4.5.4 Anforderungen an ein IT-System für das PM aus der Sicht der Praxis.. 101

4.5 VORSCHLAG EINES ANFORDERUNGSSATZES AN EIN PMS 104

III. TEIL ORGANISATORISCHE KONZEPTION EINES PMS

5 BEZUGSRAHMEN FÜR EIN PERFORMANCE-MEASUREMENT-SYSTEM 111

5.1 WESEN UND ZWECK EINES BEZUGSRAHMENS

5.2 ABDECKUNGSGRAD EXISTIERENDER PM-KONZEPTE BEZÜGLICH DER

IDENTIFIZIERTEN ELEMENTE DES BEZUGSRAHMENS

5.2.1 Diskutierte Ansätze aus Kapitel drei ....................................................... 113

5.2.2 Das Performance-Management-System von Hoffmann............................. 114 
5.2.3 Zusammenfassende Gegenüberstellung der existierenden Ansätze ............116

5.3 VORSCHLAG EINES EIGENEN BEZUGSRAHMENS ..............................................117

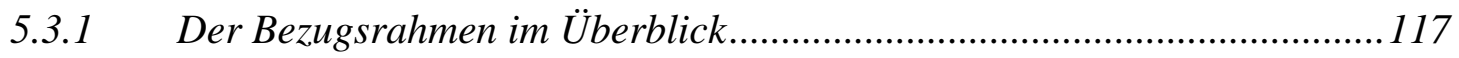

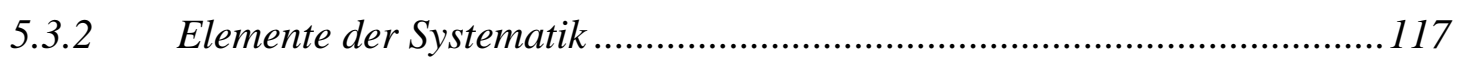

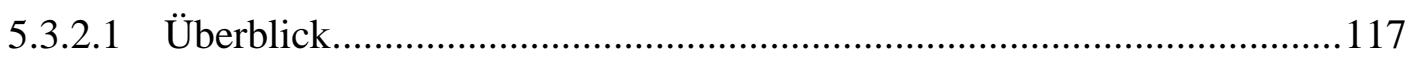

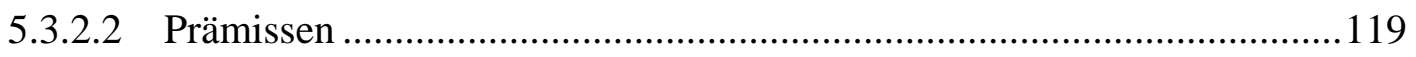

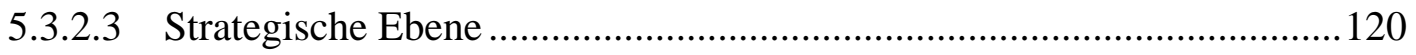

5.3.2.4 Operative Ebene .................................................................................. 120

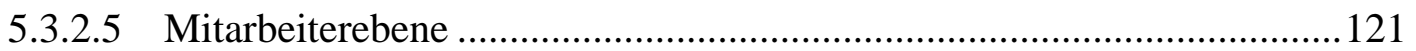

5.3.2.6 Beziehungen zwischen den Elementen der Systematik .......................... 121

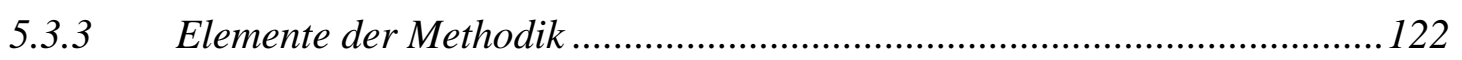

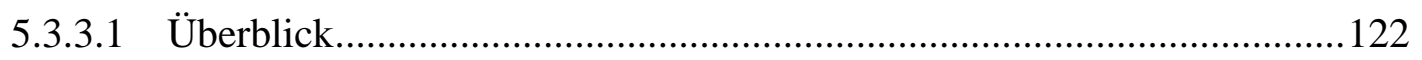

5.3.3.2 Prozess zur Erstellung der Indikatorenhierarchie .................................124

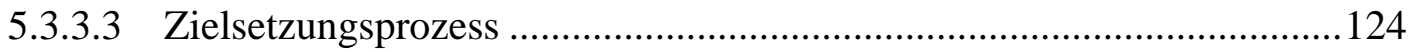

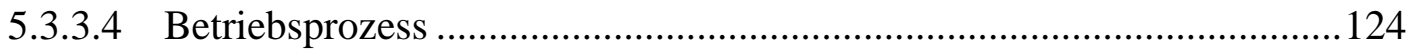

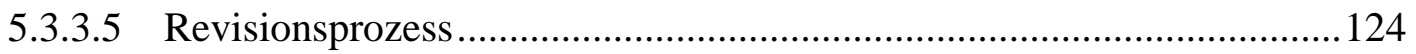

5.3.4 Elemente der Organisation ................................................................... 125

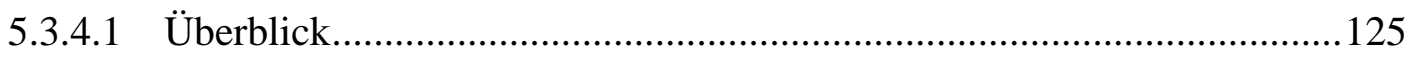

5.3.4.2 Kategorien von Informationsempfängern ............................................126

6 PROZESS ZUR ERSTELLUNG DER INDIKATORENHIERARCHIE..........127

6.1 ZIELSETZUNG UND GRENZEN DES ERSTELLUNGSPROZESSES .............................127

6.2 MÖGLICHE INPUT-FAKTOREN DES TRANSFORMATIONSPROZESSES ......................129

6.2.1 Input-Faktoren aus den strategischen Dokumenten ................................129

6.2.1.1 Ziele als Basis für den Kaskadierungsprozess .......................................130

6.2.1.2 Prämissen als Element der strategischen Kontrolle ...............................130

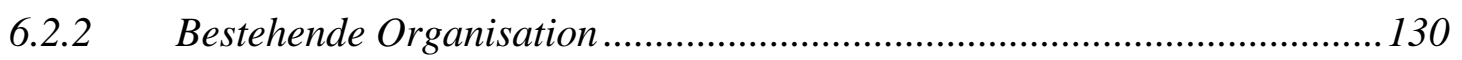

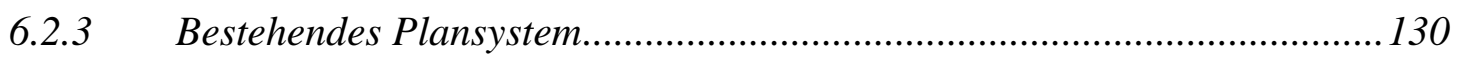

6.2.3.1 Finanzielle Pläne ..............................................................................131

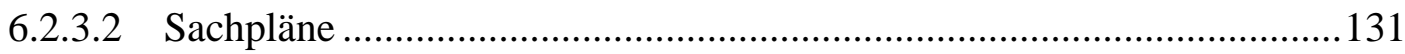

6.2.4 Zielvorgaben aus dem Management by Objectives..................................131

6.2.5 Koordination der Input-Faktoren ..........................................................131

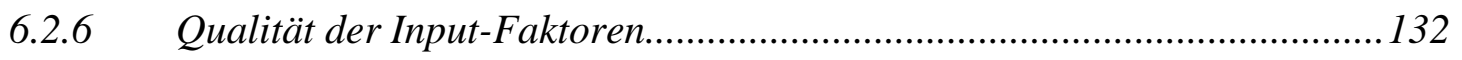

6.3 VORGEHENSSCHRITTE DES VORGESCHLAGENEN ERSTELLUNGSPROZESSES .........133

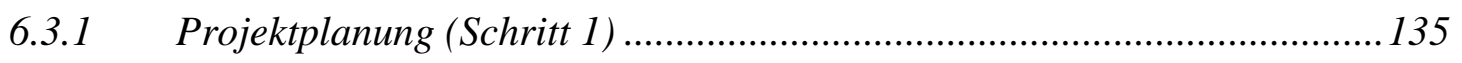


6.3.2.1 Die Zielsetzung des Performance-Measurement-Systems determiniert die relevanten Input-Informationen.... 137

6.3.2.2 Überprüfung der Machbarkeit 137

6.3.3 Erfassen der relevanten Input-Informationen (Schritt 3) .......................... 138

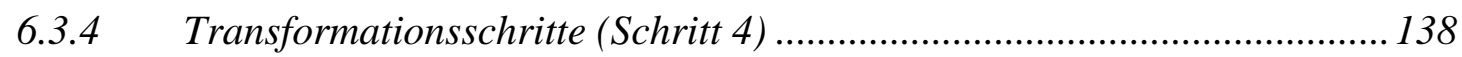

6.3.4.1 Bestimmen der Ziele der Organisationseinheit (Schritt 4.1) ................... 138

6.3.4.2 Bestimmung der Indikatoren, welche zur Messung der Ziele notwendig sind (Schritt 4.2)

6.3.4.3 Top-Down-Zuweisung der Ziele und Indikatoren an die entsprechenden Organisationseinheiten (Schritt 4.3)

6.3.4.4 Bestimmung der Mittel (Unterziele) zur Zielerreichung (Iterationen) (Schritt 4.4).

6.3.4.5 Bestimmen der Indikatoren der strategischen Programme (Schritt 4.5). 144

6.3.4.6 Erfassen der persönlichen Ziele (MbO) (Schritt 4.6) .............................. 147

6.3.4.7 Auflösen von Zielkonflikten (Schritt 4.7)............................................. 149

6.3.4.8 Bestimmung der Prämissenindikatoren (Schritt 4.8) ............................. 154

6.3.4.9 Zuordnen der Verantwortlichen der Ziele (Schritt 4.9) ........................... 158

6.3.4.10 Zuordnen der Informationsempfänger der Performance-Indikatoren

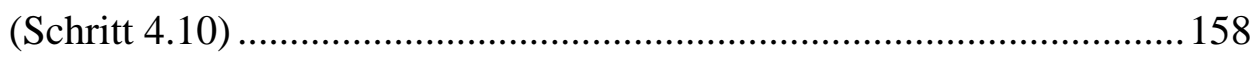

6.4 ILLUSTRATION DES VORSCHLAGES AN EINEM BEISPIEL ..................................... 161

6.4.1 Erhebung der Input-Faktoren.................................................................161

6.4.2 Transformationsschrittfolge des Erstellungsprozesses.............................165

6.4.2.1 Bestimmen der Ziele der relevanten Organisationseinheit ...................... 165

6.4.2.2 Bestimmen der Indikatoren, die zur Messung der Ziele notwendig

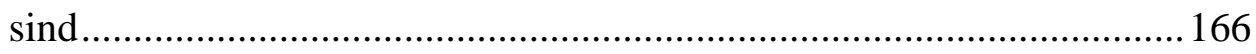

6.4.2.3 Top-Down-Zuweisung der Ziele und Indikatoren an die entsprechenden

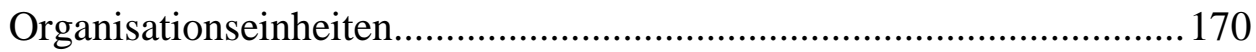

6.4.2.4 Bestimmen der Mittel zur Zielerreichung............................................. 172

6.4.2.5 Bestimmen der strategischen Programme.............................................. 175

6.4.2.6 Aufnahme der persönlichen Zielsetzungen der Mitarbeiter .................... 175

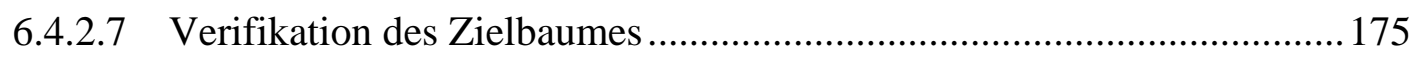

6.4.2.8 Bestimmen der Prämissenindikatoren ................................................. 176

6.4.2.9 Zuordnen der Verantwortlichen und der Informationsempfänger ...........176 


\section{PROZESSE FÜR DEN BETRIEB EINES PERFORMANCE-}

MEASUREMENT-SYSTEMS.

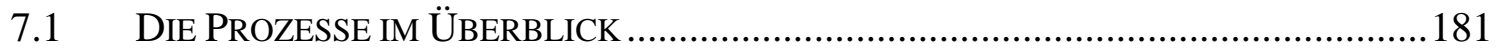

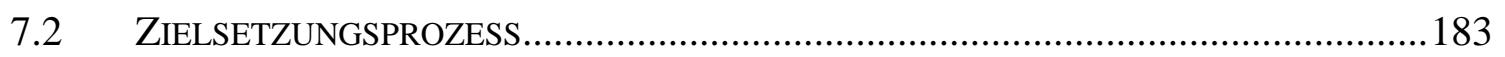

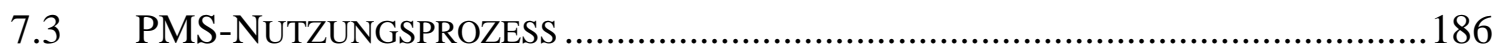

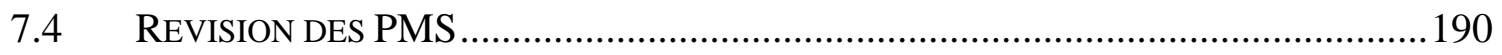

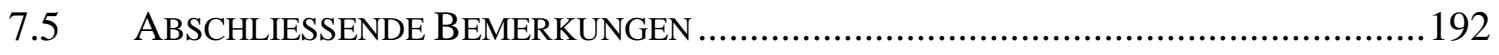

IV. TEIL TECHNISCHE KONZEPTION EINES PMS .........................................193

\section{ARCHITEKTUR DES IT-SYSTEMS FÜR DAS PERFORMANCE}

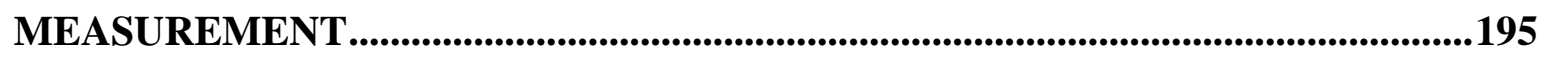

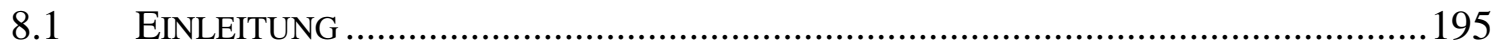

8.2 ANFORDERUNGEN AN DIE ARCHITEKTUR UND AN DAS IT-SYSTEM FÜR DAS PM 197

8.3 FUNKTIONALE ARCHITEKTUR FÜR EIN IT-SYSTEM FÜR DAS PM.........................198

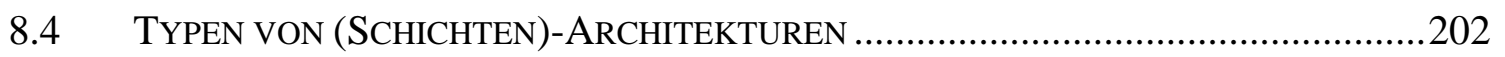

8.4.1 Vor- und Nachteile von verteilten Systemen .............................................202

8.4.2 Ein-, Zwei- und Drei-Schichten-Architekturen ........................................203

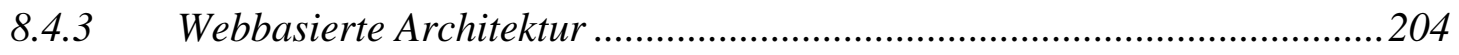

8.5 BEDEUTUNG VON NICHT-FUNKTIONALEN ANFORDERUNGEN FÜR DIE PHYSISCHE ARCHITEKTUR.

8.6 VORSCHLAG EINER PHYSISCHEN ARCHITEKTUR FÜR DAS IT-SYSTEM FÜR DAS

Performance Measurement

\section{PROZESS FÜR DEN ENTWURF EINES MULTIDIMENSIONALEN}

DATENMODELLS ZUR VERWALTUNG VON PERFORMANCE-DATEN........213

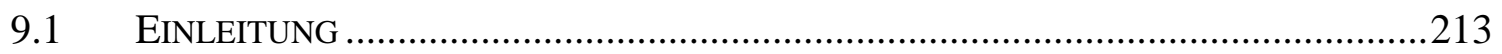

9.2 Bestimmung Der DAtenstruktur eines IT-GestütZTEN PERFORMANCE-

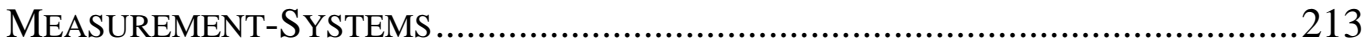

9.2.1 Anforderungen an die Datenstruktur ....................................................213

9.2.2 OLTP und OLAP als Grundtypen ..........................................................214

9.2.3 Elemente eines Data Warehouses ..............................................................215 
9.2.4 Ebenen des Data Warehouse Design........................................................2216

9.3 UNTERSUCHUNG VON VORSCHLÄGEN AUS DER LITERATUR ...............................218

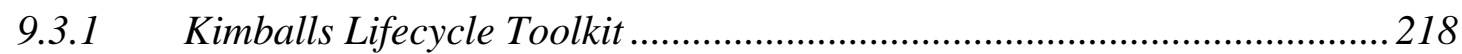

9.3.2 Der prozessorientierte Ansatz von Böhnlein und Ulbrich-vom Ende........ 219

9.3.3 Die semi-automatische Konversion von Golfarelli et al.............................221

9.3.4 Konversion eines hochnormalisierten E/R-Schemas in eine multidimensionale Datenbank nach Hüsemann et al.

9.3.5 Bewertung der Eignung zur Erstellung einer Datenstruktur für ein PMS 224

9.4 VORSCHLAG EINES VORGEHENSMODELLS ZUR ERSTELLUNG EINER

DATENSTRUKTUR FÜR EIN PERFORMANCE-MEASUREMENT-SYSTEM 225

9.4.1 Bereitstellen der Ergebnisse der organisatorischen Modellierung (Schritt 1)

9.4.2 Gruppierung identischer Indikatoren (Schritt 2)....................................228

9.4.3 Bestimmen der notwendigen Dimensionen (Schritt 3)...............................228

9.4.4 Bestimmen der Granularitätsgrade je Indikator und Informationsempfänger (Schritt 4). 230

9.4.5 Überprüfen des Aggregationspfades der einzelnen Dimensionen (Schritt 5)..

9.4.6 Verschmelzen redundanter Dimensionen (Schritt 6) ................................ 230

9.4.7 Bestimmen des höchsten Detaillierungsgrades pro Indikator (Schritt 7).. 230

9.4.8 Konsolidierung der Indikatoren mit gleichen Dimensionen und gleicher Granularität (Schritt 8)........................................................................... 231

9.4.9 Festlegen von Aggregationsbeschränkungen der Performance-Indikatoren (Schritt 9)

9.4.10 Festlegen von Standardzugriffspfaden und Berechtigungen je Informationsempfänger (Schritt 10) .................................................... 232

9.5 ILLUSTRATION DES VORGEHENSMODELLS AN EINEM BEISPIEL ............................ 232

9.5.1 Schritt 1: Bereitstellen der Ergebnisse der organisatorischen Modellierung....... 232

9.5.2 Schritt 2: Gruppierung identischer Indikatoren ...................................... 236

9.5.3 Schritt 3: Bestimmen der notwendigen Dimensionen ................................. 236

9.5.4 Schritt 4: Bestimmen der Granularitätsgrade je Indikator und Informationsempfänger 236 
9.5.5 Schritt 5: Überprüfen des Aggregationspfades der einzelnen Dimensionen ... 241

9.5.6 Schritt 6: Verschmelzen redundanter Dimensionen....................................241

9.5.7 Schritt 7: Bestimmen des höchsten Detaillierungsgrades pro Indikator ...243

9.5.8 Schritt 8: Konsolidierung der Indikatoren mit gleichen Dimensionen und gleicher Granularität.

9.5.9 Schritt 9: Festlegen von Aggregationsbeschränkungen der PerformanceIndikatoren 244

9.5.10 Schritt 10: Festlegen von Standardzugriffspfaden und Berechtigungen je Informationsempfänger.

9.6 ANSÄTZE ZUR MODELLIERUNG VON MULTIDIMENSIONALEN DATENSTRUKTUREN .248

9.6.1 Differenzierung nach Entwurfsebenen .....................................................248

9.6.2 Modellierungsansätze der semantischen Entwurfsebene..........................249

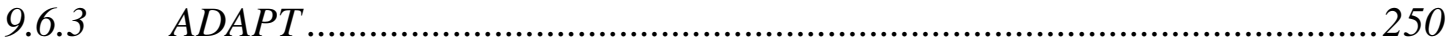

9.7 DARSTELLUNG DES FALLBEISPIELS ANHAND DER VORGESTELLTEN NOTATION ..253

9.8 EVALUATION DES VORGEHENSMODELLS .............................................................25

\section{PROTOTYP EINES RECHNERGESTÜTZTEN SYSTEMS FÜR DAS}

PERFORMANCE MEASUREMENT …......................................................................2257

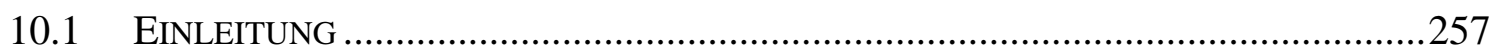

10.2 DIE VERWENDETEN SOFTWAREKOMPONENTEN IN DER ÜBERSICHT ....................257

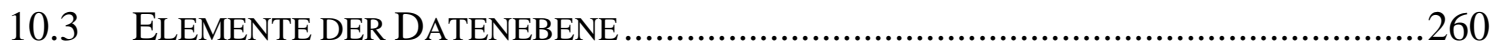

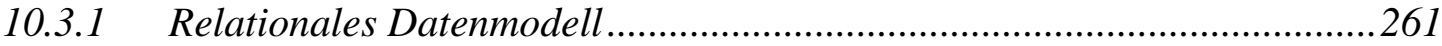

10.3.2 Repräsentation als multidimensionales Modell .......................................263

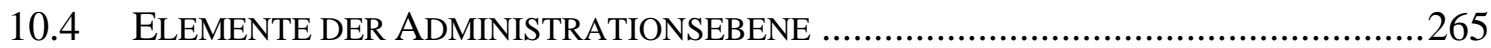

10.4.1 Festlegung von Rollen und Zugriffsrechten .............................................265

10.4.2 Repräsentation des Zielbaumes ...............................................................26

10.4.3 Festlegung der Zielwerte ..................................................................268

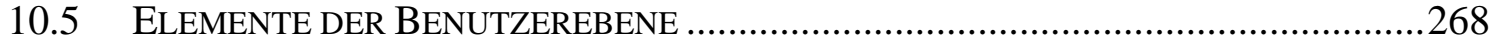

10.6 SYSTEMABGRENZUNG UND ANBINDUNG VON UMLIEGENDEN SYSTEMEN ...........270

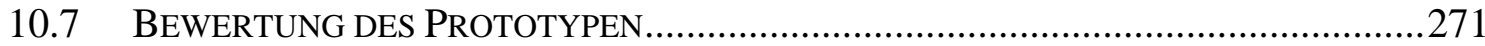


V. TEIL SCHLUSS

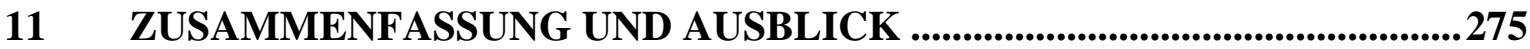

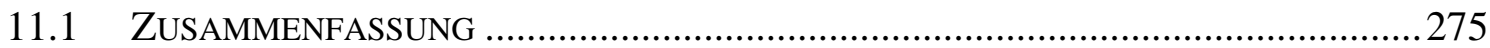

11.2 BEMERKUNGEN ZUM VORGESCHLAGENEN ANSATZ UND AUSBLICK FÜR WEITERE

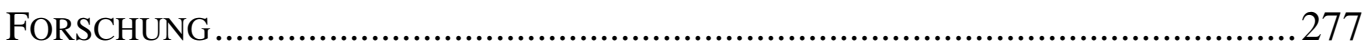

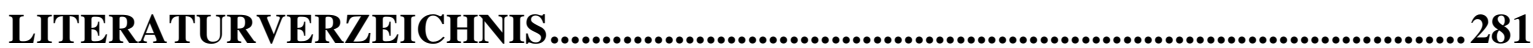

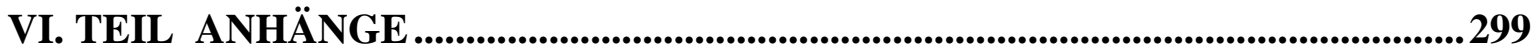

ANHANG A: ERHEBUNG ZUM STAND DES PERFORMANCE-MEASUREMENT UND DEREN IT-SYSTEME IN DER PRAXIS ............................................................301

ANHANG B: ERGEBNISSE DER ERHEBUNG ZUM STAND DES PERFORMANCE MEASUREMENT UND DEREN IT-SYSTEME IN DER PRAXIS - BERICHT FÜR TEILNEHMENDE UNTERNEHMEN............................................315 ANHANG C: ÜBERSICHT ÜBER KOMMERZIELLE IT-SYSTEME FÜR DAS PM 333

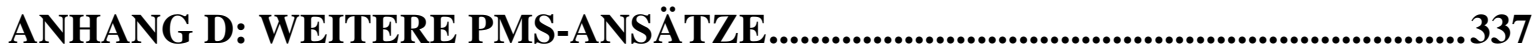




\section{Abbildungsverzeichnis}

Abbildung 1-1: Forschungsrichtungen der Betriebswirtschaftslehre (vgl. Grünig (1990))...6

Abbildung 1-2: Aussagenkategorien der praktisch-normativen BWL (vgl. Grünig und

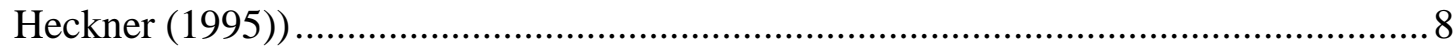

Abbildung 1-3: Überblick über die Forschungsmethodik ................................................ 11

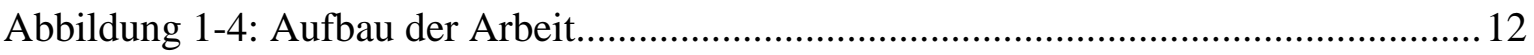

Abbildung 2-1: Regelkreis (Steinmann und Schreyögg (1993)) ......................................... 20

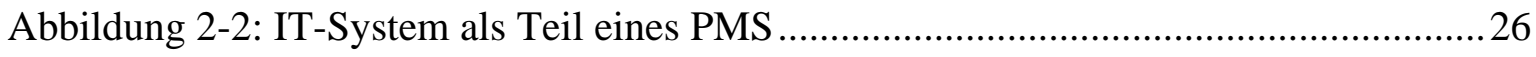

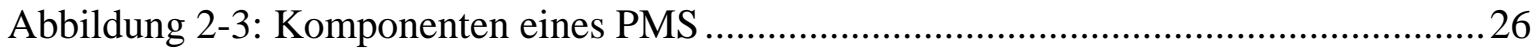

Abbildung 2-4: Unterstützungspotential eines PMS für die strategische Führung (in

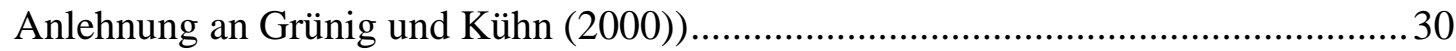

Abbildung 2-5: Arten des Benchmarking (vgl. Ortega et al. (1999)) ................................... 32

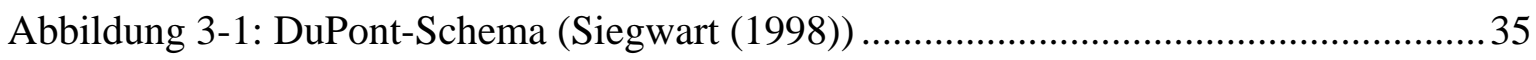

Abbildung 3-2: Ausgewogene Dimensionen nach Keegan et al. (1989)............................. 38

Abbildung 3-3: Results-Determinants-Matrix von Fitzgerald et al. (1991) ......................... 39

Abbildung 3-4: Kausalkette in einem Prozess (vgl. Brown (1996), zitiert nach Neely

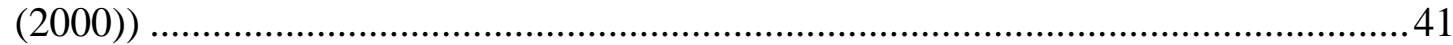

Abbildung 3-5: Performance-Pyramide (Lynch und Cross (1991)) .................................... 42

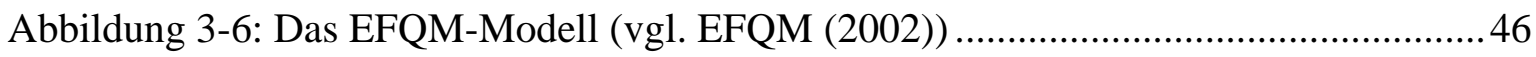

Abbildung 3-7: Kontinuierliche Verbesserung (in Anlehnung an Hakes (1996))............... 47

Abbildung 3-8: Auszug aus der Unternehmensverbesserungsmatrix der EFQM (1996)....48

Abbildung 3-9: Beispiel einer Software zur Präsentation und Kommunikation der

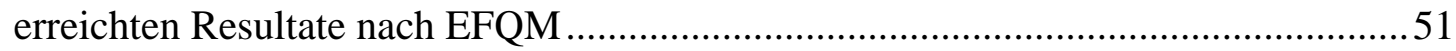

Abbildung 3-10: Strukturierung der Balanced Scorecard (vereinfachte und übersetzte

Darstellung in Anlehnung an Kaplan und Norton (1992)) .......................................52

Abbildung 3-11: Ursache-Wirkungskette in der Balanced Scorecard

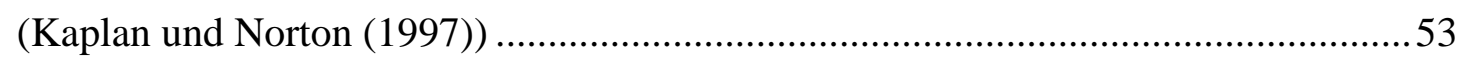

Abbildung 3-12: Management-Zyklus der Balanced Scorecard (in Anlehnung an

Kaplan und Norton (1996b) und Kaplan und Norton (1997)) ....................................55

Abbildung 3-13: Einführungsvorgehen für eine Balanced Scorecard (vgl. Kaplan

und Norton (1996b) und Kaplan und Norton (1997))..............................................56

Abbildung 3-14: Vermögensbestandteile (in Anlehnung an Sveiby (1989)) ......................63 
Abbildung 3-15: Die Komponenten des Intellectual Capital............................................64

Abbildung 3-16: Der Skandia-Navigator (vgl. Edvinsson und Malone (1997))..................65

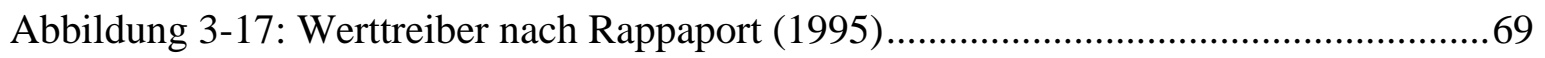

Abbildung 3-18: Zusammenhang von EVA und Shareholder Value

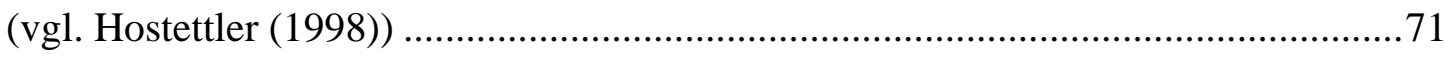

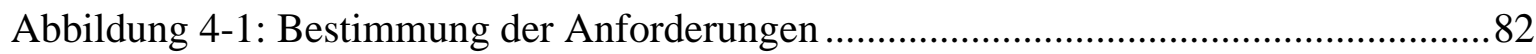

Abbildung 4-2: Screenshot aus dem elektronischen Fragebogen ....................................96

Abbildung 4-3: IT-Unterstützung der Performance-Measurement-Systeme......................100

Abbildung 4-4: Verwendete Standardsoftware und Softwarekomponenten......................101

Abbildung 4-5: Performance-relevante Daten der operativen Informationssysteme..........101

Abbildung 4-6: Anforderungen und Leistungen von Performance-Measurement-

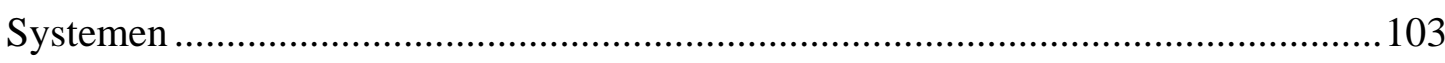

Abbildung 5-1: Generische Elemente eines PMS-Modells ............................................113

Abbildung 5-2: Kausalkette zur Einführung eines Performance-Management-

Systems (vgl. Hoffmann (1999)) …………….......................................................115

Abbildung 5-3: Systematik des Bezugsrahmens (in Anlehnung an

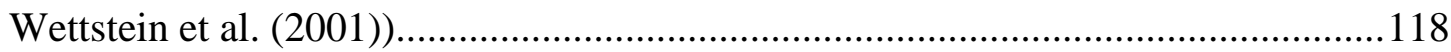

Abbildung 5-4: Elemente der strategischen Kontrolle....................................................119

Abbildung 5-5: Beziehungen zwischen den Elementen der Systematik..........................122

Abbildung 5-6: Prozesse der Methodik .............................................................................123

Abbildung 6-1: Wesentliche Elemente des Erstellungsprozesses...................................129

Abbildung 6-2: Vorgehensschritte des Erstellungsprozesses im Überblick ......................134

Abbildung 6-3: Projektplanung (vgl. Grünig und Kühn (2000)) .....................................135

Abbildung 6-4: Zu differenzierende Aspekte nach Küng (2000) ...................................139

Abbildung 6-5: Anforderungen an Performance-Indikatoren..........................................141

Abbildung 6-6: Ziel-Mittel-Denken (Haberfellner und Daenzer (1992)) ..........................144

Abbildung 6-7: Massnahmenmatrix eines Informatikdienstleisters (Konzeption in

Anlehnung an Colletti (1996), zitiert bei Hoffmann (1999))....................................146

Abbildung 6-8: Ergebnisportfolio der Zielbeziehungsanalyse ......................................151

Abbildung 6-9: Abgrenzung der Früherkennung vom strategischen Controlling .............156

Abbildung 6-10: Die Kommunikationspyramide (vgl. Wettstein et al. (2001)) ................160

Abbildung 6-11: Ausschnitt der vereinfachten Aufbauorganisation von SIDL ................163

Abbildung 6-12: Affinitätsdiagramm für die Indikatoren der Mitarbeiterzufriedenheit ...167 
Abbildung 6-13: Cluster-Bildung als Mittel zur Komplexitätsreduktion der

Zielhierarchie-Verifikation

Abbildung 7-1: Die Betriebsprozesse im Überblick

Abbildung 7-2: Grundtypen für Entwicklungsfolgen von Zielen (Scholz (1984)) 184

Abbildung 7-3: Beispiel einer koordinierten Plananpassung

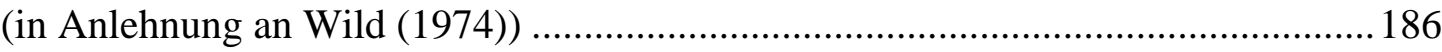

Abbildung 7-4: Einordnung der Prozesse in den Deming-Cycle ..................................... 187

Abbildung 8-1: Design einer Software-Architektur (vgl. Bosch (2000)) .......................... 196

Abbildung 8-2: Funktionale Architektur eines IT-Systems für das PM............................ 199

Abbildung 8-3: Schichten-Architekturen........................................................................ 204

Abbildung 8-4: Drei Architekturvarianten (in Anlehnung an Kimball et al. (1998)

und Kurz (1999))

Abbildung 8-5: Physische Drei-Schichten-Architektur eines IT-Systems für das

Performance Measurement

Abbildung 9-1: Darstellung der multidimensionalen Datenstruktur als Würfel

Abbildung 9-2: Modellierungs- und Implementierungsebenen (vgl. Totok (2000))

Abbildung 9-3: Vorgehensmodell zur Erstellung einer multidimensionalen

Datenstruktur 227

Abbildung 9-4: Kernelemente der ADAPT-Notation (vgl. Bulos (1996)) ......................... 250

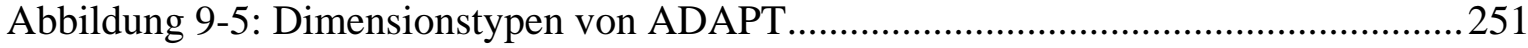

Abbildung 9-6: Dimensionselemente von ADAPT

Abbildung 9-7: Berechnungen im multidimensionalen Datenmodell mittels Formeln

(Beispiel in Anlehnung an Felden und Florek (2001))

Abbildung 10-1: Funktionsweise und Architektur von Active Server Pages.....................259

Abbildung 10-2: Architektur des Prototypen.................................................................... 260

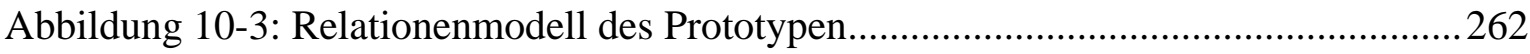

Abbildung 10-4: Übersicht über die Datenwürfel und Dimensionen des Prototypen ........263

Abbildung 10-5: Beispiel Datenwürfel Kundenzufriedenheit ......................................... 264

Abbildung 10-6: Beispiel der Dimension Organisation ...................................................... 264

Abbildung 10-7: Definition der Rollen (Informationsempfänger) .................................... 265

Abbildung 10-8: Berechtigungen für Datenwürfel und Dimensionen bestimmen ............. 265

Abbildung 10-9: Definition von zulässigen Aggregationshierarchien .............................. 266

Abbildung 10-10: Verwaltung des Ziel- und Indikatorenbaumes .....................................267

Abbildung 10-11: Performance Cockpit für den Bereichsleiter Application Services...... 269 
Abbildung 10-12: Performance-Indikator-Analyse: Kundenzufriedenheits-Index

.270 


\section{Tabellenverzeichnis}

Tabelle 2-1: Definitionen des Begriffes „Performance“...................................................... 16

Tabelle 2-2: Definitionen des Begriffes „Performance Measurement“................................ 19

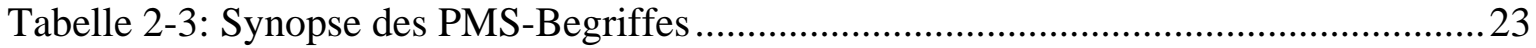

Tabelle 3-1 Anforderungen an ein Informationssystem für eine Balanced Scorecard (vgl.

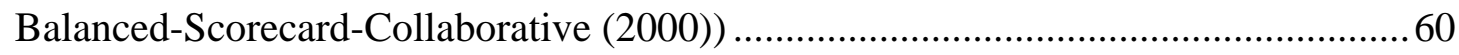

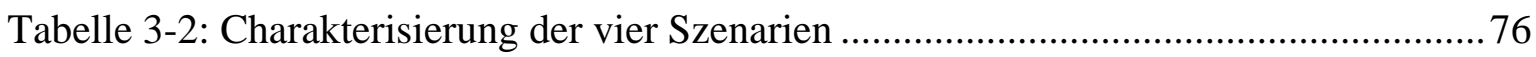

Tabelle 3-3: Typisierung der vier PMS-Ansätze ............................................................... 77

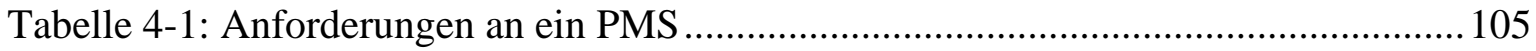

Tabelle 4-2: Anforderungen an das IT-System für das PM..............................................106

Tabelle 5-1: Abdeckungsgrad der Ansätze bezüglich der Elemente eines Bezugsrahmens

Tabelle 5-2: Nutzer eines PMS und deren Zielsetzung nach Lebas (1995)....................... 125

Tabelle 5-3: Unterschiedliche Aufgabenträger-Rollen eines PMS................................... 126

Tabelle 6-1: Aus den Anforderungen abgeleitete Input-Faktoren .................................... 128

Tabelle 6-2: Minimalanforderungen für den Transformationsprozess (in Anlehnung an

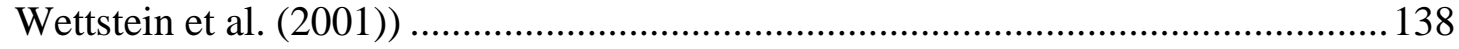

Tabelle 6-3: Muster für die schriftliche Dokumentation der Zielvereinbarungen und

Ergebnisse der gemeinsamen Bewertung der Zielerreichung (vgl. Brunner (1999)) 147

Tabelle 6-4: Matrix des Papiercomputers am Beispiel eines Serviceprozesses (Zielsystem

in Anlehnung an Küng et al. (2001)).................................................................... 149

Tabelle 6-5: Präferenz-Zielanalyse am Beispiel eines Serviceprozesses (Zielsystem in

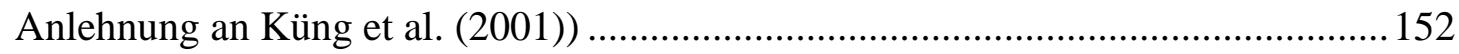

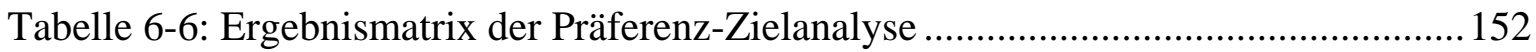

Tabelle 6-7: Beispiele der Ziele eines Früherkennungssystems einer schweizerischen

Elektrizitätsgesellschaft (vgl. Bergamin (2001)) .................................................. 156

Tabelle 6-8: Vier Grundtypen von Früherkennungssystemen (in Anlehnung an Bergamin

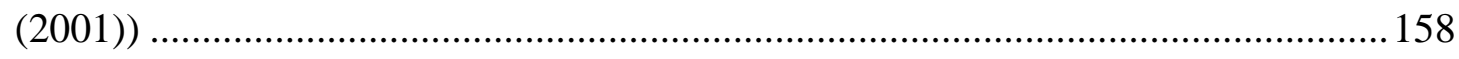

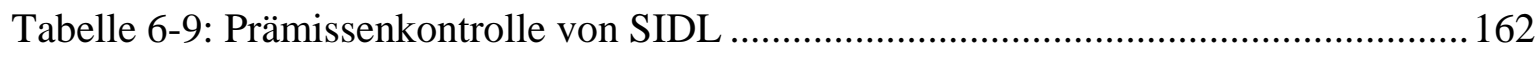

Tabelle 6-10: Vereinfachtes Budget-Schema von SIDL nach Konzern-Strukturen (US-

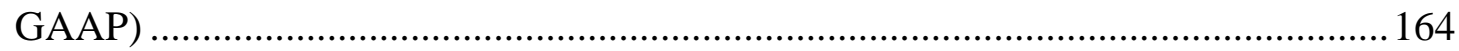

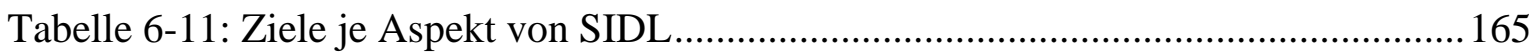


Tabelle 6-12: Identifizierte Performance-Indikatoren für das Ziel der hohen

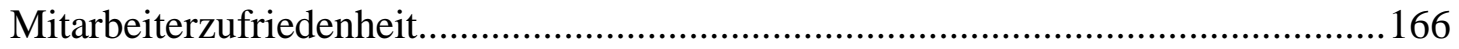

Tabelle 6-13: Definition des Performance-Indikators P252 / Anzahl Krankheitstage ......167

Tabelle 6-14: Bewertung der analysierten Indikatoren anhand der definierten Anforderungen

Tabelle 6-15: Auszug der Ziele und Performance-Indikatoren auf Unternehmensebene von SIDL AG..... 170

Tabelle 6-16: Ziele und Performance-Indikatoren auf der Ebene des Bereiches „Application Services“

Tabelle 6-17: Ziele und Performance-Indikatoren auf der Ebene des Prozesses „Support \& Maintenance“

Tabelle 6-18: Ziel- und Indikatorenhierarchie für den betrachteten Ausschnitt von SIDL

Tabelle 6-19: Ausschnitt der Zuordnung der Verantwortlichen und der Informationsempfänger Performance-relevanter Daten der SIDL AG. 178

Tabelle 8-1: Anforderungen an das IT-System für das PM

Tabelle 8-2: Vor- und Nachteile von verteilten Systemen (vgl. Coulouris und Dollimore (1994) und Noack et al. (2000)) 202

Tabelle 8-3: Bewertung der drei Architekturoptionen anhand der nicht-funktionalen Anforderungen 210

Tabelle 9-1: Anforderungen an ein Datenmodell für das Performance Measurement ......213

Tabelle 9-2: Charakterisierung von OLTP- und OLAP-Informationssystemen 214

Tabelle 9-3: Zusammenfassende Gegenüberstellung der diskutierten Ansätze. .224

Tabelle 9-4: Anforderungen an das Vorgehensmodell zur Erstellung einer Datenstruktur für ein PMS .225

Tabelle 9-5: Grade von Aggregationsbeschränkungen (vgl. Hüsemann et al. (2000))......231

Tabelle 9-6: Ziel- und Indikatorenhierarchie für den betrachteten Ausschnitt von SIDL .233

Tabelle 9-7: Ausschnitt aus der Zuordnung der Verantwortlichen und der Informationsempfänger Performance-relevanter Daten der SIDL...........................235

Tabelle 9-8: Performance-Indikatoren und mögliche Dimensionen.................................236

Tabelle 9-9: Granularitätsgrad je Indikator, Informationsempfänger und Dimension ......240

Tabelle 9-10: Aggregationspfade der Dimensionen .........................................................241

Tabelle 9-11: Auf den Dimensionen ausgeführte Aktionen ...........................................242

Tabelle 9-12: Ergebnis der bereinigten Dimensionen......................................................242 
Tabelle 9-13: Höchster Detaillierungsgrad je Indikator und Dimension.

Tabelle 9-14: Konsolidierungen der Indikatoren mit gleichen Dimensionen und gleicher Granularität

Tabelle 9-15: Aggregationsgrade der Indikatoren

Tabelle 9-16: Festlegen von Standardzugriffspfaden und Berechtigungen je Informationsempfänger.

Tabelle 9-17: Entwurfsebenen und diskutierte Ansätze 248 


\section{Abkürzungsverzeichnis}

ADAPT

ADO MD

AFQM

AG

API

ARIS

AS

ASP

BCG

BfS

BSC

Bsp.

bspw.

BWL

bzgl.

CAPM

CFO

CGI

CHF

COPPA

CORBA

COS

CS

CSI

CWQC

DB

DBMS

DCOM

DF

DIN

DSN

DSO

DTD

DTS

DWH

EAT
Application Design for Analytical Processing Technologies

Active X Data Objects Multidimensional

Austrian Foundation for Quality Management

Aktiengesellschaft

Application Programming Interface

Architektur integrierter Informationssysteme

Aktivsumme

Active Server Page

Boston Consulting Group

Bundesamt für Statistik

Balanced Scorecard

Beispiel

beispielsweise

Betriebswirtschaftslehre

bezüglich

Capital Asset Pricing Model

Chief Financial Officer

Common Gateway Interface

Schweizer Franken

Computer Supported Process Performance

Common Object Request Broker Architecture

Common Object Services resp. konzeptionelles Objekt-

Schema

Customer Satisfaction

Customer Satisfaction Index

Company Wide Quality Control

Datenbank

Database Management System / Datenbank-ManagementSystem

Distributed Component Object Mode

Dimensional-Fact (-Modell)

Deutsche Industrienorm

Data Source Name

Decision Support Objects

Document Type Definition

Data Transformation Service

Data Warehouse

Earnings after Taxes 
EBIT

EBITA

EBT

EDP

EFQM

ER, resp. E/R

ERP

ESPRIX

ETH

ETL

EVA

FA

FCF

FDA

FI

FK

Fzg.

GE

GM

GMNF

HBR

HBS

HR

HTML

HTTP

i.d.R.

IC

IDL

IDS

IE

IEEE

IIS

IMVP

IS

ISO

IT
Earnings before Interest and Taxes

Earnings before Interest, Taxes and Amortisation

Earnings before Taxes

Electronic Data Processing

European Foundation for Quality Management

Entity Relationship (-Modell)

Enterprise Resource Planning

EFQM-Initiative der Schweiz

Eidgenössische Technische Hochschule, Zürich

Extraction Transforming Loading / Extraktion

Transformation Laden

Economic Value Added

Fixed asset

Free Cash Flow

Food and Drug Administration

Modulbezeichnung von SAP R/3 für den Finanzbereich

Foreign Key

Fahrzeug

General Electric

General Motors

Generalisierte multidimensionale Normalform

Harvard Business Review

Harvard Business School

Modulbezeichnung von SAP R/3 für die Personalwirtschaft

(Human Resources)

Hyper Text Markup Language

Hyper Text Transfer Protocol

in der Regel

Intellectual Capital

Interface Definition Language

Hersteller von Prozess-Management-Software: IDS-Scheer,

Saarbrücken

Internet Explorer (von Microsoft)

Institute of Electrical and Electronics Engineers

Internet Information Server (Webserver von Microsoft)

International Motor Vehicle Program

Informationssystem

International Organisation for Standardization

Information Technology / Informations-Technologie 
JDBC

KCHF

kfr.

KMU

KOF

KPI

LBS

lfr.

MA

MBNQA

$\mathrm{MbO}$

MDX

$\mathrm{ME} / \mathrm{R}$

$\mathrm{mfr}$.

Mio.

MIS

MIT

MOLAP

NFA

NOA

NOPAT

NOPLAT

o.S.

ODBC

$\mathrm{OE}$

OLAP

OLE

OLTP

OMA

org.

PC

PDA

PDCA

PI

PIMS

PJ

PK

Pkte.

PM
Java Data Base Connectivity

Tausend Franken

kurzfristig

kleine und mittlere Unternehmen

Konjunkturforschungsstelle der ETH Zürich

Key Performance Indicator

London Business School

langfristig

Mitarbeiter

Malcom Baldrige National Quality Award

Management by Objectives

Multidimensional Expressions Language

multidimensionales Entity-Relationship (-Modell)

mittelfristig

Millionen

Management Information System

Massachusetts Institute of Technology

Multidimensional OLAP

Nicht-Funktionale Anforderungen

Net Operating Assets

Net Operating Profit After Taxes

Net Operating Profit Less Adjusted Taxes

ohne Seitenangabe

Open Data-Base Connectivity

Organisationseinheit

Online Analytical Processing

Object Linking and Embedding (Services)

Online Transaction Processing

Object Management Architecture

organisatorisch

Personal Computer

Persönliche Digitale Assistenten

Plan-Do-Check-Act

Performance Indicator / Performance-Indikator

Profit Impact on Market Strategies

Personenjahre

Primary Key

Punkte

Performance Measurement / Personenmonate 
PMS

PS

QM

QS

QUASAR

$\mathrm{R} \& \mathrm{D}$

$\mathrm{R} / 3$

resp.

RI

ROCE

ROI

ROLAP

S\&P

SAP

SAS

SDWM

Seco

SEI

SERVQUAL

SIDL

SLA

SMI

SMS

SMTP

SOM

SPC

SPI

SPI

SQC

SQL
Performance-Measurement-System

Passivsumme

Qualitätsmanagement

Qualitätssicherung

Quality attribute-oriented software architecture

Research \& Development

Produktebezeichnung der SAP

respektive

Residual Income

Return on Capital Employed

Return on Investment

Relational OLAP

Standard \& Poor

Systeme, Anwendungen und Produkte in der

Datenverarbeitung; Hersteller von betriebswirtschaftlicher

Software $^{1}$

Statistical Application System; Hersteller von Statistik-

Software

Semantisches Data Warehouse-Modell

Staatssekretariat für Wirtschaft der Schweiz

Software Engineering Institute der Carnegie Mellon

Universität

Service Quality

Schweizerischer Informatik Dienstleister; Akronym für die

in der Fallstudie verwendete Unternehmung

Service Level Agreement

Swiss Market Index

Short Message Service

Simple Mail Transfer Protocol

Semantisches Objekt Modell

Statistical Process Control

Strategic Planning Institute of Cambridge MA

Swiss Performance Index

Statistical Quality Control

Structured Query Language

1 Unmittelbar nach der Gründung, in der Zeit von 1972 bis 1976 wurde „Systemanalyse und Programmentwicklung“ verwendet. 
STAR

SVD

SW

SWX

TCO

TQC

TQM

Tsd

u.U.

US-GAAP

VBM

VDA

WACC

WfMC

WfMS

WWW

XML

ZVEI
Strategische Trend Analyse Report

Schweizerischer Verband der Datenverarbeiter

Software

Swiss Stock Exchange

Total Cost of Ownership

Total Quality Control

Total Quality Management

Tausend

unter Umständen

United States - General Accepted Accounting Principles

Value Based Management

Verband der Automobilindustrie

Weighted Average Cost of Capital

Workflow Management Coalition

Workflow-Management-System

World Wide Web

Extended Markup Language

Zentralverband Elektrotechnik- und Elektronikindustrie e.V. 


\section{Teil \\ Grundlagen}




\section{Einführung}

Die Performance hat für Organisationen in jüngster Zeit stark an Bedeutung gewonnen. Gründe hierfür sind die fortschreitende Globalisierung, die damit verbundene erhöhte Wettbewerbsintensität, immer kürzere Innovationszyklen und der zunehmende Einfluss der Stakeholder auf die Organisationen. Um in diesem anspruchsvollen Umfeld erfolgreich sein zu können, muss die Performance einer Organisation in sämtlichen relevanten Bereichen bezüglich ihres Zielerreichungsgrades ständig überwacht werden. In der Literatur wurden hierzu eine Reihe von Vorschlägen, von so genannten PerformanceMeasurement-Systemen (PMS) präsentiert. PMS überwachen und kommunizieren die Ziele und den Zielerreichungsgrad einer Organisation gesamtheitlich.

In diesem Kapitel werden die dieser Arbeit zu Grunde liegende Problemstellung erläutert und die gesetzten Ziele dargestellt. Es wird aufgezeigt, inwiefern die Problemstellung als relevant eingestuft werden kann und wie die wissenschaftstheoretische Positionierung der Arbeit verstanden werden soll. Auf Grund dieser Positionierung können die angestrebten Aussagen kategorisiert und die angewandten Forschungsmethoden bestimmt werden. Abschliessend wird der Aufbau der Arbeit erläutert.

\subsection{Problemstellung}

Verschiedene Untersuchungen zeigen, dass die in der Theorie vorgeschlagenen Konzepte des Performance Measurement in der Praxis nur mangelhaft umgesetzt wurden. Nachstehend werden hierzu einige Studien referiert:

- Bitici und Carrie (1998) untersuchten die PMS von über 30 europäischen Unternehmen. Sie stellten fest, dass die Mehrheit der PMS vergangenheitsorientiert und statisch sind. Daraus folgt, dass die gemessenen Informationen häufig nicht relevant, aktuell und akkurat sind. Ein weiterer in der Studie erwähnter Punkt betrifft die fehlende IT-Unterstützung der Performance-Measurement-Systeme. Dadurch wird die Datensammlung, -verwaltung und -berichterstattung schwerfällig.

- Die Studie von Bourne et al. (2000) zeigt, dass der Zugriff auf die operativen Informationssysteme essenziell ist. In jenen Unternehmen, in denen dies nicht der Fall 
war, konnten die Performance-Resultate nicht innerhalb der geforderten Zeit produziert werden.

- Brunner und Roth (1999) ermitteln in ihrer Studie, dass 80 Prozent der Manager mit ihren gegenwärtigen Führungsinformationssystemen nicht zufrieden sind. Die verfügbaren Informationen seien zu finanzorientiert und Aussagen hinsichtlich der kritischen Unternehmensprozesse sowie der strategischen Erfolgsfaktoren fehlten. Des Weiteren ermittelten sie, dass integrierte informationstechnische Lösungen, welche sowohl die Datenerhebung und die mehrdimensionale Datenanalyse als auch die Kommunikation von quantitativen und qualitativen Daten ermöglichen, nur selten zum Einsatz kommen.

- Hudson et al. (1999) untersuchten PMS in KMUs. Sie erwähnen drei Hauptpunkte für das Scheitern solcher Projekte: (1) Die Performance-Daten sind veraltet und irrelevant. (2) Der Aufbau und Unterhalt von PMS ist sehr aufwändig und (3) die PMS werden vom Top-Management zu wenig unterstützt.

- Küng (2001) ermittelt in seiner Studie, dass (1) die Kennzahlen nicht immer aktuell sind, (2) die Mitarbeiter mangelhaft einbezogen, (3) die Prozesse nur bruchstückhaft gemessen, und (4) die Performance-Daten verstreut gespeichert werden.

- Marr und Neely (2000) kommen zum Schluss, dass eine der grössten Schwachstellen in der Erfassung der Performance-relevanten Daten liegt. Einerseits ist es schwierig, die notwendigen Daten zu lokalisieren, und andererseits ist der Aufwand zur Sammlung der relevanten Performance-Daten erheblich.

Aus obigen Studien wird deutlich, dass wichtige Arbeiten (z.B. Sveiby (1989), Keegan et al. (1989), Eccles (1991), Fitzgerald et al. (1991), Lynch und Cross (1991b)) nur bruchstückhaft in der Praxis umgesetzt wurden. Ein Hauptproblem lässt sich auf der Schnittstelle der betriebswirtschaftlich orientierten Konzepte und der Umsetzung mittels Informationstechnologie identifizieren. Ein zweites Problemfeld ist darin zu sehen, dass es den in der Literatur vorgeschlagenen Konzepten an Konkretisierungen fehlt. 


\subsection{Zielsetzung}

Das Ziel der vorliegenden Arbeit ist es, die Anforderungen an ein rechnergestütztes Performance-Measurement-System zu bestimmen. Darauf aufbauend soll ein heuristisches Verfahren zur Herleitung der Performance-Indikatoren aus der Strategie und deren Implementierung in ein rechnergestütztes Informationssystem erarbeitet werden.

Aus der dargelegten Zielsetzung lassen sich die folgenden Forschungsfragen ableiten:

1. Was sind PMS, welche Ideen liegen zu Grunde, welche Systeme wurden in der Literatur vorgeschlagen?

2. Welche Anforderungen bestehen an PMS und an IT-Systeme für das Performance Measurement?

3. Wie könnte ein heuristisches Vorgehensmodell aussehen, welches die Herleitung der Performance-Indikatoren aus der Strategie und deren Implementierung in ein rechnergestütztes Informationssystem umfasst?

4. Wie könnte eine Architektur für ein IT-System für das Performance-Measurement aussehen?

Nachfolgend wird die Forschungsrelevanz des Themas aufgezeigt.

\subsection{Relevanz des Forschungsthemas}

In der wissenschaftlichen Literatur lassen sich eine ganze Reihe von Hinweisen zur Erstellung von Performance-Measurement-Systemen finden. Der Aspekt der Umsetzung mittels Informationstechnologie wurde jedoch bisher vernachlässigt.

Empirische Untersuchungen zum Thema des Performance Measurement zeigen deutlich, dass ohne rechnergestützte Informationssysteme PMS-Projekte kaum realisierbar sind, und dass der Gewinnung der Daten aus den operativen Informationssystemen eine herausragende Bedeutung zugesprochen wird.

Entsprechend kommt einem durchgängigen Ansatz, d.h. eine von der organisatorischen über die technische Konzeption anwendbare Vorgehensweise, ein hohes Nutzenpotenzial $\mathrm{zu}$. 


\subsection{Wissenschaftstheoretische Positionierung}

Wissenschaften können als theoretische resp. angewandte Wissenschaften verstanden werden: Theoretische Wissenschaften schaffen ihre Probleme selbst („l'art pour l'art“) und haben einen deskriptiven, wertfreien Charakter. Das oberste Forschungsregulativ ist die Wahrheit (vgl. Thommen (1992a)). Forschungskriterien können Allgemeingültigkeit, Bestätigungsgrad, Erklärungs- oder Prognosekraft der Theorien sein. Demgegenüber beschäftigen sich angewandte Wissenschaften mit Problemen aus der Praxis, sind normativ-wertend und verfolgen das Forschungsregulativ der Nützlichkeit. Im Vordergrund steht die praktische Problemlösungskraft von Modellen und Regeln (vgl. Ulrich (1988), zitiert nach Thommen (1992a)). Nach Grünig (1990) besteht die Zielsetzung einer angewandten Wissenschaft in der Erleichterung des menschlichen Handelns.

Grünig (1990) differenziert die angewandte Wissenschaft für das Beispiel der Betriebswirtschaftslehre (BWL) in normensetzende (ethisch-normative) und technologische (praktisch-normative) Betriebswirtschaftslehre. Normensetzung bedeutet die Festlegung und die Rechtfertigung verfolgter Ziele, während die technologische BWL die Gewährleistung eines optimalen Zielerreichungsgrades in realen Entscheidungssituationen vor dem Hintergrund nicht mehr zu hinterfragender Zielsetzungen anstrebt (vgl. Abbildung 1-1).

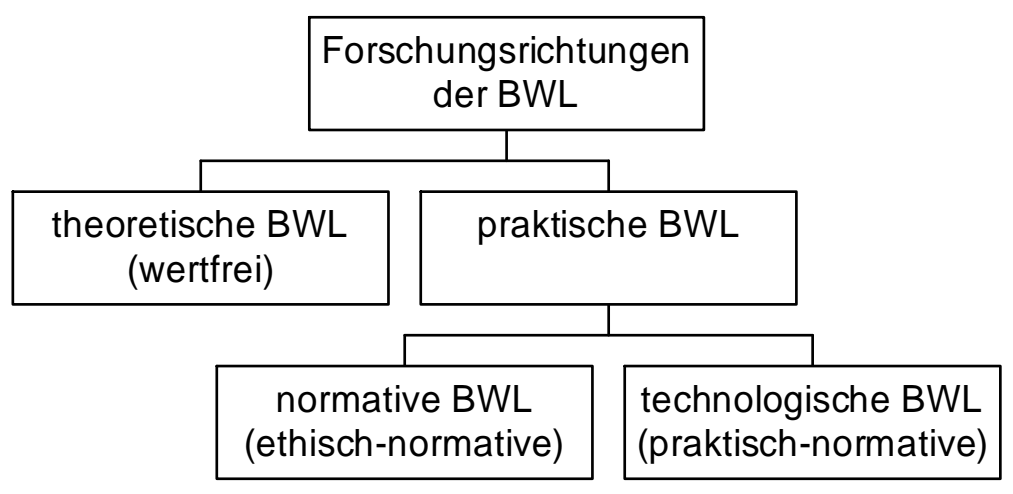

Abbildung 1-1: Forschungsrichtungen der Betriebswirtschaftslehre (vgl. Grünig (1990))

Bevor eine wissenschaftstheoretische Positionierung erfolgen kann, muss das Erfahrungsobjekt bestimmt werden. Der Begriff des Erfahrungsobjektes geht auf Ammon (1911) zurück, der eine Unterscheidung von Erfahrungs- und Erkenntnisobjekt fordert (vgl. Thommen (1992a)). Das Erfahrungsobjekt - welches den Bereich der Wirklichkeit, der in der wissenschaftlichen Arbeit betrachtet werden soll, eingrenzt (vgl. Thommen (1992a)) - 
umfasst in der vorliegenden Arbeit Organisationen, die ein IT-gestütztes PerformanceMeasurement-System einsetzen möchten. Es kann sich dabei sowohl um Unternehmen als auch um öffentliche Betriebe und Verwaltungen sowie um nicht-gewinnorientierte Organisationen handeln. Erfahrungsobjekte der BWL sind die Akteure in einer Wirtschaft, also die Konsumenten, Arbeitnehmer und insbesondere die Unternehmen. Damit umfasst das Erfahrungsobjekt der vorliegenden Arbeit dasjenige der BWL, weshalb eine entsprechende Einordnung vorgeschlagen wird. Erkenntnisobjekt der BWL ist der optimale Umgang mit knappen Gütern. (vgl. Thommen (1992a), Raffée und Abel (1979))

Denkbar wäre auch eine Einordnung in die Wirtschaftsinformatik, die sich nach einer Untersuchung von Heinzl et al. (2001) im Allgemeinen als angewandte Wissenschaft versteht, deren wichtigstes Erkenntnisziel die Beherrschung von Komplexität in Informations- und Kommunikationssystemen darstellt (vgl. Heinzl et al. (2001)). Da sich jedoch bisher wenige Arbeiten mit der wissenschaftstheoretischen Positionierung der Wirtschaftsinformatik beschäftigten, und die Grenzen zur BWL fliessend sind, wird auf die entsprechende BWL-Literatur abgestellt.

Eine Unterstellung unter die theoretische BWL würde implizieren, dass für das Untersuchungsobjekt Hypothesen aufgestellt und diese empirisch validiert würden. Nach Grünig (1990) bedingt dies einen hohen Abstraktionsgrad, da sonst keine einwandfreie empirische Überprüfung stattfinden kann. Es besteht dadurch jedoch die Gefahr, dass die komplexe Problemstellung derart reduziert wird, dass nur noch „banale“ Erkenntnisse gewonnen werden können, die wenig zur Lösung der Aufgabenstellung beitragen.

Eine ethisch-normative Auffassung, d.h. eine Aufstellung und Rechtfertigung zu verfolgender Zielsetzungen muss verworfen werden, da keine intersubjektive Überprüfung möglich ist (vgl. Steinmann und Braun (1979), zitiert bei Lanner (2000)).

Demzufolge wird die vorliegende Arbeit der praktisch-normativen BWL zugeordnet. Dem Hauptkritikpunkt nach Grünig (1990), der weniger guten Begründbarkeit der Aussagen, da auch weniger gut abgestützte Erkenntnisse verwertet werden dürfen, steht der Vorteil einer wahrscheinlich grösseren Praxisrelevanz gegenüber. Der Nachteil wird damit bewusst in Kauf genommen. 


\subsection{Angestrebte Aussagenkategorien}

Im Rahmen der gewählten Forschungsrichtung unterscheiden Grünig und Heckner (1995) sieben Aussagenkategorien der praktisch-normativen Betriebswirtschaftslehre (vgl. Abbildung 1-2). In einem ersten Schritt sollen diese vorgestellt werden, um daraufhin die vorliegende Arbeit darin präziser zu positionieren.

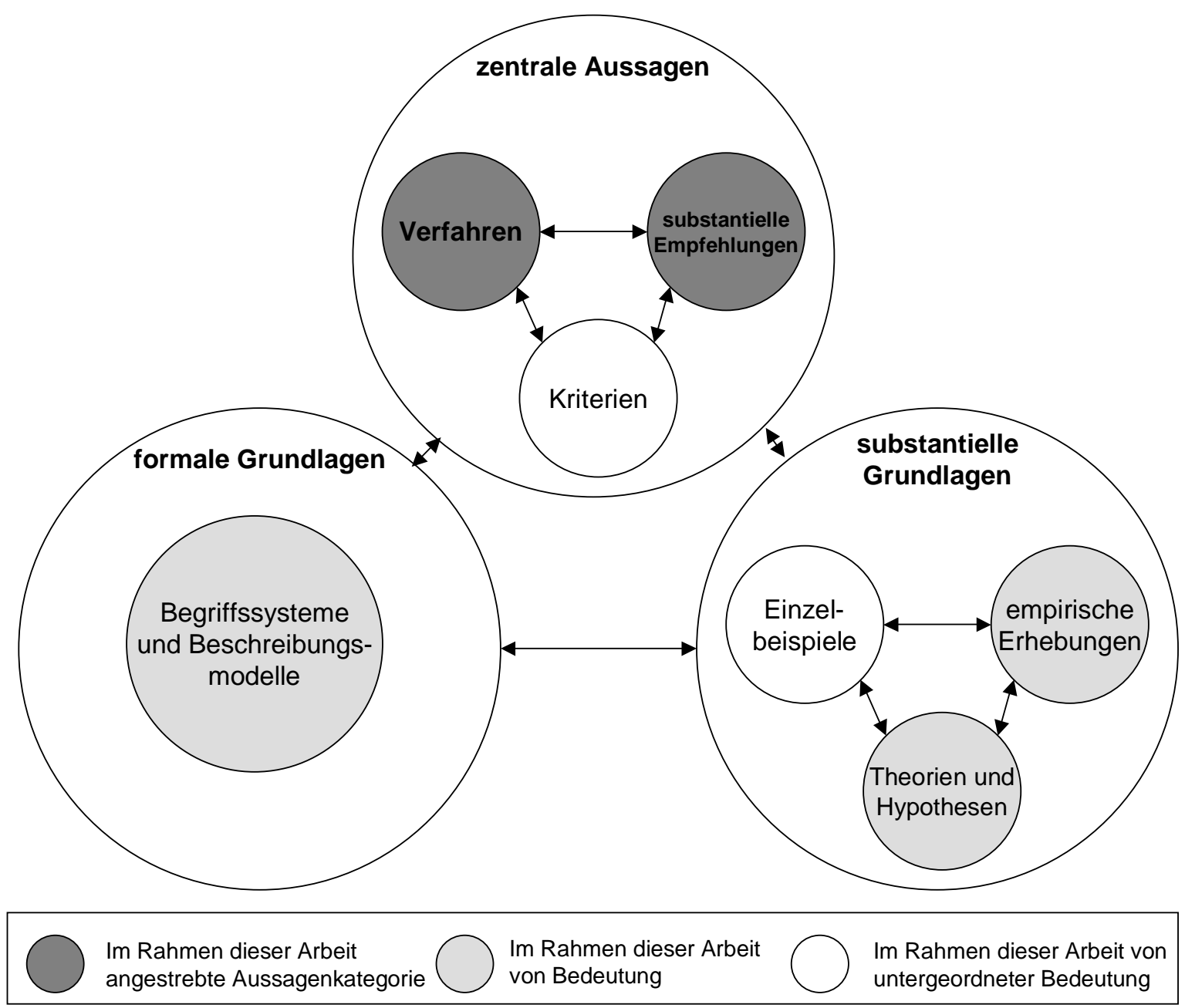

Abbildung 1-2: Aussagenkategorien der praktisch-normativen BWL (vgl. Grünig und Heckner (1995))

Unterschieden werden formale und substanzielle Grundlagen sowie zentrale Aussagen. Die formalen Grundlagen dienen der Erfassung und dem Verstehen des Untersuchungsgegenstandes und ermöglichen eine stringente Kommunikation mit allen Beteiligten. Die formalen Grundlagen bilden den Befähiger zur Gewinnung substanzieller Grundlagen. Dabei ist die Verwendung verschiedener Quellen denkbar: Einzelbeispiele 
(Case studies), empirische Erhebungen sowie bestehende Theorien und Hypothesen aus der Literatur. Die zentralen Aussagen bilden das Ziel resp. den Output der praktischnormativen BWL. Die wichtigste Kategorie ist jene der „formalen Empfehlungen in Form von Verfahren“; daneben sind auch substanzielle Empfehlungen und Kriterien denkbar (vgl. Grünig (1990)).

Für die im Rahmen dieser Arbeit zu bewältigende Problemstellung werden die folgenden Aussagenkategorien angestrebt:

- Die Kategorie der „Begriffssysteme und Beschreibungsmodelle“ kommt zur Anwendung, um die grundlegenden Begriffe von PMS und die in der Literatur vorgeschlagenen PMS-Konzepte zu erfassen.

- Die Kategorie der „Theorien und Hypothesen“ wird verwendet, um die Anforderungen an PMS und deren IT-Systeme zu erfassen. Mit einer empirischen Studie werden diese Anforderungen verifiziert. Ergebnis sind substanzielle Empfehlungen an die Ausgestaltung der Anforderungen an PerformanceMeasurement-Systeme und ihre IT-Systeme.

- Das Hauptziel gehört zur Aussagenkategorie des „Verfahrens“. Es wird ein heuristisches Vorgehensmodell, welches die Herleitung der PerformanceIndikatoren aus der Strategie und deren Implementierung in ein rechnergestütztes Informationssystem umfasst, vorgeschlagen.

\subsection{Forschungsmethodik}

Zur Erreichung der angestrebten Ziele wird folgende Forschungsmethodik verfolgt (vgl. Abbildung 1-3). Die einzelnen Schritte werden kurz diskutiert.

Im ersten Teil werden die formalen Grundlagen erarbeitet. Dabei werden die wichtigsten Begriffe definiert.

Der zweite Teil baut auf dem ersten formalen Teil auf und erarbeitet substanzielle Grundlagen, indem die einschlägige Literatur bezüglich bestehender methodischer Ansätze untersucht wird. Aus dieser Analyse werden Erkenntnisse bezüglich der Anforderungen und des herzuleitenden heuristischen Verfahrens gewonnen. 
Teil drei erarbeitet die Anforderungen an das zu erstellende Verfahren unter Einbezug der in Teil zwei durchgeführten Analyse, einer Literaturrecherche bezüglich existierender empirischer Erhebungen und ersten prototypenartigen Implementierungsversuchen. Die wichtigsten Anforderungen werden mittels einer empirischen Umfrage validiert. Das Resultat dieses Schrittes kann als erste präskriptive Aussage im Sinne einer substanziellen Empfehlung aufgefasst werden.

Im vierten Teil werden die erarbeiteten Grundlagen angewendet, um ein geeignetes heuristisches Verfahren zur Herleitung der Performance-Indikatoren aus der Strategie und deren Implementierung in ein multidimensionales Datenmodell zu entwickeln. Wie bei Problemen der Wirtschaftsinformatik üblich, wird das Verfahren in ein organisatorisches und ein technisches Element (informationstechnische Ausgestaltung) unterteilt. Der organisatorische Teil wird mittels eines Beispiels auf seine Tauglichkeit hin untersucht, und der technische Teil wird durch einen Prototypen auf die Praxistauglichkeit geprüft.

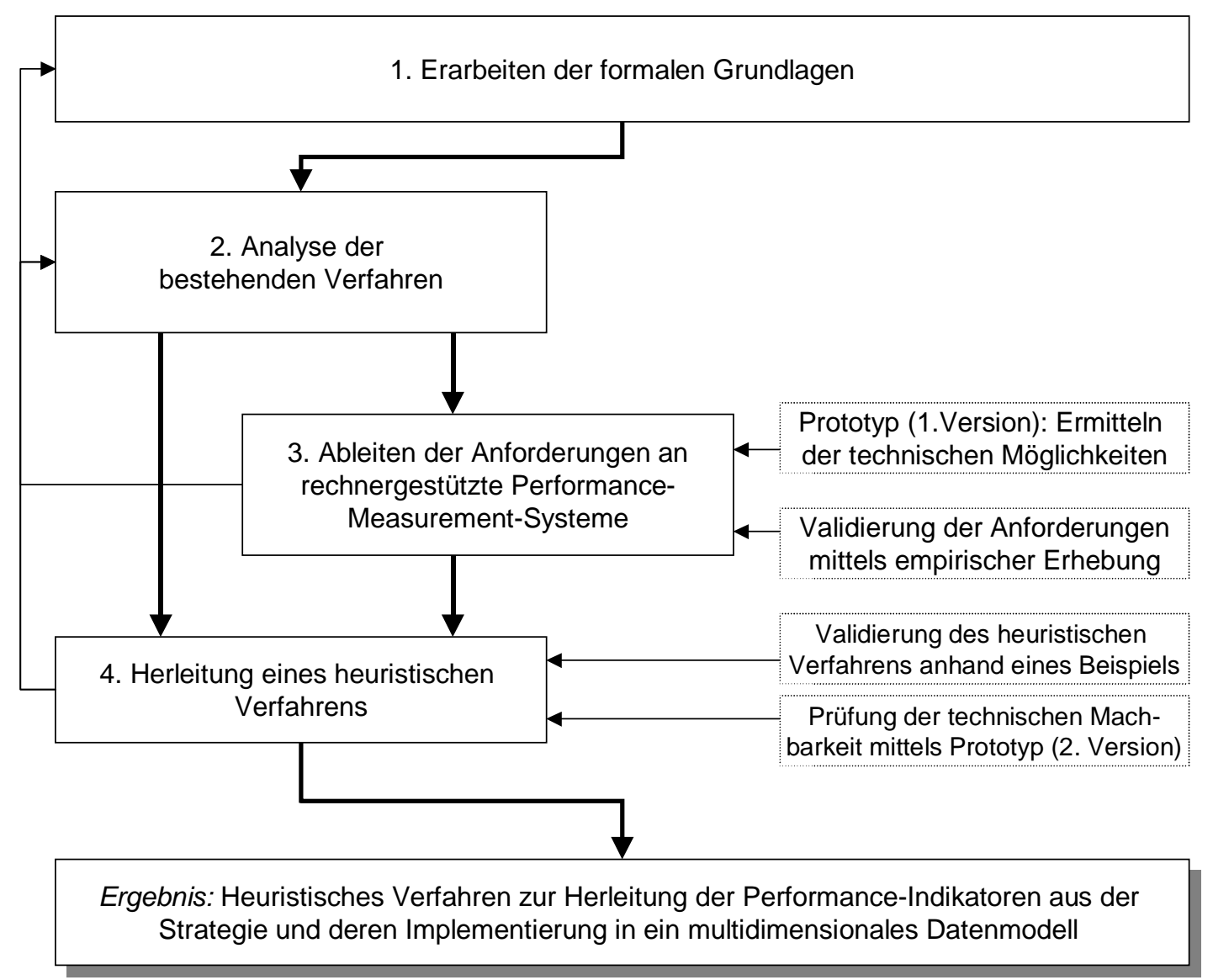

Abbildung 1-3: Überblick über die Forschungsmethodik 
Wie aus Abbildung 1-3 ersichtlich, ist das Vorgehen keineswegs deterministisch. Einzelne Schritte werden mehrfach durchlaufen. Dabei ist der Fall denkbar, dass der Output eines Schrittes als Input für den Nächsten ungenügend ist oder aber, dass im Verlaufe der Arbeit neue Erkenntnisse eine weitere Iteration für nötig erscheinen lassen.

\subsection{Aufbau der Arbeit}

Der Aufbau der Arbeit ist in Abbildung 1-4 dargestellt. Die Fläche der Rechtecke der einzelnen Kapitel zeigt ungefähr den Umfang der einzelnen Kapitel.

Die Arbeit gliedert sich in fünf Teile. Der erste Teil behandelt die Grundlagen. Im ersten Kapitel wird die Problemstellung auf Grund von Hinweisen aus der Literatur und empirischen Untersuchungen identifiziert und der geplante Beitrag begründet. Die wissenschaftstheoretische Einordnung bestimmt die gewählte Forschungsmethodik, die schlussendlich auch den Aufbau bestimmt.

Kapitel zwei befasst sich mit dem Begriffssystem. Die wesentlichen Begriffe werden charakterisiert und gegenüber verwandten Konzepten abgegrenzt. Besonderes Augenmerk wird dabei auf ein ganzheitliches Verständnis des Performance-Measurement-SystemBegriffes und einer klaren Abgrenzung zu einem IT-System für das Performance Measurement gelegt.

Im dritten Kapitel werden einige wichtige bestehende Performance-Measurement-Ansätze diskutiert und kritisch gewürdigt. Speziell betrachtet wird, in welchem Umfang die Informationstechnologie in die Ansätze Eingang gefunden hat.

Der zweite Teil widmet sich der Bestimmung der Anforderungen. Im vierten Kapitel werden auf Grund der Erfahrungen aus Kapitel drei, einer Analyse von empirischen Studien und eines ersten Prototypen die Anforderungen hergeleitet. Diese werden mittels einer empirischen Studie validiert. Ergebnis des vierten Kapitels ist eine Liste von relevanten Anforderungen an ein PMS.

Der dritte Teil befasst sich mit der organisatorischen Konzeption. Das fünfte Kapitel baut auf den Anforderungen von Kapitel vier und den Erkenntnissen der untersuchten Performance-Measurement-Konzepte (Kapitel drei) auf, um daraus einen einfachen 
Bezugsrahmen für das Performance Measurement herzuleiten. Die einzelnen Elemente wie Systematik, Methodik und Organisation werden diskutiert.
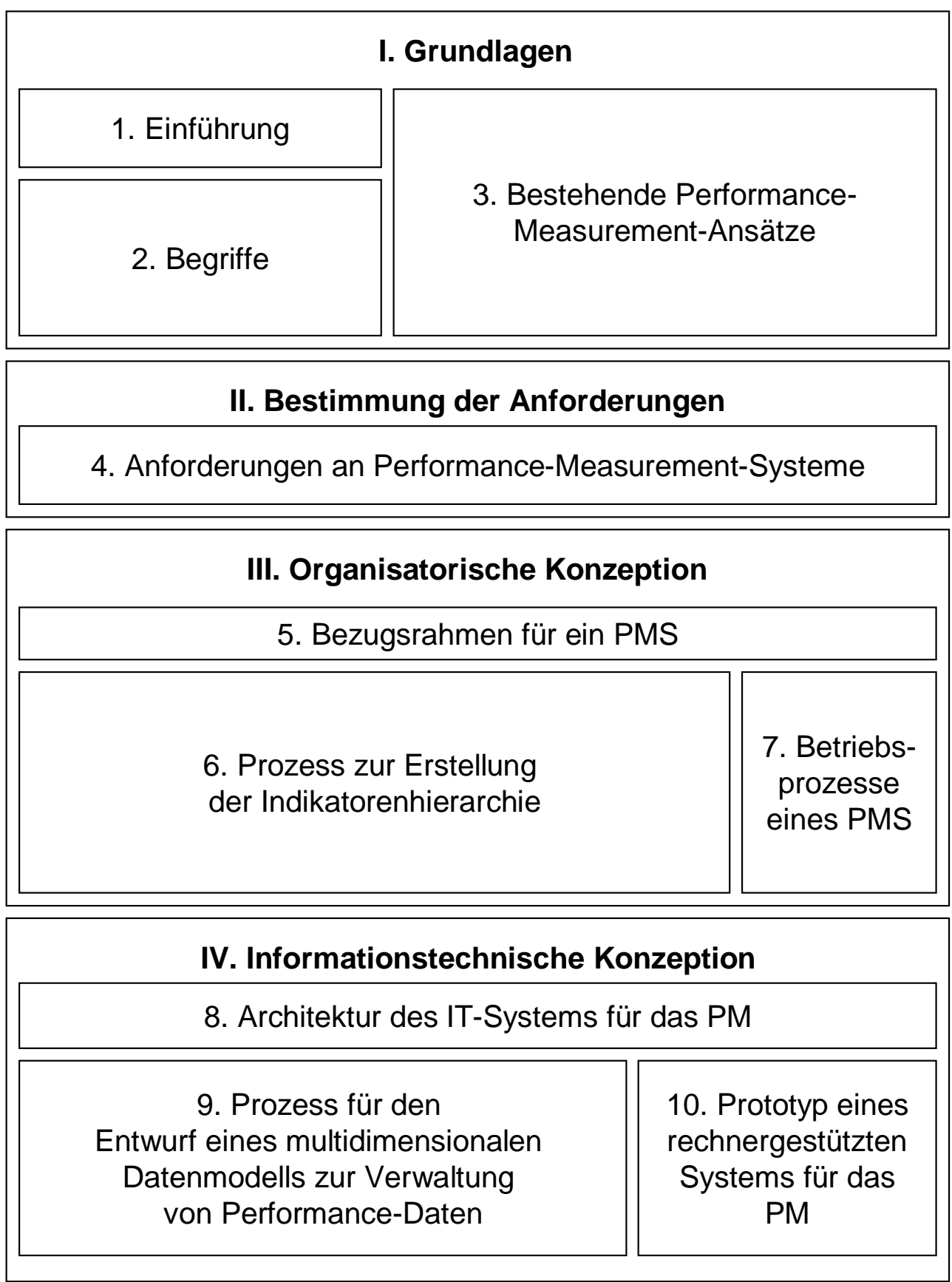

\section{Schluss}

11. Zusammenfassung und Ausblick

Abbildung 1-4: Aufbau der Arbeit 
Im sechsten Kapitel wird ein wesentliches Element der Methodik aus Kapitel fünf im Detail besprochen. Es handelt sich um den Erstellungsprozess, der eine Heuristik zur Erstellung einer Ziel- und Indikatorenhierarchie aus der Organisationsstrategie aufzeigt.

Kapitel sieben beschäftigt sich mit den notwendigen Prozessen für den Betrieb eines Performance-Measurement-Systems. Das im Rahmen von empirischen Studien festgestellte Defizit bezüglich der Aktualität der Daten soll damit erfolgreich vermieden werden.

Der vierte Teil, welcher die informationstechnische Konzeption beinhaltet, beginnt mit dem achten Kapitel, welches sich mit der Architektur von Informationssystemen im Allgemeinen und einem geeigneten Architekturvorschlag für ein IT-System für das PM auf Grund der ermittelten Anforderungen auseinander setzt.

Im Kapitel neun wird der Erstellungsprozess auf der informationstechnischen Ebene fortgesetzt, indem eine Heuristik für die Erstellung der projektspezifischen Datenstruktur in einem PMS-Projekt aufgezeigt wird.

Kapitel zehn beschreibt den Prototypen, der die Erkenntnisse von Kapitel acht und neun praktisch umsetzt und auf seine Brauchbarkeit hin untersucht.

Im fünften Teil resp. elften Kapitel, erfolgt eine kritische Beurteilung des vorgeschlagenen Vorgehensmodells. Die erreichten Ergebnisse werden kurz zusammengefasst. Ein Ausblick weist auf die Ansatzpunkte möglicher weiterführender Forschung hin. 


\section{Begriffe}

In diesem Kapitel werden die formalen Grundlagen erarbeitet. Es werden die wichtigsten Begriffe im Bereich des Performance Measurement vorgestellt. Welches ist der Betrachtungsgegenstand (Performance), was umfasst das Measurement, was ist ein Performance-Measurement-System, wie unterscheidet sich dieses von einem IT-System für das Performance Measurement? Welche Funktionen werden von den beiden Systemen abgedeckt? Welches sind die Gegenstandsbereiche? Besonderen Wert wird dabei auf eine Differenzierung der Begriffsauffassungen zwischen den angelsächsischen und den kontinentaleuropäischen Autoren gelegt. Es wird begründet, weshalb sich die Arbeitsdefinitionen an angelsächsischen Autoren orientieren. Abschliessend erfolgt eine Abgrenzung zu verwandten Konzepten wie Performance Management, strategische Führung, Controlling und Benchmarking. Damit leistet dieses Kapitel einen Beitrag zur Beantwortung der ersten Forschungsfrage „Was sind PMS, welche Ideen liegen zu Grunde, welche System wurden in der Literatur vorgeschlagen?“.

\subsection{Performance}

Gegenstand dieser Arbeit sind Performance-Measurement-Systeme. Es ist daher angezeigt, auf den Begriff der Performance einzugehen. Andersen und Fagerhaug (2002) schreiben zum Ursprung des Wortes „...performance is believed to have originated in the fifteenth century to mean a play or exhibition of some type“. Konsultieren wir ein Wörterbuch, so wird deutlich, dass die ursprüngliche Bedeutung noch heute ihre Gültigkeit hat. Es sind jedoch neue Bedeutungen hinzugekommen. Der Wordsmyth (2002) beschreibt den Begriff Performance mit „a particular entertainment presented before an audience” und mit „way of working or functioning“. Als Synonyme von Performance werden unter anderem die Begriffe „accomplishment“, „efficiency“, „capability“ und „satisfaction“ aufgeführt. Merriam-Webster-Dictionnary (2002) definiert Performance als „the execution of an action“ und mit „the ability to perform“. Es erstaunt daher nicht, dass die Literatur bezüglich der Begriffsdefinition nicht einheitlich ist.

Gleiches gilt für das Fachgebiet des Performance Measurement. Neely (1999) weist darauf hin, dass zwischen 1994 und 1996 über 3000 Artikel zum Thema Performance Measurement publiziert wurden. Umso mehr erstaunt, dass die Grundbegriffe selten 
definiert werden und diesbezüglich in der Forschergemeinde keine Einigkeit herrscht. Neely et al. (1995) schreiben in ihrem Literaturreview treffend: „Performance measurement is a topic which is often discussed but rarely defined. “ Donnell und Duffy (2001) erwähnen Meyer und Gupta (1994) mit der Aussage: „there is a massive disagreement as to what performance is and that the proliferation of performance measures has led to the paradox of performance, i.e. that organizational control is maintained by not knowing exactly what performance is. “

In der Performance-Measurement-Literatur findet sich eine grosse Breite an Definitionen. Tabelle 2-1 zeigt einige Definitionen und den Kontext, in dem sie entstanden sind. Die Reihenfolge der Nennungen erfolgt nach dem Publikationsdatum.

\begin{tabular}{|c|c|c|}
\hline Autor I Quelle & Definition des Begriffes „Performance” & Kontext \\
\hline Cordero (1989) & $\begin{array}{l}\text { Effectiveness (i.e. measuring output to determine if they } \\
\text { help accomplish objectives) } \\
\text { Efficiency (i.e. measuring resources to determine } \\
\text { whether minimum amounts are used in the production of } \\
\text { these outputs). }\end{array}$ & $\begin{array}{l}\text { Forschung und } \\
\text { Entwicklung / } \\
\text { Organisationslehre }\end{array}$ \\
\hline Lebas (1995) & $\begin{array}{l}\text { Performance is about deploying and managing well the } \\
\text { components of the causal model that lead to the timely } \\
\text { attainment of stated objectives within constraints } \\
\text { specific to the firm and to the situation. }\end{array}$ & Betriebswirtschaft \\
\hline $\begin{array}{l}\text { Neely et al. } \\
(1996)\end{array}$ & Efficiency and Effectiveness of purposeful action. & Betriebswirtschaft \\
\hline $\begin{array}{l}\text { Rolstadas } \\
\text { (1998) }\end{array}$ & $\begin{array}{l}\text { A complex interrelationship between seven performance } \\
\text { criteria: } \\
\text { - effectiveness } \\
\text { - efficiency } \\
\text { - quality } \\
\text { - quality of work life } \\
\text { - innovation } \\
\text { - profitability/budgetability }\end{array}$ & Organisationslehre \\
\hline Dwight (1999) & The level to which a goal is attained & Grundlagen \\
\hline Hoffmann (1999) & $\begin{array}{l}\text { Unter Performance/Leistung wird der bewertete Beitrag } \\
\text { zur Erreichung der Ziele einer Organisation verstanden. } \\
\text { Dieser Beitrag kann von Individuen und Gruppen von } \\
\text { Mitarbeitern innerhalb der Organisation sowie von } \\
\text { externen Gruppen (z.B. Lieferanten) erbracht werden. }\end{array}$ & Organisationslehre \\
\hline $\begin{array}{l}\text { Andersen und } \\
\text { Fagerhaug } \\
\text { (2002) }\end{array}$ & $\begin{array}{l}\text { We believe it is sufficient to have reached a point where } \\
\text { performance has replaced productivity and is generally } \\
\text { accepted to cover a wide range of aspects of an } \\
\text { organization - from the old productivity to the ability to } \\
\text { innovate, to attract the best employees, to maintain an } \\
\text { environmentally sound outfit, or to conduct business in } \\
\text { an ethical manner. }\end{array}$ & Organisationslehre \\
\hline
\end{tabular}

Tabelle 2-1: Definitionen des Begriffes „Performance“ 
Die beiden Begriffe Effizienz (Verhältnis zwischen Mitteleinsatz und Zielerreichung; Brockhaus (2001)) und Effektivität (Wirksamkeit, Leistungsfähigkeit; Brockhaus (2001)) sind die dominierende Begriffe in den Definitionen. Rolstadas (1998) schlägt jedoch noch fünf weitere Kriterien zur Bestimmung der Performance vor, und Dwight (1999) vermeidet die Nennung von konkreten Kriterien. Er bestimmt lediglich das Ausmass der Zielerreichung. Wird zusätzlich der Kontext dieser Definition betrachtet, so wird deutlich, dass die Definition der Performance vom Anwendungszusammenhang abhängt. Diese Meinung teilt auch Lebas (1995).

Im Rahmen dieser Arbeit interessiert die Performance von Organisationen. Wird eine ganzheitliche Sichtweise auf das Zielsystem einer Organisation herangezogen, so kann das Ausmass der Zielerreichung der Stakeholder der Organisation - für diejenigen Ziele, welche mit denjenigen der Organisation übereinstimmen - als Performance aufgefasst werden. Als Stakeholder werden alle Personen, Gruppierungen, Interessengemeinschaften oder Institutionen innerhalb oder ausserhalb eines Unternehmens verstanden, die einen Beitrag („stake“) zur betrieblichen Wertschöpfung erbringen, hieraus aber auch Ansprüche an das Unternehmen ableiten (vgl. Schmid (1997)). In ähnlichen Worten drückt dies Mendelow (1983) aus. Für ihn sind die Stakeholder einer Unternehmung jene Personen, welche vom Unternehmen abhängig sind, damit sie einen Teil ihrer Ziele erreichen können. Gleichzeitig ist das Unternehmen von diesen Personen (Stakeholder) ebenfalls abhängig, damit es seine unternehmerischen Ziele erreichen kann. Es existiert also eine wechselseitige Abhängigkeit zwischen Stakeholder und Unternehmen. Die Stakeholder eines Unternehmens sind bspw. die Mitarbeiter, die Kunden, die Lieferanten, die Investoren, die Steuerzahler (bei gewissen staatlichen Organisationen), die öffentliche Verwaltung sowie die Gesellschaft. An Stelle des Begriffes Stakeholder werden ebenfalls die Bezeichnungen Interessensgruppen oder Anspruchsgruppen verwendet.

Aus obigen Überlegungen leitet sich die folgende Arbeitsdefinition ab:

Performance kann aufgefasst werden als Grad der Zufriedenheit der relevanten Anspruchsgruppen (degree of stakeholder satisfaction). ${ }^{2}$

${ }^{2}$ Die Definition ist aus der Sicht der Organisation zu sehen. 
Laut Andersen und Fagerhaug (2002) lässt sich der Begriff der Performance auch aus der wirtschaftsgeschichtlichen Entwicklung begründen. Von 1800 bis 1850 produzierten die englischen Manufakturen möglichst akkurat; Qualität war oberstes Ziel. Am Ende des 19. Jahrhunderts rückte mit dem Taylorismus (Scientific Management) die Überwachung der Produktivität der Arbeiter ins Zentrum. Maschinenauslastung und Arbeitsproduktivität waren die relevanten Kenngrössen. Die daraus resultierende Formel - Produktivität als Verhältnis von Output zu Input - wird seither akzeptiert. Erst mit dem Wandel vom Produzenten- zum Konsumentenmarkt in den 80er-Jahren genügte der Produktivitätsbegriff zur Messung nicht mehr. Mit dem Begriff der Performance wird der ursprüngliche Produktivitätsbegriff um zusätzliche Elemente ergänzt.

Abschliessend soll noch kurz auf den deutschen Begriff „Leistung“ eingegangen werden. Dieser unterliegt ebenfalls einer starken Mehrfachverwendung und -bedeutung. Einzig in der Physik ist er eindeutig definiert als Arbeit (Kraft x Weg) pro Zeiteinheit. In sämtlichen anderen Bereichen ist der Begriff mehrfach besetzt (vgl. Gleich (2001)). Im Rahmen dieser Arbeit wird auf die Verwendung des Begriffes verzichtet.

\subsection{Performance Measurement}

Der Merriam-Webster-Dictionnary (2002) beschreibt Measurement als „the act or process of measuring “, wobei measuring als (a) „to choose or control with cautious restraint“, (b) „to regulate by a standard“ oder (c) „to estimate or appraise by a criterion“ aufgefasst werden kann. Die Definition macht deutlich, dass „Measurement” als ein Prozess begriffen wird, der das Messen, Analysieren, Kommunizieren und Initiieren von Handlungen umfasst.

Die meisten englischsprachigen Autoren (wie z.B. Sinclair und Zairi (1995); Neely et al. (1995)) teilen diese Auffassung und verstehen demzufolge unter Performance Measurement einen Prozess, die Performance zu erfassen, d.h. zu quantifizieren. Drongelen und Cook (1997) unterscheiden explizit die Elemente Datenakquisition und analyse, und implizit auch die Kommunikation und das Initiieren von Handlungen. (vgl. Tabelle 2-2) 


\begin{tabular}{|l|l|l|}
\hline Autor I Quelle & Definition & Kontext \\
\hline $\begin{array}{l}\text { Drongelen und } \\
\text { Cook (1997) }\end{array}$ & $\begin{array}{l}\text { The acquisition and analysis of } \\
\text { information about the actual attainment of } \\
\text { company objectives and plans, and about } \\
\text { factors that may influence this attainment. }\end{array}$ & Allgemein \\
\hline $\begin{array}{l}\text { Evangelidis } \\
(1992)\end{array}$ & $\begin{array}{l}\text { The process of determining how } \\
\text { successful organizations or individuals } \\
\text { have been in attaining their objectives. }\end{array}$ & $\begin{array}{l}\text { Organisationslehre / } \\
\text { Personallehre }\end{array}$ \\
\hline $\begin{array}{l}\text { Neely et al. } \\
(1995)\end{array}$ & $\begin{array}{l}\text { Performance measurement can be } \\
\text { defined as the process of quantifying the } \\
\text { efficiency and effectiveness of action }\end{array}$ & Produktionswirtschaft \\
\hline $\begin{array}{l}\text { Sinclair und } \\
\text { Zairi (1995) }\end{array}$ & $\begin{array}{l}\text { The measurement of performance at all } \\
\text { levels within an organization }\end{array}$ & Organisationslehre \\
\hline
\end{tabular}

Tabelle 2-2: Definitionen des Begriffes „Performance Measurement“

Unter dem Begriff Performance Measurement wird im Rahmen dieser Arbeit das Messen, Analysieren und Kommunizieren der Performance sowie das Planen von Aktionen und Massnahmen verstanden.

Die Performance eines Gegenstandsbereiches wird mittels Kennzahlen gemessen. Bürkeler (1977) definiert Kennzahlen als betrieblich relevante, numerische Informationen. Der Begriff der Kennzahl ist nicht einheitlich (vgl. Siegwart (1998)). Meyer (1994) nennt neben dem Begriff Kennzahl eine Reihe von synonym verwendeten Ausdrücken: Kennziffern, Kontrollzahlen, Kontrollziffern, Messzahlen, Ratios, Richtzahlen, Schlüsselgrössen, Schlüsselzahlen und Standardzahlen. Ein aus dem Englischen stammender Begriff ist der „Performance-Indikator“. Er bringt zum Ausdruck, dass Performance nicht etwas Absolutes ist, und dass Performance nicht immer ganz präzise erfasst und quantifiziert werden kann. Ein Performance-Indikator gibt einen Hinweis darüber (engl. to indicate), wie es um die Performance steht. Hält man sich vor Augen, dass Performance-Indikatoren nur eine beschränkte Aussage bezüglich der wahren oder tatsächlichen Performance machen, so ist nahe liegend, dass die wirkliche Performance nicht mit einem einzigen Indikator erfasst und beurteilt werden kann. Um eine fundierte Beurteilung vornehmen zu können, braucht es immer mehrere Performance-Indikatoren: ein so genanntes „Set“ von Performance-Indikatoren (vgl. Wettstein et al. (2001)).

Der Begriff des „Performance-Indikators“ bringt nach Meinung des Verfassers die Problematik gut zum Ausdruck, weshalb in der vorliegenden Arbeit dieser Begriff weiterverwendet werden soll. 
Performance Measurement kann ebenfalls im Sinne eines kybernetischen Regelkreises aufgefasst werden (vgl. Abbildung 2-1), hier dargestellt an der Kontrolle in einem Regelkreis. Dieser weist die folgenden Phasen auf (vgl. Steinmann und Schreyögg (1993)):

1. Kontrolle setzt die Existenz eines Vergleichsmassstabs voraus. Es muss somit ein SollWert bestimmt werden, der sich in der Regel aus der Planung ergibt. Dieser wird mittels Performance-Indikatoren operationalisiert.

2. Die tatsächlich realisierten Ausprägungen des Performance-Indikators - die Messwerte - müssen ermittelt werden.

3. Der Soll/Ist-Vergleich wird durchgeführt und daraus die Abweichung ermittelt.

4. Die Abweichungsanalyse sucht nach den Ursachen. Wurden der Ist-Wert und die Abweichung korrekt ermittelt, so sind gemeinhin drei Ursachen denkbar: (1) Planungsfehler, (2) Störgrössen (i.d.R. Veränderungen der Unternehmensumwelt), (3) Mehr- oder Minderleistungen, Fehlentscheidungen und Fehlverhalten.

5. Berichterstattung: Damit der Prozess Wirkung zeigt, ist eine Rückkoppelung notwendig.

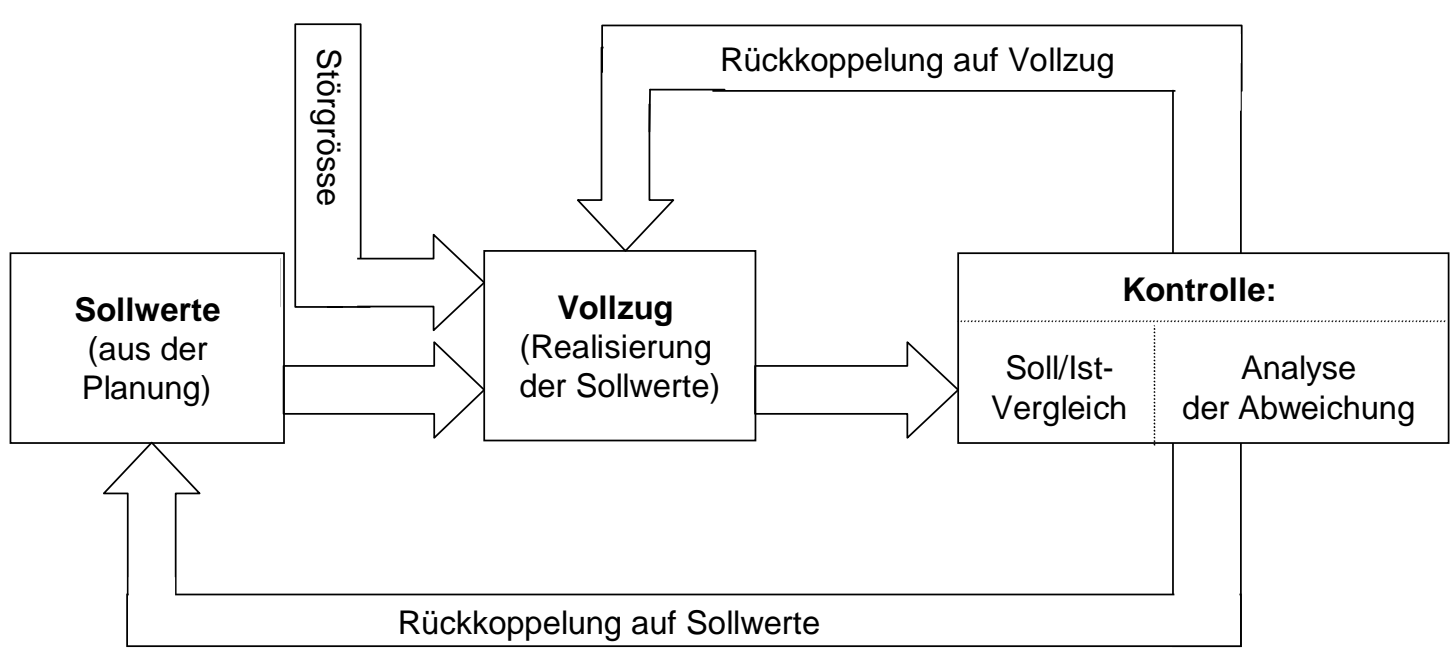

Abbildung 2-1: Regelkreis (Steinmann und Schreyögg (1993)) 


\subsection{Performance-Measurement-System}

Ein System kann definiert werden als ein einheitlich geordnetes Ganzes. Es besitzt ein gewisses Mass an Integration und Geschlossenheit im Verhältnis seiner Elemente zueinander (Struktur), eine es von anderen Systemen, d.h. von der Umwelt abhebende Grenze, eine gewisse Ordnung in den Beziehungen mit anderen Systemen sowie eine gewisse Kontinuität und Regelmässigkeit (Persistenz) in den Beziehungen zwischen den Elementen des Systems (Hartfiel und Hillmann (1982)).

Entsprechend sollte die Definition eines PMS Informationen enthalten über die

- Struktur (Elemente und Beziehungen zwischen diesen),

- Systemgrenzen (Grenze und Beziehung zur Systemumwelt) und

- Persistenz des Systems

Im Folgenden sollen einige Definitionen von PMS bezüglich dieser Elemente untersucht werden.

Ein elementarer Anspruch von Performance-Measurement-Systemen besteht in der angestrebten Transparenz sowohl der Unternehmensziele als auch der nachfolgenden Ebenen. Als weiteres charakteristisches Merkmal von Performance-MeasurementSystemen wird auf den intensiven Einsatz von nicht-monetären Indikatoren verwiesen (vgl. Klingebiel (1999)).

Gleich (1997) definiert ein PMS als den „Aufbau und Einsatz meist mehrerer quantifizierbarer Massgrössen verschiedenster Dimensionen (z.B. Kosten, Zeit, Qualität, Innovationsfähigkeit, Kundenzufriedenheit), die zur Beurteilung der Effektivität und Effizienz der Leistung und Leistungspotentiale unterschiedlichster Objekte im Unternehmen (Organisationseinheiten unterschiedlichster Grösse, Mitarbeiter, Prozesse) herangezogen werden.“

Blankenburg (1999) definiert ein PMS über die Charakterisierung der Elemente Zeit, Objekt und Verhalten. Der Zeithorizont soll die Entwicklung, Planung und Kontrolle der Strategie eines Unternehmens umfassen. An Objekten werden zusätzlich zu den klassischen internen Elementen (Zeit, Qualität und Kosten), die Bedürfnisse der externen Stakeholder einer Organisation miteinbezogen. Die Legitimation liegt in der Überzeugung, dass diese Faktoren als Frühwarnindikatoren für finanzielle Ergebnisse der 
Unternehmenstätigkeit dienen können. Bei einer konsequenten Anwendung müssen damit die vermuteten ursächlichen Indikatoren überwacht werden. Dabei besteht die Problematik, dass diese ursächlichen Faktoren häufig qualitativer Natur sind. Konsequenterweise kommt daher die Forderung, dass sowohl qualitative als auch quantitative Aspekte berücksichtigt werden sollten. Mit dem PMS möchte man des Weiteren das Verhalten der Mitarbeiter und Kader beeinflussen. So werden nach Gleich (1997) eine erhöhte Mitarbeitermotivation angeregt sowie zusätzliche Lerneffekte erzeugt.

Neely et al. (1995) definieren ein PMS als ein „system that can be defined as the set of metrics used to quantify both the efficiency and effectiveness of actions“. Die Metrik bezieht sich hier auf mehr als nur die Formel, welche benötigt wird, um die Indikatoren zu berechnen. Neely et al. (1995) subsumieren darunter ebenfalls die Definition eines Performance-Indikators, die genaue Bezeichnung, die Berechnungsvorschrift, wer die Berechnung durchführt, und woher die Daten stammen.

Simons (1999) definiert ein PMS als „...formal, information-based routines and procedures managers use to maintain or alter patterns in organizational activities. “ Zum Zweck eines PMS sagt er Folgendes: „The purpose of a PMS is to convey information: (1) The PMS focus on data - financial and non-financial - that influences decision making and managerial action, (2) PMS represent formal routines and procedures, (3) Information is written down or entered into computer systems and captured in standard formats, (4) The recording, analyzing, and distributing of this information is embedded in the rhythm of the organization, (5) PMS are designed to be used by managers, (6) Managers use PMS to maintain or alter patterns in organizational activities“.

Simons (1999) beschliesst seine Ausführungen mit der folgenden Definition: „Performance measurement systems: information systems that managers use to track the implementation of business strategy by comparing actual results against strategic goals and objectives. A performance measurement system typically comprises systematic methods of setting business goals together with periodic feedback reports. “

Die vorgestellten Definitionen sollen nun einerseits bezüglich der Merkmale des Systembegriffs (vgl. Hartfiel und Hillmann (1982)) untersucht und andererseits anhand der relevanten Schlüsselbegriffe einander gegenübergestellt werden. Dem Verfasser ist bewusst, dass sowohl die Wahl der Schlüsselbegriffe als auch die Zuordnung zu den 
Systemmerkmalen in subjektiver Weise erfolgt. Trotz dieser Nachteile ist die Darstellung für eine sinnvolle Arbeitsdefinition eines PMS hilfreich.

Die drei als wichtig identifizierten Systemmerkmale werden als (S) Struktur (Elemente und Beziehungen zwischen diesen), (G) Systemgrenzen (Grenze und Beziehung zur Systemumwelt) und (P) Persistenz des Systems bezeichnet (vgl. Tabelle 2-3).

\begin{tabular}{|c|c|c|c|c|c|c|c|c|}
\hline \multirow[t]{2}{*}{ Kriterien } & \multicolumn{3}{|c|}{ Systembegriff } & \multirow{2}{*}{$\begin{array}{l}\text { Klinge- } \\
\text { biel } \\
\text { (1999) }\end{array}$} & \multirow[t]{2}{*}{$\begin{array}{l}\text { Gleich } \\
\text { (1997) }\end{array}$} & \multirow{2}{*}{$\begin{array}{l}\text { Blanken- } \\
\text { burg } \\
(1999)\end{array}$} & \multirow{2}{*}{$\begin{array}{l}\text { Neely } \\
\text { et al. } \\
\text { (1995) }\end{array}$} & \multirow[t]{2}{*}{$\begin{array}{l}\text { Simons } \\
\text { (1999) }\end{array}$} \\
\hline & S & G & $P$ & & & & & \\
\hline $\begin{array}{l}\text { Transparenz der } \\
\text { Unternehmensziele }\end{array}$ & $\nabla$ & & & $\checkmark$ & & & & $\checkmark$ \\
\hline $\begin{array}{l}\text { Messgrössen } \\
\text { verschiedenster } \\
\text { Dimensionen }\end{array}$ & V & & & $\checkmark$ & $\checkmark$ & $\checkmark$ & $\checkmark$ & $\checkmark$ \\
\hline $\begin{array}{l}\text { Beurteilung der } \\
\text { Performance im } \\
\text { Unternehmen auf } \\
\text { verschiedenen Ebenen }\end{array}$ & V & & & & $\checkmark$ & & $\checkmark$ & \\
\hline $\begin{array}{l}\text { Entwicklung, Planung } \\
\text { und Kontrolle der } \\
\text { Strategie }\end{array}$ & & & $\nabla$ & & & $\checkmark$ & & \\
\hline $\begin{array}{l}\text { Anforderungen der } \\
\text { Stakeholder }\end{array}$ & & $\square$ & & & & $\checkmark$ & & \\
\hline $\begin{array}{l}\text { Verhaltensbeeinflussung } \\
\text { der Mitarbeiter }\end{array}$ & V & & & & & $\checkmark$ & & $\checkmark$ \\
\hline Lerneffekte & & & $\nabla$ & & $\checkmark$ & & & $\checkmark$ \\
\hline $\begin{array}{l}\text { Formale Routinen und } \\
\text { Prozeduren }\end{array}$ & & & $\nabla$ & & & & $\checkmark$ & $\checkmark$ \\
\hline $\begin{array}{l}\text { Begrenzung auf } \\
\text { relevante Informationen }\end{array}$ & & $\square$ & & & & & & $\checkmark$ \\
\hline $\begin{array}{l}\text { Kommunikation der } \\
\text { Performance-Resultate }\end{array}$ & & & $\nabla$ & & & & & $\checkmark$ \\
\hline
\end{tabular}

Tabelle 2-3: Synopse des PMS-Begriffes

Bei der Betrachtung der Tabelle 2-3 wird deutlich, dass bezüglich der begriffsbildenden Elemente sowohl im deutsch- als auch im englischsprachigen Raum erhebliche Differenzen besteht.

Im Rahmen dieser Arbeit soll das PMS in Anlehnung an die Definition von Simons verwendet werden. Ergänzend werden die Elemente „Beurteilung der Performance im Unternehmen auf verschiedenen Ebenen“ und „Anforderungen der Stakeholder“ berücksichtigt. Damit wird im Rahmen dieser Arbeit die folgende Definition verwendet: 


Ein Performance-Measurement-System (PMS) kommuniziert die
operationalisierte Strategie und überwacht die ganzheitliche Performance einer
Organisation auf sämtlichen Ebenen. Das PMS unterstützt die effektive
Kommunikation der Performance mit allen Stakeholdern, bietet Managern
sowohl operative als auch strategische Entscheidungsunterstützung, sammelt
Wissen der Organisation und vereinfacht das organisationelle Lernen. Um dieses
Ziel zu erreichen, definiert das PMS geeignete Prozesse und bedient sich
geeigneter Informationssysteme.

Diese Definition impliziert nicht, dass das PMS ein Instrument zur Festlegung der Strategie ist. Das PMS ist weiter kein Garant für den Erfolg einer Organisation. Ein PMS kann jedoch helfen, eine definierte Strategie systematisch umzusetzen.

\subsection{IT-System für das Performance Measurement}

Ein Informationssystem ist ein Mensch/Aufgabe/Technik-System zum Beschaffen, Herstellen, Bevorraten und Verwenden von Information, kurz: ein System zur Informationsproduktion und Kommunikation für die Deckung von Informationsnachfrage (Heinrich (1997)). Dabei kann es sich durchaus um ein papiergestütztes Informationssystem handeln (vgl. Andersen und Fagerhaug (2002)). Es ist jedoch einleuchtend, dass eine derartig grosse Menge von Daten, mit zahlreichen Benutzern, u.U. geographisch weit verteilt nur schwer mit einem manuellen Informationssystem verwaltet werden kann. Im Allgemeinen ist daher davon auszugehen, dass der Einsatz eines ITgestützten Informationssystems zu bevorzugen ist (vgl. Küng und Wettstein (2001)).

In Anlehnung an den Begriff eines Datenbank-Management-Systems (vgl. Conolly und Begg (1998)) soll der IT-gestützte Teil eines Performance-Measurement-Systems definiert werden. Nach Conolly und Begg besteht ein System aus den Elementen Menschen, Prozeduren, Daten, Software und Hardware. Menschen konzipieren, betreiben und nutzen das System. Prozeduren sind als Prozesse aufzufassen. Sie definieren, wie das System aufgebaut wird, wie Daten gesammelt, wie sie analysiert werden, etc.. 
Ein IT-System für das PM umfasst jenen Anteil der Prozeduren, die mittels IT unterstützt werden können, sowie die Software, die Daten und die Hardware.

Das Ziel eines IT-Systems für das PM ist es, die Aufgaben eines PMS mittels Informationstechnologie zu unterstützen. Ein zentrales Element ist dabei eine Plattform, bestehend aus Hard- und Software (z.B. Datenbank-Management-System), zur Speicherung und Kommunikation von Performance-relevanten Daten. Eventuell werden einzelne Prozeduren, die in einem PMS ablaufen, mittels Informationstechnologie unterstützt (z.B. das Erarbeiten einer Zielhierarchie, um die Strategie zu operationalisieren). Die Abgrenzung zwischen einem IT-System für das PM und einem PMS ist in Abbildung 2-2 dargestellt. (vgl. Küng und Wettstein (2001))

Wie Abbildung 2-2 zeigt, ist es schwierig, den IT-Anteil genau zu bestimmen. Einzelne ITSysteme für das PM werden kaum Prozeduren für das PMS unterstützen. Sie bestehen vielleicht aus einen PC mit einer Tabellenkalkulation. Alle Daten werden von Hand eingetragen und aufbereitet. Auf der anderen Seite ist es denkbar, dass ein IT-System für das PM sehr ausgereift ist. In einem solchen Fall würden die meisten Prozeduren (z.B. Definition der Performance-Indikatoren, Sammeln der Performance-relevanten Daten, Datenanalyse, Datenkommunikation, etc.) von der Informationstechnologie unterstützt. Es ist selbstverständlich, dass ein solches System eine leistungsfähige Informatikinfrastruktur und ausgereifte Programme bedingt. Neben dem Nutzen eines solchen Systems sind jedoch auch die damit verbundenen Kosten in Betracht zu ziehen. (vgl. Küng und Wettstein (2001)) 


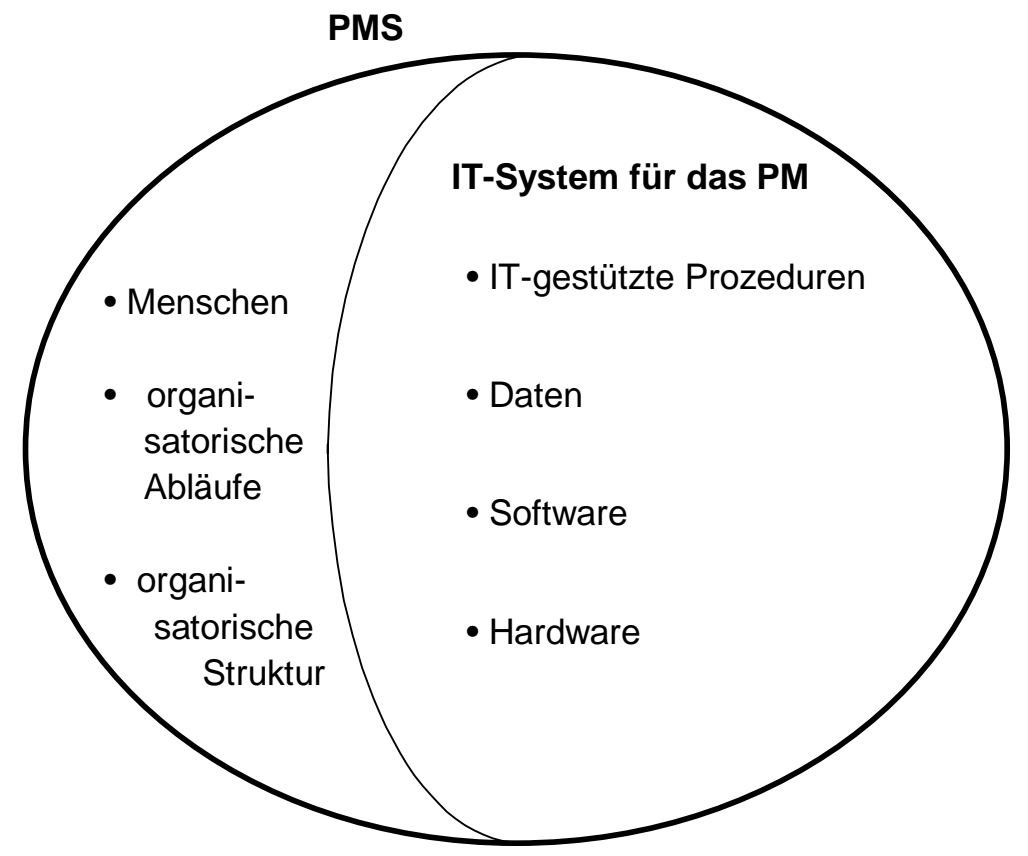

Abbildung 2-2: IT-System als Teil eines PMS

Abbildung 2-3 zeigt die fünf Komponenten eines PMS und charakterisiert diese anhand einiger wichtigen Elemente (vgl. Küng und Wettstein (2001)).

\begin{tabular}{|c|c|c|c|c|}
\hline Menschen & Prozeduren & Daten & Software & Hardware \\
\hline $\begin{array}{l}\text { Gesamtverantwortliche } \\
\text { für das PMS } \\
\text { Personen, die das PMS } \\
\text { aufbauen und betreuen } \\
\text { Datenlieferanten } \\
\text { Nutzer des PMS } \\
\text { Interne und externe } \\
\text { Stakeholder }\end{array}$ & $\begin{array}{l}\text { Definition der Indikatoren } \\
\text { Organisation des } \\
\text { Datenmanagements } \\
\text { Organisation der } \\
\text { Datenverwendung }\end{array}$ & $\begin{array}{l}\text { Performance-relevante } \\
\text { Daten } \\
\text { Zielwerte (Sollwerte) } \\
\text { der Indikatoren } \\
\text { Performance-Resultate } \\
\text { (berechnete Daten) } \\
\text { Metadaten: } \\
\text { Beschreibung } \\
\text { der Indikatoren }\end{array}$ & $\begin{array}{l}\text { Software für das } \\
\text { Extrahieren, } \\
\text { Transformieren } \\
\text { und Laden der } \\
\text { Datenbank (ETL) } \\
\text { Datenverwaltungssystem } \\
\text { Datenanalyse-Software } \\
\text { Präsentations- und } \\
\text { Kommunikations- } \\
\text { Software }\end{array}$ & $\begin{array}{l}\text { Personal Computer } \\
\text { oder sonstiger } \\
\text { Electronic Display } \\
\text { Datenbank und } \\
\text { Applikations-Server } \\
\text { Kommunikations- } \\
\text { infrastruktur }\end{array}$ \\
\hline
\end{tabular}

\section{Abbildung 2-3: Komponenten eines PMS}




\subsection{Begriffsabgrenzungen}

In diesem Abschnitt soll der Begriff des Performance Measurement resp. PMS von einigen wichtigen Begriffen abgegrenzt werden.

\subsubsection{Performance Management}

Insbesondere von deutschsprachigen Autoren werden Performance-Measurement-Systeme zu Performance-Management-Konzepten erhoben. So schreibt Brunner (1999): Performance Measurement beschränkt sich auf die „Messung“ der Performance mittels Kennzahlen, beim Performance Management steht das „Managen“ und damit die Planung, Steuerung und Kontrolle der Performance im Vordergrund. Auch Klingebiel (1999) reduziert das Performance Measurement teilweise auf den Messvorgang. An anderer Stelle schreibt Klingebiel (1999), eine begriffliche Abgrenzung und inhaltliche Zuordnung der Termini Performance Measurement und Performance Management liege bisher - wenn überhaupt - nur unzureichend vor.

In Anlehnung an die englischsprachige Literatur soll im Rahmen dieser Arbeit Performance Management als Thema der Personalwirtschaft aufgefasst werden und Performance Measurement in der weitgefassten Bedeutung verwendet werden. (Im Widerspruch dazu führt Lebas (1995) aus: „performance management is a philosophy which is supported by performance measurement“).

Die Abgrenzung von Management (Führung) und Measurement wird in Anlehnung an den Führungsbegriff von Rühli bestimmt. Rühli (1985) definiert: „Unter Führung verstehen wir die Gesamtheit der Institutionen, Prozesse und Instrumente, welche im Rahmen der Problemlösung durch eine Personengemeinschaft (mit komplexen zwischenmenschlichen Beziehungen) der Willensbildung (Planung und Entscheidung) und der Willensdurchsetzung (Anordnung und Kontrolle) dient. “ Bei der formalen Betrachtung der Führung stehen die vier konstitutiven Elemente Planung, Entscheidung, Anordnung und Kontrolle im Vordergrund. Die Kontrolle und teilweise die Planung können dem Performance Measurement zugeordnet werden, während die Entscheidung und Anordnung ganz klar dem Managementbegriff zugeordnet werden müssen. 


\subsubsection{Controlling}

Horváth (2001) versteht unter Controlling ein „Subsystem der Führung, das Planung und Kontrolle sowie Informationsversorgung systembildend und systemkoppelnd koordiniert und auf diese Weise die Adaption und Koordination des Gesamtsystems unterstützt“. Damit betont Horváth die Koordinationsfunktion. Häufig wird aber unter Controlling auch die Durchführungsfunktion verstanden, d.h. die Planung und Kontrolle selbst. Daraus lassen sich zwei Hautaufgaben ableiten: (1) Das Schaffen einer Infrastruktur zur Unterstützung der Planung und Kontrolle mittels Informationen und (2) die Koordination und Durchführung von Planung und Kontrolle. Das Ziel des Controlling ist somit die bestmögliche Unterstützung des Managements mit Instrumenten, Konzepten und Informationen.

Controlling ist ein Managementkonzept zur zielgerichteten Steuerung von Unternehmen auf der Grundlage von Plänen. Man versteht darunter auch alle Massnahmen der betrieblichen Planung, Steuerung und Kontrolle zur Erreichung der Unternehmensziele (vgl. Huch et al. (1997)). Da der Begriff sehr breit aufgefasst wird, werden darunter eine Vielzahl von Ansätzen subsumiert (Horváth (2001)).

Traditionelle Controllingsysteme werden häufig ex post benutzt, um die Umsetzung von Plänen der Unternehmensführung zu überprüfen (vgl. Blankenburg (1999)). Simons (1991) ordnet diese Systeme den diagnostischen Kontrollsystemen, PMS demgegenüber den interaktiven Kontrollsystemen zu.

\subsubsection{Total Quality Management}

Die European Foundation for Quality Management (EFQM) versteht unter Total Quality Management (TQM) eine „auf der Mitwirkung aller ihrer Mitglieder beruhende Führungsmethode einer Organisation, die Qualität in den Mittelpunkt stellt und durch die Zufriedenheit der Kunden langfristigen Geschäftserfolg sowie auf Nutzen für die Mitglieder der Organisation und für die Gesellschaft zielt. “ (EFQM (2002), vgl. auch ISO8402 (1994)).

Nach Malorny (1996) ist die genaue Herkunft des Begriffes ungeklärt. Der Ursprung kann ansatzweise auf einen von Feigenbaum ((1956), zitiert nach Malorny (1996)), publizierten Begriff Total Quality Control (TQC) und den vom Japaner K. Ishikawa ((1985), zitiert 
nach Malorny (1996)), geprägten Begriff Company Wide Quality Control (CWQC) zurückgeführt werden.

Der Ausdruck „alle ihre Mitglieder“ meint jegliches Personal aller Stellen und HierarchieEbenen der Organisationsstruktur. Der Begriff Qualität bezieht sich beim umfassenden Qualitätsmanagement auf das Erreichen aller geschäftlichen Ziele. Die Aussage „Nutzen für die Gesellschaft” bedeutet Erfüllung der an die Organisation gestellten Forderungen der Gesellschaft. (vgl. ISO-8402 (1994))

Seghezzi (1996) sieht TQM als (1) Ausrichtung der Organisation an den Bedürfnissen aller Anspruchsgruppen, (2) dem Nullfehlerprinzip, (3) den ständigen Verbesserungsaktionen, in die alle Mitarbeiter miteinbezogen werden. (4) Das Prinzip des internen Kunden / Lieferanten lässt jeden Mitarbeiter Qualitätsverantwortung für seine Arbeit tragen. (5) Die traditionellen Stärken und Erfolgspositionen des Unternehmens werden in das Konzept einbezogen, und (6) TQM wird von der Geschäftsleitung geführt.

TQM-Modelle können als PMS angesehen werden (Blankenburg (1999)). Die Operationalisierung der Strategie ist jedoch kein Kernpunkt von TQM. Ebenso werden TQM-Modelle häufig nur in unregelmässigen Abständen zur Selbstdiagnose verwendet. Im Zentrum der Aktivitäten steht das Qualitätsdenken, währenddessen PMS eine ausgewogene Auswahl der Dimensionen anstreben.

Eine Reihe der in TQM-Modellen angewandten Werkzeuge und Methoden (z.B. UrsacheWirkungs-Analysen (Fischgräten-, Ishikawa-Diagramme)) werden auch bei PMS eingesetzt.

\subsubsection{Strategische Führung}

Grünig und Kühn (2000) definieren die strategische Planung als systematischen Prozess, mit dem Zweck, unternehmensrelevante Erfolgspotenziale zu definieren und Vorstellungen von Wegen zu deren Aufbau und Erhaltung zu entwickeln. Ergebnis ist die Strategie, die in Form von Dokumenten und Plänen die langfristigen Vorgaben zum Aufbau und zur Sicherung von Erfolgspotenzialen enthält. Die Umsetzung und Kontrolle bilden zusammen mit der strategischen Planung die strategische Führung. Die Strategieumsetzung und Kontrolle ist ein kontinuierlicher Prozess. Die Kontrolle erlaubt mittels regelmässiger SollIst-Vergleiche und einer laufenden Prämissenüberwachung eine Aussage bezüglich des 
Umsetzungsfortschrittes. Der Schwerpunkt der Forschung lag bisher auf der strategischen Planung. Umsetzung und Kontrolle wurden bis vor kurzem wenig beachtet (Kreikebaum (1989)). Hammer (1992) kritisiert, dass die Kontrolle meistens ex post erfolgt und damit eine Beeinflussung der laufenden Umsetzung verunmöglicht wird.

PMS sollen hier einen Beitrag leisten. Moderne PMS zeigen Wege auf, wie die erarbeitete Strategie umgesetzt und ständig kontrolliert werden kann (vgl. bspw. Kaplan und Norton (1996a)). Einen Beitrag hierzu kann insbesondere die Verankerung des PMS in der Organisation bieten. Abbildung 2-4 fasst das Ausmass des Unterstützungspotenzials für die strategische Führung durch ein PMS zusammen.

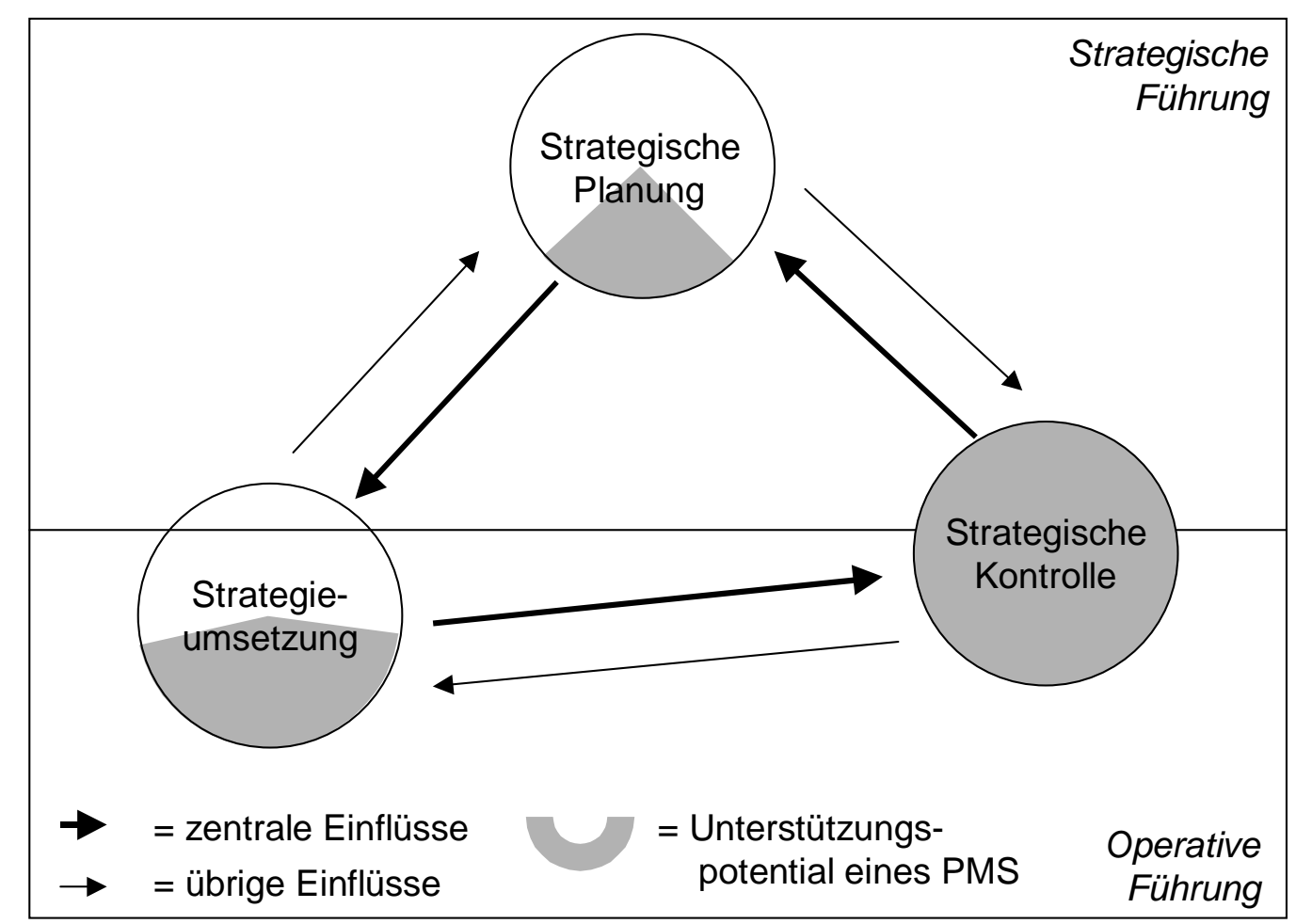

Abbildung 2-4: Unterstützungspotenzial eines PMS für die strategische Führung (in Anlehnung an Grünig und Kühn (2000)) 


\subsubsection{Benchmarking}

Der englische Begriff Benchmarking stammt ursprünglich aus dem Vermessungswesen und bezeichnet dort eine Vermessungsmarkierung, einen Bezugspunkt und Standard, an dem etwas gemessen und beurteilt wird (vgl. Camp (1994)). In der Managementlehre kann Benchmarking definiert werden als ein Leistungsvergleich zwischen Institutionen mit gleichem oder ähnlichem Aufgabenbereich mit dem Ziel, in einem qualitativen Prozess von den Besten zu lernen, um die eigene Leistungsfähigkeit zu erhöhen (vgl. Camp (1994)).

Harrington und Harrington (1996) definieren Benchmarking als „a systematic way of collecting information about equipment, product and/or process performance, then analyzing why some items outperform others and applying this knowledge to improve your organization's performance”.

Der Begriff Benchmarking wurde erstmals durch die Firma Xerox im Jahre 1979 geprägt, welche Benchmarking im Produktionsbereich einführte. Oben zitierter Robert C. Camp, war Benchmarking-Projektleiter bei Xerox. Die Wurzeln von Benchmarking reichen jedoch noch weiter zurück. Das Vergleichen mit der Konkurrenz und Adoptieren von gelungenen Ideen gehört seit langem zur Strategie von Unternehmen. Henry Ford hatte bspw. im Jahre 1916 die Fliessbänder für die Automobilherstellung nach einem Besuch in einer Grossschlachterei eingeführt.

Benchmarking kann danach unterschieden werden, ob es innen- oder aussenorientiert ist (vgl. Abbildung 2-5). Beim internen Benchmarking versuchen Organisationen von anderen Abteilungen oder Schwesterfirmen zu lernen, indem ähnliche Prozesse miteinander verglichen werden. Der Zugang zu den Prozessdaten ist in der Regel einfach, da aber in jeder Organisation eine bestimmte Kultur herrscht, sind sowohl die Unterschiede als auch das Verbesserungspotenzial beschränkt. Externes Benchmarking führt einen Vergleich mit ähnlichen Organisationen und Prozessen ausserhalb der eigenen durch. Der Zugang der Daten ist in der Regel schwierig. Wichtig erscheint, dass vergleichbare Prozesse analysiert werden. 


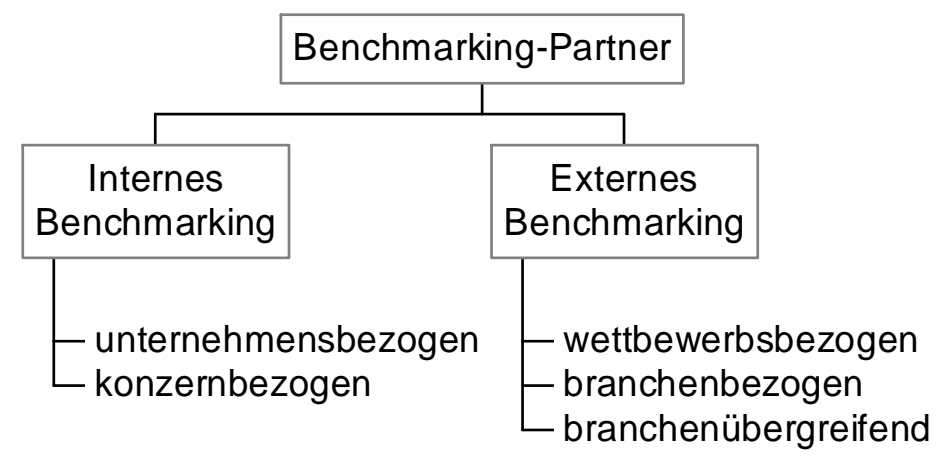

Abbildung 2-5: Arten des Benchmarking (vgl. Ortega et al. (1999))

Benchmarking ist vom Prozess her durchaus mit dem Performance Measurement vergleichbar. Betrachtet man die sechs von Watson (1992) vorgeschlagenen Schritte eines Benchmarking (Planung, Auswahl des Benchmarking-Partners, Beobachtung, Analyse, Umsetzung und Verbesserung), so sind diese dem Performance Measurement ähnlich. Unterschiede bestehen in der Institutionalisierung, der Ganzheitlichkeit (bezogen auf alle Ebenen der Organisation) und der Strategieorientierung.

Nachdem in diesem Kapitel die wichtigsten Begriffe erarbeitet wurden, erfolgt im nächsten Kapitel ein Überblick über die historische Entwicklung des Performance Measurement und eine Analyse von vier aktuellen Ansätzen. 


\section{Bestehende Performance-Measurement-Ansätze}

Die Zielsetzung dieses Kapitels besteht darin, die historische Entwicklung des Performance Measurement aufzuarbeiten und eine Auswahl von vier aktuellen Ansätzen genauer vorzustellen. Diese werden mittels einer einheitlichen Struktur erläutert. Zuerst wird das Grundkonzept dargestellt. Es erfolgt eine kritische Beurteilung des Ansatzes auf Grund einer Literaturanalyse. Daraufhin werden Weiterentwicklungen des Konzeptes referiert und die Berücksichtigung der Informationstechnologie untersucht. Eine eigene Beurteilung bedingt eine Vergleichsgrösse. Diese soll geschaffen werden, indem vier Fallbeispiele skizziert werden und die Ansätze an deren Erfordernissen gemessen werden. Damit wird der zweite Teil der Forschungsfrage eins „Was sind PMS, welche Ideen liegen zu Grunde, welche Systeme wurden in der Literatur vorgeschlagen?” beantwortet.

\subsection{Historische Entwicklung des Performance Measurement}

Wie in vielen Bereichen der Betriebswirtschaft, so sind auch die Ursprünge des Themas „Performance” schwierig zu bestimmen. Verschiedene Teilaspekte wurden schon seit längerer Zeit intensiv bearbeitet, so z.B. Überlegungen zu Produktivität, Qualität und Finanzen, aber auch in der Personalwirtschaft zur zielorientierten Führung von Mitarbeitern. Die entscheidende Frage stellt sich, wann die ersten ganzheitlichen Systeme zur systematischen Erfassung mit der elementaren Zielsetzung einer kontinuierlichen Verbesserung und Transparenz vorgeschlagen wurden (vgl. Klingebiel (2000)). Im Kontext dieser Arbeit ist ebenfalls von Interesse, wann derartige Systeme erstmals mit Informationstechnologie in Verbindung gebracht wurden.

Um die Wurzeln der aktuellen Systeme aufzeigen zu können, wird kurz auf die beitragenden Disziplinen eingegangen: (a) Finanzen, (b) Produktion, (c) Personal. Daraufhin sollen die ersten ganzheitlichen Systeme vorgestellt und der Aspekt der Informationstechnologie untersucht werden. 


\subsubsection{Ein Ansatz aus dem Finanzbereich}

Im Finanzbereich werden seit Anfang des letzten Jahrhunderts Kennzahlensysteme zur Überwachung der Unternehmensleistung eingesetzt. Kennzahlensysteme können definiert werden als „logische und/oder rechnerische Verknüpfung mehrerer Kennzahlen, die zu einander in einem Abhängigkeitsverhältnis stehen. Es handelt sich um eine aufbauende, zweckorientierte Gliederung einzelner Kennzahlen.” (Siegwart (1998)). Das wohl bekannteste Instrument, das direkt aus der Bilanz und Erfolgsrechnung abgeleitet wird, stammt aus dem Jahre 1919, entwickelt von der Eleuthère Irénée du Pont de Nemours and Company, Wilmington, Delaware, einer Sprengstofffabrik, die sich heute unter dem Namen DuPont als Chemiekonzern mit 27 Mrd. US\$ Umsatz und 94'000 Angestellten positioniert (vgl. DuPont (2002)). Das als DuPont-Schema bezeichnete Instrument hat eine Baumstruktur. An der Spitze steht der Return on Investment (ROI), also die Rendite des eingesetzten Kapitals. Die Kennzahl wird in mehreren Stufen verfeinert, bis auf die Stufe einzelner Bilanz- und Erfolgsrechnungspositionen. Dies erlaubt eine Analyse der finanziellen Haupteinflussfaktoren auf den Unternehmenserfolg, insbesondere dann, wenn innerbetriebliche oder Branchen-Vergleiche angestellt werden. So können Schwachstellen erkannt und entsprechende Gegenmassnahmen eingeleitet werden. In Abbildung 3-1 ist das Schema dargestellt.

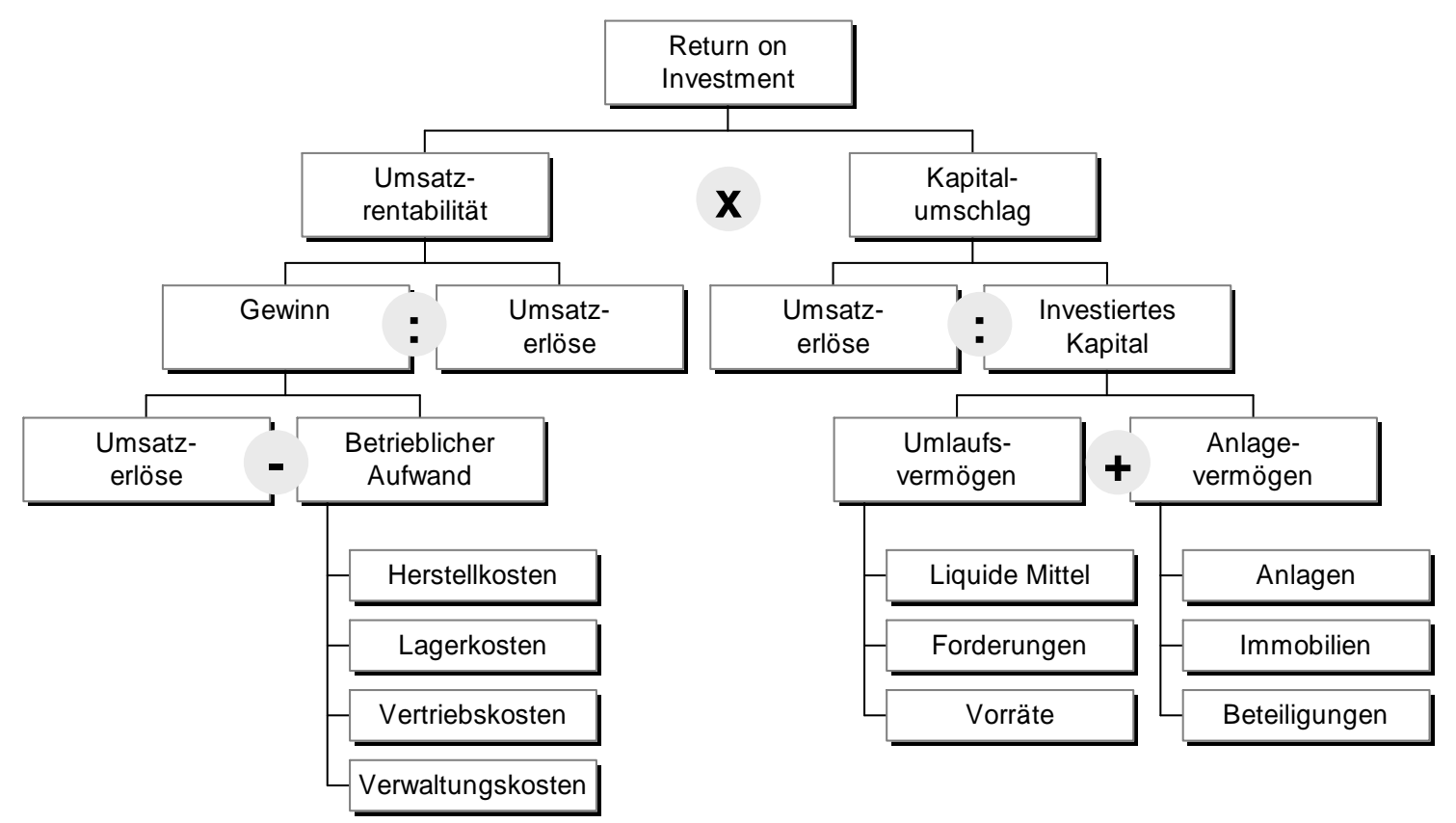


Durch die Anschaulichkeit der Wirkungszusämmenhänge im Unternehmen, geniesst das DuPont-Schema einen hohen didaktischen Wert (vgl. Thommen (1992b), Siegwart (1998)). Die formale Geschlossenheit erlaubt eine klare, multikausale und widerspruchsfreie Kennzahlenanalyse (Gladen (2001)).

Seine Popularität verdankt das DuPont-Schema auch der Tatsache, dass dem Rentabilitätsziel die grösste Bedeutung zugeordnet wird. Der Artikel von Reece und Cool (1978) bestätigt diese Aussage mit einer empirischen Studie, zeigt aber auch eindrücklich die Grenzen auf. Während der Ansatz für Profit Center verwendbar ist, versagt er für so genannte Cost Center, da sich der Gewinn nicht aufspalten lässt. Ein weiterer Problempunkt des DuPont-Schemas ist die „Kurzfristorientierung” (vgl. Gladen (2001)).

Daneben existiert eine ganze Reihe ähnlicher Systeme; eine Übersicht ist in Siegwart (1998) zu finden.

\subsubsection{Beiträge aus der Produktion}

In der Fertigung standen primär nicht-finanzielle Faktoren im Vordergrund. Diese wurden schon sehr früh erkannt. Mit der frühen Industrialisierung kam die Erkenntnis, dass mit der Spezialisierung die Produktivität erhöht werden kann. Smith (1776) zeigte dies in seinem Werk „The Wealth of Nations” eindrücklich. Er beschreibt eine Nadelfabrik in der alle Arbeitsschritte zur Produktion einer Nadel jeweils von sämtlichen Arbeitern ausgeführt wurden und wie die Produktivität massiv gesteigert werden konnte, indem jeweils ein Arbeiter nur einen einzigen Arbeitsgang ausführte. In seinem Werk „The Principles of Scientific Management” zeigt Frederick Winslow Taylor (1919) sehr eindrücklich auf, wie mit einer „Arbeitsweise auf wissenschaftlicher Grundlage”, d.h. mittels Studien (Beobachten und Analysieren der Tätigkeit der Arbeiter), der Einführung von normalisierten Werkzeugen und Geräten, die Produktivität ohne Mehrbelastung der Arbeiter gesteigert werden kann. Taylor (1919) gibt folgende konkrete Anleitung: „Im folgenden gebe ich eine Zusammenstellung der Schritte, die im allgemeinen zur Ableitung eines einfachen ... Gesetzes nötig sind: (1) Man suche 10 oder 15 Leute ... (2) Man studiere die genaue Reihenfolge der grundlegenden Operationen .... (3) Man messe mit der Stoppuhr die Zeit... (4) Man schalte alle falschen, zeitraubenden und nutzlosen Bewegungen aus ... (5) ... stelle man die schnellsten und besten Bewegungen, ebenso die besten Arbeitsgeräte tabellarisch in Serien geordnet zusammen." 
Aspekte wie das Vorschlagswesen stellte Taylor (1919) ebenfalls in seinem Werk vor: „Es soll jedoch auf jede Weise dazu ermuntert werden, Verbesserungen in den Methoden und Werkzeugen vorzuschlagen."

Neben der Produktivität steht auch die Qualität im Zentrum des Interesses der Fertigung. Für diesen Bereich wurden auch schon sehr früh eine Reihe von Instrumenten vorgeschlagen. Eines, das Statistical Process Control (SPC) auch als Statistical Quality Control (SQC) bezeichnet, ist ein Instrument zur Messung und Steuerung der Qualität (als Teil von Performance) von industriellen Prozessen. Es soll hier kurz vorgestellt werden.

Juran und Gryna (1993) definiert SPC als „the application of statistical methods to the measurement and analysis of variation in any process”. Typische Attribute, die mittels SPC gemessen werden sind laut Juran und Gryna (1993) die Folgenden: „(a) first-time yield: percentage of output meeting specifications at inspection; (b) warranty costs due to manufacturing defects; (c) amount of downgraded output; (d) amount of downtime of manufacturing equipment."

Mit dem statistischen Instrument „statistical control charts” wird die Zielsetzung verfolgt, aussergewöhnliche Variationen des Prozesses zu erkennen. Beim Überschreiten von definierten Limiten, festgesetzt auf Grund von bekannten Daten, wird der Prozess analysiert und die Ursache bestimmt (vgl. Juran und Gryna (1993)). Als Instrument zur Analyse kommen Fischgräte-Diagramme (Ishikawa-Diagramme; vgl. Ishikawa (1985)) resp. cause-and-effect diagrams (vgl. z.B. Juran und Gryna (1993) oder Department-ofNavy (1999a)) zum Einsatz. Aufbauend auf den Analyseergebnissen können geeignete Massnahmen getroffen werden.

Damit werden die zwei Ziele des SPC deutlich: Einerseits soll der Prozess stabilisiert werden und sein Verhalten vorhergesagt werden können. Damit wird auch die Voraussetzung geschaffen, dass die gewünschten Produktspezifikationen mit Bestimmtheit erreicht werden können (vgl. Juran und Gryna (1993)). Andererseits bietet das Vorgehen die Grundlage, den Prozess ständig zu verbessern (Process Improvement). Gemäss Deming (2000) ist ein stabiler Prozess nicht unbedingt ein guter Prozess: „Control charts provide information about how the process is running, not about how it should run.”

Das Instrument SPC konzentriert sich auf Daten aus der Fertigung (primär Qualitätsaspekte) und basiert auf Vergangenheitsdaten. 


\subsubsection{Der Beitrag aus dem Personalbereich}

Die Personalführung nutzt ebenfalls eine Art Performance Measurement für ihre Zwecke. Das Führungsmodell Management by Objectives (MbO) ist das wohl umfassendste und am häufigsten praktizierte Führungssystem der Gegenwart. Es wird heute in drei Varianten diskutiert (vgl. Wunderer und Grunwald (1980)): (a) Management durch Zielvorgabe (autoritäre Variante), (b) Management durch Zielvereinbarung (kooperative Variante), (c) Management durch Zielorientierung (neutrale Variante). In diesen Bezeichnungen drückt sich das geplante Ausmass der Beteiligung der Mitarbeiter an der Festsetzung der Ziele aus.

Dem MbO-Ansatz liegt folgende Idee oder Annahme zu Grunde: Ohne Ziele kann nicht optimal geplant, entschieden, realisiert und kontrolliert werden. Sind die Ziele nicht klar und quantitativ festgelegt, so leidet darunter die erreichte Leistung. Im Weiteren kommt beim MbO-Ansatz der Zufriedenheit der eine hohe Bedeutung zu, denn sie beeinflusst die Leistung ganz wesentlich. Um dies zu erreichen, werden die Ziele der Organisation und die Ziele des Mitarbeiters in einem Vereinbarungsprozess auf einander abgestimmt. Des Weiteren sollten die Ziele operationalisiert werden, d.h. präzis, wenn möglich quantitativ, terminiert und so formuliert sein, dass der Mitarbeiter selbst beurteilen kann, ob er sich dem Ziel nähert oder nicht. Durch die mögliche Selbstkontrolle kann der Mitarbeiter selbst korrigierend eingreifen. (vgl. Stroebe und Stroebe (1992))

\subsubsection{Erste ganzheitliche Systeme}

Ein wichtiger Kritikpunkt an den bisherigen Performance-Measurement-Ansätzen war die weit gehende Eindimensionalität - sprich Konzentration auf eine Messgrösse (z.B. Finanzen, Qualität, Produktivität). Dieser wird in neueren Vorschlägen durch die Kombination von finanziellen und nicht-finanziellen Indikatoren entkräftet. Welche nichtfinanziellen Dimensionen in Betracht gezogen werden, hängt stark vom vorgeschlagenen Ansatz ab. Einigkeit besteht einzig darin, dass die Auswahl der Dimensionen ausgewogen sein soll. In einer Arbeit von Keegan et al. (1989) wird bspw. ein Portfolio vorgeschlagen, das einerseits zwischen finanziellen und nicht-finanziellen Indikatoren und andererseits zwischen internen und externen Aspekten unterscheidet (siehe Abbildung 3-2). Für jedes der vier Felder werden beispielhaft einige Indikatoren aufgeführt. 


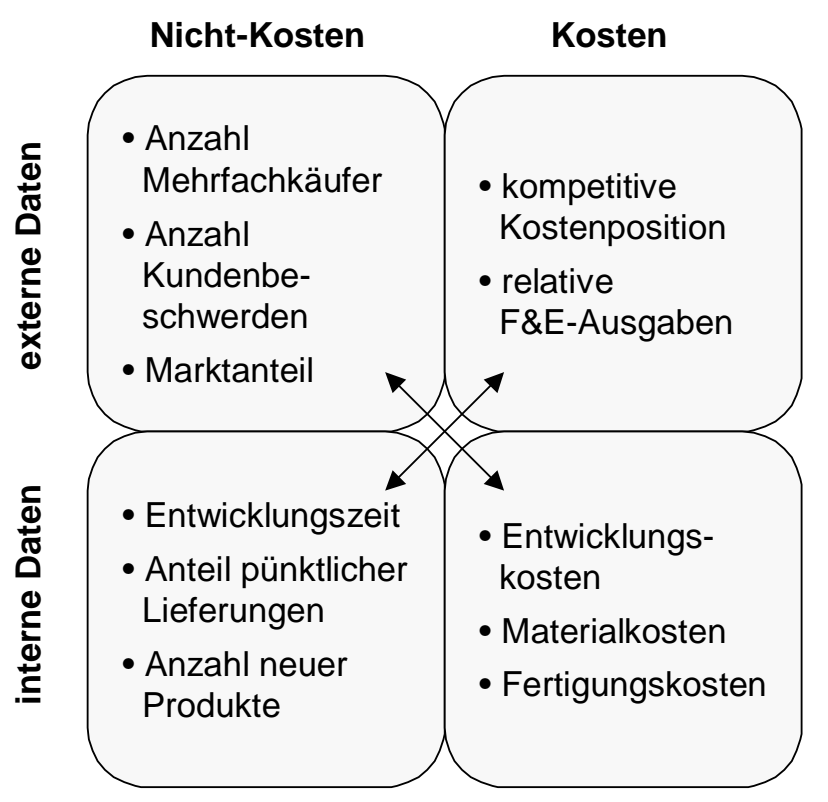

Abbildung 3-2: Ausgewogene Dimensionen nach Keegan et al. (1989)

In den Jahren 1987-89 untersuchten Wissenschaftler unter Fitzgerald et al. (1991) die Ausgestaltung des Performance Measurement in serviceorientierten Dienstleistungsunternehmen in England. Anhand verschiedener Klassifikationskriterien wurden Dienstleistungsunternehmen in Gruppen eingeteilt. Als primäres Differenzierungsmerkmal wurde die Anzahl der Kundenkontakte herangezogen, um drei Cluster zu definieren.

Neben finanziellen werden auch service-typische Kriterien (Service quality, Flexibility, Comfort, etc.) als Messgrössen vorgeschlagen. Diese Ergebnisse decken sich mit einer Studie (vgl. Küng und Wettstein (1999)) die vom Verfasser bei der Firma Hoffmann La Roche im Jahre 1999 unter der Leitung von Ambros Lüthi und Peter Küng durchgeführt wurde. ${ }^{3}$ Fitzgerald et al. (1991) schlagen einen Rahmen für die Leistungsmessung vor, in dem zwischen Treiber (Determinants) und Ergebnissen (Results) unterschieden wird.

3 Dabei wurde die Service-Qualität des Konzernrechnungswesens bestimmt. Die erstellten Produkte sind typische Service-Produkte. Mittels einer Analyse der Zielsetzungen der Organisationseinheit und semistrukturierten Interviews mit Mitarbeitern und Kunden der Abteilung wurden relevante Items bestimmt. 


\begin{tabular}{|c|c|c|}
\hline & Dimensions of performance & Types of measures \\
\hline \multirow{7}{*}{$\begin{array}{l}\frac{n}{5} \\
\frac{5}{5} \\
\stackrel{y}{x}\end{array}$} & \multirow{3}{*}{ Competitiveness } & $\begin{array}{l}\text { Relative market share } \\
\& \text { position }\end{array}$ \\
\hline & & Sales growth \\
\hline & & $\begin{array}{c}\text { Measures of the } \\
\text { customer base }\end{array}$ \\
\hline & \multirow{4}{*}{ Financial } & Profitability \\
\hline & & Liquidity \\
\hline & & Capital structure \\
\hline & & Market ratios \\
\hline \multirow{19}{*}{ 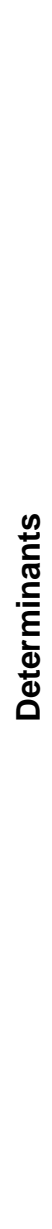 } & \multirow{12}{*}{ Service quality } & Reliability \\
\hline & & Responsiveness \\
\hline & & Esthetics/appearance \\
\hline & & Cleanliness/tidiness \\
\hline & & Comfort \\
\hline & & Friendliness \\
\hline & & Communication \\
\hline & & Courtesy \\
\hline & & Competence \\
\hline & & Access \\
\hline & & Availability \\
\hline & & Security \\
\hline & \multirow{3}{*}{ Flexibility } & Volume flexibility \\
\hline & & Delivery speed flexibility \\
\hline & & Specification flexibility \\
\hline & \multirow{2}{*}{ Resource utilisation } & Productivity \\
\hline & & Efficiency \\
\hline & \multirow{2}{*}{ Innovation } & $\begin{array}{l}\text { Performance of the } \\
\text { innovation process }\end{array}$ \\
\hline & & $\begin{array}{c}\text { Performance of } \\
\text { individual innovations }\end{array}$ \\
\hline
\end{tabular}

Abbildung 3-3: Results-Determinants-Matrix von Fitzgerald et al. (1991)

Abbildung 3-3 zeigt die vorgeschlagenen Indikatoren und deren Gliederung. Die Treiber werden von den Autoren in vier Gruppen (Dimensions of performance) klassifiziert: die Service-Qualität, die Flexibilität, die Auslastung und die Innovationskraft. Für die einzelnen Gruppen werden Indikatoren zur Operationalisierung vorgeschlagen. Als Ergebnisse (results) resultieren zwei Gruppen: die Wettbewerbsfähigkeit und die finanziellen Ergebnisse. Für diese Gruppen werden ebenfalls Indikatoren vorgeschlagen. 
Dies wird um eine Heuristik zur Bestimmung der Soll-Werte und um ein Belohnungssystem bei Zielerreichung ergänzt.

Finanzielle Daten können, im Gegensatz zu nicht-finanziellen Daten, meist ohne grössere Probleme zum gleichen Zeitpunkt bzw. im selben Zeitraum erhoben werden (Rechnungsperiode). Die Daten sind relativ einfach in einen logischen Zusammenhang zu bringen und unterliegen den gleichen Grössen (abgesehen von Währungskonvertierungen). Dadurch werden sie aggregierbar und können in den bekannten Kennzahlensystemen dargestellt werden. Siegwart (1998) definiert ein Kennzahlensystem als logische und/oder rechnerische Verknüpfung mehrerer Kennzahlen, die zueinander in einem Abhängigkeitsverhältnis stehen. Es handelt sich um eine aufbauende, zweckorientierte Gliederung einzelner Kennzahlen.

Der logische Zusammenhang zwischen finanziellen und nicht-finanziellen Indikatoren kann in der Regel nicht mehr schlüssig nachgewiesen werden. Fitzgerald et al. (1991) unterstellen eine Ursache-Wirkungsbeziehung zwischen finanziellen und nicht-finanziellen Indikatoren. Sie schreiben: „...(the investigation) highlights the fact that the results obtained are a function of past business performance with regard to specific determinants - i.e. results are lagging indicators, whereas determinants are leading indicators.”

Die vorauslaufenden Indikatoren (leading indicators) beeinflussen somit in einem unbekannten Ausmass und mit einer unbekannten Zeitverzögerung (time lag) die nachlaufenden Indikatoren (lagging indicators). Diese einleuchtende Annahme wird von sämtlichen neueren Performance-Measurement-Ansätzen aufgegriffen und bspw. auch auf den Prozessgedanken übertragen. 


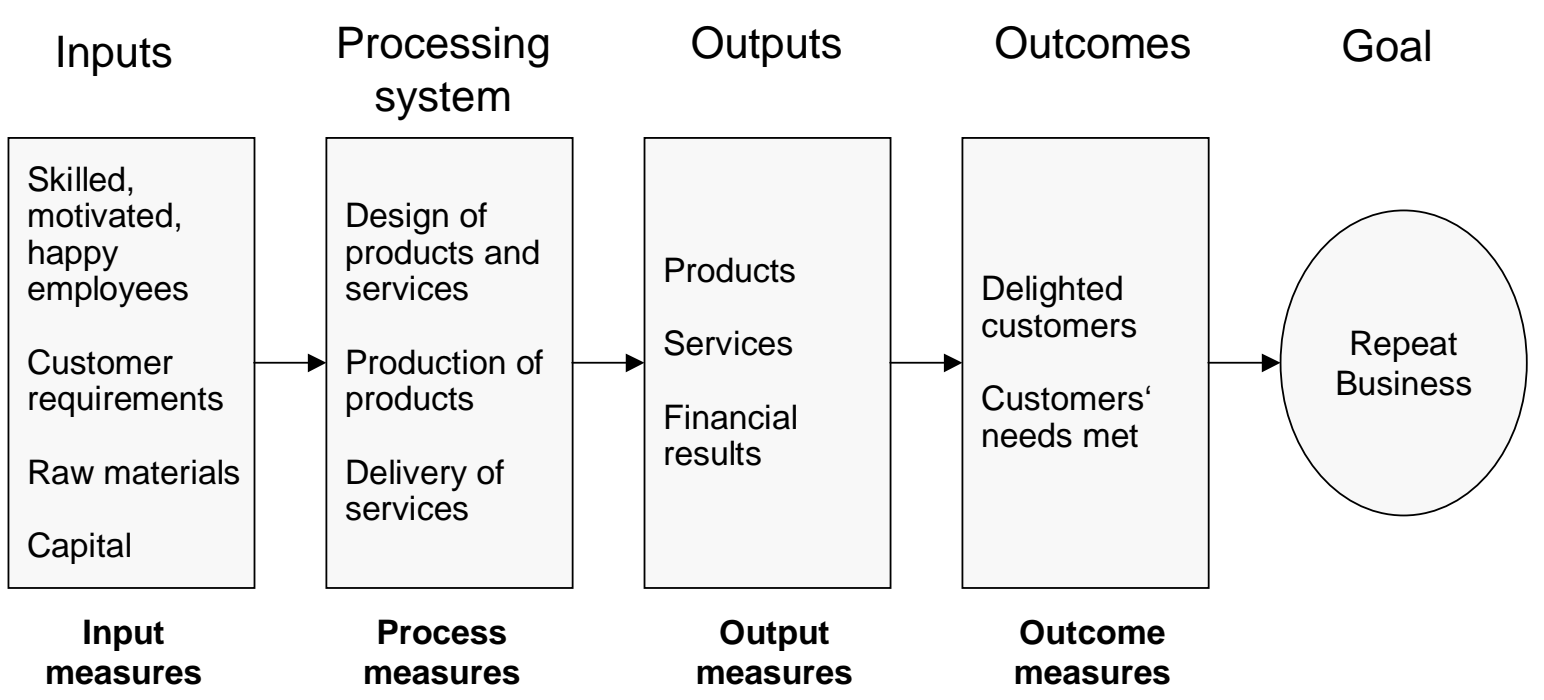

Abbildung 3-4: Kausalkette in einem Prozess (vgl. Brown (1996), zitiert nach Neely (2000))

Abbildung 3-4 zeigt eine mögliche Kausalkette in einem Prozess: Input-Grössen (z.B. Rohmaterial) beeinflussen die Produktefertigung, und diese wiederum beeinflusst den Output (z.B. Produktqualität). Der Output seinerseits bestimmt den Outcome (das wirtschaftlich relevante Ergebnis), z.B. die Kundenzufriedenheit oder die Zufriedenheit der Investoren.

Finanzielle Berichtssysteme, wie bspw. das DuPont-Schema, verfügen über verschiedene Aggregationsebenen, die in der Regel in einer Spitzenkennzahl, z.B. dem ROI, münden. Wenn die Daten im betrieblichen Rechnungswesen einen hohen Detaillierungsgrad aufweisen, so können für die unterschiedlichen organisatorischen Einheiten spezifische Daten bestimmt werden. Nicht-finanzielle Aspekte sind im Gegensatz dazu häufig schlecht aggregierbar. Zudem haben verschiedene Autoren erkannt, dass unterschiedliche Führungsebenen unterschiedliche Indikatoren - und nicht nur die gleichen Indikatoren auf einer unterschiedlichen Aggregationsstufe - benötigen. So ist es üblich, dass auf der Leitungsebene mehrheitlich monetäre Grössen zur Anwendung kommen, während auf der Ebene der Teams und Mitarbeiter mehrheitlich nicht-monetäre Grössen betrachtet werden. Ein weiterer Punkt betrifft die Interpretierbarkeit der Grössen. Monetäre Berichtsgrössen gerade aus der Kostenrechnung - setzen fundiertes Wissen voraus, welches an der Basis des Öfteren nicht gegeben ist. Ein wichtiger Beitrag zu dieser Problematik leisteten Lynch und Cross (1991b). Ihre Performance-Pyramide (vgl. Abbildung 3-5) veranschaulicht den diskutierten Sachverhalt. 


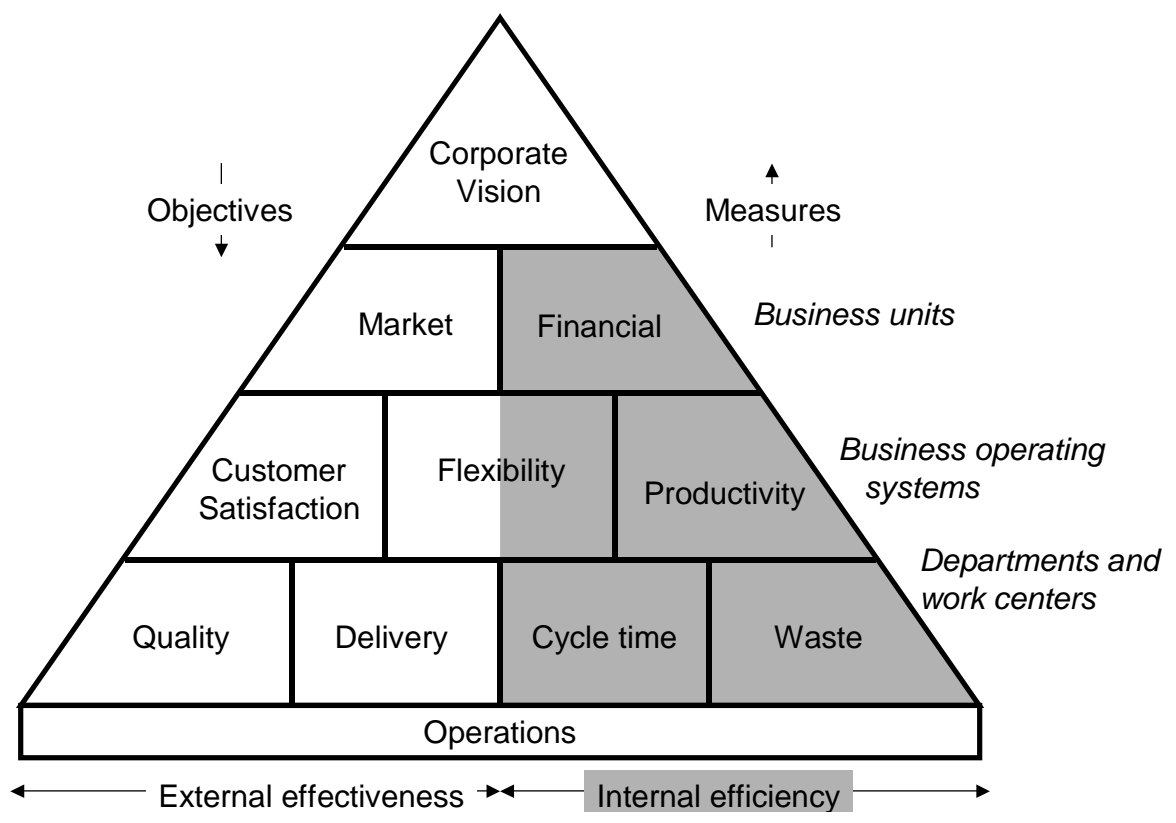

Abbildung 3-5: Performance-Pyramide (Lynch und Cross (1991))

Gemäss Lynch und Cross wird zwischen externen Informationen (linke Seite) und internen Informationen (rechte Seite) unterschieden. An der Unternehmensspitze interessiert primär die Strategieumsetzung. Auf der Ebene der Business Units stehen finanzielle und MarktAspekte im Vordergrund. Innerhalb der Business Units interessieren Themen wie Kundenzufriedenheit, Flexibilität, Innovation und Produktivität. Auf der untersten Ebene finden sich schliesslich extern orientierte Elemente wie Qualität und Einhaltung des Liefertermins sowie intern orientierte Faktoren wie Durchlaufzeit und Ausschussquote. Die Autoren propagieren ein Top-Down-orientiertes Vorgehen beim Entwickeln der Kennzahlen.

\subsubsection{Unterstützung der Ansätze durch Informationstechnologie}

Zur Unterstützung der verschiedenen vorgestellten Ansätze wird vielerorts Informationstechnologie eingesetzt. Es ist selbstverständlich, dass die älteren Vorschläge in ihren ursprünglichen Publikationen noch keinen Einsatz von Informationstechnologie vorsahen. Gerade im Finanzbereich sind IT-gestützte Systeme heute Standard. Analysen, wie das DuPont-Schema, werden in aktuellen Finanzsoftwarepaketen standardmässig angeboten (vgl. z.B. die ERP-Lösungen R/3 von SAP (2002), Baan (2002), JDEdwards (2002), PeopleSoft (2002), etc.). Weiterführende Analysen werden häufig mittels individueller Bürosoftware (z.B. einer Tabellenkalkulation wie Microsoft Excel) 
durchgeführt. Ähnliches gilt für die Produktion, die jedoch eher spezialisierte Anwendungen nutzt (z.B. SAS).

Bei den ersten ganzheitlichen Ansätzen (vgl. Keegan et al. (1989), Fitzgerald et al. (1991), Lynch und Cross (1991b), Brown (1996)) handelt es sich eher um konzeptionelle Arbeiten. Die Umsetzung resp. Unterstützung durch Informationstechnologie steht nicht im Vordergrund der originären Publikationen. Einzelne Ansätze wurden jedoch in späteren Arbeiten aufgegriffen und mittels Informationstechnologie unterstützt. Exemplarisch sollen einige Beispiele für den Bereich der Prozesse genannt werden:

- Im Rahmen des Projektes PROMOSYS (Process Monitoring System) wurde die Konzeption eines Monitoring-Systems zur Evaluation der Qualität von Geschäftsprozessen bearbeitet (vgl. PROMOSYS (2002)). Das Projekt wurde am Departement für Informatik der Universität Fribourg von Peter Küng unter der Leitung von Ambros Lüthi in den Jahren 1996 - 1999 bearbeitet. Ergebnis war u.a. ein Prototyp eines Prozess-Monitoring-Systems für das Debitorenmanagement. Das Nachfolgeprojekt COPPA hat die Umsetzung auf Prozessebene konkretisiert (vgl. COPPA (2002)). Das Akronym steht für Computer-based Process Performance Measurement.

- Das Projekt ACHIEVE (Business Process Benchmarking based on ERP Systems) an der Unversität St. Gallen beschäftigte sich mit dem Aufbau eines ProzessBenchmarking-Systems, welches die Daten aus R/2 resp. R/3 der SAP (2002) von verschiedenen Unternehmen exportierte (vgl. Legner (1999)).

- Im Rahmen des Projektes CONGO der Universität Münster wurde ein Prototyp mit der Bezeichnung PISA (Process Information System with Access) zur Visualisierung von Workflow-Protokolldaten konzipiert (vgl. Muehlen und Rosemann (2000)). Eine Weiterentwicklung stellt der Process Warehouse Approach von List et al. (2000) dar.

- Ein kommerzielles Produkt auf der Prozess-Ebene ist bspw. der Process Performance Manager von IDS-Scheer (vgl. Scheer (2000a) und Scheer (2000b)). 


\subsection{Vier aktuelle Ansätze des Performance Measurement}

\subsubsection{Der EFQM-Ansatz als Instrument für das Self-Assessment}

\subsubsection{Grundkonzept des Ansatzes}

Die European Foundation for Quality Management (EFQM) versteht unter TQM die Führungsmethode einer Organisation, welche auf der Mitwirkung der Mitglieder beruht und die Qualität in den Mittelpunkt stellt. Durch die Zufriedenheit der Kunden zielt sie auf langfristigen Geschäftserfolg, auf den Nutzen für die Organisationsmitglieder und für die Gesellschaft (vgl. EFQM (2002)) (vgl. Kapitel 2.5.3.). Seit der Erkenntnis, dass der Hauptunterschied von japanischen zu europäischen und amerikanischen Unternehmen damit erklärt werden kann, dass weite Teile der japanischen Führungskräfte in ihrem Handeln eine qualitätsorientierte ganzheitliche Betrachtungsweise präferieren und damit sehr kompetitiv sind, haben die Ideen des TQM eine ungebrochene Aktualität. Mit diesen Ergebnissen wurde die bisher vielfach vertretene Ansicht, wonach japanische Unternehmen „auf Grund ihres vermeintlichen Lohngefälles und hohen Arbeitseinsatzes ihrer Mitarbeiter im internationalen Vergleich zu immer deutlicheren wirtschaftlichen Spitzenwerten gelangen“ (Malorny (1996)), widerlegt. Im Rahmen des Total Quality Management findet ein derartiges Führungsverständnis seit Anfang der Fünfzigerjahre in Japan statt. (vgl. Malorny (1996))

Seit dieser Erkenntnis bemühen sich sowohl amerikanische als auch europäische Organisationen um entsprechende Qualitätssysteme. Ein vielbeschrittener Weg und erster Schritt eines ganzheitlichen TQM ist die Zertifizierung nach DIN EN ISO 9000 ff.. Bis Ende 2000 sind weltweit über 400'000 Zertifikate in insgesamt 158 Ländern ausgegeben worden (vgl. ISO (2001)). Die Überprüfung der Erfordernisse geschieht im Rahmen eines externen Audits, welches periodisch wiederholt wird (i.d.R. alle zwei Jahre). Damit zeigt ein Unternehmen auf, dass es zum Zeitpunkt des Audits die entsprechenden Anforderungen an den Standard erfüllt.

Die Normenreihe ISO 9000 ff. beschränkt sich damit auf die Dokumentation und beurteilt nicht Spitzenleistungen.

Unterschiedlich dazu verhält sich der Deming Application Prize (vgl. Deming (2002)), der von der Union of Japanese Scientists and Engineers seit 1951 vergeben wird und an den 
wesentlich höhere Anforderungen gestellt werden. Der hohe Prestigewert vermag die Unternehmen zudem zu Spitzenleistungen im Qualitätsmanagement zu motivieren. Seit 1987 wird eine imitierte Version des Deming Prizes auch in Amerika vergeben. Der Malcom Baldrige National Quality Award (vgl. Baldridge (2001)) wird jeweils jährlich prominent vom amerikanischen Präsidenten an diejenige Unternehmung vergeben, die nachweislich ein mustergültiges QM-System implementiert und betrieben hat. Beide Ansätze können als umfassende Qualitätsmanagementsysteme verstanden werden. In Abgrenzung zur ISO 9000ff. Normenreihe erlauben die beiden Systeme ein Lernen, indem die Gewinner ihr „Erfolgsrezept“ veröffentlichen müssen.

Die europäische Variante des Qualitätspreises wird von der European Foundation for Quality Management (EFQM) vergeben. Diese wurde 1988 von 14 europäischen Firmen unter der Schirmherrschaft der Europäischen Kommission gegründet. Das Ziel der EFQM besteht darin, europäische Unternehmen zur integralen Verbesserung anzuspornen. Damit sind Aktionen gemeint, welche die langfristige Konkurrenzfähigkeit der Unternehmen sicherstellen. Dazu gehören Massnahmen zur Steigerung der Kunden- und Mitarbeiterzufriedenheit sowie Verbesserungen bezüglich der Auswirkungen auf die Gesellschaft. Steigerungen hinsichtlich der finanziellen Resultate gehören ebenfalls dazu, haben jedoch nicht erste Priorität.

Das EFQM-Modell (siehe Abbildung 3-6) ist ein offenes, für jedermann zugängliches Rahmenwerk. Es trägt dem Fakt Rechnung, dass es viele Wege gibt, ein Unternehmen erfolgreich zu führen. Das Modell besteht aus neun Basis-Elementen, die ein Unternehmen aktiv bewirtschaften soll. Dabei wird unterschieden zwischen so genannten „Befähigern“ (enablers) und „Ergebnissen“ (results), wobei die Befähiger die Erfolgsvoraussetzung für die Ergebnisse bilden.

Die EFQM veranstaltet jedes Jahr einen Wettbewerb, um besonders erfolgreiche Unternehmen auszuzeichnen. Dabei bewerten sich die Unternehmen in einem ersten Schritt anhand eines Leitfadens selbst (self-assessment). Beurteilt wird der Reifegrad (maturity level) von neun Bereichen (siehe Abbildung 3-6). Im Bereich Kundenzufriedenheit (Customer Satisfaction) wird bspw. beurteilt, ob diese gemessen wird, wie die erhobenen Daten verwendet werden, wie mit Kundenreklamationen verfahren wird, wie sich die Kundenzufriedenheit gegenüber der letzten Periode verbessert hat, etc.. In einem zweiten Schritt werden die Finalisten von einer Jury besucht und 
bewertet. Aus den Selbst- und Fremdbewertungen werden die Sieger der einzelnen Kategorien (grosse Unternehmen, öffentlicher Sektor, KMUs als Tochterfirma eines Konzerns und unabhängige KMUs) ermittelt.

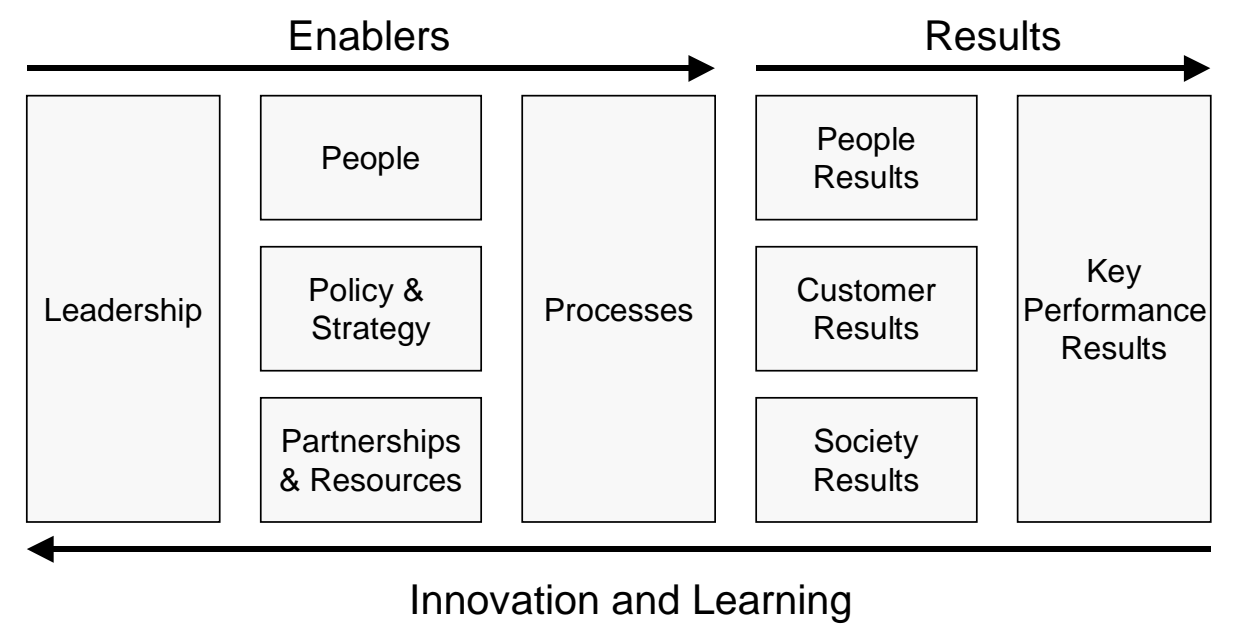

Abbildung 3-6: Das EFQM-Modell (vgl. EFQM (2002))

Diesen Qualitätsmodellen gemeinsam sind eine Reihe von Eigenschaften (vgl. Hakes (1996)): (1) Kundenorientierung, (2) kontinuierliche Verbesserung durch Lernen, (3) Mitarbeiterbeteiligung und -entwicklung, (4) Prozessmanagement, (5) Management by Facts, (6) sichtbarer Leader, (7) Entwicklung von Partnerschaften, (8) langer Planungshorizont, (9) unternehmerische Verantwortung und Beachtung der Auswirkungen auf die Umwelt und (10) Cycle-time Management.

Der zweite Punkt - das kontinuierliche Verbessern durch Lernen - soll an dieser Stelle näher dargestellt werden, da es sich um ein zentrales Anliegen des Performance Measurement handelt. Das Erreichen eines hohen Leistungsniveaus bedingt gut geplante, ausgeführte und kontrollierte Aktivitäten in einer Organisation, die kontinuierlich auf Verbesserungsmöglichkeiten hin untersucht werden. Veranschaulicht werden kann dieser Sachverhalt anhand des so genannten Deming-Rades (Deming Cycle), das in Abbildung 3-7 dargestellt ist. Es besteht aus folgenden Phasen: (1) Plan: Entwerfe einen Plan für das, was erreicht werden soll. Der Plan basiert auf dem gegenwärtigen System. (2) Do: Führe den Plan aus. (3) Check: Messe das Ergebnis und überprüfe, ob es dem erwarteten Resultat 46 
entspricht. (4) Act: Überdenke das Ergebnis, überlege, was sich verändert hat und entscheide, was zu tun ist.

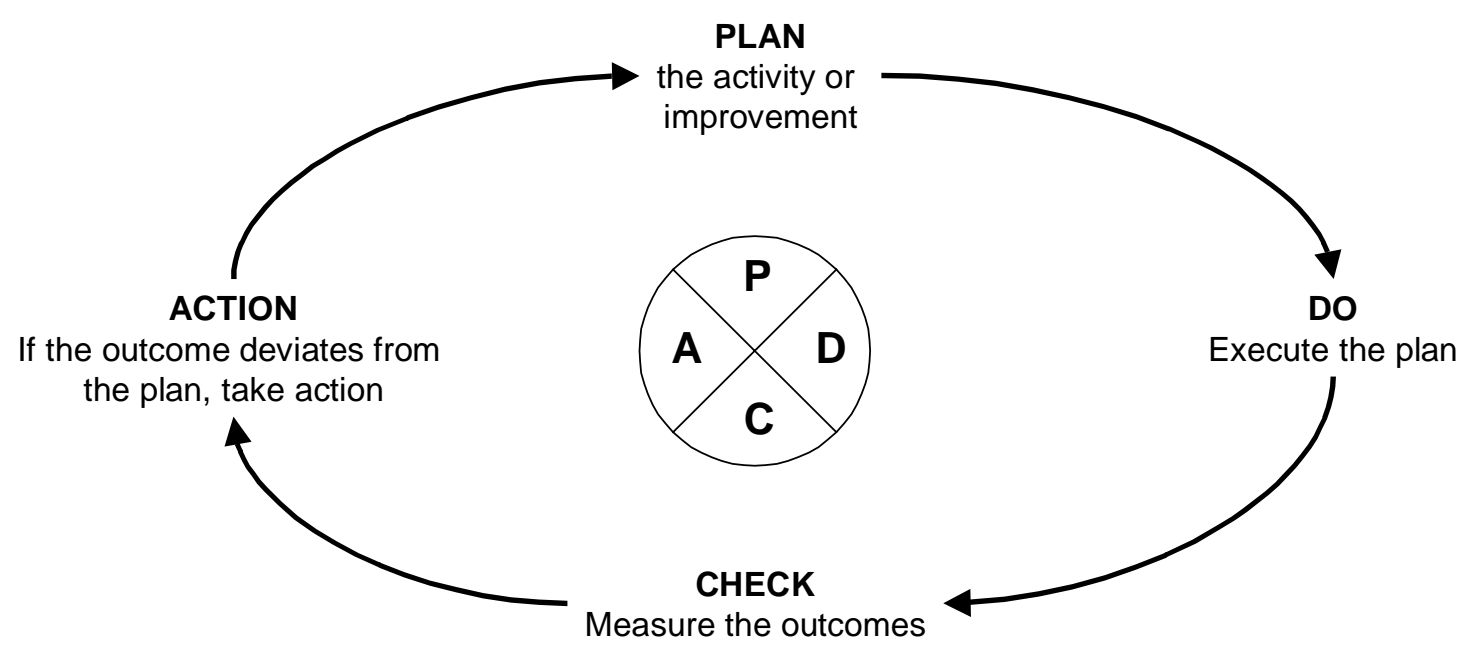

Abbildung 3-7: Kontinuierliche Verbesserung (in Anlehnung an Hakes (1996))

Das iterative Durchlaufen ermöglicht eine langfristige Verbesserung des Systems.

Der EFQM-Ansatz geht sogar noch einen Schritt weiter, indem er den Reifegrad (zur Reifegraddiskussion vgl. Gibson und Nolan (1974), Paulk et al. (1993), Paulk et al. (1994), SEI (2002), Wettstein und Küng (2002)) einer Organisation bestimmt und aufzeigt, wie dieser verbessert werden kann. Die nachfolgende Abbildung 3-8 zeigt einen Ausschnitt der Dimension „Prozesse“ der Unternehmens-Verbesserungsmatrix. Ein Unternehmen bestimmt seinen aktuellen Reifegrad (z.B. Schritt zwei) und analysiert, wie es den Schritt drei erreichen kann. Dies geschieht offensichtlich dadurch, dass die Organisation auf Grund von zu überwachenden Indikatoren überprüft, wie wirksam der Prozess die Gesamtzielsetzung der Organisation unterstützt. 


\begin{tabular}{|c|c|}
\hline Schritt & Prozesse \\
\hline 10 & $\begin{array}{l}\text { Die Schlüsselprozesse werden verstanden, formell geführt und } \\
\text { kontinuierlich verbessert. }\end{array}$ \\
\hline 9 & $\begin{array}{l}\text { Die Existenz eines formellen Qualitätsmanagementsystems lässt } \\
\text { sich nachweisen. }\end{array}$ \\
\hline 8 & $\begin{array}{l}\text { Die Leistungen der Prozesse stehen nachweislich in Bezug zu } \\
\text { den Kundenanforderungen. }\end{array}$ \\
\hline 7 & $\begin{array}{l}\text { Es gibt einen Mechanismus zur Entwicklung und Verwendung } \\
\text { entsprechender Messgrössen zur Bewertung von } \\
\text { Schlüsselprozessen. }\end{array}$ \\
\hline 6 & $\begin{array}{l}\text { Die Prozessergebnisse werden überprüft und fliessen in den } \\
\text { Verbesserungszyklus ein. }\end{array}$ \\
\hline 5 & $\begin{array}{l}\text { Ein Verbesserungsmechanismus für Schlüsselprozesse ist } \\
\text { eingeführt. }\end{array}$ \\
\hline 4 & $\begin{array}{l}\text { Ein Verbesserungsmechanismus ist identifiziert worden; Ziele für } \\
\text { Verbesserungen sind gesetzt. }\end{array}$ \\
\hline 3 & Die Wirksamkeit bestehender Schlüsselprozesse ist beurteilt. \\
\hline 2 & $\begin{array}{l}\text { Schlüsselprozesse sind identifiziert, als Flussdiagramm } \\
\text { dargestellt und/oder dokumentiert. Die Zuständigkeit ist } \\
\text { bestimmt. }\end{array}$ \\
\hline 1 & Die Hauptprozesse innerhalb der Einheit sind identifiziert. \\
\hline
\end{tabular}

Abbildung 3-8: Auszug aus der Unternehmensverbesserungsmatrix der EFQM (1996)

Entsprechende Reifegradeinschätzungen sind für sämtliche Bereiche des EFQM gegeben (vgl. EFQM (1999)).

\subsubsection{Charakterisierung des EFQM-Ansatzes auf Grund von Sekundärliteratur}

Viele Unternehmen verwenden das EFQM-Modell nur in langen Abständen zur Selbstdiagnose. Erst bei einer regelmässigen Verwendung und bei einem in den Planungsund Kontrollprozess des Unternehmens integrierten Instrument kann dagegen von einem PMS gesprochen werden (vgl. Blankenburg (1999)).

Das Verfahren zur Erhebung des „Qualitätsniveaus“ basiert auf einer Selbstbewertung. Steht die Identifikation von Verbesserungspotenzialen im Vordergrund, so genügt das Verfahren. Wird jedoch ein Vergleich angestrebt, so ist dieses verbesserungswürdig, da die Subjektivität der Assessoren einen zu grossen Spielraum enthält (vgl. Seghezzi (1997)).

Das EFQM-Modell umfasst eine Benchmarking-Komponente (vgl. Kapitel 2.5.5) indem die Gewinner verpflichtet werden, „good practice guides“ zu veröffentlichen. Seit 1996 
unterstützt EFQM eine Reihe von zusätzlichen Initiativen im Bereich Benchmarking. So wurden eine Reihe von Forschungsprojekten zu spezifischen Aspekten durchgeführt. EFQM organisiert auch so genannte „Benchmarking Groups“. Unter diesem Label verpflichten sich 15 - 20 Unternehmen einen bestimmten Prozess während einer bestimmten Dauer zu analysieren (regelmässige Erhebung von Messdaten). Diese werden im Rahmen der Gruppe diskutiert, um Verbesserungsmöglichkeiten zu entdecken. EFQM unterhält zudem eine „Benchmarking Good Practises Database“, die es Mitgliedern erlaubt, Informationen über spezifische Aspekte zu erhalten. (vgl. EFQM (2001))

Das EFQM in der Grundkonzeption unterstützt die Planung kaum und wird häufig nur für eine Ex-Post-Kontrolle eingesetzt (vgl. Blankenburg (1999)). Keine klaren Aussagen macht das EFQM ebenfalls zu Punkten wie Kommunikation der Strategie und die Verknüpfung von Zielen mit Anreizen.

Wichtigstes Endziel ist ein ganzes Bündel von Zielen: jene der Aktionäre, der Gesellschaft, der Umwelt, der Mitarbeiter und der Kunden. Dabei können konkurrenzierende Ziele bei der Priorisierung auftreten.

\subsubsection{Weiterentwicklungen und Varianten des Ansatzes}

Die meisten europäischen Länder vergeben zwischenzeitlich zusätzlich nationale Qualitätspreise, die sich ebenfalls am EFQM-Modell orientieren. In der Schweiz wird diese Auszeichnung durch die ESPRIX (2002) vergeben. In Deutschland gibt es den Ludwig Erhard Preis (vgl. Erhard (2002); Erhard war Wirtschaftsminister im 1. Kabinett Adenauers von 1949. Er spielte als Wirtschaftsminister während fast dreissig Jahren eine wichtige Rolle). In Österreich kümmert sich die AFQM (Austrian Foundation for Quality Management; AFQM (2002)) um diese Aufgabe.

Blankenburg (1999) untersucht in seiner Arbeit drei EFQM-Finalisten. Es handelt sich um die Firmen Xerox, British Telecom und Algroup Lonza. Die Untersuchung zeigt, dass in der Praxis jedes Unternehmen das Basis-EFQM-Modell entsprechend seiner Vorgeschichte und seinen Bedürfnissen anpasst. Ein identischer Eindruck entstand durch die vom Verfasser analysierten Dokumente der Schweizer Award-Winner und Preisträger (vgl. ESPRIX (2002)). Jedes Unternehmen setzt individuelle Schwerpunkte. Aus diesen Überlegungen erscheint es nicht sinnvoll, allgemeine Entwicklungen aufzuzeigen. 


\subsubsection{Berücksichtigung der Informationstechnologie im EFQM-Ansatz}

Die Informationstechnologie wird indirekt im Kriterium „Partnerschaften und Ressourcen“ (vgl. das entsprechende Basiselement unter den Enablers in Abbildung 3-6) mit den Aspekten „Technologie wird gemanagt“ und „Informationen und Wissen werden gemanagt“ berücksichtigt (vgl. EFQM (1996)). Inwiefern Informationstechnologie oder -systeme den EFQM-Ansatz unterstützen können, ist in der Basisliteratur nicht behandelt.

Auf der Seite der Softwarehersteller finden sich eine Reihe von Softwarepaketen für das Erfassen der Bewertungsbogen und zur Verwaltung (Erfassung, Freigabe, Publikation) der qualitätsrelevanten Dokumente, sowie Programme zur Präsentation und Kommunikation der erreichten Resultate. Nachfolgend sind die Produkte von zwei Herstellern aufgeführt:

- Intraware AG offeriert ein so genanntes „wissensaktives, prozessorientiertes Dokumentenmanagement“. Das Dokumentenmanagement-Modul unterstützt die Erstellung, Pflege und Verwaltung von gelenkten Dokumenten und Handbüchern. Prozesszugehörigkeiten, Wiedervorlagetermine und Gültigkeits-Zeiträume sollen zu einer hohen Aktualität der veröffentlichten Dokumente führen. Das Modul erfüllt die Formalkriterien nach DIN EN ISO 9000:2000, QS 9000, VDA 6.1, FDA (Food and Drug Administration). (vgl. Intraware (2001))

- QPR Software AG: Die Software unterstützt Unternehmen darin, die EFQMMethodik anzuwenden, indem es deren Abbildung und Kommunikation der Ergebnisse erlaubt. Die Software ist weit gehend unabhängig vom verwendeten PMS-Konzept. (vgl. QPR-Software (2002)). Abbildung 3-9 zeigt einen Bildschirmausschnitt der QPR-Software zur Präsentation und Kommunikation der erreichten Resultate nach EFQM. In der Statuszeile ist die relevante zeitliche Periode (4. Quartal 2001) sichtbar. Im Fenster ist das EFQM-Modell als Baumstruktur dargestellt. In der ersten Ebene sind links drei Enablers (People, Partnership \& Resources, Processes) und rechts die Results dargestellt. Die einzelnen Elemente gliedern sich in Subkategorien. Unterhalb der Stichworte wird mit einem Balken der aktuelle Zielerreichungsgrad dargestellt. 


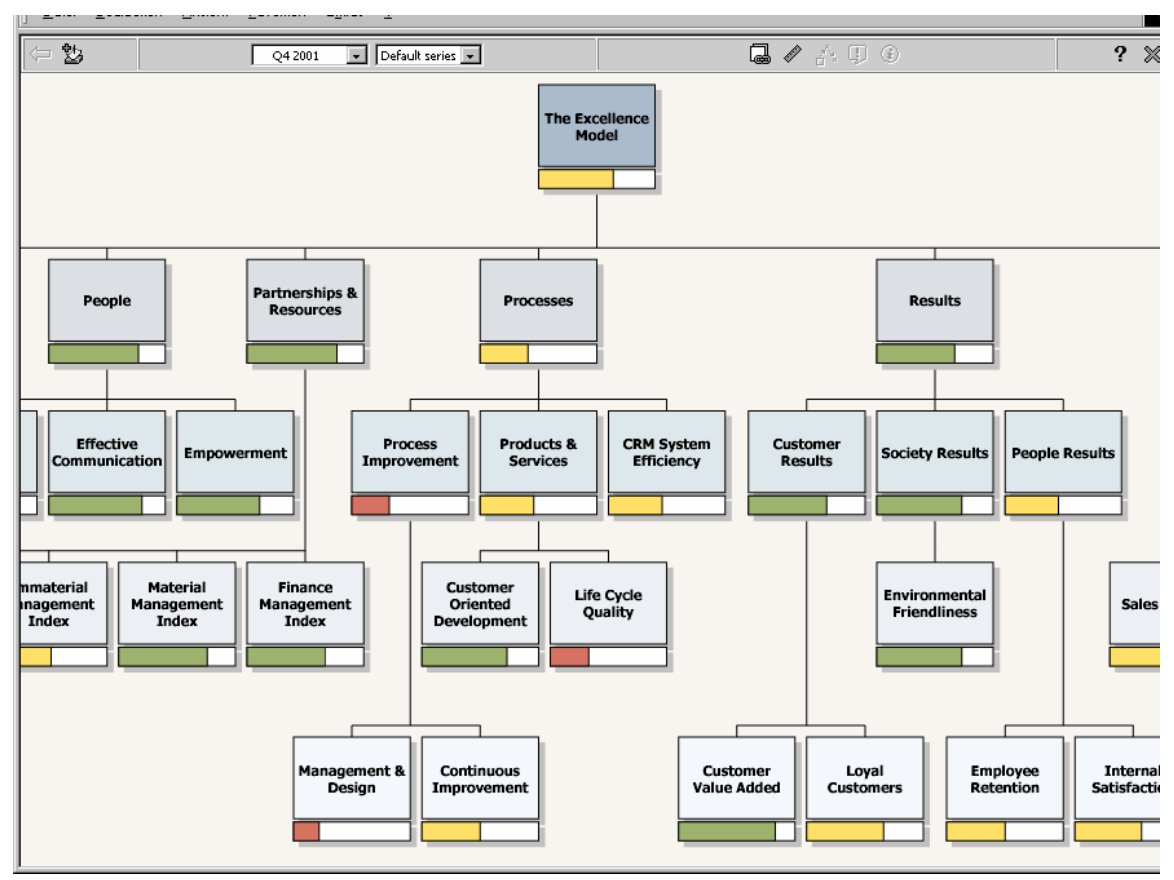

Abbildung 3-9: Beispiel einer Software zur Präsentation und Kommunikation der erreichten Resultate nach EFQM

\subsubsection{Balanced Scorecard}

\subsubsection{Grundkonzept des Ansatzes}

Balanced Scorecard ist ein Ansatz, der von Kaplan und Norton (1992) entwickelt wurde. $\mathrm{Zu}$ Grunde liegt ein Forschungsprojekt des Nolan Norton Institute (der Forschungszweig der Firma KPMG), bei dem Kaplan von der Harvard Business School mitwirkte. Untersucht wurde die Performance-Messung bei einer Reihe amerikanischer Grossunternehmen. Vorläufer des Ansatzes ist die mehrdimensionale PerformanceMessung bei der Firma Analog Devices (vgl. Kaplan und Norton (1997)). Der Ansatz wurde 1992/93 veröffentlicht (vgl. Kaplan und Norton (1992); Kaplan und Norton (1993); Kaplan und Norton (1996a); Kaplan und Norton (1996b)).

Die Autoren postulieren eine Strukturierung der verschiedenen Kennzahlen mit so genannten Ergebnistafeln (Scorecards). Vier Ergebnistafeln werden vorgeschlagen, um einen ausgewogenen (balanced) Einblick in die Leistungsfähigkeit des Unternehmens zu erhalten. Dabei sollen die einzelnen Tafeln Antworten auf grundlegende Fragen liefern. Als Analogie wird das Führen eines Unternehmens mit dem Steuern eines Flugzeuges verglichen: „Think of the balanced scorecard as the dials and indicators in an airplane 
cockpit. For the complex task of navigating and flying an airplane, pilots need detailed information about many aspects of the flight. They need information on fuel, air speed, altitude, bearing, destination, and other indicators that summarize the current and predicted environment. Reliance on one instrument can be fatal. Similarly, the complexity of managing an organization today requires that managers be able to view performance in several areas simultaneously" (Kaplan und Norton (1992)).

Abbildung 3-10 zeigt die vier Perspektiven (oder Dimensionen) und die zu stellenden Fragen.

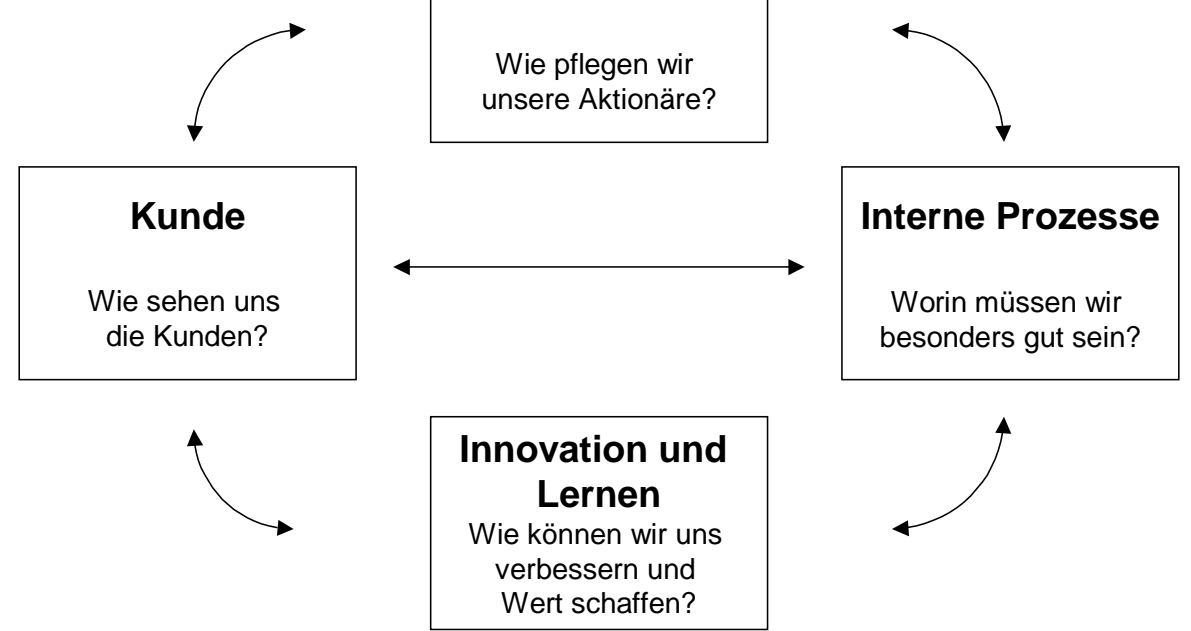

\begin{abstract}
Abbildung 3-10: Strukturierung der Balanced Scorecard (vereinfachte und übersetzte Darstellung in Anlehnung an Kaplan und Norton (1992))
\end{abstract}

Die einzelnen Fragen provozieren Ziele, die erreicht werden müssen, um die Vision zu erreichen. Diese Ziele können je Hierarchiestufe anders ausgestaltet sein, müssen aber aufeinander abgestimmt werden. Mittels Kennzahlen werden sie operationalisiert. Die Balanced Scorecard unterstellt eine Kausalkette zwischen den verschiedenen Perspektiven. Sie geht davon aus, dass die finanziellen Ziele erreicht werden, wenn die anderen Perspektiven ebenfalls erreicht werden. Anders formuliert: Die finanziellen Ziele (financial perspective) stehen in der Balanced Scorecard zuoberst. Die übrigen Perspektiven und Ziele müssen die notwendigen Beiträge leisten, um die finanziellen Ziele zu erreichen. 
In Abbildung 3-11 wird eine solche Kausalkette dargestellt. Die Kapitalrendite (ROCE; Return on Capital Employed) kann eine Messgrösse für die finanzielle Perspektive sein. Der Treiber dieser Messgrösse kann ein wiederholter und erweiterter Verkauf beim existierenden Kundenstamm sein, d.h. das Ergebnis einer grösseren Kundenzufriedenheit und -treue. Kundentreue wird in diesem Beispiel durch pünktliche Lieferung (on-time delivery) erreicht. Diese wird wiederum erreicht, wenn die Prozessqualität hoch und die Prozessdurchlaufzeit kurz ist. Dies kann unter anderem durch intensive Schulung der Mitarbeiter erreicht werden. Das Beispiel zeigt, wie sich Kaplan und Norton die UrsacheWirkungskette quer durch die vier Perspektiven vorstellen. (vgl. Kaplan und Norton (1997))

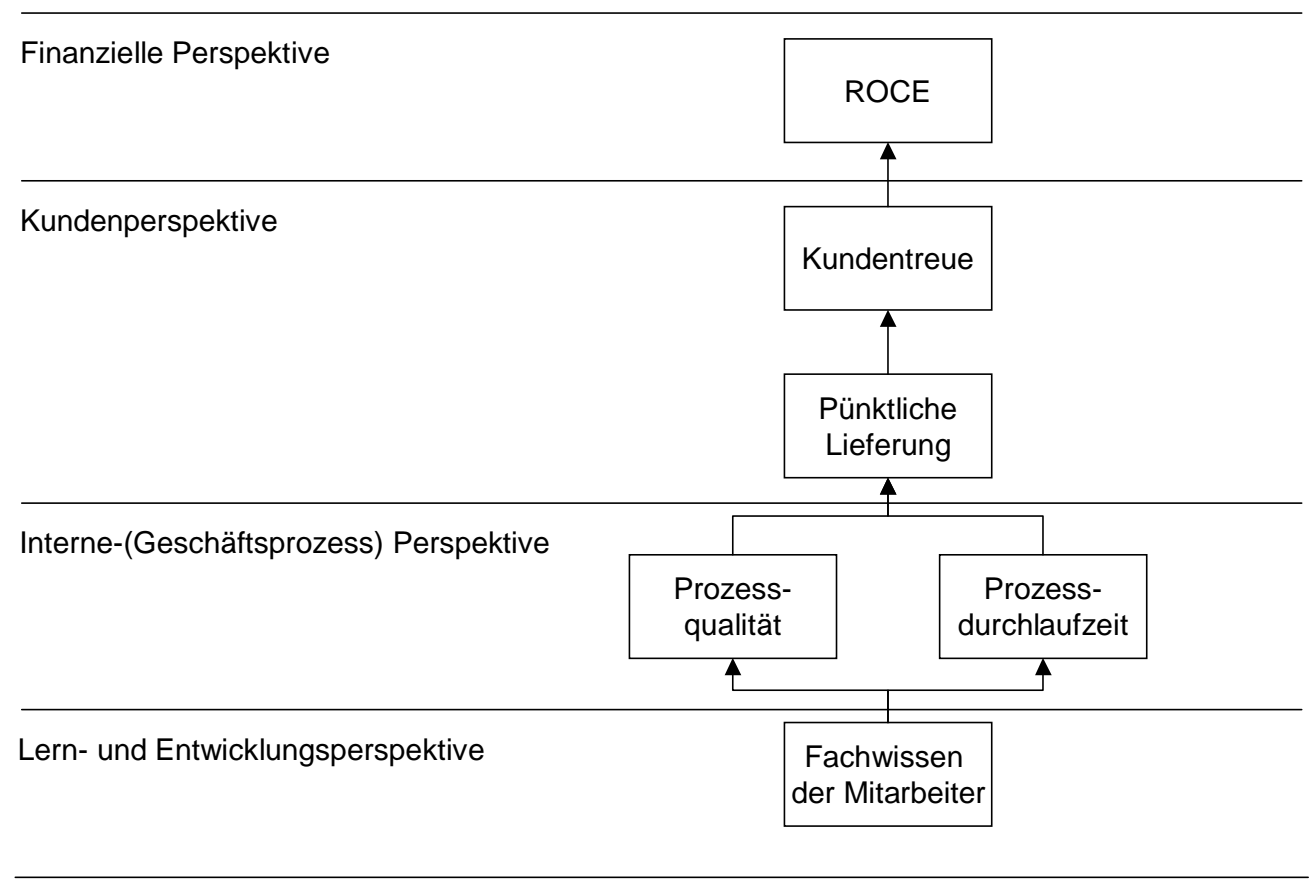

\section{Abbildung 3-11: Ursache-Wirkungskette in der Balanced Scorecard (Kaplan und Norton (1997))}

Eine gute Scorecard umfasst gemäss den Autoren etwa 15 bis 20 Indikatoren (vgl. Kaplan und Norton (1993)).

Für den Aufbau einer Balanced Scorecard werden folgende Schritte empfohlen (vgl. Kaplan und Norton (1993)): 
1. Auswahl der geeigneten Business Unit, die über eigene Kunden, Absatzkanäle, Produktionsstätten sowie finanzielle Kennzahlen verfügen.

2. Erste Interviewrunde: Nachdem die 6-12 höchsten Manager der Business Unit mit Grundlagenmaterial über die Balanced Scorecard, aber auch mit den internen Strategiedokumenten versehen worden sind, führt der Projektleiter Interviews mit diesen Managern durch und befragt sie nach ihrer Meinung bezüglich Zielen und Ideen für Performance-Indikatoren. Denkbar ist eine Erweiterung des Kreises um Shareholder und Kunden.

3. Erster Managementworkshop: Unter der Moderation des Projektleiters wird eine erste Version der Scorecard entwickelt.

4. Zweite Interviewrunde: Die Ergebnisse des ersten Managementworkshops werden mit den Mitgliedern einzeln diskutiert und ihre Meinung eingeholt.

5. Zweiter Managementworkshop: In einem grösseren Rahmen (unter Beizug des mittleren Managements) wird die Scorecard nochmals diskutiert. In Gruppenarbeiten versuchen die Teilnehmer, die erarbeiteten Ziele mit Indikatoren zu operationalisieren und sich Gedanken zur Umsetzung und den zu erreichenden Zielwerten zu machen.

6. Dritter Managementworkshop: Im kleineren Rahmen wird die Scorecard verabschiedet, die Zielwerte festgelegt und die notwendigen Massnahmen bestimmt.

7. Implementierung: Ein speziell dazu bestimmtes Team erarbeitet einen konkreten Umsetzungsplan, definiert die Datenquellen der Performance-Indikatoren, legt die Kommunikation fest und stellt sicher, dass die Scorecards auf der untergeordneten Führungsebene detailliert werden können.

8. Periodische Reviews: Quartals- oder wochenweise wird ein Bericht der Indikatoren der Balanced Scorecard für das Management erstellt. Jährlich werden im Rahmen der strategischen Planung, die Zielsetzungen der Balanced Scorecard überarbeitet.

Die Balanced Scorecard von 1992/93 wurde später erweitert und als Managementsystem ausgebaut: „recently, we have seen some companies move beyond our early vision for the scorecard to discover its value as the cornerstone of a new strategic management system “ (Kaplan und Norton (1996b)). Dabei wird dieser Ansatz als strategischer Handlungsrahmen verwendet, um kritische Managementprozesse zu meistern. Hierzu gehören: (a) Klären und Herunterbrechen von Vision und Strategie, (b) Kommunikation und Verknüpfung von strategischen Zielen und Massnahmen, (c) Planung und Festlegung 
von Zielen und Abstimmung strategischer Initiativen und (d) Verbesserung von strategischem Feed-back und Lernen (vgl. Kaplan und Norton (1996b) und Abbildung 3-12). Hierzu zeigen die Autoren einen detaillierten Einführungsplan auf (vgl. Abbildung 3-13).

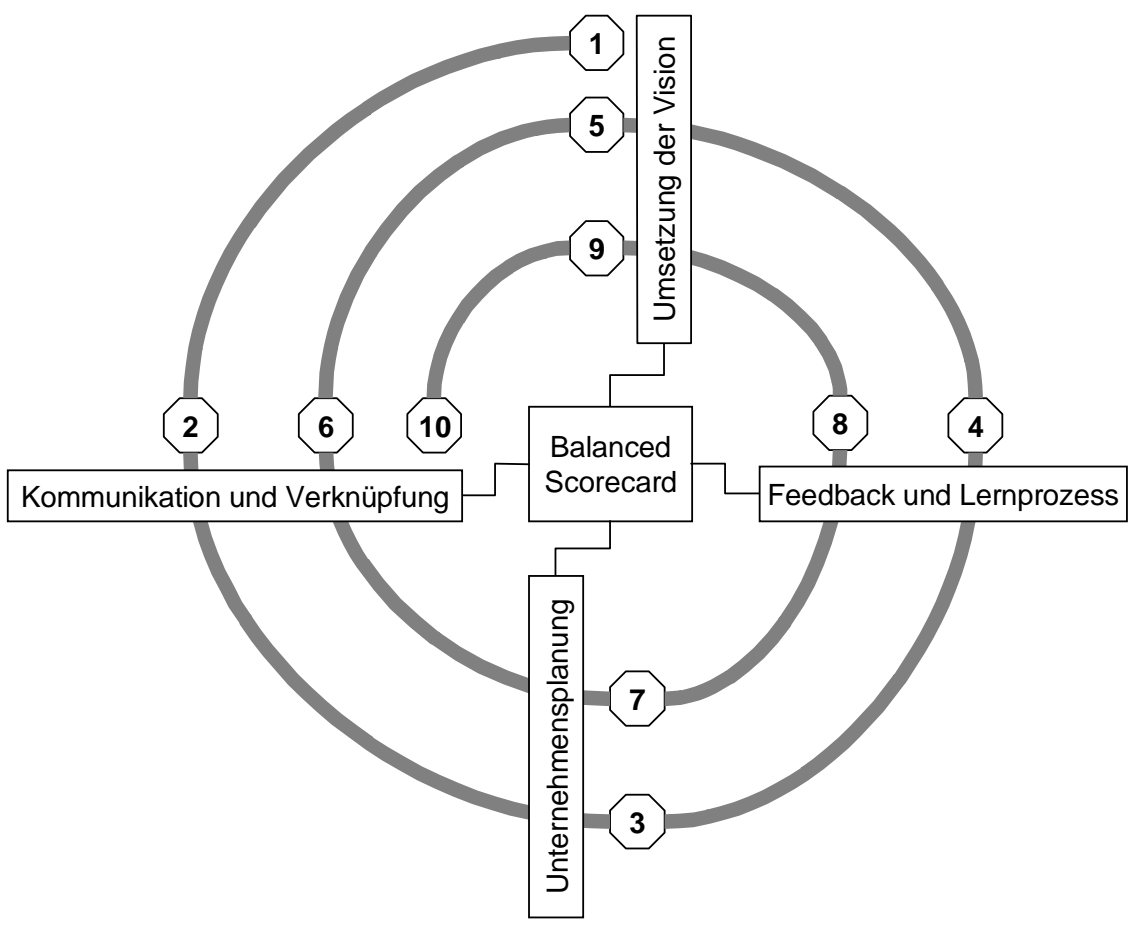

Abbildung 3-12: Management-Zyklus der Balanced Scorecard (in Anlehnung an Kaplan und Norton (1996b) und Kaplan und Norton (1997)) 


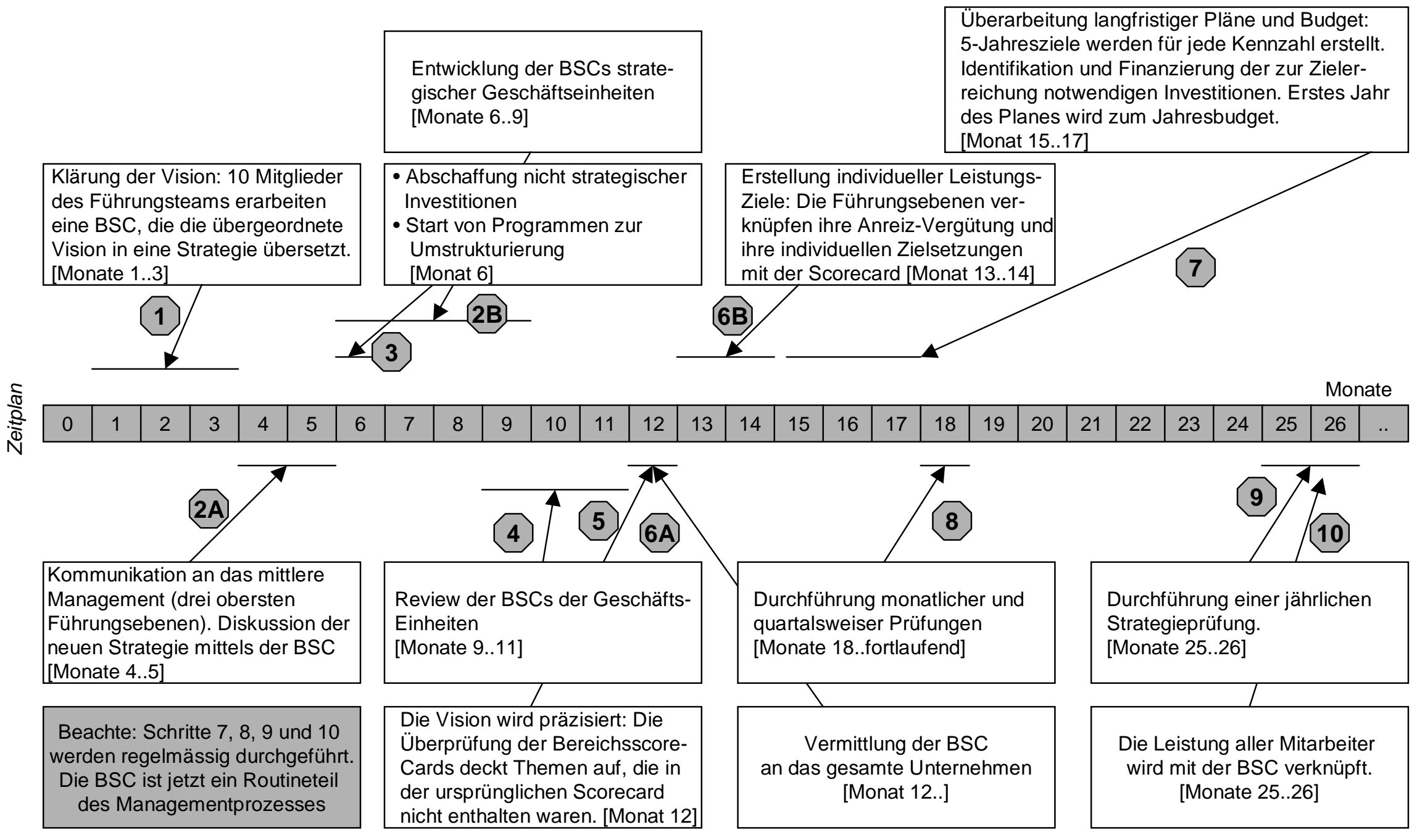

Abbildung 3-13: Einführungsvorgehen für eine Balanced Scorecard (vgl. Kaplan und Norton (1996b) und Kaplan und Norton (1997)) 


\subsubsection{Charakterisierung der Balanced Scorecard auf Grund von Sekundärliteratur}

Ein Kritikpunkt der Balanced Scorecard besteht darin, dass die Kausalketten ex ante (auf Grund von Vermutungen bezüglich des Zusammenhangs zwischen den Elementen) erstellt, jedoch in den wenigsten Fällen ex post verifiziert werden können. Dies scheint insbesondere deshalb fragwürdig, da unzählige Interdependenzen zwischen den Leistungsebenen und Zielen bestehen (vgl. Weber (1998)).

Die Balanced Scorecard fördert zwar das mehrdimensionale Performance-Verständnis indem vier Perspektiven berücksichtigt werden, doch bleibt anzumerken, dass sich die nicht-finanziellen Perspektiven unterordnen müssen. Anders ausgedrückt: Nicht-finanzielle Indikatoren werden nur insofern berücksichtigt als sie einen Beitrag zur Verbesserung der finanziellen Indikatoren leisten (vgl. Klingebiel (2000)).

Erstaunlich ist die Flut an Publikationen zum Konzept der Balanced Scorecard einerseits und die Wenigen zum praktischen Einsatz andererseits. Gemäss Gleich (2001) existieren demzufolge noch viele Unklarheiten, besonders bezüglich der Ablauforganisation, den Verantwortlichkeiten, der Perspektivenfestlegung, den Messzyklen, der Massgrössengültigkeit und den möglichen Hilfsmitteln zur Massgrössenfestlegung sowie zur Messung und Interpretation der Massgrössenausprägung.

Weber (1998) kritisiert die fehlende Differenzierung zwischen Wirkungs-, Durchführungsund Prämissenkontrolle. Das Konzept der Balanced Scorecard beschränke sich auf eine Durchführungskontrolle. Demgegenüber meinen Kaplan und Norton, dass die Auseinandersetzung mit den Hypothesen des Modells im strategischen Lernprozess die Funktion einer Prämissenkontrolle erfülle (vgl. Kaplan und Norton (1997)).

Kritisiert wird ebenfalls die ausschliessliche Konzentration auf das Top-Management (vgl. Mountfield (1998)) und das Top-Down orientierte Vorgehen (vgl. Kanji und Moura e Sà (2001)). Die Stakeholderorientierung der Balanced Scorecard ist nicht ausgewogen. Die primäre Ausrichtung auf die Shareholder ist unübersehbar (Gleich (2001)). Der Beitrag von Mitarbeitern und Lieferanten wird vernachlässigt (vgl. Kanji und Moura e Sà (2001)).

Als grösste Stärke wird von zahlreichen Autoren die Fähigkeit des Ansatzes hervorgehoben, die Strategie einer Organisation in konkrete Ziele und Zielvorgaben zu transformieren. Durch die Beschränkung der Anzahl der Indikatoren besteht keine Gefahr eines Information Overloads. Der Ansatz ist für zahlreiche Organisationen anwendbar und 
entsprechend konfigurierbar. Die als Schwäche dargestellten Ursache-Wirkungsketten können auch als Stärke aufgefasst werden, indem der Ansatz das ganzheitliche Verständnis und das Verständnis der Interdependenzen zwischen den einzelnen Organisationseinheiten fördert (vgl. Kanji und Moura e Sà (2001)).

Dadurch, dass der Balanced-Scorecard-Ansatz im „Endausbau“ jeden Mitarbeiter miteinbeziehen will, besteht ein erheblicher Aufwand für deren Schulung. Zudem wird eine offene Kommunikationskultur vorausgesetzt (vgl. Hoffmann (1999)).

\subsubsection{Weiterentwicklungen und Varianten des Balanced-Scorecard-Ansatzes}

Die Diskussion betrifft insbesondere die Anzahl der Ergebnistafeln. Nach Meinung zahlreicher Autoren ist sie als Vorschlag zu verstehen, strategierelevante Informationen zu strukturieren. Hoffmann (1999) erwähnt eine ganze Reihe von Unternehmen, welche die Scorecard entsprechend ihren spezifischen Bedürfnissen angepasst haben, z.B. ABB Schweden, die eine zusätzliche Personaldimension eingeführt haben, und Swisscom, die fünf Leistungsdimensionen differenziert (Kunden, Mitarbeiter, operative Performance, Entwicklung des Shareholder Values und übrige finanzielle Einflussgrössen).

Entsprechend den Überlegungen von Lynch und Cross (1991b) unterscheiden sich die Arten der Kennzahlen auf den unterschiedlichen Hierarchiestufen. Diesem Umstand wird von einigen Unternehmen Rechnung getragen, indem auf der obersten Ebene teilweise Indizes verwendet werden, auf unteren Ebenen jedoch vermehrt operationelle Grössen zum Einsatz kommen.

Insgesamt ist festzuhalten, dass das ursprüngliche Konzept weitgehend beibehalten wurde. Die Anstrengungen der Harvard Business School und der Renaissance Inc. (Unternehmensberatungsfirma von Norton, die Beratungen zur Balanced Scorecard anbietet) haben dazu beigetragen.

\subsubsection{Berücksichtigung der Informationstechnologie im BSC-Ansatz}

Die erste Publikation von Kaplan und Norton (1992) hatte ganz generell die Neuausrichtung von Management-Informations-Systemen (MIS) zum Thema. Insofern berücksichtigt dieser Ansatz die Informationstechnologie seit ihrem Ursprung. Konkrete Hinweise finden sich jedoch erst im 1993er-Artikel, der bei den Umsetzungsschritten meint: „...including linking the measures to databases and information systems ... “ 
(Kaplan und Norton (1993)) und weiter „...an entirely new executive information system that links top-level business unit metrics down through shop floor and site-specific operational measures could be developed“ (Kaplan und Norton (1993)). Die Autoren sprechen also explizit an, dass sie sich ein Informationssystem für die Umsetzung vorstellen. Darüber, wie dieses konkret ausgestaltet werden sollte, schweigen sie sich aus.

In jüngeren Publikationen wird die Bedeutung der IT-Unterstützung bei der Implementierung des Balanced-Scorecard-Ansatzes zunehmend erkannt (vgl. Creelman (1998); Klaus et al. (1998); Mountfield (1998)). Konkret bemüht sich die Balanced Scorecard Collaborative, Inc. um die Standardisierung von rechnergestützten Informationssystemen, die den Balanced-Scorecard-Ansatz unterstützen. Im September 1999 und später in einer überarbeiteten Version vom Mai 2000 veröffentlichten sie einen Report mit dem Titel „Balanced Scorecard Functional Standards“ (vgl. BalancedScorecard-Collaborative (2000)), der die minimalen funktionalen Anforderungen an ein entsprechendes Informationssystem beschreibt. Die Anforderungen wurden gemäss der Firma aus den Publikationen von Kaplan und Norton, Erfahrungen von BalancedScorecard-Beratern in über 300 Projekten und Best-Practices-Konferenzen hergeleitet. Die im Rahmen dieser Publikation definierten Anforderungen sind als Minimalstandards zu verstehen (vgl. Tabelle 3-1). 


\begin{tabular}{|c|c|}
\hline \multicolumn{2}{|l|}{ Balanced Scorecard Design } \\
\hline - $\quad$ Perspektiven & $\begin{array}{l}\text { Mindestens vier Dimensionen/Perspektiven } \\
\text { mit der Möglichkeit, diese individuell zu } \\
\text { benennen. }\end{array}$ \\
\hline - $\quad$ Ziele & $\begin{array}{l}\text { Verwalten von einem oder mehreren Zielen } \\
\text { je Perspektive. }\end{array}$ \\
\hline - Kennzahlen & $\begin{array}{l}\text { Verwalten von einer oder mehreren } \\
\text { Kennzahlen je Ziel. }\end{array}$ \\
\hline - $\quad$ Ziel-Werte der Kennzahlen & $\begin{array}{l}\text { Verwaltung von Ziel-Werten (Soll-Werte) je } \\
\text { Kennzahl und Zeitpunkt oder-rahmen. }\end{array}$ \\
\hline - Ursache- und Wirkungsketten & $\begin{array}{l}\text { Verschiedene Ziele sollen in Ursache- } \\
\text { Wirkungsketten miteinander verknüpft } \\
\text { werden können. Dieses Wirkungsnetz kann } \\
\text { grafisch dargestellt und einfach modifiziert } \\
\text { werden. }\end{array}$ \\
\hline - $\quad$ Strategische Massnahmen & $\begin{array}{l}\text { Eine oder mehrere strategische } \\
\text { Massnahmen sollen mit einem oder } \\
\text { mehreren Zielen verknüpft werden können. }\end{array}$ \\
\hline \multicolumn{2}{|l|}{$\begin{array}{l}\text { Strategische Ausbildung \& } \\
\text { Kommunikation }\end{array}$} \\
\hline - Dokumentation der Attribute & $\begin{array}{l}\text { Die oben aufgeführten sechs Attribute sollen } \\
\text { jeweils mit detaillierten Informationen (z.B. in } \\
\text { Form von Notizen) versehen werden können. }\end{array}$ \\
\hline \multicolumn{2}{|l|}{ Business Execution } \\
\hline $\begin{array}{l}\text { - Verknüpfung von Massnahmen und } \\
\text { Zielen }\end{array}$ & $\begin{array}{l}\text { Die Verknüpfung von einer oder mehreren } \\
\text { Massnahmen mit einem oder mehreren } \\
\text { Zielen sollte dargestellt werden können. }\end{array}$ \\
\hline \multicolumn{2}{|l|}{ Feed-back und Lernen } \\
\hline $\begin{array}{ll}\text { - } & \text { Darstellung der aktuellen } \\
& \text { Performance von Kennzahlen }\end{array}$ & $\begin{array}{l}\text { Für jede Kennzahl sollte der aktuelle Wert } \\
\text { dargestellt werden können. }\end{array}$ \\
\hline $\begin{array}{ll}\text { - } & \text { Subjektive Beurteilung der erreichten } \\
\text { Performance }\end{array}$ & $\begin{array}{l}\text { Jede Kennzahl kann mittels eines } \\
\text { Ampelsystems subjektiv eingestuft werden } \\
\text { und mittels Kommentaren versehen werden. }\end{array}$ \\
\hline - $\quad$ Visueller Status eines Indikators & $\begin{array}{l}\text { Kennzahlen können mit ihrem Ist- und Soll- } \\
\text { Wert einander gegenübergestellt werden. }\end{array}$ \\
\hline
\end{tabular}

Tabelle 3-1 Anforderungen an ein Informationssystem für eine Balanced Scorecard (vgl. Balanced-Scorecard-Collaborative (2000))

Ende 2001 sind insgesamt 14 Produkte von der Balanced Scorecard Collaborative, Inc. als zertifizierte Lösungen gemäss den obigen Anforderungen registriert worden. Es handelt sich um die Produkte von CorVu, Crystal Decisions, Fiber, Gentia, Hyperion, InPhase, Oracle, Panorama Business Views, Peoplesoft, Procos, proDacapo, QPR, SAP und SAS. (vgl. Balanced-Scorecard-Collaborative (2001))

Im Jahre 2001 hat die oben erwähnte Organisation ein Schema für den Austausch von BSC-Daten im XML-Format im Draft-Status veröffentlicht. Damit wird der Austausch zwischen verschiedenen Scorecards erleichtert (vgl. Balanced-Scorecard-Collaborative (2001)). Insbesondere soll damit auch die Möglichkeit geschaffen werden, Scorecards 
zwischen Design- und Ausführungsprogrammen auszutauschen (vgl. hierzu die vergleichbaren Ideen der Workflow-Management Coalition, die schon weiter vorangeschritten sind (vgl. Workflow-Management-Coalition (2001))).

\subsubsection{Intellectual Capital}

\subsubsection{Grundkonzept des Ansatzes}

Das Leistungsangebot eines Unternehmens wird wesentlich durch dessen Wissen geprägt. Wissen kann somit als wichtige Ressource einer Organisation aufgefasst werden. Es existiert auf verschiedenen Ebenen einer Organisation. Eine Differenzierung, die von sämtlichen Autoren in diesem Fachbereich (einige wichtige sind: Bontis (2001); Davenport und Prusak (1998); Edvinsson und Malone (1997); Nonaka (1991); Senge (1990); Stewart (1997); Sveiby (1989)) getragen wird, sieht eine Unterteilung in individuelles und organisationelles Wissen vor. Individuelles Wissen umfasst persönliches Wissen, individuelle Kenntnisse und Fähigkeiten. Organisationelles Wissen betrifft die Infrastruktur, Geschäftsbeziehungen, Technologien, Prozesse und die Organisationskultur (vgl. Marr und Schiuma (2001)).

Folgt man den Argumenten der Verfechter des Wissensmanagements, so wird klar, dass die Ressource „Wissen“ geeignet bewirtschaftet werden muss. Oder anders formuliert: Eine wissensorientierte Organisation, die ihren Wettbewerbsvorteil ausnutzt und diesen bewusst entwickeln möchte, muss ihr Wissen und dessen konkrete Zusammensetzung kennen. Dies wiederum verlangt nach einer Operationalisierung, da Wissen, welches nicht gemessen werden kann, auch nicht aktiv gemanagt werden kann (Analogie zu: „if you can’t measure it, you can’t manage it“ (Harrington und Harrington (1996)).

Ausgangspunkt des Wissensmanagements ist die wissenschaftliche Diskussion um das strategische Management. Dabei dominieren zwei Paradigmen:

- Market-based View: Dieser aus den 60er und 70er-Jahren stammende Ansatz konzentriert sich auf das Finden von attraktiven Branchen. Durch die geschickte Bewirtschaftung der Elemente des Five-Forces-Modells von Porter wird zudem eine möglichst ideale Position innerhalb der Branche angestrebt. Hauptproblem dieses Ansatzes ist die Imitierbarkeit der eigenen Strategie durch Konkurrenten und 
damit das Verlorengehen von Wettbewerbsvorteilen. Porters Werk gehört hier zur Standardliteratur (vgl. Porter (1999)).

- Resource-based View: Dieser Ansatz versucht Wettbewerbsvorteile durch Unterschiede in der Ausstattung und Kombination von kritischen Unternehmensressourcen zu erklären. Wissen scheint nur sehr beschränkt imitierbar und substituierbar zu sein. Dadurch kann das Wissensmanagement unternehmerisch gerechtfertigt werden. Im Sinne der Erhaltung und des Ausbaus der besetzten Erfolgspositionen scheint Wissensmanagement sinnvoll .

Intellectual Capital als Theorie ist dem Resource-based View-Ansatz zuzuordnen. In der Forschung sind zwei wesentliche Richtungen auszumachen. Einerseits die Vertreter, die Wissen als Ganzes betrachten (Epistemologie des Wissens mit den drei Ansätzen: Kognitiv, Konnektiv und Autopoietiv). Sie beschäftigen sich nicht mit der konkreten Operationalisierung von Wissen, sondern damit, wie Wissen geschaffen werden kann. Ergebnis dieser Forschung, die nach wie vor andauert, sind Heuristiken, um Wissensmanagement-Prozesse erfolgreich einzuführen und $\mathrm{zu}$ bewirtschaften. Kernergebnisse der bisherigen Forschung betreffen die Aggregatzustände des Wissens (vgl. Nonaka (1991); Nonaka und Takeuchi (1995)):

- Explizites Wissen

- Implizites Wissen

- Individualwissen

- Kollektivwissen:

o Fähigkeiten einer Organisation, die über die Fähigkeiten einer einzelnen Person hinausgehen und

o Wissen, welches bei mehreren Mitarbeitern in der gleichen Form vorliegt.

Die zweite Forschungsrichtung beschäftigt sich mit der Ressource Wissen als wesentlicher Wertbestandteil einer Organisation. Die bedeutendsten Ideen stammen von Sveiby (1989), Stewart (1997) und Edvinsson und Malone (1997). Von diesen Autoren stammt das Konzept des Intellectual Capital (IC), welches Führungskräften hilft, Wissenskomponenten in einer Organisation zu identifizieren, zu klassifizieren und zu valorisieren. Es werden Erklärungsansätze geliefert, inwiefern Wissen den Erfolg eines Unternehmens beeinflusst, 
wie weit Wissen die Investitionsneigung von Aktionären determiniert und somit den Wert eines Unternehmens bestimmt.

Der Schwede Sveiby (1989) machte als einer der ersten Autoren den Vorschlag, wie die nicht sichtbaren Vermögensbestandteile (intangible assets) zu messen seien. Er wendet sich in seinem Buch vor allem an Unternehmen im Wissensbereich (Know-how Companies). Seine Argumentation: Know-how Companies verwenden wenig traditionelles Rohmaterial, und das finanzielle Kapital in diesen Firmen ist oft nicht der entscheidende Investitionsfaktor. Entsprechend bildet das Know-how-Kapital den entscheidenden Produktionsfaktor und „Ertrags-Generator“.

Sveiby unterscheidet grundsätzlich zwischen dem traditionellen finanziellen Kapital (welches in der Bilanz sichtbar ist) und dem Know-how-Kapital. Letzteres umfasst zwei Kategorien: Kapital, welches an das Individuum gebunden ist (individual capital), und Kapital, welches an das Unternehmen gebunden ist (structural capital) (vgl. Abbildung 3-14). Das individuelle Kapital (individual capital) verlässt beim Ausscheiden eines Mitarbeiters die Firma ebenfalls. Beim strukturellen Kapital (structural capital) hingegen, ist dies nicht der Fall.

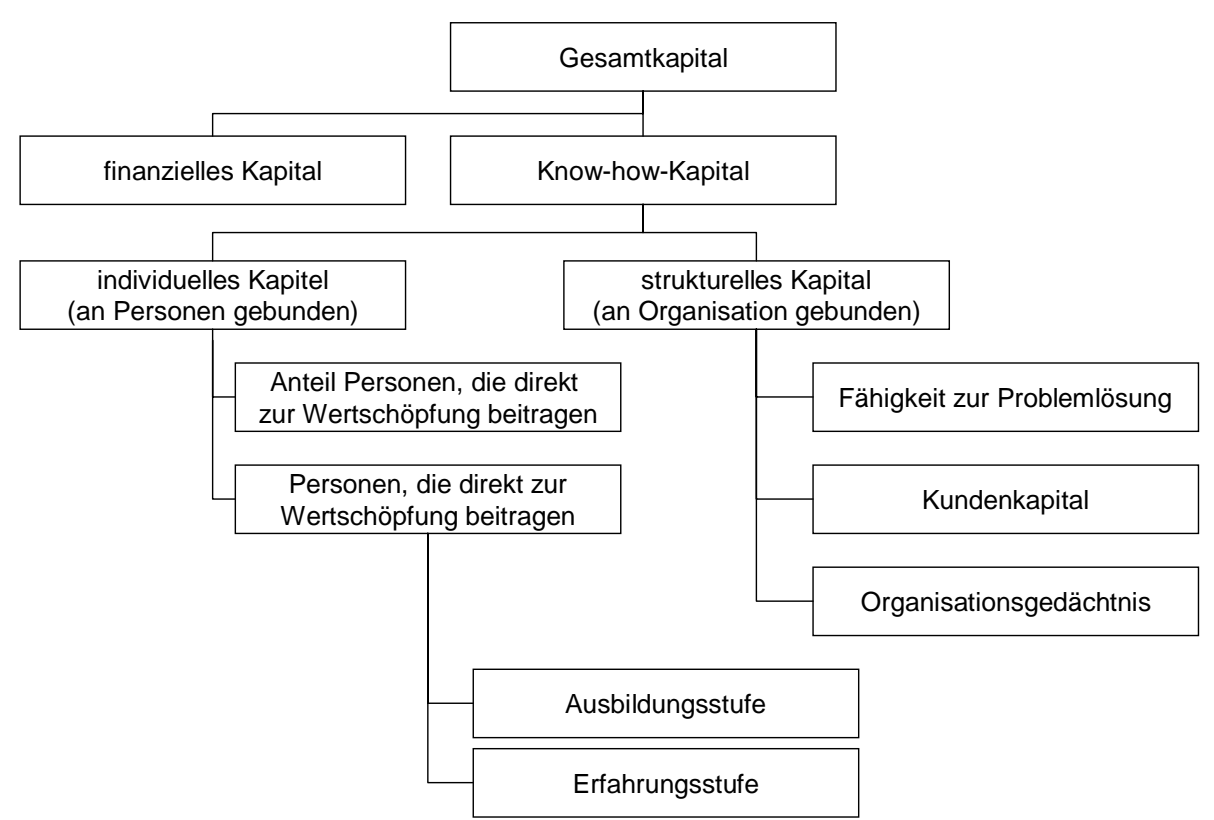

Abbildung 3-14: Vermögensbestandteile (in Anlehnung an Sveiby (1989)) 
Einen ähnlichen Ansatz verfolgen Edvinsson und Malone (1997). Sie prägten den später sehr populär gewordenen Begriff „Intellectual Capital“. Doch was bedeutet Intellectual Capital? Aus Abbildung 3-15 geht hervor, dass sich das intellektuelle Kapital (manchmal auch intangible assets genannt) aus dem Humankapital und dem strukturellen Kapital zusammensetzt. Die wichtigsten Elemente des strukturellen Kapitals sind die Organisationsstrukturen (Aufbau- und Ablauforganisationen), die Patente, welche das Unternehmen besitzt, die Marken, über die das Unternehmen verfügt, sowie die Beziehungen zu den Schlüsselkunden.

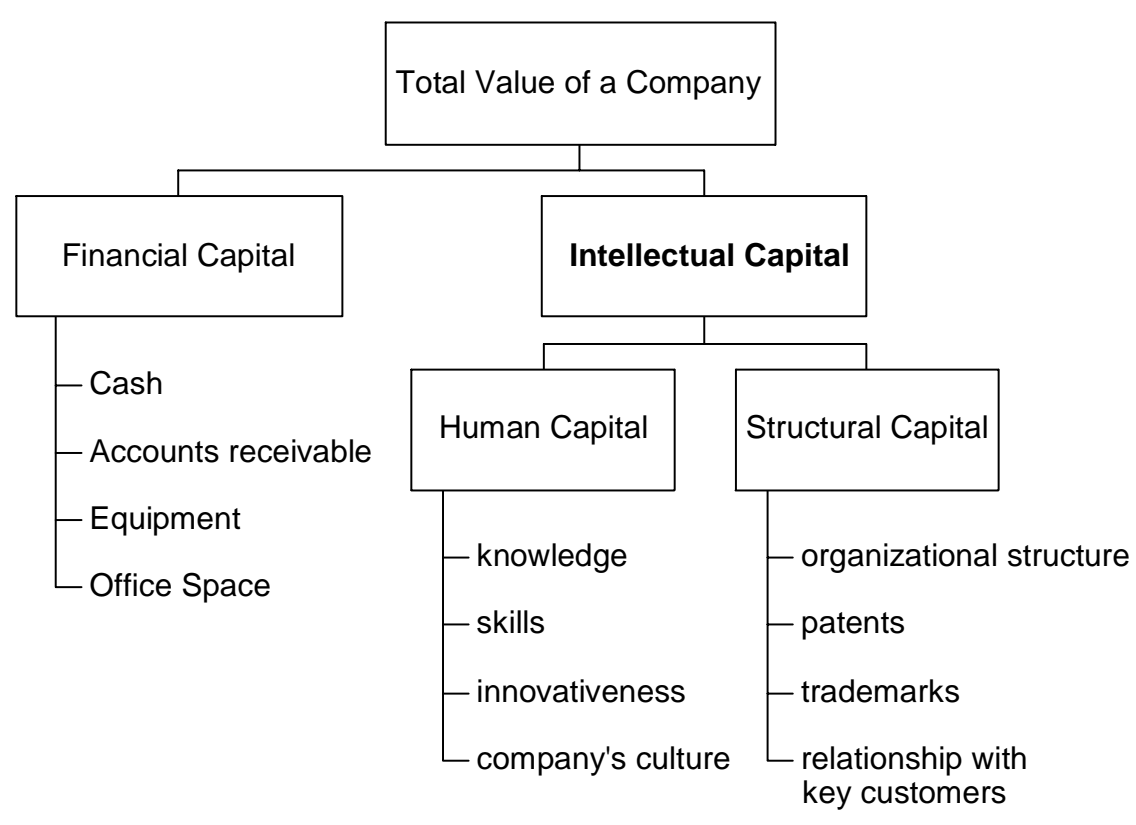

Abbildung 3-15: Die Komponenten des Intellectual Capital

Edvinson war Intellectual Capital Director bei der skandinavischen Firma Skandia, einem Unternehmen im Bereich Versicherungen und Vermögensverwaltung (siehe Skandia (2002)). Das von Skandia verwendete Werkzeug zur Bestimmung des Humankapitals heisst Navigator (vgl. Abbildung 3-16). 


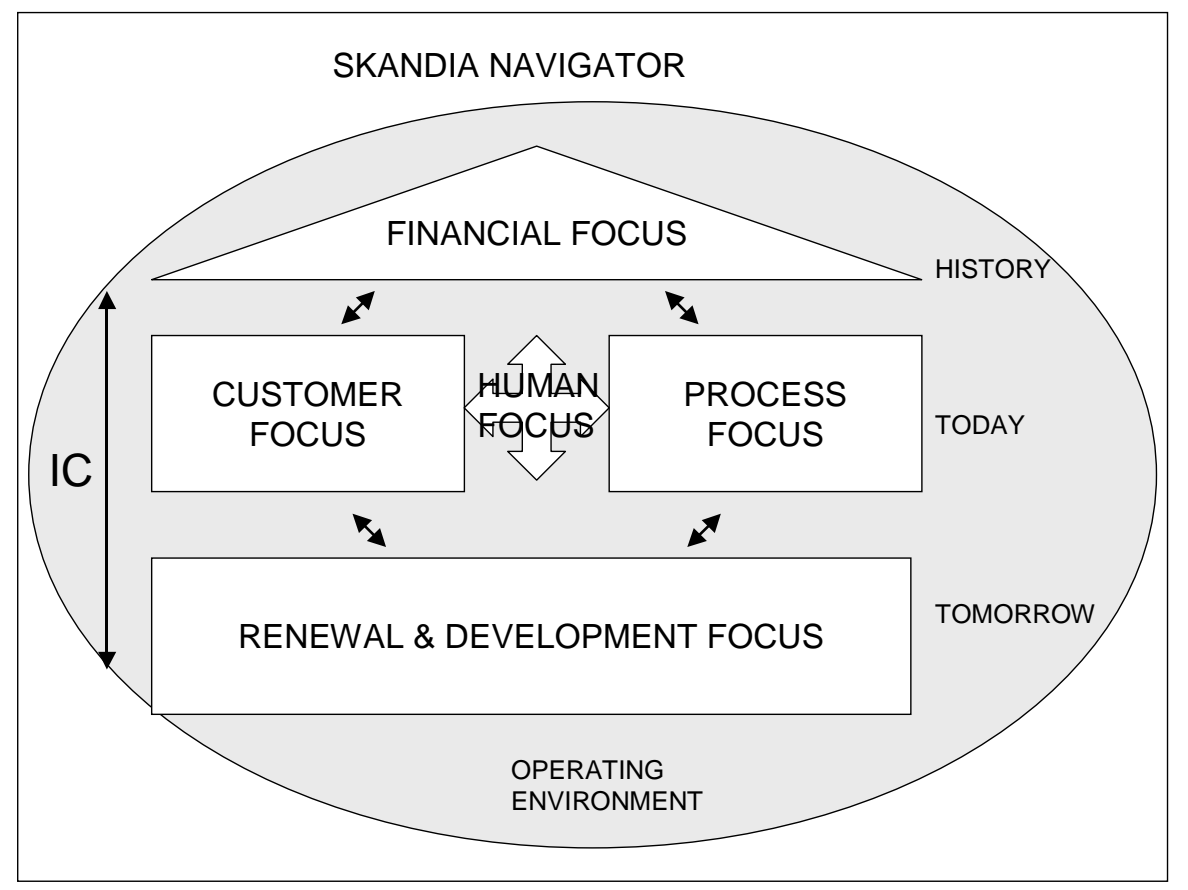

Abbildung 3-16: Der Skandia-Navigator (vgl. Edvinsson und Malone (1997))

\subsubsection{Charakterisierung des IC-Ansatzes auf Grund von Sekundärliteratur}

Der Schwerpunkt des IC-Ansatzes liegt bei der Erklärung des Humankapitals. Dies leuchtet ein, wenn man sich vergegenwärtigt, dass der Ansatz primär für wissensorientierte Unternehmen gedacht ist. Die Fokussierung auf einige wenige Elemente hat Ähnlichkeiten mit der Balanced Scorecard. Allerdings unterstellt der IC-Ansatz keine direkte Kausalkette, sondern weist lediglich auf die Zeitdimension hin, indem die finanziellen Werte mit der Vergangenheit, die Kunden, Mitarbeiter und Prozesse mit dem Heute und die Erneuerung und Entwicklung mit der Zukunft assoziiert werden. Ein zusätzliches, wesentliches Unterscheidungsmerkmal besteht in der Zielgruppe des Ansatzes. Da der IC-Ansatz den Versuch unternimmt, den unterschiedlichen Marktwert von vergleichsmässig ähnlich mit Eigenkapital ausgestatteten Unternehmen zu erklären, wird klar, dass nicht nur das obere Management eines Unternehmens, sondern auch Externe (Analysten, Aktionäre) zu den Adressaten gehören.

\subsubsection{Weiterentwicklungen und Varianten des Ansatzes}

Das ursprüngliche Grundkonzept wurde von zahlreichen Autoren bearbeitet und erweitert. Entsprechend sind eine ganze Reihe von Vorschlägen entstanden, die auf der ursprünglichen Idee basieren. Zwei der Ansätze sollen hier kurz dargestellt werden: 
Einen interessanten Ansatz verfolgen Roos et al. (1997) mit ihrem IC-Index. Die Autoren schlagen vor, die verschiedenen IC-Measures in einem einzigen Index zu konsolidieren. Damit möchten sie eine ganzheitliche Sicht auf das IC einer Organisation erlauben. Zudem sehen sie Vergleiche zwischen verschiedenen Organisationen vor (Benchmarking). Die Autoren geben eine konkrete Anweisung, wie der Index zu bestimmen sei. Die Aggregation erfolgt über dimensionslose Kennzahlen.

Noch konkreter ist der Ansatz von Brooking (1996). Dieser erlaubt die Bestimmung eines Betrages für das IC. Die vier betrachteten Hauptkategorien des IC sind market assets, human-centred-assets, intellectual property assets und infrastructure assets. Die Erhebung erfolgt mittels spezifischer Fragebogen im Rahmen eines Audits. Die erhobenen Daten werden mittels drei Verfahren in einen konkreten Betrag transformiert: (1) Kostenansatz: Dabei werden die Kosten für eine Ersatzbeschaffung der IC-assets bestimmt. (2) Marktansatz: Es werden die Kosten bestimmt, falls diese Komponenten extern beschafft würden. (3) Einkommen-Ansatz: Es wird der Ertrag bestimmt, welcher mit dieser Komponente generiert werden könnte.

\subsubsection{Berücksichtigung der Informationstechnologie im Intellectual-Capital-Ansatz}

Edvinsson und Malone (1997) gehen in ihrem Buch kurz auf die Bedeutung der IT ein; sie schreiben „...MIS director may be shaking his or her head and saying, do you know how much it will cost to install an IS system to capture all of this data? It would have to be all custom too, because there's nothing off the shelf that matches it” und weiter "the most competitive ... of these systems will be those that link together the existing databases of the company (sales information, management information, production control,...), probably through an intranet-based architecture and Java applets, to quickly capture information that is already there.” (Edvinsson und Malone (1997)). Ähnlich dem Balanced-ScorecardAnsatz sehen die Autoren den Einsatz eines Management-Informations-Systems vor. Wie dieses ausgestaltet werden soll, bleibt jedoch offen. Sveiby (1989) geht in seinem Buch „The invisible Balance Sheet“ nicht auf die informationstechnologische Implementierung ein.

Zwischenzeitlich hat sich die Situation wesentlich geändert. Die Firma Skandia, dessen Intellectual Capital Director Edvinsson war, hat eine Firma namens IC Visions AB gegründet, die ein internetbasiertes Tool entwickelte, um die Umsetzung mittels IT zu 
unterstützen. Das Tool trägt den Namen Dolphin Navigator Systems (vgl. ICVisions (2002)).

Sveiby hat ebenfalls eine Firma aufgebaut (vgl. Sveiby (2002)), die ein Softwareprodukt auf seinen ursprünglichen Ideen basierend, anbietet. Das Produkt trägt den Namen The Sveiby Toolkit (vgl. Sveiby-Toolkit (2002)).

\subsubsection{Value-Based Management}

\subsubsection{Grundkonzept des Ansatzes}

Dieser Ansatz rückt den Eigenkapitalgeber und dessen Erwartungen in den Mittelpunkt des unternehmerischen Zielsystems. Sämtliche Aktivitäten sind auf die Messung sowie das Management der wertbeeinflussenden Faktoren ausgerichtet. Im Vordergrund steht also die Maximierung des Unternehmenswertes. Bei einer Kapitalgesellschaft kann dies gleichgesetzt werden mit der Maximierung des Aktionärsnutzens.

Nach Helbling (1995) kann der Unternehmenswert definiert werden als „Barwert aller dem Unternehmen künftig entziehbarer Gewinne, zuzüglich des Barwertes aller Vermögensteile, die zur Erzielung dieser Gewinne nicht absolut notwendig sind“. In der Minimalform also der „Gegenwartswert aller zukünftigen Netto-Einnahmen des Investors“ (Hostettler (1998)).

Es können mehrere Ansätze zur Portfoliosteuerung und der systematischen Ableitung von Kennzahlen unterschieden werden (vgl. Brunner (1999)). Der Ansatz von Rappaport definiert den Shareholder Value als den ökonomischen Wert einer Investition dadurch, dass er die prognostizierten Cash-flows mittels des Kapitalkostensatzes diskontiert (Rappaport (1995)). Der Ansatz von Stern bestimmt einen Economic Value Added (EVA) als „residual income left over from operating profits after the cost of capital has been subtracted"(Stern (1994), zitiert nach Hostettler (1998)).

\section{Ansatz von Rappaport}

Der klassische Ansatz definiert den Wert des Unternehmens als Barwert der Free Cash Flows. Der Free Cash Flow wird bestimmt als den um notwendige Investitionen ins Anlagevermögen und dem Working Capital korrigierten Cash Flow. Es handelt sich somit 
um den potenziell an die Eigner ausschüttbaren Zahlungsmittelüberschuss. Geistiger Vater dieser wertorientierten Beurteilungsgrössen ist Rappaport (1986).

Rappaport nutzt zur Bestimmung des Shareholder Value die Kapitalwertmethode. Dies bedeutet, dass eine Organisation dann wertschaffend ist, wenn die Summe der abgezinsten freien Cash-flows grösser als Null ist. Zur Ermittlung der freien Cash-flows bedient sich der Autor der Value-Drivers (Werttreibern). Es handelt sich dabei um Bewertungsparameter mit Hilfe derer die Zahlungsströme bestimmt werden können (vgl. Abbildung 3-17).

Der zum Abzinsen der Cashflow Ströme verwendete Diskontierungsfaktor berechnet sich als gewichtetes Mittel der Kosten von Fremd- und Eigenkapital (Weighted Average Cost of Capital - WACC). Die Bestimmung der Eigenkapitalkosten erfolgt anhand des Capital Asset Pricing Model (CAPM):

$$
E K_{\text {Kosten }}=\text { risikofreier_Zinssatz }+\beta \times(\text { erwartete _ Marktrendite }- \text { risikofreier_Zinssatz })
$$

Als risikofreier Zinssatz wird in der Regel derjenige für langlaufende Bundesanleihen herangezogen. Die Risikoklasse wird mittels dem ß-Faktor bestimmt, der die Volatilität eines Wertpapiers angibt und somit das systematische Risiko eines Unternehmens misst. Ein ß-Wert von grösser (kleiner) eins gibt an, dass sich die Aktienrendite bei Marktschwankungen im Vergleich zur Marktrendite überproportional (unterproportional) verändert. Die Risikoprämie des Marktes, gemessen als Differenz zwischen Marktrendite und risikofreiem Zinssatz, zeigt die zusätzliche Abgeltung in Prozent, welche die Investoren für die Übernahme des Risikos im Vergleich zu sicheren Anlageformen fordern. 
Zielsetzung des Unternehmens

Bewertungs-
komponenten

Führungsentscheidungen

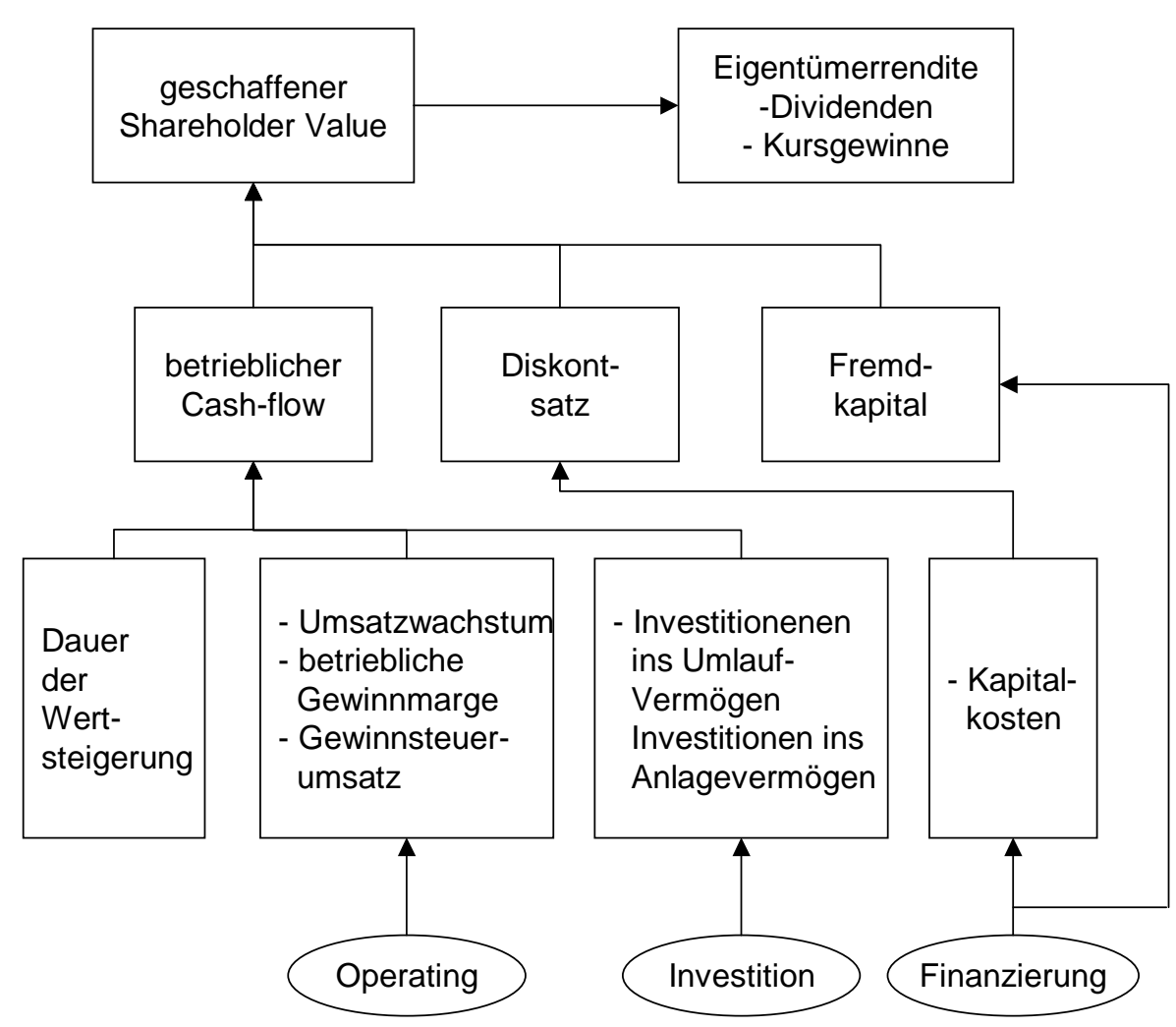

Abbildung 3-17: Werttreiber nach Rappaport (1995)

Gemäss Boemle (1998) hat das Shareholder-Value-Konzept von Rappaport das Renditeund Risikobewusstsein der Investoren wesentlich gestärkt. Aktionäre wünschen demnach eine aktive Rolle zu übernehmen und selbstbewusst ihre Ansprüche und Rechte gegenüber der Unternehmensleitung zu vertreten. In diesem Zusammenhang hat der Begriff der Corporate Governance Bedeutung erlangt. Sie beschäftigt sich mit den Zusammenhängen zwischen Unternehmensleitung, Leitungskontrolle und Unternehmenserfolg aus der Sicht der Anteilseigner .

\section{Economic Value Added nach Stern Stewart \& Co.}

Der Economic Value Added (EVA) wurde von der New Yorker Unternehmensberatungsfirma Stern Stewart \& Co. 1991 vorgeschlagen. Die Idee war, ein Instrument für die Führung und Bewertung von Unternehmen zu liefern. EVA kann als Differenz zwischen dem gewichteten Kapitalkostensatz (WACC $=$ weighted average cost of capital) und der realisierten Rendite einer Investition (ROCE = return on capital employed) multipliziert mit dem eingesetzten Kapital ( $\mathrm{NOA}=$ net operating assets) 
aufgefasst werden. Wie der Gewinn oder der Cashflow ist der Residualgewinn eine absolute finanzielle Grösse, der auf Jahresbasis berechnet wird. In einer Formel ausgedrückt ist EVA:

$$
E V A=(R O C E-W A C C) * N O A
$$

Die Rendite auf das investierte Kapital (ROCE) ist wiederum der Quotient aus operativem Ergebnis nach Steuern (NOPAT = net operating profit after taxes) und investiertem Kapital (NOA = net operating assets):

$$
R O C E=N O P A T / N O A
$$

Nach Umformung der obigen Gleichung erhält man folgenden Ausdruck:

$$
E V A=N O P A T-N O A * W A C C
$$

Erwirtschaftet die Organisation einen positiven EVA, so erlaubt die betriebliche Tätigkeit, die gesamten Finanzierungskosten (Fremd- und Eigenkapital) zu decken und einen Überschuss im Umfang des EVA-Betrages zu erwirtschaften. Ist der EVA negativ, so kann der betriebliche Gewinn die Kapitalkosten nicht decken. In diesem Falle werden aus der Sicht der Kapitalgeber Werte vernichtet, d.h. der Anleger hätte das Kapital bei einer anderen Unternehmung mit ähnlichem Risikoprofil angemessener verzinsen können. (vgl. Hostettler (1998))

Stewart (1991) definiert den EVA ganz kurz als „operating profits less the cost of all capital employed to produce those earnings".

Auf Grund der Elemente der Formel haben Führungskräfte drei Möglichkeiten den EVA ihres Unternehmens zu steigern (vgl. Ehrbar (1999); Abbildung 3-18):

- Steigerung des operativen Ergebnisses ohne zusätzliches Kapital zu binden. Dies führt zu einer höheren Rendite auf dem investierten Kapital.

- Zusätzliches Kapital wird in Projekte investiert, die eine über den Kapitalkosten liegende Rendite erwirtschaften.

- Bereiche und Projekte, die nicht die Kapitalkosten verdienen, werden liquidiert, um Mittel für profitablere Vorhaben zu schaffen. 
Operative Entscheidungen

beeinflussen primär den NOPAT

(Net Operating Profit After Taxes)

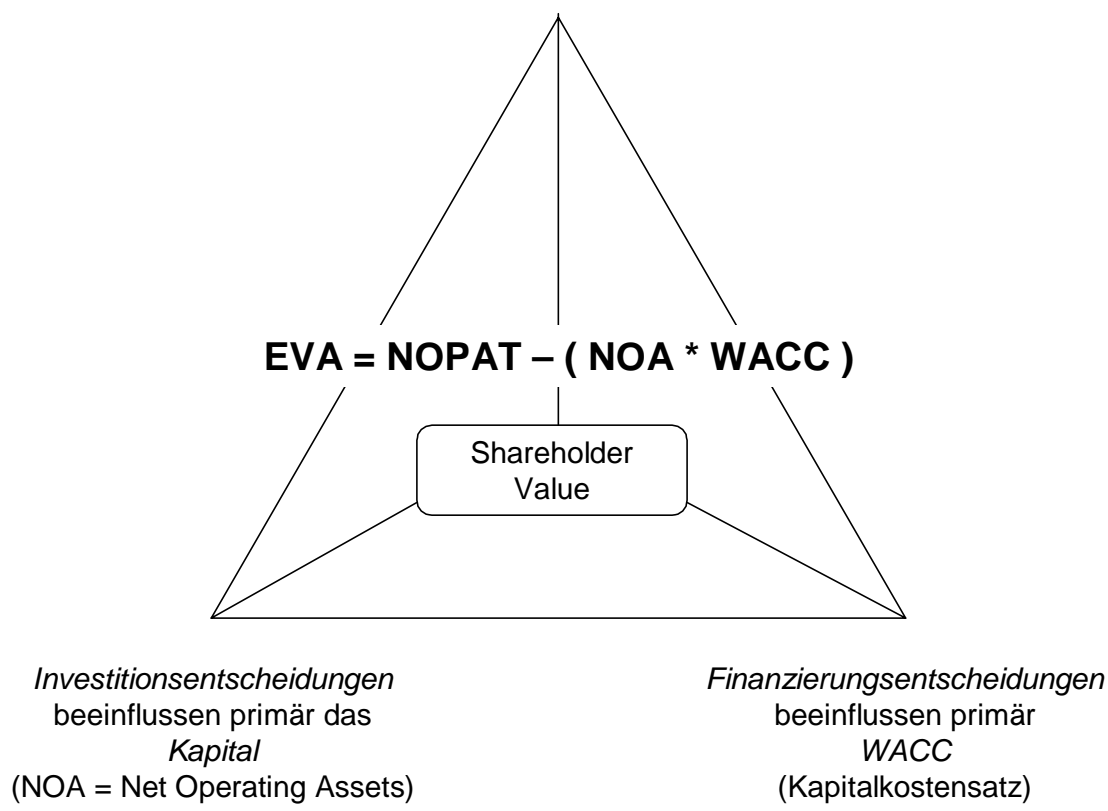

Abbildung 3-18: Zusammenhang von EVA und Shareholder Value (vgl. Hostettler (1998))

Die Berechnung des EVA bedingt die Auswahl und Anwendung verschiedener Korrekturfaktoren (adjustment). Stewart (1991) listet über Hundert solcher Faktoren auf. Da verschiedene Firmen unterschiedliche Korrekturfaktoren einsetzen, ist es kaum möglich den EVA verschiedener Firmen miteinander zu vergleichen.

Hostettler unterscheidet zwischen Finanzgrösse und Handlungsmaxime des Shareholder Value. Als Finanzgrösse entspricht der Shareholder Value der Marktkapitalisierung einer Unternehmung oder der Berechnung auf der Basis von diskontierten prognostizierten EVAs als alternativer Ansatz zur Discounted Cashflow-Methode. Ein historischer EVA kann nicht als Shareholder Value auf Jahresbasis verstanden werden, da dieser - gemessen zu Marktwerten - Ausdruck der erwarteten Ergebnisse in der Zukunft ist. Wird der Shareholder Value als Handlungsmaxime aufgefasst, so drängt sich EVA als Instrument zur finanziellen Führung auf, da operative Entscheidungen primär den NOPAT, Investitionsentscheidungen primär das NOA und Finanzierungsentscheidungen primär den Kapitalkostensatz beeinflussen. (vgl. Hostettler (1998)) 


\subsubsection{Charakterisierung des VBM-Ansatzes auf Grund von Sekundärliteratur}

Die Methoden des Value-Based Management (VBM) betrachten den Gewinn als dominierendes Ziel im Rahmen des Zielsystems der Organisation. Es werden nur finanzielle Indikatoren betrachtet. Im Gegensatz zum Ansatz des Intellectual Capital versucht das VBM die Differenz von Markt- und Buchwert über den Barwert der zukünftigen Mehrwerte zu bestimmen.

Hostettler (1998) sieht EVA als Instrument, welches (a) prioritär der Erhöhung der Gesamtrendite des Aktionärs dient, (b) die Bewertung und Analyse von Unternehmen, Unternehmensteilen und Projekten ermöglicht, (c) als Ziel- und Anreizsystem ausgestaltet und (d) leicht kommuniziert werden kann.

Die Idee des Residualgewinns ist nicht neu. General Electric (GE) und General Motors (GM) setzten entsprechende Konzepte schon in der ersten Hälfte des 20. Jahrhunderts ein: „operating profit less a capital charge” und „ten percent of all operating profits after the 15\% capital charge became the bonus tool to be shared by management” (Stern (1994), zitiert nach Hostettler (1998)).

In einer Studie bestimmten Hapeslagh et al. (2001) die Erfolgsfaktoren, um mit dem Modell des Value-Based Managements erfolgreich zu sein. Die Hauptaussage der Untersuchung besteht darin, dass ein Kulturwandel im Unternehmen stattfinden muss. Dabei unterscheiden fünf Faktoren erfolgreiche von weniger erfolgreichen Unternehmen: (1) commitment to shareholder value, d.h. die verbindliche Zusage, den Aktionärsnutzen zu steigern; (2) using training to create buy-in, d.h. die Mitarbeiter auf allen Ebenen bezüglich des VBM-Ansatzes ausbilden; (3) using incentive compensation to build change ownership, d.h. Entlohnung mit dem Unternehmenswert verknüpfen;(4) empowerment of business units to make value-creating decisions, d.h. das Unternehmertum in den Business Units zu verstärken und (5) making broad - not deep - changes to systems and processes, d.h. die Verankerung eines einfachen Reportingkonzeptes unter Berücksichtigung von wert-relevanten Performance-Indikatoren, die periodisch mit der Strategie abgestimmt werden. (vgl. Hapeslagh et al. (2001))

\subsubsection{Weiterentwicklungen und Varianten des Ansatzes}

Im Rahmen der betrieblichen Übergewinnverfahren sind neben dem Ansatz von Rappaport und demjenigen von Stern weitere Ansätze entstanden. 
Hierzu gehören der Economic Profit von McKinsey \& Company (vgl. Copeland et al. (1994)): „measures the dollars of economic value created by a company in a single year”. Das Konzept ist dem EVA sehr ähnlich, berechnet allerdings den NOPAT (hier bezeichnet als NOPLAT $=$ Net Operating Profit Less Adjusted Taxes) auf der Basis des betrieblichen freien Cash Flows. Zudem wird bei der Bestimmung des NOA auf umfangreiche Anpassungen verzichtet.

Hostettler (1998) nennt weitere Ansätze, wie der Added Value Ansatz der London Business School (LBS) der von der betrieblichen Wertschöpfung einer Unternehmung ausgeht und der Cash Value Added Ansatz der Boston Consulting Group (BCG). Auf diese Ansätze soll im Rahmen dieser Arbeit nicht weiter eingegangen werden.

\subsubsection{Berücksichtigung der Informationstechnologie im VBM-Ansatz}

In den Originalwerken findet sich kein Bezug zur Informationstechnologie. Verschiedene Organisationen bieten jedoch im Rahmen von Reporting-Tools Unterstützung: So etwa Hyperion (2002). 


\subsection{Vergleichende Beurteilung der PMS-Ansätze}

Nachfolgend werden die vier Ansätze kritisch gewürdigt. Zu diesem Zweck werden die besprochenen PMS-Ansätze anhand einiger Kriterien charakterisiert. Demgegenüber stehen vier Fallbeispiele, die nachfolgend vorgestellt und ebenfalls zusammenfassend mittels einiger Kriterien charakterisiert werden. Mit dieser komprimierten Zusammenstellung kann die Eignung der einzelnen PMS-Ansätze für die jeweiligen Fallbeispiele untersucht werden.

\subsubsection{Vier Fallbeispiele}

A) Schweizerische Landesniederlassung eines international tätigen Informatikdienstleisters (nachfolgend als SIDL AG; Schweizerischer Informatik-Dienstleister AG bezeichnet): Die organisch gewachsene, rund 10-jährige Gesellschaft ist spezialisiert auf das Angebot von Informatikgesamtlösungen (Hardware, Software und Beratung) von mittelständischen Unternehmen. Zusammen mit einer Partnerfirma entwickelt sie eine eigene ERP-Lösung für mittelständische Unternehmen. Zu den rund 100 Kunden wird eine enge, langfristige und partnerschaftliche Beziehung unterhalten. Die Kunden verstehen den Anbieter in der Regel als Einzige Anlaufstelle für ihre Informatikbelange. Dies führt zu einem breiten Produkteangebot, welches auf der Angebotspalette eines wichtigen Marktteilnehmers basiert. Entsprechend wird eine enge Geschäftsbeziehung zu diesem Lieferanten unterhalten. 1999 wurde SIDL in eine grosse börsenkotierte, europaweit tätige Informatikunternehmung in der Form einer Länderniederlassung integriert. Damit verbunden war eine Erweiterung des Kundenportfolios um Grossunternehmen und teilweise auch eine Erweiterung der Produktepalette. Durch die Einführung von Konzernstrukturen erhöhten sich die Regeldichte, der administrative Overhead und im Speziellen die Reportingverpflichtungen massiv. Die Anpassung an die neue Unternehmenskultur ist mit einigen Schwierigkeiten verbunden. 2001 wurde die Muttergesellschaft von einer international tätigen Informatikgesellschaft mit Hauptsitz in den USA übernommen. Die neuerliche Integration ist im Gange. Nachfolgend (vgl. Abschnitt 3.3.2 und Tabelle 3-2) wird diese Fallstudie mit „A“ bezeichnet. ${ }^{4}$

4 Das Fallstudie SIDL AG wird zur Illustration im Verlauf der Arbeit immer wieder aufgegriffen (vgl. Kapitel 6, 9 und 10). Zusammengesetzt ergibt sich ein durchgängiges Fallbeispiel. 
B) Unabhängiger Hersteller von qualitativ hoch stehenden Convenience-Produkten: Die über 50-jährige Firma beschäftigt rund 500 Mitarbeiter und ist spezialisiert auf die Haltbarmachung von Lebensmitteln durch Trocknung. Dabei setzt sie hochtechnologisierte Verfahren wie die Extraktion, Mischung und Trocknung ein. Die Produkte werden mehrheitlich im Inland, aber auch im Ausland abgesetzt (über 30 Länder). Einer der wichtigsten Kunden ist der grösste Detailhändler der Schweiz; aber auch Grossverbraucher (z.B. Gastronomiebetriebe, Spitäler und Heime) und die Lebensmittelindustrie, welche die Produkte weiterverarbeitet gehören zur Kundschaft. Die einzelnen Absatzkanäle werden mit unterschiedlichen Marken (Eigen- und Fremdmarken) bedient. Kernkompetenzen der Unternehmung sind die echte Kundennähe (kundenspezifische Problemlösungen, Flexibilität und Geschwindigkeit), das ausgesprochene technische Know-how und vor allem die Innovationsfähigkeit (eigene Ideen- und Entwicklungsabteilung). Nachfolgend (vgl. Abschnitt 3.3.2 und Tabelle 3-2) wird diese Fallstudie mit „B“ bezeichnet.

C) Grosse, in mehrere Geschäftsfelder segmentierte Energieversorgungsunternehmung: Neben der Produktion von Strom in verschiedenen Kraftwerktypen unterhält die Gesellschaft ein ausgedehntes Transport- und Versorgungsnetz. Zusätzlich wird ein nationaler und internationaler Handel mit Energie in erheblichem Umfang unterhalten. Der ehemalige Regiebetrieb öffnet sich im Rahmen der Liberalisierung des Energiemarktes in hohem Tempo. Damit verbunden ist ein Wandel der Unternehmenskultur von einer vormals technokratischen zu einer marktorientierten Gesellschaft. Komplett neue Geschäftsbereiche müssen in kurzer Zeit aufgebaut werden. Das Unternehmensumfeld wird wesentlich durch politische Prozesse beeinflusst. Der entstehende Wettbewerb wird voraussichtlich zu einer Konzentration der Marktteilnehmer führen. Neben den Shareholders spielen zahlreiche Stakeholder eine wichtige Rolle für das Unternehmen: Wichtig zu nennen sind Privat- und Firmenkunden, andere Stromhändler, assoziierte kleinere Kraftwerke (die ihren Strom über die Unternehmung vermarkten), das Gemeinwesen (Versorgungsauftrag), verschiedene politische Gruppierungen und speziell Umweltorganisationen. Nachfolgend (vgl. Abschnitt 3.3.2 und Tabelle 3-2) wird diese Fallstudie mit „C“ referenziert.

D) Grösseres, unabhängiges, international tätiges Hilfswerk: Das mehr als 100 Jahre alte Hilfswerk unterstützt Bedürftige im In- und Ausland. In der Schweiz unterhält die Organisation mehr als ein Dutzend Regionalbüros. Diese bieten eine ganze Reihe von Dienstleistungen und Hilfestellungen für Erwerbslose, Betroffene der „Neuen Armut“, 
Asylsuchende und Flüchtlinge an. Im Ausland setzt sich die Organisation für eine solidarische Gesellschaft ein. Gemeinsam mit örtlichen Partnerorganisationen führt sie Projekte in weit über 50 Ländern in Afrika, Asien, Lateinamerika und Europa durch. Dabei unterstützt sie marginalisierte Menschen in ihren Bestrebungen, ihre Grundrechte auf Arbeit und Einkommen, Nahrung und Wohnung, Gesundheit und Bildung einzufordern. Zudem leistet sie Not- und Wiederaufbauhilfe bei Naturkatastrophen und Kriegen. Mit der Vermittlung von Freiwilligeneinsätzen unterstützt sie Bauernbetriebe in Rand- und Bergregionen. Die Organisation finanziert sich durch Spenden, erhält aber auch Gelder von anderen staatlichen und nichtstaatlichen Organisationen. Die Schwerpunkte der Tätigkeit lassen sich längerfristig planen. Eine transparente Kommunikation mit den Geldgebern ist äusserst wichtig, da diesen aus ihrer Spende kein quantifizierbarer Nutzen zufliesst. Nachfolgend (vgl. Abschnitt 3.3.2 und Tabelle 3-2) wird die Fallstudie mit „D“ bezeichnet.

\subsubsection{Beurteilung der PMS-Ansätze bezüglich der vier Fallbeispiele}

Die vier vorgestellten Fallstudien (Szenarien) werden nachfolgend bezüglich sieben Kriterien zusammenfassend charakterisiert (vgl. Tabelle 3-2). Gleiches erfolgt für die vier vorgestellten Ansätze bezüglich einiger wesentlicher Kriterien (vgl. Tabelle 3-3):

\begin{tabular}{|l|c|c|c|c|}
\hline \multicolumn{1}{|c|}{$\begin{array}{c}\text { Fallstudiel } \\
\text { Szenario }\end{array}$} & $\begin{array}{c}\text { Fallstudie } \\
\text { A }\end{array}$ & $\begin{array}{c}\text { Fallstudie } \\
\text { B }\end{array}$ & $\begin{array}{c}\text { Fallstudie } \\
\text { C }\end{array}$ & $\begin{array}{c}\text { Fallstudie } \\
\text { D }\end{array}$ \\
\hline $\begin{array}{l}\text { Veränderungs- } \\
\text { rate der } \\
\text { Organisation }\end{array}$ & extrem hoch & klein & hoch & klein \\
\hline $\begin{array}{l}\text { Stakeholder } \\
\text { Investoren, } \\
\text { Lieferanten, } \\
\text { Kunden }\end{array}$ & $\begin{array}{c}\text { Kunden, } \\
\text { Innovationen }\end{array}$ & $\begin{array}{c}\text { Gesellschaft, } \\
\text { Investoren, } \\
\text { Kunden, Staat, } \\
\text { Lieferanten }\end{array}$ & $\begin{array}{c}\text { Spender/Geldgeber } \\
\text { Klienten, } \\
\text { Gesellschaft, } \\
\text { Staat }\end{array}$ \\
\hline $\begin{array}{l}\text { Komplexität der } \\
\text { Organisation }\end{array}$ & hoch & mittel & mittel \\
\hline $\begin{array}{l}\text { Know-how der } \\
\text { Mitarbeiter }\end{array}$ & hoch & mittel & mittel & mittel \\
\hline $\begin{array}{l}\text { Reifegrad der } \\
\text { Organisation }\end{array}$ & tief & hoch & hoch & mittel \\
\hline Führungsstil & autoritär & patriarchisch & $\begin{array}{c}\text { autoritär/ } \\
\text { partizipativ }\end{array}$ & partizipativ \\
\hline $\begin{array}{l}\text { Planungs- } \\
\text { horizont }\end{array}$ & kurzfristig & langfristig & mittelfristig & langfristig \\
\hline
\end{tabular}

Tabelle 3-2: Charakterisierung der vier Szenarien 


\begin{tabular}{|l|c|c|c|c|}
\hline \multicolumn{1}{|c|}{ PM-Ansätze } & EFQM & $\begin{array}{c}\text { Balanced } \\
\text { Scorecard }\end{array}$ & $\begin{array}{c}\text { Intellectual } \\
\text { Capital }\end{array}$ & $\begin{array}{c}\text { Value-Based } \\
\text { Management }\end{array}$ \\
\hline $\begin{array}{l}\text { Anpassungs- } \\
\text { fähigkeit bzgl. } \\
\text { veränderter } \\
\text { Informations- } \\
\text { bedürfnisse }\end{array}$ & gross & klein & gross & mittel \\
\hline $\begin{array}{l}\text { Aufwand zur } \\
\text { Implementierung } \\
\text { (techn. \& org. } \\
\text { Teil) }\end{array}$ & mittel & gross & mittel & mittel \\
\hline $\begin{array}{l}\text { Aufwand zum } \\
\text { Betrieb }\end{array}$ & gross & gross & mittel & mittel \\
\hline $\begin{array}{l}\text { Notwendiges } \\
\text { Know-how der } \\
\text { Mitarbeiter }\end{array}$ & mittel & gross & mittel & klein \\
\hline $\begin{array}{l}\text { Notwendiger } \\
\text { Reifegrad der } \\
\text { Organisation }\end{array}$ & hoch & hoch & mittel & klein \\
\hline $\begin{array}{l}\text { Stellenwert des } \\
\text { Ansatzes als } \\
\text { Führungs- } \\
\text { instrument }\end{array}$ & gross & mittel & gross & \\
\hline
\end{tabular}

Tabelle 3-3: Typisierung der vier PMS-Ansätze

Die komprimierten Daten liefern die Grundlage für eine Empfehlung bezüglich des einzusetzenden Performance-Measurement-Konzeptes für die einzelnen Szenarien:

Fallstudie A verlangt eine hohe Flexibilität und Anpassungsfähigkeit, da sich die Unternehmenssituation rasch verändert. Entsprechend muss der Aufwand für die Implementierung beschränkt sein. Der Aufwand für den Betrieb ist zweitrangig, da sich die Situation rasch verändert. Die Mitarbeiter verfügen nur über ein beschränktes Wissen zum Thema Performance Measurement, da der Führungsbereich personell bisher unterdotiert war, wie bei kleineren Unternehmen üblich. Durch die mehrfachen Reorganisationen ist der Reifegrad der Organisation eher gering. Der Konzern führt seine Töchter primär über Finanzkennzahlen, entsprechend ist dieser Bereich besonders zu gewichten. Diese Überlegungen lassen die Wahl eines Value-Based-Management-Ansatz für dieses Szenario als geeignet erscheinen.

Das B-Szenario zeigt eine stabile und unabhängige Organisation. Obwohl längerfristig eine gesunde Finanzlage angestrebt wird, stehen doch die finanziellen Ziele nicht im Vordergrund. Die Dynamik der Organisation ist beschränkt. Entsprechend ist der Aufwand für die Implementierung eher unkritisch. Das Bewusstsein für Qualität, Kosten und Zeit 
(Flexibilität, Lieferbereitschaft) der Mitarbeiter ist hoch und die Prozesse in der Organisation sind etabliert. Ein umfassendes Bild der Organisations-Performance erscheint wichtig. Diese Überlegungen legen die Wahl des Balanced-Scorecard-Ansatzes oder allenfalls des EFQM-Ansatzes nahe.

Unternehmen C richtet seine Strukturen auf die bevorstehende Marktliberalisierung aus. In Zukunft werden die finanziellen Ergebnisse eine wichtige Rolle spielen; bis dato sind jedoch die Kunden und die Gesellschaft ebenso wichtige Kommunikationspartner. Aus diesem Grunde ist der Einsatz des VBM-Ansatzes eher nicht geeignet. Als produzierendes Unternehmen ist zudem das intellektuelle Kapital der Mitarbeiter nicht das dominierende Element. Die Heterogenität der verschiedenen Geschäftsfelder und die starke Notwendigkeit zur Kommunikation der Bedürfnisse lässt einen Balanced-ScorecardAnsatz als sinnvoll erscheinen.

Beim letzten Szenario D steht die effektive Mittelverwendung und die Kommunikation mit den Geldgebern im Vordergrund. Das Wissen der Mitarbeiter um diese Punkte nimmt einen sehr hohen Stellenwert ein. Aus diesen Überlegungen heraus wäre der Einsatz des Intellectual-Capital-Ansatzes denkbar. 


\section{Teil \\ Bestimmung der Anforderungen an ein Performance-Measurement-System}




\section{Anforderungen an ein PMS}

In den zwei vorgängigen Kapiteln wurden wichtige Grundlagen von PerformanceMeasurement-Systemen (PMS) und IT-Systemen für das PM vorgestellt, sowie vier populäre PM-Konzepte analysiert. In der Literatur findet sich kein systematischer Anforderungssatz für ein Performance-Measurement-System und nur wenige Hinweise zu möglichen Anforderungen an ein IT-System für das Performance Measurement. Daher ist es notwendig aus den Erfahrungen des dritten Kapitels, einer Analyse von empirischen Studien und eines ersten Prototypen eines IT-Systems für das PM die Anforderungen herzuleiten. Dies ist Gegenstand des vierten Kapitels und dient der Beantwortung der zweiten Forschungsfrage: Welche Anforderungen bestehen an PMS und an IT-Systeme für das PM? (siehe Kapitel 1.2).

Folgendes Vorgehen wurde gewählt:

(1) In einem ersten Schritt wird kurz auf das Thema „Anforderungen“ eingegangen.

(2) In einem zweiten Schritt werden die Anforderungen an ein PMS aus der Literatur zusammengetragen. Insbesondere werden die im Kapitel drei diskutierten Systeme berücksichtigt.

(3) Sodann werden empirische Studien auf relevante Erkenntnisse hin untersucht.

(4) Erfahrungen aus kommerziellen IT-Systemen für das PM (vgl. Anhang C) und einem eigenen ersten Prototypen werden berücksichtigt.

(5) Mit einer eigenen empirischen Studie, die vom Verfasser mit der Hilfe eines Diplomanden durchgeführt wurde, werden insbesondere die Anforderungen an das IT-System für das PM bezüglich ihrer Relevanz untersucht.

(6) Es resultiert ein Satz von Anforderungen, der unterschieden wird nach Anforderungen, die für das PMS im Allgemeinen und für das IT-System für das PM im Speziellen gelten. 
Nachfolgende Abbildung 4-1 zeigt den Aufbau schematisch.

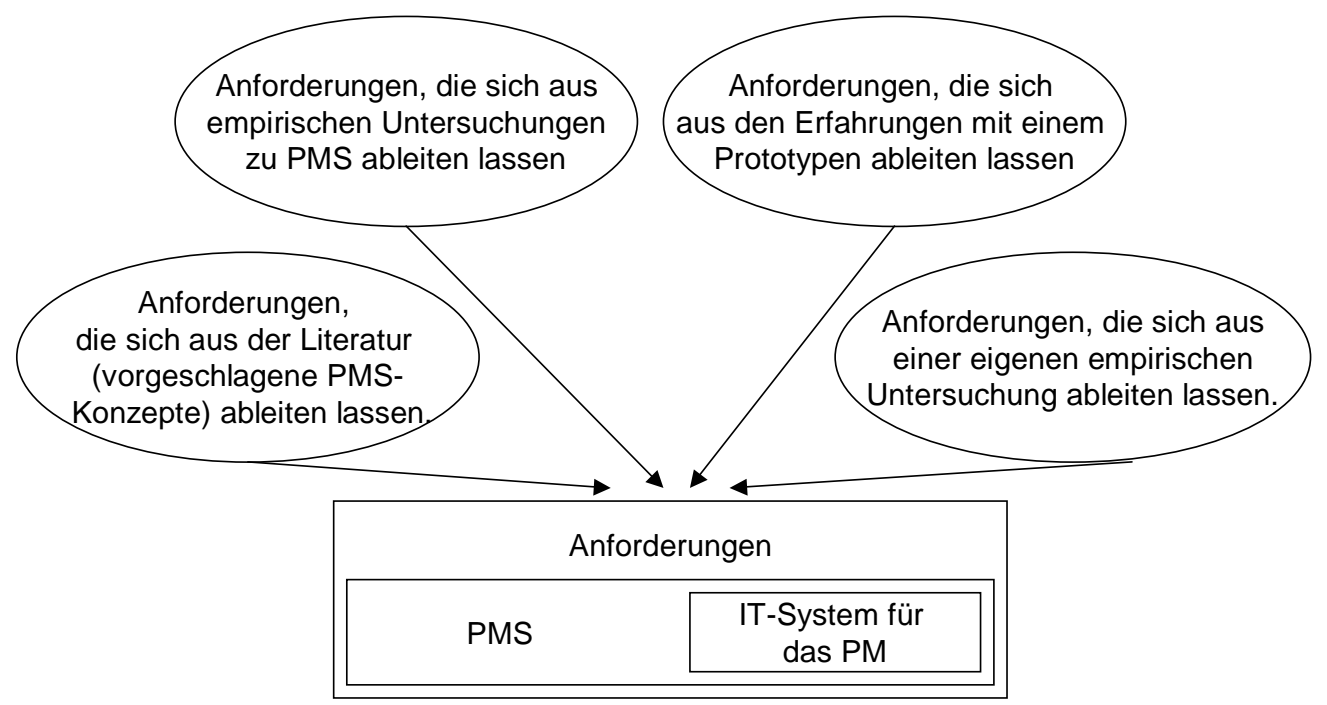

Abbildung 4-1: Bestimmung der Anforderungen

\subsection{Kategorien von Anforderungen}

Der Begriff der Anforderung wird im allgemeinen Sprachgebrauch als eine Bedingung oder Fähigkeit, die von einer Person zur Lösung eines Problems oder zur Erreichung eines Ziels benötigt wird, oder als eine Aufforderung, etwas zu erfüllen, aufgefasst (vgl. DUDEN (1999)). In betriebswirtschaftlichen Publikationen wird der Begriff häufig als allgemein verständlich angenommen und nicht näher definiert (vgl. als Beispiel: Stuber (2001), der sich in seiner Dissertation mit der Bestimmung von Anforderungen befasst).

Im Rahmen des System Engineering hat das korrekte Erheben, Dokumentieren, Verwalten und Überprüfen einen hohen Stellenwert, aus der sich eine eigene Disziplin, „Requirements Engineering“ entwickelt hat, die sich mit dieser Thematik auseinander setzt.

Im Fachbereich Requirements Engineering wird eine Anforderung wie folgt definiert: Gemäss Rupp (2001) ist „eine Anforderung eine Aussage über eine zu erfüllende Eigenschaft oder zu erbringende Leistung eines Produktes, eines Prozesses oder der am Prozess beteiligten Personen“. Andere Definitionen verwenden den Ausdruck als „eine Bedingung oder Fähigkeit, die ein System erfüllen oder besitzen muss, ...um zu erfüllen.“ (vgl. auch IEEE830 (1998)). 
Anforderungen können nach den unterschiedlichsten Kriterien gegliedert werden. Rupp (2001) erwähnt z.B. Gliederungsmöglichkeiten nach der rechtlichen Verbindlichkeit, der Priorität, der Art und der Detailstufe. Eine Unterteilung, die von den meisten Autoren getragen wird, unterscheidet funktionale und nicht-funktionale Anforderungen. Dies aus der Idee der Wiederverwendbarkeit heraus, da sich die funktionalen Anforderungen von Projekt zu Projekt verändern, während dem die nicht-funktionalen Anforderungen mindestens ihrer Form nach meistens gleich bleiben:

- Funktionale Anforderungen (functional requirements) umfassen Aktionen, die ein System ausführen soll, aber auch Interaktionen mit den Benutzern und anderen Systemen (vgl. Rupp (2001)).

- Nicht-funktionale Anforderungen (non-functional requirements) werden von einigen Autoren auch als geforderte Randbedingungen (required constraints) bezeichnet (vgl. Hatley et al. (1999)). Nicht-funktionale Anforderungen (NFA) sind Bedingungen, die an die Dienste bzw. Leistungen eines Systems gestellt werden. NFA beschreiben Systemeigenschaften und Randbedingungen, unter welchen das System arbeitet. Sie können eingeteilt werden in: (1) Eigenschaften (properties, qualities), Beispiele: Zuverlässigkeit, Erweiterbarkeit, Portabilität und (2) Randbedingungen (constraints, resources), Beispiele dazu sind: max. Speicherbedarf, max. Antwortzeiten, Einsatz gegebener Hardware.

An Anforderungen selbst werden eine Reihe von Qualitätskriterien gestellt. Nach Rupp (2001) soll jede einzelne Anforderung folgende Eigenschaften erfüllen:

- vollständig (D.h. die geforderte oder zu liefernde Funktionalität muss vollständig beschrieben werden.)

- korrekt (Eine Anforderung ist dann korrekt, wenn sie vollständig die Vorstellung des Stakeholders wiedergibt.)

- $\quad$ klassifizierbar bezüglich der juristischen Verbindlichkeit

- $\quad$ konsistent gegenüber anderen Anforderungen (widerspruchsfrei) und in sich konsistent

- testbar (Eine Anforderung muss so beschrieben sein, dass sich daraus Testfälle ableiten lassen, die zu einem konkreten Ergebnis führen.)

- für alle Stakeholder verständlich

- $\quad$ notwendig 
- verfolgbar (Dabei wird insbesondere auf die Problematik hingewiesen, dass sich Anforderungen im Zeitablauf verändern können und sodann die Verfolgbarkeit schwieriger wird.)

- bewertbar (Bei einer grossen Anzahl von Anforderungen gewinnt dieser Punkt an Bedeutung: Anforderungen sollten dann nach Priorität, Wichtigkeit, etc. bewertet werden können.)

- eindeutig (Eine eindeutige Anforderung kann nur auf eine Art und Weise verstanden werden.)

Die vorgestellten Erkenntnisse aus dem Requirements Engineering sollen nachfolgend bei der Erarbeitung der Anforderungen berücksichtigt werden.

\subsection{Anforderungen an PMS aus der Literatur}

In diesem Abschnitt sollen die Anforderungen aus dem bisher Diskutierten (vgl. Kapitel zwei und drei) abgeleitet werden. Um die einzelnen Anforderungen im weiteren Verlauf der Arbeit der Quelle zuordnen zu können, werden diese mit einem Buchstaben und einer fortlaufenden Nummer versehen. Dabei werden die Anforderungen aus der Literatur mit einem „A“, diejenigen aus den empirischen Studien mit einem „B“ und diejenigen aus der eigenen empirischen Untersuchung mit „C“ bezeichnet.

Zu den Performance-Indikatoren können folgende Anforderungen formuliert werden:

- A-1: Berücksichtigung von finanziellen und nicht-finanziellen PerformanceIndikatoren (stellvertretend für zahlreiche andere Autoren schreibt Kaplan (1984)): „In summary, the financial measures generated by traditional cost accounting systems provide an inadequate summary of a company's manufacturing operations. Today's global competition requires that non-financial measures - on quality, investory levels, productivity, flexibility, deliverability, and employees - also be used in the evaluation of a company's manufacturing performance." und in Johnson und Kaplan (1991): „More important than ... is measuring and reporting a variety of nonfinancial indicators.” (vgl. ebenfalls Keegan et al. (1989), Lynch und Cross (1991a), Kaplan und Norton (1992)). 
- A-2: Berücksichtigung von Leading und Lagging-Indikatoren resp. Treibern und Ergebnissen resp. vorauslaufenden- und nachlaufenden Indikatoren (z.B. Fitzgerald et al. (1991)).

- A-3: Berücksichtigung von internen und externen Performance-Indikatoren (vgl. Keegan et al. (1989)): der Einbezug von externen Performance-Indikatoren erlaubt ein kontinuierliches Benchmarking. Explizit unterstützt wird dies von Eccles (1991). Neely et al. (1995) erwähnen, dass die Frage „what are our competitors doing“ fundamental sei und im Rahmen eines PM-Konzeptes berücksichtigt werden müsse. Epstein und Roy (2001) schreiben „...benchmarking systems to monitor competitors ... performance should also be used ...").

- A-4: Indikatoren können danach unterschieden werden, ob sie der Wirkungs-, Durchführungs- oder Prämissenkontrolle dienen (vgl. Weber (1998)).

Performance-Indikatoren werden mit den Zielen und Zielvorgaben verknüpft:

- A-5: Indikatoren werden mit Zielen verknüpft, d.h. die Indikatoren reflektieren die Ziele der Organisation (vgl. Brown und Laverick (1994), Kaplan und Norton (1992)).

- A-6: Es werden mehrere Ebenen bei der Leistungsmessung berücksichtigt (z.B. Unternehmen, Business Units, Prozesse, Abteilungen, Projekte, Mitarbeiter) (vgl. bspw. Lynch und Cross (1991a), Rummler und Brache (1990)).

- A-7: Die Ziele werden aus der Strategie hergeleitet (vgl. z.B. Rappaport (1995): „,... letztlich muss die Performance-Messung direkt mit dem strategischen Plan verknüpft werden, um eine effektive Implementierung des Plans zu fördern. “; sowie Johnson und Kaplan (1991): „,The indicators should be based on the company’s strategy .... “ (vgl. ebenfalls Kaplan und Norton (1992)).

- A-8: Die Ziele repräsentieren die Interessen von verschiedenen Stakeholdern (vgl. z.B. Atkinson et al. (1997); Neely et al. (1995) teilen selbst die Meinung, hinterfragen aber, ob nicht der Shareholder zuoberst stehe und die anderen Stakeholder nur als Mittel zum Zweck gesehen werden.).

- A-9: Für Performance-Indikatoren können Zielvorgaben (Soll-Werte) für bestimmte Zeitperioden verwaltet werden (vgl. Kaplan und Norton (1993)). 
Die angestrebte Beeinflussung der Mitarbeiter und Manager (siehe z.B. bei Kaplan und Norton (1997): „Die Kommunikation der Zielsetzungen und Kennzahlen der BSC ist der erste Schritt zur Gewinnung des individuellen Engagements für die Strategie der Geschäftseinheit. “) und anderer Stakeholder (als Beispiel: Edvinsson und Malone (1997)), sowie der Anspruch der ständigen Verbesserung (vgl. Hakes (1996)) erfordern eine intensive Kommunikation der Performance-Daten. Daraus können die folgenden Anforderungen abgeleitet werden:

- A-10: Das PMS kann die Performance-Daten an sämtliche relevanten Stakeholder in der geeigneten Granularität kommunizieren.

- A-11: Die Erreichung der Zielvorgaben wird mit einem Anreiz- und Belohnungssystem verknüpft (vgl. Eccles (1991)).

Die Anforderung A-11 wird jedoch nicht von sämtlichen Autoren unkritisch besehen. Neely (1998) schreibt: „as soon as performance measures are used as a means of control, the people being measured begin to manage the measures rather than performance."

Zur Vermeidung eines Information Overload (im Speziellen Überschwemmung mit papierbasierten Berichten) empfiehlt sich der Einsatz von Informationstechnologie (vgl. Epstein und Roy (2001): „Undoubtedly, the development and implementation of a reliable information system for ... is essential. “) für die Kommunikation und die bekannten Pushund Pull-Verfahren (vgl. Wettstein et al. (2001)).

- A-12: Die Performance-Resultate können auf elektronischem Weg kommuniziert werden. Das IT-System für das PM unterstützt das Push-Prinzip.

- A-13: Das IT-System für das PM unterstützt das Pull-Prinzip: Die Anwender können sich im Rahmen ihrer Berechtigung frei in der Performance-Datenbank bewegen (navigieren).

- A-14: Das IT-System für das PM erlaubt es explizit auch externen Stakeholdern, elektronisch auf die für sie bestimmten Performance-Daten zuzugreifen.

- A-15: Um der Anforderung nach der geeigneten Granularität der Daten (vgl. A-10) nachzukommen, ist es notwendig, dass die Daten eines Performance-Indikators auf verschiedenen Granularitätsstufen verfügbar sind. 
Die Analyse der Performance-relevanten Daten bedingt folgende Anforderungen:

- A-16: Ein exploratives Suchen (z.B. Drill-Down, -Up, -Across) in den PerformanceDaten wird unterstützt. (Diese Anforderung kann begründet werden durch eine Suche nach Ursachen und somit entscheidungsunterstützend wirken).

- A-17: Differenzen zwischen erreichter und geplanter Performance können dargestellt werden. (Überprüfung der Zielerreichung, z.B. bei Kaplan und Norton (1996a); resp. die so genannte Abweichungskontrolle und -analyse, vgl. Horváth (2001)).

- A-18: Unterstellte Wirkungsnetze können mit geeigneten statistischen Instrumenten verifiziert werden. (Die ex ante angenommenen Wirkungsnetze sollten periodisch überprüft werden. Epstein und Roy (2001): „to applying the model to support decisionmaking”).

- A-19: Analysen verlangen die Verfügbarkeit von Performance-Daten über einen längeren Zeitraum (bspw. um Trends zu extrapolieren oder saisonale Schwankungen erklären zu können).

Implizit in jedem der Vorschläge der verschiedenen Autoren enthalten ist die Anforderung, dass eine klar dokumentierte Anleitung (Prozess) besteht, wie ein PMS aufgebaut werden kann.

- A-20: Klar definierter Prozess für den Aufbau des Performance-MeasurementSystems.

Einige Autoren (vgl. z.B. Kaplan und Norton (1996a) und Epstein und Roy (2001)) erwähnen auch explizit die Notwendigkeit, dass der Betrieb und Unterhalt eines PMS klar geregelt werden muss.

- A-21: Der Betrieb und Unterhalt eines PMS muss klar geregelt werden.

\subsection{Erkenntnisse aus empirischen Untersuchungen zu Fragen des Performance Measurement}

Neben den in verschiedenen PM-Konzepten formulierten Anforderungen, existiert eine ganze Reihe von empirischen Untersuchungen zu Fragen des Performance Measurement. 
Deren Ergebnisse sollen nachfolgend bezüglich möglicher Anforderungen untersucht werden. Die Zahl der existierenden Untersuchungen zum Thema Performance Measurement ist sehr gross. Um sich einen Überblick verschaffen zu können, werden diese nachfolgend ihrem Fokus entsprechend klassifiziert. Dabei werden folgenden Kategorien unterschieden:

- Verbreitung von bestimmten Performance-Indikatoren

- Wirkungszusammenhänge von verschiedenen Indikatoren im Allgemeinen und auf die unternehmensspezifische Performance im Speziellen

- Verbreitung von Performance-Measurement-Konzepten

- Ausgestaltung und Reifegrad von PMS

- IT-Systeme für das PM

\subsubsection{Verbreitung von bestimmten Performance-Indikatoren}

Untersuchungen dieses Typs reichen bis in die 60er-Jahre des 20. Jahrhunderts zurück. Im amerikanischen Raum sind zum Beispiel Reece und Cool (1978) zu erwähnen. Dabei handelt es sich um eine Untersuchung von 600 aus den Fortune-1000 Unternehmen bezüglich der Verbreitung von ROI (Return-On-Investment) und der RI (ResidualIncome)-Methode zur Bestimmung der Leistung von Business Units. Die Autoren der Studie differenzieren Investment- und Profit-Centers und diskutieren die Vor- und Nachteile der Berechnungsmethoden. Bei Watty und Terzioglu (1999) findet sich eine Zusammenstellung von mehr als 10 weiteren empirischen Studien, die Performance Measures zum Gegenstand haben. In ihrem Literaturreview stellen die Autoren fest, dass im zeitlichen Ablauf eine deutliche Veränderung der Art der Performance-Indikatoren festzustellen sei. Standen in den 60er-Jahren primär finanzielle Indikatoren im Zentrum (vgl. bspw. Reece und Cool (1978) und Mauriel und Anthony (1966)), interessierten sich die Forscher später vermehrt für Performance-Indikatoren aus dem Produktionsumfeld und in den späten 80ern und frühen 90ern auch für Performance-Indikatoren aus dem Dienstleistungsumfeld (vgl. z.B. Fitzgerald et al. (1991)).

- B-1: Die zitierten Studien zeigen, dass Performance-Indikatoren aus sämtlichen Funktionsbereichen der Organisation, sowohl finanzieller als auch nicht-finanzieller Natur berücksichtigt werden sollten. 


\subsubsection{Wirkungszusammenhänge von verschiedenen Indikatoren}

Studien dieser Gruppe beleuchten die Wirkungszusammenhänge zwischen verschiedenen Indikatoren (z.B. Kundenzufriedenheit und Cashflow). In diesem Bereich ist sicher das Profit Impact on Market Strategies (PIMS)-Modell zu nennen, welches in den 60er-Jahren des 20. Jahrhunderts von General Electric (GE) begründet wurde (vgl. SPI (2002)). Die Intention dieser Studie ist es, jene Faktoren zu bestimmen, welche für den Return-OnInvestment (ROI) und den Cashflow einen massgeblichen Einfluss haben. Nach anfänglich kleineren Stichproben wurde die Untersuchung von der Harvard Business School und heute vom Strategic Planning Institute (SPI) of Cambridge in Massachusetts durchgeführt. Das SPI ist im Besitz der partizipierenden Unternehmen. Sowohl die Anzahl der Indikatoren als auch die Anzahl der partizipierenden Unternehmen wurde stetig ausgeweitet. Heute werden mehr als 200 Indikatoren bei über 3000 Business Units von ca. 450 Unternehmen erhoben. Typische Fragestellungen, die die Datenbank beantworten kann, sind: (1) Zahlen zu Marktanteilen, relative Preise von Business Units, (2) durchschnittlicher ROI einer schnell wachsenden Branche, (3) Korrelation zwischen Marktanteil und Qualität für ein Unternehmen in der Investitionsgüterbranche. (4) Wie wirkt sich eine Erhöhung der Forschungs- \& Entwicklungsausgaben auf ein Produkt in ein, zwei oder drei Jahren aus?

Die beiden McKinsey-Berater Peters und Waterman (1982) befragten 20 als „excellent“ geltende Unternehmen nach ihrem „Erfolgsgeheimnis“. Aus der Studie resultierten 8 Erfolgsdimensionen (Primat des Handelns, Kundennähe, unternehmerischer Freiraum, Produktivität durch Menschen, sichtbar gelebtes Wertesystem, Fokussierung auf das angestammte Geschäft, flexible, überschaubare Aufbauorganisation, straff/lockere Unternehmensführung). Daraus wurde gefolgert, dass die Beachtung dieser Punkte wesentlich zum Erfolg des Unternehmens beiträgt.

Da sich diese Arbeit im Umfeld der Wirtschaftsinformatik bewegt, soll speziell auf den Zusammenhang von IT und Unternehmenserfolg hingewiesen werden. Seit mehr als 20 Jahren haben eine Reihe von Autoren versucht, diesen Zusammenhang empirisch nachzuweisen. Einige bekannte Arbeiten stammen von Rockart (1979) und McFarlan (1984). Der eindeutige Zusammenhang zwischen Investitionen in die IT und dem Unternehmenserfolg konnte zum Leidwesen der Informatikbranche bis dato nicht schlüssig nachgewiesen werden. Ein wesentliches Problem besteht in der fehlenden Möglichkeit, Ausseneinflüsse geeignet zu berücksichtigen. Zudem besteht eine Kontroverse darüber, ob 
die geplante (intended) oder die realisierte (emergent) Unternehmensstrategie mittels Informationstechnologie unterstützt werden soll (vgl. Marchand et al. (2001) und Expertengespräch mit William Kettinger vom 7. August 2001 in Boston).

Marchand et al. (2001) untersuchen in einer Studie, die von September 1997 bis Juli 1999 dauerte, die Auswirkung der Information Orientation auf die Business Performance. Information Orientation wird dabei von den drei Kategorien „IT practices“, „Information management practices“ und „Information behaviors and values“ bestimmt. Insgesamt beteiligten sich 376 Unternehmen mit über 1000 Personen aus dem höheren Management an der Studie. Die Unternehmen sind in insgesamt 26 Ländern domiziliert und gehören 25 verschiedenen Branchen an (vgl. Marchand et al. (2001)). Mit den Ergebnissen der Studie können die Autoren konkrete Hinweise zum Wirkungszusammenhang IT und Geschäftsergebnis geben. Sie nehmen an, damit zum effizienteren IT-Ansatz beitragen zu können. Inwiefern sich die gefundenen Zusammenhänge in der Praxis bestätigen, wird die Zukunft zeigen. Nach Auffassung des Verfassers handelt es sich um einen viel versprechenden Ansatz.

- B-2: Die vermuteten Wirkungsnetze sollten sowohl ex ante als auch ex post dargestellt und persistent gespeichert werden können.

- B-3: Zudem sollten geeignete statistische Funktionen die Überprüfung von Wirkungszusammenhängen unterstützen.

\subsubsection{Verbreitung von Performance-Measurement-Konzepten}

In einer empirischen Studie des Lehrstuhls für Controlling der Universität Dresden (vgl. Günther und Grüning (2000)) wurde der Frage nachgegangen, wie weit PMS in deutschen Unternehmen eingesetzt werden. Untersucht wurde die Struktur der Systeme, die geplante Weiterentwicklung und die Nutzung der Systeme. Fünf Themengebiete wurden in einem Fragebogen, der an 942 deutsche Unternehmen ausgesandt wurde, abgefragt:

- Wie populär sind PMS, und welche Konzepte werden eingesetzt?

- Welche Arten von Performance-Indikatoren werden verwendet und in welchem Verhältnis stehen diese zu der Strategie?

- Wie erfolgt die Anpassung des Systems an neue Herausforderungen? 
- Inwieweit ist das PMS mit einem Anreizsystem verbunden und welche hierarchischen Ebenen werden abgedeckt?

- Wie beurteilen die Unternehmen ihr PMS, wo sehen sie Probleme und Schwierigkeiten?

Die Stichprobe umfasste lediglich Unternehmen mit einem Umsatz von mehr als 400 Mio. Euro und ist demnach nicht repräsentativ. Die Rücklaufquote betrug rund 20\%, was 180 Antworten entspricht (vgl. Günther und Grüning (2000)).

37\% dieser Unternehmen setzen ein PMS ein und 16\% führen gerade ein solches ein. 15\% prüfen den Einsatz und 32\% sehen den Einsatz eines PMS nicht vor. Unternehmen, die kein PMS einsetzen, geben die folgenden vier Gründe mit abnehmender Wichtigkeit an: (a) Finanzielle Kontrollsysteme sind ausreichend, (b) hohe Kosten für ein PMS, (c) kein passendes PM-Konzept gefunden und (d) Priorität für andere Projekte höher (vgl. Günther und Grüning (2000)).

Hudson et al. (1999) untersuchten PMS in KMU’s. Sie erwähnen drei Hauptpunkte für das Scheitern solcher Projekte: Einer davon betrifft den sehr aufwändigen Aufbau und Unterhalt von PMS.

- B-4: Ein PMS sollte so ausgelegt werden, dass das Kosten-Nutzen-Verhältnis überprüft werden kann.

Das weitverbreiteste PM-Konzept ist die Balanced Scorecard. Danach folgen die ValueBased-Management-Konzepte und das ROI-Konzept. Eine Reihe von Unternehmen setzt das ZVEI-Kennzahlensystem ein (vgl. Siegwart (1998) zum ZVEI-System). Sowohl das ROI- als auch das ZVEI-Konzept geniessen eine sehr grosse Bekanntheit, was sich auch durch die weite Verbreitung in deutschen Lehrbüchern erklären lässt. Zahlreiche BalancedScorecard- und Value-Based-Management-Projekte befinden sich zurzeit in der Implementierungsphase. Es ist daher davon auszugehen, dass in Zukunft deren Nutzung noch zunehmen wird. Die Zahl der selbstentwickelten PM-Konzepte ist nach wie vor dominierend. Es ist jedoch in Zukunft von einer Substitution mit oben erwähnten Ansätzen auszugehen. (vgl. Günther und Grüning (2000))

69\% der Unternehmen entwickeln ihr eigenes PMS basierend auf dem gewählten Konzept. Weitere 23\% erwerben ein PMS und passen es ihren Bedürfnissen an. Damit wird deutlich, dass PMS keine „ready-to-use products“ sind. (Günther und Grüning (2000)) 
In einer 1997 durchgeführten Studie von Horváth setzten 36\% aller deutschen Unternehmen kein PMS ein (vgl. Horváth (1999)). Die gleiche Studie weist ein Anteil von 8.9\% aus die Balanced Scorecard einsetzten; 20\% planten den Einsatz einer BSC.

In einer 1998 in den USA durchgeführten Studie des Institute of Management Accountants (vgl. Frigo und Krumwiede (1999)) gaben 22\% der Unternehmen an, eine Balanced Scorecard zu nutzen. Eine Studie von Fleischhauer (1998) findet für die Balanced Scorecard in Deutschland eine Penetration von 17.4\%. Seidenschwarz und Gleich ((1998), zitiert nach Hoffmann (1999)) erwähnen, dass die Verbreitung der Balanced Scorecard in Deutschland nicht sehr stark ausgeprägt war.

- B-5: Ein IT-System für das Performance Measurement sollte mehrere PM-Konzepte unterstützen (z.B. das Balanced Scorecard, das Value-Based Management, das EFQMModell und das Intellectual-Capital-Modell).

- B-6: Ein PMS wird nur in Ausnahmefällen das einzige Führungssystem einer Organisation sein. Aus diesem Grunde muss das PMS mit anderen Führungssystemen verknüpfbar sein.

\subsubsection{Ausgestaltung und Reifegrad von PMS}

Eine dominierende Mehrheit der Unternehmen (90\%) passt ihr PMS nur bei Bedarf an. Nur wenige Unternehmen adaptieren das PMS systematisch einmal jährlich (16\%) resp. einmal pro Quartal (5\%) an die Veränderungen (vgl. Günther und Grüning (2000)). Küng (2001) findet in seiner qualitativen Studie heraus, die acht grosse Schweizer Unternehmen der Dienstleistungsbranche untersuchte, dass die Kennzahlen nicht immer aktuell sind. Bitici und Carrie (1998) untersuchten die PMS von über 30 europäische Unternehmen. Sie stellten fest, dass die Mehrheit der PMS vergangenheitsorientiert und statisch sind. Daraus folgt, dass die gemessenen Informationen häufig nicht relevant, aktuell und akurat sind. Hudson et al. (1999) finden in ihrer Studie ebenfalls, dass die Performance-Daten häufig veraltet und irrelevant sind.

- B-7: Ein PMS muss geeignete Mechanismen für den Unterhalt umfassen.

Weiter findet Küng (2001), dass die Mitarbeiter mangelhaft einbezogen werden, und die Prozesse nur bruchstückhaft gemessen werden. 
- B-8: Ein PMS berücksichtigt mehrere organisatorische Ebenen (Mitarbeiter, Abteilungen, Prozesse, Business Units, etc.), die in einem Verhältnis zu einander stehen.

Marr und Neely (2000) kommen zum Schluss, dass eine der grössten Schwachstellen in der Erfassung der Performance-relevanten Daten liegt. Einerseits ist es schwierig, die notwendigen Daten zu lokalisieren, und andererseits ist der Aufwand zur Sammlung der relevanten Performance-Daten erheblich.

- B-9: Ein PMS muss geeignete Verfahren bereitstellen, um Performance-relevante Daten zu identifizieren und zu sammeln.

\subsubsection{IT-Systeme für das PM}

Bitici und Carrie (1998) erwähnen in ihrer Studie den Punkt der fehlenden ITUnterstützung der Performance-Measurement-Systeme. Dadurch wird die Datensammlung, -verwaltung und -berichterstattung schwerfällig.

- B-10: Ein effizientes PMS muss mittels IT unterstützt werden.

Weiter erkennt Küng (2001), dass die Performance-Daten verstreut gespeichert werden, und dies zu mehreren Nachteilen führt.

- B-11: Das IT-System für das PM sollte in einer zentralen Datenbank gespeichert werden.

Die Studie von Bourne et al. (2000) zeigt, dass der Zugriff auf die operativen Systeme essenziell ist. In denjenigen Unternehmen, in welchen der Zugriff des PMS auf die operativen Systeme nicht gegeben war, konnten die Performance-Resultate nicht innerhalb der geforderten Zeit produziert werden.

- B-12: Das IT-System für das PM verfügt über standardisierte Schnittstellen (z.B. XML) und ETL-Mechanismen (Extraction-Transforming-Loading), um Performancerelevante Daten effizient aus den operativen Informationssystemen übernehmen zu können. 


\subsection{Empirische Studie zum Thema „Stand des Performance Measurement und deren IT-Systeme in der Praxis“}

Im Bereich der Anforderungen an ein IT-System für das PM existieren wenig Daten. Aus diesem Grund, und mit der Absicht, einige der gefundenen Anforderungen zu verifizieren, entschloss sich der Verfasser, die vorhandenen Untersuchungsergebnisse im Rahmen einer eigenen empirischen Studie geleitet durch den Autor und unter Mithilfe eines Diplomanden am Departement für Informatik ${ }^{5}$ zu verifizieren und allenfalls zu ergänzen.

\subsubsection{Gegenstandsbereich und Methode der empirischen Studie}

Die Anforderungen an ein PMS wurden in der Literatur im Wesentlichen vor zehn Jahren erarbeitet. Die vorgeschlagenen Konzepte wurden von Wissenschaft, Beratern und Unternehmenspraxis intensiv untersucht, angewandt und eingesetzt. Auch wenn einige Teilaspekte nach Ansicht des Verfassers noch nicht befriedigend behandelt wurden, so sollen diese hier dennoch nicht hinterfragt werden. Interessant erscheint vielmehr der Entwicklungsstand in den Schweizer Unternehmen, d.h. in welchem Umfang diese Anforderungen umgesetzt wurden. Diese Fragestellung bildet ein Schwerpunkt der Studie.

Bezüglich der Anforderungen an ein IT-System für das Performance Measurement bestehen weit grössere Unsicherheit. Wie die zitierten empirischen Untersuchungen zeigen, haben die Unternehmen im Allgemeinen noch wenig Erfahrungen mit entsprechenden Systemen. Zudem ist die technologische Entwicklung von Bedeutung. Einige wichtige Basistechnologien (OLAP-Tools, Internet) sind in der Breite erst seit kurzem verfügbar, weshalb sich in diesem Bereich eine Validierung der Anforderungen aus der Literatur und eine Bestandesaufnahme der unternehmerischen Praxis anbietet.

Gewählt wurde für diese Untersuchung die Form eines Fragebogens, um eine grössere Stichprobe mit beschränkten Ressourcen zu erreichen. Um eine möglichst hohe Datenqualität zu Gewähr leisten, den Rücklauf zu maximieren und die Zeit für die Datenerfassung zu minimieren, wurde der Fragebogen in Papierform und als elektronischer Fragebogen aufbereitet. Wie in Küng und Wettstein (1999) ausgeführt, eignet sich die vorliegende Untersuchung sehr gut für eine elektronische Umsetzung.

5 An dieser Stelle sei Christian Beyeler für die wertvolle Mitarbeit gedankt. 
Die Umfrage erfolgt personalisiert; dies aus der Überlegung heraus, dass bei Unklarheiten Rückfragen möglich sein sollten.

\subsubsection{Aufbau des Fragebogens}

Der insgesamt zehnseitige Fragebogen (siehe Anhang A, Abbildungen A-1 bis A-11) besteht aus einem Vorspann und vier Teilen. Der Vorspann erklärt die wichtigsten Begriffe, verweist auf weiterführende Informationen und klärt administrative Fragen. Der erste Teil überprüft den Stand der Performance-Measurement-Systeme mit insgesamt zehn Fragen. Untersucht werden die eingesetzten Führungsinstrumente, die PerformanceMessung auf der strategischen Ebene (Strategieumsetzung, strategische Projekte, Risiken, Unternehmensumwelt) und auf der operativen Ebene, die Kommunikation, der Nutzen und die Qualität der Performance-Daten. Der zweite Teil widmet sich dem Stand der ITSysteme für das PM. Es wird abgefragt, ob und welche Softwarepakete und/oder Entwicklungswerkzeuge eingesetzt werden. Zudem wird überprüft, inwiefern die operativen Informationssysteme Daten für das IT-System für das PM liefern.

Der dritte Teil untersucht die Anforderungen und Leistungsvermögen der IT-Systeme für das PM mittels einer SERVQUAL-Metrik (vgl. Parasuraman et al. (1988)). SERVQUAL steht für Service-Quality und wurde von den oben erwähnten Autoren entwickelt, um die Service-Qualität aus Kundensicht erheben zu können. Dabei bewertet der Befragte Aussagen bezüglich der eingetretenen und erwarteten Leistung. In dieser Form wurden insgesamt 20 Aussagen formuliert. Sie gruppieren sich nach den Themen PerformanceIndikatoren, Datensammlung, -speicherung, -analyse und -kommunikation. Der letzte Teil bietet Raum für Anmerkungen und die Möglichkeit, den Studienbericht zu bestellen.

\subsubsection{Elektronische Umsetzung des Fragebogens}

Unternehmen werden in neuerer Zeit mit einer grossen Anzahl von Umfragen belastet (mitunter mehrere jede Woche). Um eine Antwort zu erhalten, muss also einerseits das Interesse geweckt werden, aber auch der Komfort bei der Teilnahme an der Untersuchung maximiert werden. Es besteht die Hoffnung, mit einem attraktiven Internet-Fragebogen diese Anforderungen zu erfüllen. Für die Gestaltung des Fragebogens wurden die Erfahrungen aus mehreren ähnlich gelagerten Projekten verwendet (so hat der Verfasser selbst mehrere Untersuchungen mit elektronischem Fragebogeneinsatz durchgeführt, u.a. bei Roche Basel (vgl. Küng und Wettstein (1999)), an der Universität Fribourg (vgl. Clark 
und Wettstein (2000)) sowie ohne wissenschaftlichen Hintergrund bei einer Informatikunternehmung in den Jahren 1999 und 2000; zudem wurden vom Verfasser Diplomarbeiten in diesem Bereich betreut: Huber (2001), Villalobos (2000)). Die technische Umsetzung wurde vom erwähnten Diplomanden im Rahmen seiner Diplomarbeit vorgenommen (vgl. Beyeler (2002)). Abbildung 4-2 zeigt beispielhaft einen Screenshot.

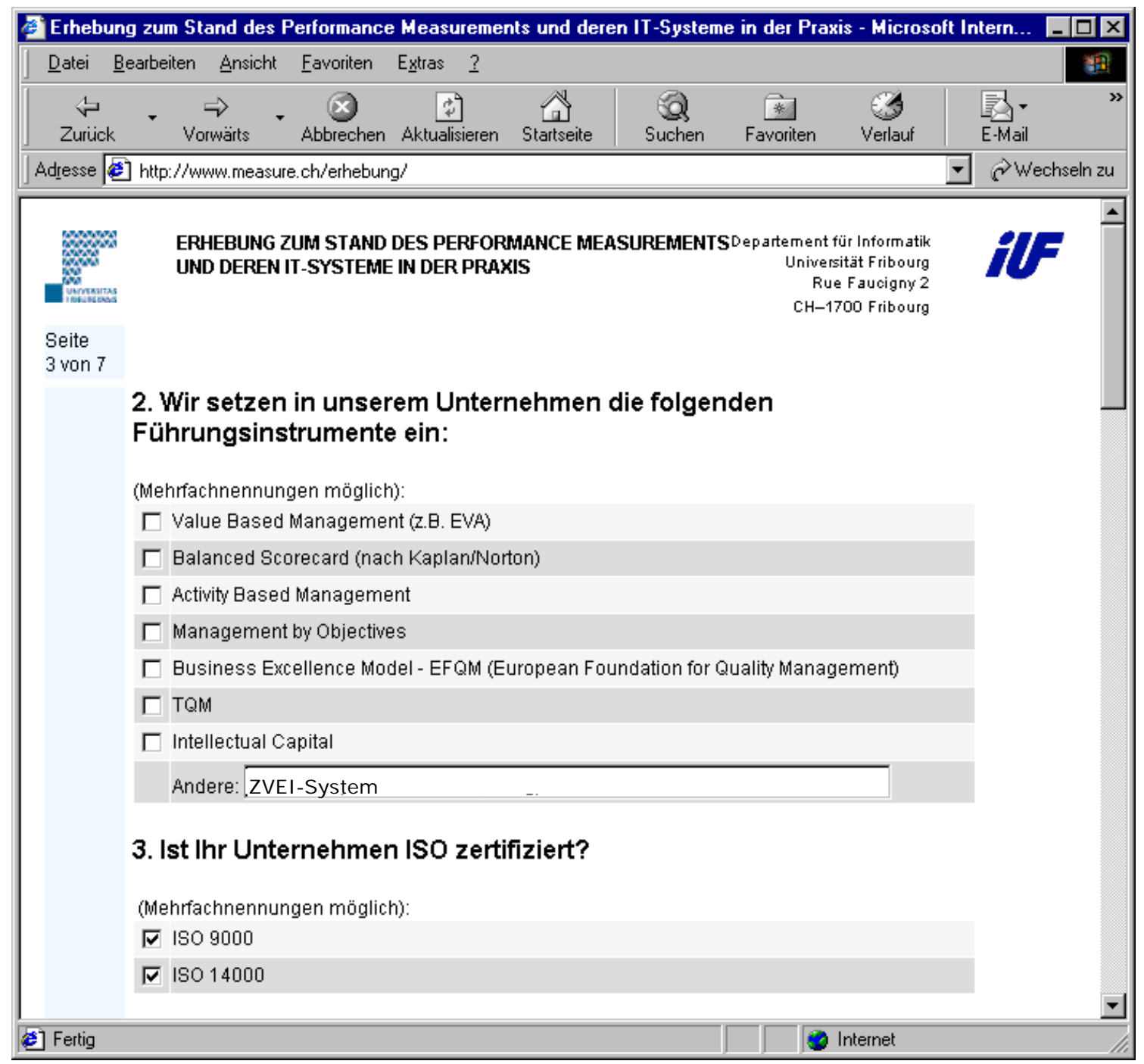

\section{Abbildung 4-2: Screenshot aus dem elektronischen Fragebogen}

Die Identifikation der Antwortenden erfolgt mit einem eigens für die Teilnehmer eingerichteten Zugangsnamen und Passwort, die mittels Brief mitgeteilt wurden (vgl. Anhang A, Abbildung A-12). Die Daten werden direkt in einer Microsoft SQL ServerDatenbank gespeichert und stehen mehreren Personen in der Organisation zur Verfügung. Der Fragebogen kann zudem bei Bedarf überarbeitet werden. Für Details sei auf die 
Diplomarbeit von Christian Beyeler verwiesen, die voraussichtlich Ende 2002 am Departement für Informatik abgeschlossen wird. Eine ausführlichere Dokumentation des elektronischen Fragebogens findet sich zudem im Anhang A.

\subsubsection{Stichprobe der Umfrage}

Es wurden bewusst grössere schweizerische Unternehmen für die Untersuchung berücksichtigt, da davon auszugehen ist, dass IT-Systeme für das PM noch nicht sehr weit verbreitet sind (vgl. Kapitel 4.3.3) und deren Aufbau kostenintensiv ist. Da bereits eine qualitative Studie zum Thema Performance Measurement bei den acht grössten Schweizer Dienstleistungs-Unternehmen von einem Mitarbeiter des Departementes für Informatik (vgl. Küng (2001)) im Jahr 2000 durchgeführt wurde, sind diese Unternehmen für die Umfrage nicht berücksichtigt worden.

Bei den Grossunternehmen wurde von Küng (2001) ermittelt, dass die einzelnen Business Units häufig eine grosse Autonomie bezüglich des Performance Measurement haben. Aus diesem Grunde entschloss sich der Verfasser, sämtliche deutschschweizerischen Unternehmen, die an der Schweizer Börse kotiert sind und im Swiss Performance Index (SPI) nicht aber im Swiss Market Index (SMI, dieser umfasst rund 28 grosse Unternehmen, wobei die Grösse über das Handelsvolumen und die Marktkapitalisierung bemessen wird) enthalten sind, für die Umfrage zu berücksichtigen. Durch die erhöhte Publizitätspflicht dieser Unternehmen ist es verhältnismässig einfach, korrekte Anschriften, Ansprechpersonen, Telefonnummern und E-Mail-Adressen zu erhalten. Die Wahl dieser Stichprobe wurde zudem beeinflusst durch die Absicht des Verfassers, in einer eigenen Publikation zu untersuchen, inwiefern der Einsatz von PMS mit dem Erfolg von Unternehmen korreliert.

Insgesamt sind 276 Schweizer Unternehmen an der Schweizer Börse kotiert. Abzüglich der 28 SMI-Titel verbleiben 242 (Stand November 2001). Diese wurden um Unternehmen aus der Westschweiz und der italienischen Schweiz korrigiert. Ingesamt wurden 160 Fragebogen versandt. Als Ansprechperson wurde die für Investor Relation bezeichnete Stelle gewählt. 


\subsubsection{Ergebnisse der Studie}

\subsubsection{Beteiligung}

Die Fragebogen wurden Anfang Dezember 2001 versandt. Zeitgleich wurde auch die Internetversion bereitgestellt. Die Rücklaufquote betrug Ende Dezember 19\%, nach einer telefonischen Erinnerung konnte der Rücklauf bis Mitte Januar 2002 auf 24\% erhöht werden (absolut 39 gültige Antworten). Die Fragebogen wurden am häufigsten durch den Chief Financial Officer (CFO), den Leiter Corporate Controlling sowie in einigen Fällen durch Mitglieder des Verwaltungsrates ausgefüllt.

Die teilnehmenden Unternehmen stammen gemäss Brancheneinteilung der SWX aus den Sektoren „Industrial“ (38\%), „Financial“ (18\%), „Basic Materials“ (13\%), „Technology“ (10\%), „Consumer, Non-Cyclical“ (8\%), „Healthcare“ (5\%), „Consumer Cyclical“ (5\%), Utilities (3\%). Gemäss der Zusammensetzung des SPI haben damit über 50\% der „Basic Materials“ Unternehmen, sowie je rund 30\% der Industrial und Utilities-Unternehmen geantwortet. Die weiteren Branchen haben mit 25\% und weniger Beteiligungsrate partizipiert. Von den teilnehmenden Unternehmen sind etwas mehr als die Hälfte (59\%) nach ISO 9000 zertifiziert, 28\% verfügen über ein Zertifikat nach ISO 14000.

Mehr als die Hälfte der teilnehmenden Unternehmen haben den Fragebogen mit der Internetversion bearbeitet (absolut 21 von 39 gültigen Antworten).

\subsubsection{Stand der Performance-Measurement-Systeme}

Details zum Stand der Performance-Measurement-Systeme finden sich im Anhang B. Dieser enthält den Bericht, welcher den teilnehmenden Unternehmen nach Abschluss der Auswertung zugesandt wurde. Des Weiteren sei auf die Diplomarbeit von Christian Beyeler verwiesen, die im Rahmen dieser Untersuchung entstanden ist (vgl. Beyeler (2002)). Im Folgenden soll nur stichwortartig auf die einzelnen Punkte eingegangen werden:

- Führungsinstrumente: Über 80\% setzen Management by Objectives, rund 45\% setzen Value-Based-Management (VBM) und/oder Balanced Scorecard (BSC) ein. Ein Drittel setzt auf TQM. Gegenüber einer Studie von Brunner und Roth (1999) besteht die Vermutung, dass die Verbreitung solcher Instrumente zugenommen hat. Diese fanden 
einen Anteil von 28\% für VBM und 33\% für BSC. ${ }^{6}$ Die Anforderung B-5, wonach der Einsatz mehrerer PM-Konzepte unterstützt werden soll, entspricht der betrieblichen Praxis.

- Aspekte des Performance Measurement: Alle Unternehmen überprüfen finanzielle Aspekte, $~ 85 \%$ berücksichtigen Kundenaspekte und 75\% prüfen Mitarbeiteraspekte. Lieferanten-, Innovations- und Gesellschaftsaspekte werden von 50\% bis 60\% der Unternehmen berücksichtigt. Die Erhebungsfrequenzen verhalten sich ähnlich der Verbreitung der jeweiligen Aspekte. Die Anforderungen A-1\&2 und B-1, wonach verschiedene Aspekte von Performance-Indikatoren berücksichtigt werden sollen, werden schon von mehr als der Hälfte der Unternehmen abgedeckt.

- Rund $80 \%$ der Unternehmen führen eine systematische Umsetzungskontrolle ihrer strategischen Projekte durch und $70 \%$ überwachen ihre strategischen Risiken systematisch. Auf der operativen Ebene sind es $~ 90 \%$, die ihre Risiken überprüfen, 70\% setzen ein Frühwarnsystem ein, 85\% bedienen sich des Benchmarking, über $60 \%$ davon mehr als einmal jährlich. (vgl. A-3 \& A-4)

- Kommuniziert werden die Daten primär dem höheren und mittleren Kader sowie den Investoren. Nur etwa die Hälfte der Unternehmen teilen diese den Mitarbeitern mit. Nur noch 12 resp. 10 von 39 informieren ihre Kunden resp. Lieferanten über die erhobenen Performance-Daten. Das Schlusslicht mit 9 von 39 Nennungen bildet die Kommunikation von Performance-Daten an öffentliche Stellen. Entsprechend dienen die Performance-Daten vor allem höheren und mittleren Kadern für ihre Arbeit. Die Anforderung A-10, wonach die Performance-Daten sämtlichen relevanten Stakeholdern zugänglich gemacht werden sollen, wird nur teilweise berücksichtigt.

- Im Allgemeinen decken die Performance-Indikatoren die verfolgten Ziele ab. In rund 80\% der teilnehmenden Unternehmen besteht Konsens über die Bedeutung der benutzten Indikatoren, und diese werden in etwa im gleichen Ausmass als ausgewogen und präzise definiert beurteilt. Der Anforderung A-5, wonach die Performance-

${ }^{6}$ Die Studien sind nicht direkt vergleichbar, da unterschiedliche Stichproben und Grundgesamtheiten zugrundeliegen und keine Repräsentativität gegeben ist. 
Indikatoren die Ziele der Organisation widerspiegeln, wird somit weitgehendst Rechnung getragen.

\subsubsection{Stand der IT-Systeme für das Performance Measurement}

In $\sim 75 \%$ der Unternehmen wird für das Performance Measurement Informationstechnologie eingesetzt. Zwei Drittel setzen dabei auf StandardSoftwareprodukte, ein Drittel setzt auf Eigenentwicklungen (vgl. Abbildung 4-3). Sämtliche Unternehmen mit mehr als 10'000 Mitarbeiter setzen IT ein. In einer Studie von grossen deutschen Unternehmen von Günther und Grüning (2000) setzen 93 von 180 Unternehmen Informationstechnologie für ihr PMS ein. 36 von 93 setzen auf Eigenentwicklungen, 57 auf Standardsoftware (vgl. Günther und Grüning (2000)).

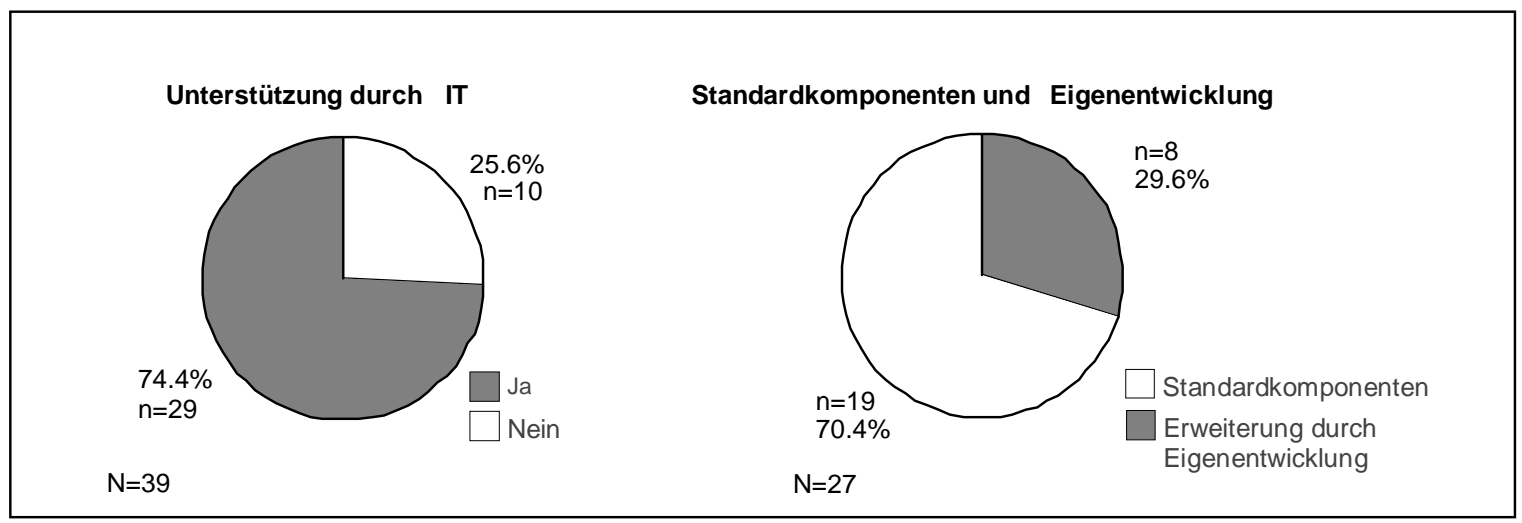

\section{Abbildung 4-3: IT-Unterstützung der Performance-Measurement-Systeme}

Am meisten werden Produkte von Cognos und SAP (je 7 Nennungen), Frango und Insight (je 4 Nennungen) und Oracle (2 Nennungen) eingesetzt. Vier Unternehmungen setzen Excel ein. Die meisten der eingesetzten Eigenlösungen basieren auf relationalen Datenbanken (7 versus 1 Nennung für eine multidimensionale Datenbank). In der Studie von Günther und Grüning (2000) finden sich 34 (37\%), die auf SAP R/3 teilweise mit Datenexporten auf Microsoft Excel setzen. 6 Unternehmen setzen Hyperion als PMS ein. 18 Unternehmen setzen auf Produkte wie Gentia, ALEA, Oracle, Lotus, etc.

Die verschiedenen Produkte wurden in der Umfrage nach fünf Kategorien gegliedert (vgl. Anhang A, Seite 7; und Anhang B). Danach werden mit 18 Nennungen IT-Systeme für das PM eingesetzt, die ein breites Spektrum unterstützen, und mit 14 Nennungen Tools, die mit ERP-Systemen gekoppelt sind. Weniger zum Einsatz kommen Produkte, die die 
Datenanalyse (8 Nennungen), die Datenakquisition, das Datenmanagement und den ETLProzess (8 Nennungen) unterstützen (vgl. Abbildung 4-4).

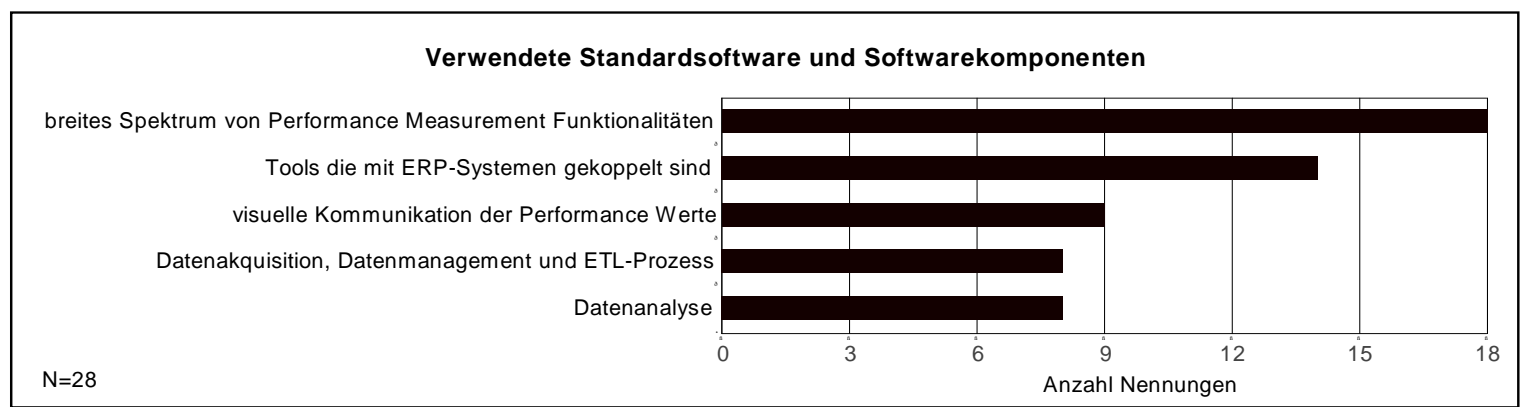

\section{Abbildung 4-4: Verwendete Standardsoftware und Softwarekomponenten}

Nur zu rund 50\% der operativen Buchungs- und Abrechungssysteme bestehen automatisierte Schnittstellen. Andere operative Informationssysteme sind noch weniger mittels automatischer Schnittstellen mit dem Performance-Measurement-System verbunden. Keines der teilnehmenden Unternehmen verfügt über eine automatisierte Schnittstelle zu unternehmensexternen Informationssystemen (vgl. Abbildung 4-5).

\begin{tabular}{|r|r|r|r|r|}
\hline \multicolumn{1}{|c|}{ Performance-relevante Daten der operativen Informationssysteme } & \\
Buchungs- und Abrechnungssysteme \\
Mitarbeiterinformationssysteme
\end{tabular}

\section{Abbildung 4-5: Performance-relevante Daten der operativen Informationssysteme}

\subsubsection{Anforderungen an ein IT-System für das PM aus der Sicht der Praxis}

Im dritten Teil der Umfrage wurde abgefragt, inwiefern einzelne Anforderungen von den bestehenden IT-Systemen für das PM abgedeckt werden und inwiefern diese Anforderung 
als wichtig beurteilt wird. Die abgefragten Kriterien orientieren sich weitgehend an Küng und Wettstein (2001). Es werden 6 Aussagen-Blöcke differenziert:

- Berücksichtigte Performance-Indikatoren (Aussagen: C2, C3, C6, C9)

- Sammlung der Performance-Daten (Aussagen: C7, C8, C13)

- Speicherung der Performance-Daten (Aussagen: C11, C12, C15)

- Analyse der Performance-Daten (Aussagen: C1, C4, C5, C16-18)

- Kommunikation der Performance-Daten (Aussagen: C10, C14, C19-20).

In der nachfolgenden Abbildung 4-6 sind die Aussagen nach der Wichtigkeit der Anforderung geordnet dargestellt. Neben der Erwartung werden auch die Bewertung der aktuellen Leistung und die daraus resultierende Leistungslücke (Darstellung nach links) aufgezeigt. Die Skalierung zeigt eine 5-Punkt-Likert-Skala, die für die Bewertung der Bedeutung einer Anforderung von unwichtig (0) bis sehr wichtig (4) reicht. Die aktuelle Leistungsbeurteilung reicht von nicht erfüllt (0) bis vollständig erfüllt (4).

Mit Ausnahme der Anforderung (C20) „Unternehmensexterne Stakeholder können auf einfache Art und Weise auf die für sie bestimmten Daten zugreifen“ wurden alle Anforderungen im Mittelwert mit grösser 2.0 bewertet. Diese Anforderungen wurden in die Anforderungsliste an ein PMS übernommen.

Die grössten Leistungslücken wurden für die Anforderung C11 „Sämtliche PerformanceDaten werden in einer Datenbank gespeichert“ und C17 „Ursache-Wirkungsketten können identifiziert und verifiziert werden“ festgestellt. Eine fehlende Funktionalität, die als wichtig eingestuft wird, ist die automatische Anbindung von operativen Informationssystemen. Dieser Schluss wird von den Ergebnissen aus Abbildung 4-5 bestätigt. 
C1: Performance-Daten können verdichtet/aggregiert werden.

C2: Die Indikatoren reflektieren die Ziele der Organisation und der Stakeholder.

C3: Sowohl finanzielle als auch nicht-finanzielle Indikatoren werden gemessen.

C4: Von einem höheren Aggregationsgrad kann auf einen kleineren Aggregationsgrad gewechselt werden (drill-down).

C5: Das Informationssystem berechnet die Lücken (performance gap) zwischen den Soll- und Ist-Werten.

C6: Die Performance wird auf verschiedenen organisatorischen Ebenen gemessen

C7: Das System ermöglicht das automatische Importieren und Konvertieren von Daten aus anderen Informationssystemen.

C8: Das System erlaubt die manuelle Erfassung von Daten.

C9: Für jede organisatorische Einheit und für jeden Kerngeschäftsprozess existiert eine Reihe definierter Performance-Indikatoren.

C10: Das Informationssystem erlaubt die Darstellung der Performance in graphischer Form.

C11: Sämtliche Performance-Daten werden in einer Datenbank gespeichert.

C12: Die Performance-Daten sind über einen langen Zeitraum hinweg verfügbar.

C13: Die Anbindung unternehmensexterner Informationsquellen ist möglich.

C14: Das System ermöglicht die Publikation der Performance Resultate über das Intranet und Internet. C15: Die Daten werden in einem integrierten IT-System verwaltet.

C16: Es können Indizes gebildet werden.

C17: Ursache-Wirkungsketten können (mittels statistischer Analyse) identifiziert und verifiziert werden.

C18: Das System ermöglicht die Berechung von Trends.

C19: Das System ermöglicht den automatischen Versand der Resultate via E-Mail, SMS (Push-Dienst).

C20: Unternehmensexterne Stakeholder können auf einfache

Art und Weise auf die für sie bestimmten Daten zugreifen.

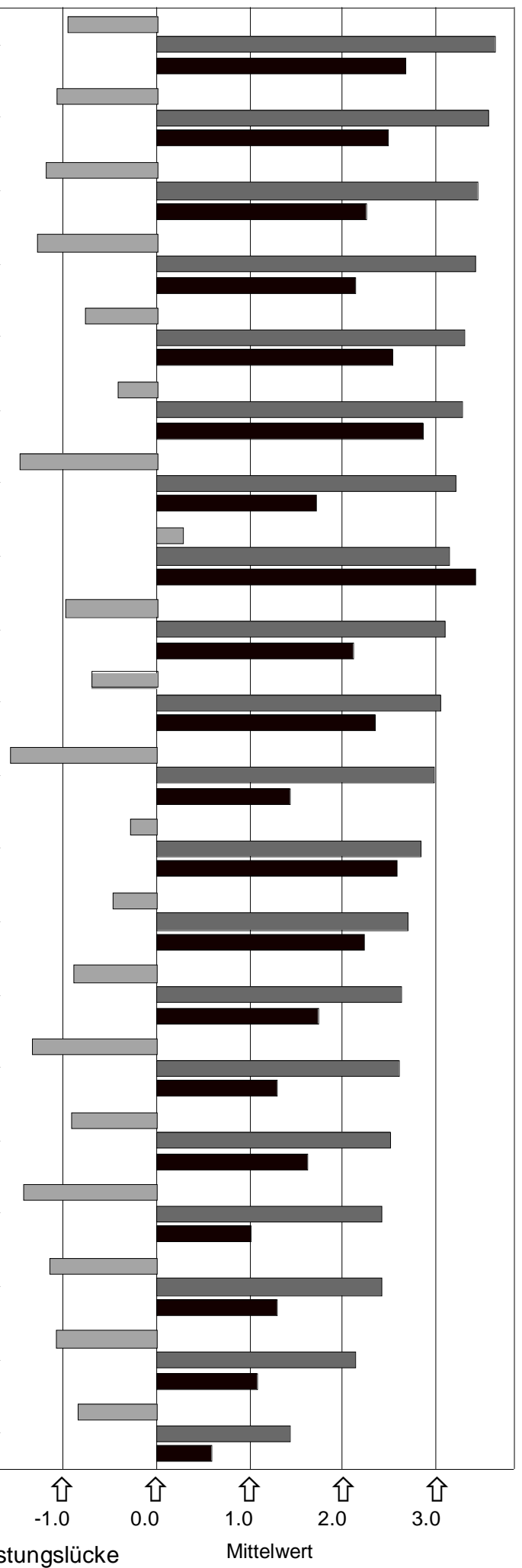

Das leistet das heutige

PMS

Das wird von

Leistungslücke

Mittelwert

erwartet

Abbildung 4-6: Anforderungen und Leistungen von Performance-Measurement-Systemen 


\subsection{Vorschlag eines Anforderungssatzes an ein PMS}

Nachfolgend werden die erarbeiteten Anforderungen zusammengefasst und bezüglich Redundanzen bereinigt. Zudem erfolgt eine Trennung nach den Anforderungen an ein PMS und denjenigen an ein IT-System für das PM. Innerhalb dieser beiden Hauptkategorien werden verschiedene Gruppierungen unterschieden:

Die Anforderungen an ein PMS (siehe Tabelle 4-1) werden unterteilt in aufbauende und prozessuale sowie wirtschaftliche Aspekte. Erstere umfassen im Wesentlichen die Verknüpfung von Aufbau-Organisation, Zielen und Performance-Indikatoren und die Einbettung des PMS in die Organisation. Die prozessualen Aspekte umfassen die notwendigen Prozesse zur Erstellung, Wartung und dem Betrieb des PMS. Der letzte Aspekt stellt die Wirtschaftlichkeit eines PMS sicher.

Die Anforderungen an ein IT-System für das PM (siehe Tabelle 4-2) behandeln die wichtigsten, berücksichtigten Entitäten ohne auf deren Beziehungen einzugehen. Die funktionalen Aspekte sind in einem zeitlichen Ablauf in vier Gruppen zusammengefasst: die Datenakquisition, -speicherung, -analyse und -kommunikation. Abschliessend werden noch einige nicht-funktionale Anforderungen aufgeführt.

Mit dem Ziel eines besseren Überblicks bezüglich der Anforderungen wurde darauf verzichtet, diese allzu detailliert auszuführen. Die Quelle der einzelnen Anforderungsstichworte ist jeweils rechts der Anforderung zu finden und verweist auf die früher in diesem Kapitel formulierten Anforderungen A1 - A19, B1 - B12 und C1 - C20.

Wie aus Tabelle 4-1 und Tabelle 4-2 ersichtlich ist, wurden einige Anforderungen umformuliert oder aber aus den referenzierten primären Anforderungen abgeleitet (z.B. leitet sich die Anforderung nach einem Sicherheitskonzept aus dem Einbezug von externen Stakeholdern und Entlohnungsaspekten ab). Einige wenige technische Anforderungen wurden ohne eingehende Besprechung aufgenommen. Sie können als generische Anforderungen an ein rechnergestütztes Informationssystem aufgefasst werden (vgl. auch Prototyp im Kapitel 10 und Anhang C). 


\begin{tabular}{|c|c|c|}
\hline Gru & ppe I Anforderung & Referenz \\
\hline Per & ormance-Indikatoren $(\mathrm{PI})$ & \\
\hline & $\begin{array}{l}\text { Berücksichtigung von finanziellen \& nicht-finanziellen } \\
\text { leading \& lagging } \\
\text { internen \& externen PI's }\end{array}$ & $\begin{array}{r}\mathrm{A} 1, \mathrm{~B} 1, \mathrm{C} 3, \\
\mathrm{~A} 2, \\
\mathrm{~A} 3\end{array}$ \\
\hline & Gliederung der PI nach Wirkung-, Durchführung- und Prämissenindikatoren & A4 \\
\hline & Indikatoren sind operationalisierte Ziele & $\mathrm{A} 5, \mathrm{C} 2$ \\
\hline & Zielvorgaben (Soll-Werte von PI) & A9 \\
\hline Ziel & & \\
\hline & Ziele werden mittels Indikatoren operationalisiert & A5 \\
\hline & Ziele werden aus der Strategie hergeleitet & A7 \\
\hline & Es werden nur Ziele berücksichtigt, die einen Stakeholder haben & A8 \\
\hline Org & anisation (Einbettung, Aufbau, Beteiligte) & \\
\hline & Die Ziele sind mit der Organisation und den Mitarbeitern verknüpft & A5, A11 \\
\hline & Die individuelle Organisationsstruktur kann abgebildet werden & $\begin{array}{l}\text { A6, B8, } \\
\text { C6, C9 }\end{array}$ \\
\hline & Das PMS kann in die bestehenden Führ & B6 \\
\hline & Die Verwaltung der Performance-relevanten Daten erfolgt zentral & B11 \\
\hline $\operatorname{Pro}_{2}$ & zesse & \\
\hline & Prozess für den Aufbau des PMS & A20 \\
\hline & Prozess für die Akquisition von unternehmensinternen und -externen Daten & B12 \\
\hline & Stakeholdergerechte Kommunikation der Performance-relevanten Daten & A10 \\
\hline & $\begin{array}{l}\text { Prozesse zur Erarbeitung von Ex-Ante-Wirkungsketten und zu deren Ex-Post- } \\
\text { Überprüfung }\end{array}$ & $\begin{array}{r}\text { A18, B2, } \\
\text { B3, C17 }\end{array}$ \\
\hline & Prozess für den Unterhalt (Review) des PMS & A21, B7 \\
\hline Wirt & schaftlichkeit & \\
\hline & Überprüfbarkeit der Kosten-Nutzensituation eines PMS & B4 \\
\hline & & \\
\hline $\mathrm{Ax}$ & forderung, die aus in der Literatu & \\
\hline $\mathrm{Bx}$ & Anforderung, die sich aus empirischen Unte & \\
\hline $\mathrm{Cx}$ & Anforderung, die mit der eigenen empirischen Untersuchung verifiziert wurd & \\
\hline
\end{tabular}

Tabelle 4-1: Anforderungen an ein PMS 


\begin{tabular}{|c|c|c|}
\hline \multicolumn{2}{|c|}{ Gruppe I Anforderung } & Referenz \\
\hline \multirow{2}{*}{\multicolumn{2}{|c|}{$\begin{array}{l}\text { Berücksichtigte Entitäten } \\
\text { Performance-Indikatoren }\end{array}$}} & \\
\hline & Performance-Indikatoren & A1-A4,B1 \\
\hline & Ziele & A5 \\
\hline & Aufbauorganisation & A6,B8 \\
\hline & Mitarbeiter & A6 \\
\hline & Stakeholder & A8 \\
\hline & $\begin{array}{l}\text { Zielvorgaben (Soll-Werte) von Performance-Indikatoren für einen bestimmten } \\
\text { Zeitpunkt oder -perioden. }\end{array}$ & A9 \\
\hline & Wirkungsketten & A18,B3 \\
\hline \multicolumn{3}{|c|}{ Funktionale Anforderungen } \\
\hline \multicolumn{3}{|c|}{ Datenakquisition } \\
\hline & Manuelle Erfassung von Daten & $\mathrm{C} 8$ \\
\hline & Interne \& externe Datenquellen & $A 3, C 13$ \\
\hline & $\begin{array}{l}\text { Geeignete Mechanismen für die automatische Gewinnung von Performance- } \\
\text { relevanten Daten aus den operativen Systemen }\end{array}$ & B9, B12, C7 \\
\hline & Ankoppelung von externen Datenquellen & $\mathrm{B} 9, \mathrm{C} 13$ \\
\hline \multicolumn{3}{|c|}{ Datenspeicherung } \\
\hline & Zentrale, einheitliche Datenstruktur & $\begin{array}{l}\text { A13, A16, } \\
\text { C15, C11 }\end{array}$ \\
\hline & $\begin{array}{l}\text { Speicherung der Performance-Indikatoren auf verschiedenen } \\
\text { Granularitätsstufen }\end{array}$ & A15, C16 \\
\hline & Zielvorgaben & A9+A17 \\
\hline & Langfristige Speicherung der Performance-Daten & A19,C12 \\
\hline & $\begin{array}{l}\text { Performance-Daten sollten für andere Führungssysteme im Unternehmen zur } \\
\text { Verfügung stehen }\end{array}$ & B6 \\
\hline \multicolumn{3}{|c|}{ Datenanalyse } \\
\hline & Werkzeuge zur Analyse der Ursache-Wirkungsketten & A18,B2,C17 \\
\hline & Das System ermöglicht die Berechnung von Trends & C18 \\
\hline & $\begin{array}{l}\text { Das Informationssystem berechnet die Lücken (performance gap) zwischen } \\
\text { Ist- und Soll-Werten }\end{array}$ & A17, C5 \\
\hline \multicolumn{3}{|c|}{ Datenkommunikation } \\
\hline & Freie Navigation in der Datenstruktur & $\begin{array}{r}\text { A13, A16, } \\
\text { C1, C4 }\end{array}$ \\
\hline & Stakeholdergerechte Kommunikation der Performance-relevanten Daten & $\begin{array}{r}\text { A10, } \mathrm{A} 12, \\
\text { A13, C10, } \\
\text { C19 }\end{array}$ \\
\hline & Darstellung der Performance-Lücke & A17 \\
\hline \multicolumn{3}{|c|}{ Nichtfunktionale Anforderungen } \\
\hline & Sicherheitskonzept, hohe Datensicherheit & $\begin{array}{r}\mathrm{A} 10, \mathrm{~A} 12, \\
\mathrm{~A} 13, \mathrm{~A} 14\end{array}$ \\
\hline & Basierend auf standardisierter Technologie (z.B. Internet) & $\mathrm{A} 14, \mathrm{C} 14$ \\
\hline & Gute Antwortzeiten & \\
\hline & Gute Erweiterbarkeit und Skalierbarkeit & \\
\hline & Hohe Verfügbarkeit und kurze Wiederanlaufzeit & \\
\hline \multicolumn{3}{|c|}{ Weitere Anforderungen } \\
\hline & $\begin{array}{l}\text { Weit gehende Unabhängigkeit vom angewandten Performance-Measurement- } \\
\text { Konzept }\end{array}$ & B5 \\
\hline \multicolumn{3}{|c|}{ Legende: } \\
\hline $\mathrm{Ax}$ & \multicolumn{2}{|l|}{ Anforderung, die aus in der Literatur vorgeschlagenen PM-Konzepten ableitbar ist } \\
\hline $\mathrm{Bx}$ & \multirow{2}{*}{\multicolumn{2}{|c|}{$\begin{array}{l}\text { Anforderung, die sich aus empirischen Untersuchungen zu PMS ableiten lassen } \\
\text { Anforderung, die mit einer eigenen empirischen Untersuchung verifiziert wurden. }\end{array}$}} \\
\hline$C x$ & & \\
\hline
\end{tabular}

Tabelle 4-2: Anforderungen an das IT-System für das PM 
Die in diesem Kapitel erarbeiteten Anforderungen bilden die Basis für den in den nachfolgenden Teilen vorgeschlagenen Lösungsansatz. 


\section{Teil \\ Organisatorische Konzeption eines PMS}




\section{Bezugsrahmen für ein Performance-Measurement-System}

Im vierten Kapitel wurden die Anforderungen an ein PMS resp. IT-System für das PM erarbeitet. Um eine ganzheitliche Sicht für die definierten Anforderungen zu erhalten, wird in diesem Kapitel ein Bezugsrahmen für das PMS vorgeschlagen. Dieser soll erlauben, in einem Modell (resp. Bezugsrahmen) die einzelnen Elemente eines PMS referenzieren zu können. Kapitel 5.2. untersucht den Abdeckungsgrad bereits in der Literatur vorgeschlagener Konzepte. Da gemäss dieser Analyse keines der Systeme für den vorliegenden Zweck geeignet erscheint, wird im nachfolgenden Kapitel ein eigenes Konzept eingeführt und detailliert diskutiert.

\subsection{Wesen und Zweck eines Bezugsrahmens}

Die verschiedenen in der Literatur vorgeschlagenen PM-Konzepte fussen auf unterschiedlichen Grundideen (vgl. die vorgestellten Konzepte im dritten Kapitel). Es besteht keine Einheitlichkeit bezüglich der Begriffsverwendung. Zudem gewichten die Autoren jeweils einzelne Elemente ganz unterschiedlich (vgl. den ausführlichen Vergleich bei Gleich (2001)). Dies erschwert den Anwendern das Verständnis und die Auswahl ganz erheblich. Erschwerend kommt hinzu, dass fast jedes Beraterhaus ein eigenes PM-Konzept mit eigenen Begrifflichkeiten postuliert (vgl. die Zusammenstellung bei Klingebiel (2000)).

Für diese Arbeit wird daher vorgeschlagen, einen Bezugsrahmen resp. ein Modell des Performance Measurement als übergeordnete Ebene einzuführen. Darin lassen sich die einzelnen PM-Konzepte einordnen und die einzelnen Elemente wieder erkennen, obwohl deren Bezeichnungen differieren. Ein Bezugsrahmen (resp. Bezugssystem) kann dabei als ein zu Grunde liegendes System von Beziehungen, Überzeugungen etc. aufgefasst werden (vgl. DUDEN (1999)). In der Physik versteht man unter einem Bezugssystem ein Koordinatensystem auf das eine Bewegung bezogen wird (vgl. Brockhaus (2001)). Angewandt auf die Informationstheorie gibt das Bezugssystem der Nachricht eine Bedeutung. Ein Bezugsrahmen für ein PMS erlaubt somit, für grundlegende Elemente eines Systems und deren Beziehungen untereinander ein gemeinsames Verständnis zu 
schaffen. Teilweise wird auch der englische Begriff framework synonym für das Bezugssystem verwendet (vgl. z.B. Hoffmann (1999); Merriam-Webster-Dictionnary (2002) definiert den Begriff als a basic conceptual structure).

Die generischen Elemente eines Bezugsrahmens umfassen in Anlehnung an Grünig (1996) die drei Subsysteme Systematik, Methodik und Organisation. Gemäss den erarbeiteten Anforderungen aus Kapitel vier können den einzelnen Subsystemen bestimmte Aufgaben zugeordnet werden:

- Die Systematik soll dabei die einzelnen Ebenen des Performance Measurement sowie die Verknüpfung mit den Unternehmenszielen beinhalten. Zudem wird die Verknüpfung zwischen den einzelnen Zielen festgelegt.

- Die Methodik beinhaltet die prozessualen Aspekte, d.h. wie ein PMS aufgebaut, betrieben und verbessert werden soll (vgl. Wettstein und Küng (2001)).

- In der Organisation werden sodann die Verantwortlichkeiten und die Empfänger der Performance-relevanten Daten definiert.

Kurz gesagt, legt die Systematik das Was, die Methodik das Wie und die Organisation das Wer fest. Abbildung 5-1 zeigt die generischen Elemente im Überblick. 


\section{SYSTEMATIK}

- zu berücksichtigende Objekte (z.B. Prämissen, strategische, operative und Mitarbeiter-Ebene)

- Verknüpfung zwischen den einzelnen Objekten

- Verknüpfung mit der Organisationsstrategie

\section{METHODIK}

- Prozess für den Aufbau des PMS

- Prozesse für den Betrieb (z.B. Zielfestlegung)

- Prozesse für die laufende Weiterentwicklung/Verbesserung

ORGANISATION

- zu berücksichtigende Subjekte

(z.B. Verantwortliche und Empfänger)

Abbildung 5-1: Generische Elemente eines PMS-Modells

\subsection{Abdeckungsgrad existierender PM-Konzepte bezüglich der identifizierten Elemente des Bezugsrahmens}

In diesem Abschnitt soll untersucht werden, inwiefern bestehende Konzepte Antworten auf einzelne Elemente des Bezugsrahmens liefern. Hierzu werden die in Kapitel drei diskutierten Ansätze sowie ein zusätzlicher Ansatz berücksichtigt.

\subsubsection{Diskutierte Ansätze aus Kapitel drei}

Der EFQM-Ansatz offeriert einen Rahmen für die relevanten Kennzahlen und zeigt, wie sich ein Unternehmen in den einzelnen Bereichen verbessern kann. Werden die Erkenntnisse aus der TQM-Literatur verwendet, so wird ein sehr breiter Bogen aufgespannt. Die Systematik ist nur in Teilen zu erkennen und innerhalb der Methodik fehlt ein konkreter Prozess für den Aufbau. Keine klaren Aussagen macht das EFQM auch 
zu Punkten wie Kommunikation der Strategie und der Verknüpfung von Zielen mit Anreizen. (vgl. Kapitel 3.2.1)

Die Balanced Scorecard (BSC) ist sehr weit ausgereift. Es existiert eine Systematik und eine Methodik. Die Elemente der Systematik sind - mit Ausnahme einer Prämissenkontrolle - umfassend. Die Verknüpfung zwischen den betrachteten Objekten wird klar aufgezeigt. Innerhalb der Methodik wird sowohl die Herleitung der Indikatoren als auch der Ablauf eines Projektes detailliert erläutert. Es fehlen weder ein Vorschlag für die Kommunikation noch ein Ansatz für die Verknüpfung von Zielen mit Anreizen. Weniger konkret ist der Ansatz jedoch im Bereich des Betriebs. Die Nutzung und die Überarbeitung sind nur sehr vage formuliert und im Bereich der Verantwortlichkeiten (Organisation) bestehen Unklarheiten. (vgl. Kapitel 3.2.2)

Der Intellectual-Capital-Ansatz (IC) zeigt ähnlich den beiden ersten Ansätzen einen Rahmen für die relevanten Kennzahlen auf. Dieser wird ebenso ausführlich besprochen wie die Beziehungen zwischen den einzelnen Elementen (Kapitalarten) erläutert. Hinweise, wie die Indikatoren konkret ausgewählt werden sollen, fehlen jedoch (resp. diesbezüglich fassen sich die Autoren sehr kurz, vgl. Edvinsson und Malone (1997)). Gleiches gilt für die Nutzung und die Überarbeitung des Systems. Insgesamt lässt sich festhalten, dass der Ansatz keinen ganzheitlichen Bezugsrahmen darstellt und nur einzelne Aspekte diskutiert. (vgl. Kapitel 3.2.3)

Die Ansätze des Value-Based-Management (VBM) bieten wie die anderen Ansätze einen Rahmen für die relevanten Kennzahlen, konzentriert sich jedoch weitgehend auf finanzielle Aspekte. Eine übergeordnete Systematik wird für den Ansatz nicht explizit vorgestellt. Sowohl der Aufbau als auch der Betrieb (Management der Einflussfaktoren) ist klar erläutert. Zudem wird ein Modul zur Leistungsbewertung und Entlohnung von Führungskräften berücksichtigt. Zur organisatorischen Gestaltung finden sich demgegenüber wenige Hinweise. (vgl. Kapitel 3.2.4)

\subsubsection{Das Performance-Management-System von Hoffmann}

Hoffmann (1999) schlägt in seiner Dissertation ein koordiniertes Vorgehen bei der Einführung eines Performance-Management-Systems zur Vermeidung von Dysfunktionalitäten und Inkonsistenzen vor. Dabei orientiert er sich an einer Kausalkette, die in Abbildung 5-2 dargestellt ist. 


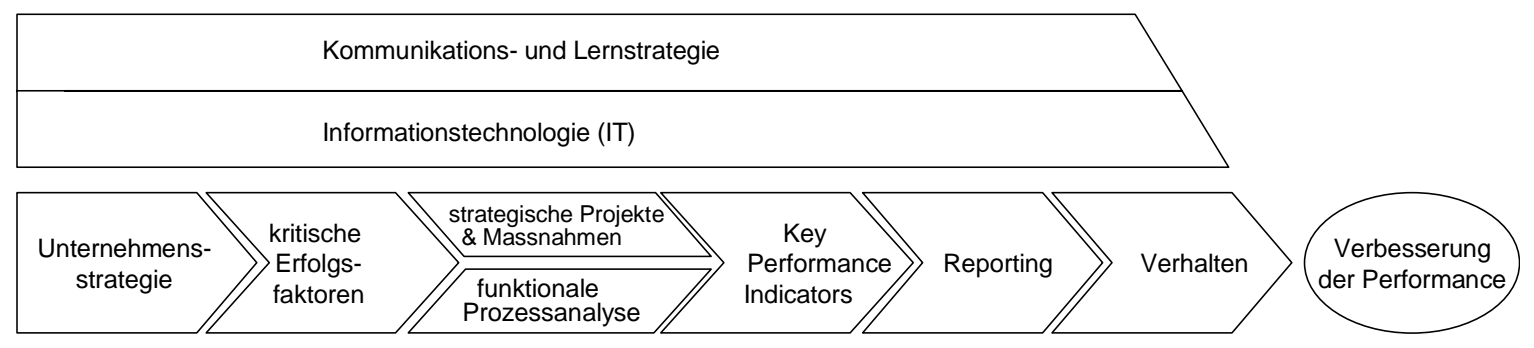

\section{Abbildung 5-2: Kausalkette zur Einführung eines Performance-Management-Systems (vgl. Hoffmann (1999))}

Die Unternehmensstrategie wird als Startpunkt verwendet, um daraus über das Geschäftsmodell die kritischen Erfolgsfaktoren abzuleiten. Dabei erfolgt eine Gliederung in strategische und operative Elemente. Erstere leiten sich aus den Massnahmenplänen ab, während sich zweitere aus den Geschäftsprozessen ergeben.

Hoffmann schlägt ein Verfahren für das Herunterbrechen der Strategie (Strategy Deployment) nach Colletti (1996) vor. Dabei handelt es sich um einen partizipativen Ansatz.

Für die operative Ebene verwendet Hoffmann ein prozessorientiertes Unternehmensmodell. Wie die Prozessziele konkret ermittelt werden, kann dem Vorschlag nicht entnommen werden. Er verweist hierfür auf das Vorgehen von Lüthi et al. (1998), nicht ohne den Hinweis auf die damit verbundenen Schwierigkeiten zu liefern. Als einer von wenigen Autoren beschreibt er auch die Revision der operativen Indikatoren, wobei er sich an den Vorschlag von Roberts (1996) anlehnt.

Hoffmann beschäftigt sich zudem ausführlich mit dem Reporting. Dabei werden der Aufbau und der Betrieb des Reporting sowie teilweise auch die Empfänger der Reports erläutert. Implizit geht er vom Einsatz von Informationstechnologie aus, ohne deren Möglichkeiten für die Kommunikation genauer zu analysieren. Des Weiteren unterstreicht er die Nützlichkeit von Drill-Down-Möglichkeiten. An dieser Stelle ebenfalls ohne näher zu erläutern, wie diese Funktion realisiert werden soll. In einem Kapitel unterstreicht er den Einfluss der Informationstechnologie für das Performance Management und erläutert 
die grundlegenden Techniken. Ein letzter Teil beschäftigt sich mit der möglichen Ausgestaltung der individuellen Leistungshonorierung.

Das von Hoffmann vorgeschlagene Performance-Management-System umfasst alle wesentlichen Elemente, die ein Bezugsrahmen gemäss den erarbeiteten Anforderungen beinhalten sollte und ist damit als wertvoll zu betrachten. Es werden zahlreiche Vorschläge aus der Literatur innerhalb der einzelnen Module erläutert. Dies führt jedoch dazu, dass die einzelnen Lösungsvorschläge nur schwer zu einem Ganzen zusammengefügt werden können. Zudem fehlt es teilweise an Konkretisierung, um die vorgeschlagenen Ansätze direkt anwenden zu können.

\subsubsection{Zusammenfassende Gegenüberstellung der existierenden Ansätze}

Eine zusammenfassende Einschätzung des Verfassers bezüglich der Abdeckung der einzelnen Elemente eines Bezugsrahmens der einzelnen Ansätze findet sich in Tabelle 5-1.

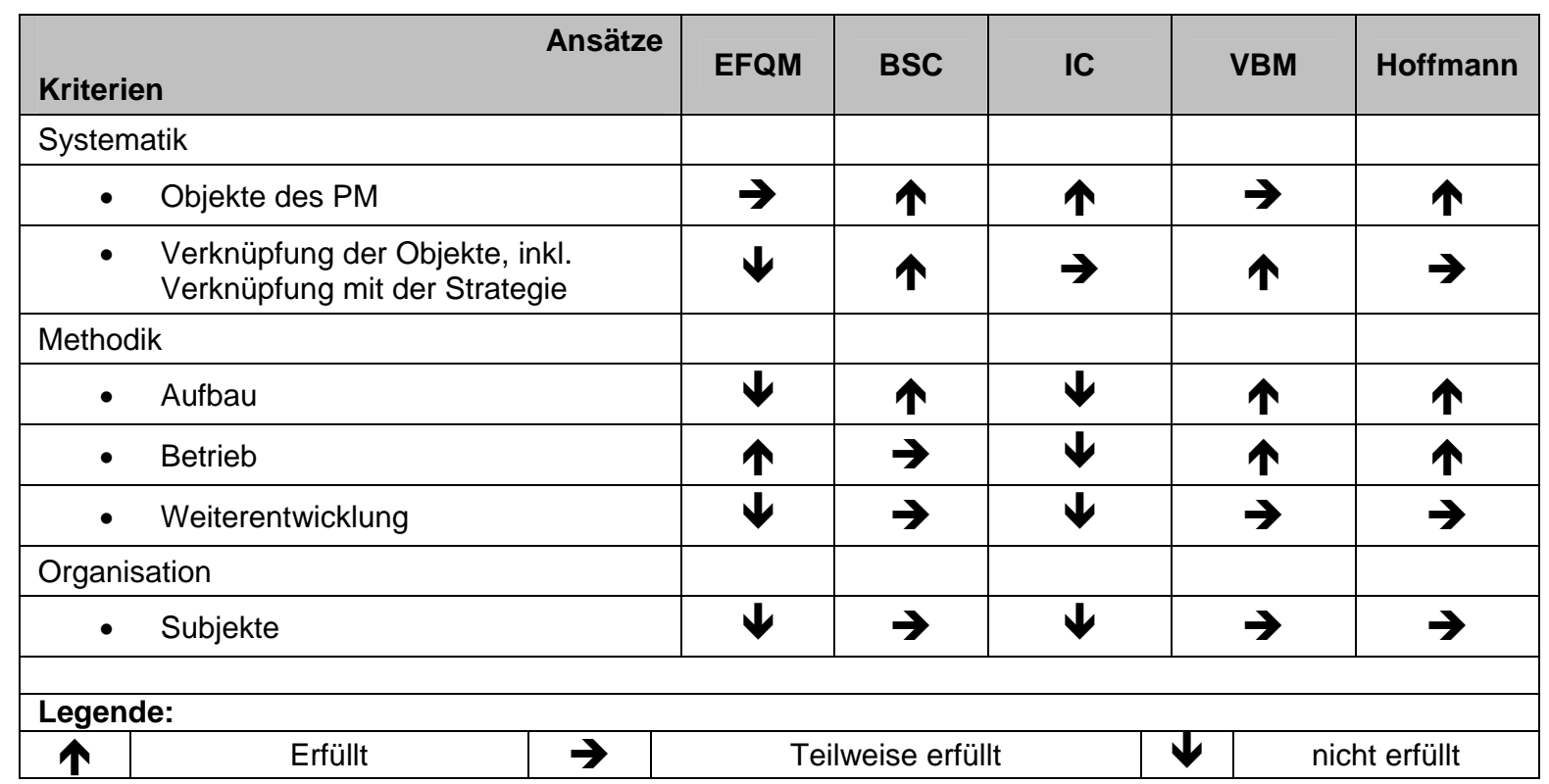

Tabelle 5-1: Abdeckungsgrad der Ansätze bezüglich der Elemente eines Bezugsrahmens

Die Tabelle 5-1 zeigt, dass jeder Ansatz über individuelle Stärken und Schwächen verfügt. Kein Ansatz deckt jedoch sämtliche Elemente des Bezugsrahmens ab. Grundsätzlich stehen somit zwei Varianten zur Verbesserung offen. Entweder kann ein bestehender Ansatz in den Bereichen, für welche er keine Lösungen anbietet, weiterentwickelt werden, oder ein neuer, in sich geschlossener Ansatz kann entwickelt werden. Bei der Verwendung eines bestehenden Ansatzes müssen Konzessionen bezüglich der bestehenden 
Begrifflichkeiten und der Systematik eingegangen werden. Zudem besteht die Gefahr, durch Erweiterungen Inkonsistenzen und eine hohe Komplexität zu schaffen.

Wird ein eigener Bezugsrahmen erstellt, so können diese Nachteile vermieden werden. Aus diesem Grunde wird nachfolgend ein eigener Vorschlag eines Bezugsrahmens erläutert.

\subsection{Vorschlag eines eigenen Bezugsrahmens}

\subsubsection{Der Bezugsrahmen im Überblick}

Wie vorgängig bereits vorgestellt, umfasst der hier vorgeschlagene Bezugsrahmen drei Subsysteme:

- Systematik: Sie definiert die zu messenden Objekte des Performance Measurement und deren Beziehungen untereinander. Gemäss den in Kapitel vier definierten Anforderungen ist eine strategische, operative und Mitarbeiterebene sowie eine Prämissen-Ebene zu schaffen.

- Methodik: Sie definiert inhaltlich die einzelnen Prozesse für die Erstellung, den Betrieb und die Überarbeitung des Performance-Measurement-Systems sowie die Kommunikation der Performance-relevanten Daten (vgl. die Anforderungen aus Kapitel vier).

- Organisation: Sie definiert die Träger der einzelnen Teilaufgaben und regelt die Empfänger und Lieferanten der Performance-relevanten Daten (vgl. die Anforderungen aus Kapitel vier).

Nachfolgend werden die einzelnen Elemente näher erläutert.

\subsubsection{Elemente der Systematik}

\subsubsection{1 Überblick}

Wie aus Abbildung 5-3 ersichtlich, werden insgesamt vier Ebenen, die strategische, die operative, die Mitarbeiter-Ebene sowie die Prämissenebene vorgeschlagen. Diese ergeben sich aus den erarbeiteten Anforderungen aus Kapitel vier. 


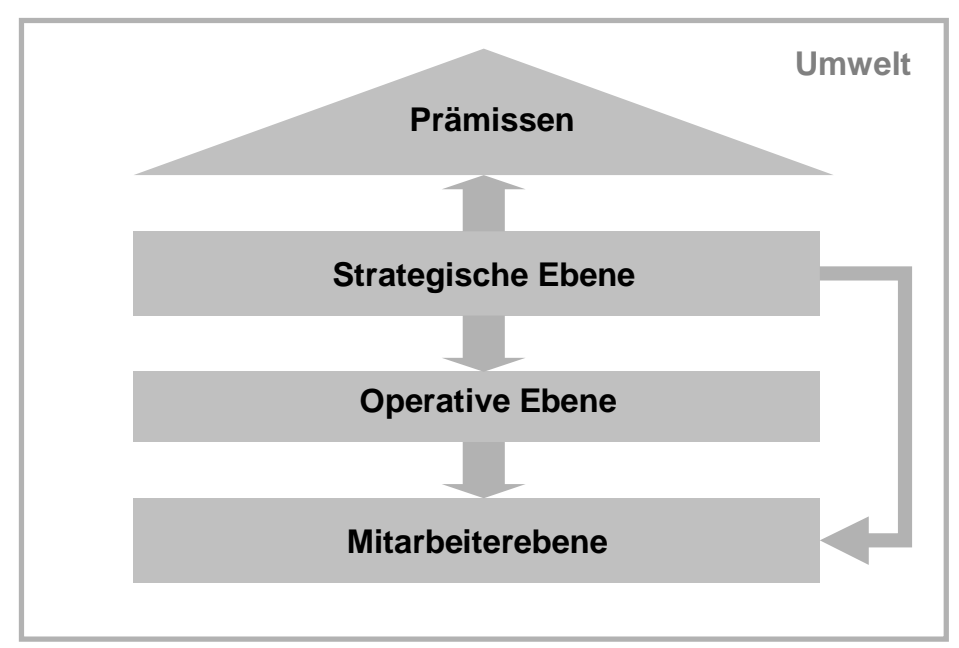

Abbildung 5-3: Systematik des Bezugsrahmens (in Anlehnung an Wettstein et al. (2001))

Die strategische Ebene beinhaltet zwei Kategorien. Einerseits die Kategorie der strategischen Projekte (Massnahmen resp. Programme), die zur Umsetzung der Strategie abgeleitet wurden. Dabei werden in der Regel wesentliche Verbesserungen angestrebt (vgl. ebenfalls die Ideen zum Business Process Reengineering von Hammer und Champy (1994)). Andererseits werden die strategischen Ziele auf der operativen Stufe weiter konkretisiert.

Die operative Ebene resultiert - wie erwähnt - aus der strategischen Ebene, leitet sich jedoch nicht aus den strategischen Projekten ab, sondern aus der Strategie im Allgemeinen. Die Notwendigkeit der Differenzierung liegt darin, dass auf der operativen Ebene der Wunsch nach einer gewissen Konstanz der Ziele besteht. Mit den strategischen Projekten werden dagegen meist grössere Veränderungen angestrebt, die nach erfolgreicher Umsetzung ebenfalls einen Einfluss auf die operativen Ziele haben können.

Die Ziele der Mitarbeiter leiten sich aus der operativen Ebene sowie aus Zielen von strategischen Projekten und strategischen Zielen ab. Diese offene Regelung (Missachtung einer rein hierarchischen Gliederung) erlaubt es bspw., Mitarbeiter auch für jene Aufgaben zu fördern, die nicht in ihrer organisatorischen Einheit nachgefragt werden (strategische Ziele) und sie in strategische Projekte einzubinden, deren Ziele im Widerspruch zu operativen Zielen stehen.

Auf den drei besprochenen Ebenen wird die Performance überwacht. Es ist jedoch zu berücksichtigen, dass die Wahl der Ziele unter bestimmten Prämissen erfolgt, die ebenfalls 
beachtet werden sollten. Hierbei wird die tatsächliche Entwicklung von Umweltvariablen (Umwelt) mit den im Rahmen der Planung getroffenen Annahmen verglichen.

\subsubsection{Prämissen}

Die Prämissenkontrolle kann als eine von drei Aufgaben der strategischen Kontrolle begriffen werden. Die Prämissenkontrolle richtet sich auf die laufende Überprüfung der wichtigsten Annahmen auf ihre weitere Gültigkeit aus. Gegenstand der Prämissenkontrolle sind sowohl externe als auch interne Faktoren. Externe Faktoren beziehen sich auf relevante Entwicklungen in der Unternehmensumwelt (Eine ausführliche Zusammenstellung möglicher Gegenstände der globalen Umweltanalyse findet sich bei Kühn und Grünig (1998; Grünig und Kühn (2000), die sich an Pümpin (1992) anlehnen.). Interne Faktoren betreffen Potenziale und Prozesse in Geschäfts- und Funktionsbereichen bzw. Regionen. Teilweise werden auch Annahmen über das Wertsystem der Unternehmung berücksichtigt (vgl. Renggli (1996), zitiert nach Kühn und Grünig (1998)).

Neben der Prämissenkontrolle unterscheiden Schreyögg und Steinmann (1985) noch die Durchführungskontrolle und die strategische Überwachung als Elemente der strategischen Kontrolle. Die Durchführungskontrolle dient der Überwachung der schrittweisen Realisierung eines strategischen Plans und wird im vorgeschlagenen Modell auf der strategischen Ebene berücksichtigt (vgl. Abbildung 5-4).

\begin{tabular}{|c|}
\hline Elemente der strategischen Kontrolle \\
\hline Prämissenkontrolle (stimmen die Annahmen noch?) \\
\hline Durchführungskontrolle (können wir den Umsetzungsplan einhalten?) \\
\hline Strategische Überwachung (gibt es neue Chancen/Gefahren?) \\
\hline
\end{tabular}

\section{Abbildung 5-4: Elemente der strategischen Kontrolle}

Sowohl die Prämissenkontrolle als auch die Durchführungskontrolle sind gerichtete Aktivitäten, d.h. sie beziehen sich auf ein konkretes Ziel resp. Objekt. Demgegenüber versucht die strategische Überwachung durch ungerichtete Beobachtungsaktivitäten die gewählte Strategie abzusichern. Die strategische Überwachung weist somit Ähnlichkeiten 
mit Früherkennungs- oder Frühwarnsystemen aus. Diese bestimmen jedoch meist mit ihrer Konstituierung bestimmte Beobachtungsfelder und Indikatoren und sind somit den gerichteten Verfahren zuzuordnen. Zudem zielen Früherkennungssysteme im Gegensatz zur strategischen Überwachung meist darauf ab, neue strategische Chancen zu erkennen. (vgl. Schreyögg und Steinmann (1985))

Im vorgeschlagenen Modell erfolgt auf der Ebene der Prämissen eine Fokussierung auf die Überwachung der Planungsprämissen. Die ungerichtete strategische Überwachung wird nicht berücksichtigt, da eine Operationalisierung schwer fällt. In der Praxis wird jedoch eine gewisse Durchmischung der beiden strategischen Kontrollarten auftreten. Die Prämissen leiten sich - wie aus Abbildung 5-5, Pfeil „A“ ersichtlich - von der strategischen Ebene ab.

\subsubsection{Strategische Ebene}

Voraussetzung für die Anwendung der vorgestellten Systematik ist es, dass Klarheit über die Unternehmensstrategie - mindestens im Sinne einer Vision - besteht (Die Vision beschreibt den finalen Zustand, die Strategie den Weg zum Ziel.). Ziele auf der strategischen Ebene beschreiben eine konkretisierte Vision, d.h. es wird eine Aussage bezüglich des angestrebten Zielerreichungsgrades gemacht. Dabei ist auf die Ausgewogenheit der Ziele zu achten. Ausgewogenheit bedeutet in diesem Kontext, dass sowohl finanzielle als auch nicht-finanzielle Ziele berücksichtigt werden. (vgl. auch die Anforderungen im Kapitel 4)

Die Zielsetzungen werden dahingehend unterteilt, ob es sich um Ziele handelt, die über eine längere Zeit in der bestehenden Organisation existent sind, oder ob es sich um wesentliche Veränderungen im Sinne von strategischen Projekten resp. Massnahmen oder Programmen handelt. Erstere sind die Grundlage der operativen Zielsetzungen. Dabei wird eine gewisse Konstanz bezüglich der Ziele intendiert. Zweitere ergeben sich auf Grund der strategischen Planung. Deren Ziele werden mit entsprechenden Massnahmen umgesetzt. Diese beeinflussen wiederum die operativen Ziele.

\subsubsection{Operative Ebene}

Die operativen Zielsetzungen leiten sich aus der ersten Kategorie der strategischen Zielsetzungen ab (vgl. Abbildung 5-5, Pfeil „B“). Es erfolgt die Gliederung nach der gewählten Organisationsform. Eine Kombination von Prozess- und Abteilungsgliederung 120 
oder die Berücksichtigung einer zusätzlichen Gliederungsstufe im Sinne von Business Units ist möglich. Dieser Schritt ist jedoch mit der Gefahr verbunden, dass sich einzelne Ziele konkurrenzieren und eine Abkehr vom hierarchischen Zielbaum erfolgen muss (An deren Stelle treten dann Wirkungsnetze. Eine Hilfestellung zur Gewichtung der einzelnen Wirkungen und einer Rückführung in eine baumähnliche Struktur bietet bspw. der Papiercomputer von Vester (1987). ${ }^{7}$ ).

\subsubsection{Mitarbeiterebene}

Die Ziele der Mitarbeiter bestimmen sich aus vier verschiedenen Quellen (vgl. Abbildung 5-5): (1) Ist der Mitarbeiter einer operativen Organisationseinheit zugehörig, so werden sich seine Ziele mitunter aus den Zielen der entsprechenden Organisationseinheit ableiten. (2) Denkbar ist des Weiteren, dass der Mitarbeiter in gewissen strategischen Projekten engagiert ist, und sich seine Ziele daraus ableiten. (3) Besteht die Option, dass für den Mitarbeiter Ziele vereinbart werden, die sich an den strategischen Zielen (der ersten Kategorie) orientieren, nicht aber denjenigen der operativen Einheit entsprechen (Ein Beispiel wäre bspw. die Marketingweiterbildung einer Mitarbeiterin, die im Finanzbereich arbeitet, längerfristig aber ins Marketing wechseln möchte). (4) Als letztes Element sind schliesslich noch die individuellen Ziele der Mitarbeiter zu berücksichtigen. Diese stehen in keiner direkten Abhängigkeit mit den erwähnten Zielkategorien, sollten sich jedoch in einem gewissen Umfang vereinen lassen, um die Zufriedenheit der Mitarbeiter sicherzustellen.

\subsubsection{Beziehungen zwischen den Elementen der Systematik}

Abbildung 5-5 zeigt die vorgestellte Systematik mit den vier Ebenen, den relevanten Elementen und den Beziehungen zwischen diesen. Das System steht nicht isoliert da, vielmehr interagiert jedes einzelne Element mit der Organisationsumwelt, wie der Rahmen um die Abbildung 5-5 veranschaulicht.

7 Der Papiercomputer ist ein Verfahren zur Bestimmung der Art und Stärke der Einflussnahme eines Ziels auf ein Anderes (vgl. auch die konkrete Anwendung im Kapitel 6.3.4.5 Auflösung von Zielkonflikten). 


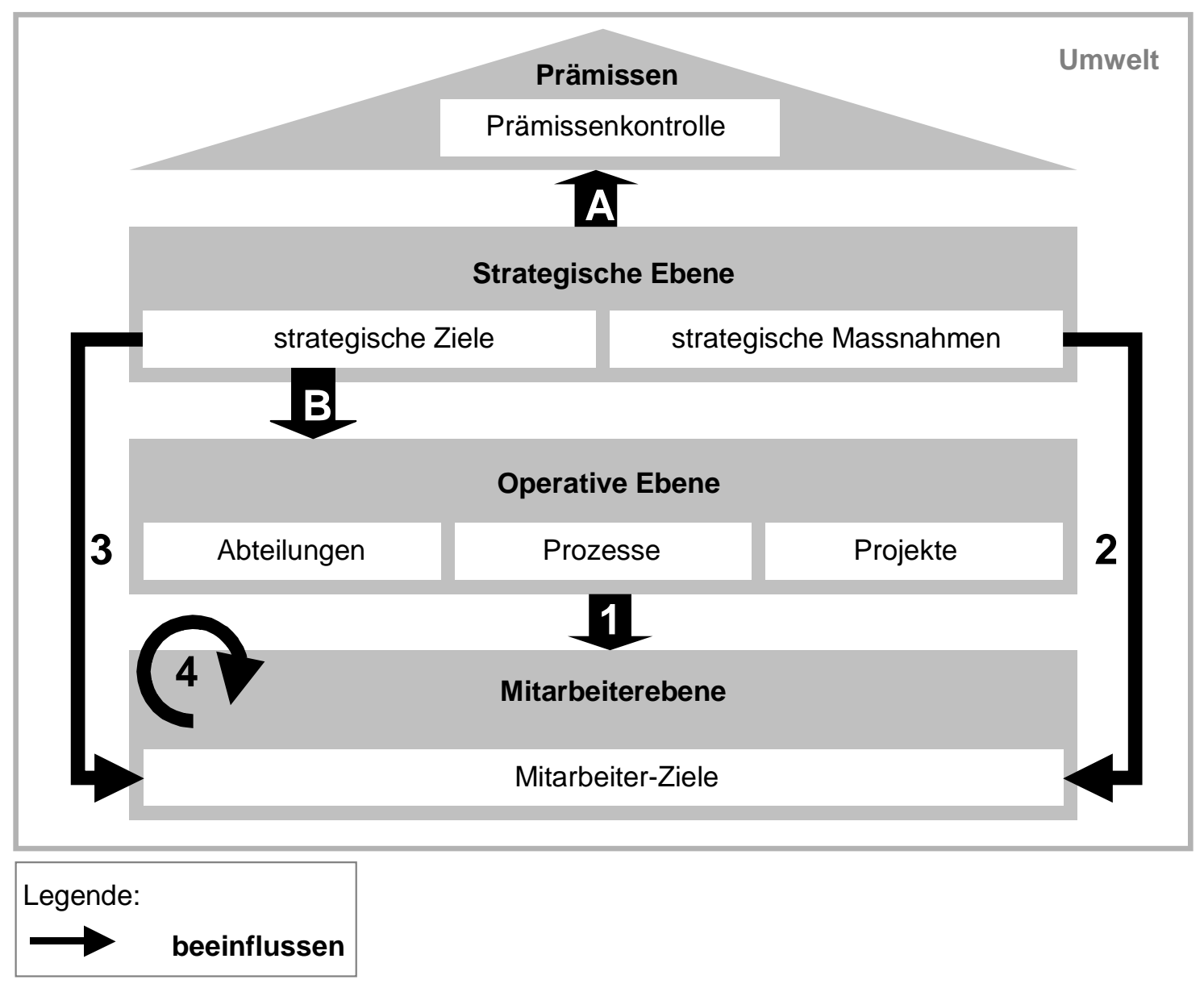

Abbildung 5-5: Beziehungen zwischen den Elementen der Systematik

\subsubsection{Elemente der Methodik}

\subsubsection{1 Überblick}

Die Methodik beantwortet die Frage nach dem „wie“, d.h. wie das PMS aufgebaut, betrieben und überarbeitet wird. Im nachfolgenden sechsten und siebten Kapitel werden hierzu für einzelne Prozesse konkrete organisatorische Aktivitäten (Vorgehensschritte) erläutert. Kapitel acht und neun gehen sodann auf die technische Umsetzung ein.

Gemäss den Anforderungen von Kapitel 4 sind Prozesse für das Datenmanagement, die Kommunikation, das Handling der Wirkungsketten, den Aufbau und den Unterhalt des PMS zu definieren. Die drei erstgenannten Prozesse werden in einem Betriebsprozess zusammengefasst. Dieser kann in Abhängigkeit der konkreten Ausgestaltung in verschiedene Teilprozesse (z.B. Nutzung, Unterstützung, technischer Betrieb, etc.) gegliedert werden. Die Konzeption des PMS kann in einen organisatorischen und 122 
technischen Prozess unterteilt werden. Zudem ist vorzusehen, dass das PMS periodisch an neue Rahmenbedingungen angepasst werden muss. Der Revisionsprozess kann durch eine Reihe zu definierender Ereignisse ausgelöst werden. Die entsprechenden Prozesse sind in Abbildung 5-6 unter Berücksichtigung ihrer gegenseitigen Wirkungen und dem zeitlichen Ablauf schematisch dargestellt.

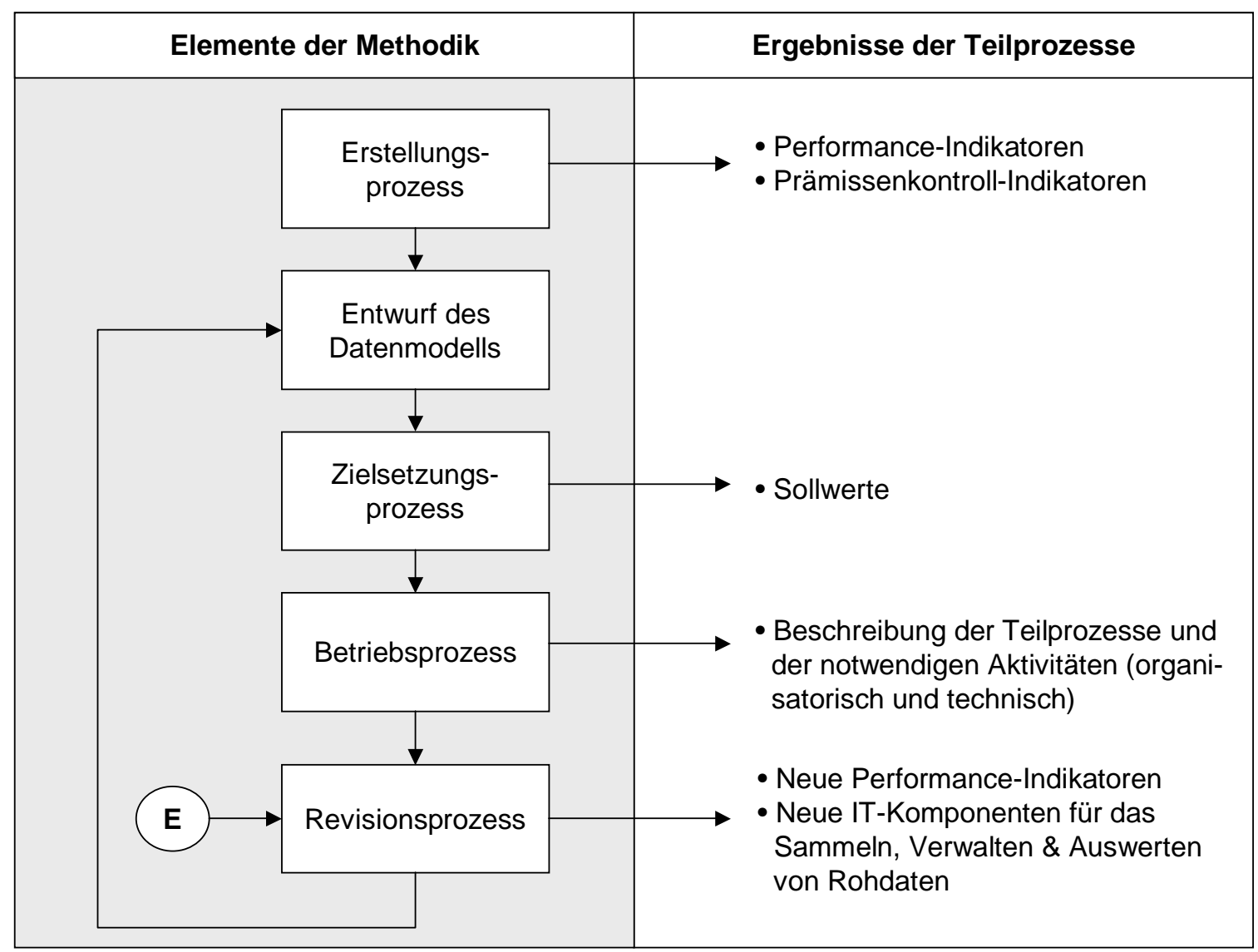

\section{E Ereignis}

Abbildung 5-6: Prozesse der Methodik

Nachfolgend soll kurz auf die einzelnen Prozesse eingegangen werden. Eine detaillierte Besprechung der organisatorischen Prozesse erfolgt in den Kapiteln sechs und sieben resp. neun für den technischen Prozess. 


\subsubsection{Prozess zur Erstellung der Indikatorenhierarchie}

Der Erstellungsprozess umfasst eine Reihe von Aktivitäten, die die Organisationsstrategie mittels Zielen und Indikatoren operationalisieren. Dabei werden für jede organisatorische Leistungsebene die notwendigen Performance-Indikatoren definiert. Somit bestehen die Grundlagen zur strategischen und operativen Durchführungskontrolle. Der Erstellungsprozess generiert zudem eine Prämissenkontrolle.

Auf der technischen Ebene des Erstellungsprozesses wird die Umsetzung in ein rechnergestütztes Informationssystem diskutiert.

\subsubsection{Zielsetzungsprozess}

Der Zielsetzungsprozess umfasst das Festlegen der Ziel-Werte (Soll-Werte) der einzelnen Performance-Indikatoren auf den einzelnen Leistungsebenen der Organisation. Dabei sind die Werte aufeinander abzustimmen. Der Zielsetzungsprozess ist in die Jahresplanung der Organisation zu integrieren.

\subsubsection{Betriebsprozess}

Der Betriebsprozess definiert die einzelnen Teilprozesse, die für die reibungslose Nutzung des PMS notwendig sind. Dabei können bspw. (1) ein Nutzungsprozess bestehend aus dem Sammeln, Speichern, Analysieren und Kommunizieren der Performance-relevanten Daten, (2) ein Prozess zur Unterstützung und (3) ein Prozess für den Unterhalt des Systems differenziert werden.

\subsubsection{Revisionsprozess}

Beim Eintreten von bestimmten Ereignissen ist es notwendig, dass das PMS überarbeitet wird. Der Revisionsprozess beschreibt die dann durchzuführenden Schritte. Eine Überprüfung des PMS ist bspw. notwendig, wenn (1) sich die Business-Strategie verändert, (2) Stakeholder neue Bedürfnisse an das PMS anmelden, (3) sich einzelne Performance-Indikatoren als nicht nützlich erweisen, (4) neue operative Informationssysteme in Betrieb genommen werden, die Performance-relevante Daten verwalten oder (5) sich neue informationstechnische Möglichkeiten für die Nutzung des PMS ergeben (vgl. Küng und Wettstein (2001)). 


\subsubsection{Elemente der Organisation}

\subsubsection{1 Überblick}

Die Organisation legt die Träger der einzelnen Teilaufgaben fest. Zudem umfasst dieser Teilschritt auch die für die Erstellung und die jeweiligen Revisionen benötigte Projektorganisation. Auf diese soll im Rahmen dieser Arbeit nur überblicksartig eingegangen werden, da Projekte dieser Art (d.h. Projekte mit organisatorischer und informationstechnischer Komponente) gängig sind (bei Grünig (1996) findet sich bspw. eine Basisvariante einer möglichen Projektorganisation).

Zur Vereinfachung der Zuordnung der Aufgabenträger wird ein Rollenkonzept eingeführt. Eine Rolle kann dabei von mehreren Personen wahrgenommen werden, und eine Person kann ihrerseits mehrere Rollen wahrnehmen. Dies ist daher viel versprechend, da eine Rolle in der Regel unterschiedliche Zielsetzungen verfolgt, was bei einer direkten Verknüpfung nicht zu Tage treten würde. Zudem ergeben sich Vorteile beim Unterhalt, da bei Wechseln von Personen nur die Rollen, nicht jedoch die Zuordnung in den Prozessen verändert werden müssen.

Lebas erwähnt eine Reihe von Rollen (siehe Tabelle 5-2), die unterschiedliche Zielsetzungen verfolgen:

- For the manager/measurer: learning and self improving

- For others in lateral partnerships: dynamic coordination of actions and continuous improvement

- For supervisors: (1) integration of local measures to create aggregated or eventually corporate wide measures; (2) monitoring of actions delegated to others for continuous improvement and control; (3) feeding the reward system

- For all actors in the organization: (1) creating a sense of belonging; (2) feeding discussions as a basis for continuous improvement

- For some external stakeholders: customers, suppliers, and some financial institutions as well as some regulatory agencies may require some measures about how well the organization is and will be doing.

Tabelle 5-2: Nutzer eines PMS und deren Zielsetzung nach Lebas (1995) 


\subsubsection{Kategorien von Informationsempfängern}

In Anlehnung an die von Lebas definierten Rollen der Informationsempfänger werden folgende Rollen vorgeschlagen:

\begin{tabular}{|l|l|}
\hline Träger & Aufgabe \\
\hline Bei der Erstellung und Überarbeitung des PMS \\
\hline Auftraggeber/Geldgeber & $\begin{array}{l}\text { Person oder Gremium, die die Erstellung des PMS in } \\
\text { Auftrag gibt (meistens identisch mit dem Sponsor). }\end{array}$ \\
\hline Projektleiter & $\begin{array}{l}\text { Leiter der Neuerstellung oder Überarbeitung des PMS. } \\
\text { Wahrnehmung der Führungs- und Koordinationsaufgabe. }\end{array}$ \\
\hline $\begin{array}{l}\text { Mitarbeiter für die organisatorische } \\
\text { Konzeption \& Implementation }\end{array}$ & $\begin{array}{l}\text { Neben allfälligen Beratern (intern oder extern) sind } \\
\text { insbesondere die für die entsprechenden organisatorischen } \\
\text { Einheiten verantwortlichen Personen beizuziehen. }\end{array}$ \\
\hline $\begin{array}{l}\text { Mitarbeiter für die technische } \\
\text { Konzeption \& Implementation }\end{array}$ & $\begin{array}{l}\text { Für die technische Konzeption und Umsetzung kommen } \\
\text { neben allällig eingesetzten Beratern primär Informatiker } \\
\text { zum Einsatz. }\end{array}$ \\
\hline Beim Zielsetzungsprozess & \multicolumn{2}{|l|}{} \\
\hline Erarbeiter der Ziel-Werte & $\begin{array}{l}\text { Für den Zielsetzungsprozess sollten die Leiter der } \\
\text { entsprechenden organisatorischen Einheiten einbezogen } \\
\text { werden. }\end{array}$ \\
\hline $\begin{array}{l}\text { Koordinator des Zielsetzungs- } \\
\text { prozesses }\end{array}$ & $\begin{array}{l}\text { Stellt die Abstimmung zwischen den einzelnen } \\
\text { Leistungsebenen und Zielen sicher. }\end{array}$ \\
\hline Genehmiger der Ziel-Werte & $\begin{array}{l}\text { Im Sinne einer Gewaltentrennung empfiehlt es sich, dass } \\
\text { der Erarbeiter einer Zielgrösse nicht mit dem Genehmiger } \\
\text { dieser Grösse übereinstimmt. }\end{array}$ \\
\hline Informationslieferanten & $\begin{array}{l}\text { Die für bestimmte Performance-Indikatoren } \\
\text { Verantwortlichen. }\end{array}$ \\
\hline Bei der Nutzung des PMS & $\begin{array}{l}\text { Beobachtung, Analyse, allenfalls Kommentierung der } \\
\text { Daten, für die der Empfänger verantwortlich ist und sofern } \\
\text { notwendig das Einleiten von entsprechenden Massnahmen }\end{array}$ \\
\hline $\begin{array}{l}\text { Verantwortlicher für die } \\
\text { Performance-relevanten Daten }\end{array}$ & $\begin{array}{l}\text { Personen, die für die Erhebung resp. die ordnungsmässige } \\
\text { Lieferung (auch elektronische) von bestimmten } \\
\text { Performance-relevanten Daten verantwortlich sind. }\end{array}$ \\
\hline Informationsempfänger & $\begin{array}{l}\text { Personen, die für den Betrieb des IT-Systems für das PM } \\
\text { verantwortlich sind. }\end{array}$ \\
\hline $\begin{array}{l}\text { Diese Rolle ist zuständig dafür, dass der im } \\
\text { Planungsprozess festgelegte Ablauf eingehalten wird. }\end{array}$ \\
\hline
\end{tabular}

Tabelle 5-3: Unterschiedliche Aufgabenträger-Rollen eines PMS

In Abhängigkeit der Ausgestaltung der Betriebsprozesse sind weitere Rollen denkbar (z.B. Personen zur Unterstützung der Nutzer (Helpdesk-Mitarbeiter)). 


\section{Prozess zur Erstellung der Indikatorenhierarchie}

Eine der Zielsetzungen der vorliegenden Arbeit besteht darin, ein heuristisches Vorgehensmodell zu erarbeiten, welches die Herleitung der Performance-Indikatoren aus der Strategie und deren Implementierung in ein rechnergestütztes Informationssystem umfasst. Diese Zielsetzung lässt sich gemäss dem Aufbau der Arbeit in einen organisatorischen Teil (Ableiten der Performance-Indikatoren aus der Unternehmensstrategie) und einen technischen Teil (Implementierung in ein rechnergestütztes Informationssystem) unterteilen. Das erste Teilziel soll im vorliegenden Kapitel bearbeitet werden, welches wiederum aus zwei Hauptteilen besteht. Im Ersten werden die Input-Faktoren und der Transformationsprozess theoretisch vorgestellt, im Zweiten wird der vorgeschlagene Prozess an einem konkreten Beispiel illustriert.

Der vorgeschlagene Transformationsprozess ist gemäss der gewählten praktischnormativen Forschungsrichtung, als heuristisches Verfahren einzuordnen. Heuristische Verfahren versuchen unter Zuhilfenahme von heuristischen Prinzipien eine für die Praxis taugliche Lösung zu erarbeiten. In der Regel erfolgt eine Dekomposition der Problemstellung in eine Sequenz von Einzelproblemen (vgl. Kühn (1978)). Dabei besteht kein Anspruch auf eine optimale Lösung (vgl. Grünig und Kühn (2000)). Demgegenüber stehen die abschätzbaren Kosten, der benötigte Zeitrahmen und eine gute Akzeptanz durch die mit den Entscheidungen beauftragten Personen im Vordergrund (vgl. Heckner (1998)).

Als Basis für den zu erarbeitenden Erstellungsprozess dienen methodische Vorschläge in der Literatur zur Problembewältigung (vgl. bspw. Grünig und Kühn (2000) oder Sommerville (2001)), grundlegende Arbeiten zum Thema des Performance Measurement (vgl. Kapitel 2-3 und Teile von Kapitel vier) sowie praktische Erkenntnisse (vgl. empirische Studien im Kapitel vier).

\subsection{Zielsetzung und Grenzen des Erstellungsprozesses}

Wie in Kapitel vier gezeigt wurde, bekunden Organisationen grosse Probleme darin, einen ausgewogenen und durchgängigen Satz von Zielen zu erarbeiten und mittels Indikatoren zu operationalisieren. Der Erstellungsprozess soll hierzu einen Beitrag leisten und demzufolge 
als Output die Ziele einer Organisation und die zugehörigen Performance-Indikatoren in einem geordneten Rahmen darstellen (vgl. Abbildung 6-1).

Hierzu sind zwei wesentliche Elemente notwenig. Erstens müssen die notwendigen InputFaktoren bestimmt und erfasst werden. Zweitens muss ein Verfahren entwickelt werden, welches es erlaubt, die Input-Faktoren mittels verschiedener Instrumente in den angestrebten Zielzustand zu überführen.

Die möglichen Input-Faktoren ergeben sich aus den Anforderungen, die an ein PMS gestellt werden (vgl. Tabelle 6-1):

\begin{tabular}{|l|l|}
\hline Anforderung (siehe Tabelle 4.1) & daraus abgeleiteter Input-Faktor \\
\hline Ziele werden aus der Strategie hergeleitet & Strategie \\
\hline Ziele werden mittels Indikatoren operationalisiert & Ziele \\
\hline $\begin{array}{l}\text { Gliederung der PI nach Wirkung-, Durchführung- und } \\
\text { Prämissenindikatoren }\end{array}$ & Prämissen \\
\hline $\begin{array}{l}\text { Die individuelle Organisationsstruktur kann abgebildet } \\
\text { werden }\end{array}$ & Bestehende Organisation \\
\hline $\begin{array}{l}\text { Das PMS kann in die bestehenden Führungssysteme } \\
\text { integriert werden }\end{array}$ & Bestehende Plansysteme \\
\hline $\begin{array}{l}\text { Die Ziele sind mit der Organisation und den Mitarbeitern } \\
\text { verknüpft }\end{array}$ & $\begin{array}{l}\text { Zielvorgaben aus dem Management } \\
\text { by Objectives }\end{array}$ \\
\hline
\end{tabular}

Tabelle 6-1: Aus den Anforderungen abgeleitete Input-Faktoren

Nachfolgende Abbildung 6-1 zeigt den Kontext des Erstellungsprozesses schematisch. Im Kern handelt es sich um einen einfachen Transformationsprozess, der ausgehend von einem angestrebten Ergebnis, die notwendigen Input-Faktoren und Transformationsschritte auf Grund der theoretischen Erkenntnisse, den heuristischen Prinzipien und den Einzelfallbeispielen sowie der durchgeführten Umfrage (vgl. Kapitel 4) ableitet. 


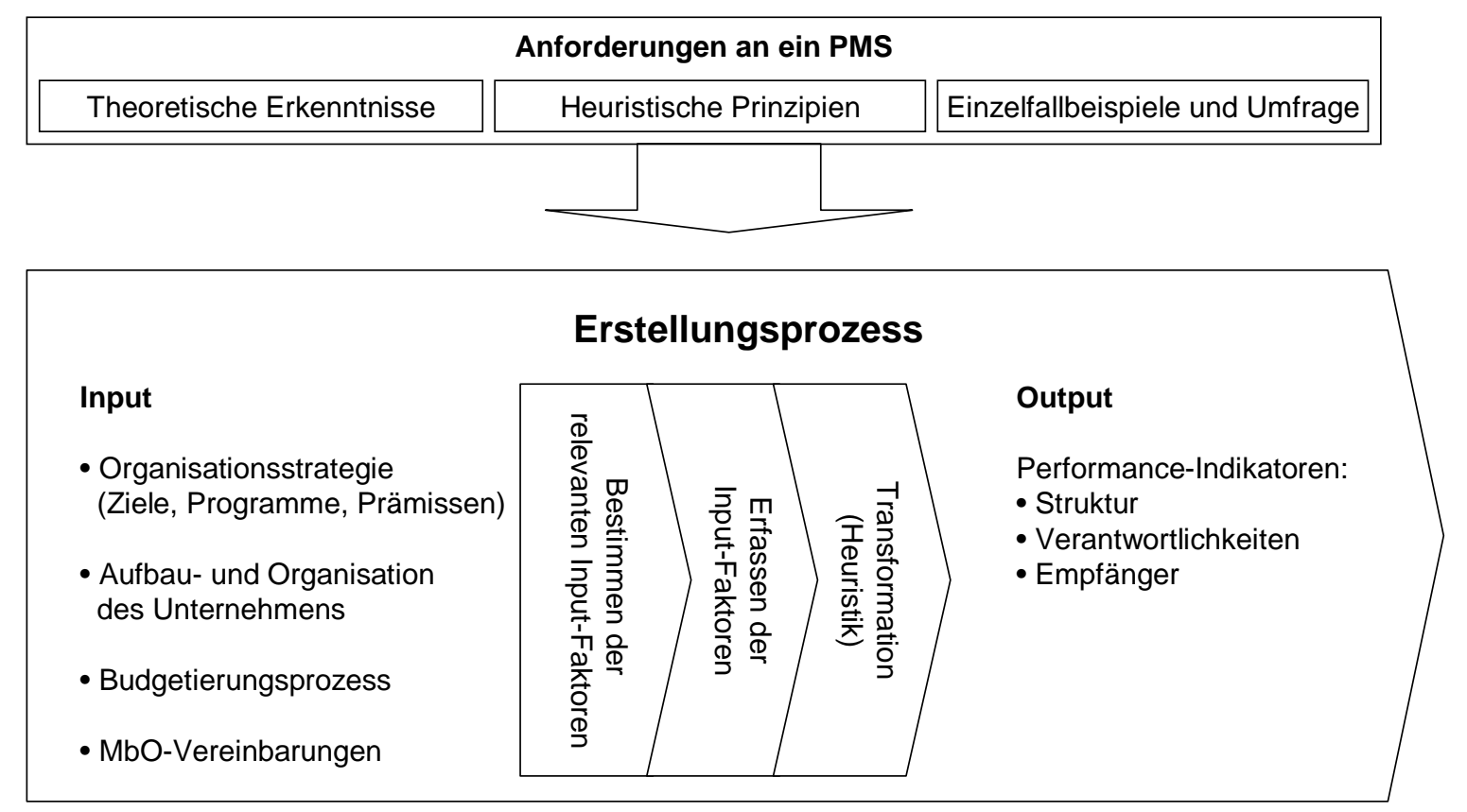

\section{Abbildung 6-1: Wesentliche Elemente des Erstellungsprozesses}

Der Erstellungsprozess soll die strategische Planung, die Definition der strategischen Programme (vgl. Grünig und Kühn (2000)), den Budgetierungsprozess (vgl. Horváth (2001)) oder das Führungsinstrument Management by Objectives (vgl. Wunderer und Grunwald (1980)) nicht ersetzen. Vielmehr soll das Performance-Measurement-System, welches mit dem Erstellungsprozess aufgebaut wird, relevante Führungsinformationen geordnet zusammentragen und die Basis für eine informationssystemtechnische Implementierung bieten.

Nachfolgend werden die einzelnen Elemente des Erstellungsprozesses diskutiert und die vorgeschlagene Schrittfolge präsentiert.

\subsection{Mögliche Input-Faktoren des Transformationsprozesses}

\subsubsection{Input-Faktoren aus den strategischen Dokumenten}

Im Kapitel 2.5.4. wurde der Begriff der strategischen Führung im Sinne einer Abgrenzung zum Performance Measurement diskutiert. An dortiger Stelle wurde gezeigt, dass die strategische Planung in ihrem Ergebnis zur Strategie führt, die in Form von Dokumenten und Plänen die langfristigen Vorgaben zum Aufbau und zur Sicherung von 
Erfolgspotenzialen enthält (vgl. Grünig und Kühn (2000)). Die Strategie bildet somit einen wesentlichen Input-Faktor des angestrebten Erstellungsprozesses, da sie die Grundlage der Zielbildung darstellt.

\subsubsection{Ziele als Basis für den Kaskadierungsprozess}

Die langfristigen Vorgaben der Strategie werden in der Regel in Form von Zielen formuliert. Diese bilden die Basis für die Zuordnung von Leistungszielen zu den einzelnen organisatorischen Einheiten (Beispiele: Kostenführerschaft im Bereich des xy bei definiertem Qualitätsniveau, Marktanteil von 30\% im Bereich yz).

\subsubsection{Prämissen als Element der strategischen Kontrolle}

Unter Prämissen im Kontext der strategischen Dokumente einer Organisation werden Annahmen verstanden, auf deren Basis Ziele für die Organisation festgelegt wurden (vgl. Grünig (1996)). Die Festlegung der Prämissen erfolgt in der Regel auf der Basis der im strategischen Planungsprozess getroffenen Annahmen. In einem ersten Schritt wird eine optimale Transparenz bezüglich der getroffenen Annahmen angestrengt. Dies geschieht meist durch schriftliches Festhalten der Prämissen (vgl. Stufe eins des Reifegradmodells bei SEI (2002); Beispiele: Die Anzahl der Marktteilnehmer wird sich bis zum Jahre 2008 von heute 875 auf 600 reduzieren, das Preisniveau wird sich für das Kundensegment KMU bis 2008 um 30\% ermässigen).

\subsubsection{Bestehende Organisation}

Berücksichtigt werden muss neben Zielen und Prämissen die bestehende Aufbau- und Ablauforganisation. Sie zeigt auf, in welcher Organisationsform angestrebt wird, die vorgegebenen Ziele zu realisieren. Die Erfassung der bestehenden Organisation stellt in der Praxis kaum ein Problem dar.

\subsubsection{Bestehendes Plansystem}

Unternehmen operationalisieren ihre Strategie in Form eines Plansystems. Die konkrete Ausgestaltung ist abhängig von der Grösse, dem Produktesortiment und den anvisierten Märkten. In der Regel umfasst es eine Beschreibung der einzelnen Pläne mit ihrem Planungshorizont. Unterschieden werden finanzielle Pläne (Investitions-, Erfolgs- und Finanzplan, respektive -budget (für die unterjährige Planung)) und Sach-, Ziel- und 
Massnahmenpläne (z.B. für das Marketing, die Entwicklung, den Verkauf, den Einkauf, die Produktion und das Personal). Die zeitliche Abfolge der Erstellung und Überprüfung der einzelnen Pläne wird im Planungskalender geregelt (vgl. Grünig et al. (1996)). Das Plansystem umfasst zudem die Verantwortlichkeiten für die einzelnen Teilaufgaben und die Informationsempfänger.

\subsubsection{Finanzielle Pläne}

Traditionell erfolgt die Finanzplanung (bei der unterjährigen Finanzplanung spricht man in der Praxis von Budgetierung; vgl. Horváth (2001)) sehr detailliert. Wie in Kapitel vier gezeigt, bestehen für finanzielle Performance-Indikatoren meist ausgereifte Kontrollinstrumente. Nachteilig wirkt sich aber deren isolierte Betrachtung aus. Aus diesen Überlegungen heraus erscheint es nicht sinnvoll, die Informationen im gleichen Detaillierungsgrad in das Performance-Measurement-System zu übernehmen. Vielmehr erfolgt eine Beschränkung auf eine Reihe von Kern-Kennzahlen.

\subsubsection{Sachpläne}

Die Sach-, Ziel- und Massnahmenpläne sind die den finanziellen Zielen vorangehenden Pläne. Sie beschäftigen sich, wie der Name zum Ausdruck bringt, mit der Sache. Ein Verkaufsplan beinhaltet daher bspw. die geplanten Absatzmengen je Produkt in den verschiedenen Teilmärkten, die Verkaufspreise, usw.. Dieser bildet wiederum die Grundlage für den Produktions- und Beschaffungsplan.

\subsubsection{Zielvorgaben aus dem Management by Objectives}

Das Führungsmodell Management by Objectives (MbO) wurde im Kapitel 3.1 .3 vorgestellt. Die im Rahmen des MbO festgelegten Ziele, die in einem Vereinbarungsprozess zwischen den Zielen des Mitarbeiters und den Zielen der Organisation zu Stande gekommen sind, sollten bei der Erstellung des PMS berücksichtigt werden.

\subsubsection{Koordination der Input-Faktoren}

Der Koordination kommt eine grosse Bedeutung zu. Einerseits sind die Instrumente in sich zu koordinieren, andererseits sind die Instrumente untereinander abzustimmen. Dieses Koordinationsproblem ist unabhängig vom hier betrachteten Performance-Measurement- 
System. Aus diesem Grunde sollen nur einige grundlegende Überlegungen gemacht und auf eine detaillierte Besprechung verzichtet werden.

Innerhalb der Instrumente muss aus der Sicht der Organisation eine horizontale und vertikale Koordination vorgenommen werden (vgl. z.B. Wunderer und Grunwald (1980)). Betrachtet man den Planungshorizont, so sind einzelne Elemente über dieses Objekt in Einklang zu bringen (vgl. z.B. Mintzberg (1994)). In der zeitlichen Abfolge kommt meist ein Kalender zum Einsatz, um die Abstimmung zwischen den einzelnen Aktivitäten Gewähr leisten zu können (vgl. Küpper (2001)). Hierbei stehen verschiedene Verfahren für den Aufbau des Planungskalenders zur Auswahl. Gängig sind Schachtelungs- und Reihungsverfahren (vgl. Grünig (1996)). Dabei handelt es sich um Bottom-Up- resp. TopDown-Verfahren. Neuere Ansätze schlagen kombinierte Verfahren vor, so etwa das insbesondere im angelsächsischen Raum bekannte Hoshin-Verfahren (vgl. die ausführliche deutschsprachige Darstellung bei Hoffmann (1999)). Als formales Instrument zur Förderung der Koordination wird in der Regel ein Planrahmen eingeführt (vgl. Kapitel 5).

Die Abstimmung der einzelnen Instrumente umfasst die Begriffshomogenisierung und die Abstimmung der Zielsetzungen der eingesetzten Instrumente. Darauf soll hier nicht näher eingetreten werden.

\subsubsection{Qualität der Input-Faktoren}

Das Ergebnis des Erstellungsprozesses ist wesentlich abhängig von der Güte der InputFaktoren. Wie verschiedene empirische Untersuchungen zeigen, verfügen Organisationen über ganz unterschiedliche Reifegrade bezüglich der oben dargestellten Plan- und Kontrollsysteme. Ohne schriftlich vorhandene Strategie ist es bspw. kaum möglich, ein stringentes Zielsystem herzuleiten. Gleiches gilt bei umfassenden Reorganisationen, wenn die bestehende Organisation nicht mehr (oder noch nicht) mit den gesetzten Zielen korrespondiert. Eine gewisse Flexibilität besteht bei sekundären Elementen, wie z.B. nicht explizit erarbeiteten Prämissen. 


\subsection{Vorgehensschritte des vorgeschlagenen Erstellungsprozesses}

Abbildung 6-2 zeigt die Vorgehensschritte des vorgeschlagenen Erstellungsprozesses im Überblick. Er gliedert sich in vier Hauptschritte und eine Reihe von Unterschritten. Die Hauptschritte umfassen:

\section{Projektplanung}

2. Bestimmung der relevanten Input-Informationen

3. Erfassen der relevanten Input-Informationen

4. Transformationsschritte

Am Ende von Hauptschritt zwei ist explizit eine Abbruchbedingung vorgesehen, falls die benötigten Input-Informationen nicht verfügbar sind. Für die Schritte $4.2-4.4$ ist eine Iteration bis zur Erreichung des definierten Detaillierungsgrades eingeplant.

Nachfolgend werden die einzelnen Schritte im Detail besprochen und der Einsatz möglicher Instrumente zur Unterstützung der Problembearbeitung bei den einzelnen Schritten eingeführt. 


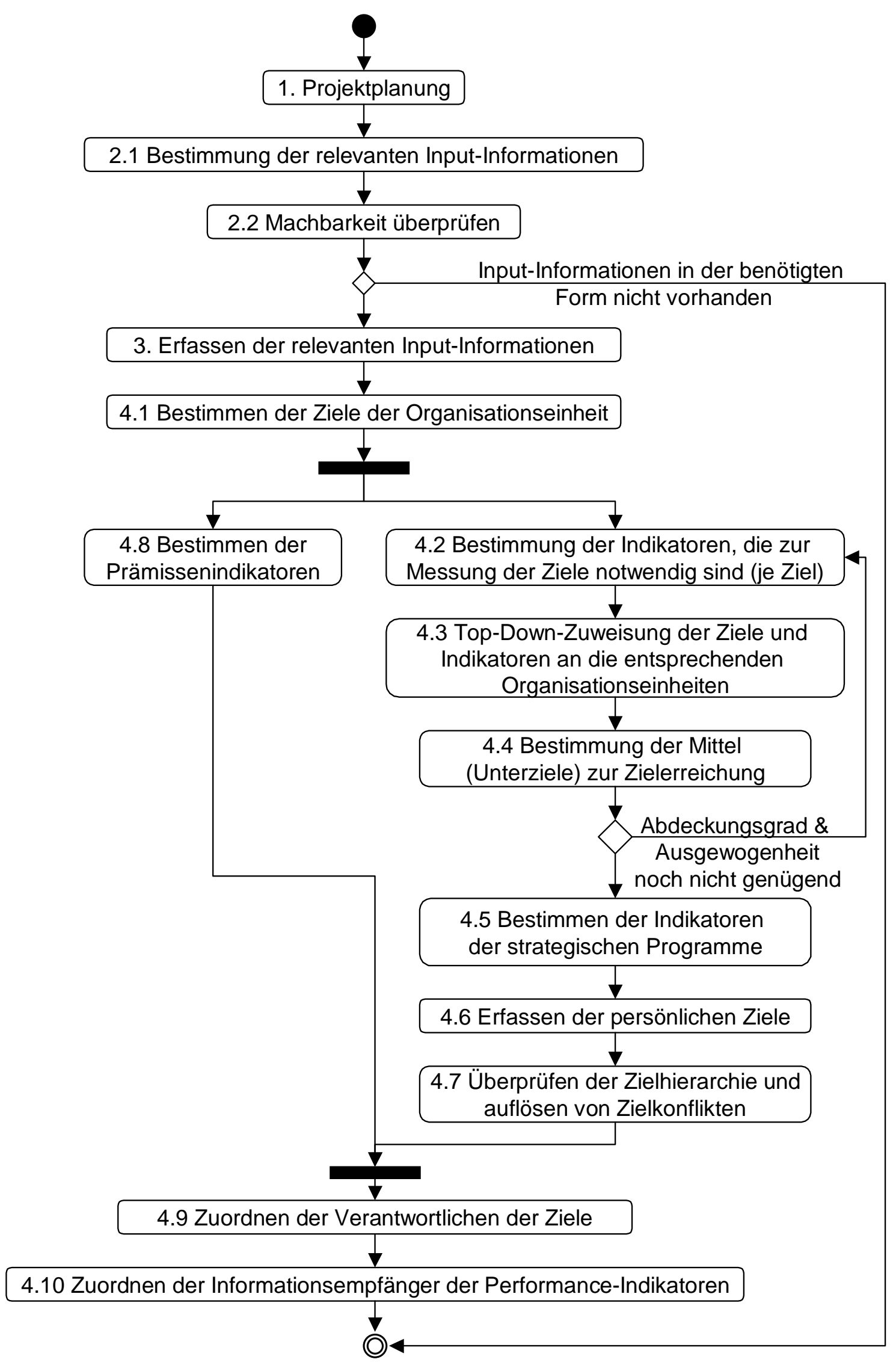

Abbildung 6-2: Vorgehensschritte des Erstellungsprozesses im Überblick 


\subsubsection{Projektplanung (Schritt 1)}

Der Prozess zur Erstellung der Indikatorenhierarchie kann als Projekt aufgefasst werden. In einem ersten Schritt geht es darum, dieses Projekt zu planen. In Anlehnung an Grünig und Kühn (2000) sind folgende Planungsschritte zu durchlaufen (vgl. Abbildung 6-3):

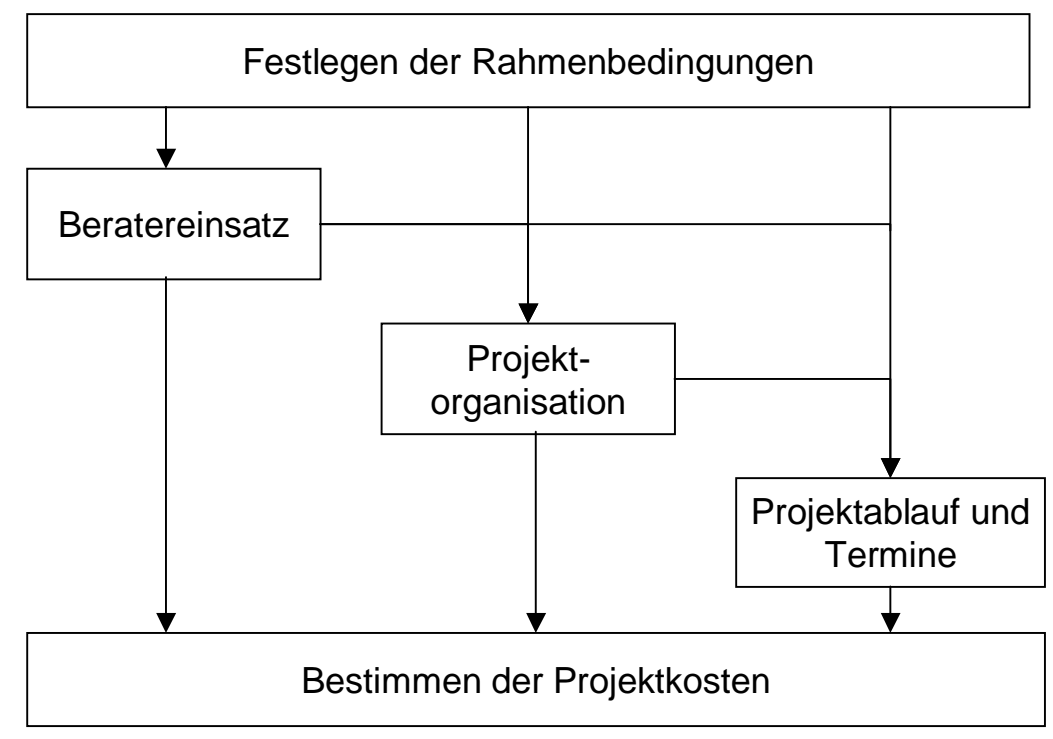

Abbildung 6-3: Projektplanung (vgl. Grünig und Kühn (2000))

Die Rahmenbedingungen umfassen die Festlegung des Projektgegenstandes und der Projektziele. Der Gegenstand des Projektes im Allgemeinen bedarf keiner Klärung. Bezüglich der zu integrierenden Bereiche (Abgrenzung) besteht jedoch Handlungsbedarf. Grundsätzlich kann ein PMS für alle Ebenen und organisatorischen Einheiten eingesetzt werden: von der obersten Ebene eines Konzerns, über Business Units und Projekte bis hinunter zur Mitarbeiterebene. Wollte man jedoch in einem Grossunternehmen ein PMS konstruieren, welches sämtliche Bereiche abdecken würde, so wäre die Komplexität eines solchen Systems hoch. Diese hohe Komplexität würde dazu führen, dass die Systemerstellung enorm aufwändig und die Anpassung an neue Gegebenheiten kaum durchführbar wäre. Hinzu käme, dass ein solches PMS von kaum jemandem mehr begriffen und gedanklich erfasst werden könnte. Es ist daher zu empfehlen, den Umfang des Projektes nicht zu überladen. Im Kapitel fünf wurden im Rahmen der Systematik die einzelnen Gegenstandsbereiche diskutiert. Diese sollen an dieser Stelle nicht erneut aufgegriffen werden. 
Bei Projekten, in denen das benötigte Fachwissen im Unternehmen nicht vorhanden ist, können externe Personen beigezogen werden. Grünig und Kühn (2000) weisen darauf hin, dass eine präzise Auftragsbeschreibung und Klarheit bezüglich der Kosten notwendig sind, um mit Externen zusammen zu arbeiten. Nicht alle Aufgaben eignen sich, outgesourct zu werden (vgl. z.B. Brenner et al. (1999)). Aufgaben, die von Externen wahrgenommen werden können, sind bspw. Sitzungsmoderationen, Methodenbeiträge oder Marktkenntnisse. Sowohl die Auslagerung der Projektleitung als auch die Auslagerung der konkreten Erarbeitung von Zielen und Indikatoren eignen sich hingegen nicht. Einerseits kann die Verantwortung kaum delegiert werden, andererseits muss die Lösung auch getragen werden. Letzteres ist eher der Fall, wenn sie durch interne Personen erarbeitet wurde. (vgl. Grünig und Kühn (2000))

Unter der Projektorganisation versteht man die Gesamtheit der Organisationseinheiten sowie die aufbau- und ablauforganisatorischen Regelungen zur Abwicklung eines bestimmten Vorhabens (vgl. Jenny (1998)). Die Ausgestaltung ist von der Komplexität des Projektes abhängig. Es existiert ausführliche Literatur zum Thema, weshalb dieser Punkt nicht näher besprochen wird (vgl. bspw. die Literatur im Informatik-Bereich: Zehnder (1991), Jenny (1998)) .

Ein weiteres Planelement umfasst die Festlegung der Projektphasen und der Termine. Die einzelnen Projektphasen werden in diesem Kapitel diskutiert. Es handelt sich um ein mögliches Vorgehen. Individuell festgelegt werden muss hingegen die zeitliche Terminierung.

Schlussendlich erfolgt die Budgetierung des Projektes. Dabei müssen interne und allfällige externe Kosten berücksichtigt werden.

Aus Abbildung 6-2 ist ersichtlich, dass nach Schritt 2 eine Abbruchmöglichkeit vorgesehen ist, da bestimmte Daten in einer geeigneten Qualität notwendig sind, um das Vorhaben realisieren zu können. Selbstverständlich kann auch der in Abbildung 6-3 vorgeschlagene Ablauf Iterationen umfassen, die aber aus Gründen der Übersichtlichkeit in der Abbildung nicht eingezeichnet wurden. 


\subsubsection{Bestimmen der relevanten Input-Informationen (Schritt 2)}

\subsubsection{Die Zielsetzung des Performance-Measurement-Systems determiniert die relevanten Input-Informationen}

Auf Grund der in Schritt eins festgelegten Projektziele erfolgt nun die Bestimmung der benötigten Input-Informationen. Dabei ist in diesem Schritt ausser Acht zu lassen, ob einzelne Input-Informationen im Unternehmen bereits existieren oder nicht. Das Ergebnis dieses Schrittes ist eine Liste mit den benötigten Input-Informationen.

\subsubsection{2 Überprüfung der Machbarkeit}

In einem zweiten Schritt wird überprüft, inwiefern die einzelnen benötigten InputInformationen in der geforderten Qualität und Aktualität existieren. Falls diese nicht vorhanden sind, stellt sich die Frage, wie diese wirtschaftlich gewonnen werden können:

- Existenz: Nicht alle gewünschten Informationen sind tatsächlich in der betroffenen Organisation verfügbar. Es muss also überprüft werden, ob das entsprechende Informationsobjekt existiert. Dabei soll festgestellt werden, ob das Informationsobjekt für jeden gewünschten Betrachtungsgegenstand vorhanden ist (Wenn bspw. auf Business-Unit-Stufe ein MbO vorgeschrieben wird - aus welchem im vorliegenden Fall die persönlichen Zielsetzungen extrahiert werden könnten, so ist damit noch nicht sichergestellt, dass dieses auch in der Abteilung X existiert).

- Vollständigkeit: Es sollte zudem verifiziert werden, inwiefern sämtliche Objekte, die das Instrument umfasst, abgedeckt sind (Bspw. wird in einer Organisation MbO als Führungsinstrument auf jährlicher Basis eingesetzt, wobei Abteilung $\mathrm{X}$ als Einzige dieses nicht einsetzt.).

- Qualität: Die benötigten Informationen sind in ausreichender Qualität bereitzustellen. Obwohl diese Abklärung auf der hohen Aggregationsstufe schwierig durchzuführen ist, sollte dieser Punkt beachtet werden, um bei schlechter Qualität reagieren zu können.

- Aktualität: Letztlich ist die Aktualität zu prüfen. Immer wieder trifft man auf Instrumente, die in einer hohen Qualität eingeführt wurden, jedoch nicht - oder 
nicht mehr - benutzt werden. Damit würden sie für die beabsichtigten Zwecke unbrauchbar.

- Wirtschaftlichkeit: Ist bei einem der oben erwähnten Punkte ein erhebliches Defizit auszumachen, so sollte überprüft werden, mit welchem Aufwand und in welcher Zeitspanne dieses behoben werden kann, und ob es sich um ein zwingendes Element handelt. In Abhängigkeit vom Ergebnis ist eine Iteration zum Schritt eins angezeigt, in welcher allenfalls die Zielsetzung des Projektes überdacht resp. die Zeit- und/oder Kostenplanung modifiziert werden muss.

Für die weiteren Schritte ist es notwendig, dass zumindest die Aufbauorganisation und die Ziele ihrer Einheiten bekannt sind. Auf diesen Elementen baut die vorgestellte Methodik auf. Dies bedeutet konkret:

- Die betrachtete Organisationseinheit muss mit ihren über- und untergeordneten Einheiten dargestellt sein.

- $\quad$ Für die höchste betrachtete organisatorische Einheit müssen deren Ziele bekannt sein.

Tabelle 6-2: Minimalanforderungen für den Transformationsprozess (in Anlehnung an Wettstein et al. (2001))

\subsubsection{Erfassen der relevanten Input-Informationen (Schritt 3)}

Stehen die zu nutzenden Input-Informationen fest, so sind diese im Teilschritt drei zu erfassen und geordnet zusammenzustellen. Damit sind die vorbereitenden Arbeiten abgeschlossen und der eigentliche Transformationsschritt kann in Angriff genommen werden.

\subsubsection{Transformationsschritte (Schritt 4)}

\subsubsection{Bestimmen der Ziele der Organisationseinheit (Schritt 4.1)}

Der erste Schritt der Transformation besteht darin, die Ziele der organisatorischen Einheit zu erfassen. Dabei empfiehlt es sich, darauf zu achten, dass die Ziele ausgewogen sind. Ausgewogen bedeutet in diesem Kontext, dass sowohl finanzielle als auch nicht-finanzielle Ziele berücksichtigt werden (vgl. Tabelle 4-1: Anforderungen an ein PMS). Bei Küng (2000) werden bspw. die Aspekte nach den Stakeholdern in Anlehnung an das EFQMModell (vgl. EFQM (2002)) vorgeschlagen. Unterschieden werden die Aspekte Investoren, 138 
Mitarbeiter, Kunden (Käufer \& Lieferanten), Gesellschaft und Innovation (vgl. Abbildung 6-4). Gleichzeitig ist darauf zu achten, dass die Zahl der Ziele je Organisationseinheit nicht zu gross wird. Hoffmann zitiert Mulligan et al. und nennt eine Obergrenze von vier (vgl. Mulligan et al. (1996), Hoffmann (1999)). Treffend drücken dies Simons und Davila (1998) aus: „Too often, managers succumb to a form of political correctness in determining performance factors. In order not to offend any division or constituency within the company, they compile long lists of critical performance variables - such as information processing productivity, employee satisfaction, and revenue growth - but do not differentiate what is supposedly nice to have from what is truly critical to the company's success. As a result, people don't know exactly where they should focus their time."

Ergebnis ist eine Liste von Zielen für die zu messenden Einheiten. (vgl. Wettstein und Küng (2001))

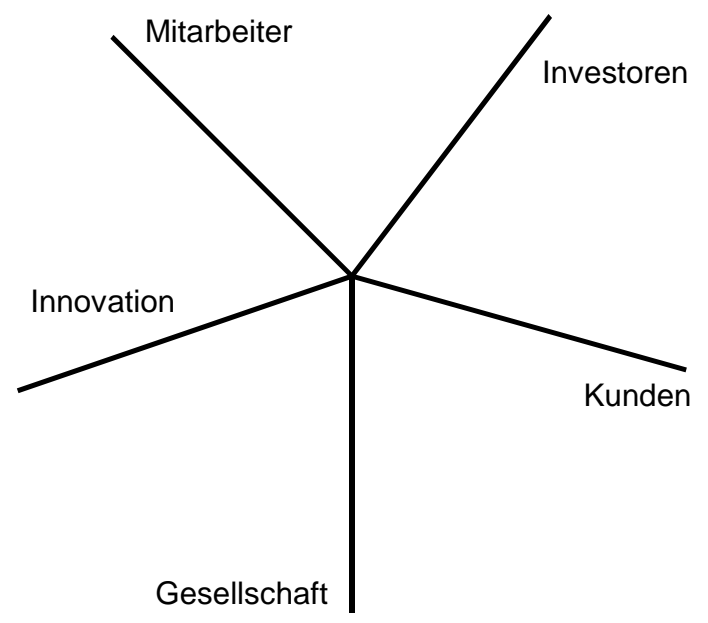

Abbildung 6-4: Zu differenzierende Aspekte nach Küng (2000)

\subsubsection{Bestimmung der Indikatoren, welche zur Messung der Ziele notwendig sind} (Schritt 4.2)

Der zweite Schritt der Transformation umfasst die Operationalisierung der definierten Ziele mittels Performance-Indikatoren. Es handelt sich dabei gemäss der Meinung von zahlreichen Autoren um einen der schwierigsten Schritte. Dementsprechend breit ist das Angebot an Vorschlägen zur Lösung dieser Aufgabe. Allgemein anwendbar erscheint nach 
Meinung des Verfassers die von Fries (1994) vorgeschlagenen drei Phasen: Identifikation, Analyse und Auswahl.

Identifikation möglicher Indikatoren: In einer ersten Phase werden mögliche Performance-Indikatoren gesammelt. Dies geschieht durch Beantwortung folgender Fragen: „Mit welchen Performance-Indikatoren können die definierten Ziele gemessen werden?“. Dabei kommen häufig Kreativitätstechniken zum Einsatz (z.B. Brainstorming (vgl. Department-of-Navy (1999a))).

Analyse und Bereinigung der Performance-Indikatoren: In einer nächsten Phase sollten die entwickelten Performance-Indikatoren auf ihren Zusammenhang und ihren Zielbezug hin untersucht werden. So kann bei den Beteiligten ein Verständnis über die Bedeutung und Wirkung der einzelnen Indikatoren erreicht werden. Die genaue Definition der Indikatoren ist ein wichtiger Punkt. Mit Vorteil wird dafür ein vorgefertigter Raster verwendet, damit keine wichtigen Eigenschaften vergessen gehen. Dieser sollte neben einem Namen eine möglichst präzise Beschreibung, eine Berechnungsformel, Angaben über die zu verwendenden Einheiten sowie allfällige Angaben zur Erhebungsfrequenz enthalten (vgl. die detaillierte Auflistung von Attributen zu Performance-Indikatoren bei Brunner (1999)).

Zur Analyse können Instrumente, wie Ursache- und Wirkungsdiagramme (vgl. Department-of-Navy (1999c)), teilweise auch bekannt als Fischgräte-Diagramm (Ishikawa oder cause-and-effect diagram (vgl. Ishikawa (1985))), Netzwerkdiagramme (vgl. Vester (1987), Probst und Gomez (1995)) und Affinitätsdiagramme (vgl. Department-of-Navy (1999b)) eingesetzt werden.

- Affinitätsdiagramme: Sie werden häufig verwendet, um Ideen, die aus dem Brainstorming resultieren gemäss ihren natürlichen Beziehungen zu gruppieren (vgl. Department-of-Navy (1999d)).

- Ursache- und Wirkungsdiagramme: Sie erlauben die weitere Analyse der einzelnen Indikatoren dadurch, dass diese in Beziehung zueinander gebracht werden. Fischgräte-Diagramme münden in einer einzigen Grösse, währenddessen Netzwerkdiagramme multilaterale Beziehungen erlauben. 
Nach Erfahrung des Verfassers kann durch die präzise Beschreibung und die Analyse der Performance-Indikatoren mit obigen Instrumenten bereits eine erste Bereinigung erfolgen. Zudem hilft die kritische Auseinandersetzung bei den Beteiligten die Akzeptanz bezüglich der vorgeschlagenen Performance-Indikatoren zu festigen.

\section{Auswahl:}

Sind die Performance-Indikatoren identifiziert und definiert, erfolgt die schwierigste Phase: die Auswahl. Ein mögliches Auswahlverfahren kann dadurch erreicht werden, indem überprüft wird, ob von den einzelnen Indikatoren einige allgemein gültige Prinzipien eingehalten werden. In der Literatur werden zahlreiche Anforderungen an Performance-Indikatoren aufgeführt (vgl. z.B. Winchell (1996), Kitchenham (1996)). Abbildung 6-5 zeigt eine mögliche Auswahl.

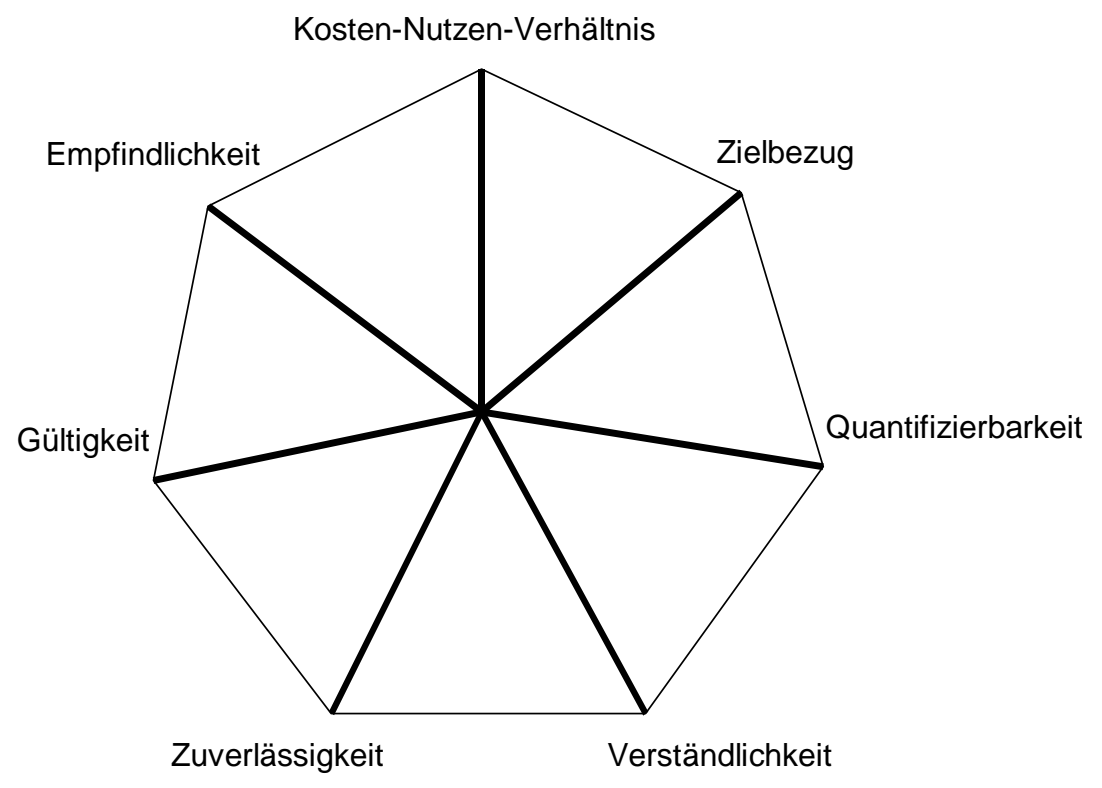

\section{Abbildung 6-5: Anforderungen an Performance-Indikatoren}

Die erwähnten Anforderungen sollen nachfolgend kurz charakterisiert werden:

Der Zielbezug sollte durch die vorgeschlagene Methodik ohne weiteres Zutun erreicht werden, da die Zielhierarchie als Grundlage dient und der Zielbezug damit unmittelbar hergestellt wird. 
Durch die präzise Beschreibung der Performance-Indikatoren (siehe Analyse und Bereinigung der Performance-Indikatoren) sollte die Quantifizierbarkeit ebenfalls Gewähr leistet sein. Ansonsten ist eine Transformation von qualitativen Indikatoren in quantitative zu prüfen. Ein Beispiel: Der qualitative Indikator „Zahlungsverhalten der Schuldner“ könnte z.B. in einen quantitativen Indikator transformiert werden der „Anzahl Tage von Rechnungsstellungsdatum bis Zahlungsdatum“ lautet.

Ein wichtiges Kriterium stellt auch die Verständlichkeit der Performance-Indikatoren dar. Dabei ist davon auszugehen, dass der Indikator von denjenigen Personen begriffen werden muss, an die der Indikator kommuniziert wird.

Drei eher technische Kriterien sind die Zuverlässigkeit, die Gültigkeit und die Empfindlichkeit. Ein Indikator ist dann zuverlässig, wenn ein mehrmaliges Messen (unter den gleichen Bedingungen) zum gleichen Ergebnis führt. Die Gültigkeit eines Performance-Indikators ist dann hoch, wenn dasjenige gemessen wird, was zu messen beabsichtigt war. Die Empfindlichkeit drückt aus, wie der Indikator auf Veränderungen der Performance reagiert. Sensitive Indikatoren reagieren stark. Dies ist von grosser Bedeutung, wenn es sich um schwache Signale handelt.

Nicht alle Performance-Indikatoren stiften einen gleich hohen Nutzen für die Entscheidungsträger und Stakeholder. Die Kosten (Datenakquisition, Berechnung, Kommunikation) sind ebenfalls von Indikator zu Indikator verschieden. Indikatoren, welche ein günstiges Kosten-Nutzen-Verhältnis aufweisen werden daher bevorzugt. Ob diese Anforderung erfüllt ist, kann erst annähernd beurteilt werden, wenn die Datenquelle identifiziert ist. Dieses Beispiel verdeutlicht, dass das Definieren ein iterativer Prozess ist und ein einmaliges Durchlaufen der Phasen auch bei guter Planung nicht ausreicht.

Eine weiterer Ansatz könnte darin bestehen, die Ausgewogenheit der Indikatoren bezüglich eines Zieles zu untersuchen und auf Grund der Analyseergebnisse eine Auswahl zu treffen. Um zu prüfen, inwiefern diese Anforderung erfüllt ist, kann es lohnend sein, die identifizierten Indikatoren nach verschiedenen Kriterien $\mathrm{zu}$ klassieren. Dadurch wird schnell deutlich, welche Forderungen besser und welche weniger gut abgedeckt sind. Nachstehend werden einige mögliche Gliederungskriterien aufgeführt, um diese Art der Qualitätsprüfung durchführen zu können. 
Performance-Indikatoren können nach folgenden Gesichtspunkten gegliedert werden:

- nach systemtechnischer Sicht: Unterteilung in Input-, Ablauf-, Output- und OutcomeIndikatoren.

- nach den Anspruchsgruppen (Stakeholder): Eine mögliche Unterteilung wäre die in Investoren/Geldgeber, Mitarbeiter, Kunden, Lieferanten, Behörde.

- nach der Art der Messgrösse. Eine relativ grobe Unterteilung ist bspw. die Kategorisierung in finanzielle und nicht-finanzielle Indikatoren.

- nach der zeitlichen Verzögerung: Messgrössen können unterteilt werden in vorlaufende Indikatoren (leading indicators) und in Ergebnis-Indikatoren (lagging indicators).

- nach der zeitlichen Fristigkeit, welche die Indikatoren unterstützen: strategische, taktische und operative Indikatoren.

Mit Hilfe der vorgestellten Instrumente sollte eine qualitativ hoch stehende Auswahl an Indikatoren erreicht werden. Nicht zu unterschätzen ist gemäss der Erfahrung des Verfassers der Aufwand und die Komplexität. Der Einsatz von computergestützten Informationssystemen wird daher rasch unumgänglich.

\subsubsection{Top-Down-Zuweisung der Ziele und Indikatoren an die entsprechenden Organisationseinheiten (Schritt 4.3)}

Mit einer weiteren Frage erfolgt die Zuweisung $\mathrm{zu}$ den entsprechenden Organisationseinheiten: „Für welche Ebenen sind die identifizierten Ziele und Performance-Indikatoren relevant?“ Das Resultat ist eine Liste von Zielen und Performance-Indikatoren für alle zu messenden Organisationseinheiten. (vgl. Wettstein et al. (2001)) 
6.3.4.4 Bestimmung der Mittel (Unterziele) zur Zielerreichung (Iterationen) (Schritt 4.4)

Im vierten Schritt wird untersucht, mit welchen Mitteln die Ziele erreicht werden können. Das Resultat ist eine Liste von Unterzielen. Die Darstellung in

Abbildung 6-6 verdeutlicht, dass - je nach Betrachtungsebene - ein Ziel sowohl Ziel als auch Mittel sein kann. Entscheidend ist dabei der Betrachtungswinkel, wie folgende einfachen Fragen beantworten: Die Frage Warum oder Wozu weist in der Ziel-MittelHierarchie nach oben (d.h. zum Ziel); die Frage Womit? weist nach unten (d.h. zum Mittel bzw. zur Massnahme) (vgl. Haberfellner und Daenzer (1992)).

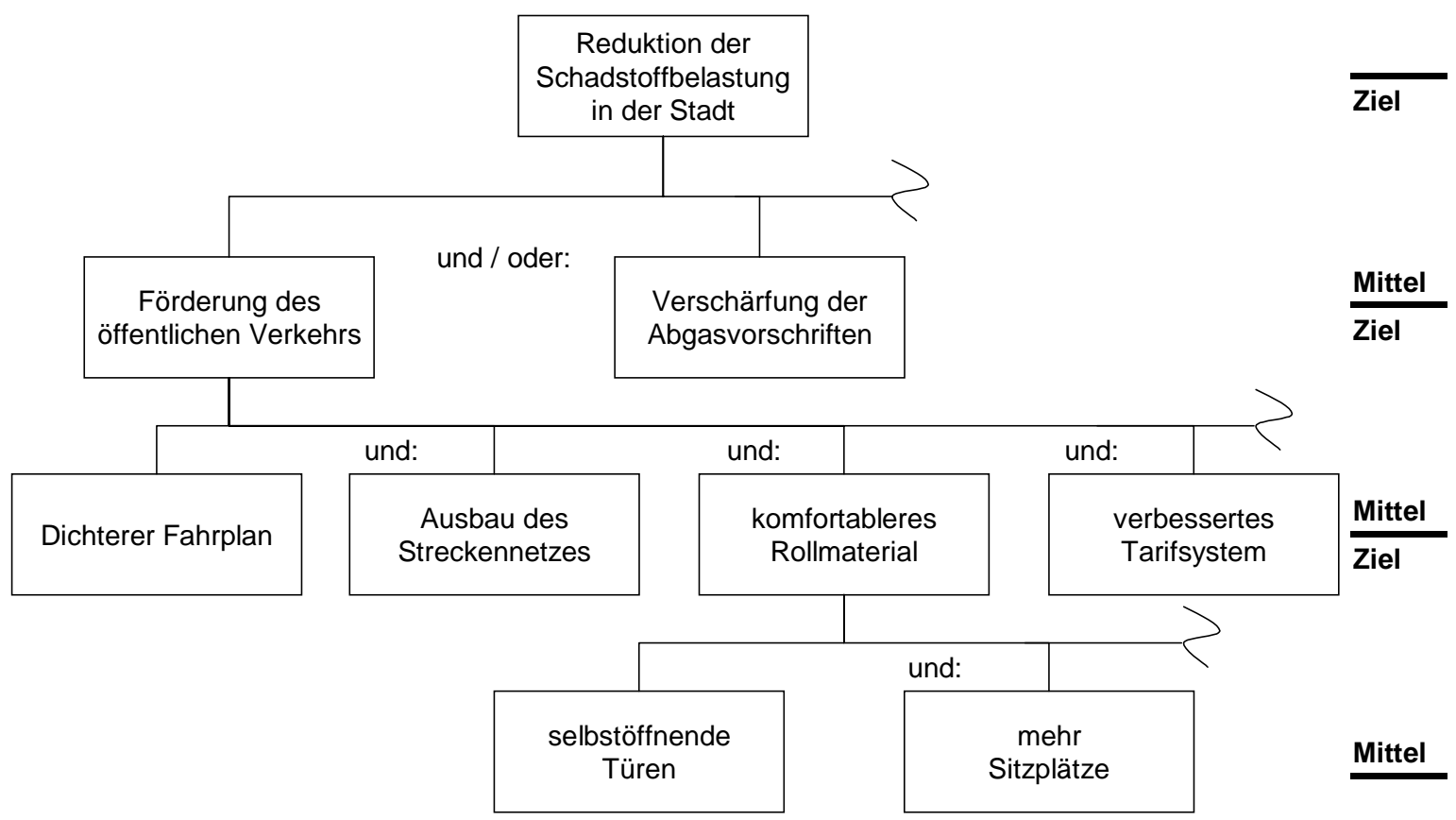

Abbildung 6-6: Ziel-Mittel-Denken (Haberfellner und Daenzer (1992))

Die Schritte 4.2 bis 4.4 werden so lange wiederholt, bis der Detaillierungsgrad der Performance-Indikatoren ausreichend ist. Das Resultat ist eine Zielhierarchie mit den zugehörigen Performance-Indikatoren.

\subsubsection{Bestimmen der Indikatoren der strategischen Programme (Schritt 4.5)}

Wie in Kapitel fünf ausgeführt, werden die Organisationszielsetzungen im Vorschlag des Verfassers dahingehend unterteilt, ob es sich um Ziele handelt, die über längere Zeit in der 
bestehenden Organisation existent sind, oder ob es sich um wesentliche Veränderungen im Sinne von strategischen Projekten resp. Massnahmen oder Programmen handelt.

In diesem Abschnitt wird nun die Berücksichtigung der strategischen Programme im PMS diskutiert. Typisch an diesen ist ihr Projektcharakter, d.h.

- die Aktionen sind zeitlich begrenzt

- und in aller Regel einmalig.

- Die Verantwortlichen werden in der Regel losgelöst von der bestehenden Organisationsstruktur festgelegt.

- Die allozierten Ressourcen werden in der Planung separat ausgewiesen und kontrolliert.

Diese Punkte haben den Verfasser dazu geleitet, die strategischen Programme als separate Position im PMS zu berücksichtigen. Zur Festlegung der strategischen Programme sei auf die entsprechende Spezialliteratur verwiesen (siehe z.B. Grünig und Kühn (2000) oder Lanner (2000)). Abbildung 6-7 zeigt einen Ausschnitt des strategischen Massnahmenportfolios eines schweizerischen Informatikdienstleistungsunternehmens (vgl. Fallbeispiel im Kapitel 3.3.1). Die einzelnen strategischen Massnahmen können strategischen Zielen zugeordnet werden. Dabei wird unterschieden, wo der Haupteinfluss unterstellt wird und bei welchen Zielen die Massnahmen unterstützend wirken. Zusätzlich sind die geplanten internen personellen und die finanziellen Ressourcen sowie die Verantwortlichkeiten geregelt. Die letzten drei Spalten weisen die PerformanceIndikatoren zur Überprüfung der strategischen Massnahme mit den Soll- resp. Ziel-Werten und der Messhäufigkeit aus.

Der Festlegung der Performance-Indikatoren kommt eine grosse Bedeutung zu. Es gelten grundsätzlich die gleichen Kriterien, wie an früherer Stelle bereits besprochen wurde (Zielbezug, Quantifizierbarkeit, Verständlichkeit, Zuverlässigkeit, Gültigkeit, Empfindlichkeit und Kosten-Nutzen-Verhältnis) (vgl. Schritt 4.2). Dort, wo keine geeignete stetige Messgrösse gefunden werden kann, werden oft zeitliche Meilensteine festgelegt, bis zu denen die Massnahmen umzusetzen sind (vgl. Hoffmann (1999)). 


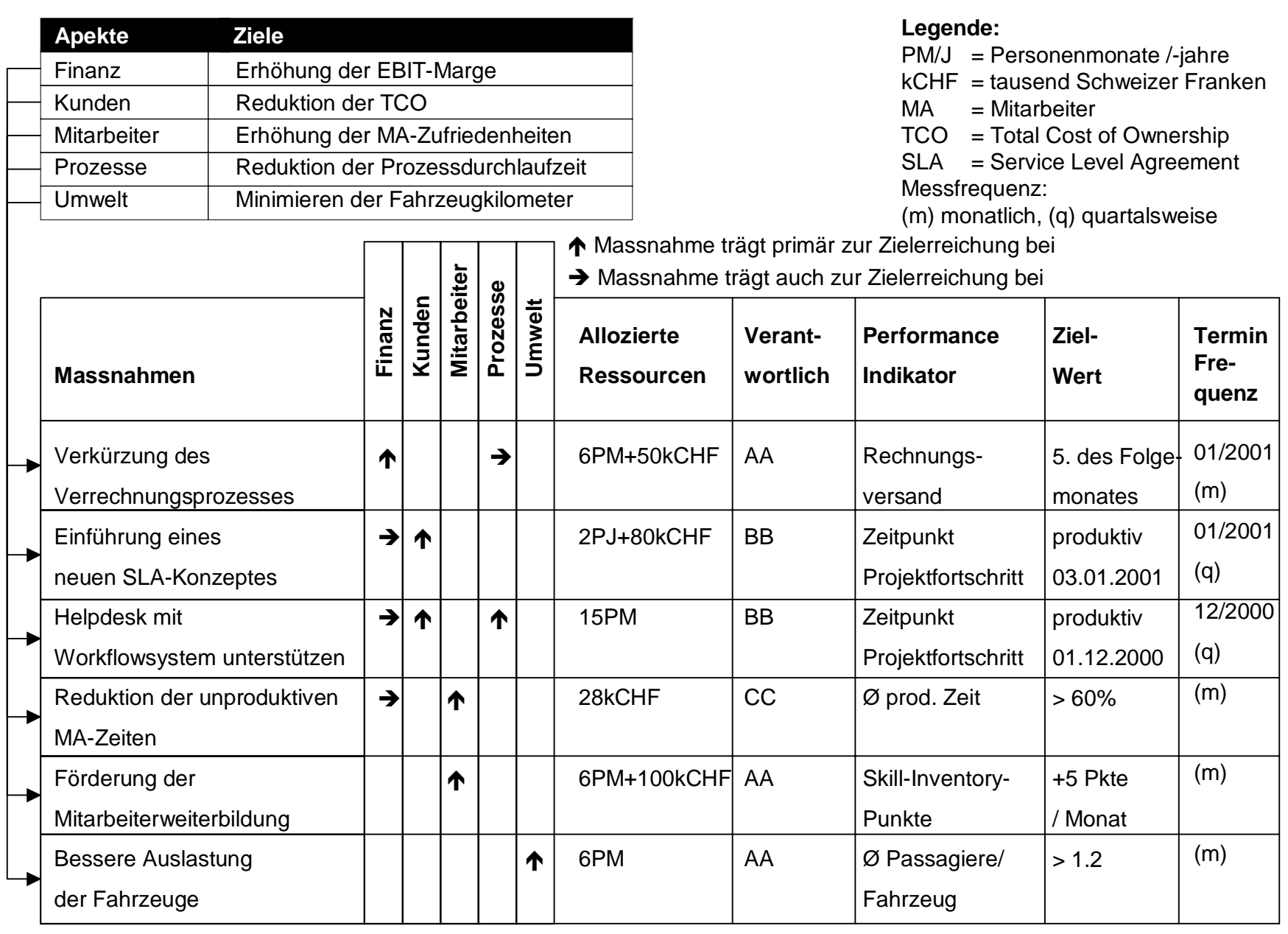

Abbildung 6-7: Massnahmenmatrix eines Informatikdienstleisters (Konzeption in Anlehnung an Colletti (1996), zitiert bei Hoffmann (1999)) 


\subsubsection{Erfassen der persönlichen Ziele (MbO) (Schritt 4.6)}

Die in den Schritten 4.1 - 4.5. festgelegten Ziele können bis auf die Ebene der Mitarbeiter heruntergebrochen werden. Es erscheint jedoch wichtig, dass die Erarbeitung der Ziele in einem dem Objekt angemessenen Rahmen erfolgt. Die Zielerarbeitung sollte die Motivationsfaktoren eines Mitarbeiters (vgl. Wunderer (1997)) explizit bei der Festlegung berücksichtigen. Wunderer nennt (a) interessante, sinnvolle und spassmachende Arbeit, (b) Entwicklungsmöglichkeiten, (c) Übertragung von Mitverantwortung, (d) Einkommen und (e) Arbeitsgestaltung. Zudem umfassen die so erarbeiteten Ziele nur einen Teil der gemeinhin persönlichen Ziele (in Abhängigkeit der Ausgestaltung des PMS können bspw. Personalentwicklungsziele fehlen).

Tabelle 6-3 zeigt ein Beispiel für ein Zielvereinbarungsformular, welches neben den administrativen Daten (Mitarbeiter, Vorgesetzter, Funktion, Daten, etc.) die Ziele der einzelnen Kategorien enthält.

\begin{tabular}{|c|c|c|c|c|c|}
\hline \multicolumn{6}{|c|}{ Zielvereinbarung für das Jahr 200x } \\
\hline \multirow{3}{*}{$\begin{array}{l}\text { Mitarbeiter: } \\
\text { Funktion: }\end{array}$} & \multicolumn{5}{|c|}{ Vorgesetzter: } \\
\hline & \multirow{2}{*}{$\begin{array}{l}\text { Zu } \\
\text { erreichen } \\
\text { bis }\end{array}$} & \multirow[t]{2}{*}{ Messgrössen } & \multirow[t]{2}{*}{ Gewichtung } & \multicolumn{2}{|c|}{ Ergebnisbewertung } \\
\hline & & & & $\begin{array}{l}\text { Zwischen- } \\
\text { ergebnis }\end{array}$ & Jahresende \\
\hline \multicolumn{6}{|l|}{ Finanzziele } \\
\hline \multicolumn{6}{|c|}{ Kundenziele } \\
\hline \multicolumn{6}{|c|}{ Gesellschaftsziele } \\
\hline \multicolumn{6}{|c|}{ Innovationsziele } \\
\hline \multicolumn{6}{|c|}{ Mitarbeiterziele } \\
\hline \multicolumn{6}{|c|}{ Beurteilungsgespräch: } \\
\hline \multicolumn{2}{|l|}{ 1. Termin: } & \multicolumn{2}{|l|}{ Vorgesetzter: } & \multicolumn{2}{|l|}{ Mitarbeiter: } \\
\hline \multicolumn{2}{|l|}{ 2. Termin: } & \multicolumn{2}{|l|}{ Vorgesetzter: } & \multicolumn{2}{|l|}{ Mitarbeiter: } \\
\hline
\end{tabular}

Tabelle 6-3: Muster für die schriftliche Dokumentation der Zielvereinbarungen und Ergebnisse der gemeinsamen Bewertung der Zielerreichung (vgl. Brunner (1999))

Die Ziele eines Mitarbeiters aus den einzelnen Kategorien können sich aus verschiedenen Ebenen der PMS-Systematik zusammensetzen: 
- Operative Ziele (Prozess-/Abteilungsziele): In Abhängigkeit der Funktion, welche der Mitarbeiter wahrnimmt, können hier die Ziele des verantwortlichen Prozess-Managers, Abteilungsleiter oder die aus der übergeordneten organisatorischen Einheit abgeleiteten Mitarbeiterziele umfassen (Beispiel: Die acht Vertriebsmitarbeiter zeichnen in der Vertriebsabteilung verantwortlich für 16 Mio. CHF Umsatz. Jeder Mitarbeiter erhält ein Verkaufsziel von 2 Mio. CHF).

- Strategische Projekte/Massnahmen: Ziele aus diesem Bereich ergeben sich aus den strategischen Massnahmen, in Abhängigkeit der Funktion in Form der Gesamtverantwortung für eine Massnahme oder aber daraus abgeleitete Individualziele (Beispiel aus Abbildung 6-7: Verantwortlich für die Massnahme „Helpdesk mit Workflowsystem unterstützen“).

- Strategische Ziele: Sie umfassen jene Ziele, welche in der Strategie des Unternehmens vorgesehen wurden, sich jedoch nicht aus der operationellen Ebene ableiten lassen (Beispiel: Eine Mitarbeiterin einer Organisationsabteilung möchte mittelfristig in die Marketingabteilung wechseln. Um ihre Qualifizierung zu erhöhen, absolviert sie ein Nachdiplomstudium in Marketing. Aus den Weiterbildungszielsetzungen der Organisationsabteilung lässt sich diese Massnahme nicht ableiten. Auf der Stufe der Gesamtunternehmung wird jedoch die Zielsetzung der „Förderung der Weiterbildung unserer Mitarbeiter“ erwähnt.).

- Persönliche und Mitarbeiter-Ziele: Dem Management-by-Objectives-Ansatz liegt die Annahme zu Grunde, dass die Motivation eines Mitarbeiters nur dann erreicht werden kann, wenn seine persönlichen Ziele mit denjenigen der Organisation in Einklang gebracht werden können. Diese Abstimmung mit den persönlichen Zielen unterstützen die ersten drei genannten Kategorien nur indirekt. Diese Rubrik soll hierzu explizit einen Rahmen bereitstellen. (Beispiel: Reduktion der Vollzeit- auf eine Teilzeitbeschäftigung, um den Interessen der Familie Rechnung tragen zu können.)

Die Kaskadierung der Zielhierarchie bis auf die Mitarbeiterebene beinhaltet den Vorteil, das dysfunktionale Verhalten der Mitarbeiter bezüglich der Ziele durch eine geeignete Abstimmung verhindern zu können. Auf der anderen Seite können ex ante bestimmte Anreize zu ungewolltem Verhalten führen. Aus diesem Grunde ist der Festlegung der 
Indikatoren höchste Aufmerksamkeit zu schenken. Ein weiterer zu beachtender Punkt besteht darin, die kurz- und langfristigen Ziele aufeinander abzustimmen.

\subsubsection{Auflösen von Zielkonflikten (Schritt 4.7)}

Die so erstellte Zielhierarchie muss in einem nächsten Schritt auf ihre Qualität hin geprüft werden. Es stellt sich die Frage, ob die in mehreren Iterationen identifizierten Indikatoren mit den Input-Informationen positiv korrelieren. Zwei Instrumente, welche die Beseitigung von Zielkonflikten unterstützen, sollen hier vorgestellt werden. Ein erstes Instrument zur Lösung dieses Problems ist der Papiercomputer von Vester (1987). Ein zweites Instrument ist der Zielvergleich (Präferenzanalyse).

Der Papiercomputer steht für ein Gruppen-Rating-Verfahren über einer Matrix. Mit Hilfe des Papiercomputers wird die Frage nach der Stärke der Einflussnahme im Zielsystem beantwortet. Hierzu wird jedes Ziel des Zielsystems mit jedem anderen Ziel in Beziehung gesetzt und die Stärke der Beeinflussung auf einer Skala von 0 (keine Beeinflussung) bis 3 (starke Beeinflussung) bewertet. Sind alle Beeinflussungen in der beschriebenen Form festgehalten, so werden die so genannte Aktivsumme (AS) und die Passivsumme (PS) gebildet, indem die Werte in der Matrix für jede Grösse horizontal, sowie vertikal aufsummiert werden (vgl. Tabelle 6-4).

\section{Wirkung jeder Zeile auf jedes Spaltenelement bestimmen}

( 0 = keine Beeinflussung, 1=geringe Beeinflussung, 2=mittlere Beeinflussung, $3=$ starke Beeinflussung)

\begin{tabular}{|c|c|c|c|c|c|c|c|c|c|c|c|c|c|}
\hline \multicolumn{2}{|c|}{ Ziele } & \multirow[t]{2}{*}{ A } & \multirow{2}{*}{$\begin{array}{l}\text { B } \\
0\end{array}$} & \multirow{2}{*}{$\begin{array}{l}\mathbf{C} \\
0\end{array}$} & \multirow{2}{*}{$\begin{array}{l}\text { D } \\
0\end{array}$} & \multirow{2}{*}{$\begin{array}{l}\mathbf{E} \\
0\end{array}$} & \multirow{2}{*}{$\begin{array}{l}\mathbf{F} \\
0\end{array}$} & \multirow{2}{*}{$\begin{array}{l}\mathbf{G} \\
0\end{array}$} & \multirow{2}{*}{$\begin{array}{l}\mathbf{H} \\
0\end{array}$} & \multirow{2}{*}{$\begin{array}{l}\mathbf{I} \\
0\end{array}$} & \multirow{2}{*}{$\begin{array}{l}\mathbf{J} \\
0\end{array}$} & \multirow{2}{*}{$\begin{array}{l}\mathbf{K} \\
0\end{array}$} & \multirow{2}{*}{$\begin{array}{c}\text { AS } \\
0\end{array}$} \\
\hline$A$ & hohe Profitabilität & & & & & & & & & & & & \\
\hline $\mathrm{B}$ & tiefe Kosten & 3 & & 0 & 1 & 0 & 0 & 0 & 0 & 0 & 0 & 0 & 4 \\
\hline C & tiefe Wartungspreise & 3 & 0 & & 0 & 0 & 0 & 0 & 0 & 0 & 0 & 0 & 3 \\
\hline $\mathrm{D}$ & tiefer Reparaturmaterialverbrauch & 2 & 3 & 3 & & 0 & 0 & 0 & 0 & 0 & 0 & 0 & 8 \\
\hline$E$ & wenige Reisekilometer & 2 & 3 & 0 & 0 & & 0 & 0 & 0 & 0 & 0 & 0 & 5 \\
\hline $\mathrm{F}$ & hohe Produktqualität & 1 & 1 & 2 & 2 & 0 & & 0 & 0 & 0 & 0 & 1 & 7 \\
\hline G & tiefe Löhne & 2 & 3 & 3 & 0 & 0 & 0 & & 0 & 0 & 0 & 3 & 11 \\
\hline $\mathrm{H}$ & Ausbildung der Servicemitarbeiter & 0 & 0 & 0 & 1 & 0 & 0 & 2 & & 2 & 0 & 1 & 6 \\
\hline I & hohe Servicequalität & 0 & 0 & 1 & 3 & 0 & 0 & 0 & 0 & & 0 & 0 & 4 \\
\hline $\mathrm{J}$ & moderne Ausrüstung & 0 & 0 & 0 & 1 & 0 & 0 & 0 & 0 & 3 & & 1 & 5 \\
\hline $\mathrm{K}$ & motivierte Servicemitarbeiter & 0 & 0 & 0 & 1 & 0 & 0 & 0 & 0 & 3 & 0 & & 4 \\
\hline & PS & 13 & 10 . & 9 & 9 & 0 & 0 & 2 & 0 & 8 & 0 & 6 & \\
\hline
\end{tabular}

Tabelle 6-4: Matrix des Papiercomputers am Beispiel eines Serviceprozesses (Zielsystem in Anlehnung an Küng et al. (2001)) 
Die aufsummierten Aktiv- und Passivsummen können daraufhin in einem Portfolio dargestellt werden (vgl. Abbildung 6-8). Unterschieden werden können vier Gruppen:

- Die „aktive Grösse“ (höchste Aktivsumme [AS]) beeinflusst andere Ziele stark, wird jedoch selbst schwach beeinflusst (im Beispiel: tiefe Löhne, tiefer Reparaturmaterialverbrauch, hohe Produktequalität, Ausbildung der Servicemitarbeiter).

- Die „passive Grösse“ (höchste Passivsumme [PS]) beeinflusst andere Elemente schwach, wird selbst stark beeinflusst (im Beispiel: hohe Profitabilität, tiefe Kosten, tiefe Wartungspreise, tiefer Reparaturmaterialverbrauch).

- Die „kritische Grösse“ (hohe Aktiv- und Passivsumme) beeinflusst stark, wird aber selber ebenfalls stark beeinflusst (im Beispiel: tiefer Reparaturmaterialverbrauch).

- Die „träge Grösse“ (tiefe Aktiv- und Passivsumme) beeinflusst schwach und wird ebenfalls schwach beeinflusst (im Beispiel: moderne Ausrüstung, wenig Reisekilometer, Ausbildung der Servicemitarbeiter). 


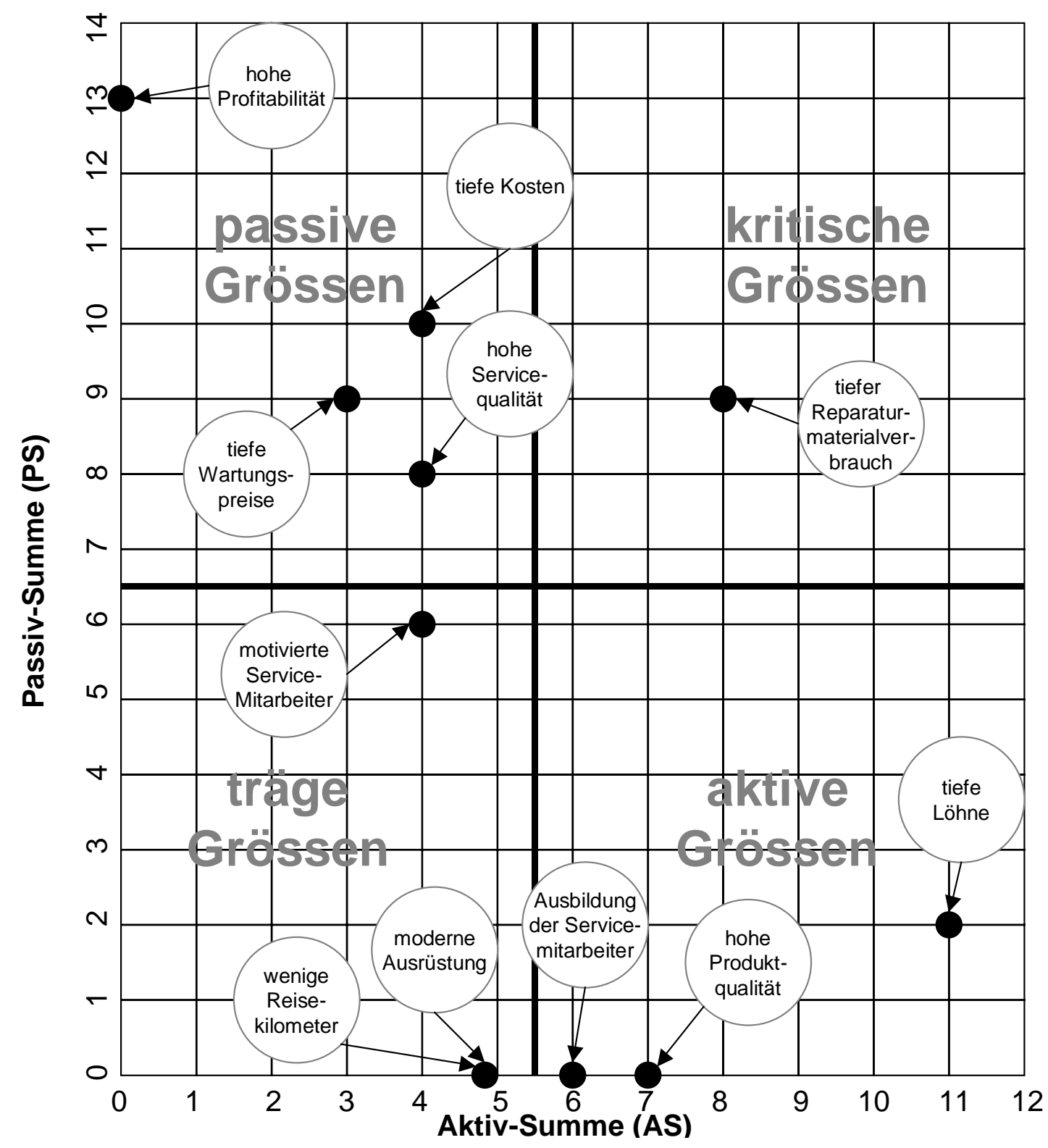

Abbildung 6-8: Ergebnisportfolio der Zielbeziehungsanalyse

Bezüglich des Zielbaumes wird deutlich, dass das Top-Element als passive Grösse, die Bottom-Elemente als aktive Grössen resultieren müssen. Der Verlauf der restlichen Ziele im Zielbaum von oben nach unten sollte im Ergebnisportfolio der Zielbeziehungsanalyse von Abbildung 6-8 von links oben nach rechts unten verlaufen. Trifft dies zu, so bestätigt die Zielbeziehungsanalyse den Zielbaum. Scheren einzelne Elemente aus, so ist deren Position im Zielbaum nochmals zu hinterfragen.

Als zweites Instrument soll auf den Zielvergleich (Präferenzanalyse) eingegangen werden. Diese erlaubt es, die Ziele je nach Wichtigkeit in eine erste Reihenfolge zu bringen. Sie basiert auf einem paarweisen Vergleich der Ziele. Für jedes mögliche Zielpaar erfolgt die 
Bewertung danach, welches der beiden Ziele wichtiger erscheint. Das Ergebnis ist eine Präferenzmatrix, welche die ermittelte Rangfolge mit der Wichtigkeit der einzelnen Ziele und der absoluten und relativen Anzahl der erzielten „Stimmen“ anzeigt. Unten stehend (Tabelle 6-5 und Tabelle 6-6) ist die Präferenzanalyse für einen Serviceprozess dargestellt.

\section{Präferenz-Zielvergleich}

(jedes Ziel wird mit jedem verglichen: 1=linkes Ziel ist wichtiger; 0=rechtes Ziel ist wichtiger)

\begin{tabular}{|c|c|c|c|c|c|c|c|c|c|c|c|c|c|}
\hline & Ziele & A & B & C & D & $E$ & $\mathbf{F}$ & G & $\mathrm{H}$ & $\mathbf{I}$ & $\mathbf{J}$ & $\mathbf{K}$ & S1 \\
\hline$A$ & hohe Profitabilität & & 1 & 1 & 1 & 1 & 1 & 1 & 1 & 1 & 1 & 1 & 10 \\
\hline$B$ & tiefe Kosten & 0 & & 1 & 1 & 1 & 1 & 1 & 1 & 1 & 1 & 1 & 9 \\
\hline $\mathrm{C}$ & tiefe Wartungspreise & 0 & 0 & & 1 & 1 & 1 & 1 & 1 & 1 & 1 & 1 & 8 \\
\hline $\mathrm{D}$ & tiefer Reparaturmaterialverbrauch & 0 & 0 & 0 & & 1 & 1 & 1 & 1 & 0 & 1 & 0 & 5 \\
\hline $\mathrm{E}$ & wenige Reisekilometer & 0 & 0 & 0 & 0 & & 0 & 0 & 0 & 0 & 0 & 0 & $\begin{array}{lll}0 & 0 \\
\end{array}$ \\
\hline$F$ & hohe Produktqualität & 0 & 0 & 0 & 0 & 1 & & 1 & 1 & 0 & 1 & 1 & 5 \\
\hline$G$ & tiefe Löhne & 0 & 0 & 0 & 0 & 1 & 0 & & 0 & 0 & 1 & 0 & 2 \\
\hline $\mathrm{H}$ & Ausbildung der Servicemitarbeiter & 0 & 0 & 0 & 0 & 1 & 0 & 1 & & 0 & 1 & 0 & 3 \\
\hline I & hohe Servicequalität & 0 & 0 & 0 & 1 & 1 & 1 & 1 & 1 & & 1 & 1 & 7 \\
\hline $\mathrm{J}$ & moderne Ausrüstung & 0 & 0 & 0 & 0 & 1 & 0 & 0 & 0 & 0 & & 0 & 1 \\
\hline \multirow[t]{2}{*}{$\mathrm{K}$} & motivierte Servicemitarbeiter & 0 & 0 & 0 & 1 & 1 & 0 & 1 & 1 & 0 & 1 & & 5 \\
\hline & \multicolumn{12}{|l|}{ TOTAL } & 55 \\
\hline
\end{tabular}

Tabelle 6-5: Präferenz-Zielanalyse am Beispiel eines Serviceprozesses (Zielsystem in Anlehnung an Küng et al. (2001))

\begin{tabular}{|l|l|c|c|}
\hline & Ziele & $\begin{array}{c}\text { Absolutes } \\
\text { Stimmengewicht }\end{array}$ & $\begin{array}{c}\text { Relatives } \\
\text { Stimmengewicht }\end{array}$ \\
\hline A & hohe Profitabilität & $\mathbf{1 0}$ & $\mathbf{1 8 \%}$ \\
\hline B & tiefe Kosten & $\mathbf{9}$ & $\mathbf{1 6 \%}$ \\
\hline C & tiefe Wartungspreise & $\mathbf{8}$ & $\mathbf{1 5 \%}$ \\
\hline I & hohe Servicequalität & $\mathbf{7}$ & $\mathbf{1 3 \%}$ \\
\hline D & tiefer Reparaturmaterialverbrauch & $\mathbf{5}$ & $\mathbf{9 \%}$ \\
\hline K & motivierte Servicemitarbeiter & $\mathbf{5}$ & $\mathbf{9 \%}$ \\
\hline F & hohe Produktqualität & $\mathbf{5}$ & $\mathbf{9 \%}$ \\
\hline H & Ausbildung der Servicemitarbeiter & $\mathbf{3}$ & $\mathbf{5 \%}$ \\
\hline G & tiefe Löhne & $\mathbf{2}$ & $\mathbf{4 \%}$ \\
\hline J & moderne Ausrüstung & $\mathbf{1}$ & $\mathbf{2 \%}$ \\
\hline E & wenige Reisekilometer & $\mathbf{0}$ & $\mathbf{0 \%}$ \\
\hline Total & & $\mathbf{5 5}$ & $\mathbf{1 0 0 \%}$ \\
\hline
\end{tabular}

Tabelle 6-6: Ergebnismatrix der Präferenz-Zielanalyse 
In der Ergebnismatrix von Tabelle 6-6 ist das absolute und relative Stimmengewicht jedes Zieles ersichtlich. Für die Verifikation des Zielbaumes bedeutet dies, dass Ziele mit hohem Stimmengewicht oben im Zielbaum, Ziele mit tiefem Stimmengewicht unten im Zielbaum angesiedelt sein müssen.

Die Zahl der bei der Präferenz-Zielanalyse vorzunehmenden Vergleiche erhöht sich mit einer steigenden Anzahl von Zielen stark (Es müssen bei n Zielen n*(n-1)/2 Vergleiche angestellt werden.). Daher wird der Einsatz eines rechnergestützten Hilfsmittels rasch notwendig. Eine Software, welche das vorgestellte Verfahren unterstützt, ist der Zielsystem-Editor, der am Fraunhofer Institut für Produktionsanlagen und Konstruktionstechnik, Bereich Planungstechniken, entwickelt wurde (vgl. UM.IPK.FHG (1996)).

Bei der hier vorgestellten Methodik wurde stillschweigend davon ausgegangen, dass die Präferenz-Zielanalyse nur von einer Person oder aber einem einstimmig handelnden Kollektiv durchgeführt wird. In Organisationen sind in der Regel jedoch zahlreiche Individuen betroffen, welche unterschiedliche Präferenzen aufweisen. Es könnte wünschenswert sein, das Verfahren in der Hinsicht zu erweitern, dass mehrere Personen ihre Präferenz-Zielanalyse durchführen können und diese anschliessend aggregiert und mit unterschiedlichen Gewichten für die einzelnen Ziele ausgewertet werden können.

Mit den beiden vorgestellten Instrumenten kann die erarbeitete Zielhierarchie bezüglich ihrer Konsistenz überprüft werden. Es handelt sich jedoch nicht um Methoden, welche die Hierarchie eindeutig prüfen lassen. Ein gewisser Interpretationsspielraum bleibt. Es empfiehlt sich daher, Entscheidregeln bei Konfliktsituationen im Voraus aufzustellen. Diese könnten wie folgt aussehen: Besteht ein Konflikt in der Zielhierarchie, so kann dieser, wenn eine direkte Beziehung zwischen den Zielen besteht, immer auf zwei Ziele auf unterschiedlichen Hierarchiestufen reduziert werden. Die höher angesiedelte Entität gewinnt gegenüber der Untergeordneten. Zweitere muss derart angepasst werden, dass der Zielkonflikt entfällt.

In einer Zielhierarchie werden auch bei der Anwendung der obigen Regel fast immer Konflikte bestehen bleiben. Daher werden Konflikte von Zielen auf gleicher Ebene ohne direkte Verknüpfung nicht aufgelöst. Es wird davon ausgegangen, dass die beabsichtigte Wirkung stärker ist als die unerwünschte Nebenwirkung. 


\subsubsection{Bestimmung der Prämissenindikatoren (Schritt 4.8)}

Das bis zu diesem Schritt aufgebaute Zielsystem basiert auf bestimmten Annahmen. Diese als Prämissen bezeichneten Annahmen sind jedoch nicht konstant. Es handelt sich um exogene Grössen, die demnach vom System nicht (oder kaum) beeinflusst werden können. Damit das „Auseinanderdriften“ von PMS und der Unternehmensumwelt rechtzeitig erkannt wird und gegebenenfalls Korrekturmassnahmen ergriffen werden können, sieht der Vorschlag den Einbezug der Prämissen vor.

Es genügt nicht, die Prämissen der strategischen Planung lediglich qualitativ explizit darzustellen. Um eine Überwachung der Planungsannahmen Gewähr leisten zu können, muss das PMS um die entsprechenden operationalisierten Prämissen (Kennzahlen) erweitert werden (vgl. Weber und Schäffer (1999), sowie Schreyögg und Steinmann (1985)). Dabei ist darauf zu achten, dass nur die wesentlichen Kennzahlen berücksichtigt werden. Das Ziel muss sein, den „Kontrollaufwand“ zu minimieren. Sinnvollerweise werden daher nicht alle Prämissen mit der gleichen Intensität überwacht. Es empfiehlt sich der Einsatz einer Dringlichkeitsordnung. „Einer besonders hohen Kontrollintensität bedürfen jene Prämissen, die auf schwachen Prognosen gründen, damit dem eigenen Einflussfeld voll entzogen sind und im strategischen Konzept einen kritischen Stellenwert haben, etwa weil bereits geringe Abweichungen weitreichende Konsequenzen nach sich ziehen. “ (Schreyögg und Steinmann (1985)).

$\mathrm{Zu}$ beachten ist ebenfalls, dass grundsätzlich keine abschliessende Liste der Prämissen erstellt werden kann. Die Umwelt ist nicht vollständig beschreib- und prognostizierbar. Zudem ist es schwierig zu wissen, welche Elemente relevant sind. Insofern kann der Informationsbedarf nur beschränkt erfasst werden. Daher besteht die Absicht, dies mit der strategischen Überwachung zu kompensieren. Im englischen Sprachraum findet man den Begriff environmental scanning. (Diese muss ihrem Wesen nach ungerichtet sein, d.h. sie hat keinen ex ante identifizierbaren Kontrollgegenstand) (vgl. Weber und Schäffer (1999)).

Gemäss Steinmann und Schreyögg (1986) ist die Umsetzung der strategischen Überwachung jedoch mit einigen Schwierigkeiten behaftet. So schreiben die Autoren „...dass die strategische Kontrolle auf mehr oder weniger informellem Weg betrieben wird...“ und weiter „...vertraute auf die strategische Wachsamkeit der Akteure...“ Steinmann und Schreyögg (1986). Weber und Schäffer (1999) schlagen vor, eine Art Leitmotiv für die ungerichtete Suche nach Risiken und Chancen vorzugeben. Dies 
entspricht dem von Steinmann und Schreyögg (1986) erwähnten „strategischen Denkklima“. Konsequenterweise wird die strategische Überwachung im PMS nicht direkt berücksichtigt. Es wird jedoch organisationsweit allen Mitarbeitern die Möglichkeit gegeben, einen Prozess zu instanziieren, wenn kritische Signale auf eine strategische Bedrohung oder Chance hinweisen. Dies bedingt eine entsprechende Kommunikationsplattform. Simons und Davila (1998) dazu: „...managers mobilize the entire organization to scan the environment for early warnings of either changes in the marketplace or competitive threats."

Neben der Prämissenkontrolle und der strategischen Überwachung finden sich auch die Begriffe der Früherkennung resp. Frühwarnung und Frühaufklärung in der Literatur. Die Früherkennung ist eine spezielle Art der Information, die sich auf zukünftige Entwicklungen oder Zustände bezieht (vgl. Horváth (2001)). Löhneysen (1982) definiert das Frühwarnsystem wie folgt: „Ein Frühwarnsystem soll im unternehmungsinternen wie unternehmungsexternen Bereich potentielle, d.h. mögliche, aber noch nicht real vorhandene oder bereits eingetretene Gefährdungen in einem möglichst frühen Stadium so rechtzeitig aufzeigen, dass der jeweilige Benutzer oder Entscheidungsträger in die Lage versetzt wird, drohende Gefahren abzuwenden oder eingetretene Gefahren in ihren Auswirkungen zu mildern. “ Neben dieser sehr engen Definition, die nur die Warnung vor Unternehmensrisiken umfasst, existieren auch Auffassungen, die das Frühwarnsystem um den Aspekt der entgangenen Chancen erweitern: „Die Frühwarnung hat die Aufgabe, Veränderungen in der Umwelt sowie in der Unternehmung selber so frühzeitig aufzuzeigen, dass geeignete Massnahmen ergriffen werden können, bevor der Unternehmung ein Schaden erwächst oder eine Chance entgeht. “(Gomez (1983)).

Im Gegensatz dazu umfasst der Begriff der Früherkennung nicht nur den Aspekt der entgangenen Chancen und Gefahren, sondern haben allgemein zum Ziel, Informationen über zukünftige Chancen und Gefahren zu generieren (vgl. Muchna (1988)).

Die als strategisches Controlling zusammenfassbare Prämissenkontrolle, strategische Überwachungskontrolle und strategische Durchführungskontrolle (vgl. auch Kapitel fünf) kann gegenüber der Früherkennung bestehend aus der Frühwarnung und Frühaufklärung wie in Abbildung 6-9 dargestellt - abgegrenzt werden. Die Früherkennung ist somit Teil jedes der Elemente des strategischen Controlling, umfasst aber zusätzlich die Ebenen des operativen und taktischen Controlling. 


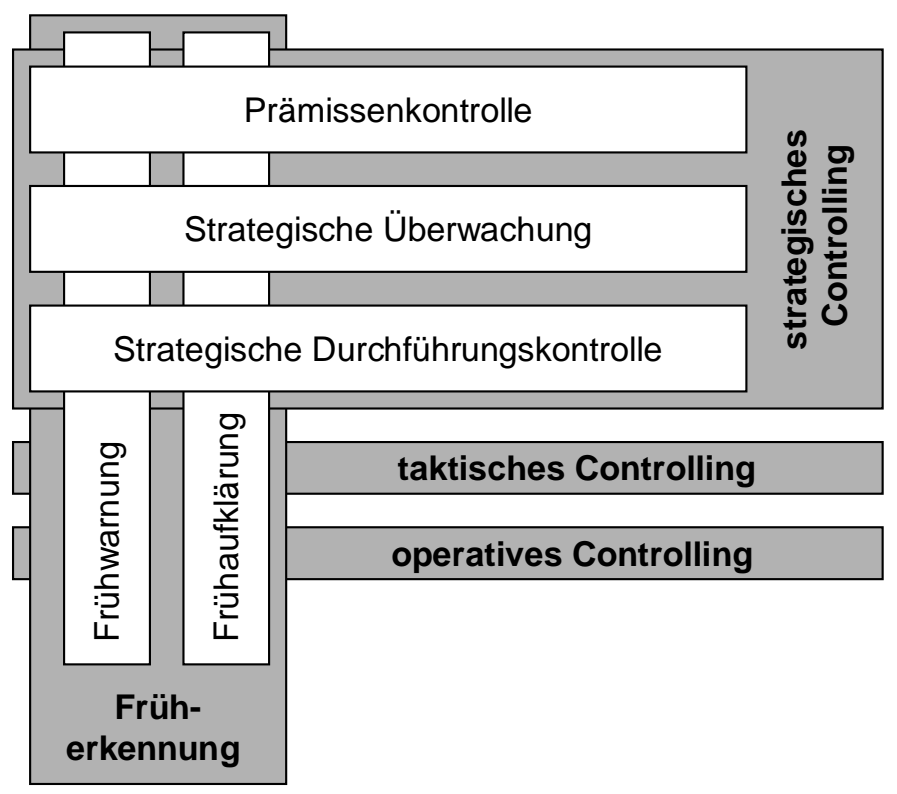

Abbildung 6-9: Abgrenzung der Früherkennung vom strategischen Controlling

Nach den gemachten Ausführungen wird deutlich, dass das PMS seinem Wesen nach primär die gerichtete Kontrolle unterstützen kann. Allgemein gültige Hinweise zu geben, welche Elemente ins PMS übernommen werden sollen, erscheint schwierig. Einigkeit besteht darüber, dass die Ziele des Kontrollsystems aus den Unternehmenszielen abgeleitet werden müssen (vgl. Bergamin (2001)).

Wie breit die Zielfestlegung eines Früherkennungssystems sein kann, zeigt das nachfolgende Beispiel einer schweizerischen Elektrizitätsgesellschaft (vgl. Tabelle 6-7):

- Erstellen eines Überblicks der dominierenden Systemelemente der strategischen Geschäftseinheiten. Dieser soll erlauben, systematisch und frühzeitig zu erkennen, bei welchem dominierenden Systemelement im Zusammenspiel mit anderen Systemelementen Handlungsbedarf entsteht.

- Reduktion der Ungewissheit, welche Folgen die Liberalisierung des Elektrizitätsmarktes für die einzelnen Geschäftseinheiten haben wird.

- Gewinnen von Informationen, um aus Gesamtunternehmensperspektive entscheiden zu können, welches Verständnis für die Funktion der Geschäftsfelder Produktion und Übertragung in Zukunft Gültigkeit haben soll.

- Aufzeigen von dominierenden Systemelementen, die für Lenkungseingriffe geeignet sind.

- Die vernetzte Darstellung der dominierenden Systemelemente soll den Mitarbeitern zur Veranschaulichung der unternehmerischen Zusammenhänge dienen.

Tabelle 6-7: Beispiele der Ziele eines Früherkennungssystems einer schweizerischen Elektrizitätsgesellschaft (vgl. Bergamin (2001)) 
Die Breite der oben definierten Ziele ergibt sich durch die Art des angestrebten Kontrollsystems. Bergamin unterscheidet in seiner Dissertation vier Typen von Frühwarnsystemen, wobei obige Zielsetzungen auf den vierten Typ zugeschnitten sind. Die ersten drei Typen (es wird auch von Generationen gesprochen, da sie in einer zeitlichen Abfolge stehen) deckt sich mit der Darstellung bei Horváth (2001). Die vier Typen sind in nachfolgender Tabelle 6-8 kurz charakterisiert:

\begin{abstract}
Kennzahlenorientierte Früherkennungssysteme: Erste Informationssysteme mit Frühwarncharakter tauchen im angelsächsischen Raum Ende der Sechzigerjahre auf (vgl. Aguilar (1967)). Im deutschsprachigen Raum prägte Szyperski den Begriff der Frühwarnung 1973 als spezielle Form der Informationsgewinnung aus computergestützten Planungs- und Kontrollsystemen (vgl. Szyperski (1973)). Das Konzept der ersten Form der Frühwarnsysteme bestand darin, Toleranzbänder für bestimmte, zuvor definierte Messgrössen zu bestimmen, deren Über- oder Unterschreitung durch das Frühwarnsystem gemeldet wurde (Soll-Ist-Vergleich) (vgl. Krystek und Müller-Stewens (1993)).
\end{abstract}

Indikatorenorientierte Früherkennungssysteme: Der Grundgedanke dieser Form der Früherkennung ist, dass Risiken und Gefahren noch nicht offensichtlich sein müssen, sich aber an anderer Stelle bereits in irgend einer Form ankündigen (vgl. Klausmann (1983)). Als Konsequenz generierte man eine ganze Reihe von Frühindikatoren, die umfassend Entwicklungen beschreiben und gegenüber der Referenzgrösse einen zeitlichen Vorlauf aufweisen (vgl. Müller (1981)). Nach Hahn (vgl. Hahn (1996)) handelt es sich dabei um einen iterativen Prozess, der zuerst Beobachtungsbereiche zur Erkennung von Risiken und Chancen identifiziert und darauf aufbauend innerhalb dieser Bereiche Frühwarnindikatoren generiert.

Früherkennungssysteme auf der Basis schwacher Signale: Strategische Überraschungen sind aus der Sicht der Unternehmung plötzliche, dringende und ungewöhnliche Veränderungen, die entweder einen bedeutenden Erfolgsrückschlag oder den Ausfall einer grösseren Chance ankündigen ( $v g l$. Ansoff (1976)). Nach Ansoff sind zwei Reaktionsmöglichkeiten auf dieses Szenario denkbar. Entweder verfügt die Organisation über ein wirksames Krisenmanagement, welches erlaubt, effizient auf Umweltveränderungen zu reagieren. Oder es kann versuchen, mutmassliche strategische Überraschungen bereits vor ihrem eigentlichen Auftreten anzugehen. Im zweiten Fall sieht Ansoff zwei konkurrenzierende Dimensionen, die zeitliche, in Form der Geschwindigkeit der Reaktion, und die zur Verfügung stehende Information über sich abzeichnende Chancen und Gefahren. Ansoff sieht den Ausweg darin, dass die Reaktion mit zunehmender Informationssicherheit (er unterscheidet fünf Stadien der Ignoranz bei Diskontinuitäten) konkreter wird und empfiehlt mögliche Vorgehensweisen (Normstrategien: Beobachtung der Umwelt bis direkte Aktion). Die praktische Ausgestaltung erfolgt in der Regel durch Beobachtung, Sammlung und Bewertung von verschiedenen Informationen, die sich als 
Quellen für schwache Signale herauskristallisiert haben (z.B. sich häufende Ereignisse, die im Zusammenhang mit der verfolgten Strategie stehen, Expertenmeinungen, Medien). Es existieren Systeme, die auf einzelne Organisationen zugeschnitten sind und andere, die übergreifend (z.B. der STAR - strategische Trend Analyse Report des Institutes für Strategie und Zukunftsforschung) operieren. Der Ansatz der schwachen Signale überwindet die Einschränkung des Beobachtungsbereiches und kann demzufolge als Ergänzung zu den oben beschriebenen Früherkennungssystemen verstanden werden.

Ganzheitlich-vernetzende Früherkennungssysteme: Kernelement der ganzheitlichen Früherkennungssysteme ist die Abbildung der Unternehmung in einem Erklärungsmodell, welches Rückschluss auf die geschäftsbestimmenden Faktoren sowie auf deren gegenseitige Beeinflussung gibt (vgl. Gomez (1981)). Aus den ermittelten Systemelementen werden unternehmensspezifische Indikatoren abgeleitet, welche die relevanten Entwicklungen in der Umwelt abbilden. Gomez (1983) intendiert, überraschende Entwicklungen und Trendbrüche über schwache Signale ebenfalls in sein Modell aufzunehmen. Das Erklärungsmodell kann hierzu Hilfestellung bieten, die bestehende Grundproblematik jedoch nicht überwinden.

Tabelle 6-8: Vier Grundtypen von Früherkennungssystemen (in Anlehnung an Bergamin (2001))

Es erscheint zudem wichtig, aufzuzeigen, dass die Prämissen vom Betrachtungsort abhängig sind. Dargestellt an einer grösseren Unternehmensgruppe bestehend aus mehreren Geschäftseinheiten bedeutet dies, dass geltende Randbedingungen für die Business Unit für den Konzern unter Umständen keine Gültigkeit haben. Dieser Sachverhalt ist entsprechend zu berücksichtigen.

\subsubsection{Zuordnen der Verantwortlichen der Ziele (Schritt 4.9)}

Auf der Ebene der einzelnen Organisationseinheiten müssen für die einzelnen Ziele die Verantwortlichkeiten festgelegt werden. Meist sind diese identisch mit den jeweiligen Leitern der organisatorischen Einheiten. So wird der Geschäftsprozess-Manager des Prozesses „X“ in der Regel auch verantwortlich sein für die Einhaltung der entsprechenden Ziele. Einige Details zur Organisation wurden bereits im fünften Kapitel besprochen.

\subsubsection{Zuordnen der Informationsempfänger der Performance-Indikatoren (Schritt} 4.10)

Neben den Verantwortlichkeiten sollen an dieser Stelle auch die Informationsempfänger der Performance-Daten bestimmt werden. Als Informationsempfänger kommen 
grundsätzlich sämtliche Stakeholder des Unternehmens in Frage. Dabei ist zu beachten, dass die Informationsmenge auf den Empfänger zugeschnitten ist (vgl. Horváth (2001)). Eine Zuordnung kann auf Grund der folgenden Überlegungen erfolgen:

Stakeholder, die von einem Ziel betroffen sind, d.h. deren eigene Zielerreichung durch die Erreichung resp. Nicht-Erreichung des Zieles beeinflusst wird, sollten als Empfänger der Performance-Daten in Betracht gezogen werden. Das Ergebnis sollte darauf hin mit der Informationskultur der Organisation abgestimmt werden. Das nachfolgende Kommunikationskonzept basiert auf Wettstein et al. (2001):

Wird restriktiv kommuniziert, werden die Informationsempfänger nicht mit Daten überflutet und die Orientierung der Stakeholder ist einfacher. Zudem sind die Kosten der Informationsverarbeitung und -verbreitung kleiner.

Eine umfassende Kommunikationspolitik hat zur Folge, dass sich die Stakeholder stärker mit dem Unternehmen identifizieren. Daraus lässt sich die Erwartung ableiten, dass sie einen höheren Beitrag zur Steigerung der Performance leisten. Des Weiteren ist anzunehmen, dass das Informationsbedürfnis oder die optimale Informationsmenge von Externen nicht bestimmt werden kann. Aus diesem Grund sollte die Filterung nicht durch den Informationsproduzenten, sondern durch den Informationskonsumenten vorgenommen werden.

Eine Gliederung kann nach dem in Abbildung 6-10 dargestellten Schema erfolgen. Diejenigen Informationen, für welche die Stakeholder verantwortlich zeichnen, müssen auf jeden Fall kommuniziert werden (Stufe 1). Bei den Mitarbeitern wären dies bspw. jene Ziele, für deren Zielerreichung sie verantwortlich sind (Management by Objectives). Andere Stakeholder müssen auf dieser Stufe Informationen erhalten, für die sie gemäss Vertrag oder Gesetz verantwortlich sind (z.B. Steuerbehörde zur Berechnung der Warenumsatzsteuer, Kunden die Chargennummer für das Produkt-Haftpflichtgesetz, etc.).

Auf der zweiten Stufe werden Informationen zur Performance kommuniziert, welche die Stakeholder direkt beeinflussen können. Bspw. wird den Mitarbeitern die Ausschussquote in der Fertigung mitgeteilt oder dem Lieferanten die Produktqualität und Lieferpünktlichkeit.

Auf Stufe drei werden zusätzlich jene Daten kommuniziert, welche die Stakeholder nur indirekt beeinflussen können. Ein Beispiel könnte sein, dass den Mitarbeitern nicht nur die 
direkt zurechenbaren Kosten, sondern auch die Gemeinkosten (z.B. Heizkosten) kommuniziert werden. Auf Stufe 4 werden schliesslich all jene Daten kommuniziert, welche die Stakeholder interessieren (sofern die Daten aus plausiblen Gründen nicht nur einem bestimmten Kreis zugänglich gemacht werden können). Unternehmen, die auf Stufe vier kommunizieren, haben ein grosses Datenvolumen zu bewältigen und brauchen dafür eine entsprechende Infrastruktur.

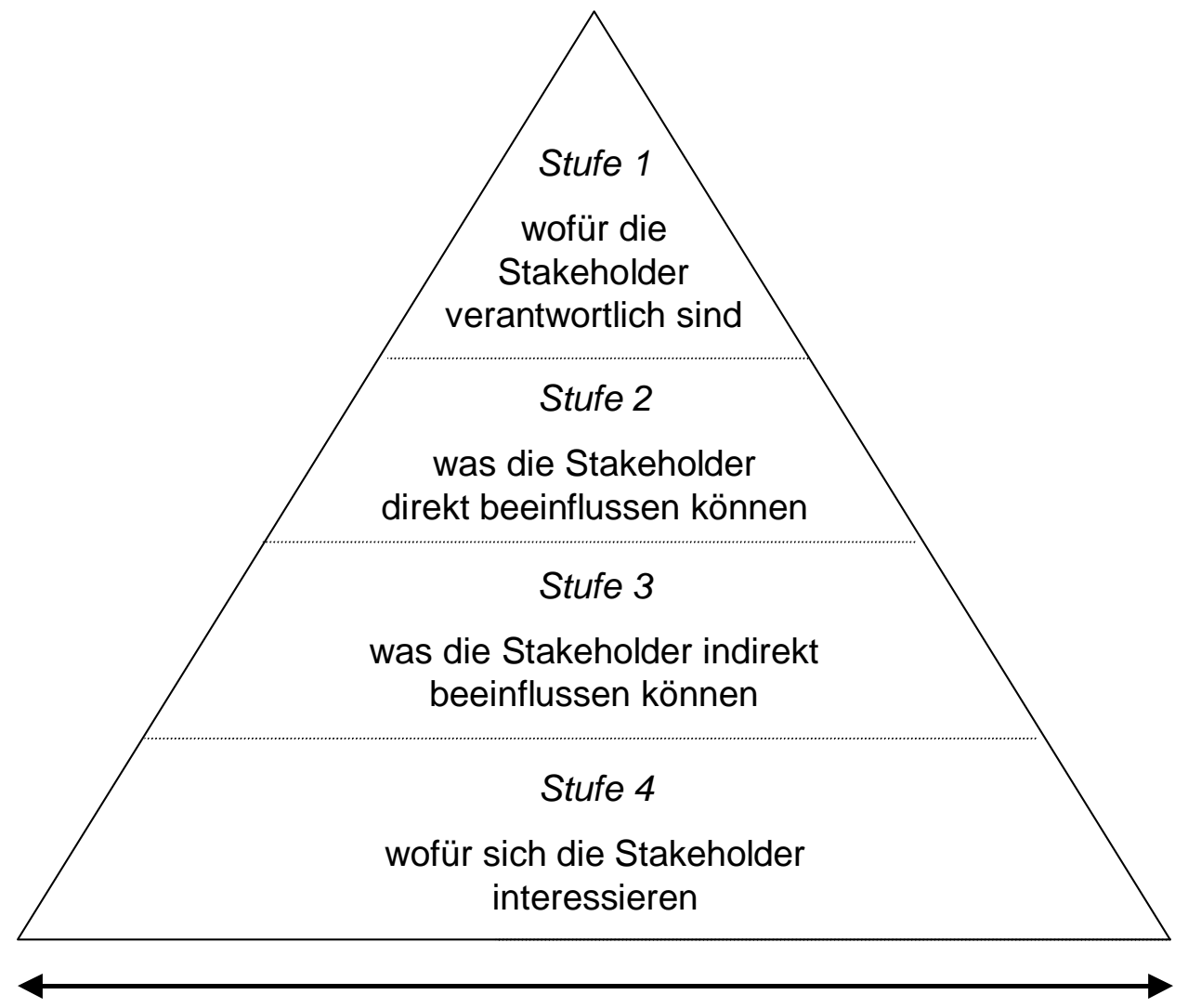

zu kommunizierendes Datenvolumen

Abbildung 6-10: Die Kommunikationspyramide (vgl. Wettstein et al. (2001))

Es ist zu bedenken, dass gewisse Stakeholder mit einer zielgerichteten Kommunikation beeinflusst werden können: Eine gezielte Auswahl der Performance-Daten kann die Erwartungen seitens der Kunden beeinflussen. Je nachdem, welche Daten kommuniziert werden, haben die Investoren ein höheres oder geringeres Vertrauen in das Unternehmen. Schliesslich kann durch eine gezielte Auswahl der kommunizierten Performance-Daten Einfluss, auf die Wahrnehmung einer Firma durch die Gesellschaft, den Arbeitsmarkt oder die Lieferanten genommen werden. 


\subsection{Illustration des Vorschlages an einem Beispiel}

\subsubsection{Erhebung der Input-Faktoren}

Die vorgestellte Heuristik soll nachfolgend an einem Beispiel dargestellt werden. Bei der betrachteten Unternehmung handelt es sich um einen schweizerischen Informatikdienstleister (SIDL) mittlerer Grösse, der als Tochterfirma eines börsenkotierten und weltweit tätigen Unternehmens für die schweizerische Marktbearbeitung zuständig ist (vgl. die Einführung zum Fallbeispiel in Kapitel 3.3.1). SIDL besitzt neben einem Hauptsitz in der Region Bern Niederlassungen in drei grösseren Schweizer Städten. Das Beispiel wurde, um die Interessen der Firma zu schützen, anonymisiert. Zudem wurden verschiedene wesentlichen Angaben verändert.

Das Performance-Measurement-System soll unter der Zielsetzung aufgebaut werden, dass dieses die bestehenden Führungssysteme für ein einheitliches, integrales Reporting zusammenfasst. Insbesondere soll die Transparenz für die Mitarbeiter bezüglich der angestrebten Ziele erhöht werden, die verschiedenen Führungssysteme bezüglich der Kontrolle zusammengeführt werden, um der Informationspflicht gegenüber dem Hauptlieferanten besser Rechnung $\mathrm{zu}$ tragen. Zudem soll das dynamische Unternehmensumfeld besser beobachtet werden können.

Um die Machbarkeit überprüfen zu können, müssen zuerst die verfügbaren InputFaktoren vorgestellt werden.

Der Unternehmenszweck von SIDL lässt sich in einem Satz formulieren: „Erbringen von hochwertigen Dienstleistungen rund um die Produkte eines führenden InformatikprodukteHerstellers im schweizerischen Markt.“ Dabei hat sich SIDL auf drei Bereiche spezialisiert:

1. Realisation von Informatik-Komplettlösungen für mittelständische Unternehmen basierend auf der eigenen ERP-Lösung.

2. Spezialisierte Informatikdienstleistungen im entsprechenden HerstellerProdukteumfeld.

3. Outsourcen der Hardwarebeschaffung (primär der entsprechenden HerstellerProduktepalette) für grössere Unternehmen und Informatikunternehmen, die sich auf Softwareentwicklung, -vertrieb oder Dienstleistung spezialisieren. 
Zielsetzungen: Für das erste Tätigkeitsfeld wird angestrebt, auf der relevanten ServerPlattform das grösste Kundenportfolio und die meisten Neu-Installationen im relevanten Marktsegment zu halten. Für das zweite Tätigkeitsfeld wird ein Boutique-Ansatz verfolgt. Es werden für ausgewählte hochspezialisierte Bereiche im entsprechenden HerstellerProdukteumfeld - welche einer periodischen Evaluation unterzogen werden - Leistungen angeboten. Dabei wird primär ein Kompetenzdemonstrationseffekt gegenüber dem Markt und dem entsprechenden Hersteller angestrebt. Im dritten Bereich wird angestrebt, zu einem der fünf wichtigsten Geschäftspartner des Herstellers bezüglich Umsatz innerhalb der nächsten drei Jahre zu werden.

Vision: SIDL strebt ein Wachstum bezüglich Umsatz und Ertrag (EBIT) an, welches langfristig über dem Marktwachstum liegt. Dabei soll dieses nicht auf Kosten einer unsicheren Kapitalstruktur erreicht werden. Bedingt durch die hohe Abhängigkeit vom Know-how der Mitarbeiter sind diese als wertvollstes Aktivuum entsprechend zu behandeln. Sowohl mit den Mitarbeitern als auch den Kunden und Lieferanten wird eine faire, langfristige Beziehung angestrebt. Der Wahrnehmung der sozialen und gesellschaftlichen Verantwortung soll im Rahmen der Leistungsfähigkeit des Unternehmens Rechnung getragen werden.

Institutionalisiert ist eine Prämissenkontrolle (gerichtete Kontrolle) bezüglich einiger weniger Indikatoren. Tabelle 6-9 gibt einen Einblick.

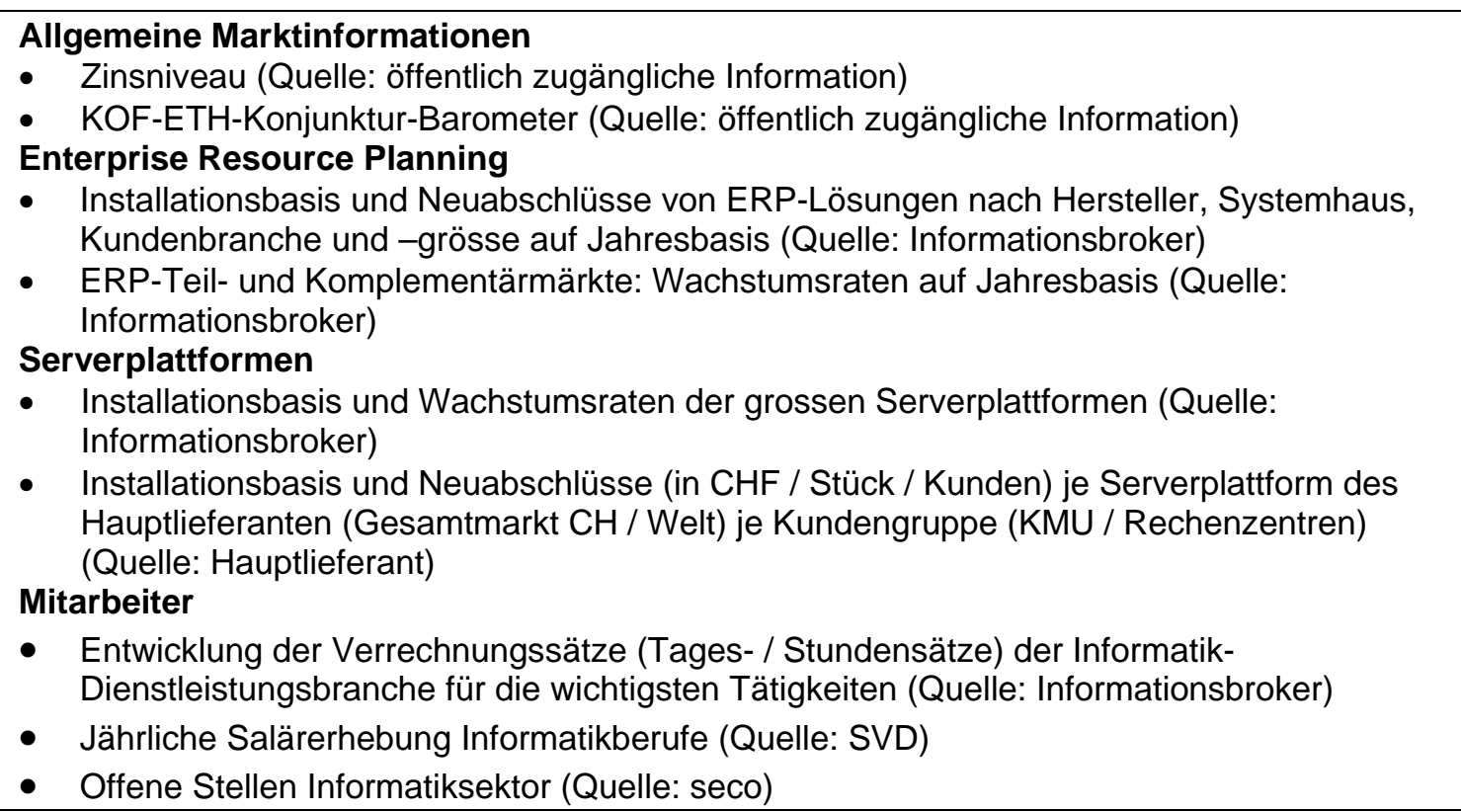

Tabelle 6-9: Prämissenkontrolle von SIDL 
Die Aufbauorganisation gliedert sich wie in Abbildung 6-11 dargestellt. Grob betrachtet unterteilt sich SIDL in vier Bereiche. Der Bereich Management Services stellt die gesamten Administrationsdienste (Finanzen, Reporting, Verrechnung, Infrastruktur, Personalwesen, etc.) bereit. Hardware \& Software Business kümmert sich um den Handel, d.h. die Beschaffung von Handelsprodukten für die eigenen Projekte, wie auch für Unternehmen, die ihre Beschaffung ausgelagert haben. Hardware \& Networking Services erbringt Dienstleistungen auf der Ebene der Produkte des Hauptlieferanten als Boutiqueleistungen (siehe oben) und für die eigenen ERP-Projekte. Der Bereich Application Services kümmert sich um die angesprochene ERP-Lösung. Dabei lässt sich dieser Bereich bei näherer Betrachtung in vier Hauptprozesse untergliedern: Research \& Development kümmert sich um die (Weiter)-Entwicklung des ERP-Produktes, Sales stellt die Generierung von Interessenten bis zum Vertragsabschluss von Projekten sicher, Fulfillment erledigt die Implementierung und Einführung der ERP-Lösungen, während sich Support \& Maintenance um die Unterstützung nach dem produktiven Einsatzstart kümmert.

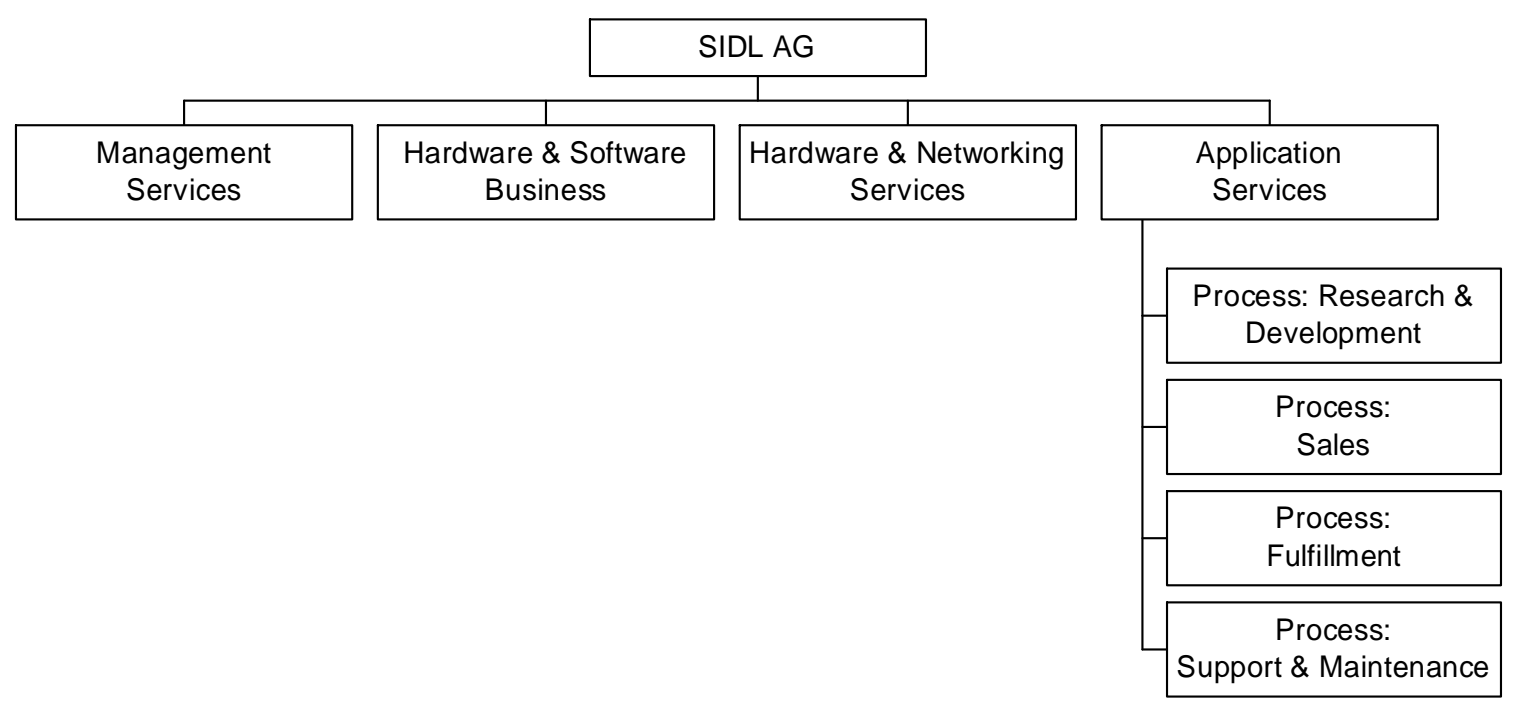

\section{Abbildung 6-11: Ausschnitt der vereinfachten Aufbauorganisation von SIDL}

Tabelle 6-10 zeigt ein vereinfachtes Budget von SIDL. Es handelt sich dabei um eine aggregierte Sicht, die aus den Sachplänen resultiert. Die Planung verfügt über einen Horizont von fünf Jahren, wobei der Detaillierungsgrad mit der Gegenwartsnähe zunimmt. Die Gliederung erfolgt nach den Posten der Absatzkosten und wird auch als Erfolgsrechnung nach dem Umsatzkostenverfahren bezeichnet (vgl. Boemle (1992)). 
SIDL AG

\section{Income statement}

Mio CHF

\begin{tabular}{|l|}
\hline Sales \\
\hline Hardware \\
Software \\
Service \\
Cost of sales \\
Cost of goods sold \\
\hline purchased goods \\
\hline Gross Margin \\
Cost of operating departments \\
\hline personnel costs \\
\hline salary \\
\hline social security contributions \\
\hline depreciation on FA and SW \\
\hline other costs \\
\hline Gross profit \\
\hline Selling expenses \\
\hline personnel costs \\
\hline fixed salary \\
\hline Social security contributions \\
\hline depreciation on FA and SW \\
\hline other costs \\
\hline General and admin. expenses \\
\hline personnel costs \\
\hline fixed salary \\
\hline social security contributions \\
\hline depreciation on FA and SW \\
\hline other costs \\
\hline Depreciation on goodwill \\
\hline Operating income \\
\hline Other income \\
\hline Other expenses \\
\hline Income from Equity Method \\
\hline EBITDA \\
EBIT \\
Interest income \\
\hline Interest expenses \\
\hline EBT \\
\hline Income taxes \\
\hline EAT \\
\hline
\end{tabular}

$\sum$ Expenses (Oper.,Sell.,Admin.)

Personnel costs

Other Costs

Depreciation

\begin{tabular}{|c|c|c|}
\hline \multicolumn{3}{|c|}{$200 x$} \\
\hline $\begin{array}{c}\text { E- } \\
\text { solution }\end{array}$ & $\begin{array}{l}\text { System- } \\
\text { Services }\end{array}$ & $\Sigma$ \\
\hline \multirow[t]{2}{*}{105} & 902 & 1,007 \\
\hline & 604 & 604 \\
\hline 10 & 95 & 105 \\
\hline 95 & 203 & 298 \\
\hline 75 & 760 & 835 \\
\hline 19 & 626 & 645 \\
\hline 19 & 626 & 645 \\
\hline 87 & 275 & 362 \\
\hline 56 & 134 & 190 \\
\hline 46 & 102 & 147 \\
\hline 40 & 89 & 129 \\
\hline 6 & 13 & 19 \\
\hline 3 & 9 & 12 \\
\hline 7 & 24 & 31 \\
\hline 31 & 141 & 172 \\
\hline 8 & 65 & 73 \\
\hline 7 & 50 & 57 \\
\hline 6 & 43 & 50 \\
\hline 1 & 6 & 7 \\
\hline 0 & 3 & 4 \\
\hline 1 & 12 & 13 \\
\hline 12 & 45 & 57 \\
\hline 8 & 29 & 37 \\
\hline 7 & 25 & 32 \\
\hline 1 & 3 & 4 \\
\hline 1 & 3 & 4 \\
\hline \multirow[t]{2}{*}{4} & 13 & 17 \\
\hline & & 0 \\
\hline 11 & 32 & 42 \\
\hline \multirow[t]{3}{*}{1} & 4 & 5 \\
\hline & & 0 \\
\hline & & 0 \\
\hline 16 & 51 & 67 \\
\hline \multirow[t]{2}{*}{12} & 36 & 48 \\
\hline & & 0 \\
\hline 1 & 4 & 5 \\
\hline \multirow[t]{2}{*}{11} & 32 & 43 \\
\hline & & 0 \\
\hline 11 & 32 & 43 \\
\hline
\end{tabular}

\begin{tabular}{|c|c|c|}
\hline 76 & 243 & 320 \\
60 & 180 & 241 \\
12 & 48 & 60 \\
4 & 15 & 19 \\
\hline
\end{tabular}

Tabelle 6-10: Vereinfachtes Budget-Schema von SIDL nach Konzern-Strukturen (US-GAAP) 
Auf den Einbezug der bestehenden Zielvereinbarung (Management by Objectives) wird verzichtet.

Überprüfen der Machbarkeit: Die für die vorgängig vorgestellte Zielsetzung notwendigen Informationsobjekte sind verfügbar. Eine Herausforderung bezüglich der Koordination der Input-Faktoren besteht darin, dass das finanzielle Reporting-Schema auf das Konzernreporting ausgerichtet ist und nicht der Struktur von SIDL entspricht. Die Aktualität der bestehenden Prämissenkontrolle scheint nicht Gewähr leistet zu sein, da diese nur bei Bedarf aktualisiert wird. Das finanzielle Reporting ist demgegenüber monatsaktuell verfügbar (bedingt durch die Reportingverpflichtungen gegenüber dem Konzern) und von hoher Qualität. Die gemäss Tabelle 6-2 definierten Minimalanforderungen sind somit gegeben.

\subsubsection{Transformationsschrittfolge des Erstellungsprozesses}

\subsubsection{Bestimmen der Ziele der relevanten Organisationseinheit}

Nachdem die relevanten Input-Informationen erfasst sind, wird nun die unter Kapitel 6.3.4 vorgestellte Schrittfolge auf das Beispiel angewandt. In einem ersten Schritt werden die Ziele der zu messenden Organisationseinheit - hier die SIDL AG - bestimmt.

\begin{tabular}{|l|l|}
\hline Aspekt (vgl. Abbildung 6-4) & Ziele \\
\hline Investoren & $\begin{array}{l}\text { - Hohe Eigenkapitalquote } \\
\text { - Umsatz und Ergebniswachstum, welches langfristig die } \\
\text { Leistung der Branche übertrifft }\end{array}$ \\
\hline Kunden (Käufer \& Lieferanten) & • Langfristig, beidseitig nutzbringende Beziehung \\
\hline Gesellschaft & $\begin{array}{l}\text { Wahrnehmung der sozialen und gesellschaftlichen } \\
\text { Verantwortung }\end{array}$ \\
\hline Innovation & $\begin{array}{l}\text { Robuste und bewährte Produkte und Dienstleistungen, die } \\
\text { mit der technischen Entwicklung Schritt halten }\end{array}$ \\
\hline Mitarbeiter & - Hohe Mitarbeiterzufriedenheit \\
\hline
\end{tabular}

Tabelle 6-11: Ziele je Aspekt von SIDL 


\subsubsection{Bestimmen der Indikatoren, die zur Messung der Ziele notwendig sind}

Der zweite Schritt besteht darin, die definierten Ziele mittels Performance-Indikatoren zu operationalisieren. Dabei wird ein dreistufiges Vorgehen angewandt: Zuerst werden potenzielle Indikatoren identifiziert, welche analysiert und schliesslich ausgewählt werden. Dies soll am Beispiel der hohen Mitarbeiterzufriedenheit demonstriert werden. In einem Brainstorming wurden folgende möglichen Indikatoren identifiziert unter Beizug der Frage „Mit welchen Performance-Indikatoren kann die Mitarbeiterzufriedenheit gemessen werden?“

- Anzahl Krankheitstage pro Mitarbeiter

- Index der Mitarbeiter-Zufriedenheitsumfrage

- Zufriedenheit mit dem Gehalt (aus Mitarbeitersicht)

- Zufriedenheit mit Betreuung und Karriereplanung (aus Mitarbeitersicht)

- Fluktuationsrate

- Durchschnittliches Gehalt pro Mitarbeiter

- Anzahl Stunden, die ein Vorgesetzter für einen Mitarbeiter für Beurteilungsgespräche und Karriereplanung aufwendet.

- Qualität der Zusammenarbeit zwischen den Mitarbeitern (aus Mitarbeitersicht)

- Qualität der Kommunikation zwischen verschiedenen hierarchischen Ebenen

- Investition in Mitarbeiterausbildung (in Tage und CHF)

- Verbleibquote (Anteil der Mitarbeiter, die ein Jahr nach Anstellung noch im Unternehmen sind)

Tabelle 6-12: Identifizierte Performance-Indikatoren für das Ziel der hohen Mitarbeiterzufriedenheit

In einem zweiten Schritt werden die identifizierten Indikatoren mittels eines Affinitätsdiagramms gemäss ihren natürlichen Beziehungen gruppiert (vgl. Abbildung 6-12).

Nach der Bereinigung wurden die Indikatoren „Fluktuationsrate“, „Anz. Krankheitstage pro Mitarbeiter“, „Index der Mitarbeiter-Zufriedenheitsumfrage“, „Investition in Mitarbeiterausbildung“ und „Durchschnittliches Gehalt pro Mitarbeiter“ als viel versprechend identifiziert.

Die identifizierten Performance-Indikatoren werden in einem nächsten Schritt präzise definiert. Tabelle 6-13 zeigt beispielhaft ein Definitionsformular für den Indikator Anzahl Krankheitstage (P252). 


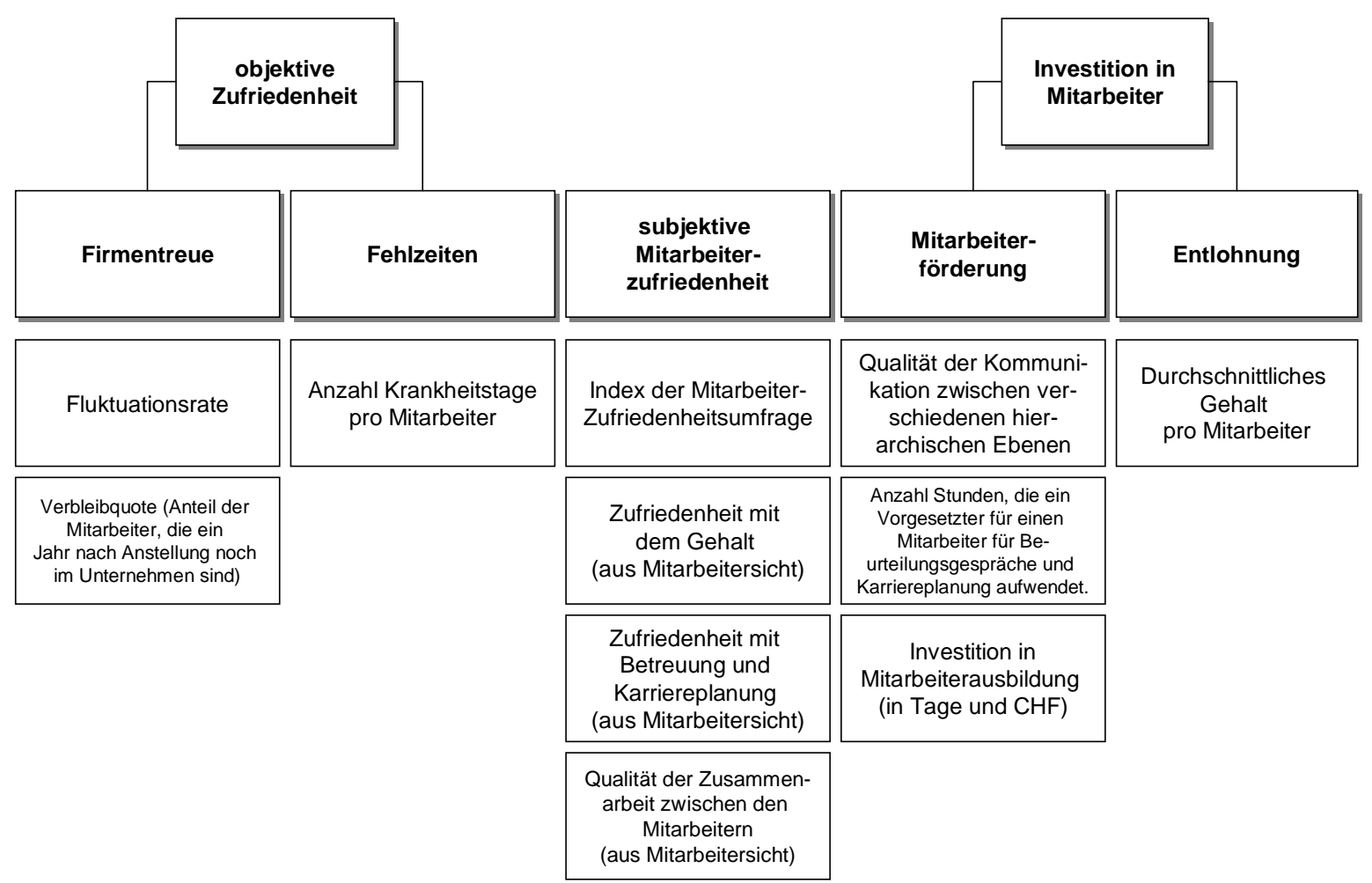

Abbildung 6-12: Affinitätsdiagramm für die Indikatoren der Mitarbeiterzufriedenheit

\begin{tabular}{|l|l|}
\hline P252 I Anzahl Krankheitstage & \\
\hline PI-Kennung & P252 \\
Performance-Indikator & Anzahl Krankheitstage / Mitarbeiter \\
Zugehörige Ziel-Kennung & Z48 \\
Zugehöriges Ziel & hohe Mitarbeiterzufriedenheit \\
Beschreibung & Anzahl ganzer Fehltage auf Grund von Krankheit oder Unfall. \\
& Nicht darunter fallen Arztbesuche und Mutterschaftsurlaub. \\
& Es werden nur ganze Tage berücksichtigt. \\
Berechnungsformel & Anzahl ganze Krankheitstage / Anzahl Mitarbeiter) \\
Einheit & ganze Arbeitstage \\
Erhebungsfrequenz & Monatlich \\
Stichtag & 12. des Folgemonates für Daten des abgelaufenen Monats \\
Datenquelle & R/3-HR (\#P252) \\
Verantwortlich für Datenqualität & Personalbereichsleiter \\
\hline
\end{tabular}

Tabelle 6-13: Definition des Performance-Indikators P252 I Anzahl Krankheitstage

In einem letzten Schritt kann nun die Auswahl der identifizierten Kandidaten auf Grund der in Abbildung 6-5 erwähnten Kriterien erfolgen.

Im Bereich der objektiven Mitarbeiterzufriedenheit wurden zwei Indikatoren ausgewählt. Die Fluktuationsrate weist gegenüber der Anzahl Krankheitstage den Nachteil auf, dass der 
Indikator später reagiert und weniger empfindlich ist. Die Zuverlässigkeit der Fluktuationsrate wird demgegenüber präziser sein. Der Indikator der Anzahl Krankheitstage birgt die Gefahr, dass Mitarbeiter „krank“ zur Arbeit erscheinen, wenn dieser mit Anreizen verknüpft wird. Dies kann nicht der Sinn sein (vgl. bzgl. der negativen Auswirkungen Saldo (2002)). Zum Schutz des einzelnen Mitarbeiters wird zudem darauf verzichtet, den Indikator detaillierter als auf Abteilungsebene aufzuschlüsseln. Wegen der höheren Empfindlichkeit und der Frühwarnfunktion wird jedoch trotzdem der Indikator „Anzahl Krankheitstage“ ausgewählt.

Die subjektive Mitarbeiterzufriedenheit umfasste ursprünglich vier Vorschläge. Der Zufriedenheitsindex der Mitarbeiterumfrage wurde ausgewählt, da dieser als Index auch die drei anderen Indikatoren umfassen kann.

Die unter der Rubrik „Investition in Mitarbeiter“ zusammengefassten Vorschläge umfassen das „durchschnittliche Gehalt pro Mitarbeiter“ und die „Investition in die Mitarbeiterausbildung“. Beide Informationen können verhältnismässig einfach aus den operativen Informationssystemen gewonnen werden. Die Quantifizierbarkeit und die Verständlichkeit ist bei beiden Indikatoren gegeben. Den Ausschlag für den Entscheid für den Indikator „Investition in die Mitarbeiterausbildung“ gab die Überlegung, dass mit dem Salär auf einer tieferen organisatorischen Ebene (z.B. Arbeitsgruppe) schützenswerte Informationen von Mitarbeitern preisgegeben werden.

Um die Auswahl zu unterstützen, kann ein Kriterienraster, wie in Tabelle 6-14 dargestellt, verwendet werden. Die schattierten Performance-Indikatoren-Kandidaten markieren die getroffene Auswahl. Selbstverständlich ist auch diese Zuordnung subjektiv.

Bereits die hier gezeigte Operationalisierung eines einzigen Ziels macht deutlich, dass es sich um einen aufwändigen Prozess handelt. Organisationen werden sich daher rasch die Frage stellen, warum nicht generische Performance-Indikatoren verwendet werden sollen. Entsprechende Kataloge wurden in verschiedenen Projekten entwickelt (vgl. z.B. Neely (2002) oder Ortega et al. (1997), Flores und Ortega (1998), SPI (2002)). Grundsätzlich ist nichts gegen deren Verwendung einzuwenden. Es besteht jedoch die Gefahr, dass diese unkritisch übernommen, von den Betroffenen nicht akzeptiert oder nur beschränkt verstanden oder nicht auf die organisationsspezifische Problemstellung angepasst werden. Der damit verbundene Zeitgewinn würde sich somit längerfristig nicht auszahlen. Das 
Hauptproblem ist jedoch darin zu sehen, dass die angestrebte Zielorientierung verloren gehen kann.

\begin{tabular}{|l|c|c|c|c|c|c|c|}
\hline \multicolumn{1}{|c|}{ Kriterien } & $\begin{array}{c}\text { Ziel- } \\
\text { bezug }\end{array}$ & $\begin{array}{c}\text { Quanti- } \\
\text { fizier- } \\
\text { barkeit }\end{array}$ & $\begin{array}{c}\text { Ver- } \\
\text { ständ- } \\
\text { lichkeit }\end{array}$ & $\begin{array}{c}\text { Zuver- } \\
\text { lässig- } \\
\text { keit }\end{array}$ & $\begin{array}{c}\text { Gültig- } \\
\text { keit }\end{array}$ & $\begin{array}{c}\text { Empfind- } \\
\text { lichkeit }\end{array}$ & $\begin{array}{c}\text { Kosten- } \\
\text { Nutzen- } \\
\text { Verhältnis }\end{array}$ \\
\hline Fluktuationsrate & + & + & + & 0 & + & 0 \\
\hline $\begin{array}{l}\text { Anz. Krankheitstage } \\
\text { pro Mitarbeiter }\end{array}$ & 0 & + & 0 & + & 0 & + & + \\
\hline $\begin{array}{l}\text { Index der Mitarbeiter- } \\
\text { Zufriedenheitsumfrage }\end{array}$ & + & 0 & + & 0 & + & 0 & 0 \\
\hline $\begin{array}{l}\text { Investition in } \\
\text { Mitarbeiterausbildung }\end{array}$ & 0 & + & + & + & 0 & 0 & + \\
\hline $\begin{array}{l}\text { Durchschnittliches } \\
\text { Gehalt pro Mitarbeiter }\end{array}$ & 0 & + & + & + & 0 & - & 0 \\
\hline \\
Legende:
\end{tabular}

Tabelle 6-14: Bewertung der analysierten Indikatoren anhand der definierten Anforderungen

Tabelle 6-15 zeigt die Performance-Indikatoren für sämtliche Ziele auf der Ebene des Unternehmens von SIDL AG. Diese werden für die weiteren Schritte benötigt. 


\begin{tabular}{|l|l|}
\hline Ziele & Performance-Indikator \\
\hline Hohe Eigenkapitalquote & Eigenkapitalquote \\
\hline $\begin{array}{l}\text { Umsatz und Ergebniswachstum, welches } \\
\text { langfristig die Leistung der Branche übertrifft }\end{array}$ & $\begin{array}{l}\text { Veränderung des relativen Marktanteils } \\
\text { gegenüber dem Vorjahresquartal }\end{array}$ \\
\cline { 2 - 2 } & EBITA-Marge \\
\hline $\begin{array}{l}\text { Kunden und Lieferanten: Langfristig, } \\
\text { beidseitig nutzbringende Beziehung }\end{array}$ & CS-Index ${ }^{8}$ (Customer Satisfaction-Index) \\
\cline { 2 - 2 } & $\ldots$ \\
\hline Hohe Mitarbeiterzufriedenheit & Index der Mitarbeiter-Zufriedenheitsumfrage \\
\cline { 2 - 3 } & Investition in Mitarbeiterausbildung \\
\cline { 2 - 2 } & Anzahl Krankheitstage pro Mitarbeiter \\
\hline \multirow{2}{*}{$\begin{array}{l}\text { Wahrnehmung der sozialen und } \\
\text { gesellschaftlichen Verantwortung }\end{array}$} & $\ldots$ \\
\hline$\ldots$ & $\ldots$ \\
\hline
\end{tabular}

Tabelle 6-15: Auszug der Ziele und Performance-Indikatoren auf Unternehmensebene von SIDL AG

\subsubsection{Top-Down-Zuweisung der Ziele und Indikatoren an die entsprechenden Organisationseinheiten}

In einem nächsten Schritt (Schritt 4.3, vgl. Kap. 6.3.4.3) erfolgt die Top-Down-Zuweisung der Ziele und Indikatoren an die entsprechenden Organisationseinheiten. Dieser Vorgang soll hier am Beispiel des Bereiches „Application Services“ und des Prozesses „Support \& Maintenance“ dargestellt werden (vgl. Organigramm in Abbildung 6-11).

Es ist zu prüfen, welche der Ziele und Indikatoren auf den Bereich „Application Services“ übernommen werden können. Die Ziele der „hohen Mitarbeiterzufriedenheit“ und des „CS-Index“ können mitsamt den Indikatoren übernommen werden. Die Übernahme der hohen Eigenkapitalquote ist nicht möglich, da das Eigenkapital dem entsprechenden Bereich nur schwierig zugerechnet werden kann. Die EBITA-Marge wird auf

8 Beim vorliegenden CS-Index handelt es sich um eine standardisierte, weltweit einmal jährlich durchgeführte Vollerhebung sämtlicher Kunden des Konzern. Dabei wird die Kundenzufriedenheit mittels Telefoninterviews von einer externen Forschungsfirma erhoben. Die Erhebung umfasst 15 Aussagen die nach dem SERVQUAL-Prinzip erhoben werden (zu SERVQUAL vgl. Parasuraman et al. (1988)). Die Daten werden zentral ausgewertet und für jede betroffene Organisationseinheit aufbereitet. Die Auswertung umfasst insbesondere auch Vergleiche im Zeitablauf (Vorjahr) sowie BenchmarkingInformationen. 
Bereichsebene ausgewiesen (Umlage der Management-Services auf die „general and administration expenses“, vgl. Tabelle 6-10). Der Performance-Indikator „Veränderung des relativen Marktanteils gegenüber dem Vorjahresquartal“ lässt sich zwar technisch auf den Bereich transformieren, macht jedoch wenig Sinn. Die Top-Down zuweisbaren Ziele und Indikatoren sind in der Tabelle 6-16 dargestellt.

\begin{tabular}{|l|l|}
\hline Ziele & Performance-Indikator \\
\hline $\begin{array}{l}\text { Umsatz und Ergebniswachstum, welches } \\
\text { langfristig die Leistung der Branche übertrifft }\end{array}$ & EBITA-Marge \\
\hline $\begin{array}{l}\text { Kunden und Lieferanten: Langfristig, } \\
\text { beidseitig nutzbringende Beziehung }\end{array}$ & CS-Index (Customer Satisfaction-Index) \\
\hline \multirow{2}{*}{ Hohe Mitarbeiterzufriedenheit } & Index der Mitarbeiter-Zufriedenheitsumfrage \\
\cline { 2 - 2 } & Investition in Mitarbeiterausbildung \\
\cline { 2 - 2 } & Anzahl Krankheitstage pro Mitarbeiter \\
\hline
\end{tabular}

Tabelle 6-16: Ziele und Performance-Indikatoren auf der Ebene des Bereiches „Application Services"

Des Weiteren soll die Zuweisung auf den Prozess „Support \& Maintenance“ innerhalb des Bereiches „Application Services“ überprüft werden. Die Indikatoren des Zieles „hohe Mitarbeiterzufriedenheit“ können sicher übernommen werden. Eine weitere Kaskadierung ist in Abhängigkeit der Informationspolitik des Unternehmens zu überdenken. Der Kundenzufriedenheitsindex kann ebenfalls übernommen werden. Es ist jedoch zu bedenken, dass sich die Zufriedenheit der Kunden des Bereichs „Application Services“ aus verschiedenen Prozessen zusammensetzt, also nicht ursächlich von diesem Prozess determiniert wird. Gleiches Argument liesse sich jedoch bereits auf der Ebene des Bereiches anführen, da unter anderem der Bereich „Management Services“ für die Verrechnung zuständig ist und dieser die Kundenzufriedenheit ebenfalls beeinflussen $\mathrm{kann}^{9}$. Dieser Argumentation folgend wird der Indikator auf der Ebene des Prozesses berücksichtigt. Die EBITA-Marge kann auf der Ebene des Prozesses nicht mehr erhoben werden.

9 Bei SIDL AG wurde genau dies anlässlich der Kundenzufriedenheitsumfrage festgestellt. Der bei den Fakturas, die für sämtliche Bereiche von „Management Services“ erstellt werden, gegebene Informationsgehalt erwies sich als unbefriedigend. 


\begin{tabular}{|l|l|}
\hline Ziele & Performance-Indikator \\
\hline $\begin{array}{l}\text { Kunden und Lieferanten: Langfristig, } \\
\text { beidseitig nutzbringende Beziehung }\end{array}$ & CS-Index (Customer Satisfaction-Index) \\
\hline \multirow{2}{*}{ Hohe Mitarbeiterzufriedenheit } & Index der Mitarbeiter-Zufriedenheitsumfrage \\
\cline { 2 - 2 } & Investition in Mitarbeiterausbildung \\
\cline { 2 - 2 } & Anzahl Krankheitstage pro Mitarbeiter \\
\hline
\end{tabular}

Tabelle 6-17: Ziele und Performance-Indikatoren auf der Ebene des Prozesses „Support \& Maintenance"

\subsubsection{Bestimmen der Mittel zur Zielerreichung}

Der vierte Schritt der Transformationsschrittfolge besteht in der Untersuchung der Mittel zur Erreichung jener Ziele, die nicht auf die unteren Ebenen kaskadiert werden konnten. Die gemäss Kapitel 6.3.4.4 zu stellende Frage lautet: „Mit welchen Mitteln können die Ziele erreicht werden?“

Ein Ziel auf der Unternehmensebene, welches nicht kaskadiert werden konnte, ist die „hohe Eigenkapitalquote“. Mit welchen Mitteln kann eine hohe Eigenkapitalquote erreicht werden? Die Eigenkapitalquote definiert sich als Eigenkapital über dem Gesamtkapital. Die Finanzierung der Geschäftstätigkeit kann als Tochterfirma eines Konzerns nur sehr beschränkt selbst beeinflusst werden, jedoch kann die notwendige Menge an Kapital beeinflusst werden. Mögliche Mittel hierzu wären bspw.:

- Weit gehende Kongruenz von Einnahmen- und Ausgabenströmen

- Minimierung des Anlagevermögens

- Optimale Bewirtschaftung des Umlaufvermögens

Für den Bereich „Application Services“ könnte die Zielsetzung lauten: (1) „Zahlungsmodalitäten mit der Leistungserbringung korrespondieren“, (2) „grössere Neuentwicklungen der ERP-Lösung werden mit Kostenbeteiligungsmodellen der Kunden realisiert“".

Das Ziel „Umsatz und Ergebniswachstum, welches langfristig die Leistung der Branche übertrifft“ kann im Bereich des „Application Services“ mit einer relativ wachsenden Installationsbasis und einer relativ höheren Neuabschlussquote (mengenmässig) erfasst 
werden. Die zugehörigen Performance-Indikatoren können den „relativen Marktanteil der ERP-Lösung im relevanten Segment“ und den „mengenmässige Anteil an Neuabschlüssen“ darstellen.

Auf der Ebene des Prozesses „Support \& Maintenance“ kann das Ziel „Umsatz und Ergebniswachstum, welches langfristig die Leistung der Branche übertrifft“ bspw. mit dem Mittel „kompetitive SLA-Konditionen“ oder „hohe Wartungsqualität“ erreicht werden.

Diese Unterziele sind nun wiederum mit geeigneten Performance-Indikatoren zu operationalisieren (vgl. Kapitel 6.4.2.2). Die neuen, zusätzlichen Ziele (beziehungsweise Unterziele) bilden die Basis, um erneut nach den Mitteln (Unter-Unter-Ziele) zu fragen. Der vorliegende Schritt wird somit rekursiv ausgeführt. Die Abbruchbedingung ist dadurch gegeben, dass für die relevante Organisationseinheit genügend Ziele und PerformanceIndikatoren vorhanden sind, um diese zielgerichtet führen und verbessern zu können.

Nachfolgende Tabelle 6-18 zeigt die Ziel- und Indikatorenhierarchie für den betrachteten Ausschnitt von SIDL. In den ersten drei Spalten ist die Aufbauorganisation mit den Ebenen Unternehmen, Geschäftsbereich und Prozess dargestellt. In der vierten Spalte sind die Ziele erkennbar, die einem Organisationselement zugeordnet sind. Die Ziele werden entweder in einer Ziel-Mittelbeziehung heruntergebrochen oder mittels PerformanceIndikatoren operationalisiert. Ziele auf tieferen Organisationsebenen, die sich aus Mitteln einer höheren Ebene ableiten sind entsprechend referenziert. Das Ziel „Z05 Zahlungsmodalitäten korrespondieren mit der Leistungserbringung“ ist mit dem Kürzel „M1“ versehen. Dies bedeutet, dass sich dieses Ziel aus dem Mittel „M01 - Kongruenz von Einnahmen- und Ausgabenströmen“ ableitet. 


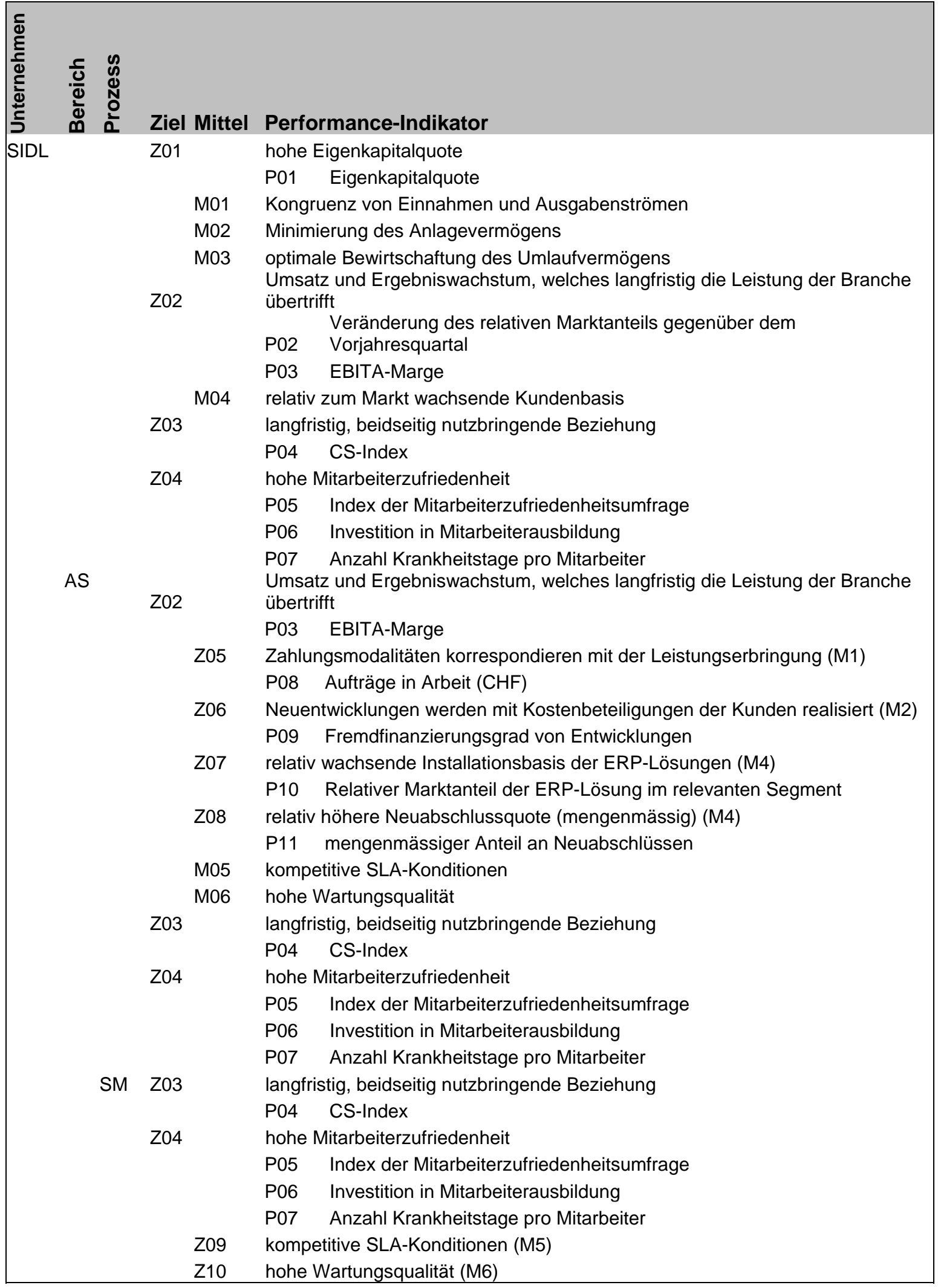

Tabelle 6-18: Ziel- und Indikatorenhierarchie für den betrachteten Ausschnitt von SIDL 


\subsubsection{Bestimmen der strategischen Programme}

Beim vorliegenden Fallbeispiel von SIDL AG wurde bei der Bestimmung der Mittel zur Zielerreichung (vgl. Kapitel 6.4.2.4) ein mögliches Mittel „Optimale Bewirtschaftung des Umlaufvermögens“ zur Reduktion des betriebsnotwendigen Kapitals identifiziert. Nach Abklärung mit dem lokalen Rechnungswesen wurde festgestellt, dass dieses ein entsprechendes Projekt nicht aus der eigenen Organisation heraus durchführen kann. Die Idee wurde an die Konzernfinanzstelle (Corporate Finance) herangetragen, welche diese aufgriff und SIDL AG in das Netting- und Cash-Pooling-Konzept ${ }^{10}$ des Konzerns integrierte.

Nach Vorabklärungen wird die Massnahme „Netting + Cash-Pooling“ mit den Zielen „Reduktion der eigenen Liquiditätsreserve um 60\% bis in 6 Monaten“ und „Erhöhung der Rendite auf der durchschnittlich „abgetretenen“ Liquiditätsreserve um 1.5\% p.a. nach Abschluss des Projektes“, einem Verantwortlichen (Bereichsleiter „Management Services“) und einem Endtermin für die Umsetzung zugewiesen.

\subsubsection{Aufnahme der persönlichen Zielsetzungen der Mitarbeiter}

Auf eine Aufnahme der persönlichen Zielsetzungen wurde im Rahmen dieses Fallbeispiels verzichtet.

\subsubsection{Verifikation des Zielbaumes}

In einem nächsten Schritt sollte die Qualität des so erarbeiteten Zielbaums sichergestellt werden. Im Kapitel 6.3.4.7 wurden zwei Instrumente zum Aufdecken von Zielkonflikten vorgestellt sowie Entscheidregeln zum Auflösen von Zielkonflikten diskutiert. Die Zielsetzung der Verifikation des Zielbaumes besteht in der Überprüfung, inwiefern die einzelnen Ziele im Zielbaum an der richtigen Stelle angesiedelt sind. Um den Rahmen nicht zu sprengen, werden die vorgestellten Instrumente nicht auf das hier vorgestellte Fallbeispiel von SIDL angewandt.

10 Unter Cash-Pooling wird die tägliche Bewirtschaftung und Steuerung der Geldein- und ausgänge mit dem Ziel, Soll- und Habensaldi von Konzerngesellschaften untereinander auszugleichen, verstanden. Beim Netting handelt es sich um die Kompensation von Debitoren- und Kreditorenpositionen, um die Überweisung von einer Gesellschaft zur anderen möglichst zu vermeiden. (Boemle (1998)) 
Aus dem behandelten Fallbeispiel SIDL wird deutlich, dass die Komplexität und damit der Aufwand für die Verifikation der Zielhierarchie in praxisnahen Umgebungen rasch ansteigt. Ein mögliches Vorgehen, um die Komplexität zu bewältigen, besteht in einem Top-Down-Clustern des Baumes (vgl. Abbildung 6-13). Konkret bedeutet dies, dass in einem ersten Schritt die Ziele im Cluster eins, darauf diejenigen des Zweiten, etc. verifiziert werden. Für die einzelnen Cluster werden die Instrumente zur Verifikation des Zielbaumes (siehe Kapitel 6.3.4.7) angewandt. Die Schnittstellen-Ziele (Ziel06 resp. Ziel18 in Abbildung 6-13) werden jeweils im über- und untergeordneten Cluster berücksichtigt. Die Clusterbildung erfolgt derart, dass die Beziehungen zwischen den Clustern nur über das Schnittstellenelement erfolgen.

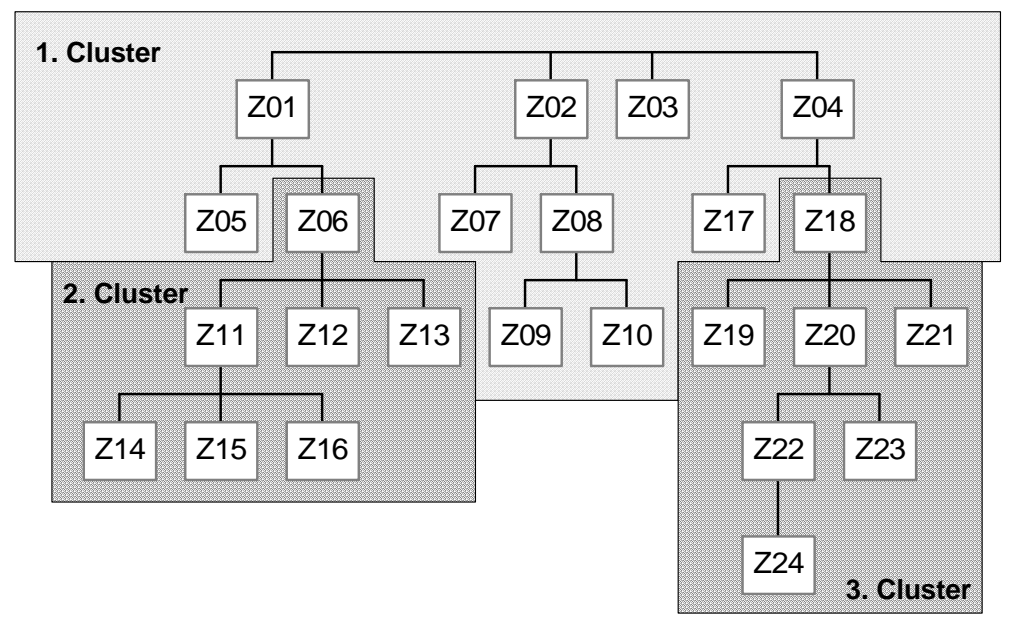

Abbildung 6-13: Cluster-Bildung als Mittel zur Komplexitätsreduktion der ZielhierarchieVerifikation

\subsubsection{Bestimmen der Prämissenindikatoren}

Das Kontrollziel von SIDL in diesem Bereich beschränkt sich auf die gerichtete Kontrolle von quantifizierbaren Indikatoren, um für die Analyse Relationen zur Unternehmensumwelt herstellen zu können (vgl. Kapitel 6.3.4.8 und Tabelle 6-9).

\subsubsection{Zuordnen der Verantwortlichen und der Informationsempfänger}

Im fünften Schritt sind für die einzelnen Ziele die Verantwortlichkeiten festzulegen. Dieser Schritt wird zur Vereinfachung mit Schritt sechs zusammengefasst, der die Informationsempfänger der Performance-relevanten Daten bestimmt. 
Die Heuristik zur Erstellung des Zielbaumes schlägt vor, dass ein Ziel, sofern anwendbar, auf mehreren organisatorischen Ebenen zur Anwendung kommt. Damit muss die Frage der Bezugsgrössen der Ziele berücksichtigt werden. Die Mitarbeiterzufriedenheit auf der Unternehmensebene bezieht sich demnach auf sämtliche Mitarbeiter, auf der Ebene des Bereiches „Application Services“ nur mehr auf diesen, etc. Es entsteht somit ein Aggregationspfad über einem Ziel entlang der Aufbauorganisation.

Die Zuordnung der Informationsempfänger erfolgt gemäss dem in Kapitel 6.3.4.10 vorgestellten Vorgehen, wobei zur Vereinfachung nur die wichtigsten Empfänger aufgeführt wurden. In Tabelle 6-19 wird je Indikator und Organisationseinheit ein Verantwortlicher für die Zielerreichung sowie die relevanten Informationsempfänger ausgewiesen. Die Liste der Empfänger zeigt lediglich einen Ausschnitt. Der PerformanceIndikator „CS-Index“ (Kundenzufriedenheit) auf der Stufe des Prozesses „Service \& Maintenance“ zeigt für den Empfänger ein „X/Kunde“. Damit wird angedeutet, dass jeder Kunde lediglich seinen individuellen Zufriedenheitswert auf dieser Stufe erhält. Auf der Stufe des Bereichs und der Unternehmung erhält er demgegenüber den Mittelwert des CSIndexes. In Tabelle 6-19 nicht enthalten ist die Kommunikationsart (Information Push oder Pull). Diese sollte vom „Informationskonsumenten“ selbst festgelegt werden können und wird deshalb im Rahmen der informationstechnischen Umsetzung aufgegriffen (vgl. den Prototypen im Kapitel elf). In diesem Sinne handelt es sich hierbei um eine MaximalVariante der verfügbaren Informationen. 


\begin{tabular}{|c|c|c|c|c|c|c|c|}
\hline \multirow{2}{*}{ 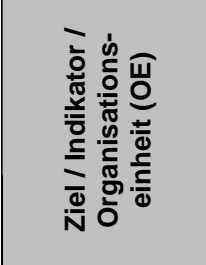 } & \multirow{2}{*}{ 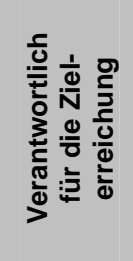 } & \multicolumn{6}{|c|}{ Empfänger } \\
\hline & & 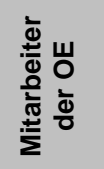 & 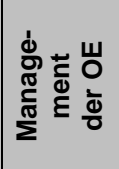 & 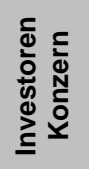 & 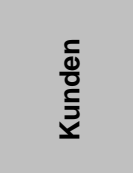 & 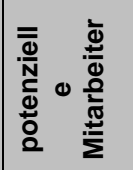 & 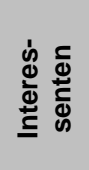 \\
\hline Z01.P01.SIDL & UL & & $x$ & $x$ & & & \\
\hline Z02.P02.SIDL & UL & & $x$ & $x$ & & & \\
\hline Z02.P03.SIDL & UL & & $x$ & $x$ & & & \\
\hline Z03.P04.SIDL & UL & $x$ & $x$ & $x$ & $x$ & & $x$ \\
\hline Z04.P05.SIDL & UL & $x$ & $x$ & & & $x$ & \\
\hline Z04.P06.SIDL & UL & $x$ & $x$ & & & $x$ & \\
\hline Z04.P07.SIDL & UL & $x$ & $x$ & & & & \\
\hline Z02.P03.AS & BL-AS & & $x$ & $x$ & & & \\
\hline Z05.P08.AS & BL-AS & & $x$ & $x$ & & & \\
\hline Z06.P09.AS & BL-AS & & $x$ & $x$ & & & \\
\hline Z07.P10.AS & BL-AS & & $x$ & $x$ & $x$ & & \\
\hline Z08.P11.AS & BL-AS & & $x$ & $x$ & & & \\
\hline Z03.P04.AS & BL-AS & $x$ & $x$ & & $x$ & & $x$ \\
\hline Z04.P05.AS & BL-AS & $x$ & $x$ & & & & \\
\hline Z04.P06.AS & BL-AS & $x$ & $x$ & & & & \\
\hline Z04.P07.AS & BL-AS & $x$ & $x$ & & & & \\
\hline Z03.P04.SM & PM-SM & $x$ & $x$ & & X/Kunde & & \\
\hline Z04.P05.SM & PM-SM & & $x$ & & & & \\
\hline Z04.P06.SM & PM-SM & & $x$ & & & & \\
\hline Z04.P07.SM & PM-SM & & $x$ & & & & \\
\hline Z20.P20.S01 & OD & $x$ & $x$ & $x$ & & & \\
\hline Z21.P21.S01 & $O D$ & $x$ & $\mathrm{x}$ & $\mathrm{x}$ & & & \\
\hline
\end{tabular}

\section{Legende:}

UL Unternehmensleitung

BL-AS Bereichsleiter „Application Services”

PM-SM Prozessmanager „Support \& Maintenance”

SIDL Unternehmen SIDL AG

AS Bereich „Application Services”

SP Prozess „Support \& Maintenance”

OD Konzernentwicklung

Sxx Strategische Massnahme $x$

Zxx Ziel $x x$

Pxx Performance-Indikator $x$

Tabelle 6-19: Ausschnitt der Zuordnung der Verantwortlichen und der Informationsempfänger Performance-relevanter Daten der SIDL AG. 


\subsection{Zusammenfassung}

Die Ausführungen zur Erstellung der Indikatorenhierarchie haben die Komplexität des Prozesses aufgezeigt. Es ist eine ganze Reihe von Instrumenten notwendig, um das angestrebte Ziel einer geordneten, in sich geschlossenen Indikatorenhierarchie zu erreichen. Gegenüber anderen in der Literatur beschriebenen Ansätzen unterscheidet sich der Vorliegende durch seine Durchgängigkeit und Anwendbarkeit. Speziell verhält sich auch die Tatsache, dass der vorliegende Ansatz auf dem Subsidiaritätsprinzip aufbaut, d.h. kein neues Führungssystem propagiert, sondern vielmehr Bestehende zu integrieren versucht. Zudem ist die Einordnung ins PM-Modell einfach nachvollziehbar. 


\section{Prozesse für den Betrieb eines Performance-Measurement-Systems}

\subsection{Die Prozesse im Überblick}

Ziel des vorliegenden Kapitels ist es, aufzuzeigen, welche Prozesse definiert werden müssen, um den Betrieb eines Performance-Measurement-Systems sicherzustellen. Es können verschiedene Arten von Prozessen unterschieden werden (vgl. Abbildung 7-1). Die primäre Differenzierung leitet sich aus den Anforderungen aus Kapitel vier und der Methodik des Bezugsrahmens im fünften Kapitel ab:

- Zielsetzungsprozess: Der Zielsetzungsprozess umfasst das Festlegen der ZielWerte (Soll-Werte) der einzelnen Performance-Indikatoren auf den einzelnen Leistungsebenen der Organisation. Dabei sind die Ziel-Werte aufeinander abzustimmen. Der Zielsetzungsprozess ist in die bestehenden Planungs- und Budgetierungsprozesse und im Besonderen in die Jahresplanung der Organisation zu integrieren.

- PMS-Nutzungsprozess: Dieser beschreibt den Weg des Datenflusses, d.h. welche einzelnen Schritte die Performance-relevanten Daten durchlaufen. Dieser Prozess orientiert sich an dem in Küng und Wettstein (2001) vorgeschlagenen Use-Cycle, bestehend aus den Phasen (1) Datensammlung, (2) Kommunikation, (3) Analyse und Diskussion und (4) daraus abgeleiteten Handlungen.

Die Definition der nachfolgenden unterstützenden Prozesse, welche sich nicht direkt aus den Anforderungen ableiten lassen, erfolgen in Anlehnung an ISO (1998), welche für den Betrieb einen Prozess für den Unterhalt des Systems (operational use process) und einen Prozess zur Unterstützung der Anwender (customer support process) unterscheiden.

- Prozess zur Unterstützung der Anwender: Die in Kapitel fünf definierten Anwender, d.h. die Rollen, die das PMS benutzen, müssen bei Problemen geeignet unterstützt werden. Hierfür muss ein entsprechender Prozess definiert werden.

- Prozesse für den Unterhalt des Systems: Das PMS muss organisatorisch und technisch (betrifft das IT-System für das PM) unterhalten werden. 
o Organisatorischer Unterhalt: Das PMS als Abbild eines Teilbereichs einer Organisation ist entsprechend der realen Umwelt ständigen Veränderungen ausgesetzt. Dabei erfolgt eine Differenzierung gemäss dem Aufwand zur Umsetzung der Veränderungen. Während grosse Veränderungen separat, als Revision resp. Überarbeitung des PMS, aufgefasst werden, werden kleinere Veränderungen im vorliegenden Vorschlag dem Systemunterhalt zugerechnet. Auf der organisatorischen Ebene betrifft dies bspw. die Aufnahme von neuen Benutzern, das Administrieren von Rollen, etc..

o Technischer Unterhalt: Der technische Unterhalt betrifft primär das ITSystem für das PM. Dabei ist bspw. an ein Performance Monitoring (Antwortzeitverhalten) des IT-Systems für das Performance Measurement zu denken.

- Querschnittsprozesse: Eine Reihe von Aufgaben sind unabhängig vom verwendeten System wahrzunehmen. Dabei handelt es sich bspw. um ein Datenschutz- oder Datensicherheitskonzept. Denkbar ist ebenfalls, dass der Prozess zur Unterstützung der Anwender als Querschnittsprozess aufgefasst wird.

- Revisionsprozess: Beim Eintreten von bestimmten Ereignissen ist es notwendig, das PMS zu überarbeiten. Der Revisionsprozess beschreibt die entsprechend durchzuführenden Schritte. Eine Überprüfung des PMS ist bspw. notwendig, wenn (1) sich die Business-Strategie verändert, (2) Stakeholder neue Bedürfnisse an das PMS anmelden, (3) sich einzelne Performance-Indikatoren als nicht nützlich erweisen, (4) neue operative Informationssysteme in Betrieb genommen werden, welche Performance-relevante Daten verwalten oder (5) sich neue informationstechnische Möglichkeiten für die Nutzung des PMS ergeben (vgl. Küng und Wettstein (2001)).

Nachfolgend wird auf die wichtigsten Prozesse detaillierter eingegangen. 
Prozess für die Datensammlung, -speicherung, -analyse und -kommunikation

\section{Prozess zur Unterstützung der Anwender}
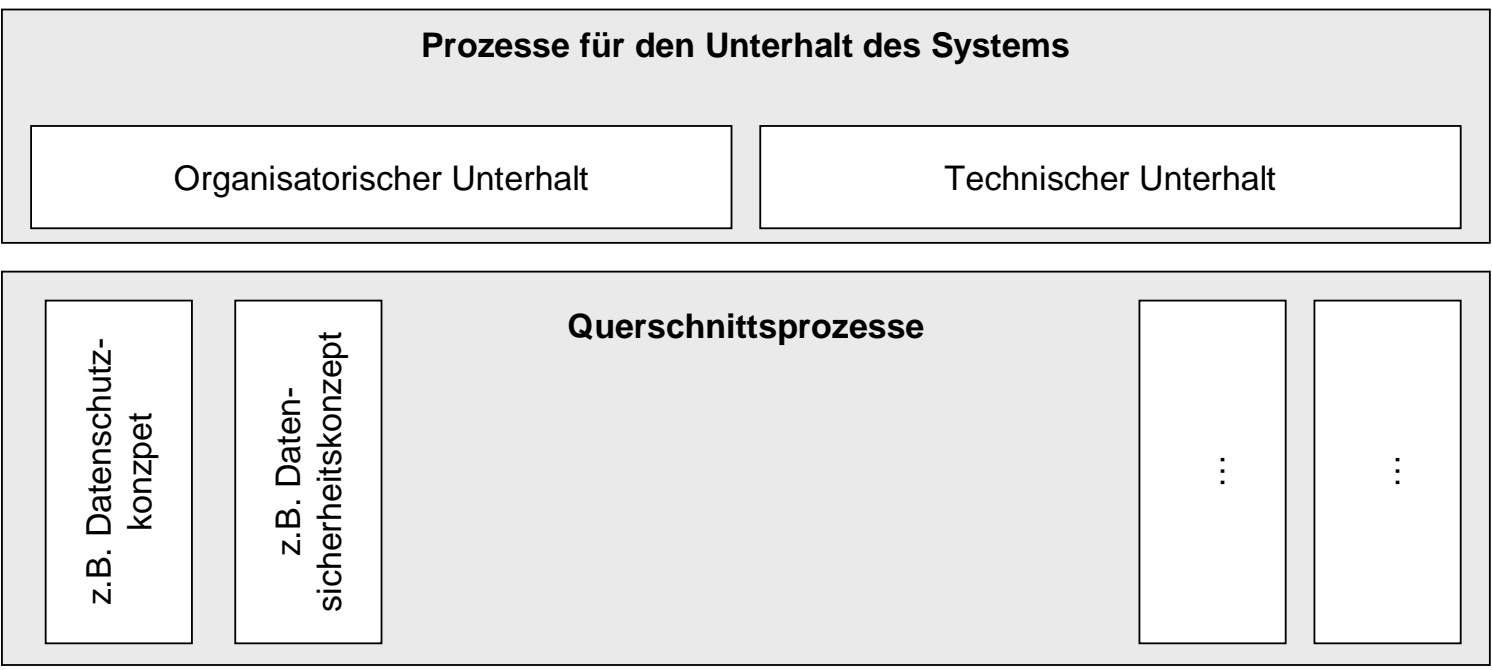

Revisionsprozess: Überarbeitung des PMS

Abbildung 7-1: Die Betriebsprozesse im Überblick

\subsection{Zielsetzungsprozess}

Soll-Werte werden für die einzelnen Performance-Indikatoren benötigt, um das angestrebte Ziel der aktuellen Performance gegenüberstellen zu können und damit eine Rückwirkung auf das Verhalten zu erreichen, resp. zu ergreifende Massnahmen bestimmen zu können.

Wie die in Kapitel sechs aufgebaute Zielhierarchie, dessen Elemente aufeinander abgestimmt wurden, müssen auch die Ziel-Werte ein in sich geschlossenes System darstellen. Zur Lösung dieses Koordinationsproblems werden Verfahren benötigt, die festlegen, welche Stellen der einzelnen Leistungsebenen, in welcher Reihenfolge, mit welchen Funktionen und Kompetenzen am Prozess zur Festlegung der Soll-Werte der Performance-Indikatoren mitwirken (vgl. Wild (1974)). Das Problem wird unter dem Begriff der Hierarchiedynamik in der Literatur behandelt (vgl. Horváth (2001)). Es werden darin drei Grundtypen von möglichen Vorgehensweisen diskutiert (vgl. Abbildung 7-2): 
Der Top-Down-Ansatz beschreibt einen Prozess, bei welchem die obersten Ziele festgelegt werden und die nächste Leistungsebene diese als Rahmenbedingungen betrachtet und darauf aufbauend ihre Ziele festlegt. In der umgekehrten Variante werden auf der untersten Leistungsebene die Ziele festgelegt. Diese werden nach oben hin lediglich konsolidiert (Bottom-Up). Das Gegenstromverfahren ist in zwei Varianten denkbar. Bei der ersten Variante wird zuerst der Top-Down-Ansatz verfolgt und daraufhin mit dem Bottom-UpAnsatz die Ziel-Werte schrittweise koordiniert. Die oberste Ebene schliesst mit der endgültigen Festlegung der Ziel-Werte den Prozess ab. Bei der zweiten Variante wird der Prozess von „unten“ angestossen. Laut Scholz (1984) dominiert in Deutschland das Gegenstromverfahren mit Anstoss von „oben“. Horváth (2001) weist daraufhin, dass die Grundtypen in der Praxis mit Pufferebenen und Planungskomitees erweitert werden müssen.

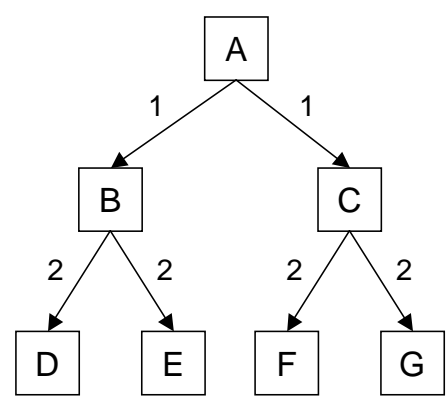

Top-Down (retrograd)

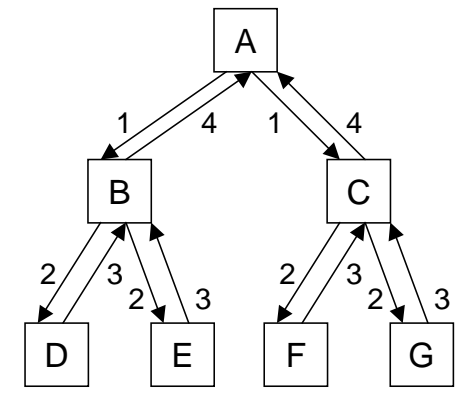

Gegenstrom (Anstoss von „oben“)

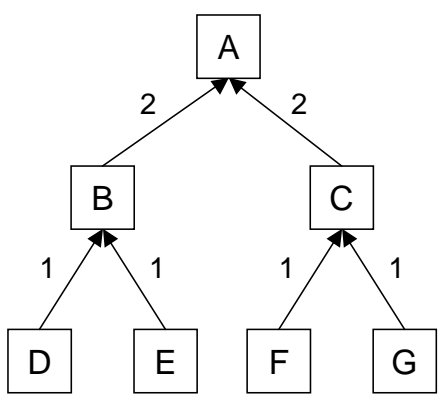

Bottom-Up (progressiv)

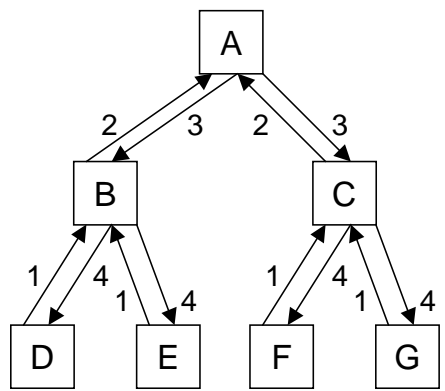

Gegenstrom (Anstoss von „unten“)

Die Zahlen beziehen sich auf die Reihenfolge der Soll-Wert-Festlegungs-Schritte.

\section{Abbildung 7-2: Grundtypen für Entwicklungsfolgen von Zielen (Scholz (1984))}

Das Gegenstromverfahren mit Anstoss von „oben“ soll nachfolgend an einem kleinen Beispiel veranschaulicht werden. Der Konzern „A“ besteht aus zwei Geschäftsfeldern „B“ und „C“, welche ihrerseits aus je zwei Geschäftseinheiten („D“ und „E“ resp. „F“ und „G“) 
bestehen. Konzern „A“ stösst den Zielsetzungsprozess an, indem er für die oberste Stufe des Konzerns Ziele, z.B. bezüglich des Umsatzes, des EBIT und der Anzahl Kunden definiert und diese für die Geschäftsfelder „B“ und „C“ operationalisiert. Die Geschäftsfelder „B“ und „C“ operationalisieren die Ziele für ihre Geschäftseinheiten. Die Geschäftseinheiten können sodann die Zielvorgaben auf ihre Machbarkeit hin untersuchen und ihren Geschäftsfeldern allfällige Unstimmigkeiten melden, welche diese an den Konzern zurückmelden. Entgegen einer unidirektionalen Entwicklungsfolge von Zielen enthalten die Gegenstromverfahren eine „Vernehmlassung“, welche die Chancen der Erreichbarkeit der Zielvorgaben erhöhen.

Eine wesentliche Schwierigkeit besteht darin, dass die Gültigkeitsdauer der einzelnen SollWerte ganz unterschiedlich ist. Diese ist abhängig von verschiedenen Faktoren wie dem Planungshorizont, der wiederum durch die Leistungsebene, in der sich der Indikator befindet, die gewählte Messfrequenz sowie der Reaktionsgeschwindigkeit der Indikatoren beeinflusst wird. Letztendlich wird aber nur eine Überprüfung der Plan-Werte die Notwendigkeit der Änderung offenbaren. Zudem ist der Abweichungsvergleich hilfreich für eine sinnvolle Festlegung von neuen Ziel-Werten. Sinnvoll ist somit eine geeignete Koordination der Erstellung, Kontrolle und Überarbeitung der Soll-Werte über die verschiedenen Ebenen. Abbildung 7-3 zeigt ein Beispiel einer koordinierten Plananpassung nach Wild ((1974), siehe auch Wild (1982) und Grünig (1996)). Wild unterscheidet in diesem Beispiel vier Formen von Anpassungsmassnahmen und drei Planungsstufen. Die Planungsstufen sind mit kurz-, mittel- und langfristig bezeichnet, könnten sich aber auch auf die Leistungsebenen (operativ, taktisch und strategisch) beziehen. Die Planungsstufen werden mit den möglichen Anpassungsmassnahmen verknüpft. Abbildung 7-3 sagt somit für die Kontrolle aus, dass diese auf der kurzfristigen Planungsstufe monatlich, auf der mittelfristigen halbjährlich und auf der langfristigen jährlich zu erfolgen hat. Der Planungshorizont beträgt auf der kurzfristigen Planungsstufe ein Jahr, auf der mittelfristigen drei Jahre und auf der langfristigen sechs Jahre. Entsprechendes gilt für die weiteren Formen der Anpassungsmassnahmen.

Für die Anwendung in der Praxis muss das Schema in stufengerechte Terminkalender (z.B. für Corporate Controller, für Geschäftsfeldcontroller, etc.) übersetzt werden. Damit wird ein kompatibles Vorgehen sichergestellt. 


\begin{tabular}{|c|c|c|c|c|c|c|c|c|c|c|c|c|c|c|}
\hline Anp & lahr & $20 \times 1$ & & & & & & $20 \times 2$ & & $20 \times 3$ & & $20 \times 4$ & $20 \times 5$ & $20 \times 6$ \\
\hline$A$ & Monat & 1 & 3 & 5 & 7 & 9 & 11 & 1 & 7 & 1 & 7 & 1 & 4 & 1 \\
\hline
\end{tabular}

\begin{tabular}{|c|c|c|c|c|c|c|c|c|c|c|c|c|c|c|}
\hline \multirow{3}{*}{ Überprüfung } & KFR & $M$ & $M$ & $M$ & M & $\mathrm{M}$ & $M$ & $M$ & $M$ & $M$ & $M$ & \multirow[t]{2}{*}{$M$} & \multirow[t]{2}{*}{$M$} & \multirow[t]{2}{*}{$M$} \\
\hline & MFR & $\mathrm{H}$ & & & $\mathrm{H}$ & & & $\mathrm{H}$ & $\mathrm{H}$ & $\mathrm{H}$ & $\mathrm{H}$ & & & \\
\hline & LFR & $\mathrm{J}$ & & & & & & $\mathrm{J}$ & & $\mathrm{J}$ & & $\mathrm{J}$ & $\mathrm{J}$ & $\mathrm{J}$ \\
\hline
\end{tabular}

\begin{tabular}{|cllllllll|}
\hline Konkretisierung & KFR & & & & & & & \\
& MFR & & & & & & \\
\cline { 3 - 8 } & LFR & $\mathrm{J}$ & & $\mathrm{J}$ & $\mathrm{J}$ & $\mathrm{J}$ & $\mathrm{J}$ & $\mathrm{J}$ \\
\hline
\end{tabular}

\begin{tabular}{|ll|}
\hline Änderung & KFR \\
& MFR \\
& LFR bei Bedarf \\
\hline
\end{tabular}

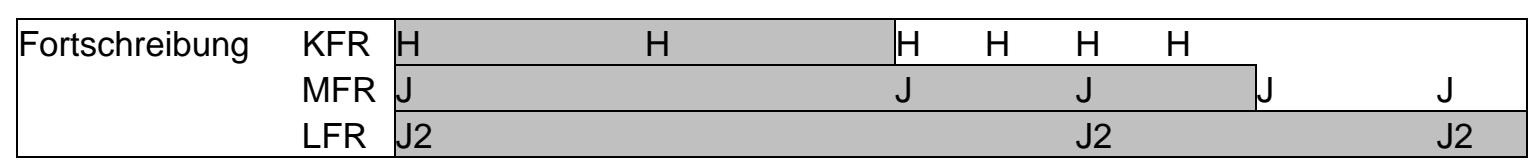

\section{Legende:}

\begin{tabular}{|c|c|c|c|c|}
\hline \multicolumn{2}{|l|}{-ege } & & & \multirow[b]{2}{*}{ Planhorizont } \\
\hline $20 \times 1$ & Planjahr & & monatlich & \\
\hline KFR & kurzfristig & $\mathrm{H}$ & halbjährlich & \\
\hline MFR & mittelfristig & $\mathrm{J}$ & jährlich & \\
\hline LFR & langfristig & $\mathrm{J} 2$ & zweijährlich & \\
\hline
\end{tabular}

Abbildung 7-3: Beispiel einer koordinierten Plananpassung (in Anlehnung an Wild (1974))

Der Prozess zur Festlegung der Soll-Werte ist somit als ein zeitlicher und inhaltlich verzahnter, mehrstufiger, kombiniert retrograd-progressiv durchzuführender rollender Prozess zu verstehen (vgl. Horváth (2001)).

Werden einzelne Informationsobjekte des PMS als subsidiär begriffen, d.h. wird die Bewirtschaftung der Soll-Werte, z.B. in den strategischen Dokumenten, dem bestehenden Plansystem, dem MbO-System vorgenommen, so muss lediglich sichergestellt werden, dass die Überarbeitungen der Ziel-Werte ins PMS übertragen werden.

\subsection{PMS-Nutzungsprozess}

Der Betriebsprozess definiert die einzelnen Vorgehensschritte, die zur Nutzung des PMS aus der Sicht der Performance-relevanten Daten notwendig sind. Schwerpunkte stellen das Sammeln, Speichern, Analysieren und Kommunizieren der Performance-relevanten Daten dar. Darauf aufbauend können Massnahmen ergriffen werden. (Phasen in Anlehnung an Küng und Wettstein (2001)) 


\begin{tabular}{|c|c|c|c|c|}
\hline $\begin{array}{c}\text { Erstellungs } \\
\text { prozess }\end{array}$ & Erstellungspr & & & \\
\hline $\begin{array}{c}\text { Zielsetzungs } \\
\text { prozess }\end{array}$ & Zielsetzungsp & & & \\
\hline $\begin{array}{l}\text { Betriebs- } \\
\text { prozess }\end{array}$ & & & $\left(\begin{array}{c}\text { Performance- } \\
\text { Daten sammeln }\end{array}\right)>\left(\begin{array}{c}\text { Ergebnisse } \\
\text { kommunizieren }\end{array}\right.$ & Hand \\
\hline $\begin{array}{l}\text { Deming- } \\
\text { Cycle }\end{array}$ & Plan & Do & Check & Act \\
\hline
\end{tabular}

\section{Abbildung 7-4: Einordnung der Prozesse in den Deming-Cycle}

Der Prozess kann in Anlehnung an den Deming-Cycle (vgl. Abbildung 3-7) aufgefasst werden. Dieser unterscheidet die Phasen Plan, Do, Check, Act. Plan meint dabei, dass in einem ersten Schritt ein Plan entworfen wird für das, was erreicht werden soll. Do bezeichnet die Ausführung des Planes. Mit Check wird das Ergebnis bestimmt und überprüft, inwiefern es dem erwarteten Resultat entspricht. Act bezeichnet die Handlungsphase nach Analyse des Ergebnisses.

Während die Planphase der Erstellung des PMS und dem Festlegen der Soll-Werte der Performance-Indikatoren zuzuordnen ist, bildet der Betriebsprozess die Phasen Check und Act ab (vgl. Abbildung 7-4).

Eine erste Aktivität besteht darin, die im Erstellungsprozess je Indikator definierten Performance-relevanten Daten zu erheben. Dabei sind verschiedene Datenquellen denkbar. Einerseits kann unterschieden werden nach dem Ort der Datenquelle, d.h. unternehmensintern resp. -extern. Andererseits ist eine Differenzierung nach der Speicherungsform der Daten denkbar, d.h. ob die Performance-relevanten Daten in einem rechnergestützten Informationssystem oder in Papierform vorliegen. Die manuelle Erhebung von Performance-relevanten Daten kann mit erheblichem Aufwand verbunden sein. Eine Anforderung an einen Performance-Indikator ist jedoch ein günstiges KostenNutzenverhältnis (vgl. Kapitel 6.3.4.2). Dieser Anforderung kann dadurch Rechnung getragen werden, indem versucht wird, die bestehenden operativen Informationssysteme als Datenquellen für die Performance-relevanten Daten zu nutzen. Mögliche operative Informationssysteme sind Enterprise Resource Planning Systems (ERP-Systems) (z.B. R/3 
von SAP (2002), Baan (2002), ERP von JDEdwards (2002), PeopleSoft (2002)), Workflow-Management-Systeme (z.B. WebSphere MQSeries Workflow von IBM (2002), Workflow von Lotus (2002)) oder andere operative betriebliche Informationssysteme (vgl. Küng et al. (2000)). Denkbar ist auch die Einbeziehung von unternehmensexternen Datenbeständen, z.B. von Marktforschungsinstituten (z.B. GartnerGroup (2002)), Finanzmarktdatenlieferanten (z.B. Reuters (2002)) oder spezialisierten Unternehmen für anonymisierte Benchmarking-Daten (z.B. SPI (2002)).

Eine Hauptherausforderung liegt bei digital vorhandenen Daten darin, diese in die gewünschte Form zu transformieren. Im Bereich des Data Warehousing hat sich für die Problemstellung des Extrahierens, Transformierens und Ladens der externen Daten in die analytischen Datenbestände des Unternehmens ein eigener Forschungszweig gebildet. Gemäss empirischen Befunden beträgt der Aufwand für diesen als ETL (Extraction Transforming \& Loading) bezeichneten Prozess bis zu 80\% des Aufwandes beim Bau eines entsprechenden Informationssystems. (vgl. Kurz (1999) und Bauer und Günzel (2001))

Sind die Daten nicht in digitaler Form verfügbar, so besteht einerseits die Möglichkeit diese Daten digital verfügbar zu machen oder diese zu erheben. Es handelt sich hierbei häufig um nicht-finanzielle Daten (vgl. z.B. Küng (2001)). Ein des Öfteren beschrittener Weg besteht darin, die Daten an einer zentralen Erfassungsstelle in das PMS einzutippen. Eine Reihe von Herstellern von Performance-Measurement-Software-Systemen, welche sich an das Top-Management richten, verfolgen diesen Weg (z.B. Promeasys). Nachteil dieser Methode ist der damit verbundene Arbeitsaufwand und die demotivierende Wirkung. Ein hoher Nutzen kann erzielt werden, wenn die Daten direkt am Entstehungsort erhoben und die betroffenen Mitarbeiter in die Auswahl der Indikatoren und die Auswertungen direkt miteinbezogen werden. Es ist darauf zu achten, dass der Arbeitsfluss nicht zu stark gestört wird. Handelt es sich um Computer-Arbeitsplätze, so werden im Idealfall die entsprechenden Applikationen um die Performance-relevanten Daten ergänzt. Denkbar ist auch der Einsatz von spezialisierten Applikationen, bspw. ein elektronischer Fragebogen der über das Intra- oder Internet verfügbar gemacht wird (vgl. Kapitel 4 oder Huber (2001), Villalobos (2000), Küng und Wettstein (1999)).

Entscheidend ist, dass die Informationsentstehung und -verwendung in der Regel auseinander fallen. Zwischen den beiden Stellen müssen deshalb Informations- 
Übermittlungsvorgänge stattfinden. Traditionell wird dies mit dem betrieblichen Berichtswesen bewerkstelligt (vgl. Horváth (2001)). Nach Blohm (1974) (zitiert bei Horváth (2001)) sind im Zusammenhang mit dem Berichtswesen vier Grundfragen zu klären: (1) Wozu soll berichtet werden? (2) Was soll berichtet werden? (3) Wer soll berichten, und wer soll unterrichtet werden? (4) Wann soll berichtet werden? Nachfolgend werden die einzelnen Punkte kurz diskutiert.

- Wozu und Was soll berichtet werden: Diese zentrale Fragestellung, die den Informationsbedarf bestimmt, kann beantwortet werden, indem auf die Definition des PMS (vgl. Kapitel zwei) und die im konkreten PMS-Projekt zu integrierenden Bereiche (vgl. Kapitel 6.3.1) zurückgegriffen wird. Allgemein wurde festgehalten, dass das PMS die operationalisierte Strategie stufengerecht kommuniziert und die Performance der Organisation auf sämtlichen Ebenen überwacht. Zudem soll sie Managern Entscheidungsunterstützung bieten, aber auch das Wissen der Organisation sammeln und somit das organisationelle Lernen unterstützen. Aus dieser Definition wird deutlich, dass der konkrete Informationsbedarf vom Empfänger, der Art der Performance-Indikatoren und einem schwer voraussagbaren Element, der Intention des Empfängers, abhängt. Es empfiehlt sich somit, eine Reihe von Basisinformationen zur Verfügung zu stellen und die Option vorzusehen, dass der Empfänger weitere Informationen, je nach zu bearbeitender Aufgabenstellung, nachfragen kann. Gladen (2001) schlägt vier Basistypen mit jeweils unterschiedlichem Zweck vor: Dokumentation, Planung, Kontrolle und Steuerung.

- Was soll berichtet werden, und Wer soll unterrichtet werden: Zur Bestimmung der Empfänger der Informationen wurde in Kapitel sechs ein Vorschlag zur Festlegung mittels der Informationspyramide diskutiert (vgl. Kapitel 6.3.4.10 und Wettstein et al. (2001)).

- Wann soll berichtet werden: Die Bestimmung von Terminen für die Überprüfung von bestimmten Performance-relevanten Daten wurde im Erstellungsprozess (vgl. Kapitel 6.3.4.5. resp. 6.4.2.2) diskutiert. Neben dieser als Push-Variante zu bezeichnenden Kommunikationsart ist zusätzlich eine Pull-Variante vorzusehen. Diese erlaubt dem Informationsempfänger, bei Bedarf auf die entsprechenden Informationen zuzugreifen. 
Für die Gestaltung von Plan- und Kontrollinformationen sind eine Reihe von Empfehlungen erarbeitet worden (vgl. Horváth (2001) und Deyhle (1978), zitiert nach Gladen (2001)):

- Plan- und Ist-Werte sollten präsentiert werden, damit entsprechende Vergleiche möglich sind.

- Die Information sollen geeignet verdichtet werden, um den Empfänger zu entlasten.

- Zur Entlastung kann auch eine Selektion oder Verknüpfung der Informationen erfolgen.

- Ein Berichtssystem soll einen formal einheitlichen Aufbau besitzen.

- Der Überblick und das Detail eines Berichtes sind in der Darstellung deutlich voneinander zu trennen.

- Aussergewöhnliche Sachverhalte sind in Berichten hervorzuheben.

- Grafische Darstellungen übertreffen tabellarische Darstellungen an Aussagekraft.

- Künstlich eingebaute Redundanzen können die Verständlichkeit erhöhen.

- Es sollten eindeutige Termini verwendet werden.

- Um Missverständnissen vorzubeugen und nicht direkt Involvierten Hilfestellungen zu bieten, empfiehlt es sich den Grundsatz „Keine Performance-Daten ohne Kommentar!“ einzuhalten. Performance-Daten sollten von den sie Verantwortenden oder ihren Controllern kommentiert werden.

Die dritte Aktivität des Betriebsprozesses betrifft die Analyse der Performance-relevanten Daten. Welche Aktivitäten die Analyse umfasst, ist abhängig vom verfolgten Ziel des Analysten. Auf Grund der Erkenntnisse können sodann die notwendigen Massnahmen beschlossen werden und somit gehandelt werden (Schritt vier).

\subsection{Revision des PMS}

Die Umwelt eines Performance-Measurement-Systems verändert sich im Zeitablauf. In bestimmten Zeitabständen und beim Eintreten bestimmter Ereignisse ist es daher angezeigt, das PMS bezüglich seiner Struktur zu überprüfen. Folgende Ereignisse sind denkbar (Ereignisse in Anlehnung an Küng und Wettstein (2001)): 
- Beendigung von strategischen Massnahmen mit Auswirkungen auf die organisatorische Struktur oder die strategischen Ziele selbst führen zu einer Überarbeitung des PMS. Einerseits müssen die Auswirkungen auf die Zielhierarchie überprüft und bei Bedarf angepasst werden; andererseits ist das beendete strategische Programm aus dem PMS zu eliminieren.

- Überarbeitung der Strategie: Erfolgt eine Anpassung der Strategie, so sind damit in der Regel neue oder veränderte Ziele verbunden. Dies kann zu neuen strategischen Massnahmen führen, die mit verhältnismässig wenig Aufwand aufgenommen werden können. Denkbar ist ebenfalls, dass die Zielhierarchie komplett überarbeitet werden muss.

- Stakeholder haben neue Informationsbedürfnisse: Ein weiterer Anlass zur Überarbeitung kann darin bestehen, dass bspw. Lieferanten im Rahmen einer neuen Preispolitik, die in Abhängigkeit der Lieferpünktlichkeit definiert wird, neu über diese Performance-Grösse informiert werden müssen. Entsprechend müsste ein neuer Indikator aufgenommen werden.

- Benutzte Performance-Indikatoren erweisen sich als ungenügend: Gerade bei neuen PM-Systemen müssen erst Erfahrungen mit der Nützlichkeit der einzelnen Performance-Indikatoren gesammelt werden. Wird erkannt, dass ein Indikator ungenügend ist, so muss dieser durch einen Besseren ersetzt werden, was eine Revision des PMS nach sich zieht.

- Performance-relevante Daten werden neu verfügbar oder sind plötzlich nicht mehr verfügbar (z.B. externer Informationslieferant, Austausch eines operativen Informationssystems, welches bisher als Datenlieferant funktioniert hat, etc.).

- Innovationen im IT-Bereich begründen neue Möglichkeiten: Bspw. erlauben neue technologische Möglichkeiten den Einsatz von besser geeigneten Indikatoren (z.B. mobile Performance-Daten-Erfassung). Dies führt zu einer Überarbeitung des PMS. 


\subsection{Abschliessende Bemerkungen}

Das Design und die Implementierung der Betriebsprozesse sind wesentlich für den Erfolg eines PMS (vgl. die übereinstimmenden Aussagen von Bitici und Carrie (1998), Hudson et al. (1999), Küng (2001), Günther und Grüning (2000) und Kap 4.3.4, im Speziellen Anforderung B-7). Der Nutzungsprozess stellt die systematische Anwendung des Plan-DoCheck-Act-Zyklus sicher, der Zielsetzungsprozess sorgt für eine periodische Aktualisierung der Ziel-Werte, und der Revisionsprozess stellt sicher, dass eine Anpassung an die sich verändernde Umwelt erfolgt.

Der Betrieb eines PMS ist in Abhängigkeit zur konkreten Ausgestaltung mit erheblichen Aufwendungen verbunden. Um diese $\mathrm{zu}$ minimieren, ist eine Integration in die bestehenden Plan- und Kontrollsysteme notwendig. Diese Integration bewirkt z.B., dass Performance-Indikatoren und ihre Soll-Werte nicht redundant erhoben werden.

Entsprechend Kapitel sechs, welches die Erstellung der Indikatorenhierarchie behandelt, ordnet sich auch dieses Kapitel, welches die Betriebsprozesse eines PMS beschreibt, in den vorgeschlagenen Bezugsrahmen ein. Die beiden Kapitel beschreiben die Prozesse der Methodik. Implizit wird auch die vorgestellte Systematik und Organisation berücksichtigt. Damit sollen die Überlegungen zur organisatorischen Konzeption eines PMS abgeschlossen und im nächsten Teil die informationstechnische Ausgestaltung untersucht werden. 


\section{Teil \\ Technische Konzeption eines PMS}




\section{Architektur des IT-Systems für das Performance Measurement}

\subsection{Einleitung}

Ziel dieses Kapitels ist es, eine mögliche Architektur für ein IT-System für das PM aufzuzeigen. Bosch (2000) definiert die Aufgaben der Architektur folgendermassen: „.. is concerned with rational and the top-level decomposition of the system into its main components and the design process leading to that decomposition." Der Einsatz einer Architektur erlaubt somit die Aufteilung eines „Monolithen“ in seine Komponenten. Damit wird einerseits die Komplexität reduziert und andererseits der Aufbau und die Wartung des Systems vereinfacht. Um die Struktur des „Bauwerks“ zu erhalten, ist auch die Beziehung der Komponenten untereinander und die Einbettung in die Umgebung relevant. Da sich die einzelnen Komponenten im Zeitablauf verändern, umfasst die Architektur ebenfalls Prinzipien, welche Entwicklung und Evolution eines Systems lenken (vgl. IEEE1471 (2000)).

Zweck der Architektur ist es, einen objektiven Ausgangspunkt für die Implementierung eines konkreten Systems zu bilden und ein eindeutiges Verständnis bezüglich der Komponenten, Funktionen und Begriffe bei den involvierten Personen zu schaffen (vgl. Sommerville (2001)).

Nach Bosch (2000) können drei Hauptphasen bei der Erstellung einer Architektur differenziert werden (vgl. Abbildung 8-1): (1) die funktionale Architektur, (2) die Transformation der funktionalen Architektur in eine technische Architektur durch die Evaluation von qualitativen resp. nicht-funktionalen Kriterien und (3), sofern notwendig, deren Transformation. Das zur Bestimmung der IT-Architektur des IT-Systems für das PM gewählte Vorgehen orientiert sich im Wesentlichen an diesen drei Teilschritten. 


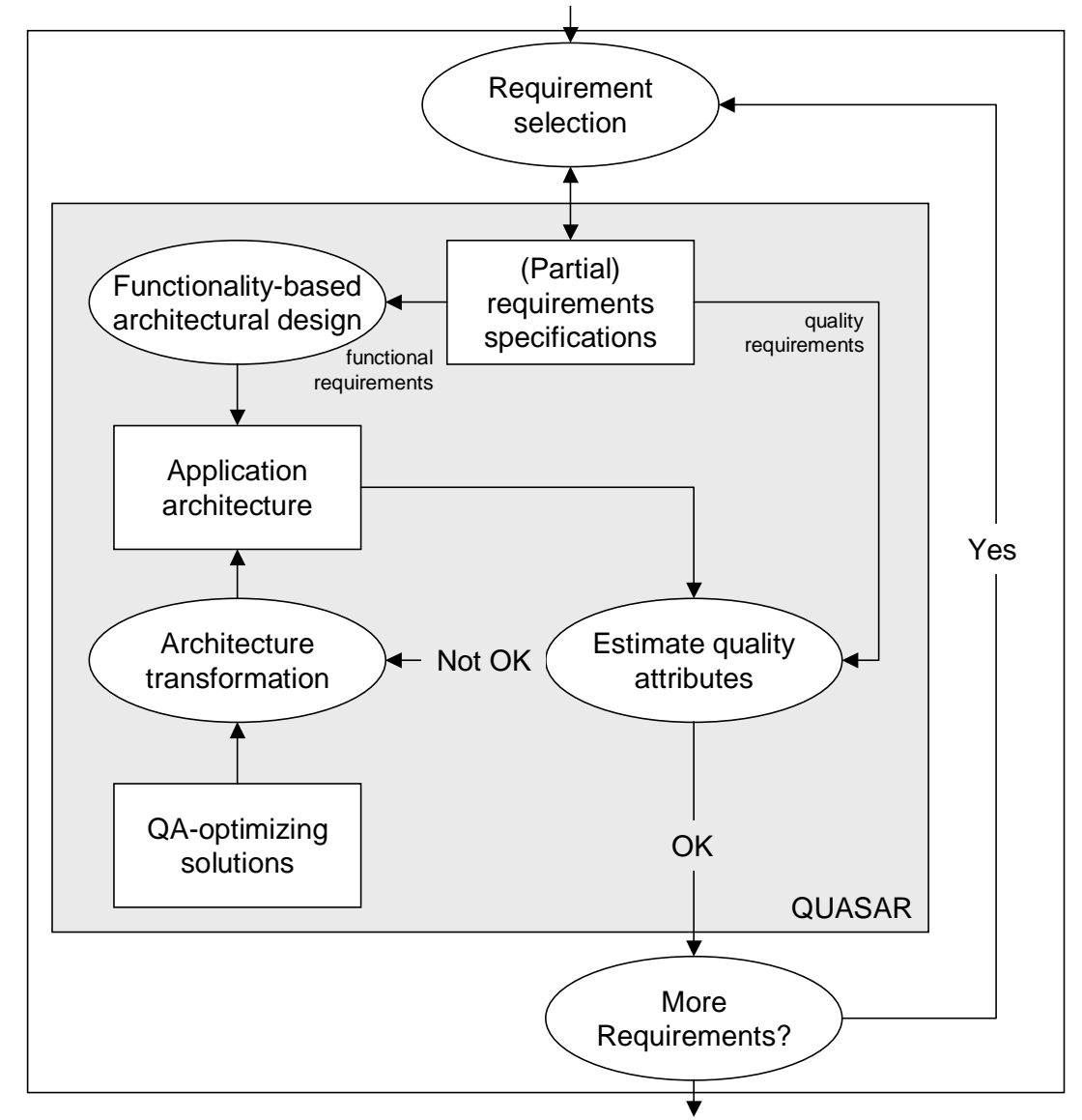

Abbildung 8-1: Design einer Software-Architektur (vgl. Bosch (2000))

Bosch (2000) bezeichnet das vorgeschlagene Modell als quality attribute-oriented software architecture design method (QUASAR). In einem ersten Schritt wird eine funktionale Architektur (von Bosch als „application architecture“ (vgl. Abbildung 8-1) bezeichnet) auf Grund der funktionalen Anforderungen und allgemeinen Anforderungen an eine Referenzarchitektur hergeleitet. Dabei wird ein iteratives Vorgehen vorgeschlagen. Vorab werden nur die wichtigsten Anforderungen ausgewählt und in die Applikationsarchitektur transformiert. Das Vorgehen wird mit einem umfassenderen Anforderungssatz solange wiederholt, bis sämtliche Anforderungen berücksichtigt worden sind. Für den jeweils berücksichtigten Anforderungssatz wird die technische Architektur hergeleitet. Dies indem anhand der funktionalen Architektur und den allgemeinen Überlegungen zu technischen Architekturen verschiedene technische Architekturen hergeleitet werden, welche mit den qualitativen (nicht-funktionalen) Anforderungen bewertet werden. 


\subsection{Anforderungen an die Architektur und an das IT-System für das PM}

Die Anforderungen an das IT-System für das PM wurden im Kapitel vier erarbeitet und sind hier zur Erinnerung noch einmal in der Tabelle 8-1 dargestellt:

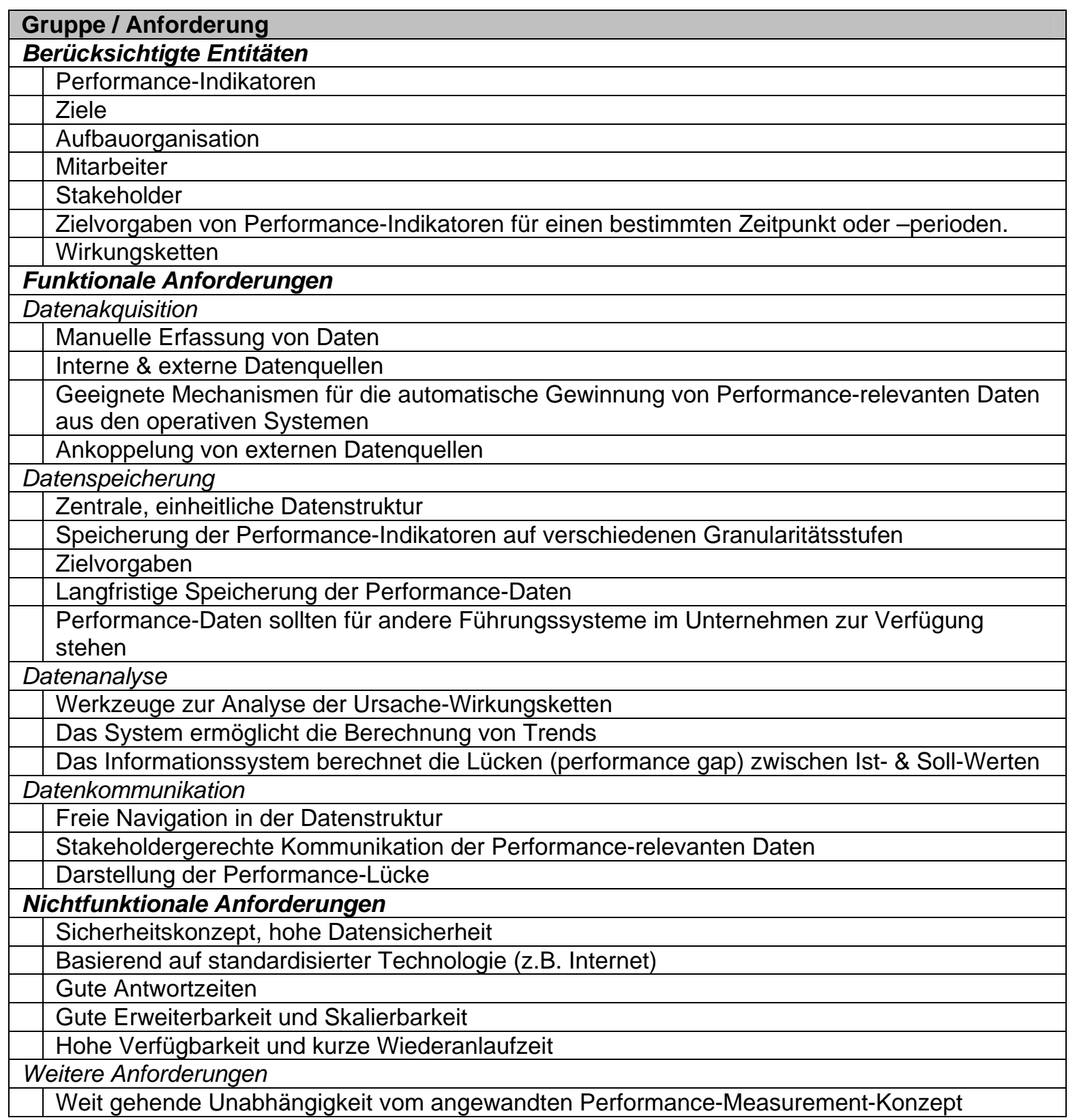

Tabelle 8-1: Anforderungen an das IT-System für das PM 
Die Architektur selbst sollte einer Reihe von Prinzipien genügen. Bauer und Günzel (2001) nennen im Kontext von Data Warehouses ${ }^{11}$ u.a. die Prinzipien:

- der Abstraktion (technische Details, Inhalte und spezifischer Zweck),

- der Hierarchisierung, d.h. es besteht eine Rangordnung zwischen den einzelnen Elementen des Systems und

- der Modularisierung, d.h. das Dekomponieren des Systems in einzelne Elemente.

\subsection{Funktionale Architektur für ein IT-System für das PM}

Aufbauend auf den funktionalen Anforderungen an ein IT-System für das PM und den Anforderungen an eine Architektur wird nachfolgend eine funktionsorientierte Architektur hergeleitet. Dabei werden die Funktionen des PMS einzelnen Komponenten der Architektur zugeordnet (vgl. Bauer und Günzel (2001)).

Bereits die Anforderungen von Tabelle 8-1 wurden in mehrere Kategorien aufgeteilt. Wichtige Gruppen, die differenziert werden, sind die Datenakquisition und die Datenspeicherung, sowie die Analyse und Kommunikation. Sie enthalten funktionale Anforderungen, die einen starken Zusammenhang aufweisen. Zudem besteht zwischen diesen Gruppen eine logische Abhängigkeit bezüglich des Datenflusses. Entsprechend werden daraus die drei funktionalen Hauptgruppen der Architektur gebildet (vgl. Abbildung 8-2). Die Kommunikation und Analyse werden dabei in der Hauptgruppe „Verwertung“ zusammengefasst.

11 Ein Data Warehouse ist eine integrierte, themenorientierte Datensammlung auf welche in der Regel nur lesend zugegriffen wird. Die darin enthaltenen Daten werden über eine längere Zeitperiode verfügbar gemacht (zum Begriff siehe Kapitel 9.2.2.). 


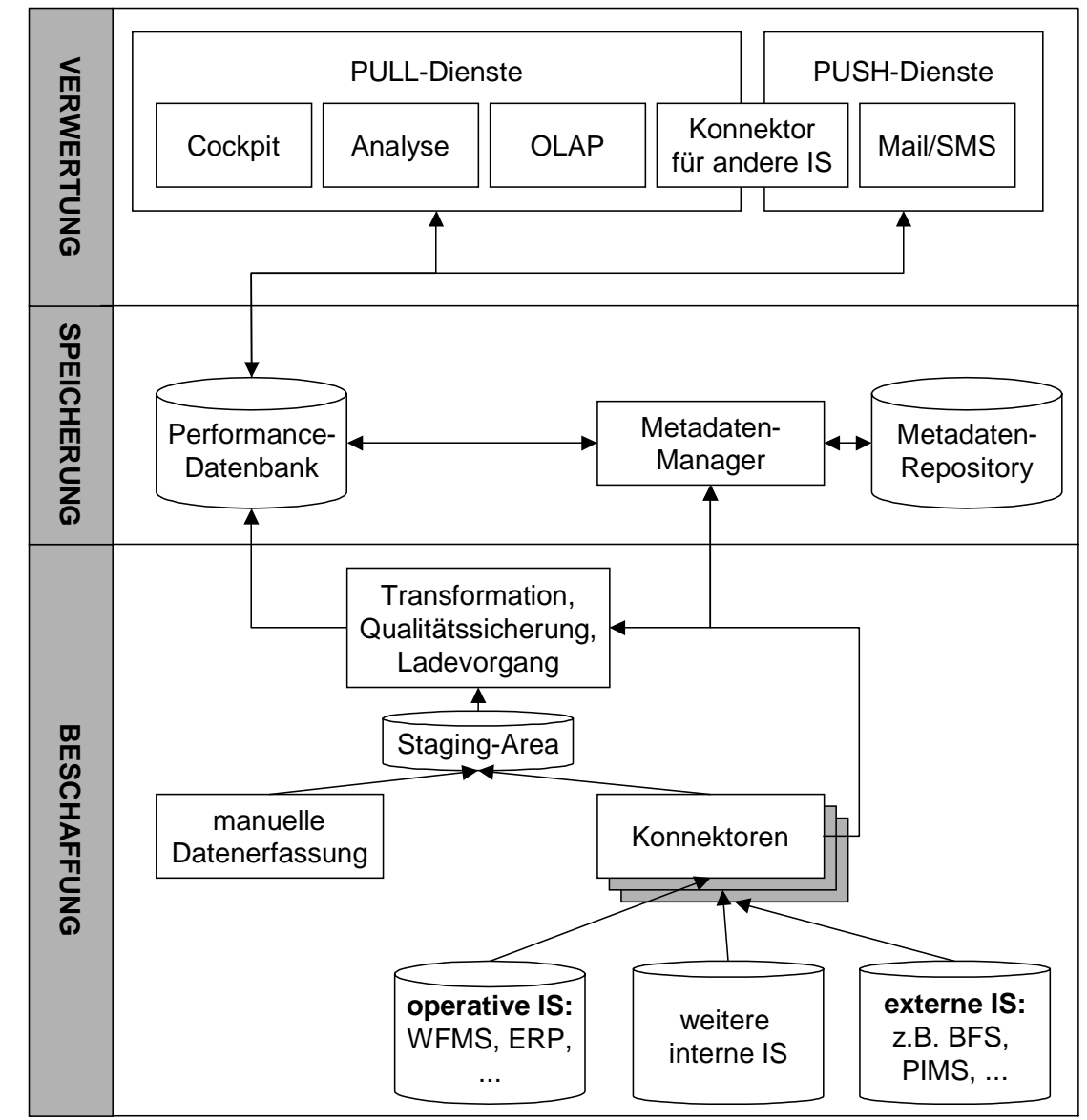

Abbildung 8-2: Funktionale Architektur eines IT-Systems für das PM

Innerhalb der Beschaffung wird die manuelle und automatische Aufnahme von internen und externen Daten abgebildet. Für die manuelle Datenerfassung wird eine entsprechende Funktionalität vorgesehen. Für die automatische Gewinnung von Daten wird die Funktion von so genannten Konnektoren verwendet. Das Ausgabeformat dieser Funktionseinheiten ist eindeutig definiert und muss zwingend respektiert werden. Für jedes unterschiedliche operative Informationssystem kommt ein eigener Konnektor zum Einsatz, der die Charakteristiken des Informationssystems berücksichtigt. Im Idealfall wird der Konnektor vom Hersteller der Datenquelle bereitgestellt, da dieser die internen Strukturen seines Informationssystems am besten kennt. Konnektoren können $\mathrm{zu}$ operativen Informationssystemen wie Workflow-Management-Systemen (WFMS; z.B. Lotus (2002), Fabasoft (2002), etc.), Enterprise-Ressource-Planning-Systemen (ERP, z.B. SAP (2002), Baan (2002), JDEdwards (2002), etc.), zu allgemeinen internen Informationssystemen oder zu externen Informationssystemen, wie z.B. Datenbanken des Bundesamtes für Statistik 
(vgl. BFS (2002)) oder der PIMS-Datenbank (vgl. SPI (2002)) bestehen (siehe Kapitel vier).

Die Daten aus beiden Gewinnungsarten werden in einen Zwischenspeicher, die Staging Area, geladen. Dieser Schritt ist notwendig, um die Integrität der Daten in der Performance-Datenbank sicherzustellen. Es muss sichergestellt werden, dass die Daten nur einmal in die Performance-Datenbank gelangen, deren Qualität ausreichend ist und die notwendigen Transformationsschritte ausgeführt werden. Diese Aufgaben werden von der Funktionseinheit „Transformation, Qualitätssicherung und Ladevorgang“ wahrgenommen.

Auf der Ebene der Speicherung der Performance-Daten wird zwischen Nutz- und Steuerungsdaten differenziert. Die Nutzdaten werden in der Performance-Datenbank gespeichert und enthalten die Performance-relevanten Daten. Die Steuerungsdaten enthalten die Ausprägungen der berücksichtigten Entitäten, d.h. die Aufbauorganisation, die Zielhierarchie und die Ziel-Werte sowie die berücksichtigten Stakeholder. Sie umfassen auch die berücksichtigten Auswertungsdimensionen. Sie werden im MetadatenRepository gespeichert. Die Bewirtschaftung des Metadaten-Repository erfolgt durch einen Metadaten-Manager. Dieser wird von den Konnektoren (bezüglich der zu berücksichtigenden Daten) und der Funktionseinheit für die Transformation, Qualitätssicherung und Ladevorgang herangezogen. Dies bedeutet, dass sowohl Informationen zu den operativen Quellsystemen (z.B. Datenbank-Schemata, Konnektionspfade, verwendete Protokolle, etc.) als auch dem ETL-Prozess (z.B. Transformationsregeln, Regeln zur Prüfung der Datenqualität, Frequenzen, etc.) im Metadaten-Repository gespeichert werden.

Es stellt sich die Frage, inwiefern es sinnvoll und realistisch ist, die manuelle Datenerfassung über die Staging-Area vorzunehmen. Die Überlegungen, die zu diesem Vorschlag führten, liegen in der Sicherung der Datenqualität und -integrität. Zudem müssen mit diesem Verfahren keine zusätzlichen Umsetzungsregeln und Plausibilitätstest bei der manuellen Erfassung vorgesehen werden. Vielmehr werden die Daten roh erfasst und dann mittels eines Stapelverarbeitungslaufes (Batchlaufs), sofern valid, in die Performance-Datenbank übernommen. Entsprechende Verfahren werden bei den ERPHerstellern für Systemkonsolidierungen, z.B. nach einer Fusion, angewandt (SAP untersagt ihren Kunden bspw. direkte Manipulationen auf der Datenbank. Bei Migrationen 
werden die Daten üblicherweise über die Standardfunktionen mittels Batchjobs eingelesen, um die Integrität zu sichern.).

Die Verwertung der Daten kann in einer ersten Stufe grob in Pull- und Push-Dienste unterteilt werden. Damit wird allgemein bezeichnet, ob die Information vom Informationslieferant, d.h. der Performance-Datenbank zum Informationsempfänger geschoben wird (Push) oder der Informationsempfänger die Daten beim Informationslieferant abholt (Pull). Im ersten Fall wird die Aktion vom Sender, im zweiten Fall vom Empfänger initiiert.

Innerhalb der Pull-Dienste werden drei Funktionen unterschieden:

- Das Cockpit erlaubt die Darstellung der Ist-, der Soll-Werte und der Performance-Lücke der relevanten Performance-Indikatoren für eine Person.

- Die Analyse erlaubt die Anwendung von verschiedenen analytischen Operationen auf den Performance-Daten, bspw. die Berechnung von Trendsoder die Untersuchung von Wirkungszusammenhängen verschiedener Indikatoren. Die Behandlung von statischen, d.h. vordefinierten Analysen wird in diesem Teilbereich behandelt, während die dynamischen Analysen in unten stehendem Punkt besprochen werden.

- OLAP (On-Line Analytical Processing) ist ein Ansatz, der die dynamische, multidimensionale Analyse von Daten bezeichnet, mit dem Ziel, neue oder unerwartete Beziehungen zwischen den Variablen zu erkennen (vgl. Bauer und Günzel (2001)). Es handelt sich, wie oben erwähnt, um einen Teilbereich der Analyse, der wegen der besonderen Bedeutung (siehe Anforderungen) extra besprochen wird. Mit einer spezialisierten Software - dem OLAP-Client besteht die Möglichkeit, frei innerhalb der Performance-Daten zu navigieren, um bspw. Ursachen für Abweichungen oder Wirkungszusammenhänge zu erkennen. Im Gegensatz zur statischen Darstellung eines Cockpits und der vordefinierten Funktionalität der Analysekomponente bestehen beim OLAPClient nur Grenzen hinsichtlich des Analysegegenstandes, den Performancerelevanten Daten und deren Beziehungen.

Neben den beschriebenen Pull-Diensten ist auch ein Push-Dienst denkbar. Beim Eintreten bestimmter zu definierender Bedingungen (z.B. dem Überschreiten des Toleranzwertes 
eines Performance-Indikators) wird das System selbst aktiv und informiert den Verantwortlichen. Dabei sind verschiedene Kommunikationskanäle, wie z.B. E-Mail oder Short Message Service (SMS) denkbar.

Die funktionale Architektur des IT-Systems für das PM bildet die Ausgangsbasis zur Bestimmung der technischen Architektur. Diese bestimmt sich auf Grund von nichtfunktionalen resp. qualitativen Anforderungen sowie den Vor- und Nachteilen von verschiedenen Architekturtypen.

\subsection{Typen von (Schichten)-Architekturen}

\subsubsection{Vor- und Nachteile von verteilten Systemen}

Bei den meisten heute verfügbaren Informationssystemen handelt es sich um verteilte Systeme. Der hier aufgezeigte Vorschlag für die technische Architektur sieht ebenfalls ein verteiltes System vor. Aus diesem Grunde sollen kurz einige Charakteristika verteilter Systeme diskutiert werden (vgl. Tabelle 8-2):

\begin{tabular}{|c|c|}
\hline 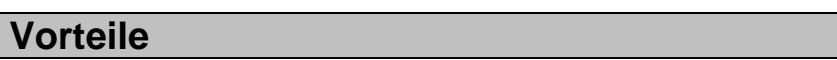 & \\
\hline $\begin{array}{l}\text { - Ressourcenteilung: gemeinsame Benutzung } \\
\text { verschiedener Ressourcen. } \\
\text { - Offenheit: das System ist erweiterbar mit } \\
\text { zusätzlichen Komponenten. } \\
\text { - Nebenläufigkeit: in einem verteilten System } \\
\text { können verschiedene Prozesse gleichzeitig } \\
\text { auf mehreren Systemteilen ablaufen. Diese } \\
\text { Prozesse können während ihrer normalen } \\
\text { Aktivität miteinander kommunizieren. Dies ist } \\
\text { jedoch nicht zwingend. } \\
\text { - Skalierbarkeit: Verteilte Systeme sind durch } \\
\text { Hinzufügen weiterer Ressourcen (z.B. } \\
\text { zusätzliche Rechner, Netzbandbreite, etc.) } \\
\text { skalierbar. } \\
\text { Fehlertoleranz: Durch das Vorhandensein } \\
\text { verschiedener Systeme, der Möglichkeit der } \\
\text { Replikation von Daten, besteht eine gewisse } \\
\text { Toleranz von verteilten Systemen gegenüber } \\
\text { Fehlern. } \\
\text { Transparenz ist die Eigenschaft, die die } \\
\text { verteilte Struktur des Systems für den } \\
\text { Benutzer unsichtbar macht. }\end{array}$ & $\begin{array}{l}\text { - Zugriffsschutz: Der } \\
\text { Zugriffsschutz ist aufwändiger } \\
\text { in der Realisierung, da mehrere } \\
\text { Systeme zusammenarbeiten. } \\
\text { - Verwaltbarkeit: Das } \\
\text { Zusammenspiel von } \\
\text { unterschiedlichen Teilsystemen } \\
\text { und die unerwarteten } \\
\text { Auswirkungen bei der } \\
\text { Zusammenarbeit erhöhen den } \\
\text { Aufwand. } \\
\text { - Unvorhersagbarkeit: Die } \\
\text { Vorhersagbarkeit hängt von der } \\
\text { allgemeinen Auslastung des } \\
\text { Systems, seiner Organisation } \\
\text { und der Netzbelastung ab. Das } \\
\text { Zusammenspiel der Faktoren } \\
\text { ist äusserst komplex und } \\
\text { schwer abschätzbar, was z.B. } \\
\text { die Vorhersagbarkeit der } \\
\text { Antwortzeit stark erschwert. }\end{array}$ \\
\hline
\end{tabular}

Tabelle 8-2: Vor- und Nachteile von verteilten Systemen (vgl. Coulouris und Dollimore (1994) und Noack et al. (2000)) 
Ein grundlegendes Muster, welches bei verteilten Systemen angewendet wird, ist die Zerlegung der Anwendung in überschaubare Einheiten auf Basis einer Schichtenarchitektur. Jede Schicht abstrahiert einen definierten Problembereich und besitzt dokumentierte Schnittstellen. Eine hierarchische Anordnung Gewähr leistet, dass eine höherliegende Schicht mit Hilfe der Dienste realisiert werden kann, welche die tieferliegenden Dienste bereitstellen. Bei der Betrachtung von Software-Architekturen muss zwischen logischen und physischen Softwareschichten unterschieden werden. Während die logischen Softwareschichten die Modularisierung einer Anwendung darstellen, repräsentieren physische Softwareschichten die Einheiten, die auf unterschiedliche Rechnerklassen verteilt werden können (vgl. Noack et al. (2000)). Nachfolgend erfolgt eine Beschränkung der Diskussion auf physische Softwareschichten. In der Regel werden drei Typen von Schichten differenziert: Präsentation, Anwendungslogik und Datenhaltung.

\subsubsection{Ein-, Zwei- und Drei-Schichten-Architekturen}

Grundsätzlich ist es denkbar, sämtliche Komponenten der funktionalen Architektur auf einem einzigen System zu implementieren (vgl. Abbildung 8-3). In diesem Fall spricht man von einer Ein-Schichten-Architektur (one-tier architecture).

Bei der Zwei-Schichten-Architektur (two-tier architecture) werden die Komponenten auf zwei Schichten verteilt. Betrachtet man die drei Grundkomponenten Präsentation, Anwendungslogik und Datenhaltung, so ist eine Aufteilung der Präsentationsschicht auf den Client und die Anwendungslogik sowie die Datenhaltung auf dem Server denkbar. Die Hauptlast liegt beim Server. Die Verbindung zwischen Datenhaltung und Anwendungslogik ist eng gekoppelt, während diejenige zwischen Anwendungslogik und Präsentationsschicht lose gekoppelt ist und nur beschränkte Volumen transferieren muss. Die Disposition entspricht einem Zugriff über das Internet mittels WWW-Technologie. Die Vorteile liegen darin, dass keine oder nur wenig Software auf den Clients installiert werden muss. Nachteilig ist, dass der Server rasch zum Flaschenhals werden kann. Befinden sich die Präsentationsschicht und die Anwendungslogik auf der Client-Seite und nur mehr die Datenhaltung auf dem Server, so wird zwar der Server entlastet, jedoch steigen die zu transferierenden Datenvolumen stark an. Neben der in der Regel aufwändig zu installierenden Software auf der Client-Seite ist das Augenmerk auf die 
Anfragestrategie an die Datenhaltungskomponente zu legen, um die Transfervolumen zu minimieren (vgl. Abbildung 8-3).

Bei einer Drei-Schicht-Architektur werden die drei erwähnten Komponenten auf unterschiedlichen Ebenen gehalten. Wie eingangs erwähnt, resultieren hier Vorteile im Hinblick auf die Wartbarkeit und Erweiterbarkeit. Zudem können solche Systeme durch redundantes Auslegen einzelner Schicht-Komponenten fehlertolerant und skalierbar gemacht werden. Nachteilig sind die höheren Aufwendungen für den erstmaligen Aufbau einer entsprechenden Architektur anzuführen (vgl. Abbildung 8-3).

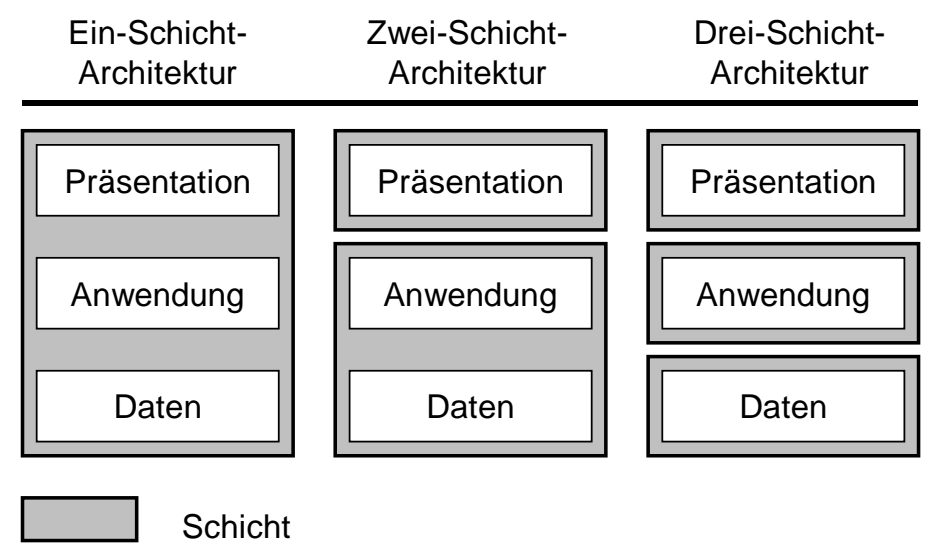

\section{Abbildung 8-3: Schichten-Architekturen}

Als viel diskutierte Variante einer mindestens Zwei-Schichten-Architektur sind die webbasierten Architekturen zu bezeichnen. Sie gewinnen in der Praxis zunehmend an Bedeutung, weshalb dieser Typ nachfolgend noch speziell besprochen werden soll.

\subsubsection{Webbasierte Architektur}

Bei webbasierten Architekturen handelt es sich mindestens um Zwei-SchichtenArchitekturen. Bezüglich der verwendeten Bausteine einer Web-Architektur kann differenziert werden nach Systemkomponenten, die standardmässig gewisse Dienste zur Verfügung stellen und Komponenten, welche individuell für den jeweiligen Anwendungszweck entwickelt werden. $\mathrm{Zu}$ den Systemkomponenten gehören etwa die Browser, die Web-Server (z.B. Apache, Microsoft Internet Information Server), Transaction-Processing-Monitore und Datenbank-Management-Systeme (DBMS). Die zweite Gruppe umfasst den Präsentationsbaustein der Hypertext-Markup-Language- 
Dokumente (HTML) sowie Programmbausteine wie etwa Java-Applets (vgl. Noack et al. (2000)).

Für die Kommunikation zwischen den einzelnen Komponenten steht eine Reihe von Protokollen und Schnittstellen zur Verfügung. Hyper Text Transfer Protocol (HTTP) ist das Standardprotokoll zwischen Web-Client und -Server. Common Gateway Interface (CG), Servlet Application Programming Interfaces (Servlet-APIs) und Active Server Pages (ASP) sind drei bekannte Schnittstellen. CGI ist Bestandteil der HTTP-Spezifikation und hat sich als Standard für Internet-Anwendungen etabliert. Er verfügt jedoch über eine schlechte Skalierbarkeit. Für den Zugriff auf Daten in standardisierter Form ist Structured Query Language (SQL), Open Data Base Connectivity (ODBC) und Java Data Base Connecitivity (JDBC) von Bedeutung. (vgl. Noack et al. (2000))

Die Bausteine einer Web-Architektur zeichnen sich insbesondere auf der Client-Seite durch ein hohes Mass an Unabhängigkeit vom verwendeten Betriebssystem aus. Die Verwendung von standardisierten Techniken erlaubt eine wesentliche Reduktion bei der Gestaltung der Benutzer-Interfaces sowie der Wartungsaufwendungen auf der Client-Seite. Demgegenüber stehen Nachteile wie die Sicherheit und die Verfügbarkeit der Übertragung. (vgl. Bauer und Günzel (2001))

Neuere Webapplikations-Architekturen unterstützen die Realisierung von Anwendungen auf der Basis von Mehr-Schichten-Architekturen, die eine explizite Trennung der drei Schichten (1) Benutzeroberfläche, (2) Anwendungslogik und (3) Datenhaltung unterstützen (vgl. bspw. Weitz (2002)).

\subsection{Bedeutung von nicht-funktionalen Anforderungen für die physische Architektur}

Nicht-funktionale Anforderungen (non-functional requirements) werden von einigen Autoren auch als geforderte Randbedingungen (required constraints) bezeichnet (vgl. Hatley et al. (1999)). Nicht-funktionale Anforderungen (NFA) sind Bedingungen, die an die Dienste bzw. Leistungen eines Systems gestellt werden. NFA beschreiben Systemeigenschaften und Randbedingungen, unter welchen das System arbeitet. Sie können eingeteilt werden in: (1) Eigenschaften (properties, qualities): Beispiele: Zuverlässigkeit, Erweiterbarkeit, Portabilität; und (2) Randbedingungen (constraints, 
resources): Beispiele: max. Speicherbedarf, max. Antwortzeiten, Einsatz gegebener Hardware.

Die expliziten nicht-funktionalen Anforderungen an ein IT-System für das PM umfassen u.a. ein Sicherheitskonzept und den Einsatz einer Web-basierten Technologie (vgl. Kapitel 8.2). Ersteres leitet sich aus der Art der Informationen ab, die durchaus sensitiven Charakter haben können. Zweiteres stammt aus der Anforderung, einen breiten Benutzerkreis einzubeziehen, der u.U. auch ausserhalb der Organisation steht. Dabei kann die zweite Anforderung als Randbedingung aufgefasst werden. Eine Schichten-Architektur erlaubt, sensitive Inhalte in einer unteren Schicht zu halten und diese mit einem hohen Schutz auszustatten. Bei hohen Sicherheitsanforderungen sollte die Architektur so ausgelegt werden, dass die relevanten Funktionen in einem oder möglichst wenigen Teilsystemen abgedeckt werden, um die Kosten der Systemvalidierung zu beschränken.

Die Leistungsfähigkeit des Systems hat insofern einen hohen Stellenwert, als dass eine grosse Menge an Performance-relevanten Daten verwaltet werden muss, und die interaktive Analyse der Daten (OLAP-Client) gute Antwortzeiten verlangt. Zudem ist von einem grösseren Benutzerkreis auszugehen. Hohe Performance wird klassisch mit möglichst wenigen Teilsystemen erreicht: Je kleiner die Anzahl der Teilsysteme, desto kleiner ist die notwendige Kommunikation zwischen diesen. Zu berücksichtigen ist jedoch, dass auch die Leistungsfähigkeit eines einzelnen Systems beschränkt ist, und somit ein Zielkonflikt auftreten kann (vgl. Sommerville (2001)). Auf der anderen Seite gehen neuere Entwicklungen gerade in die gegenteilige Richtung. Durch den massiven Einsatz von parallel arbeitenden Rechnern werden sehr hohe Leistungen erreicht. Allgemein lässt sich somit ableiten, dass die Leistungsfähigkeit eines Systems durch das schwächste Element determiniert wird, was für ein Design von ausgewogenen Kompositionen spricht.

Das IT-System für das PM bezieht die wesentlichen Daten aus den operativen Informationssystemen der Organisation sowie externen Systemen. Diese Systeme sind erfahrungsgemäss einem ständigen Wandel unterworfen. Dies ist bei der Wartbarkeit des Systems entsprechend zu berücksichtigen. Kleine, unabhängige Teilsysteme begünstigen die Wartbarkeit eines Systems. Datengenerierung und -auswertung sollten getrennt und gemeinsame Datenstrukturen vermieden werden.

Die notwendige Verfügbarkeit des Systems ist abhängig vom Kontext des konkreten Einsatzes. Im Allgemeinen werden eine Verfügbarkeit während der Arbeitszeiten und 
gewisse Unterbrechungen für Wartungsarbeiten vertretbar sein. Eine erhöhte Verfügbarkeit wird in der Regel mit redundanten Teilsystemen erreicht.

Die vorgängig diskutierten nicht-funktionalen Anforderungen können lediglich als allgemeine Hinweise für die Bestimmung der Architektur dienen. Im Einzelfall sind zahlreiche Zusatzinformationen, wie der Detaillierungsgrad der Performance-Daten, die Transformationsregeln, die Anzahl der Benutzer und vieles mehr zu berücksichtigen. Zudem ist zu beachten, dass die Architektur der Entwicklung eines PMS über die Zeit Rechnung tragen muss (vgl. Kimball et al. (1998)).

\subsection{Vorschlag einer physischen Architektur für das IT-System für das Performance Measurement}

Nachfolgend soll, basierend auf der funktionalen Architektur eines IT-Systems für das PM, den diskutierten Schicht-Architektur-Typen und den nicht-funktionalen Anforderungen, eine physische Architektur hergeleitet werden.

In einem ersten Schritt werden sich die Überlegungen auf die physische Organisation des OLAP-Clients und der Performance-Datenbank konzentrieren. Hierbei handelt es sich um eine Data-Warehouse-Konzept. Die nachfolgend diskutierten Szenarien gehen von einem Data Warehouse (DWH) aus, welches auf einem relationalen Datenbanksystem basiert (vgl. Bauer und Günzel (2001)). In Abbildung 8-4 sind drei mögliche Varianten dargestellt.

Variante eins und zwei stellen Zwei-Schichten-Architekturen dar. In der ersten Variante erfolgt die gesamte Berechnung und Aufbereitung auf der Client-Seite. Dies umfasst die Benutzerschnittstelle, die Applikationslogik, die Analyse-Funktionen, die SQLGenerierung, die Kommunikation im Allgemeinen und die Kommunikation mit der Datenbank im Speziellen. Die Datenbank führt lediglich den empfangenen SQL-Befehl aus und sendet die Ergebnisrelation an den Client zur weiteren Bearbeitung. Dadurch, dass die Berechnungen auf der Client-Seite erfolgen, ist das SQL-Statement in der Regel einfach. Nachteilig kann sich die grosse Ergebnis-Datenmenge auswirken, welche über das Netzwerk transportiert werden muss. 
In der zweiten Variante werden die analytischen Funktionen der relationalen Datenbank benutzt (Verwendung der Stored Procedures ${ }^{12}$ des DBMS). Mit dem Absetzen einer komplexen SQL-Anfrage werden die Berechnungen bereits zu einem grossen Teil von der Datenbank ausgeführt. Dies führt zu einer kleineren Ergebnis-Relation, welche über das Netzwerk transportiert werden muss. Zudem können die bereits im DBMS implementierten Funktionen benutzt werden und müssen Client-seitig nicht nochmals umgesetzt werden.

Variante drei zeigt eine Drei-Schichten-Architektur: Neben Client und Datenbank-Server wird hier ein Server zwischengeschaltet, der die Dekomposition und Aufbereitung der multidimensionalen Datenstruktur übernimmt. Der Client kommuniziert mit einer spezialisierten, für multidimensionale Datenbankanfragen ausgerichteten Sprache mit dem Server. Dieser setzt die spezialisierte Anfrage in eine komplexe Datenbankabfrage um, welche vom Datenbank-Server verarbeitet wird. Das Ergebnis in Form einer gezielten Ergebnismenge wird auf dem Server aufbereitet und zur Präsentation an den Client übermittelt. kann. Im Allgemeinen werden zwei Typen unterschieden: Datenbank-Prozeduren und Trigger: Datenbank-Prozeduren können direkt von einem Anwendungsprozess gleich einer SQL-Anweisung aufgerufen werden. Sie werden eingesetzt (1) zur Verbesserung der Effizienz, da eine DB-Prozedur durch den Datenbank-Server abgearbeitet wird, wodurch der Kommunikationsaufwand verringert wird, (2) zur Vereinfachung der Programmierung, da komplexe SQL-Anweisungsfolgen durch einen einzigen Aufruf einer DB-Prozedur ersetzt werden und aus mehreren Anwendungsprogrammen heraus zentral genutzt werden können und (3) zur Vereinfachung der Privilegien, da es nicht notwendig ist, neben dem Aufrufprivileg einer DB-Prozedur auch noch Privilegien für die angesprochenen Datenbankobjekte zu erteilen. Trigger sind demgegenüber immer an eine bestimmte Tabelle gebunden. Sie werden implizit von der Datenbank im Anschluss an INSERT-, UPDATE- oder DELETE- Anweisungen aufgerufen. Sie sind sinnvoll um Information in anderen Tabellen konsistent zu halten. (vgl. Petkovic (2001)) 


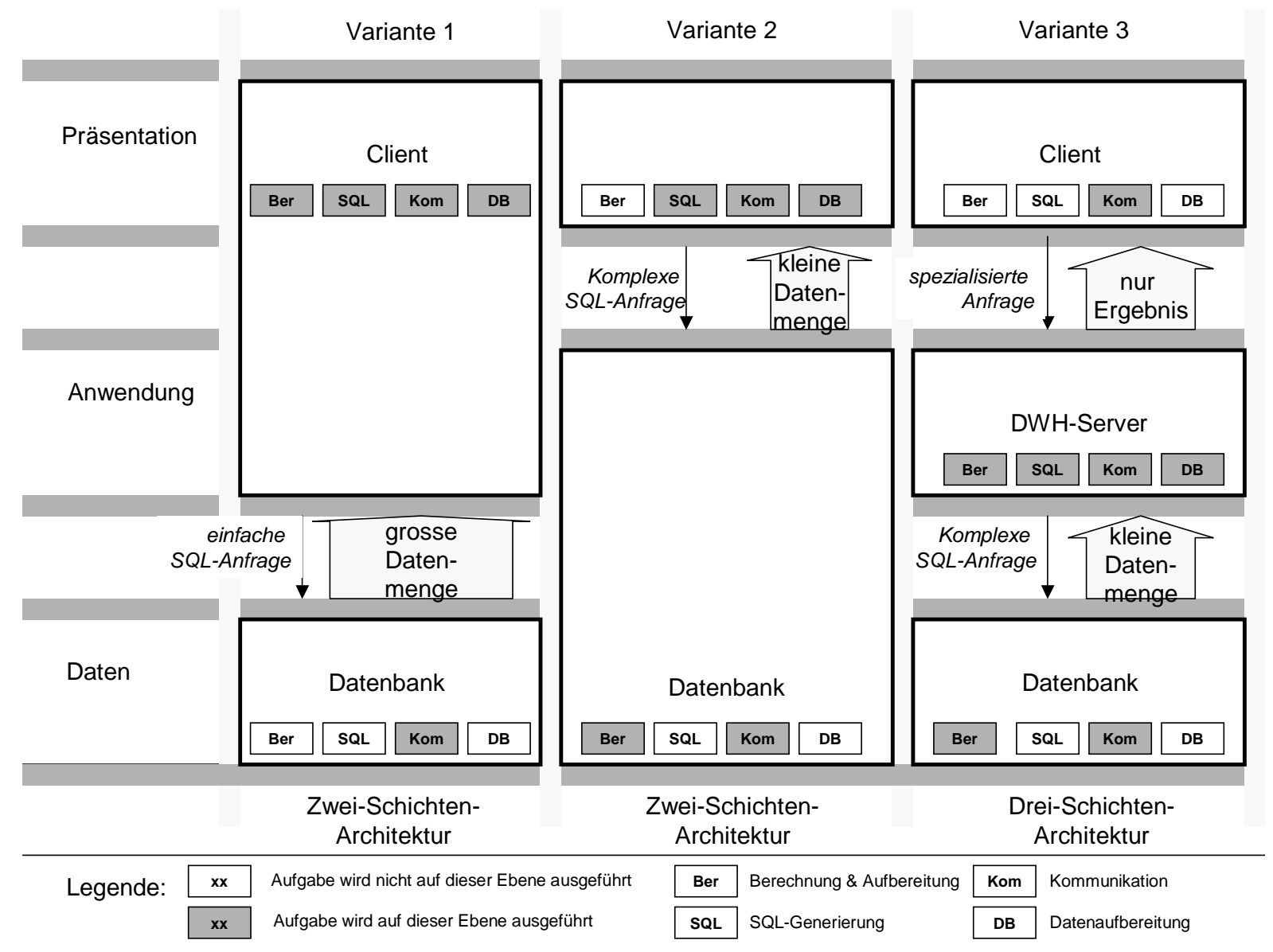

Abbildung 8-4: Drei Architekturvarianten (in Anlehnung an Kimball et al. (1998) und Kurz (1999))

Nachfolgend werden die drei Varianten bezüglich der besprochenen nicht-funktionalen Anforderungen diskutiert und in Tabelle 8-3 einander gegenübergestellt:

- $\quad$ Die Webfähigkeit wird von Variante eins schlecht unterstützt, während die Variante zwei und drei hierzu besser geeignet sind. Sie erlauben, den Client als Präsentationsschicht zu verwenden, was der Webfähigkeit entgegenkommt.

- $\quad$ Eine Massierung der dezentralen (Client)-Funktionalitäten wirkt sich negativ auf das Sicherheitsniveau aus. Umgekehrt unterstützt eine starke Zentralisierung das Sicherheitsniveau. Variante drei stellt hierbei einen Kompromiss dar. 
- $\quad$ Das Antwortzeitverhalten wird durch verschiedene Faktoren beeinflusst: Die Belastung der Server und des Netzwerkes sowie die Anzahl der gleichzeitigen Benutzer. Variante eins führt $\mathrm{zu}$ einer hohen Netzbelastung, Variante zwei zu einer starken Belastung des DatenbankServers. Variante drei stellt demgegenüber eine ausgewogene Variante dar. Zudem ist eine Skalierung bei grossen Benutzerzahlen denkbar.

- Die Modifizierbarkeit (Wartbarkeit, Flexibilität gegenüber neuen Anforderungen) wird durch Modularisierung positiv beeinflusst. Variante eins konzentriert die Funktionalität im Client, während Variante zwei diese zwischen Client und Datenbank-Server splittet. Variante drei verteilt die Funktionalität auf drei Ebenen.

- $\quad$ Die Verfügbarkeit eines Systems wird durch eine steigende Anzahl der Teilsysteme negativ beeinflusst, da bei gleicher Fehlerwahrscheinlichkeit je Teilsystem die Gesamtwahrscheinlichkeit eines Fehlers steigt (vgl. Sommerville (2001)).

\begin{tabular}{|l|c|c|c|}
\hline Kriterium & Variante 1 & Variante 2 & Variante 3 \\
\hline Randbedingung: web-fähige Architektur & 1 & 3 & 5 \\
\hline Sicherheitsniveau & 1 & 5 & 3 \\
\hline Antwortzeitverhalten & 1 & 1 & 5 \\
\hline Wartbarkeit/Modifizierbarkeit & 1 & 3 & 3 \\
\hline Verfügbarkeit & 5 & 3 & 1 \\
\hline Summe & $\mathbf{9}$ & $\mathbf{1 5}$ & $\mathbf{1 7}$ \\
\hline Legende: 1= negative Wirkung; 2=eher negativ; 3= neutral; 4=eher positiv; 5=positiv \\
\hline
\end{tabular}

Tabelle 8-3: Bewertung der drei Architekturoptionen anhand der nicht-funktionalen Anforderungen

Gemäss der erfolgten Bewertung wird die dritte Variante weiter verfolgt, da sie die nichtfunktionalen Anforderungen insgesamt am besten erfüllt. Der bis anhin isolierte Bereich soll nun in die Gesamtarchitektur eingebettet werden, die sich auf Grund der funktionalen Architektur ableiten lässt. 


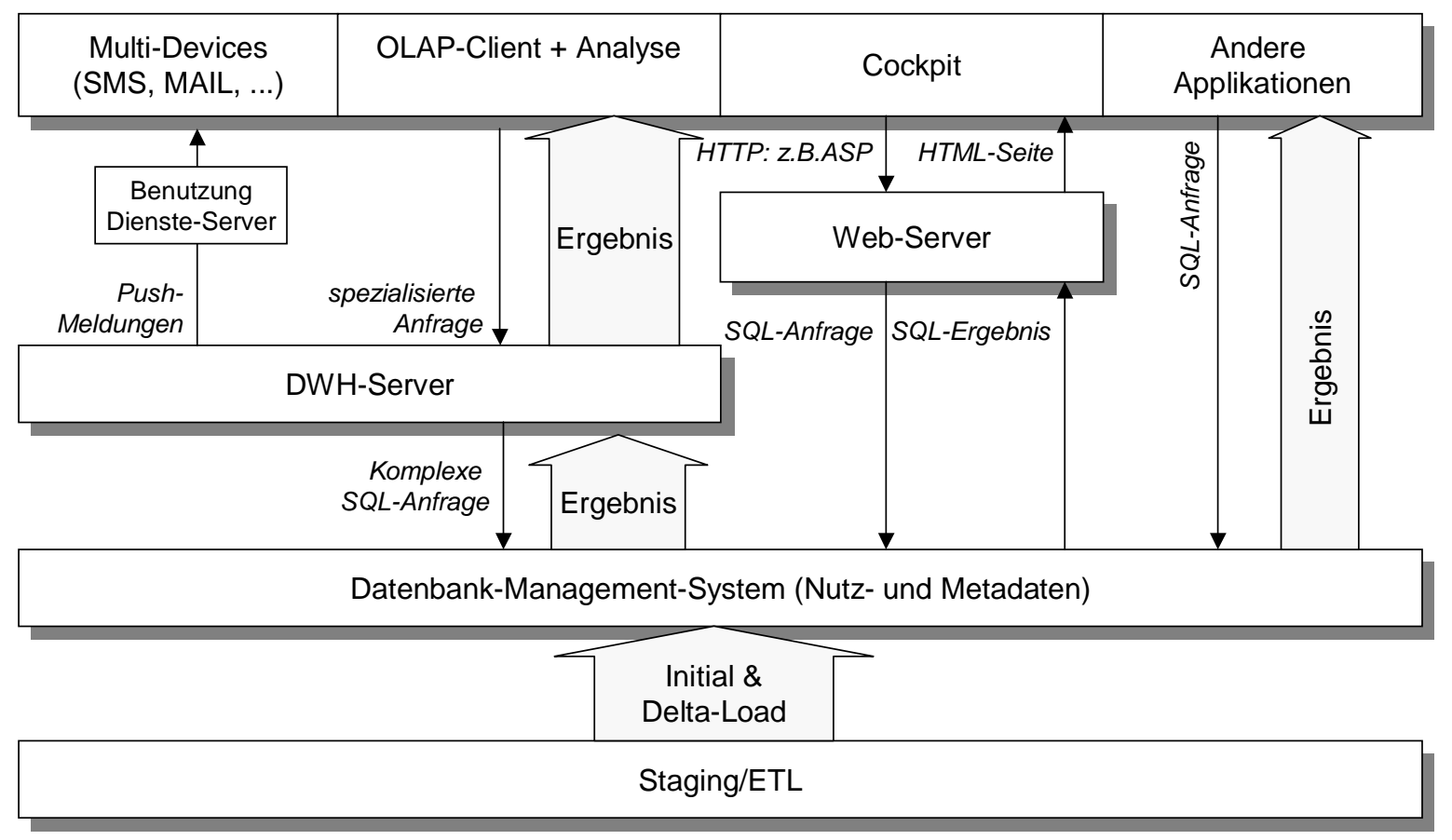

\section{Abbildung 8-5: Physische Drei-Schichten-Architektur eines IT-Systems für das Performance Measurement}

Abbildung 8-5 zeigt eine mögliche physische Gesamtarchitektur des IT-Systems für das PM. Neben der bereits besprochenen Teil-Architektur ist die Ebene für die Datenakquisition (ETL) und Zwischenspeicherung (Staging) erkennbar. Aus diesem Bereich werden die Daten aus den vorgelagerten Systemen periodisch in die PerformanceDatenbank eingespiesen. Neben dem OLAP-Client - der bereits besprochen wurde - ist zudem eine einfache SQL-Schnittstelle für andere Applikationen, ein Web-Server zur Aufbereitung der Cockpit-Daten und ein SMS-Gateway erkennbar. Damit kann die funktionale Architektur vollständig abgebildet werden.

Nach detaillierten Betrachtungen zum Entwurf des multidimensionalen Datenmodells im nächsten Kapitel, wird die vorgeschlagene Architektur im zehnten Kapitel mittels eines Prototypen validiert. 


\section{Prozess für den Entwurf eines multidimensionalen Datenmodells zur Verwaltung von Performance-Daten}

\subsection{Einleitung}

Ziel dieses Kapitels ist es, die Bestimmung des Datenmodells für ein IT-gestütztes Performance-Measurement-Systems herzuleiten. Dabei werden die in der Literatur besprochenen Modellierungsansätze auf ihre Eignung für Performance-MeasurementDaten hin untersucht. Mangels geeigneter Ansätze wird ein eigener, für die spezifische Problemstellung optimierter Ansatz entwickelt und an einem Beispiel illustriert.

\subsection{Bestimmung der Datenstruktur eines IT-gestützten Performance-Measurement- Systems}

\subsubsection{Anforderungen an die Datenstruktur}

Im vierten Kapitel wurden die Anforderungen an ein IT-gestütztes PerformanceMeasurement-System hergeleitet. Unten stehend sind in Tabelle 9-1 die für die Datenstruktur relevanten Anforderungen dargestellt.

- zentrale, einheitliche Datenstruktur

- Speicherung der Performance-Indikatoren auf verschiedenen Granularitätsstufen

- Langfristige Speicherung der Performance-Daten

- Freie Navigation in der Datenstruktur

- Stakeholdergerechte Kommunikation der Performance-relevanten Daten

- Darstellung der Performance-Lücke

- Kurze Antwortzeiten

- Hohe Datensicherheit

- Gute Erweiterbarkeit und Skalierbarkeit

- Hohe Verfügbarkeit und kurze Wiederanlaufzeit

Tabelle 9-1: Anforderungen an ein Datenmodell für das Performance Measurement

Nachfolgend wird untersucht, welcher Grundtypus von Datenmodell den erwähnten Anforderungen am besten genügt. 


\subsubsection{OLTP und OLAP als Grundtypen}

Unterschieden werden in der Regel so genannte Online Transaction Processing (OLTP) und Online Analytical Processing (OLAP) Lösungen. Erstere können den transaktionsorientierten, operativen Informationssystemen zugeordnet werden. Auf den Daten werden sowohl Lese-, Schreib- als auch Löschvorgänge ausgeführt. Die Daten sind in der Regel nur für den aktuellen Moment gültig. Zweitere können den analytischen Informationssystemen zugeordnet werden. Auf die Daten wird in der Regel nur lesend zugegriffen, und diese werden über die Zeit historisiert.

Tabelle 9-2 zeigt wichtige Charakteristiken von OLTP- und OLAP-Lösungen.

\begin{tabular}{|l|l|}
\hline OLTP & OLAP \\
\hline Daten lesen, schreiben, löschen & Meist nur lesender Zugriff \\
\hline Enthält aktuelle Daten & Daten werden historisiert \\
\hline $\begin{array}{l}\text { i.d.R. wenig übersichtlich und } \\
\text { benutzerfreundlich }\end{array}$ & Übersichtlich \\
\hline Speichert jede Transaktion & $\begin{array}{l}\text { Enthält verdichtete und nicht-verdichtete } \\
\text { Daten }\end{array}$ \\
\hline i.d.R. viele Benutzer & i.d.R. wenige Benutzer \\
\hline
\end{tabular}

Tabelle 9-2: Charakterisierung von OLTP- und OLAP-Informationssystemen

Analytische Systeme basieren häufig auf einem Data Warehouse. Inmon (1996) definiert ein Data Warehouse als „a subject oriented, integrated, non-volatile, and time-variant collection of data in support of management's decisions. “

Nach Inmon (1996) sind vier Eigenschaften charakteristisch für ein Data Warehouse:

- $\quad$ Subjekt-Orientierung (subject oriented): Die Daten werden nicht mit dem Zweck der Erfüllung einer Aufgabe, sondern nach einem eigenen Anwendungsziel hin, modelliert.

- Integrierte Datenbasis (integrated): Ziel ist es, die Daten von verschiedenen Informationssystemen bezüglich eines Themas in einer integrierten Datenbasis zusammenzufassen.

- $\quad$ Nicht-Flüchtig (non-volatile): Die Datenbasis ist bezüglich jedes Zeitpunktes als stabil zu betrachten. Daten, die einmal ins Data Warehouse eingebracht wurden, bleiben stabil. Der Zugriff erfolgt nur lesend. 
- $\quad$ Historisch (time-variant): Die Organisation der Daten ist so angelegt, dass Vergleiche über die Zeit möglich sind.

Die Ausführungen zu den vier charakteristischen Eigenschaften eines Data Warehouses und die Charakterisierung von OLTP- und OLAP-Systemen verglichen mit den Anforderungen, die an ein IT-System für das Performance Measurement gestellt werden, machen deutlich, dass ein solches System als ein Analytisches, welches auf einem Data Warehouse basiert, begriffen werden muss.

\subsubsection{Elemente eines Data Warehouses}

Auf der Datenstrukturebene werden OLAP-Lösungen nach Codd et al. (1993) multidimensional ausgelegt. Begründungen hierfür sind die effizientere (u.a. Antwortzeitverhalten) und einfachere Art der Datenauswertung. Durch die dem Verständnis der Anwender angepasste Datenstruktur können diese selbst einfache Ad-hoc Auswertungen durchführen. In traditionellen OLTP-Systemen haben Anwender ebenfalls multidimensionale Analysen durchgeführt. Als Beispiel sei eine Produkterechnung nach Gruppen und einzelnen Produkten, nach der Zeit und einzelnen Kostenpositionen (Verkaufspreis, Erlösminderung, Beschaffungskosten, etc.) aufgeführt. Diese Auswertungen sind jedoch bei traditionellen Systemen mit erheblichen Antwortzeiten verbunden und die Flexibilität der Analysen ist stark eingeschränkt.

Bei Data Warehouses werden zwei Kernelemente differenziert. Einerseits die Entscheidungsobjekte, welche die in der Regel als Mengen oder Wertgrössen auftreten (siehe Beispiel oben: Verkaufspreis, Erlösminderung, Beschaffungskosten, etc.). Sie werden als Kennzahlen, Variablen, Fakten, Facts, Measures oder sogar Measured Facts bezeichnet.

Andererseits wir von Dimensionen gesprochen, welche die Kennzahlen nach gemeinsamen Begriffen zusammenfassen. Jede Dimension besteht aus Elementen, die zu einem Typ gehören (oder einem Oberbegriff zugeordnet werden können). Tag, Monat, Jahr sind solche Elemente der Dimension Zeit. Die einzelnen Elemente stehen häufig in einem hierarchischen Verhältnis.

Die Datenanalyse geschieht durch Isolierung einzelner „Schichten“ aus dem Datenpool. Slice (Schneiden) bezeichnet die Selektion eines Ausschnittes aus einer Dimension unter 
Beibehaltung der übrigen Dimensionen. Bei dem erwähnten Würfel würde ein Slice auf das Jahr 2002 die Umsätze je Produkt und die Deckungsbeitragsrechnungsstruktur in Tabellenform darstellen (vgl. Abbildung 9-1). Dice bezeichnet das Drehen, Kippen des Würfels, d.h. es wird eine Dimension rotiert (vgl. Schinzer (1999)).

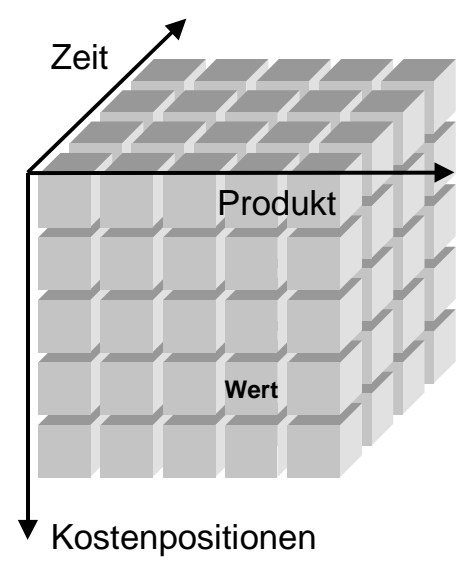

Fakten

- Wert

Dimensionen

- Zeit

- Produkt

- Kostenposition

\section{Abbildung 9-1: Darstellung der multidimensionalen Datenstruktur als Würfel}

Eine Dimension kann unter Umständen nicht nur in ihre einzelnen Elemente (z.B. das Datum 01.01.2002, 02. 01.2002, etc.), sondern ebenfalls in Hierarchiestufen dargestellt werden (z.B. Jahr - Monat - Woche - Tag). Entlang diesen auch als Aggregationspfad resp. Konsolidierungspfad bezeichneten Ebenen kann der Anwender navigieren. Der Sprung auf eine höhere Detaillierungsebene (z.B. vom Jahr 2002 auf die einzelnen Monate des Jahres 2002) wird als Drill Down, der umgekehrte Vorgang als Roll Up bezeichnet.

\subsubsection{Ebenen des Data Warehouse Design}

In Anlehnung an den Datenbankentwurf werden für den Entwurf von Data Warehouses die folgenden drei Ebenen unterschieden: (1) Semantisches Design, (2) Logisches Design, (3) Physisches Design. Abbildung 9-2 illustriert die drei Ebenen mit einem Beispiel aus dem Data-Warehouse-Bereich. Auf der semantischen Ebene kann bspw. ein multidimensionales Entity-Relationship-Modell (ME/R) erstellt werden. Das semantische Modell kann wahlweise mit einem relationalen oder einem multidimensionalen Modell auf der logischen Ebene repräsentiert werden. In Abhängigkeit davon erfolgt die physische Implementierung als Relational-OLAP (ROLAP) oder als Multidimensional-OLAP (MOLAP). Es ist zu beachten, dass in der Literatur Unterschiede bestehen bezüglich der Einordnung der Begriffe des konzeptionellen Schemas und des semantischen Modells. Die 216 
meisten Autoren verwenden die beiden Begriffe synonym (vgl. z.B. Rauh (1992)). Einige Autoren fassen die semantische und logische Ebene in der Konzeptionellen zusammen (vgl. z.B. Vetter (1990)).
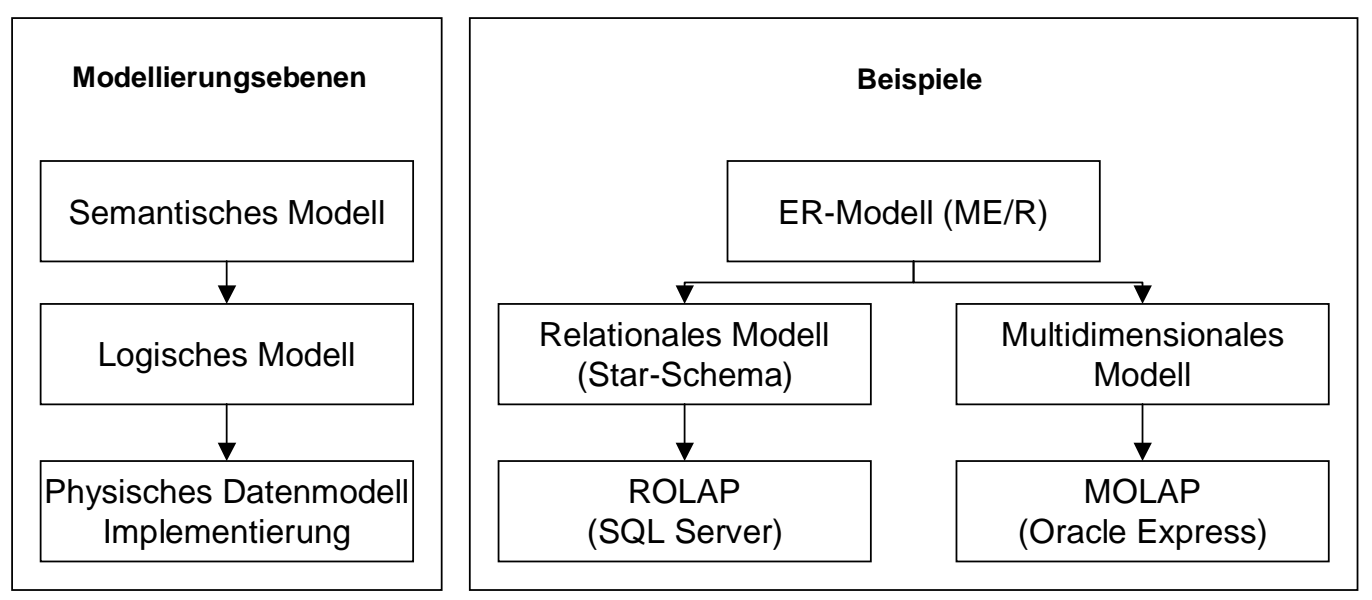

Abbildung 9-2: Modellierungs- und Implementierungsebenen (vgl. Totok (2000))

Die Erstellung eines Data Warehouses erfolgt Top-Down entlang der einzelnen Ebenen. In der ersten Phase werden die Elemente der Performance-Datenbank bestimmt und beschrieben. Diese werden in der zweiten Phase in einem semantischen Datenmodell dargestellt. Dabei ist das semantische Datenmodell als Verfahren zu verstehen, welches die Beziehungen zwischen den Elementen sowie die Regeln zu ihrer Analyse und Darstellung definiert (vgl. Ortner und Söllner (1989)). In einer dritten Phase wird aus diesem das logische Design abgeleitet. Ziel ist es, dass dieser den formalen, logischen Anforderungen von konkreten Datenmodellen bzw. Datenbanksystemen genügt. Dieser basiert entweder auf einer relationalen oder multidimensionalen Datenbank. Für ein relationales Datenmodell könnte eine Darstellung als Star- oder Snowflake-Struktur erfolgen. Die Transformation erfolgt mit Umsetzungsregeln oder mittels Gütekriterien (vgl. Rauh (1991)). Im letzten Schritt wird das logische Schema im Rahmen des physischen Designs unter Berücksichtigung der spezifischen Eigenschaften der gewählten Datenbank und der zur Verfügung stehenden Techniken umgesetzt.

Nachfolgende Überlegungen konzentrieren sich auf die Erstellung eines formalisierten multidimensionalen Modells (semantisches Modell) für die in Kapitel sechs definierten Elementen. 


\subsection{Untersuchung von Vorschlägen aus der Literatur}

Es soll untersucht werden, wie aus der Menge der Performance-Indikatoren, ihrer Struktur, ihren Verantwortlichkeiten und den Empfängern (Ergebnis der organisatorischen Konzeption) ein geeignetes Datenmodell für das Data Warehouse der Performancerelevanten Daten hergeleitet werden kann.

Im Gegensatz zum klassischen Entity-Relationship-Modelling (vgl. Chen (1976)), bei welchem die Modellierung als gefestigte Disziplin gilt, handelt es sich bei der Modellierung eines Data-Warehouse um eine junge Disziplin. Es existieren kaum etablierte Techniken und Modellierungsstandards. Hüsemann et al. (2000) äussern sich pointiert: „there is a large body of literature that discusses how to derive schemas based on intuitive principles, but no methodology is yet in sight“.

Die aus Sicht des Verfassers wichtigsten Ansätze sollen nachfolgend referiert und auf ihre Eignung für die beschriebene Problemstellung untersucht werden. Es handelt sich dabei um den Ansatz von (1) Kimball (1996), (2) Böhnlein und Ulbrich-vom-Ende (2000), (3) Golfarelli, Maio und Rizzi (vgl. Golfarelli et al. (1998)) sowie (4) Hüsemann, Lechtenbörger und Vossen (vgl. Hüsemann et al. (2000)).

Kimball ist einer der meistzitierten Autoren im Bereich des Data Warehousing und hat mehrere Standardwerke veröffentlicht. Böhnlein und Ulbrich-vom-Ende (2000) schlagen ein Vorgehensmodell basierend auf dem Semantischen-Objekt-Modell-Ansatz von Ferstl und Sinz (1998) vor. Golfarelli et al. (1998) propagieren ein semi-automatisches Verfahren zur Transformation von normalisierten Datenmodellen in Multidimensionale vor. Der Ansatz von Hüsemann et al. (2000) zielt in eine ähnliche Richtung und beschäftigt sich mit der Transformation einer operationellen Datenbank in ein multidimensionales Datenmodell.

\subsubsection{Kimballs Lifecycle Toolkit}

In ihrer umfassenden Publikation „The Data Warehouse Lifecycle Toolkit“ (vgl. Kimball et al. (1998)) präsentieren die Autoren einen Ansatz, um Data Warehouses zu erstellen, zu nutzen und diese sich verändernden Bedürfnissen anzupassen. Die nachfolgende Diskussion beschränkt sich auf die Transformation der Anforderungen - welche mittels Interviews erhoben werden - in das multidimensionale Datenmodell. Es ist zu beachten, 
dass keine semantische Modellierung erfolgt, sondern das logische Datenmodell direkt erstellt wird.

Im ersten Abschnitt werden „conformed dimensions“ definiert. Dies sind Dimensionen, die eine identische Bedeutung für sämtliche Kennzahlen haben. Der Prozess zur Erstellung, Veröffentlichung, Wartung und organisatorischen Durchsetzung dieser Dimensionen ist zu regeln. Die Kennzahlen sind ebenfalls zu standardisieren (z.B. die Stückzahl im Handel: Karton (bestehend aus 6 Stück) versus Einzelstück).

In einem zweiten Abschnitt wird eine Vier-Schritte-Methode für die Erstellung einer individuellen Faktentabelle vorgeschlagen. In einem ersten Schritt wird ein interessierender Data-Mart ${ }^{13}$ ausgewählt. Dabei empfehlen die Autoren, auf eine einzige Datenquelle abzustellen, um die Komplexität zu reduzieren. Der zweite Schritt umfasst die Festlegung der Granularität der Faktentabelle. In einem dritten Schritt werden die zugehörigen Dimensionen bestimmt. Dabei ist die gewählte Granularität zu berücksichtigen. Im vierten Schritt werden die Fakten zugeordnet. Der zweite Abschnitt kann für eine zweite Faktentabelle wiederholt werden.

Der vorgeschlagene Ansatz widerspiegelt die langjährige praktische Erfahrung der Autoren. Viele der Tipps bewahren vor möglichen Fehlerquellen in einem Data Warehouse-Projekt. Primäre Informationsquellen bilden Interviews. Wie die Dimensionen und Fakten ausgewählt werden, wird jedoch nicht detaillierter besprochen.

\subsubsection{Der prozessorientierte Ansatz von Böhnlein und Ulbrich-vom Ende}

Der Ansatz von Böhnlein und Ulbrich-vom-Ende (2000) basiert auf der Idee der Verwendung von allgemein akzeptierten Prozessmodellen zur Bestimmung der Anforderungen an ein Data Warehouse. Der Ansatz wird anhand des semantischen Objekt Modells (SOM) als Prozessmodell veranschaulicht (vgl. Ferstl und Sinz (1998)). SOM differenziert drei Ebenen: Den „Enterprise plan“, das „Business process model“ und die „Specification of resources“. Das Business process model wird mit Interaktions- und TaskEvent-Schemata konkretisiert. Ersteres betrachtet die statischen Geschäftsobjekte und

13 Kimball et al. (1998) verstehen den Data-Mart in diesem Kontext als eine Kennzahlengruppe, die in einer Faktentabelle zusammengefasst werden kann. Im Allgemeinen wird der Begriff Data-Mart für eine Teilmenge eines Data Warehouses verwendet (vgl. Bauer und Günzel (2001)). 
deren Interaktion, zweiteres fokussiert das dynamische Verhalten des Geschäftsprozesses. Die Darstellung erfolgt in einer Petrinetz-ähnlichen Struktur (vgl. zu Petrinetzen Murata (1989)).

Basierend auf dem semantischen Objekt-Modell wird ein Vier-Schritte-Plan durchlaufen, um die initiale Data Warehouse-Struktur abzuleiten. In einem ersten Schritt werden die Ziele der Organisation hergeleitet. Es handelt sich dabei um eine unstrukturierte Aktivität ${ }^{14}$. Der Argumentationskette „goal, services, business-process, metric“ folgend wird nach der Bestimmung eines Zieles das zugehörige Produkt bestimmt. Produkte werden gemäss den Autoren durch Geschäftsprozesse erstellt und werden teilweise durch Hilfsprozesse unterstützt. Entsprechend folgt im zweiten Schritt eine Analyse der Geschäftsprozesse. Die Darstellung erfolgt, wie bereits erläutert, mit Interaktions- und Task-Event-Schemata. Im dritten Schritt wird die Ressourcenausstattung erhoben. Die Darstellung erfolgt in einem so genannten konzeptionellen Objekt-Schema (COS), welches als konzeptionelles Datenmodell in der strukturierten ER-Modell-Notation (vgl. Sinz (1987)), um objektorientierte Elemente ergänzt, aufgefasst werden kann (vgl. auch Böhnlein und Ulbrich-vom-Ende (2001)). Das COS wird um entscheidungsrelevante Attribute ergänzt. Hierzu werden bspw. Interviews mit Entscheidungsträgern geführt.

Im letzten Schritt werden aus der ergänzten COS-Darstellung die initialen Data Warehouse-Strukturen abgeleitet. Basis bildet die Argumentationskette „goal-servicemetric“. Mit welcher Kennzahl kann das spezifizierte Produkt gemessen werden? Die Antwort auf diese Frage ergeben die Fakten. Die Erstellung der Dimensionen und deren Hierarchien erfolgt ohne formalen Prozess auf Grund der identifizierten Attribute.

Der Ansatz ist sehr komplex. Er stellt hohe Abstraktions-Ansprüche an die involvierten Entscheidungsträger. Zudem bedingt er eindeutig definierte Geschäftsprozesse, die mit Zielen verknüpft sind. Trotz hohem Formalismus erfolgen die wesentlichen Schritte mit viel Intuition.

14 „Finding goals is a complex and creative process“ (vgl. Böhnlein und Ulbrich-vom-Ende (2000)). 


\subsubsection{Die semi-automatische Konversion von Golfarelli et al.}

Die Autoren verfolgen in ihrem Aufsatz (vgl. Golfarelli et al. (1998)) einen datengetriebenen Ansatz zur Transformation eines relationalen Datenbank-Schemas in ein konzeptionelles Data Warehouse-Modell. Die Autoren referenzieren Kimball (1996), der ausführt „Entity relation data modells ... cannot be understood by users and they cannot be navigated usefully by DBMS software. Entity relation models cannot be used as the basis for enterprise data warehouses”, als Begründung, um eine eigene Notation, das Dimensional Fact (DF) Modell für die konzeptionelle Darstellung vorzuschlagen. Das DFModell kann als Sammlung von Fakt-Schemata, die in einer Baumstruktur dargestellt werden, aufgefasst werden. Die Basiselemente umfassen Fakten, Attribute, Dimensionen und Hierarchien. Zudem wird die Additionsfähigkeit von Fakten entlang von Dimensionen, die Optionalität von Dimensionen und die Darstellung von nicht-dimensionalen Attributen behandelt.

Die vorgeschlagene Methodik verwendet als Basis das relationale Datenbank-Schema einer einzigen transaktionsorientierten Anwendung, bestehend aus Tabellen, Attributen und Beziehungen inklusive deren Mächtigkeiten und den Attributen, welche die Beziehungen realisieren. Für die Transformation werden von den Autoren insgesamt sechs Schritte vorgeschlagen.

Attraktiv erscheint der Ansatz unter der Berücksichtigung der Tatsache, dass ein Data Warehouse rasch und semi-automatisch aufgebaut werden kann. Der Ansatz ist jedoch limitiert auf die im operationellen Schema enthaltenen Daten. Zudem ist nicht klar, inwiefern mehrere Datenquellen berücksichtigt werden. Damit werden die Unternehmensziele und die spezifischen Bedürfnisse der Organisation ignoriert. In einer neueren Publikation (vgl. Golfarelli und Rizzi (1999)) gehen die Autoren auf den gesamten Prozess zur Erstellung eines Data Warehouse ein, d.h. von der Erhebung der Anforderungen bis zum physischen Design. Sie konzentrieren sich auf bestehende Datenbank-Schemata, ohne diese bspw. mit Interviews oder einer zielbasierten Struktur zu ergänzen. 


\subsubsection{Konversion eines hochnormalisierten E/R-Schemas in eine multidimensionale Datenbank nach Hüsemann et al.}

Die Autoren schlagen eine Methodik für den konzeptionellen Data Warehouse-Design vor. Der Ansatz differenziert sich laut den Autoren von den intuitiven Ansätzen dadurch, dass eine Reihe von Zielen - die teilweise formalisiert werden können - bei der Modellierung berücksichtigt werden. Ausgangspunkt ist - wie beim Ansatz von Golfarelli et al. (1998) ein operationelles Datenbank-Schema, welches in mehreren Schritten semi-automatisch in ein Data Warehouse-Schema transformiert wird. Hierzu sind gemäss den Autoren die folgenden Schritte notwendig:

In einem ersten Schritt wird das E/R-Schema der operationellen Datenbank bezüglich interessierenden Kennzahlen, Dimensionen und Basisabfragen analysiert. Ergebnis ist eine Tabelle mit Attributen, zugehöriger Beschreibung und Angabe darüber, ob es sich um eine Kennzahl, eine zwingende oder fakultative Dimension handelt. Die Tabelle wird in einem zweiten Schritt nach individuellen Bedürfnissen ergänzt um jene Daten, die im originären Schema nicht enthalten waren. Dabei kann es sich bspw. um Klassenbildungen handeln. Für die beiden Aktivitäten offerieren die Autoren keine formale Methodik - vielmehr handelt es sich hier um einen intuitiven Vorgang.

Ausgehend vom Ergebnis der beiden ersten Schritte folgt ein formales Vorgehen zur Bestimmung des Datenmodells. Dieses startet mit der Bestimmung der funktionalen Abhängigkeit der einzelnen Granularitätsstufen der Dimensionen von den Kennzahlen. Damit können die minimalen Schlüssel für jede Kennzahl und die terminalen Elemente jeder Dimension (Anfang des Aggregationspfades; höchste Granularität) bestimmt werden. Im nächsten Schritt werden die Dimensionhierarchien (Aggregationspfade der Dimensionen) bestimmt. Zu diesem Zweck werden die funktionalen Abhängigkeiten sämtlicher zu einer Dimension zugehörigen Granularitätsstufen zum terminalen Element bestimmt. Angenommen, das terminale Element sei $d_{j}$, und zwei Granularitätsstufen seien $d_{k}$ und $d_{l}$. Wenn $d_{k}$ funktional abhängig von $d_{l}$ ist und eine (wahrscheinlich transitive), funktionale Abhängigkeit von $d_{k}$ zu $d_{j}$ besteht, so kann $d_{k} \rightarrow d_{l}$ dem Aggregationspfad hinzugefügt werden $\left(F_{\text {Dim }}=\left\{d_{j} \rightarrow d_{k} \rightarrow d_{l}\right\}\right)$. Der Aggregationspfad wird in einem zweiten Schritt noch mit Eigenschafts-Attributen besetzt (z.B. der Kundenname zur Kundennummer). Zusätzlich stellen die Autoren Regeln zur Bildung von Hierarchien mit mehreren Pfaden vor. 
In einem weiteren Schritt untersuchen die Autoren, inwiefern Aggregationen von Kennzahlen sinnvoll sind. Hierzu differenzieren sie vier Stufen mit unterschiedlichen Restriktionen. Die erste Stufe erlaubt die Anwendung sämtlicher Aggregationsfunktionen auf die Kennzahlen ( $\{\text { SUM, AVG, MIN, MAX, STDDEV, VAR, COUNT }\}^{15}$ ), die Zweite schliesst die Summierung aus (dies gilt bspw. für den Tag des Monates), die Dritte erlaubt nur noch das Zählen (\{COUNT\}), und die Vierte erlaubt keine Aggregationsfunktionen mehr.

Abschliessend zeigen die Autoren, dass die vorgestellte Methodik Schemata in so genannter „generalisierter multidimensionaler Normalform“ (GMNF) erzeugt. Die Regeln, die der GMNF zu Grunde liegen, sollen eine gewisse Modellierungsqualität Gewähr leisten.

15 SUM steht für die Summenbildung, AVG für Average/Mittelwert, MIN für den kleinsten Wert, MAX für den grössten Wert der Zahlenreihe. STDDEV bezeichnet die Standardabweichung, VAR die Varianz und COUNT die Anzahl der betrachteten Werte. 


\subsubsection{Bewertung der Eignung zur Erstellung einer Datenstruktur für ein PMS}

Nachfolgend werden die weiter vorne besprochenen Ansätze charakterisiert und einander gegenübergestellt.

\begin{tabular}{|c|c|c|c|c|}
\hline & Kimball (1996) & $\begin{array}{l}\text { Böhnlein und } \\
\text { Ulbrich-vom-Ende } \\
(2000)\end{array}$ & $\begin{array}{l}\text { Golfarelli et al. } \\
\text { (1998) }\end{array}$ & $\begin{array}{l}\text { Hüsemann et al. } \\
(2000)\end{array}$ \\
\hline \begin{tabular}{|l|} 
Quelle der \\
Requirements
\end{tabular} & Interviews & $\begin{array}{l}\text { Prozessmodell } \\
\text { (SOM) + Interviews }\end{array}$ & $\begin{array}{l}\text { Datenschema des } \\
\text { operativen Systems }\end{array}$ & $\begin{array}{l}\text { Datenschema des } \\
\text { operativen Systems } \\
+ \text { ev. Interviews }\end{array}$ \\
\hline Umsetzung & \begin{tabular}{|l} 
Heuristisches \\
Vorgehensmodell
\end{tabular} & \begin{tabular}{|l} 
Heuristisches \\
Vorgehensmodell
\end{tabular} & Semiformaler Ansatz & $\begin{array}{l}\text { Semiformaler } \\
\text { Ansatz }\end{array}$ \\
\hline Charakteristik & \begin{tabular}{|l|} 
Inkrementelles, \\
iteratives Verfahren
\end{tabular} & $\begin{array}{l}\text { Argumentations- } \\
\text { kette: goal - service } \\
\text { - business process } \\
\text { - metric }\end{array}$ & $\begin{array}{l}\text { Detaillierte } \\
\text { Vorgehens- } \\
\text { beschreibung für die } \\
\text { Konversion }\end{array}$ & $\begin{array}{l}\text { Ansatz mit } \\
\text { Rollenteilung } \\
\text { Business - IT }\end{array}$ \\
\hline $\begin{array}{l}\text { Transformations- } \\
\text { regeln }\end{array}$ & $\begin{array}{l}\text { Einige „best- } \\
\text { practices“, } \\
\text { Vorschlag einer } \\
\text { Schrittfolge, } \\
\text { teilweise } \\
\text { ungenügende Tiefe } \\
\text { zur konkreten } \\
\text { Anwendung }\end{array}$ & $\begin{array}{l}\text { Aufwändige } \\
\text { Analyseschritte, } \\
\text { Vorschlag einer } \\
\text { Schrittfolge, } \\
\text { einzelne Schritte } \\
\text { sind teilweise nur } \\
\text { vage beschrieben }\end{array}$ & $\begin{array}{l}\text { Vorschlag einer } \\
\text { Schrittfolge, einzelne } \\
\text { Teilschritte sind } \\
\text { formalisiert }\end{array}$ & $\begin{array}{l}\text { Vorschlag einer } \\
\text { Schrittfolge, erster } \\
\text { Teil ist sehr vage, } \\
\text { zweiter Teil ist } \\
\text { formal beschrieben. }\end{array}$ \\
\hline Ergebnis & \begin{tabular}{|l} 
Logisches \\
Datenmodell
\end{tabular} & $\begin{array}{l}\text { Konzeptionelles } \\
\text { Objekt-Schema } \\
\text { (COS) }\end{array}$ & $\begin{array}{l}\text { Dimensional Fact } \\
\text { Model }\end{array}$ & $\begin{array}{l}\text { Konzeptionelles } \\
\text { Schema (eigene } \\
\text { Notation) }\end{array}$ \\
\hline $\begin{array}{l}\text { Gesamtbeurteilung: } \\
\text { Stärken (+) und } \\
\text { Schwächen (-) }\end{array}$ & $\begin{array}{l}\text { (+) in zahlreichen } \\
\text { Projekten bewährt } \\
(+) \text { viele } \\
\text { Gestaltungs- } \\
\text { hinweise } \\
(-) \text { zu wenig präzise } \\
\text { Beschreibung }\end{array}$ & $\begin{array}{l}\text { (-) Anwendung in } \\
\text { einem Forschungs- } \\
\text { projekt, sehr } \\
\text { komplex } \\
\text { (-) Einbezug der } \\
\text { Benutzer schwierig }\end{array}$ & $\begin{array}{l}(+) \text { mit weiterer } \\
\text { Forschung könnte } \\
\text { ein automatisch zu } \\
\text { generierender erster } \\
\text { DWH-Entwurf } \\
\text { realisierbar sein } \\
\text { (-) Einseitige } \\
\text { Datenbasis }\end{array}$ & $\begin{array}{l}\text { (-) Grobe } \\
\text { Beschreibung der } \\
\text { ersten Schritte } \\
\text { (+) danach recht } \\
\text { detaillierte } \\
\text { Vorgehens- } \\
\text { beschreibung } \\
(+) \text { Gute } \\
\text { Anwendbarkeit, gute } \\
\text { Rollenteilung } \\
\text { (-) Einseitige } \\
\text { Datenbasis }\end{array}$ \\
\hline
\end{tabular}

Tabelle 9-3: Zusammenfassende Gegenüberstellung der diskutierten Ansätze

Keine der besprochenen Ansätze offeriert ein auf Regeln basiertes Umsetzen der Requirements in ein multidimensionales Datenmodell. Dies kann nur erreicht werden, wenn die Requirements ausreichend formal spezifiziert sind. Es ist aus dieser Überlegung heraus nachvollziehbar, dass diejenigen Ansätze, die ein bestehendes Datenschema als Basis verwenden, diesem Ziel am nächsten kommen. 
Des Weiteren differiert die Ausgangsbasis der Ansätze stark. Der Ansatz von Böhnlein und Ulbrich-vom-Ende (2000) basiert auf einem Prozessmodell. Die anderen Ansätze sind entweder stark durch das bestehende operative Datenschema oder Benutzerinterviews geprägt.

Die diskutierten Ansätze sind generisch ausgelegt. Diese im Allgemeinen positive Eigenschaft der breiten Anwendbarkeit besitzt jedoch den Nachteil, dass situative, mit der konkreten Problemstellung verbundene Eigenschaften nicht berücksichtigt werden können. Die kritisierte Ungenauigkeit der Anweisungen liesse sich damit teilweise erklären. Durch Ziehen von engen Systemgrenzen, d.h. der Optimierung der Schrittfolge für einen einzigen Anwendungsbereich, können genauere Angaben für einen Vorgehensvorschlag gemacht werden. Nachfolgend wird dieser Weg gewählt und ein eigenes Vorgehensmodell zur Erstellung einer multidimensionalen Datenstruktur für Performance-Indikatoren vorgestellt.

\subsection{Vorschlag eines Vorgehensmodells zur Erstellung einer Datenstruktur für ein Performance-Measurement-System}

An das Ergebnis (Datenmodell) und an die zu entwerfende Schrittfolge selbst, werden eine Reihe von Anforderungen gestellt. Diese lassen sich in drei Gruppen einteilen: Erstens ist der Übergang von der organisatorischen zur technischen Konzeption sicherzustellen (Pkt. eins). Zweitens sind die Anforderungen an ein PMS aus Kapitel vier zu berücksichtigen (Pkt. zwei bis vier) und drittens sind eine Reihe von Praktikabilitätsüberlegungen zum Datenmodell zu beachten (Pkt. fünf bis sieben). Die Anforderungen sind in Tabelle 9-4 zusammengefasst:

1. Schnittstellenkompatibilität, d.h. das Ergebnis der Ziel-Indikatorenhierarchie (vgl. Kapitel sechs) muss als Basis für das Vorgehensmodell des Datenmodells dienen können.

2. Berücksichtigung der spezifischen Anforderungen an ein Performance-MeasurementSystem (vgl. Kapitel vier).

3. Die Datenstruktur muss potenzielle Anfragen repräsentieren.

4. Der Informationsbedarf muss gedeckt werden.

5. Daten in einem nicht benötigten Detaillierungsgrad sollen nicht verwaltet werden.

6. Dimensionen werden nicht redundant geführt.

7. Die Anzahl der Datenwürfel soll möglichst klein gehalten werden.

Tabelle 9-4: Anforderungen an das Vorgehensmodell zur Erstellung einer Datenstruktur für ein PMS 
Aus diesen Anforderungen und den diskutierten Ansätzen (vgl. Tabelle 9-3) wurde in einem iterativen Prozess eine Schrittfolge zur Transformation erarbeitet. Zwischenversionen wurden anhand von praktischen Beispielen getestet und partiell in der Diskussion mit Experten hinterfragt. Als Ergebnis resultierte eine Schrittfolge, die aus insgesamt 10 Schritten besteht, welche sich in fünf Gruppen einteilen lässt:

1. Daten bereitstellen: Der erste Schritt dient der Bereitstellung der Daten. Er stellt die Schnittstellenkompatibilität (Anforderung eins) sicher.

2. Informationsbedarf bestimmen: Die zweite Gruppe umfasst drei Schritte (zwei vier). Es erfolgt eine geeignete Aufbereitung der bereitgestellten Daten, die Bestimmung der notwendigen Dimensionen und des Detaillierungsgrades der Performance-Indikatoren. Diese Gruppe berücksichtigt die Anforderungen zwei bis fünf.

3. Dimensionen erstellen und optimieren: In zwei Schritten (fünf und sechs) werden die Aggreationspfade der Dimensionen bereinigt und geeignete Kandidaten vereinigt (vgl. Anforderung sechs).

4. Datenwürfel erstellen und optimieren: In Schritt sieben wird die endgültige Granularität der einzelnen Indikatoren, bezogen auf die optimierten Dimensionen, bestimmt und somit der Datenwürfel definiert. Die einzelnen Datenwürfel werden im achten Schritt noch auf vereinigungskompatible Kandidaten hin untersucht. (vgl. Anforderung sieben)

5. Datenmodell mit spezifischen Informationen anreichern: In der letzten Gruppe wird das Datenmodell um spezifische Informationen, die aus den Anforderungen an das PMS resultieren, ergänzt. Es handelt sich dabei um allfällig zu definierende Aggregationsbeschränkungen (Schritt neun) und die Hinterlegung von Standardzugriffen und Berechtigungen der einzelnen Informationsempfänger (Schritt zehn).

Die Abbildung 9-3 zeigt die Schritte im Überblick. Nachfolgend werden die einzelnen Schritte theoretisch besprochen und im Anschluss daran an einem Beispiel illustriert. 


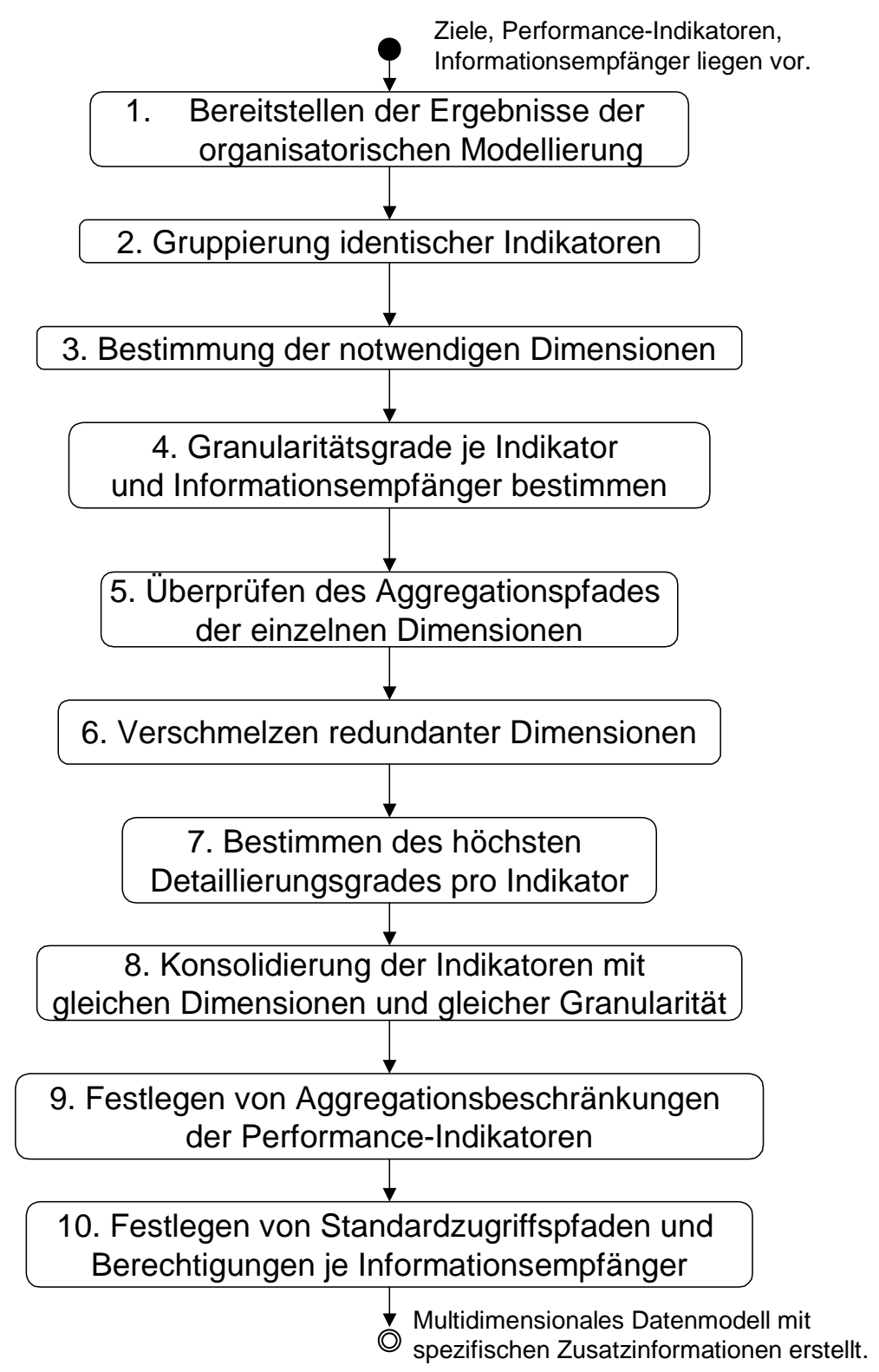

Abbildung 9-3: Vorgehensmodell zur Erstellung einer multidimensionalen Datenstruktur

\subsubsection{Bereitstellen der Ergebnisse der organisatorischen Modellierung (Schritt 1)}

Im ersten Schritt werden die Ergebnisse der organisatorischen Modellierung aus Kapitel sechs zweckmässig aufbereitet. Diese umfassen im Wesentlichen die folgenden Elemente:

\footnotetext{
- $\quad$ Zielbaum inkl. Indikatoren

- Aufbauorganisation bis auf die Mitarbeiterebene

- $\quad$ Prämissen mit Bezug zu Zielen

- $\quad$ Strategische Massnahmen mit Zielen und Indikatoren
} 
- Verantwortlichkeiten für Ziele, Strategische Massnahmen, Prämissen und Mitarbeiterziele

- $\quad$ Empfänger von Performance-Daten

Es ist zu sicherzustellen, dass die verwendeten Begriffe und Kürzel korrespondieren. Die Identifikation wird in den nächsten Schritten dadurch wesentlich vereinfacht.

\subsubsection{Gruppierung identischer Indikatoren (Schritt 2)}

Da die gleichen Indikatoren auf verschiedenen Hierarchiebenen (z.B. sowohl auf Unternehmens- als auch auf Abteilungsebene) vorkommen können, der einzelne Indikator aber in der Folge gesammelt behandelt wird, müssen diese konsolidiert werden.

\subsubsection{Bestimmen der notwendigen Dimensionen (Schritt 3)}

Die Dimensionen determinieren die Analysemöglichkeiten eines Performance-Indikators und bestimmen somit potenzielle Abfragen in der Datenstruktur. Grundsätzlich sind Dimensionen abhängig vom betriebswirtschaftlichen Anwendungsgebiet und von der Art der geplanten Analysen (vgl. Totok (2000)). Durch die vorgenommene Einschränkung auf das Anwendungsgebiet eines Performance-Measurement-Systems können jedoch eine Reihe von Dimensionen mit ihren Aggregationspfaden unabhängig von den konkreten Zielen und Indikatoren festgelegt werden:

- Zeit: Der Dimensionstyp Zeit wird in Verbindung mit fast allen Kennzahlen angewandt. Unterschieden werden muss, ob Kennzahlen auf der Basis von Bewegungs- (z.B. Anzahl Verkäufe) oder Bestandesgrössen (z.B. Lagerbestand zum Tag X) erfasst werden. Des Weiteren ist zu beachten, dass die einzelnen Hierarchieebenen nicht direkt voneinander abhängig sind (z.B. Wochen können nicht als Detaillierungsstufe des Monates dargestellt werden). Dieses Problem wird umgangen, indem mehrere Aggregationspfade für die Dimension Zeit abgelegt werden. Ein weiterer wichtiger Punkt ist die Vergleichbarkeit. Eine Reihe von Feiertagen ist unabhängig vom gregorianischen Kalender und benötigt deshalb eine separate Speicherung. Ebenso ist zu berücksichtigen, dass das Geschäftsjahr der Organisation u.U. nicht dem Kalenderjahr entspricht. Der 
Granularitätsgrad der Dimension Zeit ist abhängig von den definierten Kennzahlen und kann nicht generisch spezifiziert werden (Ein Energieversorgungsunternehmen erhebt den Verbrauch von Energie bei grösseren Abnehmern bspw. alle 15 Minuten, das Detailhandelsunternehmen interessieren die Tagesumsätze, die Umsätze in einem Grosshandelsunternehmen werden nur monatlich erhoben). Moderne Softwareprodukte unterstützen den Benutzer bezüglich der Dimension Zeit. Häufig muss diese gar nicht mehr physisch angelegt werden (so genannte virtuelle Dimension).

- Aufbauorganisation: Die Organisationsstruktur kann ebenfalls als fixe Dimension bezeichnet werden. Bezüglich ihrer konkreten Ausgestaltung (Anzahl Hierarchieebenen, Bezeichnungen, etc.) besteht keine Vorgabe. Die Dimension erlaubt, Kennzahlen mit einzelnen Organisationseinheiten resp. Stellen und Mitarbeitern zu verknüpfen.

- Ziel-Indikatoren-Hierarchie: Diese Dimension repräsentiert den Zielbaum. Damit können Beziehungen zwischen einzelnen Zielen und Indikatoren, die ex ante angenommen wurden, auf Grund von konkreten Daten ex post verifiziert werden. Zudem kann dem Anwender das mit dem Zielbaum verbundene hierarchische Wirkungsnetz visualisiert werden.

- Ebenen: Bis anhin wurde stillschweigend davon ausgegangen, dass die realisierten Performance-Werte erfasst werden. Denkbar ist jedoch auch, dass die Plan- und Soll-Werte in Form von Szenarien in der Performance-Datenbank gespeichert werden.

Neben diesen generischen Dimensionen, sind weitere auf Grund der betrachteten Kennzahl zu identifizieren. Typische sind Produkte, Geografie, Kundenarten und Währungen. Zur Bestimmung von weiteren Dimensionen können die Anwender bezüglich möglicher Analysebereiche befragt werden.

Ergebnis dieses Schrittes ist es, die in Frage kommenden Dimensionen, und damit die möglichen Abfragen, je Performance-Indikator zu bestimmen. 


\subsubsection{Bestimmen der Granularitätsgrade je Indikator und Informationsempfänger}

(Schritt 4)

Ziel des Datenmodell ist es, auf der einen Seite den potenziellen Informationsbedarf zu decken und auf der anderen Seite keine unnötigen Informationen zu speichern. Im vierten Schritt ist demzufolge für jeden einzelnen Performance-Indikator und Informationsempfänger der notwendige Granularitätsgrad bezüglich der einzelnen Dimensionen zu klären. Dabei können neue Aggregrationsebenen bei einzelnen Dimensionen auftreten. Diese sind vorzumerken und im nächsten Schritt in die Hierarchiebenen der Dimensionen einzubauen.

Ergebnis dieses Schrittes ist somit eine Matrix je Indikator, die je Informationsempfänger und Dimension die interessierenden Granularitätsgrade nennt.

\subsection{5 Überprüfen des Aggregationspfades der einzelnen Dimensionen (Schritt 5)}

Im dritten Schritt sind die einzelnen Dimensionen bestimmt worden, im vierten Schritt sind diese um die Hierarchieebenen erweitert worden. Um zukünftigen Benutzeranfragen optimal Rechnung tragen zu können, werden die Aggregationspfade nun auf ihre Durchgängigkeit hin überprüft. Bspw. könnte sich ein Stakeholder auf der Zeitdimension für die Aggregationsstufe „Tag“, ein anderer für „Quartal“ interessieren. In diesem Fall wäre es unter Umständen sinnvoll, die Aggregationsebene „Monat“ einzufügen.

\subsubsection{Verschmelzen redundanter Dimensionen (Schritt 6)}

Es ist zu prüfen, ob einzelne Dimensionen auf Grund ihrer Gleichartigkeit verschmolzen werden können. Ist dies der Fall, so können diese als gemeinsame Dimensionen (shared dimensions) definiert werden und müssen im Datenmodell nur einmal aufgebaut und unterhalten werden. Es ist ein möglichst hoher Anteil an gemeinsamen Dimensionen anzustreben, um die Komplexität des Data Warehouse beherrschbar zu machen.

\subsubsection{Bestimmen des höchsten Detaillierungsgrades pro Indikator (Schritt 7)}

Nach Bereinigung der Dimensionen kann der maximale Detaillierungsgrad je Indikator gemäss den Ergebnissen aus Schritt vier bestimmt werden. Es ist dem entsprechend pro Indikator und Dimension die tiefste Aggregationsebene (was dem höchsten Detaillierungsgrad entspricht) zu bestimmen. Für jede Kombination ist im nächsten Schritt ein „Datenwürfel“ vorzusehen. 


\subsubsection{Konsolidierung der Indikatoren mit gleichen Dimensionen und gleicher Granularität (Schritt 8)}

Um die Anzahl der Datenwürfel möglichst klein zu halten, geht es im achten Schritt darum, Performance-Indikatoren mit den gleichen Dimensionen und den gleichen Aggregationsebenen zusammenzufassen, um sie in einem einzigen Datenwürfel speichern zu können. (Als Beispiel könnte der Einzelpreis und die Menge eines verkauften Gutes angeführt werden.)

\subsubsection{Festlegen von Aggregationsbeschränkungen der Performance-Indikatoren (Schritt 9)}

Verschiedene Autoren (vgl. Lenz und Shoshani (1997), Golfarelli et al. (1998), Hüsemann et al. (2000)) haben darauf hingewiesen, dass es nicht für alle Indikatoren sinnvoll ist, auf diesen sämtliche existenten Operationen auszuführen. Dies kann bspw. an einem Kundenzufriedenheitsindex illustriert werden. Die Bestimmung des Mittelwertes für eine Kundengruppe erscheint sinnvoll, die Summierung der Werte hingegen macht keinen Sinn. Diese für den Benutzer wertvolle Information kann mittels Aggregationsbeschränkungen auf dem Datenmodell hinterlegt werden. Der Data Warehouse-Server stellt dadurch sicher, dass nur in diesem Sinne gültige Aggregationen auf den Performance-Indikatoren ausgeführt werden können.

In Anlehnung an Pedersen und Jensen ((1999), zitiert bei Hüsemann et al. (2000)) werden vier Grade von Aggregationsbeschränkungen differenziert (vgl. Tabelle 9-5):

\begin{tabular}{|c|l|}
\hline Grade & Zulässige Aggregationsfunktionen \\
\hline 1 & $\{$ SUM, AVG, MIN, MAX, STDDEV, VAR, COUNT $\}$ \\
\hline 2 & $\{$ AVG, MIN, MAX, STDDEV, VAR, COUNT $\}$ \\
\hline 3 & $\{$ COUNT $\}$ \\
\hline 4 & \{\} \\
\hline
\end{tabular}

Tabelle 9-5: Grade von Aggregationsbeschränkungen (vgl. Hüsemann et al. (2000))

Die Anwendung der Tabelle ist wie folgt vorgesehen: Für einen Performance-Indikator und eine Dimensionsebene gilt bspw. Grad 1, wenn alle Aggregationsfunktionen angewendet werden können für alle im Aggregationspfad höherliegenden Aggregationsebenen. Die Bestimmung der zulässigen Aggregationsfunktionen ist für jede 
Kombination eines Performance-Indikators und einer Dimension vorzunehmen. Komplexe Dimensionen, die über mehrere Aggregationspfade verfügen (z.B. die Zeitdimension, die das Kalenderjahr und das Geschäftsjahr differenziert), sind einzeln je Aggregationspfad abzuklären. Zudem ist es denkbar, auf einer höheren Aggregationsebene einen höheren Grad von Aggregationsbeschränkungen vorzusehen.

\subsubsection{Festlegen von Standardzugriffspfaden und Berechtigungen je Informationsempfänger (Schritt 10)}

Mit Hilfe der Informationen aus Schritt vier (Granularitätsgrad je Informationsempfänger), den Verantwortlichen je Ziel, Strategische Massnahme, Prämisse und der Mitarbeiterziele, sowie den definierten Empfängern von Performance-Daten, ist für jeden Informationsempfänger und Datenwürfel zu bestimmen, auf welcher Hierarchieebene der Zugriff standardmässig, d.h. welcher Granularitätsgrad vom Informationsempfänger bezüglich eines Indikators in der Regel als Ausgangslage gewünscht wird, erfolgen soll und welche weiteren Ebenen vom Benutzer eingesehen werden dürfen. Die erste Information wird für den Zugriff auf den Datenwürfel eines bestimmten Benutzers benötigt, die Zweite zur Regelung der Zugriffsrechte. Der Standardzugriffspfad wird in der Regel derart festgelegt, dass dieser auf das Dimensions- und Kennzahlentupel zeigt, für welches der Informationsempfänger verantwortlich ist.

\subsection{Illustration des Vorgehensmodells an einem Beispiel}

Das eingeführte Vorgehensmodell soll an einem Beispiel illustriert werden. Als Grundlage dient das Beispiel aus Kapitel sechs.

\subsubsection{Schritt 1: Bereitstellen der Ergebnisse der organisatorischen Modellierung}

Nachfolgend sind die Ergebnisse der organisatorischen Modellierung zusammengestellt (Schritt eins; vgl. Tabelle 9-6 und Tabelle 9-7). In Tabelle 9-6 sind in den linken Spalten die Organisationseinheiten der Aufbauorganisation erkennbar. SIDL als Gesamtunternehmen, bestehend aus dem Geschäftsbereich Application Services (AS), in welchem der Prozess Support und Maintenance (SM) betrachtet wird. Die einzelnen Ziele werden danach differenziert, ob es sich um Ziele handelt, die aus der Ziel- und Indikatorenhierarchie resultieren (Z) oder aus den strategischen Programmen (S). 


\begin{tabular}{|c|c|c|c|c|c|c|c|c|c|}
\hline 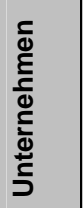 & 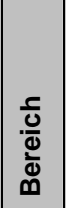 & 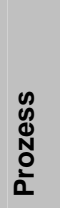 & 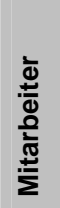 & 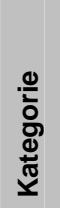 & $\frac{\underline{\frac{O}{i}}}{\frac{\mathrm{d}}{\mathrm{N}}}$ & 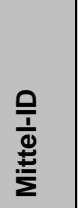 & 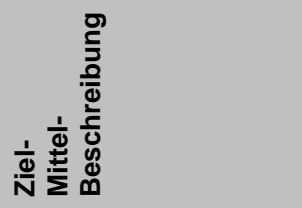 & $\frac{9}{\frac{1}{\alpha}}$ & 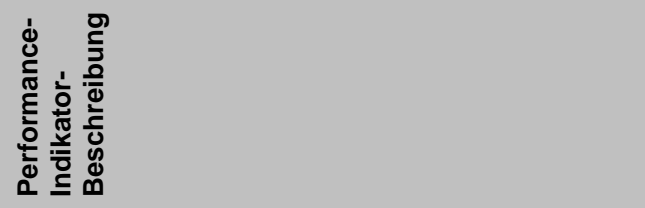 \\
\hline SIDL & & & & Z & Z01 & & \multicolumn{3}{|l|}{ Hohe Eigenkapitalquote } \\
\hline & & & & & & & & P01 & Eigenkapitalquote \\
\hline & & & & & & M01 & \multicolumn{3}{|c|}{ Kongruenz von Einnahmen und Ausgabenströmen } \\
\hline & & & & & & M02 & \multicolumn{3}{|c|}{ Minimierung des Anlagevermögens } \\
\hline & & & & & & M03 & \multirow{2}{*}{\multicolumn{3}{|c|}{$\begin{array}{l}\text { Optimale Bewirtschaftung des Umlaufvermögens } \\
\text { Umsatz und Ergebniswachstum, welches über dem Branchendurchschnitt liegt }\end{array}$}} \\
\hline & & & & Z & Z02 & & & & \\
\hline & & & & & & & & P02 & $\begin{array}{l}\text { Veränderung des relativen Marktanteils gegenüber } \\
\text { dem Vorjahresquartal }\end{array}$ \\
\hline & & & & & & & & P03 & EBITA-Marge \\
\hline & & & & & & M04 & \multicolumn{3}{|c|}{ Relativ zum Markt wachsende Kundenbasis } \\
\hline & & & & $\mathrm{Z}$ & Z03 & \multicolumn{4}{|c|}{ langfristig, beidseitig nutzbringende Beziehung } \\
\hline & & & & & & & & P04 & Kundenzufriedenheits-Index \\
\hline & & & & Z & Z04 & & \multicolumn{3}{|c|}{ hohe Mitarbeiterzufriedenheit } \\
\hline & & & & & & & & P05 & Index der Mitarbeiterzufriedenheitsumfrage \\
\hline & & & & & & & & P06 & Investition in Mitarbeiterausbildung \\
\hline & & & & & & & & P07 & Anzahl Krankheitstage pro Mitarbeiter \\
\hline & AS & & & Z & Z02 & \multicolumn{4}{|c|}{ Umsatz und Ergebniswachstum, welches langfristig die Leistung der Branche übertrifft } \\
\hline & & & & & & & & P03 & EBITA-Marge \\
\hline & & & & Z & Z05 & \multicolumn{4}{|c|}{ Zahlungsmodalitäten korrespondieren mit der Leistungserbringung (M1) } \\
\hline & & & & & & & & P08 & Aufträge in Arbeit (CHF) \\
\hline & & & & Z & Z06 & \multicolumn{4}{|c|}{ Neuentwicklungen werden mit Kostenbeteiligungen der Kunden realisiert (M2) } \\
\hline & & & & & & & & P09 & Fremdfinanzierungsgrad von Entwicklungen \\
\hline & & & & Z & Z07 & \multicolumn{4}{|c|}{ relativ wachsende Installationsbasis der ERP-Lösungen (M4) } \\
\hline & & & & & & & & P10 & $\begin{array}{l}\text { relativer Marktanteil der ERP-Lösung im relevanten } \\
\text { Segment }\end{array}$ \\
\hline & & & & & Z08 & \multicolumn{4}{|c|}{ relativ höhere Neuabschlussquote (mengenmässig) (M4) } \\
\hline & & & & & & & & P11 & mengenmässiger Anteil an Neuabschlüssen \\
\hline & & & & & & M05 & \multicolumn{3}{|c|}{ Kompetitive SLA-Konditionen } \\
\hline & & & & & & M06 & \multicolumn{3}{|l|}{ hohe Wartungsqualität } \\
\hline & & & & & Z03 & \multicolumn{4}{|c|}{ langfristig, beidseitig nutzbringende Beziehung } \\
\hline & & & & & & & & P04 & Kundenzufriedenheits-Index \\
\hline & & & & & Z04 & \multicolumn{4}{|c|}{ hohe Mitarbeiterzufriedenheit } \\
\hline & & & & & & & & P05 & Index der Mitarbeiterzufriedenheitsumfrage \\
\hline & & & & & & & & P06 & Investition in Mitarbeiterausbildung \\
\hline & & & & & & & & P07 & Anzahl Krankheitstage pro Mitarbeiter \\
\hline & & SM & & Z & Z03 & \multicolumn{4}{|c|}{ langfristig, beidseitig nutzbringende Beziehung } \\
\hline & & & & & & & & P04 & Kundenzufriedenheits-Index \\
\hline & & & & $Z$ & ZO4 & \multicolumn{4}{|c|}{ hohe Mitarbeiterzufriedenheit } \\
\hline & & & & & & & & P05 & Index der Mitarbeiterzufriedenheitsumfrage \\
\hline & & & & & & & & P06 & Investition in Mitarbeiterausbildung \\
\hline & & & & & & & & P07 & Anzahl Krankheitstage pro Mitarbeiter \\
\hline & & & & Z & Z09 & \multicolumn{4}{|c|}{ Kompetitive SLA-Konditionen (M5) } \\
\hline & & & & Z & Z10 & \multicolumn{4}{|c|}{ hohe Wartungsqualität (M6) } \\
\hline & & & & & & & & & \\
\hline & & & & $\mathrm{S}$ & Z20 & \multicolumn{4}{|c|}{ Reduktion der eigenen Liquiditätsreserve } \\
\hline & & & & & & & & P20 & Anteil des Working Capital am Umsatz \\
\hline & & & & $\mathrm{S}$ & Z21 & Erhör & ung der Rendite auf der & Liquidi & itätsreserve \\
\hline & & & & & & & & P21 & Rendite des Working Capital \\
\hline
\end{tabular}

Tabelle 9-6: Ziel- und Indikatorenhierarchie für den betrachteten Ausschnitt von SIDL

Tabelle 9-7 zeigt die Verantwortlichen und Informationsempfänger je Indikator. In der ersten Spalte ist eine Referenz auf die Tabelle 9-6 enthalten, welche das Ziel, den zugehörigen Indikator und die relevante Ebene der Organisation umfasst. Z04.P07.SIDL als Beispiel bezieht sich auf das Ziel Z04 „hohe Mitarbeiterzufriedenheit“ und den 
Indikator P07 „Anzahl Krankheitstage pro Mitarbeiter“ auf der Ebene des Gesamtunternehmens SIDL. Verantwortlich für die Zielerreichung ist die Unternehmensleitung, Die Ausprägung dieses Performance-Indikators auf dieser Granularitätsstufe erhalten sämtliche Mitarbeiter und Kader von SIDL AG. 


\begin{tabular}{|c|c|c|c|c|c|c|c|}
\hline \multirow{2}{*}{ 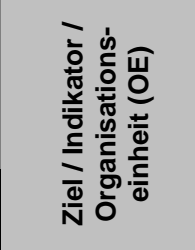 } & \multirow{2}{*}{ 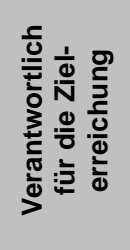 } & \multicolumn{6}{|c|}{ Empfänger } \\
\hline & & 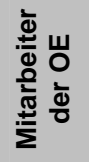 & 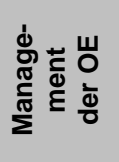 & 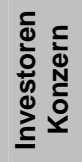 & 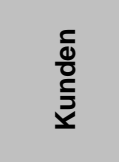 & 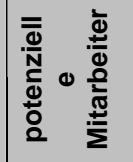 & 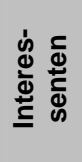 \\
\hline Z01.P01.SIDL & UL & & $x$ & $x$ & & & \\
\hline Z02.P02.SIDL & UL & & $x$ & $x$ & & & \\
\hline Z02.P03.SIDL & UL & & $x$ & $\mathrm{x}$ & & & \\
\hline Z03.P04.SIDL & UL & $x$ & $x$ & $x$ & $x$ & & $x$ \\
\hline Z04.P05.SIDL & UL & $\mathrm{x}$ & $x$ & & & $x$ & \\
\hline Z04.P06.SIDL & UL & $x$ & $x$ & & & $x$ & \\
\hline Z04.P07.SIDL & UL & $x$ & $x$ & & & & \\
\hline Z02.P03.AS & BL-AS & & $x$ & $\mathrm{x}$ & & & \\
\hline Z05.P08.AS & BL-AS & & $x$ & $x$ & & & \\
\hline Z06.P09.AS & BL-AS & & $x$ & $\mathrm{x}$ & & & \\
\hline Z07.P10.AS & BL-AS & & $x$ & $x$ & $x$ & & \\
\hline Z08.P11.AS & BL-AS & & $x$ & $\mathrm{x}$ & & & \\
\hline Z03.P04.AS & BL-AS & $x$ & $x$ & & $x$ & & $x$ \\
\hline Z04.P05.AS & BL-AS & $x$ & $x$ & & & & \\
\hline Z04.P06.AS & BL-AS & $x$ & $x$ & & & & \\
\hline Z04.P07.AS & BL-AS & $x$ & $x$ & & & & \\
\hline Z03.P04.SM & PM-SM & $x$ & $x$ & & X/Kunde & & \\
\hline Z04.P05.SM & PM-SM & & $x$ & & & & \\
\hline Z04.P06.SM & PM-SM & & $x$ & & & & \\
\hline Z04.P07.SM & PM-SM & & $x$ & & & & \\
\hline Z20.P20.S01 & $O D$ & $x$ & $x$ & $\mathrm{x}$ & & & \\
\hline Z21.P21.S01 & $\mathrm{OD}$ & $\mathrm{x}$ & $x$ & $x$ & & & \\
\hline
\end{tabular}

Legende:

UL Unternehmensleitung

BL-AS Bereichsleiter „Application Services”

PM-SM Prozessmanager „Support \& Maintenance”

SIDL Unternehmen SIDL AG

AS Bereich „Application Services”

SP Prozess „Support \& Maintenance”

OD Konzernentwicklung

Sxx Strategische Massnahme $x$

Zxx Ziel $x x$

Pxx Performance-Indikator $x$

Tabelle 9-7: Ausschnitt aus der Zuordnung der Verantwortlichen und der Informationsempfänger Performance-relevanter Daten der SIDL 


\subsubsection{Schritt 2: Gruppierung identischer Indikatoren}

Im zweiten Schritt werden die identischen Indikatoren gruppiert. Da es sich um einen ausschliesslich ordnenden Schritt handelt, soll dieser hier nicht dargestellt werden.

\subsubsection{Schritt 3: Bestimmen der notwendigen Dimensionen}

Im dritten Schritt werden die notwendigen Dimensionen bestimmt. Dabei werden die als generisch klassifizierten Dimensionen (vgl. Kapitel 9.4.3) bezüglich ihrer Relevanz geprüft. Zudem können in Brainstormings mit Anwendervertretern aus den Fachabteilungen weitere Dimensionen identifiziert werden. Das Ergebnis ist in Tabelle 9-8 dargestellt. Für die Eigenkapitalquote wurden bspw. die Dimensionen Zeit und Ziele identifiziert. Die Dimension Zeit wurde deshalb gewählt, weil die Informationsempfänger Auswertungen über diese Dimension (z.B. Veränderungen von Quartal zu Quartal) vornehmen möchten. Eine Verknüpfung mit der Organisation wurde nicht vorgenommen, da die Eigenkapitalquote nur für die SIDL AG als Ganzes berechnet werden kann. Sämtliche Indikatoren sind zudem mit der Zielhierarchie verknüpft.

\begin{tabular}{|c|c|c|c|c|c|c|c|c|c|c|}
\hline \multirow{3}{*}{\multicolumn{2}{|c|}{ Performance-Indikator }} & \multicolumn{9}{|c|}{ Dimensionen } \\
\hline & & \multicolumn{3}{|c|}{ generische } & \multicolumn{6}{|c|}{ spezifische } \\
\hline & & \multirow{2}{*}{$\begin{array}{l}\stackrel{\text { U. }}{\mathrm{N}} \\
\mathrm{X}\end{array}$} & \multirow{2}{*}{ 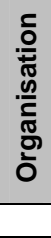 } & \multirow{2}{*}{$\begin{array}{l}\frac{0}{\frac{0}{N}} \\
X\end{array}$} & \multirow[t]{2}{*}{ 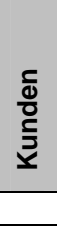 } & \multirow[t]{2}{*}{ 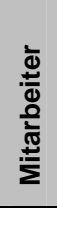 } & \multirow[t]{2}{*}{$\begin{array}{l}\frac{c}{d} \\
\frac{0}{0} \\
\frac{0}{d} \\
\frac{\pi}{\square} \\
\frac{\pi}{4}\end{array}$} & \multirow[t]{2}{*}{ } & \multirow[t]{2}{*}{ 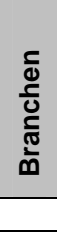 } & \multirow[t]{2}{*}{$\begin{array}{l}\frac{0}{\frac{\pi}{\sigma}} \\
\frac{0}{0} \\
\mathbb{d} \\
0\end{array}$} \\
\hline P01 & Eigenkapitalquote & & & & & & & & & \\
\hline P02 & Veränderung des relativen Marktanteils gegenüber Vorjahresquartal & $\mathrm{X}$ & & $\mathrm{X}$ & $\mathrm{X}$ & & & & & \\
\hline $\mathrm{P} 03$ & EBITA-Marge & $\mathrm{X}$ & $\mathrm{X}$ & $\mathrm{X}$ & & & & & & \\
\hline P04 & Kundenzufriedenheitsindex & $\mathrm{X}$ & $\mathrm{X}$ & $\mathrm{X}$ & $\mathrm{X}$ & & $\mathrm{X}$ & & $\mathrm{X}$ & \\
\hline P05 & Index der Mitarbeiterzufriedenheitsumfrage & $\mathrm{X}$ & $\mathrm{X}$ & $\mathrm{X}$ & & $\mathrm{X}$ & $\mathrm{X}$ & & & \\
\hline P06 & Investition in Mitarbeiterausbildung & $\mathrm{X}$ & $\mathrm{X}$ & $\mathrm{X}$ & & $\mathrm{X}$ & & & & \\
\hline $\mathrm{P} 07$ & Anzahl Krankheitstage pro Mitarbeiter & $\mathrm{x}$ & $\mathrm{x}$ & $\mathrm{X}$ & & $\mathrm{X}$ & & & & \\
\hline P08 & Aufträge in Arbeit (CHF) & $\mathrm{X}$ & & $\mathrm{X}$ & $\mathrm{X}$ & & & $\mathrm{X}$ & $\mathrm{X}$ & \\
\hline P09 & Fremdfinanzierungsgrad von Entwicklungen & $\mathrm{X}$ & & $\mathrm{X}$ & $\mathrm{X}$ & & & $\mathrm{X}$ & $\mathrm{X}$ & \\
\hline P10 & relativer Marktanteil der ERP-Lösung im relevanten Segment & $\mathrm{X}$ & & $\mathrm{X}$ & & & & & $\mathrm{X}$ & $\mathrm{X}$ \\
\hline P11 & mengenmässiger Anteil an Neuabschlüssen & $\mathrm{X}$ & & $\mathrm{X}$ & & & & & $\mathrm{X}$ & $\mathrm{X}$ \\
\hline $\mathrm{P} 20$ & Anteil des Working Capital am Umsatz & $\mathrm{X}$ & & $\mathrm{X}$ & & & & & & \\
\hline P21 & Rendite des Working Capital & $\mathrm{X}$ & & $\mathrm{X}$ & & & & & & \\
\hline
\end{tabular}

Tabelle 9-8: Performance-Indikatoren und mögliche Dimensionen

9.5.4 Schritt 4: Bestimmen der Granularitätsgrade je Indikator und Informationsempfänger

Im vierten Schritt werden für die einzelnen Performance-Indikatoren je Informationsempfänger und Dimension der Detaillierungsgrad bestimmt. Dies erfolgt 
mittels Befragung der Informationsempfänger einerseits und in Abstimmung mit dem Kommunikationskonzept der SIDL AG (vgl. Kapitel sechs; Schritt Festlegung der Informationsempfänger) andererseits. Einen wesentlichen Einfluss darauf, in welchem Detaillierungsgrad Informationen publiziert werden, hat die Kommunikationskultur des Unternehmens. Nachfolgend soll für den Kundenzufriedenheitsindex die Zuordnung detailliert besprochen werden.

Für den Kundenzufriedenheitsindex (P04) wurden bspw. die Mitarbeiter der Organisationseinheit (OE), das Management der OE, die Kunden und Interessenten der Firma als Informationsempfänger identifiziert. Dies bedeutet: Mitarbeiter und Management gehören im betrachteten Beispiel entweder der Organisation SIDL, der Business Unit AS oder dem Prozess SM an. Für die Dimension Zeit ist für die Mitarbeiter und das Management der Erhebungszeitpunkt der Umfrage von Interesse, für die Kunden und Interessierten werden hierzu Angaben auf Jahresebene gemacht. Auf der Dimension der Aufbauorganisation enthält der Kundenzufriedenheitsindex teilweise Informationen bis auf die Ebene Prozess. Mitarbeiter resp. Management, die diesem Prozess zugehörig sind oder diesem vorstehen, interessieren sich für diese Informationen. Mitarbeiter interessieren sich primär für ihren eigenen Bereich, das Management ist jedoch ebenfalls an Quervergleichen interessiert. Für Kunden und Interessierte werden Angaben auf der Ebene des Gesamtunternehmens gemacht. Der Indikator P04 ist mit dem Ziel Z03 verknüpft. Diese Information wird benötigt, um Ex-Post-Analysen der Wirkungszusammenhänge zwischen Indikatoren errechnen zu können.

Auf der Kundendimension werden Informationen zum Kundenzufriedenheitsindex P04 bis auf die Ebene einzelner Kunden von Management und Mitarbeitern gewünscht. Die Kunden können ihren eigenen Fragebogen sowie die aggregierten Werte sämtlicher Kunden einsehen. Interessierte erhalten Informationen auf der Ebene der aggregierten Werte. Für die Fragebogendimension erhalten das Management und die Mitarbeiter ebenfalls Detailinformationen bis auf die Ebene der einzelnen Fragen. Die Kunden können ihren eigenen Fragebogen bis auf die Ebene der einzelnen Fragen einsehen, für die restlichen jedoch nur die aggregierten Werte des Gesamtfragebogens (vgl. die Zelle (Spalte: Fragebogen; Zeile: P04-Kunden in der Tabelle 9-9): Dieser Sachverhalt ist vermerkt mit „Eigener: Frage; Andere: Alle Fragen“). Interessierte erhalten Informationen auf der Ebene der aggregierten Werte. Mitarbeiter und Management interessieren sich für 
die Werte der einzelnen Branchen. Die anderen Informationsempfänger erhalten hierzu keine detaillierten Informationen. 


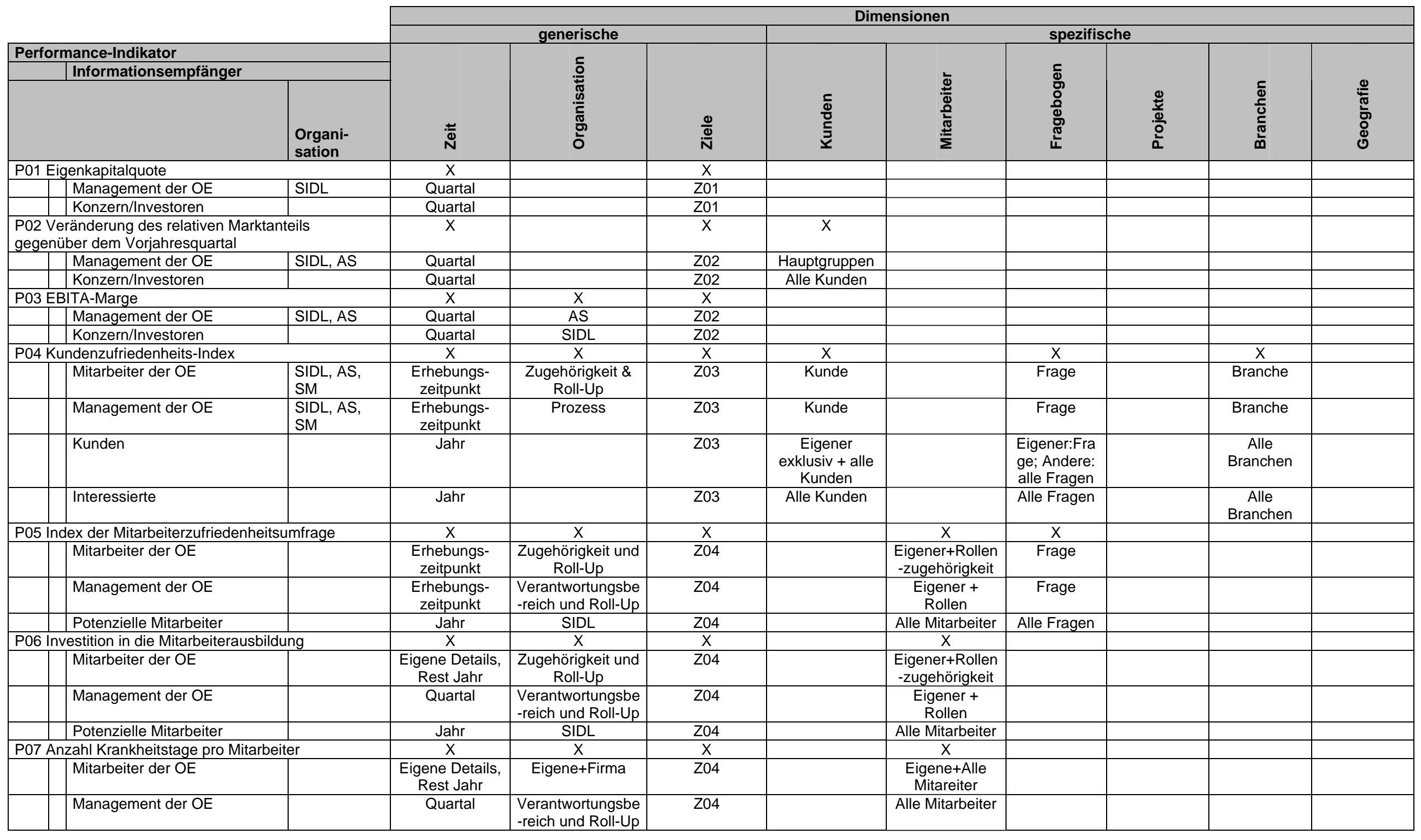




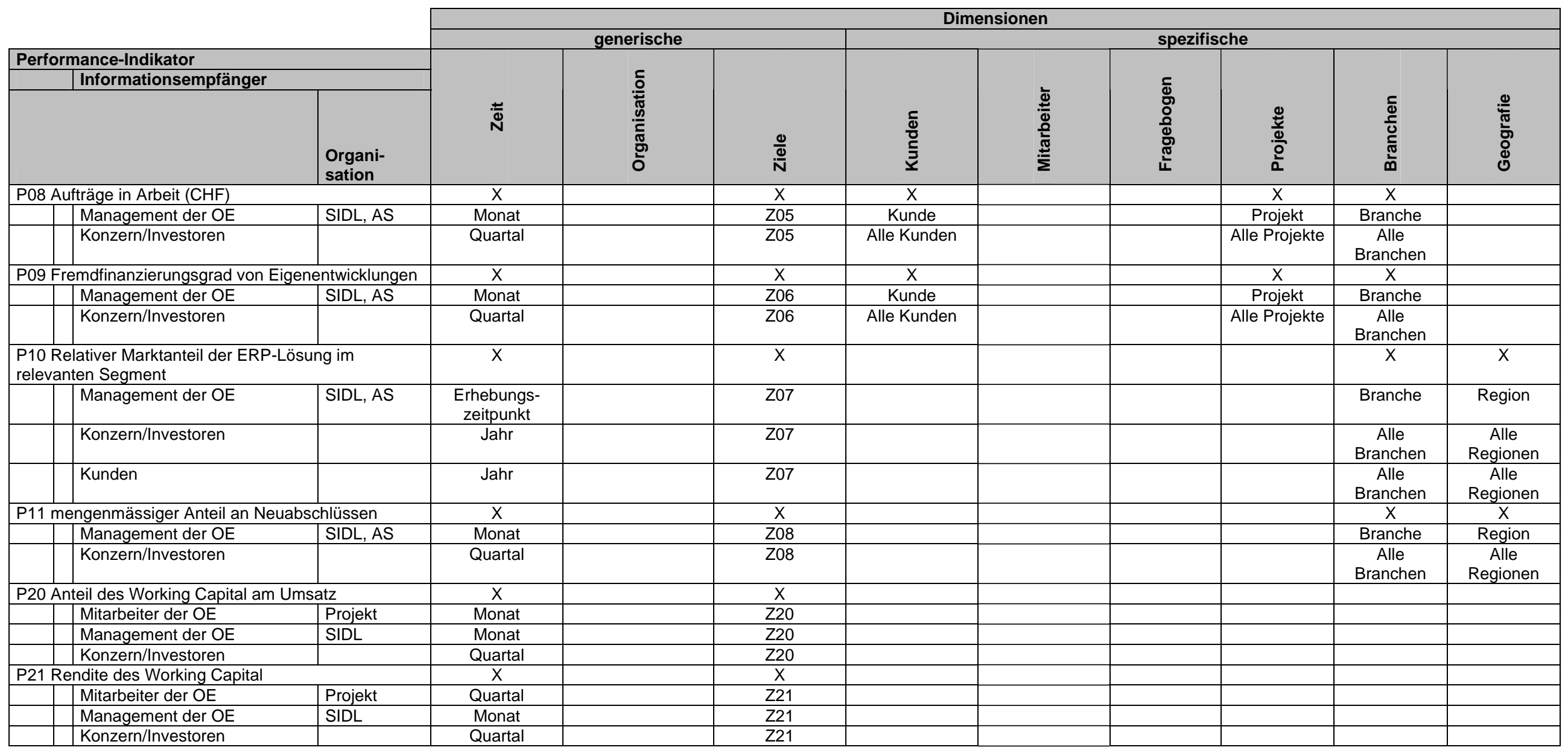

Tabelle 9-9: Granularitätsgrad je Indikator, Informationsempfänger und Dimension 
Diese Zuordnung je Informationsempfänger und Dimension wird für sämtliche Performance-Indikatoren in identischer Weise durchgeführt.

Als Ergebnis aus Schritt vier stehen für einzelne Dimensionen eine Reihe von möglichen Ausprägungen zur Verfügung.

\subsubsection{Schritt 5: Überprüfen des Aggregationspfades der einzelnen Dimensionen}

Die Dimensionen mit ihren identifizierten Ausprägungen werden im Schritt fünf bezüglich ihres Aggregationspfades untersucht. Tabelle 9-10 zeigt die erhobenen Aggregationsebenen je Dimension und dem daraus abgeleiteten Aggregationspfad. Dieser startet immer mit der Gesamtmenge der die Dimension umfassenden Elemente und wird in einer hierarchischen Beziehung detailliert, d.h. zwischen einem Element $\mathrm{n}$ und dem nachfolgenden Element $n+1$ besteht immer eine 1:n-Beziehung.

\begin{tabular}{|c|c|c|c|}
\hline $\mathrm{Nr}$ & Dimension & $\begin{array}{l}\text { Erhobene } \\
\text { Aggregationsebenen }\end{array}$ & Aggregationspfad \\
\hline 1 & Zeit & $\begin{array}{l}\text { Quartal, Erhebungszeitpunkte, } \\
\text { Jahr, Monat }\end{array}$ & Alle Tage $\rightarrow$ Jahr $\rightarrow$ Quartal $\rightarrow$ Monat $\rightarrow$ Tag \\
\hline 2 & $\begin{array}{l}\text { Aufbau- } \\
\text { organisation }\end{array}$ & $\begin{array}{l}\text { SIDL, AS, SM, Prozess, } \\
\text { Mitarbeiter }\end{array}$ & $\begin{array}{l}\text { Organisation } \rightarrow \text { Geschäftsbereich } \rightarrow \text { Prozess } \\
\rightarrow \text { Mitarbeiter }\end{array}$ \\
\hline 3 & Ziele & Z01 .. Z21 und OE & Alle Ziele $\rightarrow$ Zieltyp $\rightarrow$ Ziel \\
\hline 4 & Kunden & $\begin{array}{l}\text { Alle Kunden, Hauptgruppen, } \\
\text { Kunde }\end{array}$ & Alle Kunden $\rightarrow$ Kundengruppen $\rightarrow$ Kunde \\
\hline 5 & Mitarbeiter & $\begin{array}{l}\text { Alle Mitarbeiter, Rollen, } \\
\text { Mitarbeiter }\end{array}$ & Alle Mitarbeiter $\rightarrow$ Rollen $\rightarrow$ Mitarbeiter \\
\hline 6 & Fragebogen & Frage, alle Fragen & $\begin{array}{l}\text { Alle Fragen } \rightarrow \text { Fragebogen } \rightarrow \text { Fragengruppe } \\
\rightarrow \text { Frage }\end{array}$ \\
\hline 7 & Projekte & Alle Projekte, Projekt & Alle Projekte $\rightarrow$ Projektgruppe $\rightarrow$ Projekt \\
\hline 8 & Branchen & Alle Branchen, Branche & Alle Branchen $\rightarrow$ Branche \\
\hline 9 & Geografie & Alle Regionen, Region & Alle Regionen $\rightarrow$ Region \\
\hline
\end{tabular}

Tabelle 9-10: Aggregationspfade der Dimensionen

\subsubsection{Schritt 6: Verschmelzen redundanter Dimensionen}

Im sechsten Schritt werden die erhobenen Dimensionen bezüglich möglicher Optimierungen und Konsolidierungen untersucht. Das Ziel besteht darin, die Anzahl der Dimensionen möglichst klein und deren Ausgestaltung offen zu halten. Das Vorgehen wird nachfolgend am Beispiel des Attributes Mitarbeiter erläutert. In der Dimension zwei wurde der Mitarbeiter als zu einem Prozess zugehörig identifiziert. Gleichzeitig existiert eine 
eigenständige Dimension Mitarbeiter. Die Optimierung erfolgt nun dahingehend, dass die aufbauorganisatorische Zugehörigkeit als beschreibendes Attribut auf der Ebene des einzelnen Mitarbeiters in der Mitarbeiterdimension geführt und damit in der Dimension Aufbauorganisation obsolet wird. Gleichzeitig kann die Dimension Aufbauorganisation an Stelle der Mitarbeiter um Aktivitäten ergänzt werden. Damit wird es möglich, diese Dimension für eine Prozesskennzahl (z.B. Durchlaufzeit) heranzuziehen (ausserhalb des Beispiels). Zusätzlich werden die Dimensionen Branche und Geografie mit der Kundendimension verknüpft, da beide in direktem Zusammenhang zum Kunden stehen. Es entsteht somit kein Informationsverlust, und die Zahl der Dimensionen kann um zwei reduziert werden. Das Ergebnis der Bereinigung ist in Tabelle 9-12 dargestellt.

Zur einfacheren späteren Bezugnahme wird die Dimension mit Grossbuchstaben, die einzelnen Hierarchieebenen mit Kleinbuchstaben referenziert. [A.e] bezeichnet somit die Dimension Zeit (A) mit dem Detaillierungsgrad Tag (e).

\begin{tabular}{|l|l|l|}
\hline $\mathrm{Nr}$ & Dimension & Aggregationspfade \\
\hline 2 & Aufbauorganisation & Organisation $\rightarrow$ Geschäftsbereich $\rightarrow$ Prozess $\rightarrow$ Aktivität \\
$4+8+9$ & Kunden & $\begin{array}{l}\text { Alle Kunden } \rightarrow \text { Kundengruppen } \rightarrow \text { Kunde } \\
\text { Alle Kunden } \rightarrow \text { Branchen } \rightarrow \text { Kunde } \\
\text { Alle Kunden } \rightarrow \text { Regionen } \rightarrow \text { Kunde }\end{array}$ \\
\hline
\end{tabular}

Tabelle 9-11: Auf den Dimensionen ausgeführte Aktionen

\begin{tabular}{|c|c|c|}
\hline $\mathrm{Nr}$ & Dimension & Aggregationspfade \\
\hline A & Zeit & (a) Alle Tage $\rightarrow$ (b) Jahr $\rightarrow$ (c) Quartal $\rightarrow$ (d) Monat $\rightarrow$ (e) Tag \\
\hline B & Aufbauorganisation & (a) Organisation $\rightarrow$ (b) Geschäftsbereich $\rightarrow$ (c) Prozess $\rightarrow$ (d) Aktivität \\
\hline C & Ziel-Indikatoren & (a) Alle Ziele $\rightarrow$ (b) Zieltyp $\rightarrow$ (c) Ziel \\
\hline \multirow[t]{3}{*}{ D } & Kunden & (a) Alle Kunden $\rightarrow$ (b) Kundengruppen $\rightarrow$ (c) Kunde \\
\hline & & (a) Alle Kunden $\rightarrow$ (d) Branchen $\rightarrow$ (c) Kunde \\
\hline & & (a) Alle Kunden $\rightarrow$ (e) Regionen $\rightarrow$ (c) Kunde \\
\hline $\mathrm{E}$ & Mitarbeiter & $\begin{array}{l}\text { (a) Alle Mitarbeiter } \rightarrow \text { (b) Rollen } \rightarrow \text { (c) Mitarbeiter [aufbauorg. Zugehörigkeit] } \\
\text { (a) Alle Fragen } \rightarrow \text { (b) Fragebogentyp } \rightarrow \text { (c) Fragebogen } \rightarrow \text { (d) Fragengruppe }\end{array}$ \\
\hline$F$ & Fragebogen & $\rightarrow(\mathrm{e})$ Frage \\
\hline G & Projekte & (a) Alle Projekte $\rightarrow$ (b) Projektgruppe $\rightarrow$ (c) Projekt \\
\hline
\end{tabular}

Tabelle 9-12: Ergebnis der bereinigten Dimensionen

Damit ist die Erstellung der Dimensionen abgeschlossen. 


\subsubsection{Schritt 7: Bestimmen des höchsten Detaillierungsgrades pro Indikator}

Basierend auf den bereinigten Dimensionen und den Ergebnissen aus Schritt vier wird im Arbeitsschritt sieben für jeden Performance-Indikator der höchste Detaillierungsgrad je Dimension bestimmt. Damit wird der Datenwürfel je Performance-Indikator eindeutig definiert.

Für die Eigenkapitalquote (P01) wurden die Dimensionen Zeit und Ziel identifiziert. Da die Eigenkapitalquote quartalsweise erhoben wird, kann ein (c) eingetragen werden. Die Verknüpfung zu den Zielen erfolgt auf der Ebene der einzelnen Ziele (C). Das Ergebnis für sämtliche betrachteten Indikatoren ist in Tabelle 9-13 dargestellt.

\begin{tabular}{|c|c|c|c|c|c|c|c|}
\hline & & & & & & & \\
\hline & \multicolumn{7}{|c|}{ Dimensionen } \\
\hline & $\stackrel{+}{\mathbb{N}}$ & 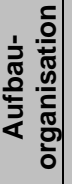 & $\frac{0}{\frac{N}{N}}$ & $\begin{array}{l}\frac{c}{d} \\
\frac{d}{c} \\
\frac{I}{J} \\
\underline{y}\end{array}$ & 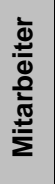 & 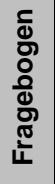 & 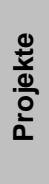 \\
\hline Performance-Indikator & A & B & C & D & E & $\mathbf{F}$ & G \\
\hline P01 Eigenkapitalquote & $\mathrm{c}$ & - & $\mathrm{c}$ & - & - & - & - \\
\hline P02 Veränderung des relativen Marktanteils gegenüber dem Vorjahresquartal & $\mathrm{c}$ & - & $\mathrm{c}$ & $\mathrm{b}$ & - & - & - \\
\hline P03 EBITA-Marge & $\mathrm{c}$ & $\mathrm{b}$ & $\mathrm{c}$ & - & - & - & - \\
\hline P04 Kundenzufriedenheits-Index & $\mathrm{e}$ & $\mathrm{c}$ & $\mathrm{c}$ & $\mathrm{c}$ & - & $\mathrm{e}$ & - \\
\hline P05 Index der Mitarbeiterzufriedenheitsumfrage & $\mathrm{e}$ & $\mathrm{c}$ & $\mathrm{c}$ & - & $c$ & $\mathrm{e}$ & - \\
\hline P06 Investition in Mitarbeiterausbildung & $\mathrm{e}$ & c & $\mathrm{c}$ & - & c & - & - \\
\hline P07Anzahl Krankheitstage pro Mitarbeiter & $\mathrm{e}$ & c & $\mathrm{c}$ & - & c & - & - \\
\hline P08Aufträge in Arbeit (CHF) & $d$ & - & $\mathrm{c}$ & $\mathrm{c}$ & - & - & $\mathrm{C}$ \\
\hline P09 Fremdfinanzierungsgrad von Entwicklungen & d & - & $\mathrm{c}$ & $\mathrm{c}$ & - & - & $\mathrm{C}$ \\
\hline P10 relativer Marktanteil der ERP-Lösung im relevanten Segment & $\mathrm{e}$ & - & $\mathrm{c}$ & $\mathrm{d} / \mathrm{e}$ & - & - & - \\
\hline P11 mengenmässiger Anteil an Neuabschlüssen & d & - & $\mathrm{c}$ & $\mathrm{d} / \mathrm{e}$ & - & - & - \\
\hline P20 Anteil des Working Capital am Umsatz & $\mathrm{d}$ & - & $\mathrm{c}$ & - & - & - & - \\
\hline P21 Rendite des Working Capital & $\mathrm{d}$ & - & $\mathrm{C}$ & - & - & - & - \\
\hline
\end{tabular}

Tabelle 9-13: Höchster Detaillierungsgrad je Indikator und Dimension.

\subsubsection{Schritt 8: Konsolidierung der Indikatoren mit gleichen Dimensionen und gleicher Granularität}

Indikatoren mit gleicher Granularität bezüglich sämtlicher Dimensionen können in einem Datenwürfel zusammengefasst werden. Dies wird im achten Schritt realisiert. Damit kann die Zahl der Datenwürfel und die Komplexität reduziert werden. Zudem erlaubt es direkte Vergleiche von Performance-Indikatoren in den gleichen Datenwürfeln. 
Wie aus Tabelle 9-14 ersichtlich ist, können P06 und P07, P08 und P09 sowie P20 und P21 je in einen Würfel zusammengefasst werden. Bei praxisorientierten Anwendungen, in welchen eine wesentlich grössere Anzahl von Performance-Indikatoren bearbeitet werden, steigt erfahrungsgemäss die Zahl der realisierbaren Konsolidierungen mit der Zahl der Performance-Indikatoren überproportional an.

\begin{tabular}{|c|c|c|c|c|c|c|c|c|c|}
\hline & & & & & & & \\
\hline & & & \multicolumn{7}{|c|}{ Dimensionen } \\
\hline & & & \multirow{2}{*}{$\begin{array}{l}\text { ․ㅡㅁ } \\
\text { A }\end{array}$} & \multirow{2}{*}{ 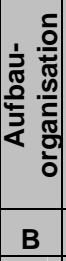 } & \multirow{2}{*}{$\begin{array}{l}\frac{0}{\frac{D}{N}} \\
\text { C }\end{array}$} & \multirow{2}{*}{ 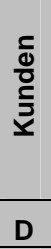 } & \multirow{2}{*}{ 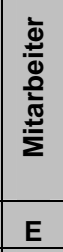 } & \multirow{2}{*}{ 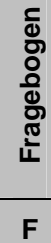 } & \multirow{2}{*}{ 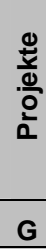 } \\
\hline & & & & & & & & & \\
\hline Würfel & \multicolumn{2}{|c|}{ Performance-Indikator } & & & & & & & \\
\hline 1 & P01 & Eigenkapitalquote & $\mathrm{c}$ & - & c & - & - & - & - \\
\hline 2 & P02 & Veränderung des relativen Marktanteils gegenüber dem Vorjahresquartal & $\mathrm{c}$ & - & $\mathrm{c}$ & $\mathrm{b}$ & - & - & - \\
\hline 3 & P03 & EBITA-Marge & $\mathrm{c}$ & $\mathrm{b}$ & $\mathrm{c}$ & - & - & - & - \\
\hline 4 & P04 & Kundenzufriedenheits-Index & $\mathrm{e}$ & $\mathrm{c}$ & $\mathrm{c}$ & $\mathrm{c}$ & - & $\mathrm{e}$ & - \\
\hline 5 & P05 & Index der Mitarbeiterzufriedenheitsumfrage & $\mathrm{e}$ & c & $\mathrm{c}$ & - & $\mathrm{c}$ & $\mathrm{e}$ & - \\
\hline \multirow[t]{2}{*}{6} & P06 & Investition in Mitarbeiterausbildung & e & c & c & - & c & - & - \\
\hline & P07 & Anzahl Krankheitstage pro Mitarbeiter & e & c & c & - & c & - & - \\
\hline \multirow[t]{2}{*}{7} & P08 & Aufträge in Arbeit (CHF) & d & 3 & c & c & 3 & 3 & c \\
\hline & P09 & Fremdfinanzierungsgrad von Entwicklungen & d & 3 & c & c & 3 & 3 & c \\
\hline 8 & P10 & relativer Marktanteil der ERP-Lösung im relevanten Segment & $\mathrm{e}$ & - & $\mathrm{c}$ & $\mathrm{d} / \mathrm{e}$ & - & - & - \\
\hline 9 & P11 & mengenmässiger Anteil an Neuabschlüssen & $\mathrm{d}$ & - & $\mathrm{c}$ & $\mathrm{d} / \mathrm{e}$ & - & - & - \\
\hline \multirow[t]{2}{*}{10} & $\mathrm{P} 20$ & Anteil des Working Capital am Umsatz & $d$ & - & $\mathrm{c}$ & - & - & - & - \\
\hline & P21 & Rendite des Working Capital & $\mathrm{d}$ & - & $\mathrm{c}$ & - & - & - & - \\
\hline
\end{tabular}

Tabelle 9-14: Konsolidierungen der Indikatoren mit gleichen Dimensionen und gleicher Granularität

\subsubsection{Schritt 9: Festlegen von Aggregationsbeschränkungen der Performance- Indikatoren}

In Schritt neun werden allfällige Aggregationsbeschränkungen festgelegt, wie dies in Kapitel 9.4.9 aufgezeigt wurde. Dieser Schritt muss im Rahmen der konzeptionellen Modellierung erfolgen, da technisch sämtliche Operationen möglich sind, praktisch jedoch keinen Sinn ergeben. Für das vorliegende Beispiel soll dies am Performance-Indikator „Aufträge in Arbeit“ (P08) dargestellt werden (vgl. Tabelle 9-15). Eine Summierung über die Zeit ist nicht zulässig, da es sich um einen Bestandeswert handelt. Das Bilden eines Mittelwertes, das Bestimmen eines Minimum oder Maximum in einer bestimmten Zeitperiode ist jedoch zulässig. Auch das Bestimmen von Streumassen und das Zählen sind 
möglich. Daher wird ein Aggregationsgrad von zwei eingetragen. Das Summieren über den Kunden zu einem bestimmten Zeitpunkt ist jedoch sinnvoll, deshalb wird hier ein Aggregationsgrad von eins eingetragen.

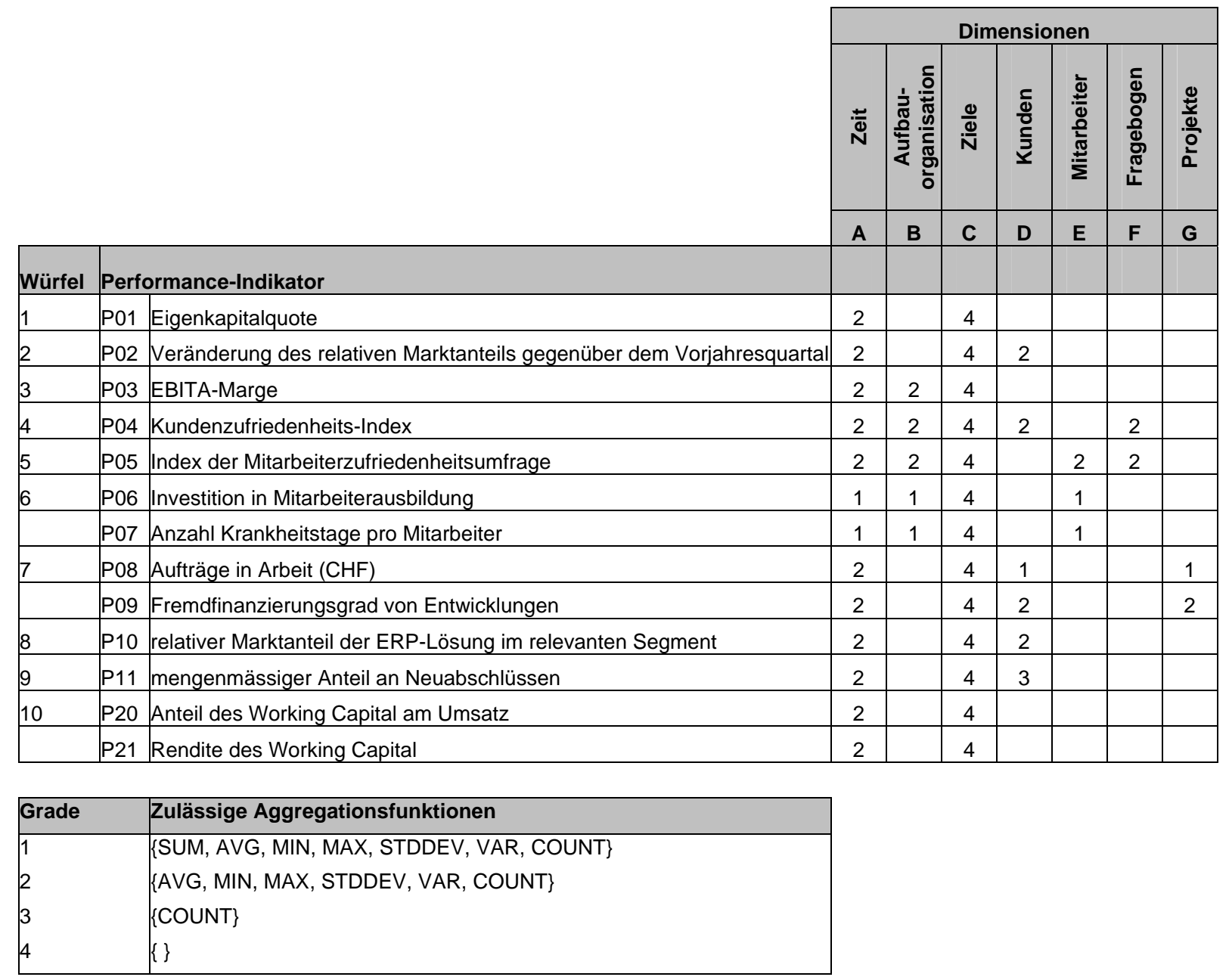

Tabelle 9-15: Aggregationsgrade der Indikatoren

\subsubsection{Schritt 10: Festlegen von Standardzugriffspfaden und Berechtigungen je Informationsempfänger}

Im zehnten Schritt wird der Standardzugriffspfad auf einen Datenwürfel je Informationsempfänger und die Berechtigungen festgelegt. Der Standardzugriff auf eine Kennzahl ist je Informationsempfänger unterschiedlich. Die Berechtigungen werden als Positiv-Liste aufgenommen. Dies bedeutet, dass nur zugänglich ist, was explizit freigegeben wurde. In den Spalten sind die einzelnen Dimensionen (mit Grossbuchstaben referenziert, siehe Tabelle 9-12), jeweils nach Standardzugriff (S) und Berechtigungen (Z) aufgeführt. In den Zeilen werden pro Indikator und Informationsempfänger die Aggregationsebenen festgelegt. 


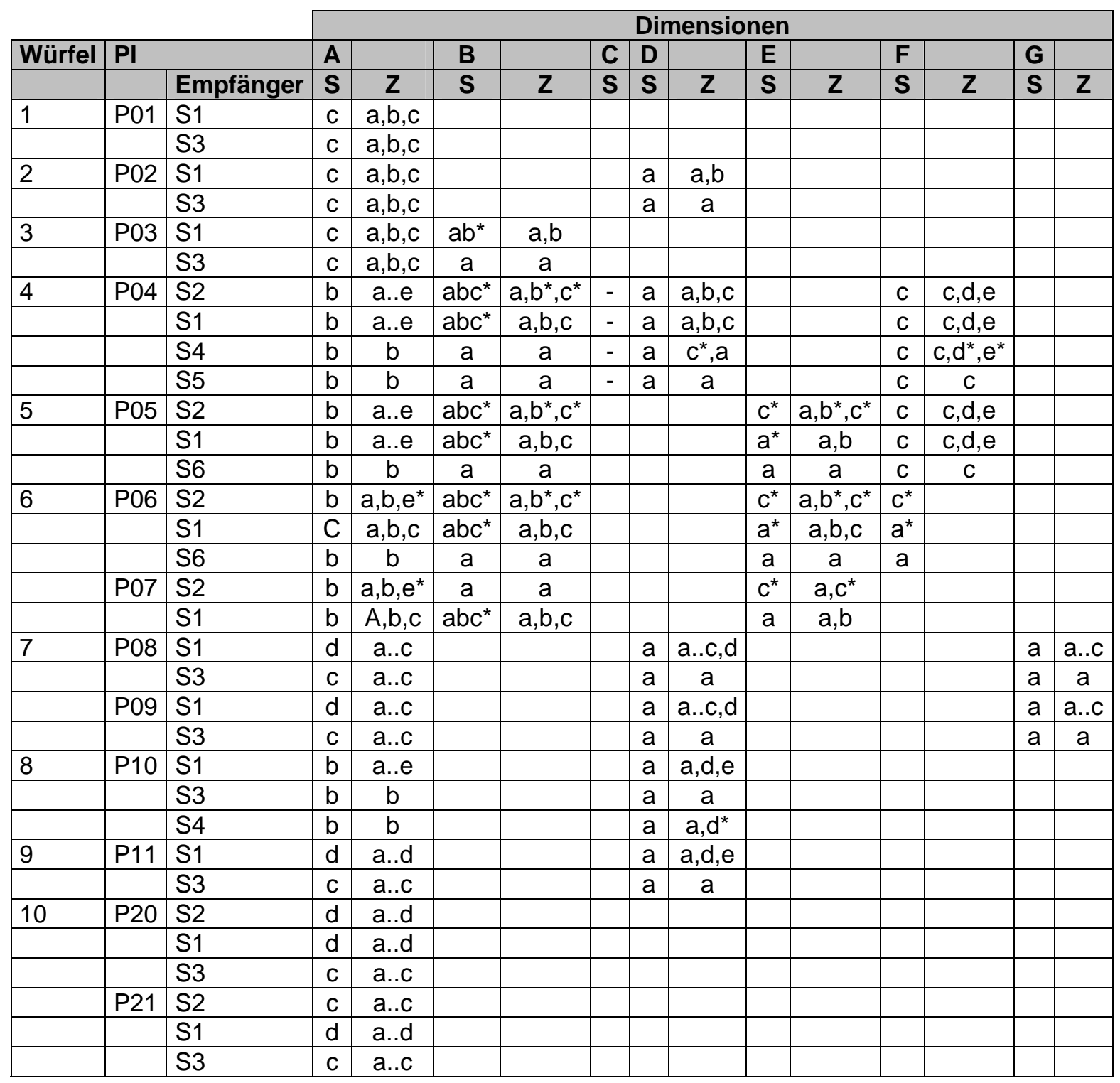

\begin{tabular}{|l|l|}
\hline \multicolumn{2}{|l|}{ Legende: } \\
\hline$S$ & Standardzugriff-Liste \\
\hline$Z$ & Berechtigung (Positivliste) \\
\hline$\star$ & für eigene Organisationseinheit oder sich selbst \\
\hline S1 & Management \\
\hline S2 & Mitarbeiter \\
\hline S3 & Konzern/Investoren \\
\hline S4 & Kunden \\
\hline S5 & Interessierte \\
\hline S6 & potenzielle Mitarbeiter \\
\hline
\end{tabular}

Tabelle 9-16: Festlegen von Standardzugriffspfaden und Berechtigungen je Informationsempfänger 


\subsection{Ansätze zur Modellierung von multidimensionalen Datenstrukturen}

Die hier vorgestellten zehn Schritte werden ohne spezifische Notation dargestellt. Meist kommen einfache Tabellen zum Einsatz. Um das Ergebnis mit den Benutzern und den Auftraggebern diskutieren zu können, empfiehlt sich eine grafische Repräsentation des Datenmodells einerseits und eine prototypische Implementierung andererseits. Die prototypische Implementierung wird im Kapitel zehn besprochen. Nachfolgend wird ein möglicher Ansatz zur Modellierung vorgestellt.

\subsubsection{Differenzierung nach Entwurfsebenen}

Wie in Kapitel 9.2.4 erwähnt, können mehrere Entwurfsebenen zur Modellierung differenziert werden. Für die einzelnen Entwurfsebenen kommen unterschiedliche Ansätze zur Anwendung (vgl. Tabelle 9-17).

\begin{tabular}{|c|c|}
\hline Entwurfsebene & Ansätze \\
\hline Semantische & $\begin{array}{ll}\text { - } & \text { Application Design for Analytical Processing Technologies (ADAPT) (vgl. } \\
\text { Bulos (1996)) } \\
\text { - } & \text { Modifizierte Objekttypenmethode (vgl. Rautenstrauch (1997)) } \\
\text { - } & \text { Multidimensionales Entity-Relationship Model (vgl. Sapia et al. (1998)) } \\
\text { - } & \text { Anwendung der Object Modeling Technique (vgl. Holthuis (1999)) } \\
\text { - } & \text { Semantisches Data Warehouse-Modell (SDWM) (vgl. Böhnlein und Ulbrich- } \\
& \text { vom-Ende (2001)) }\end{array}$ \\
\hline Logische & $\begin{array}{ll}\text { - } & \text { Dimension Modelling (vgl. Kimball (1996)) } \\
\text { - } & \text { Star Schema (vgl. Poe et al. (1997)) } \\
\text { - } & \text { Snowflake Schema (vgl. Kimball (1996)) } \\
\text { - } & \text { Dimensional Fact Model (vgl. Golfarelli et al. (1998)) }\end{array}$ \\
\hline Physische & $\begin{array}{l}\text { - Auf der physischen Ebene stehen vermehrt technische Aspekte zur } \\
\text { Diskussion. Dazu gehören z.B. Speicherstrukturen und Indexierung. Diese } \\
\text { Aspekte weren stark durch das eingesetzte Produkt determiniert. }\end{array}$ \\
\hline
\end{tabular}

Tabelle 9-17: Entwurfsebenen und diskutierte Ansätze

Für die Darstellung des Ergebnisses des Vorgehensmodells wird ein semantisches Datenmodell benötigt, da dieses noch unabhängig von der konkreten Implementierung ist. Zudem ist die logische Modellierung ungeeignet für die Diskussion mit den Fachabteilungen (vgl. Böhnlein und Ulbrich-vom-Ende (2001)). 


\subsubsection{Modellierungsansätze der semantischen Entwurfsebene}

Die in Tabelle 9-17 genannten Ansätze lassen sich in drei Gruppen einteilen:

- Eine erste Gruppe basiert auf der klassischen ER-Modellierung (vgl. Entity Relationship Model von Chen (1976)). Hierzu gehört beispielweise das multidimensionale Entity-Relationship Model von Sapia et al. (1998), welcher die Grundidee von Chen um einige Elemente zur multidimensionalen Darstellung ergänzt. Die Stärke dieser Ansätze kann darin gesehen werden, dass der Erfahrungsschatz der klassischen Datenmodellierung genutzt werden kann und die Kommunikation zwischen Datenarchitekten von OLTP- und OLAP-Systemen gefördert wird.

- Eine zweite Gruppe basiert auf objektorientierten Ansätzen (Modifizierte Objekttypenmethode, Anwendung der Object Modeling Technique). Ein Vertreter dieser Richtung ist Holthuis (1999). Er evaluiert objektorientierte Konstrukte zur Abbildung multidimensionaler Elemente wie Klassen- und Zustandsdiagramme sowie funktionale Modelle. Analog zur Architektur integrierter Informationssysteme (vgl. ARIS; Scheer (2001)) werden auf einer Makroebene Daten-, Funktions-, Organisations- und Geschäftsprozesssicht differenziert. Die Mikroebene ist innerhalb der Datensicht angesiedelt und dient der Definition von statischen Strukturen, Funktionen und Verhalten.

- Eine dritte Gruppe ist weitgehend unabhängig von bestehenden Ansätzen und wurde spezifisch für die multidimensionale Datenmodellierung entworfen (ADAPT-Ansatz von Bulos (1996), Ansatz von Kurz (1999), SDWM-Ansatz von Böhnlein und Ulbrich-vom-Ende (2001)).

In der vorliegenden Arbeit wurde der ADAPT-Ansatz von Bulos (1996) verwendet. In der engeren Auswahl wurden die Ansätze von folgenden Autoren berücksichtigt: Kurz (1999), Böhnlein und Ulbrich-vom-Ende (2001) und Bulos (1996).

- Der Ansatz von Kurz (1999) ist primär mit einem Hersteller (Microstrategy) verknüpft. Es existieren nach Wissen des Verfassers keine englischsprachigen Publikationen zum Ansatz. Er umfasst relativ wenig verschiedene Elemente. Dazu gehören die Dimension, die Kennzahlengruppe, die Kennzahl, das Hierarchieobjekt und der Aggregationspfad. Die Stärke dieses Ansatzes liegt in der leichten 
Lesbarkeit der Modelle. Eine sehr ähnliche multidimensionale Modellierungstechnik wurde von IBM (1998) vorgeschlagen. Der Ansatz wurde nicht näher verfolgt.

- Der Ansatz von Böhnlein und Ulbrich-vom-Ende (2001) ist neueren Datums. Die Reaktionen der wissenschaftlichen Community und der Praxis bleiben abzuwarten. Er wurde daher nicht berücksichtigt.

- Demgegenüber datiert der Ansatz von Bulos aus dem Jahre 1996. Er wurde in mehreren internationalen Publikationen aufgegriffen. Der Autor ist mit der Praxis (Symmetry Corp.) assoziiert. Die Stärke dieses Ansatzes kann darin gesehen werden, dass er sehr ausdrucksstark ist und von Nicht-Informatikern gut verstanden wird. Dieser Ansatz wird nachstehend detaillierter beschrieben.

\subsubsection{ADAPT}

ADAPT steht für Application Design for Analytical Processing Technologies. Der ADAPT-Ansatz von Bulos (1996) wurde speziell für die multidimensionale Datenstrukturierung entworfen und findet ihren Ursprung in der Unternehmensberatungspraxis. Motiviert wurde die Entwicklung durch die Unzulänglichkeit traditioneller Modellierungstechniken. (vgl. Bulos (1996))

Werden sämtliche von Bulos vorgeschlagenen Notationselemente benutzt, so kann der Ansatz nicht mehr nur der semantischen Ebene zugeordnet werden. Es existieren auch Elemente, die der logischen und physikalischen Ebene zuzuordnen sind.

Die grundsätzlichen Notationselemente von ADAPT bestehen aus (1) dem Würfel (Hypercube), (2) der Dimension (Dimension) und (3) der Formel (Model), welche auch als Ableitungsregel bezeichnet wird. Abbildung 9-4 zeigt, wie die drei Kernelemente grafisch dargestellt werden.

Würfel

(Hypercube)

Dimension1 Dimension2

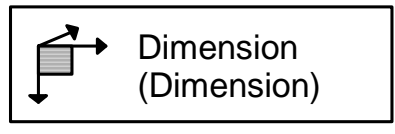

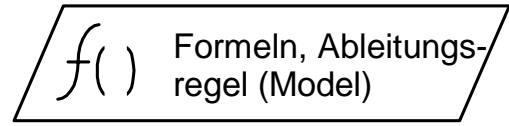


Im Zentrum steht der Hypercube, der die Basis für jede Performance-Indikatorengruppe bildet. Im oberen Teil des Elementes wird die Performance-Indikatorengruppe benannt, und im unteren Teil werden die zugehörigen Dimensionen eingetragen. Es ist zu erwähnen, dass Bulos in seiner ursprünglichen Publikation für jede Kennzahl einen eigenen Würfel vorsah. In Anlehnung an Totok und Jaworski (1998) wird von diesem Vorgehen im Sinne einer Vereinfachung abgewichen und nur ein Würfel pro Performance-Indikatorengruppe vorgesehen.

Das Dimensionselement (vgl. Abbildung 9-4 Mitte) entspricht der wortgleichen Dimension. Bulos beschreibt insgesamt sechs Dimensionstypen (vgl. Bulos (1996)). Diese Differenzierung wird hier jedoch nicht vorgenommen, da sich die einzelnen Dimensionstypen teilweise nicht eindeutig klassifizieren lassen ${ }^{16}$. Wichtig ist jedoch die Unterscheidung zwischen aggregierenden Dimensionen und Kennzahlendimensionen (vgl. Abbildung 9-5). Aggregierende Dimensionen lassen sich vertikal in Ebenen untergliedern. Die einzelnen Ebenen bilden einen zusammenhängenden Verdichtungsweg. Die Daten der untersten Dimensionsebene besitzen die höchste Detaillierung. Die Granularität der Daten nimmt vertikal nach oben zu. Die aggregierende Dimension wird mit dem Dimensionselement von Bulos dargestellt (vgl. Abbildung 9-5). Kennzahlendimensionen werden für die Performance-Indikatoren (Fakten) genutzt.

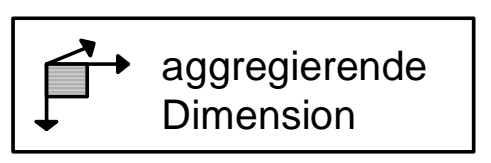

\section{Abbildung 9-5: Dimensionstypen von ADAPT}

Im ADAPT-Ansatz werden vier Dimensionselemente unterschieden (siehe Abbildung 9-6). Eine Dimension kann mehrere Hierarchien beinhalten. Eine Hierarchieebene beschreibt die Position eines Elementes innerhalb einer Hierarchie. Dimensionswerte stehen demgegenüber in keinem hierarchischen Zusammenhang (vgl. meist werden die Kennzahlen mit Dimensionswerten dargestellt, da die Kennzahlendimension in sich keine Hierarchie aufweist.). Mit Dimensionsattributen lassen sich Hierarchieebenen näher

16 In einer neueren Publikation verzichtet Bulos auf die Darstellung der verschiedenen Dimensionen (vgl. Bulos und Forsman (1998)). 
beschreiben. Sollten Performance-Daten für Geschäftsprozesse ausgewertet werden, so könnte bspw. ein Dimensionsattribut „Geschäftsprozessmanager“ lauten. Dies würde es ermöglichen, Auswertungen nicht nur nach Geschäftsprozessnamen, sondern ebenfalls nach den Namen der Geschäftsprozessmanager vornehmen zu können.
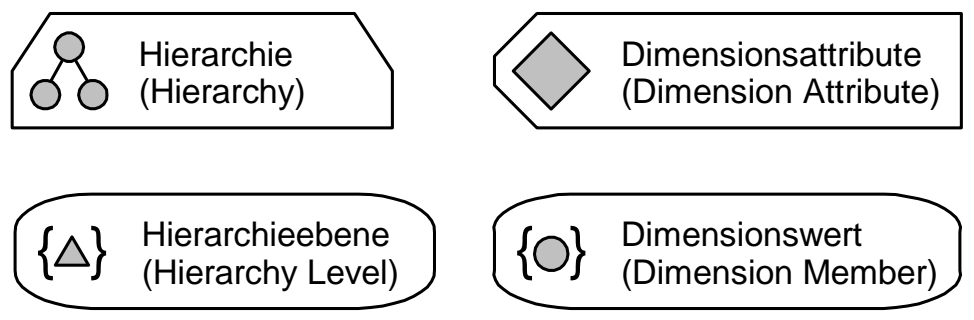

\section{Abbildung 9-6: Dimensionselemente von ADAPT}

Kennzahlen können originär oder abgeleitet sein. Um bei abgeleiteten Kennzahlen die angewendete Formel im multidimensionalen Datenmodell zum Ausdruck zu bringen, können Formeln genutzt werden (vgl. Abbildung 9-7 rechts unten). In Abweichung zum Vorschlag von Bulos wurde vom Verfasser die Kennzahlen-Dimension mit dem ursprünglichen Symbol des Massstabes belassen, da sich dieses bei verschiedenen Diskussionen mit Nicht-IT-Fachleuten bewährt hat.

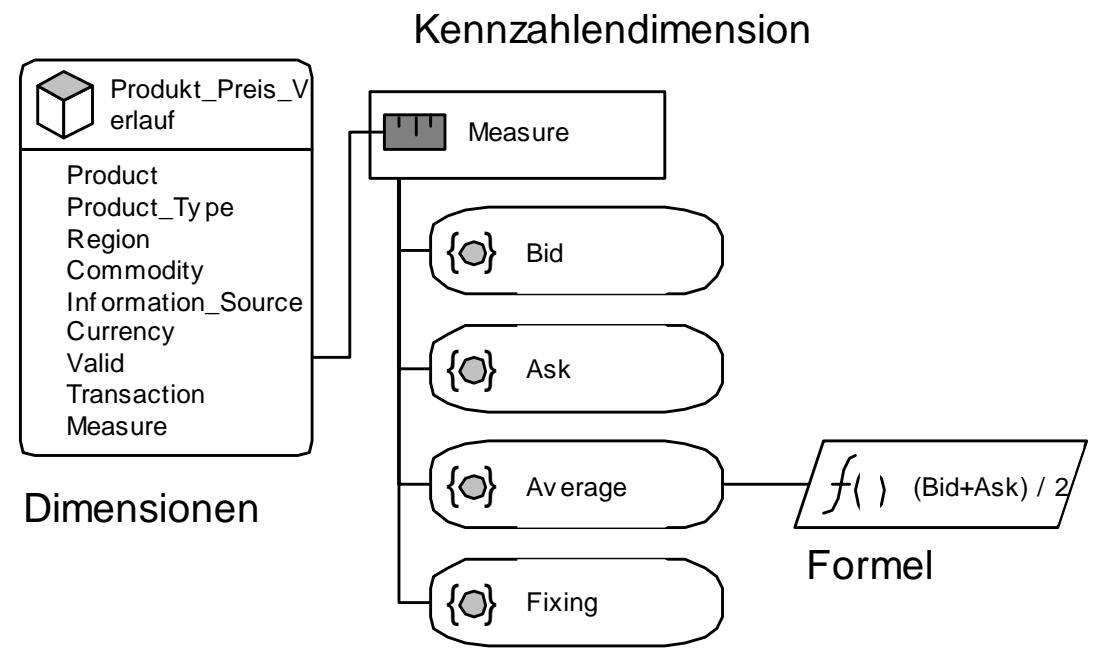

Fakten

Abbildung 9-7: Berechnungen im multidimensionalen Datenmodell mittels Formeln (Beispiel in Anlehnung an Felden und Florek (2001)) 
Bulos (1996) stellt eine ganze Reihe weiterer Elemente vor. Dazu gehören Beziehungstypen, weitere Dimensionselemente und, wie erwähnt, insgesamt sechs Dimensionstypen. Auf die Darstellung weiterer Elemente soll hier jedoch verzichtet werden. Die Firma Symmetry Corporation bietet die Elemente als Schablone für Microsoft Visio an. Seit der ersten Version 1.1. wurde der Umfang verkleinert. In der Version 2.0 fehlten die verschiedenen Dimensionstypen. Dies wurde auch in der aktuellen Version 3.0 beibehalten (vgl. www.symcorp.com).

\subsection{Darstellung des Fallbeispiels anhand der vorgestellten Notation}

Das in Kapitel 9.5 erarbeitete Ergebnis (primär Informationen aus Tabelle 9-12 und Tabelle 9-14) wird abschliessend noch in der in Kapitel 9.6.3 vorgestellten ADAPTNotation dargestellt. Dabei ist zu berücksichtigen, dass nicht alle erarbeiteten Informationen im semantischen Datenmodell Platz finden. Eine Reihe von Daten (z.B. Berechtigungen, Zugriffspfade) bleiben in Tabellenform und werden bei der konkreten Implementierung verwendet.

Mit den erarbeiteten Informationen des semantischen Datenmodells kann dieses auf die logische Ebene transformiert und auf einer Datenbank implementiert werden.

Das in Abbildung 9-8 dargestellte Datenmodell zeigt aus Gründen der Vereinfachung keine grafischen Verbindungen zwischen den Würfeln und den assoziierten Dimensionen. Diese sind im unteren Teil des Würfels jeweils in Textform aufgeführt. 


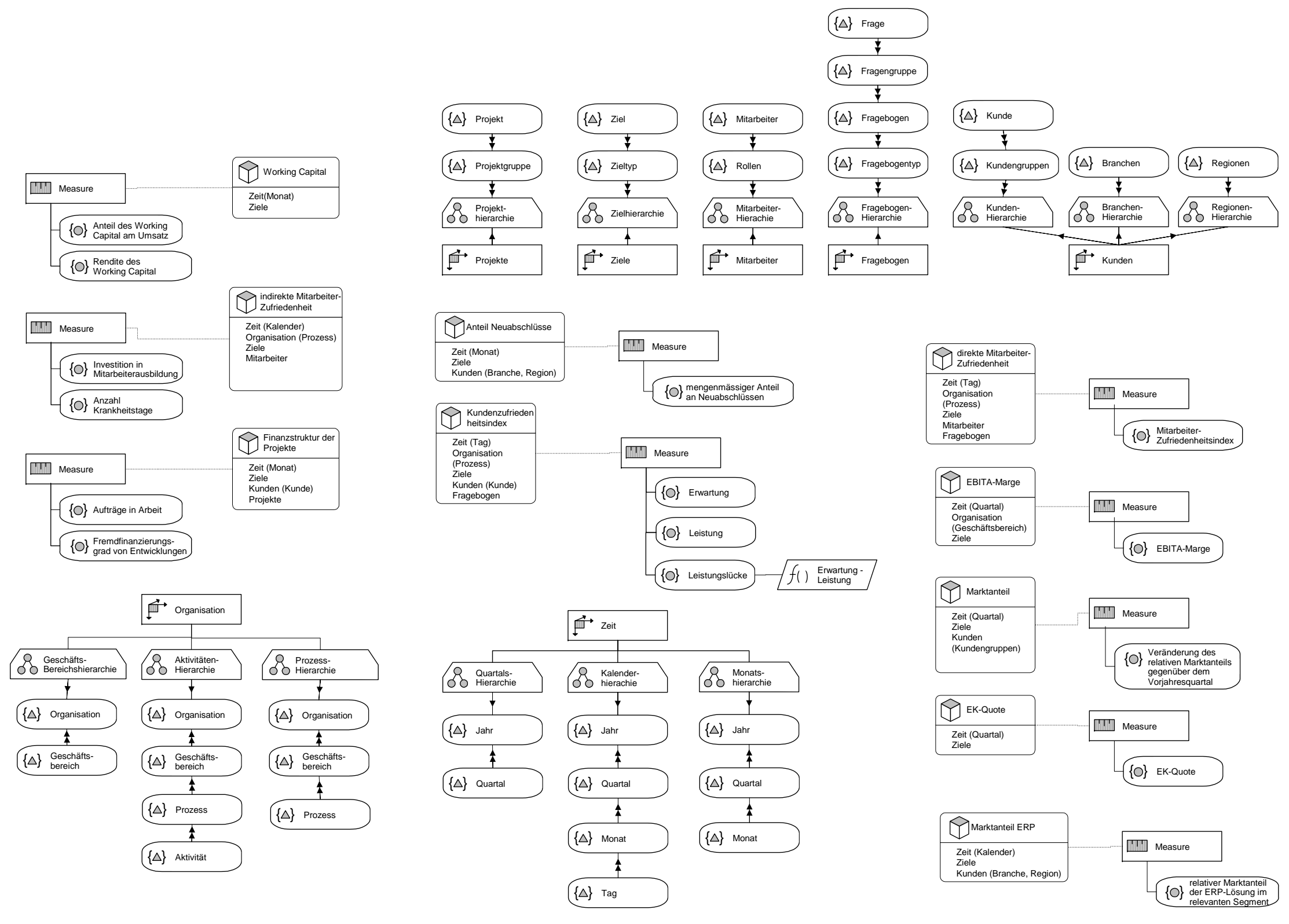

Abbildung 9-8: ADAPT-Darstellung des Beispiels 


\subsection{Evaluation des Vorgehensmodells}

Die vorgeschlagene Schrittfolge erlaubt nach Ansicht des Verfassers unter den beschriebenen Rahmenbedingungen (vgl. Anforderungen aus Tabelle 9-1) die Erstellung eines multidimensionalen Datenmodells. Der Ansatz wurde in verschiedenen Szenarien erfolgreich angewendet. Der Verfasser konnte feststellen, dass insbesondere bei grösseren Modellen durch die Anwendung der Schrittfolge gegenüber einem intuitiven Vorgehen bessere Ergebnisse resultieren.

Bei den untersuchten Ansätzen wurde teilweise die vage beschriebene Schrittfolge kritisiert. Es erscheint daher angebracht, den eigenen Vorschlag diesbezüglich kritisch zu beleuchten. Werden die Arbeitsanweisungen der einzelnen Schritte betrachtet, so bestehen nach Ansicht des Verfassers keine Unklarheiten. In jedem Arbeitsschritt wird das angestrebte Ergebnis und die dazu notwendigen Aktivitäten klar beschrieben. Einige nach Meinung des Verfassers systembedingten und kaum vermeidbaren - Unsicherheiten bestehen bezüglich des inhaltlichen Input der Betroffenen. Inwiefern in Schritt drei in der Diskussion mit den Betroffenen sämtliche relevanten Dimensionen gefunden werden, kann ex ante nicht festgestellt werden. Gleiches gilt für den Aggregationspfad einzelner Dimensionen und den Granularitätsgrad der Indikatoren. Insgesamt kann jedoch festgestellt werden, dass unter den beschriebenen Rahmenbedingungen eine Verbesserung gegenüber den bisher in der Literatur diskutierten Ansätzen erreicht werden konnte.

Im nächsten Kapitel wird das Ergebnis des Vorgehensmodells, d.h. das Datenmodell, in einem Prototypen umgesetzt und damit die technische Machbarkeit nachgewiesen. 


\section{Prototyp eines rechnergestützten Systems für das Performance Measurement}

\subsection{Einleitung}

Der in diesem Kapitel beschriebene Prototyp wurde erstellt, um zu zeigen, dass der vorgeschlagene Ansatz umsetzbar ist (proof of concept). Nachfolgend werden die einzelnen Komponenten des Prototypen, die auf der in Kapitel acht erarbeiteten Architektur basieren, diskutiert. In einem ersten Schritt werden die verwendeten SoftwareKomponenten vorgestellt. Aufbauend auf den eingeführten Komponenten wird die Daten-, die Funktions- und die Präsentationsebene dargestellt. Abschliessend erfolgt eine Abgrenzung zu den umliegenden Systemen und die Diskussion von möglichen Anbindungen.

\subsection{Die verwendeten Softwarekomponenten in der Übersicht}

Bei den verwendeten Softwarekomponenten handelt es sich um Standardprodukte der Firma Microsoft. Als Datenbank-Management-System (DBMS) wird der Microsoft SQL Server 2000 (Version 8.0) eingesetzt. Das DBMS kann nur auf der Microsoft Windows Betriebssystemfamilie (ab Windows NT Server 4.0 mit Service Pack 5) betrieben werden. Das DBMS unterscheidet zwischen internen und externen Datenbanken. Die Interne dient der Selbstverwaltung des DBMS in Form einer Metadatenbank (es handelt sich um die Datenbank dbmaster). Die externen Datenbanken enthalten die eigentlichen Nutzdaten. Der Microsoft SQL Server 2000 besteht aus mehreren Komponenten (vgl. Petkovic (2001)):

- Der Enterprise Manager erlaubt das Einrichten und Verwalten von Datenbankservern, Datenbanken, Sicherheitsaspekten und Stored Procedures, etc. Mit dem Service Manager können Datenbank-Server gestartet und gestoppt werden.

- Der Data Transformation Service (DTS) erlaubt das Erstellen und Verwalten von Datentransformationsprozeduren. Damit können Daten aus verschiedenen Quellen mittels Konnektoren mit der Zieldatenbank verbunden werden. Das Werkzeug unterstützt den Extraktions-, Transformations- und den Ladevorgang (ETL). Eine 
Reihe von Funktionen unterstützt Standardtransformationen für komplexe ETLVorgänge und kann auf eine Skriptsprache (Visual Basic Active X Skript) zurückgreifen.

- Der Query-Analyser dient der Erstellung und Ausführung von SQL-Abfragen auf den Datenbanken.

Der Microsoft Analysis Server ist Teil von Microsoft SQL Server 2000 (Version 8.0). Dieser bietet eine Reihe von Data-Warehouse-Funktionalitäten. Mittels des Analysis Manager können Datenwürfel, Dimensionen, Benutzerrollen und deren Zugriffsrechte, aber auch Push-Dienste verwaltet werden. Die Kommunikation zwischen dem Analysis Server und dem Analysis Manager erfolgt über so genannte Decision Support Objects (DSO) (vgl. Jacobson (2000)).

Als Webserver wird der Internet Information Server (IIS) von Microsoft eingesetzt. In der Version 4.0 ist er Bestandteil des Microsoft Windows NT-Optionenpaketes. Die Version 5.0 von IIS ist Bestandteil des Betriebssystems Windows 2000 Server. Neben dem Webserver beinhaltet er den Index-Server, den Zertifikate-Server und SMTP-Server. Durch die Microsoft-Erweiterung ASP (Active Server Pages) können interaktive Elemente realisiert werden. ASP ist eine Skriptsprache, die es ermöglicht Webseiten dynamisch zu erzeugen. Dies bedeutet, dass der Webserver zum Zeitpunkt des Seitenaufrufs eine HTMLSeite erstellt und diese mit Inhalt füllt. Der Inhalt kann bspw. aus einer Datenbank stammen. Die Funktionsweise der ASP-Architektur ist in Abbildung 10-1 dargestellt: (1) Der Browser ruft via HTTP eine Seite beim IIS ab. (2) Dieser interpretiert die ASP-Seite. (3) Bei entsprechend gekennzeichneten Datenabfrage-Bereichen wird über eine Data Source Name (DSN; erlaubt eine relative Beziehung zur Datenbank) und einer Open Data Base Connectivity - Schnittstelle (ODBC; Microsoft-Standard zur Verbindung mit einer beliebigen Datenbank) ein Zugriff auf den SQL-Server realisiert. (4) Das so übermittelte SQL-Statement wird auf dem SQL-Server ausgeführt. (5) Darauf werden die Daten an den IIS zurückgesandt. (6) Der IIS erstellt aus den ASP-Anweisungen und den Daten die HTML-Seite. (7) Diese wird vom IIS via HTTP an den Browser gesandt (vgl. Kauffmann (2000)). 


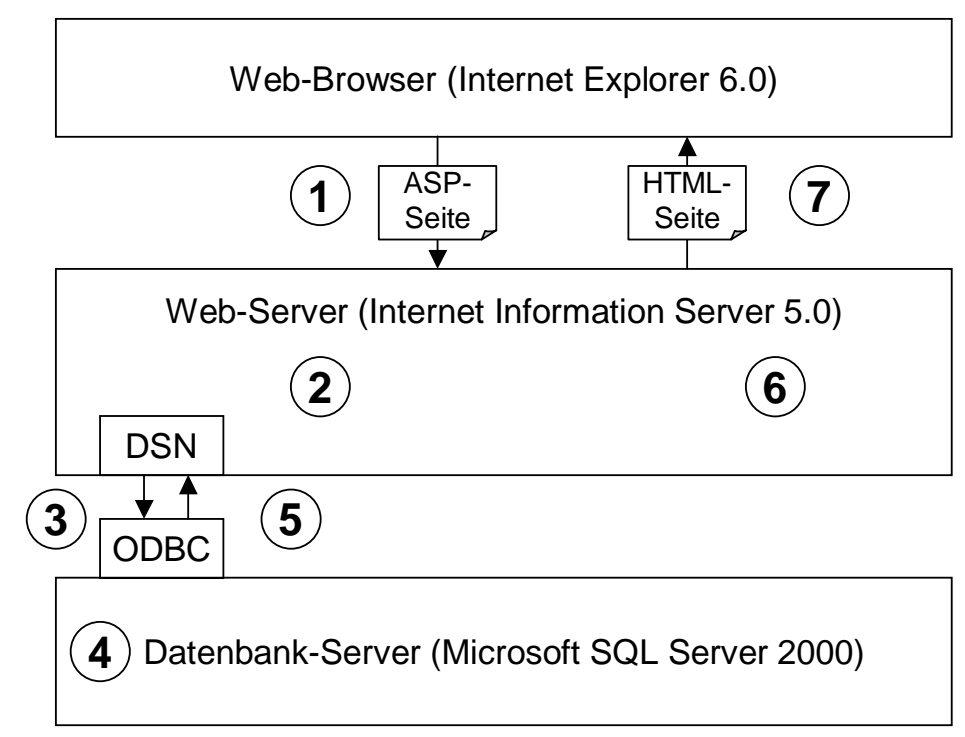

\section{Abbildung 10-1: Funktionsweise und Architektur von Active Server Pages}

Als Benutzerinterface wurde für den Prototypen der Microsoft Internet Explorer 6 Browser eingesetzt (IE 6.0). Die verwendeten Active X-Komponenten (Microsoft Office Web Components Version 9.0) stellen innerhalb des Webbrowsers OLAP-Funktionalitäten zur Verfügung. Es handelt sich dabei um das Active X Data Objects Multidimensional (ADO MD). Dieses kommuniziert mit dem Pivot Table Service, einer Komponente, die auf dem Client-Rechner installiert sein muss. Dabei ist zu beachten, dass diese nur funktionieren, wenn der Browser auf einem mit Microsoft Windows Betriebssystem betriebenen Rechner läuft. Bezüglich der Funktionen, die auf dem Analysis Server und dem Pivot Table Service zur Verfügung stehen, besteht eine gewisse Redundanz. Dies erlaubt unter anderem ein effizientes Caching und reduziert den Netzverkehr. (vgl. Microsoft (2002)) 


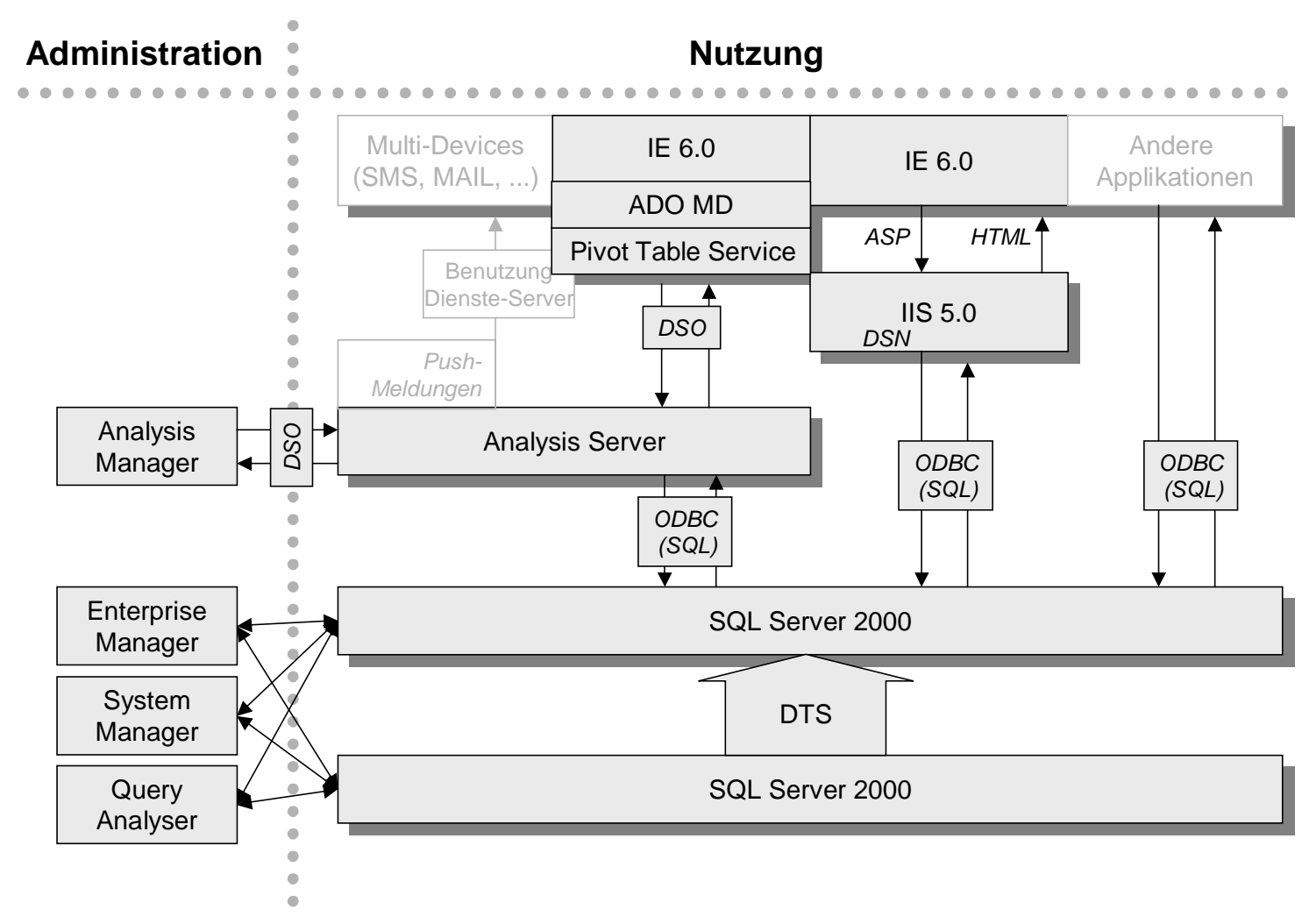

Abbildung 10-2: Architektur des Prototypen

In der Abbildung 10-2 ist die besprochene Architektur zusammenfassend dargestellt. Die Struktur basiert auf der vorgeschlagenen Architektur von Kapitel acht (vgl. Abbildung 8-5: Physische Drei-Schichten-Architektur eines IT-Systems für das Performance Measurement). Auf der linken Seite sind die Administrationskomponenten, rechts die Nutzungskomponenten dargestellt.

\subsection{Elemente der Datenebene}

Auf der Datenebene ist zwischen den Nutz- und Metadaten zu differenzieren. Auf insgesamt drei Ebenen des Prototypen werden entsprechende Daten gehalten. Die unterste Ebene, die nicht Gegenstand des Prototypen ist, enthält die Quellsysteme der Performancerelevanten Daten. Von dort gelangen die Daten mittels eines ETL-Prozesses (eingesetztes Tool: DTS) in die Staging Area, die als SQL Server 2000 Datenbank ausgestaltet ist. Auf dieser Ebene werden zwei Arten von Metadaten gehalten: Einerseits die Struktur der Staging-Area und andererseits die DTS-Routinen zur Akquisition der Performance-Daten. Von der Staging-Area werden die Daten periodisch in die Performance-Datenbank geladen, welche ebenfalls diese beiden Typen von Metadaten umfasst. Auf der Ebene des 
Analysis Server werden keine Nutzdaten gehalten. Allfällige Aggregate, Berechnungen, etc. werden in der Performance-Datenbank verwaltet. Die Metadaten des Analysis Server können wahlweise auf der Ebene des Data Warehouse (in diesem Falle werden die Daten in einer Microsoft-Access-Tabelle namens msmdrep.mdb abgelegt, vgl. Jacobson (2000)) oder auf dem SQL Server gespeichert werden.

Nachfolgend soll das relationale Datenmodell der Nutzdaten auf der Ebene des SQLServers sowie ein Ausschnitt aus der Repräsentation als multidimensionales Datenmodell im Microsoft Analysis Manager dargestellt werden.

\subsubsection{Relationales Datenmodell}

Die Abbildung 10-3 zeigt das aus dem ADAPT-Modell resultierende Relationenmodell. Dieses ist in der Star-Schema-Notation aufgebaut (vgl. Kapitel 9.5.1). Es besteht aus sieben Dimensionstabellen (abgeleitet aus den Dimensionen von Abbildung 9-8), gekennzeichnet mit einem D_, sowie zehn Faktentabellen (abgeleitet aus den Hypercubes von Abbildung 9-8), gekennzeichnet mit einem F_. Die Dimensionstabellen zeigen den Primärschlüssel (PK) fett gedruckt und etwas abgesetzt an erster Stelle. Weitere Schlüsselattribute welche die alternativen Hierarchiepfade in der gleichen Dimension repräsentieren, sind fett gedruckt im Hauptblock dargestellt (FK). Die einzelnen Hierarchieebenen mit allfälligen deskriptiven Attributen sind im Hauptblock in normalem Schriftschnitt dargestellt. Die Faktentabellen differenzieren Performance-IndikatorenAttribute, die mit $F_{-}$gekennzeichnet sind, sowie Fremdschlüsselattribute zu den Dimensionstabellen, die mit $\mathrm{D}_{-}$bezeichnet sind. Die Pfeile zwischen den Dimensionstabellen und den Faktentabellen stehen für die einfach-komplexe (1:n) Beziehung zwischen den Relationen.

Das Relationenmodell bildet die Basis für die Aufbereitung der Daten mit dem Analysis Manager. 


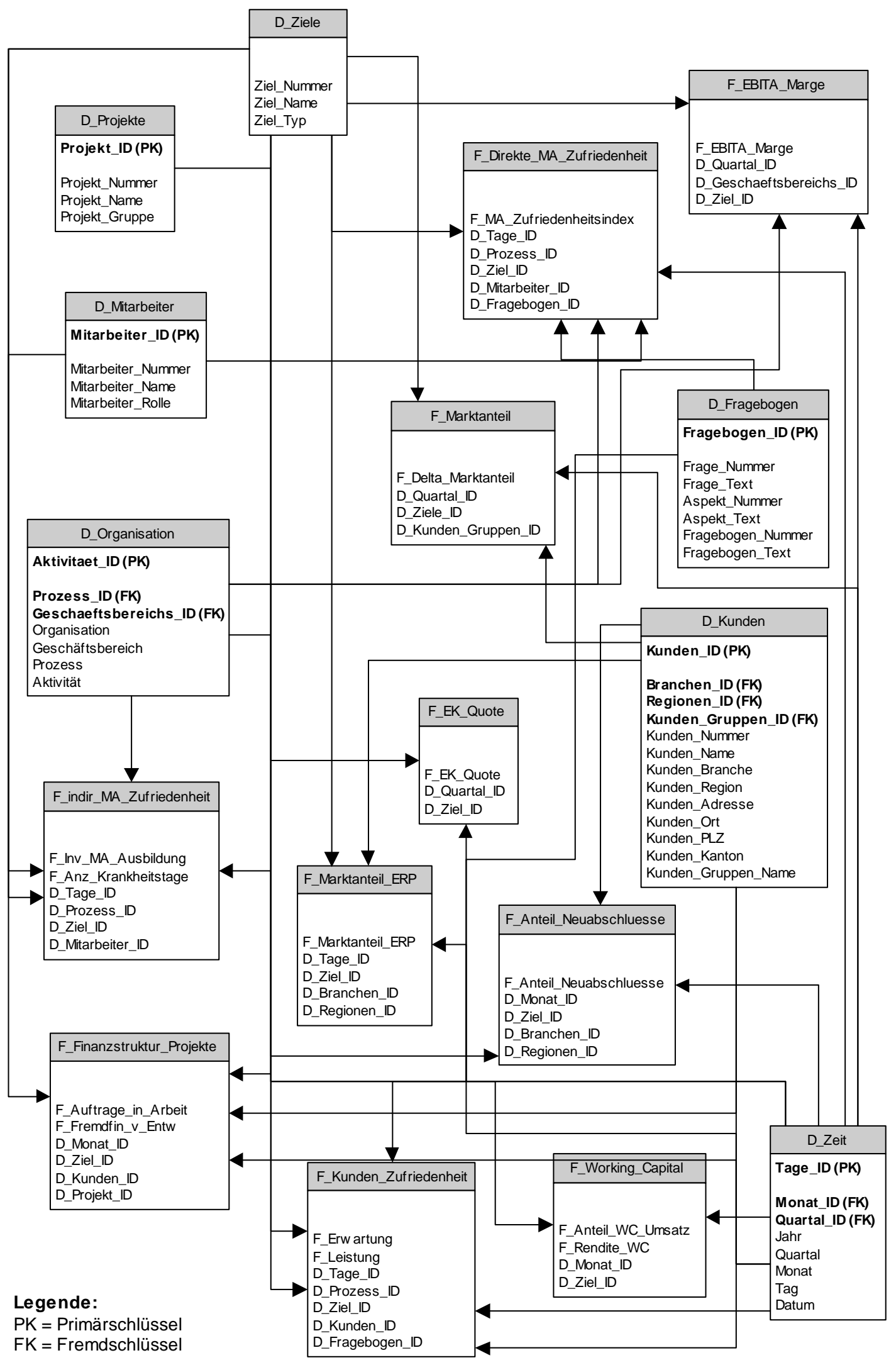

Abbildung 10-3: Relationenmodell des Prototypen 


\subsubsection{Repräsentation als multidimensionales Modell}

Auf Grund des besprochenen Relationenmodells wird die multidimensionale Struktur im Microsoft Analysis Manager aufgebaut. Dabei sind zwei Hauptelemente, die Datenwürfel (Cubes) und die Dimensionen (Shared Dimensions), zu differenzieren.

Abbildung 10-4 zeigt die Datenwürfel und die Dimensionen des Prototypen im Objektbaum des Microsoft Analysis Manager. Unter „Cubes“ sind die identifizierten Datenwürfel aus Tabelle 9-14 erkennbar. Unter „Shared Dimensions“ werden die identifizierten Dimensionen aus Tabelle 9-12 dargestellt.

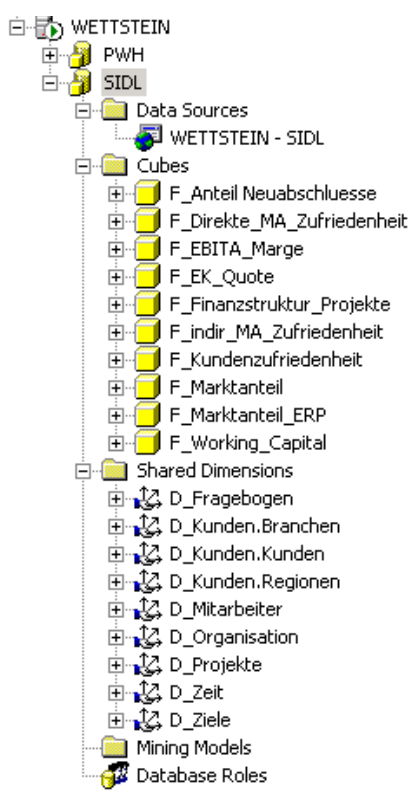

Abbildung 10-4: Übersicht über die Datenwürfel und Dimensionen des Prototypen

Abbildung 10-5 zeigt den Cube-Editor des Microsoft Analysis Manager am Beispiel des Datenwürfels Kundenzufriedenheit. Auf der rechten Seite ist das Star-Schema, mit der Faktentabelle in der Mitte und den darum herum angeordneten Dimensionen mit den Beziehungen erkennbar. Auf der linken Seite sind die assoziierten Dimensionen mit ihren Aggregationsebenen sowie die Performance-Indikatoren dargestellt (Rubrik: Calculated Members). Insgesamt sind drei Performance-Indikatoren ersichtlich: Der Erwartungs-, der Leistungswert und die Leistungslücke. 


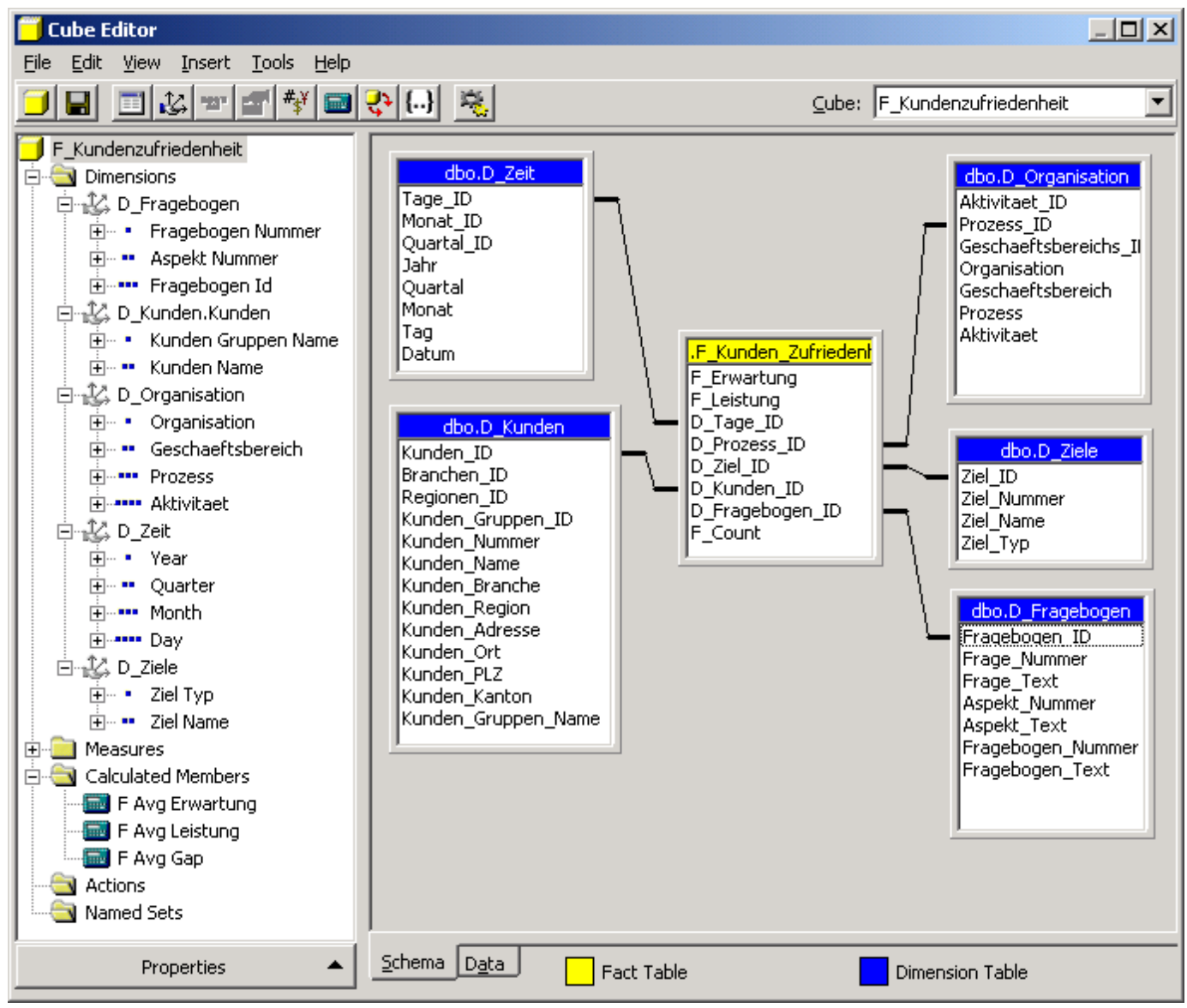

\section{Abbildung 10-5: Beispiel Datenwürfel Kundenzufriedenheit}

Abbildung 10-6 zeigt einen Ausschnitt der zur Dimension Organisation assoziierten Attribute. Auf der obersten Ebene ist die Organisation, hier die SIDL AG, dargestellt. Diese ist aufgeteilt in vier Geschäftsbereiche, wobei der Fokus im vorgestellten Beispiel auf den Bereich Application Services fällt. Dieser ist wiederum aufgeteilt in vier Hauptprozesse. Für den Prozess Sales sind die einzelnen Aktivitäten ersichtlich.

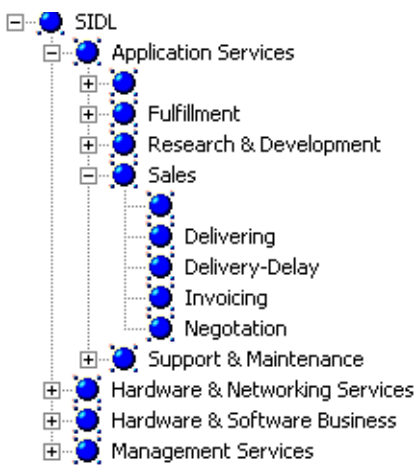




\subsection{Elemente der Administrationsebene}

\subsubsection{Festlegung von Rollen und Zugriffsrechten}

Im zehnten Schritt des Vorgehensmodells zur Erstellung des Datenmodells wurden die Berechtigungen je Informationsempfänger bestimmt. Diese werden mittels eines Rollenkonzeptes, teilweise verbunden mit dem Begriff des Datenherrs, auf die Datenwürfel übertragen.

Für jede Rolle wird festgelegt, auf welche Datenwürfel und auf welche Dimensionen zugegriffen werden darf. Kann für eine Rolle die Hierarchieebene einer Dimension über alle Datenwürfel beschränkt werden, so wird dies zusätzlich auf dieser Ebene geregelt. In einem zweiten Schritt werden die Restriktionen je Datenwürfel für die assoziierten Dimensionen verfeinert.

Die beschriebene Funktion kann mittels Standardwerkzeugen des Microsoft Analysis Managers vorgenommen werden. Abbildung 10-7 zeigt die definierten Hauptrollen (Informationsempfänger). Für die Rolle Kunden werden die berechtigten Datenwürfel und Dimensionen beschrieben (vgl. Abbildung 10-8). Für jede Dimension wird bestimmt, welche Aggregationsebenen eingesehen werden können. In Abbildung 10-9 wird für den Informationsempfänger Kunden für die Dimension Zeit festgelegt, dass nur die Aggregationsebene Jahr eingesehen werden kann.

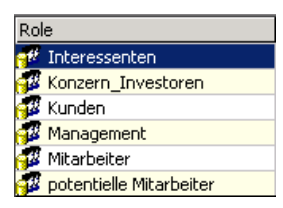

\section{Abbildung 10-7: Definition der Rollen (Informationsempfänger)}
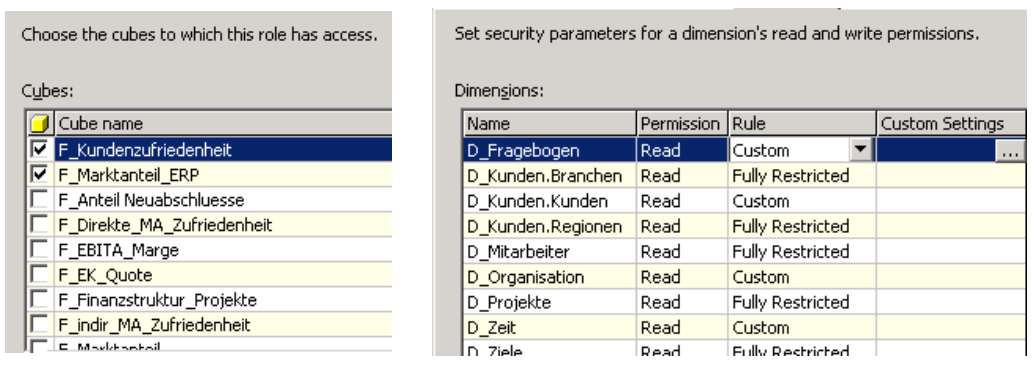

Abbildung 10-8: Berechtigungen für Datenwürfel und Dimensionen bestimmen 


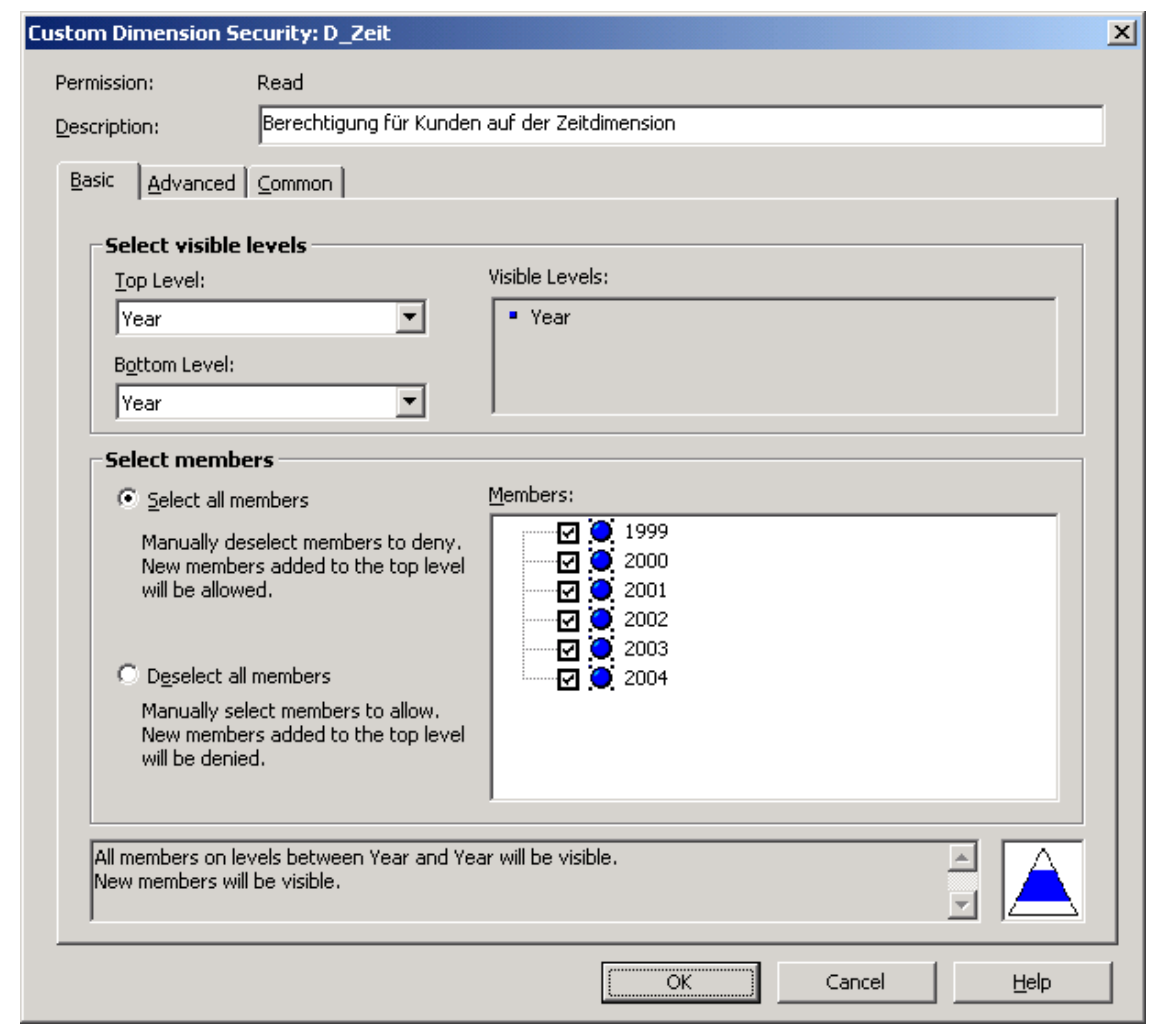

Abbildung 10-9: Definition von zulässigen Aggregationshierarchien

\subsubsection{Repräsentation des Zielbaumes}

Mit den im Microsoft Analysis Server zur Verfügung stehenden Dimensionen können keine Hierarchien mit einer undefinierten Anzahl Aggregationsebenen modelliert werden. Der in Kapitel sechs eingeführte Ziel- und Indikatorenbaum besitzt jedoch diese Eigenschaft, da die Ebenenzahl durch die Anzahl Ziel-Mittelbeziehungen definiert wird. Diese Überlegung und die Absicht, die Festlegung der Ziel-Werte je Indikator und Verantwortlichem praktischerweise auch im Ziel- und Indikatorenbaum vorzunehmen, hat zur separaten Realisierung dieser Funktionalität geführt. Ein Prototyp wurde im Rahmen einer studentischen Projektarbeit in Informatik unter der Betreuung des Verfassers entwickelt. Die Anwendung, welche mit ASP entwickelt wurde, interagiert in der oben beschriebenen Weise mit der SQL-Server-Datenbank. Abbildung 10-10 zeigt einen Ausschnitt aus dem Ziel- und Indikatorenbaum des betrachteten Beispiels. Es ist ersichtlich, dass sich die Zahlungsmodalitäten und die Neuentwicklungen als Mittel aus dem Ziel der hohen Eigenkapitalquote herleiten. Auf der nächsten Hierarchieebene sind jeweils die zugehörigen Indikatoren dargestellt. 
Durch die Verknüpfung des Ziel- und Indikatorenbaumes mit den Datenwürfeln können die ex ante unterstellten Wirkungsbeziehungen mittels statistischer Methoden ex post verifiziert werden.

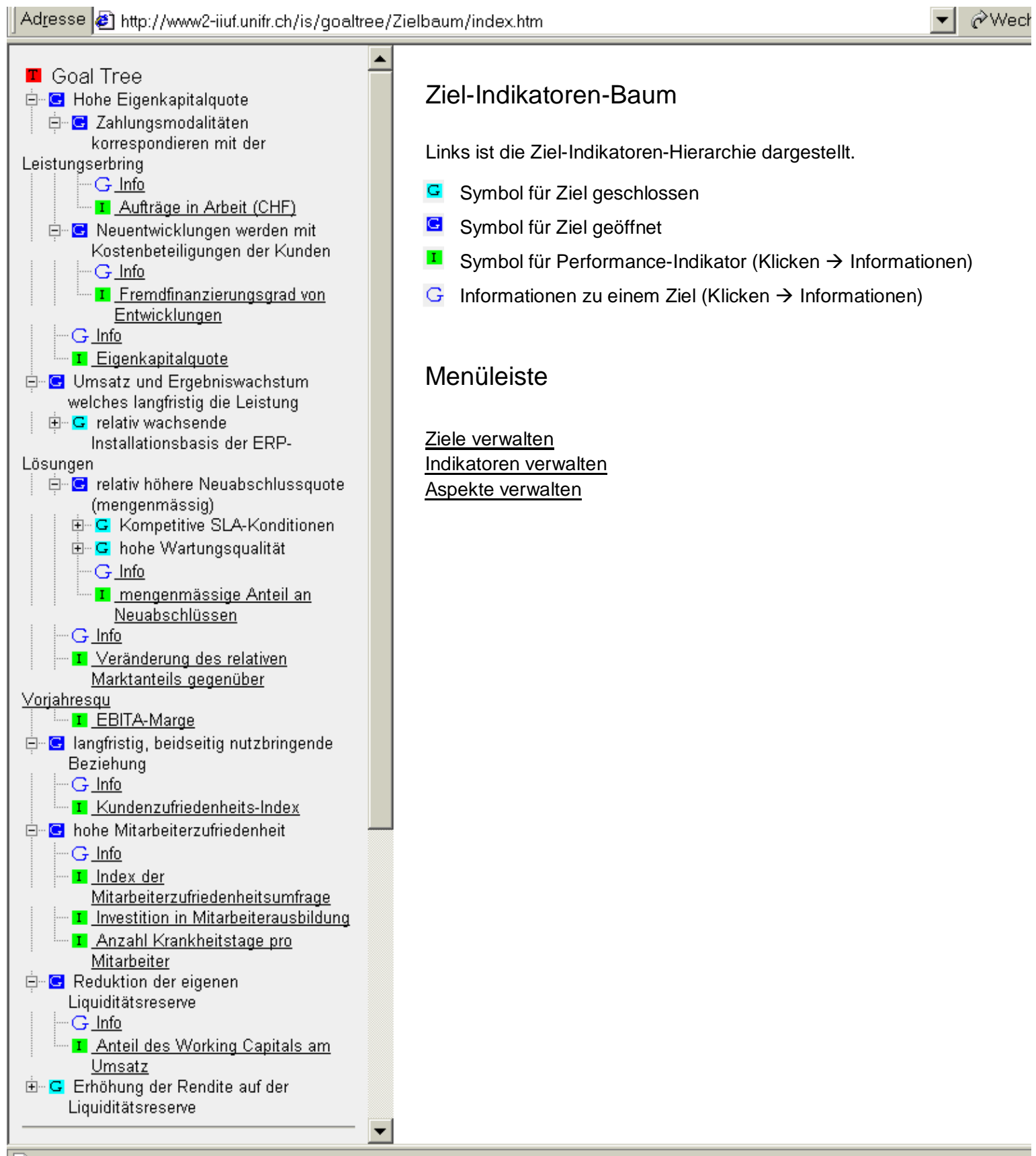

\section{Abbildung 10-10: Verwaltung des Ziel- und Indikatorenbaumes}




\subsubsection{Festlegung der Ziel-Werte}

Für einzelne Performance-Indikatoren können Ziel- und Schwellen-Werte verwaltet werden. Unter einem Ziel-Wert wird der angestrebte Zielerreichungsgrad eines Indikators zu einem bestimmten Zeitpunkt oder für eine bestimmte Zeitperiode verstanden (bspw. ein Kundenzufriedenheitsindex von 3.8 per Ende 2002 oder eine Fluktuationsrate für das Jahr 2002 von 5\%). Unter Schwellenwerten sind Ausprägungen eines Indikators zu verstehen, bei dessen Über- oder Unterschreitung bestimmte Aktionen ausgeführt werden sollen.

Wie in Kapitel 9.4.3. diskutiert, bestehen zwei grundsätzliche Möglichkeiten zur Speicherung der Ziel-Werte. Diese können entweder als Ausprägungen, d.h. als eigene Dimension im Data Warehouse gespeichert oder ausserhalb des Data Warehouse gehalten werden. Im Prototyp wurde die zweiten Variante umgesetzt.

\subsection{Elemente der Benutzerebene}

Zentrales Element aus Benutzersicht ist das so genannte Performance Cockpit, welches die Performance-relevanten Informationen rollen- resp. personenorientiert zusammenstellt. In Abbildung 10-11 ist ein Cockpit für den Leiter des Geschäftsbereiches Application Services zusammengestellt. Im ersten Bereich wird die zugehörige Rolle, der Benutzer und der Aktualisierungsstand der zu Grunde liegenden Performance-Daten dargestellt. Im zweiten Bereich sind die Ziele und Indikatoren des Geschäftsbereiches Application Services (siehe Ziel- und Indikatorenbaum als Ergebnis von Kapitel sechs) dargestellt. In der ersten Spalte wird jeweils darauf hingewiesen, um welche Art von Zielen (siehe Aspekte von Kapitel sechs) es sich handelt. Rechts vom Indikator, über welchen durch Klicken auf das kleine Dreieck zusätzliche Information abgerufen werden können, ist der Status ersichtlich. Der Status erlaubt eine schnelle Übersicht über den Stand eines Indikators mittels des Ampelprinzips. Die Ampel wird auf Grund des aktuellen Ist-Wertes und definierten Schwellenwerten bestimmt. Rechts davon ist der aktuelle Ziel- und Leistungswert aufgeführt. Der aktuelle Leistungswert wird aus der PerformanceDatenbank abgefragt. 


\begin{tabular}{|c|c|c|c|c|c|c|c|c|}
\hline File Edit & Wiew Favorites Tools Help & & & & & & & \\
\hline \multicolumn{9}{|c|}{ SIDL AG } \\
\hline Role & Manager Application Services & User & Thomas Wettstein/iuf & \multicolumn{2}{|c|}{ Last Update } & \multicolumn{3}{|c|}{12 July 2002 / ok. } \\
\hline \multicolumn{9}{|c|}{ (1) Geschäftsbereich Application Services } \\
\hline Aspect & \multicolumn{2}{|l|}{ Goal } & Performance Indicator & Status & & Target & Performance & Details \\
\hline \multirow[t]{5}{*}{ Finanziell } & \multicolumn{2}{|l|}{$\begin{array}{l}\text { Umsatz und Ergebniswachstum welches } \\
\text { langfristig die Leistung der Branche übertrifft }\end{array}$} & EBITA-Marge $>$ & (2) & & $12 \%$ & $11.5 \%$ & \\
\hline & \multicolumn{2}{|c|}{$\begin{array}{l}\text { Zahlungsmodalitäten korrespondieren mit der } \\
\text { Leistungserbringung (M1) }\end{array}$} & Aufträge in Arbeit (CHF) $>$ & & 4.0 & Mio CHF & 3.8 Mio CHF & \\
\hline & \multicolumn{2}{|c|}{$\begin{array}{l}\text { Neuentwicklungen werden mit } \\
\text { Kostenbeteiligungen der Kunden realisiert (M2) }\end{array}$} & $\begin{array}{l}\text { Fremdfinanzierungsgrad von } \\
\text { Entwicklungen }\end{array}$ & Q & & $50 \%$ & $44 \%$ & \\
\hline & \multicolumn{2}{|c|}{$\begin{array}{l}\text { relativ wachsende Installationsbasis der ERP- } \\
\text { Lösungen (M4) }\end{array}$} & $\begin{array}{l}\text { relativer Marktanteil der ERP-Lösung im } \\
\text { relevanten Segment }\end{array}$ & Q) & & $2 \%$ & $1.8 \%$ & \\
\hline & \multicolumn{2}{|l|}{$\begin{array}{l}\text { relativ höhere Neuabschlussquote } \\
\text { (mengenmässig) (M4) }\end{array}$} & $\begin{array}{l}\text { mengenmässige Anteil an } \\
\text { Neuabschlüssen }\end{array}$ & & & $30 \%$ & $15 \%$ & 1 \\
\hline Kunden & \multicolumn{2}{|c|}{ langfristig, beidseitig nutzbringende Beziehung } & $\begin{array}{l}\text { Kundenzufriedenheits-Index } \\
\text { (Leistungslücke) }\end{array}$ & & & $25 \mathrm{pt}$ & $48 \mathrm{pt}$ & \\
\hline \multirow[t]{3}{*}{ Mitarbeiter } & \multicolumn{2}{|l|}{ hohe Mitarbeiterzufriedenheit } & $\begin{array}{l}\text { Index der } \\
\text { Mitarbeiterzufriedenheitsumfrage } \\
\text { (Leistungslücke) }\end{array}$ & (0) & & $20 \mathrm{pt}$ & $22 \mathrm{pt}$ & \\
\hline & & & Investition in Mitarbeiterausbildung $>$ & Q & & $100 \mathrm{CHF}$ & $11^{\prime} 872 \mathrm{CHF}$ & a \\
\hline & & & Anzahl Krankheitstage pro Mitarbeiter & (a) & & 4 & 5.5 & \\
\hline
\end{tabular}

Administration

\begin{tabular}{|c|c|c|c|}
\hline Zielbaum & Personalisierung & Schwellenwerte & Kommentierung \\
\hline
\end{tabular}

\section{Abbildung 10-11: Performance Cockpit für den Bereichsleiter Application Services}

Im Administrations-Teil des Cockpits kann (1) der Zielbaum (siehe Kapitel 10.4.2), (2) die Personalisierungsfunktion (Der Benutzer kann die eigene Cockpit-Ansicht individuell parametrisieren.), (3) die Definition der Schwellenwerte und (4) die PerformanceKommentierung aufgerufen werden (Diese Funktion erlaubt den Mitarbeitern zuhanden der Vorgesetzten, die aktuelle Performance zu kommentieren. Dies erweist sich als wertvoll, da der alleinige quantitative Wert für einen Indikator teilweise schwer zu interpretieren ist.). Die Punkte zwei und vier wurden im vorliegenden Prototyp nicht implementiert.

Die aktuellen Performance-Werte des Cockpits werden aus den Datenwürfeln abgefragt. Möchte man diesen Wert analysieren, so besteht durch das Anwählen des entsprechenden Würfels in der letzten Spalte des Cockpits (Details) die Möglichkeit, den Datenwürfel aufzurufen. In Abbildung 10-12 sind die Detaildaten für den Indikator Kundenzufriedenheits-Index dargestellt. Für das erste Quartal für die Geschäftseinheit Application Services und über alle Kunden betrachtet, bestehen die zwei grössten Leistungslücken bei der speditiven Bestellabwicklung und einer guten Antwortzeit (siehe 
Markierungen). Können diese beiden Faktoren verbessert werden, z.B. durch eine Reorganisation der Bestellabwicklung oder aber durch die Neuorganisation der Serviceorganisation, so kann die Leistungslücke verkleinert werden.

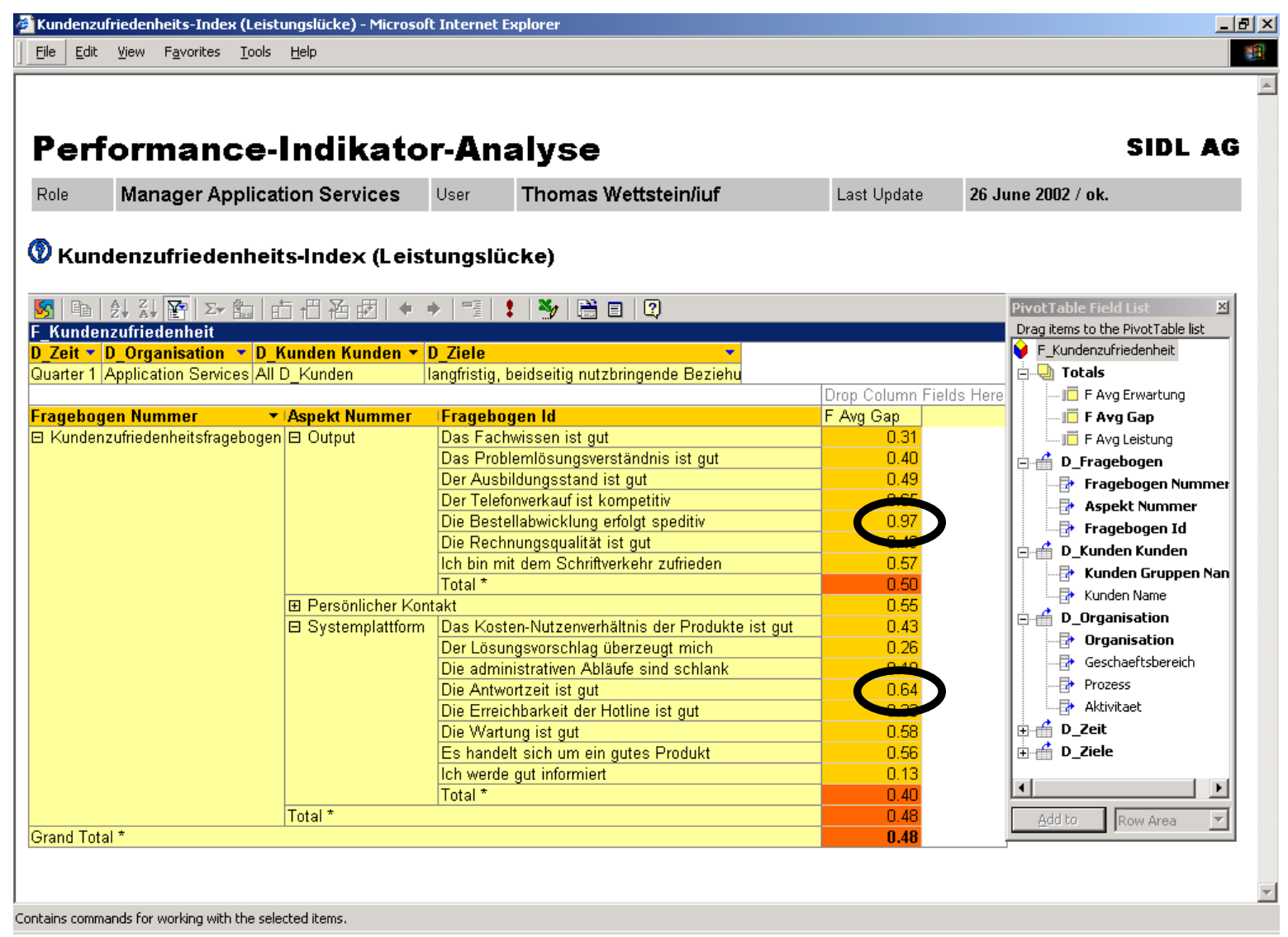

Abbildung 10-12: Performance-Indikator-Analyse: Kundenzufriedenheits-Index

\subsection{Systemabgrenzung und Anbindung von umliegenden Systemen}

Der Prototyp besitzt zur Seite der Datenakquisition hin eine Schnittstelle zur Staging-Area, die ihrerseits mit den informationsliefernden Systemen über Extraktionsroutinen (DTSPackages) verknüpft sind.

Die Performance-Datenbank liefert die Daten für das diskutierte Performance Cockpit, den OLAP-Client und die Administrationskomponente. Denkbar ist des Weiteren, dass andere Applikationen (bspw. ein Simulationstool, eine Statistiksoftware, etc.) auf die Daten zugreifen. Dabei sind zwei Zugriffsarten denkbar: Einerseits kann via SQL über die ODBC-Schnittstelle direkt auf die Datenbank zugegriffen werden. Diese wären, sollte 
diese Zugriffsart bereitgestellt werden, entsprechend zu konfigurieren. Andererseits besteht die Möglichkeit über den Pivot Table Service resp. die Decision Support Objects auf den Analysis Server zuzugreifen. Die Vorteile dieser Variante bestehen darin, (1) dass die Daten korrekt mit den Strukturinformationen zur Verfügung stehen und damit kein Wissen über die Struktur bekannt sein muss, (2) die Berechtigungen über das Rollenkonzept aufrechterhalten bleiben (3) der Endanwender mit sehr einfachen Mittel eine Verbindung von z.B. Microsoft Excel auf die Datenwürfel vornehmen kann, um weiterführende Auswertungen $\mathrm{zu}$ erstellen und (4) keine Abfragesprache erlernt werden muss. Im vorliegenden Prototypen wurde der Zugriff über den Pivot Table Service gewählt.

\subsection{Bewertung des Prototypen}

Der vorliegende Prototyp unterstützt die wichtigsten gemäss Kapitel vier hergeleiteten Anforderungen (vgl. Tabelle 4-2): Die Akquisition und zentrale Speicherung der relevanten Daten, deren Analyse sowie stakeholdergerechte Kommunikation wurde im Prototypen abgebildet. Die multidimensionale Struktur erlaubt eine freie Navigation in den Daten. Mit den implementierten Sicherheitsmassnahmen wird Gewähr leistet, dass nur Berechtigte Einblick in die Performance-relevanten Daten erhalten. Die Antwortzeiten sind durch den Einsatz einer multidimensionalen Datenstruktur sehr gut und die Applikation läuft stabil.

Die im Kapitel neun vorgeschlagene Schrittfolge zur Erstellung des Datenmodells konnte für das multidimensionale Datenmodell des Prototypen angewendet werden. Gleiches gilt für die im Kapitel acht erarbeitete Referenzarchitektur, die für den vorliegenden Prototypen als Basis diente.

Für den operativen Einsatz fehlt die Automatisierung der Delta-Loads ${ }^{17}$ zwischen den operativen Systemen und der Staging Area. Im Rahmen dieses Prototypen wurde dieser Prozess jeweils manuell durchgeführt. Zudem wäre die Verwaltung und automatische Generierung der personalisierten Cockpits noch umzusetzen. Im Prototypen sind diese Funktionen statisch realisiert.

17 Der erste Transfer der Daten aus dem operativen Informationssystem ins Data Warehouse wird als InitialLoad, der Transfer jenerDaten, die sich zwischen zwei Zeitpunkten verändert haben, wird als Delta-Load bezeichnet (vgl. Bauer und Günzel (2001)). 
Abschliessend betrachtet konnte gezeigt werden, dass der vorgeschlagene Ansatz umsetzbar ist, auch wenn einzelne Funktionen nicht komplett realisiert wurden. 
V. Teil

Schluss 


\section{Zusammenfassung und Ausblick}

In diesem Kapitel werden die wichtigsten Ergebnisse zusammengefasst, die Grenzen des vorliegenden Ansatzes erläutert und offene Forschungsfragen diskutiert.

\subsection{Zusammenfassung}

Performance-Measurement-Systeme (PMS) werden in der Wissenschaft seit mehr als zehn Jahren intensiv diskutiert. Wie Untersuchungen zeigen, wurden die vorgeschlagenen Ansätze in der Praxis jedoch erst mangelhaft umgesetzt. Die Verbreitung und die Qualität der IT-unterstützten PMS ist unbefriedigend. Die bisher vorgeschlagenen Ansätze tragen den Bedürfnissen der Unternehmen nur teilweise Rechnung. Ein wesentlicher Teil des Problems dürfte darin liegen, dass kein umfassendes und ausführliches Vorgehensmodell existiert, welches beschreibt, wie ein PMS mit IT-Unterstützung aufgebaut werden kann. Bestehende Ansätze berücksichtigen primär organisatorische Aspekte; IT-bezogene Aspekte werden nur partiell thematisiert. Wenn IT-Systeme zum Einsatz kommen, so werden die Performance-relevanten Daten häufig verstreut gespeichert, was eine integrale Sicht stark erschwert. Die Datensammlung und -bereinigung erfordert oft manuelle Eingriffe. Zudem sind entsprechende Systeme bezüglich der gespeicherten PerformanceIndikatoren einseitig. Meist werden nur finanzielle und vergangenheitsorientierte Kennzahlen gespeichert. Die verfügbaren technischen Lösungen sind von den Softwareherstellern geprägt, die eher die technischen als die betriebswirtschaftlichen Aspekte berücksichtigen. Ein weiterer Problemkreis ist darin zu sehen, dass PerformanceMeasurement-Systeme in der Praxis häufig mit wenig relevanten und veralteten Informationen operieren, was zu einer schlechten Akzeptanz führt. Die mangelnde Aktualität entsteht ferner dadurch, dass diese Systeme nicht geeignet unterhalten und betrieben werden; und dieses Manko ist unter anderem darauf zurückzuführen, dass die notwendigen Prozesse nicht definiert sind. Zu einer schlechten Akzeptanz führt ebenfalls der sehr kleine Adressatenkreis, welcher häufig nur aus der Unternehmensspitze besteht. Mit geeigneten technischen Mitteln und einem Kommunikationskonzept könnte der Kreis der PMS-Benutzer erweitert und damit der Nutzen des Systems gesteigert werden. 
Für einige der oben beschriebenen Probleme hat die vorliegende Arbeit auf der Basis von systematisch erarbeiteten Anforderungen an IT-Systeme für das Performance Measurement Lösungsansätze vorgeschlagen. Zur Gewinnung der Anforderungen wurden bestehende Ansätze analysiert, existierende qualitative und quantitative Studien zum Thema ausgewertet, technologische Möglichkeiten erfasst und wichtige Anforderungen in einer empirischen Studie validiert.

Ein Ergebnis bildet der Bezugsrahmen für das Performance Measurement, der sicher stellt, dass sämtliche relevanten Aspekte beachtet werden. Der Bezugsrahmen setzt sich aus den drei Elementen Systematik, Methodik und Organisation zusammen. Die Systematik definiert die Ebenen des Performance Measurement, ihre Elemente und Beziehungen. Die Methodik beschreibt die Prozesse zum organisatorischen und technischen Aufbau, Betrieb und Unterhalt des PMS. Der Organisationsaspekt (als dritter Teil des Bezugsrahmens) definiert die notwendigen Rollen, damit die relevanten Verantwortlichkeiten wahrgenommen und die Informationsempfänger bestimmt werden können.

Der Prozess zur Erstellung des organisatorischen Konzeptes als Element der Methodik beschreibt ausführlich, wie aus der Organisationsstrategie eine Zielhierarchie und darauf aufbauend eine Indikatorenhierarchie abgeleitet werden kann. Als Grundlage dienen die Strategie, die Aufbauorganisation, das bestehende Plansystem und allenfalls individuelle Ziele aus dem Management by Objectives. Als Kern der Transformation kann die Kaskadierung der Ziele auf untergeordnete Organisationsebenen bezeichnet werden. Diese basiert auf der Ermittlung der Ziel-Mittel-Beziehungen. Allfällige Zielkonflikte werden mittels Wirkungs-, Zielbeziehungs- und Präferenzziel-Analysen identifiziert und bereinigt. Im Anschluss daran werden die Performance-Indikatoren bestimmt. Dabei werden erste potenzielle Indikatoren gesammelt. Auf Grund bestimmter Anforderungen, die Performance-Indikatoren zu erfüllen haben, wird die definitive Auswahl getroffen. Integraler Bestandteil ist ein Kommunikationskonzept, welches festlegt, welche Gruppen von Informationsempfängern welche Performance-relevanten Daten in welcher Form kommuniziert erhalten.

Basierend auf der organisatorischen Konzeption wird ein Prozess zur Transformation in ein multidimensionales Datenmodell vorgeschlagen. Dabei wird beschrieben, wie das erarbeitete organisatorische Konzept und die spezifischen Anforderungen, die an ein Performance-Measurement-System gestellt werden, umgesetzt werden können. Es wird gezeigt, wie ein Datenmodell erstellt werden kann, welches eine genau definierte 
Informationsnachfrage und eine weniger klare Informationssuche decken kann. Neben dem multidimensionalen Datenmodell wird ein Verfahren vorgestellt, das beschreibt, wie das Kommunikationskonzept und die Sicherheit auf die technische Ebene transponiert werden können.

Für die konkrete technische Umsetzung wird eine Referenzarchitektur beschrieben, welche auf die Anforderungen eines PMS zugeschnitten wurde. Vom Prinzip her handelt es sich um eine offene Drei-Schichten-Architektur, welche eine Daten-, Funktions- und Präsentationsebene differenziert. Für die Präsentation wird Webtechnologie eingesetzt, auf der Funktionsebene wird neben dem Webserver ein Data Warehouse Server vorgeschlagen. Die Datenhaltung erfolgt in einem relationalen Datenbank-ManagementSystem.

Mit einem Prototyp eines IT-Systems für das Performance Measurement, der auf der vorgeschlagenen Referenzarchitektur basiert, wird die Funktionsfähigkeit des vorgeschlagenen Ansatzes gezeigt. Die Ziel- und Indikatorenhierarchie sowie das Datenmodell wurden auf Grund der vorgeschlagenen Prozesse realisiert.

\subsection{Bemerkungen zum vorgeschlagenen Ansatz und Ausblick für weitere Forschung}

Der vorgeschlagene Ansatz erhebt nicht den Anspruch, bestehende Instrumente zu substituieren. Vielmehr ist situativ jeweils sorgfältig abzuwägen, wie existente und bewährte Instrumente mit dem vorgeschlagenen Ansatz verknüpft werden können. Damit verbunden ist der Nachteil, dass ein zusätzliches System betrieben und unterhalten werden muss. Zusätzlich besteht die Gefahr einer erhöhten Redundanz der gespeicherten Daten, die zu Inkonsistenzen, einer erhöhten Komplexität und zu höheren Kosten führt. Längerfristig ist daher eine Entwicklung zu erwarten, welche entweder versuchen wird, die verschiedenen Führungssysteme zu integrieren und damit die bestehenden Instrumente abzulösen, oder diese so schlank wie möglich zu halten und über ein Bausteinkonzept zu integrieren. Denkbar und insbesondere aus ökonomischen Gesichtspunkten interessant könnte auch die Integration des PMS in ein ERP-System sein (vgl. z.B. Entwicklungen bei SAP und Küng et al. (2000)).

Mit der zentralen Speicherung von Performance-Daten werden mächtige Informationsgefässe sowohl über Organisationen als auch über einzelne Mitarbeiter angelegt. Die damit möglicherweise verbundenen Gefahren, die dem Verfasser durchaus 
bewusst sind, werden in der vorliegenden Arbeit nicht berücksichtigt. Hier sind weitere Aktivitäten angezeigt. Der Gedanke, dass eine ganze Reihe von Performance-relevanten Daten auf der Ebene des einzelnen Mitarbeiters gespeichert werden, kann zu Abwehrreaktionen führen. Beim Aufbau eines PMS sind die Mitarbeiter daher in einer frühen Phase einzubeziehen, und es ist offen zu legen, welche Daten warum und wie lange gespeichert werden. Auf jeden Fall sollten Mitarbeiter „ihre“ Daten uneingeschränkt einsehen können und die Möglichkeit besitzen, allfällige Korrekturen zu verlangen. Vorgesetzte sollten bezüglich des richtigen Umgangs mit den Performance-Daten sensibilisiert werden. Die Daten sollen nicht zu Kontrollzwecken, sondern vielmehr zu Motivationszwecken verwendet werden. Ein Sportler misst seine Leistung auch nicht, damit der Trainer kontrollieren kann, ob er sein Training absolviert hat, sondern um Ansatzpunkte zu entdecken, wie seine Leistung noch verbessert werden könnte. In diesem Zusammenhang wird auch deutlich, dass eine Organisation, die den Einsatz eines PMS prüft, über eine entsprechende Kultur - welche die Transparenz zulässt - verfügen muss.

Neben den inneren Widerständen, die durch die Speicherung von Performance-Daten entstehen können, besteht ebenfalls die Gefahr des Missbrauchs der Performance-Daten von ausserhalb der Organisation liegenden Stellen. Die Vorteile, die mit der elektronischen Speicherung verbunden sind, können hier auch als Nachteile erfahren werden, wenn Unberechtigte in den Besitz der Daten kommen.

Der vorgeschlagene Ansatz basiert auf heute verfügbaren Technologien. Durch die schnelle technische Entwicklung sind periodisch neue technologische Möglichkeiten für die Anwendung bei einem computergestützten Performance-Measurement-System zu prüfen. So ist der Einsatz neuer Hard- und Software für die Sammlung und Verteilung von Performance-relevanten Informationen denkbar (z.B. Persönliche Digitale Assistenten, Mobiltelefone, etc.). Mit dem Einsatz neuer Technologien werden unter Umständen neue Führungsideen - die sich jenseits von Anordnung und Kontrolle bewegen - vorstellbar. Allenfalls kann die Kommunikation mit organisationsexternen Informationsempfängern neu gestaltet werden. Dieser Aspekt wird im Rahmen des Bezugsrahmens nicht konkretisiert. (vgl. Küng und Wettstein (2001))

Wie in der Definition eines PMS im Kapitel zwei festgehalten, sammelt ein PMS Wissen der Organisation und vereinfacht das organisationelle Lernen. In einer einfachen Form berücksichtigt dies der vorliegende Ansatz. Das Zielsystem einer Organisation 
operationalisiert die Strategie und verkörpert das Geschäftsmodell. Verbunden mit den über eine längere Zeitperiode verfügbaren Performance-relevanten Daten können Wirkungsgefüge mittels entsprechender statistischer Analysen expliziert werden. Die Anwenderfreundlichkeit dieses Vorgehens ist jedoch sehr gering. Um eine breite Akzeptanz zu gewinnen, ist demzufolge zu untersuchen, wie diese Daten geeignet aufbereitet und wie Führungspersonen und Mitarbeiter für die Nutzung der Daten geschult werden können.

In der vorliegenden Arbeit endet die Performance-Messung an der Grenze der Organisation. Eine mögliche Weiterentwicklung könnte darin bestehen, zu untersuchen, inwiefern Performance-Daten entlang der Wertschöpfungskette interorganisationell ausgetauscht werden könnten und welche Opportunitäten und Gefahren damit verbunden wären. So könnten bspw. Prozesse über Organisationsgrenzen hinaus optimiert werden, oder Performance-Daten von unternehmensexternen Stakeholdern, die heute mittels spezieller Applikationen (z.B. Kundenzufriedenheit) erhoben werden müssen, günstiger gewonnen werden. Entsprechende Überlegungen könnten bspw. zu ganz neuen Preismodellen führen, die sich dynamisch auf Grund von verschiedenen PerformanceGrössen ergeben. Neben der Integration entlang der Wertschöpfungskette ist ebenfalls ein Benchmarking mit Mitbewerbern denkbar. Eine grosse Herausforderung bestünde hier in der Standardisierung der Ziele und Performance-Indikatoren, ähnlich wie dies zurzeit bei Produktkatalogen erfolgt, die auf elektronischen Marktplätzen ausgetauscht werden.

Mit der Anwendung der Heuristik an einem Fallbeispiel und der Realisierung des Prototypen konnte die Brauchbarkeit des Ansatzes nachgewiesen werden. Erfahrungen für den Betrieb und die Revision eines PMS konnten nur in beschränktem Umfang gewonnen werden. Hierbei handelte es sich um keine konkrete Zielsetzung, die an diese Arbeit gestellt wurde. Es wurde jedoch deutlich, dass in diesem Bereich noch einige offene Punkte bestehen, welche Gegenstand weiterer Forschungsarbeiten sein können. Zum Beispiel die Integration der Aktivitäten eines PMS in den Planungskalender eines Unternehmens. Auf der Ebene des Datenmodells betrifft dies bspw. temporale Aspekte der Dimensionen. Wie werden Veränderungen des Datenmodells gehandhabt, wie wird mit historischen Daten verfahren?

Das heutige Wirtschaftsleben weist immer kürzere Innovationszyklen auf. Die Reorganisation wird zum Normalzustand, Beständigkeit zur Ausnahmeerscheinung. Der 
Aufbau eines IT-gestützten Performance-Measurement-Systems ist mit beachtlichem Aufwand verbunden. Kritiker mögen deshalb einwenden, dass ein PMS veraltet sei, bevor es produktiv in Betrieb genommen wird. Dies mag eine gewisse Berechtigung haben. Auf der anderen Seite lässt sich anführen, dass das PMS Veränderungsprozesse unterstützt und sicherstellt, dass im Unternehmen alle wissen, welche Ziele erreicht werden sollen. Das PMS kann somit als Instrument zur Bewältigung der ständigen Veränderung aufgefasst werden.

Als letzter Ansatzpunkt für offene Forschungsfragen soll die Wirtschaftlichkeit eines PMS beleuchtet werden. Eine klassische Wirtschaftlichkeitsrechnung verspricht - mit den Erkenntnissen aus der IT-Paradoxon-Forschung - keine erfolgsversprechende Variante zu sein. Hier besteht ein Bedarf nach neuen Ansätzen. Denkbar ist eine subjektive Einschätzung des Nutzens durch die Benutzer des Systems, oder indem untersucht wird, welche Indikatoren in welcher Intensität für bestimmte Entscheidungen konsultiert werden. Interessant wäre auch zu erfahren, inwiefern nicht-finanzielle Indikatoren zweckdienlich sind. Diese werden von der Forschung seit Jahrzehnten propagiert, finden in den Unternehmen aber nach wie vor eine untergeordnete Beachtung.

Einen Beitrag könnte eine Untersuchung liefern, welche Unternehmen auf Grund des Entwicklungsstandes ihrer Performance-Measurement-Systeme in Reifegrade einteilt und diesen dem längerfristigen finanziellen Erfolg gegenüberstellt (vgl. Wettstein und Küng (2002)).

Die Diskussion der oben genannten Punkte macht deutlich, dass das Forschungsgebiet des Performance Measurement noch zahlreiche Herausforderungen bietet. Trotz der zahlreichen bestehenden offenen Fragen hofft der Verfasser, mit der vorliegenden Arbeit einen Beitrag zur Qualitätssteigerung bestehender und neuer PMS gemacht zu haben. Der wesentlichste Beitrag der vorliegenden Dissertation sieht der Verfasser dabei in der Durchgängigkeit des vorgeschlagenen Vorgehensmodells von der Strategie einer Organisation bis zu dem zugehörigen Datenmodell, welches die entwickelten PerformanceIndikatoren verwaltet.

Der Verfasser ist davon überzeugt, dass das Thema Performance Measurement aktuell bleiben wird. Die Beurteilung der Performance war, ist, und bleibt ein spannendes Thema. 


\section{Literaturverzeichnis}

AFQM: Homepage der Austrian Foundation for Quality Management (http://www.afqm.at; letzter Zugriff: 12. September 2002).

Aguilar, F. J.: Scanning the business environment. New York, 1967.

Ammon, A.: Objekt und Grundbegriffe der theoretischen Nationalökonomie. Wien, 1911.

Andersen, B., Fagerhaug, T.: Performance Measurement Explained. Milwaukee, 2002.

Ansoff, I.: Managing Surprise and Discontinuity - Strategic Response to Weak Signals. In: Zeitschrift für betriebswirtschaftliche Forschung 1976, Vol. 28, S. 129-152.

Atkinson, A. A., Waterhouse, J. H., Wells, R. B.: A Stakeholder Approach to Strategic Performance Measurement. In: Sloan Management Review 1997, Vol. 38(3), S. 2538.

Baan: Homepage der Baan Corp. (http://www.baan.com; letzter Zugriff: 25. August 2002).

Balanced-Scorecard-Collaborative: Balanced Scorecard Functional Standards. Release 1.0a; Version vom 5. Mai 2000 (www.bscol.com/bscoldata/Standardsv10a.pdf; letzter Zugriff: 12. September 2002). Lincoln/MA, 2000.

Balanced-Scorecard-Collaborative: BSC XML Draft Standards - Introduction Document; Version vom 18. März 2001 (http://www.bscol.com/bscoldata/XMLStandards1.pdf; letzter Zugriff:

12. September 2002).

Baldridge, M.: Homepage des Baldrige National Quality Program (http://www.quality.nist.gov/; letzter Zugriff: 12. September 2002).

Bauer, A., Günzel, H.: Data-Warehouse-Systeme: Architektur, Entwicklung, Anwendung. Heidelberg, 2001.

Bergamin, I.: Ein Gestaltungskonzept integrierter Früherkennung - Ergebnis einer systemtheoretischen Analyse von Metakonzepten der Früherkennung und von Prognoseansätzen. Berlin, 2001.

Beyeler, C.: Performance-Measurement-Systeme in der Schweizer Unternehmenspraxis eine empirische Untersuchung. (wird voraussichtlich Ende 2002 als Diplomarbeit am Departement für Informatik, Universität Fribourg eingereicht). Fribourg, 2002.

BFS: Homepage des Bundesamtes für Statistik (http://www.statistik.admin.ch; letzter Zugriff: 29. August 2002).

Bitici, U., Carrie, A. S.: Integrated Performance Measurement Systems: Structures and Relationships. Swindon, UK, EPSRC Final Research Report Grant No. GR/K 48174, 1998.

Bitici, U., Carrie, A. S., Turner, T. J., Lutz, S.: Integrated Performance Measurement Systems: Implementation Case Studies. In: Proceedings of the IFIP WG5.7 Annual 
Working Conference on Strategic Management of the Manufacturing Value Chain, Dordrecht, 1998, S. 177-186.

Blankenburg, D. A.: Evaluation von Performance Measurement Systemen - Eine empirische Analyse. Regensburg, 1999.

Blohm, H.: Die Gestaltung des betrieblichen Berichtswesens als Problem der Leitungsorganisation. Berlin, 1974.

Boemle, M.: Der Jahresabschluss - Bilanz, Erfolgsrechnung und Anhang. Zürich, 1992.

Boemle, M.: Unternehmensfinanzierung. Zürich, 1998.

Böhnlein, M., Ulbrich-vom-Ende, A.: Business Process Oriented Development of Data Warehouse Structures. In: Proceedings of Data Warehousing 2000 - Methoden, Anwendungen, Strategien. Friedrichshafen, 2000, S. 3-22.

Böhnlein, M., Ulbrich-vom-Ende, A.: Ein konzeptuelles Data Warehouse-Modell für die Erstellung multidimensionaler Datenstrukturen. In: InformationssystemArchitekturen, Rundbrief der GI-Fachgruppe 5.10, 2001, 8. Jahrgang, S. 25-58.

Bontis, N.: Assessing knowledge assets: a review of the models used to measure intellectual capital. In: International Journal of Management Reviews 2001, Vol. 3(1), S. 41-60.

Bosch, J.: Design and use of software architectures - Adopting and evolving a product-line approach. Harlow, 2000.

Bourne, M., Mills, J. F., Wilcox, M., Neely, A., Platts, K.: Designing, implementing and updating performance measurement systems. In: International Journal of Operations \& Production Management 2000, Vol. 20(7), S. 754-771.

Brenner, W., Wilking, G., Zarnekow, R.: Strategische Aspekte des Make or Buy im Informationsmanagement. In: HMD - Praxis der Wirtschaftsinformatik 1999, Vol. 206, S. 9-17.

Brockhaus: Die Enzyklopädie in 24 Bänden. Mannheim, 2001.

Brooking, A.: Intellectual Capital: Core Assets for the Third Millennium Enterprise. London, 1996.

Brown, D. M., Laverick, S.: Measuring corporate performance. In: Long range planning 1994, Vol. 27(4), S. 89-98.

Brown, M. G.: Keeping Score - Using the Right Metrics to Drive World-Class Performance. New York, 1996.

Brunner, J.: Value-Based Performance Management: Wertsteigernde Unternehmensführung: Strategien - Instrumente - Praxisbeispiele. Wiesbaden, 1999.

Brunner, J., Roth, P.: Performance-Management und Balanced Scorecard in der Praxis. In: io management 1999, Vol. 68(7/8), S. 50-55. 
Bulos, D.: A New Dimension. In: Database Programming \& Design 1996, Vol. 9(6), S. 3337.

Bulos, D., Forsman, S.: Getting Started with ADAPT. Report: Symmetry Corporation. San Rafael, 1998.

Bürkeler, A.: Kennzahlen als Führungsinstrument. Zürich, 1977.

Camp, R. C.: Benchmarking. Milwaukee, 1994.

Charnes, A., Cooper, W. W., Rhodes, E.: Measuring the Efficiency of Decision Making Units. In: European Journal of Operations Research 1978, Vol. 2, S. 429-444.

Chen, P. P.: The Entity-Relationship Model - Towards a Unified View of Data. In: ACM Transactions on Database Systems 1976, Vol. 1(1), S. 9-36.

Clark, A., Wettstein, T.: English Language Internet Survey of Students: Is English a Priority for you? Fribourg, Internal Working Paper for the Rectorate of Fribourg University, 2000.

Codd, E. F., Codd, S. B., Salley, C. T.: Providing OLAP to User-Analysts: An IT Mandate. Report: E.F. Codd Associates (http://www.hyperion.com/downloads/olap_to_useranalysts_wp.pdf; letzter Zugriff: 12.September 2002).

Colletti, J.: A field guide to focused planning: Hoshin Kanri - American Style. East Granby, 1996.

Conolly, T. M., Begg, C. E.: Database systems: a practical approach to design, implementation, and management. Essex, 1998.

Copeland, T., Koller, T., Murrin, J.: Valuation - Measuring and Managing the Value of Companies. New York, 1994.

COPPA: Computer-based Process Performance Measurement (http://www2iiuf.unifr.ch/is/peter/COPPA.htm; letzter Zugriff: 12. September 2002).

Cordero, R.: The measurement of innovation performance in firm: An overview. In: Research Policy 1989, Vol. 19, S. 185-192.

Coulouris, G., Dollimore, J.: Distributed Systems: Concepts and Design. Wokingham, 1994.

Creelman, J.: Building and implementing a balanced scorecard. London, 1998.

Davenport, T. H., Prusak, L.: Working knowledge: How organizations manage what they know. Boston, 1998.

Deming, E. W.: Out of the Crisis. Boston, 2000.

Deming, E. W.: The W. Edwards Deming Institute (http://www.deming.org; letzter Zugriff: 25. August 2002). 
Department-of-Navy: The Process Improvement Notebook (PIN). Arlington, VA / USA, Departement of the Navy - Total Quality Leadership Office, 1999a.

Department-of-Navy: The Process Improvement Notebook (PIN) - Module 2 Brainstorming. Arlington, VA / USA, Departement of the Navy - Total Quality Leadership Office, 1999b.

Department-of-Navy: The Process Improvement Notebook (PIN) - Module 4 Affinity Diagram. Arlington, VA / USA, Departement of the Navy - Total Quality Leadership Office, 1999c.

Department-of-Navy: The Process Improvement Notebook (PIN) - Module 5 Cause- andEffect-Diagram. Arlington, VA / USA, Departement of the Navy - Total Quality Leadership Office, 1999d.

Deyhle, A.: Controlling-Berichtswesen. In: Controller-Magazin 1978, Vol. 3, S. $122 \mathrm{ff.}$

Donnell, F. J. O., Duffy, A. H. B.: Modelling Design Development Performance. In: Proceedings of the International Workshop on Performance Measurement. Glasgow, 2001, S. 1-20.

Drongelen, C. K., Cook, A.: Design principles for the development of measurement systems for research and development processes. In: R\&D Management 1997, Vol. 27(4), S. 345-357.

DUDEN: DUDEN - Das grosse Wörterbuch der deutschen Sprache in 10 Bänden. Mannheim, 1999.

DuPont: Homepage der E.I. du Pont de Nemours and Company (http://www.dupont.com; letzter Zugriff: 12. September 2002).

Dwight, R.: Searching for real maintenance performance measures. In: Journal of Quality in Maintenance Engineering 1999, Vol. 5(3), S. 258-275.

Eccles, R.: The Performance Measurement Manifesto. In: Harvard Business Review 1991, Vol. 69(1), S. 131-138.

Edvinsson, L., Malone, M. S.: Intellectual Capital: Realizing Your Company's True Value by Finding Its Hidden Brainpower. New York, 1997.

EFQM: Selbstbewertung 1997 - Richtlinien für Unternehmen. Brüssel, European Foundation for Quality Management, 1996.

EFQM: Die acht Eckpfeiler der Excellence - Die Grundkonzepte der EFQM und ihr bedeutsamer Nutzen. Brüssel, EFQM, 1999.

EFQM: The EFQM in Action. Brüssel, EFQM, 2001.

EFQM: Homepage der European Foundation for Quality Management (http://www.efqm.org; letzter Zugriff: 12. September 2002).

Ehrbar, A.: EVA - Economic Value Added: Der Schlüssel zur wertsteigernden Unternehmensführung. Wiesbaden, 1999. 
Epstein, M. J., Manzoni, J. F.: The Balanced Scorecard and Tableau de Bord - Translating Strategy Into Action. In: Management Accounting 1997, Vol. 79(8), S. 28-36.

Epstein, M. J., Roy, M.-J.: Sustainability in Action: Identifying and Measuring the Key Performance Drivers. In: Long Range Planning 2001, Vol. 34(5), S. 585-604.

Erhard, L.: Homepage des Ludwig Erhard Preises e.V. (http://sun.vdi-online.de/lep/; letzter Zugriff: 25. Juli 2002).

ESPRIX: Homepage des Schweizerischen Qualitätspreises für Business Excellence (http://www.esprix.ch; letzter Zugriff: 12. September 2002).

Evangelidis, K.: Performance measured performance gained. In: The Treasurer 1992, Vol. (February), S. 45-47.

Fabasoft: Homepage der Fabasoft AG (http://www.fabasoft.at; letzter Zugriff: 29.08.2002).

Feigenbaum, A.: Total Quality Control. In: Harvard Business Review 1956, Vol. 34(6), S. 93-101.

Felden, C., Florek, S.: Zentrale Datenbasis im Energiehandel. In: Operations Research Proceedings 2001 - Selected Papers of the Symposium on Operations Research (OR 2001). Duisburg, 2001, S. 43-50.

Ferstl, O. K., Sinz, E.: SOM Modeling of Business Systems. In: Bernus, P., Mertins, K., Schmidt, G.: Handbook on Architectures on Information Systems - International Handbook on Information Systems. Berlin, 1998, S. 339-358.

Fitzgerald, L., Johnston, R., Brignall, S., Silvestro, R., Voss, C.: Performance Measurement in Service Businesses. London, 1991.

Fleischhauer, D.: Königsdiziplin der Unternehmenssteuerung: Studie zu Performance Measurement. In: Managementberater 1998, Vol. 2(11), S. 10-12.

Flores, M., Ortega, I.: Performance Indicators Structure. St. Gallen, ITEM-HSG, 1998.

Fries, S.: Neuorientierung der Qualitätskostenrechnung in prozessorientierten TQM Unternehmen. St. Gallen, Universität St. Gallen, 1994.

Frigo, M. L., Krumwiede, K. R.: 1998 CMG Survey on Performance Measurement. In: Cost Management Update 1999, Vol. 94, S. 1-4.

GartnerGroup: Homepage der Gartner Group (http://www.gartnergroup.com; letzter Zugriff: 25. August 2002).

Gibson, C., Nolan, R. L.: Managing the four stages of EDP growth. In: Harvard Business Review 1974, Vol. 52(1), S. 76-88.

Gladen, W.: Kennzahlen- und Berichtssysteme: Grundlagen zum Performance Measurement. Wiesbaden, 2001.

Gleich, R.: Performance Measurement. In: DBW - Die Betriebswirtschaft 1997, Vol. 57(1), S. 114-117. 
Gleich, R.: Das System des Performance Measurements. München, 2001.

Golfarelli, M., Maio, D., Rizzi, S.: Conceptual Design of Data Warehouses from E/R Schemes. In: Proceedings of the Hawaii International Conference on System Sciences. Kona, Hawaii, 1998.

Golfarelli, M., Rizzi, S.: Designing the Data Warehouse: Key Steps and Crucial Issues. In: Journal of Computer Science and Information Management 1999, Vol. 2(1), S. 114.

Gomez, P.: Modelle und Methoden des systemorientierten Managements. Bern, 1981.

Gomez, P.: Frühwarnung in der Unternehmung. Bern, 1983.

Grünig, R.: Verfahren zur Überprüfung und Verbesserung von Planungskonzepten. Bern, 1990.

Grünig, R.: Das Planungskonzept - Instrument zur Gestaltung der Planung und ihrer Kontrolle. Bern, 1996.

Grünig, R., Heckner, F.: Vorlesungsskript "Führung 1995/96". Fribourg, Seminar für Unternehmensführung, Universität Fribourg, 1995.

Grünig, R., Heckner, F., Zeus, A.: Methoden zur Identifikation strategischer Erfolgsfaktoren. In: Die Unternehmung 1996, Vol. 50(1), S. 3-12.

Grünig, R., Kühn, R.: Methodik der strategischen Planung - Ein prozessorientierter Ansatz für Strategieplanungsprojekte. Bern, 2000.

Günther, T., Grüning, M.: Performance Measurement Systems in Germany - A Descriptive Evaluation Report. Dresden, Department of Economics, Dresden University of Technology, 2000, S. 1-27.

Haberfellner, R., Daenzer, W. F. H.: Systems engineering: Methodik und Praxis. Zürich, 1992.

Hahn, D.: PuK. Planung und Kontrolle, Planungs- und Kontrollsysteme, Planungs- und Kontrollrechnung, Controllingkonzepte. Wiesbaden, 1996.

Hakes, C.: The Corporate Self Assessment Handbook - For measuring business excellence. London, 1996.

Hammer, M., Champy, J.: Business Reengineering: Die Radikalkur für das Unternehmen. Frankfurt, 1994.

Hammer, R.: Strategische Planung und Frühaufklärung. München, 1992.

Hapeslagh, P., Noda, T., Boulos, F.: Managing for Value - It's not just about the numbers. In: Harvard Business Review 2001, Vol. 79(7), S. 65-73.

Harrington, J. H., Harrington, J. S.: High Performance benchmarking: 20 steps to sucess. New York, 1996. 
Hartfiel, G., Hillmann, K.-H.: Wörterbuch der Soziologie. Stuttgart, 1982.

Hatley, D., Huruschka, P., Pirbhai, I.: Process for System Architecture and Requirements Engineering. New York, 1999.

Heckner, F.: Identifikation marktspezifischer Erfolgsfaktoren - Ein heuristisches Verfahren angewendet am Beispiel eines pharmazeutischen Teilmarktes. Bern, 1998.

Heinrich, L. J.: Grundlagen der Wirtschaftsinformatik In: Rechenberg, P., Pomberger, G.: Informatik-Handbuch. München, 1997, S. 859-873.

Heinzl, A., König, W., Hack, J.: Erkenntnisziele der Wirtschaftsinformatik in den nächsten zehn Jahren. In: Wirtschaftsinformatik 2001, Vol. 43(3), S. 223-233.

Helbling, C.: Unternehmensbewertung und Steuern. Düsseldorf, 1995.

Hoffmann, O.: Performance Management: Systeme und Implementierungsansätze. Bern, 1999.

Holthuis, J.: Der Aufbau von Data Warehouse-Systemen. Konzeption - Datenmodellierung - Vorgehen. Wiesbaden, 1999.

Horváth, P.: Neue Instrumente in der deutschen Unternehmenspraxis. In: Egger, A., Grün, O., Moser, R.: Managementinstrumente und -konzepte: Entstehung, Verbreitung und Bedeutung für die Betriebswirtschaft. Stuttgart, 1999, S. 289-328.

Horváth, P.: Controlling. München, 2001.

Hostettler, S.: Economic Value Added (EVA) - Darstellung und Anwendung auf Schweizer Aktiengesellschaften. Bern, 1998.

Huber, O.: Die Online-Version der Computermarktstudie: Eine Analyse unter dem Aspekt der Usability. Fribourg, Diplomarbeit, Departement für Informatik. Universität Fribourg, 2001.

Huch, B., Behme, W., Ohlendorf, T.: Rechnungswesen-orientiertes Controlling. Heidelberg, 1997.

Hudson, M., Bennett, J., Smart, A., Bourne, M.: Performance Measurement for Planning and Control in SMEs. In: Proceedings of the IFIP International Conference on Advances in Production Management Systems. Berlin, 1999, S. 219-225.

Hüsemann, B., Lechtenbörger, J., Vossen, G.: Conceptual Data Warehouse Design. In: Proceedings of the International Workshop on Design and Management of Data Warehouses. Stockholm, 2000, S. 6.1-6.11.

Hyperion: Homepage der Hyperion Solutions Corporation (http://www.hyperion.com; letzter Zugriff: 12. September 2002).

IBM: Data Modeling Techniques for Data Warehousing. San Jose, 1998.

IBM: Homepage der Websphere MQ family (http://www-3.ibm.com/software/ts/mqseries; letzter Zugriff: 25. August 2002). 
ICVisions: (http://www.icvisions.com; letzter Zugriff: 25. Juli 2002).

IEEE830: IEEE Std 830-1998: Recommended Practice for Software Requirements Specifications. New York, 1998.

IEEE1471: IEEE 1471-2000: Recommended Practice for Architectural Description of Software-Intensive Systems. New York, 2000.

Inmon, W. H.: Building the Data Warehouse. New York, 1996.

Intraware: Homepage der Intraware AG (http://www.intraware.de; letzter Zugriff: 12. September 2002).

Ishikawa, K.: What is Total Quality Control? New York, 1985.

ISO-8402: Quality management and quality assurance - Vocabulary. Geneva, International Organization for Standardization, 1994.

ISO: Information technology - Software process assessment - Part 2: A reference model for processes and process capability, TR 15504-2, ISO / IEC, 1998.

ISO: New edition of ISO 9000 Compendium includes ISO 9000:2000 series (www.iso.ch; letzter Zugriff: 10. Dezember 2001).

Jacobson, R.: Microsoft SQL Server 2000 Analysis Services Step by Step, Redmond, 2000.

JDEdwards: Homepage der J.D.Edwards (http://www.jdedwards.com/; letzter Zugriff: 12. September 2002).

Jenny, B.: Projektmanagement in der Wirtschaftsinformatik. Zürich, 1998.

Johnson, T. H., Kaplan, R. S.: Relevance Lost: The Rise and Fall of Management Accounting. Boston, 1991.

Juran, J. M., Gryna, F., M.: Quality Planning and Analysis - From Product development through Use. New York, 1993.

Kanji, G. K., Moura e Sà, P.: Kanji-Certification of Performance Measurement. Kanji Quality Culture, Ltd., An Integrated approach of organisational excellence. Sheffield, 2001.

Kaplan, R. S.: Yesterday's accounting undermines production. In: Harvard Business Review 1984, Vol. 62(4), S. 95-101.

Kaplan, R. S., Norton, D.: The Balanced Scorecard - Measures That Drive Performance. In: Harvard Business Review 1992, Vol. 70(1), S. 71-79.

Kaplan, R. S., Norton, D.: Putting the Balanced Scorecard to Work. In: Harvard Business Review 1993, Vol. 71(5), S. 134-147.

Kaplan, R. S., Norton, D.: The Balanced Scorecard. Boston, 1996a. 
Kaplan, R. S., Norton, D.: Using the Balanced Scorecard as a Strategic Management System. In: Harvard Business Review 1996b, Vol. 74(1), S. 75-85.

Kaplan, R. S., Norton, D.: Balanced Scorecard - Strategien erfolgreich umsetzen. Stuttgart, 1997.

Kauffmann, J.: Beginning ASP Databases. Birmingham, 2000.

Keegan, D. P., Eiler, R. G., Jones, C. R.: Are Your Performance Measures Obsolete? In: Management Accounting 1989, Vol. (June), S. 45-50.

Kimball, R.: The data warehouse toolkit. New York, 1996.

Kimball, R., Reeves, L., Roos, M., Thornthwaite, W.: The Data Warehouse Lifecycle Toolkit. New York, 1998.

Kitchenham, B.: Software Metrics: Measurement for Software Process Improvement. Cambridge, MA, 1996.

Klaus, A., Dörnemann, J., Knust, P.: Chance der IT-Unterstützung bei der Balanced Scorecard Einführung -Unternehmensweiter Einsatz der Renaissance Balanced Scorecard powered by Gentia. In: Controlling 1998, Vol. (6), S. 374-380.

Klausmann, W.: Betriebliche Frühwarnsysteme im Wandel. In: Zeitschrift für Führung und Organisation 1983, Vol. 52(1), S. 39 -45.

Kleingeld, P.: Performance Management in a Field Service Department: Design and Transprotatin of a Productivity Measurement and Enhancement System (ProMES). Valkenswaard, 1994.

Klingebiel, N.: Performance Measurement - Grundlagen, Ansätze, Fallstudien. Wiesbaden, 1999.

Klingebiel, N.: Integriertes Performance Measurement. Wiesbaden, 2000.

Kreikebaum, H.: Strategische Unternehmensplanung. Stuttgart, 1989.

Krystek, U., Müller-Stewens, G.: Frühaufklärung für Unternehmen. Identifikation und Handhabung zukünftiger Chancen und Bedrohungen. Stuttgart, 1993.

Kühn, R.: Entscheidungsmethodik und Unternehmenspolitik. Bern, 1978.

Kühn, R., Grünig, R.: Grundlagen der strategischen Planung - Ein integraler Ansatz zur Beurteilung von Strategien. Bern, 1998.

Küng, P.: Process performance measurement systems: a tool to support process-based organisations. In: Total Quality Management 2000, Vol. 11(1), S. 67-85.

Küng, P.: Performance-Measurement-Systeme im Dienstleistungssektor - Das Denken in Wirkungsketten ist noch wenig verbreitet. In: io management 2001, Vol. 70(1/2), S. 56-63. 
Küng, P., Meier, A., Wettstein, T.: Computer-based Performance Measurement in SMEs: Is there any option? In: Proceedings of the International Conference on Systems Thinking in Management. Geelong, Australia, 2000, S. 318-323.

Küng, P., Wettstein, T.: Measuring Customer Satisfaction using IT: A Case Study. In: Proceedings of the European Conference on Information Technology Evaluation. Brunel University, Uxbridge, 1999, S. 279-288.

Küng, P., Wettstein, T.: Performance Measurement Systems - there is a long road ahead. In: Proceedings of the International Workshop on Performance Measurement. Glasgow, 2001, S. 1-17.

Küng, P., Wettstein, T., List, B.: A Holistic Process Performance Analysis Through a Performance Data Warehouse. In: Proceedings of the Americas Conference on Information Systems. Boston, 2001, S. 349-356.

Küpper, H.-U.: Controlling - Konzeption, Aufgaben und Instrumente. Stuttgart, 2001.

Kurz, A.: Data Warehousing - Enabling Technology. Bonn, 1999.

Lanner, C.: Definition und Planung von Programmen zur Strategieumsetzung. Dissertation Universität Fribourg, Fribourg, 2000.

Lebas, M.: Managerial Accounting in France - Overview of past tradition and current practice. In: The European Accounting Review 1994, Vol. 3(3), S. 23-35.

Lebas, M.: Performance measurement and performance management. In: International Journal of Production Economics 1995, Vol. 41(9), S. 23-35.

Legner, C.: Benchmarking informationssystemgestützter Geschäftsprozesse: Methode und Anwendung. Wiesbaden, 1999.

Lenz, H., Shoshani, A.: Summarizability in OLAP and statistical databases. In: Proceedings of the 9th SSDBM, Olympia/Washington, 1997, S. 132-143.

List, B., Schiefer, J., Tjoa, A. M., Quirchmayr, G.: Multidimensional Business Process Analysis with the Process Warehouse. In: Abramowicz, W., Zurada, J.: Knowledge Discovery for Business Information Systems. Boston, 2000, S. 211-227.

Löhneysen, G.: Die rechtzeitige Erkennung von Unternehmungskrisen mit Hilfe von Frühwarnsystemen als Voraussetzung für ein wirksames Krisenmanagement. Göttingen, 1982.

Lotus: Homepage von Lotus Workflow (http://www.lotus.com/products/domworkflow.nsf; letzter Zugriff: 25. August 2002).

Lüthi, A., Krahn, A., Küng, P.: Herleitung von Indikatoren zur Messung der Geschäftsprozessqualität. In: Die Unternehmung 1998, Vol. 52(1), S. 35-47.

Lynch, R. L., Cross, K. F.: Applied Organizational Change in Industry - Structural, Technological and Humanistic Approaches. London, 1991a. 
Lynch, R. L., Cross, K. F.: Measure Up - The Essential Guide to Measuring Business Performance. London, 1991b.

Malorny, C.: TQM umsetzen: der Weg zur Business Excellence. Stuttgart, 1996.

Marchand, D. A., Kettinger, W. J., Rollins, J. D.: Information Orientation. New York, 2001.

Marr, B., Neely, A.: Measuring Business Performance in the E-Economy. In: Neely, A.: Performance Measurement - Past, Present and Future. Cranfield, 2000, S. 355-362.

Marr, B., Schiuma, G.: Defining Key Performance Indicators for Organisational Knowledge Assets. Cranfield, Cranfield School of Management, 2001, o.S.

Mauriel, J. J., Anthony, R. W.: Misvaluation of Investment Centre Performance. In: Harvard Business Review 1966, Vol. 44(2), S. 98-105.

McFarlan, W. F.: Information Technology Changes the Way You Compete. In: Harvard Business Review 1984, Vol. 62(3), S. 98-103.

Mendelow, A. L.: Information systems for organizational effectiveness - the use of the stakeholder approach. In: Proceedings of the IFIP WG8.2 Working Conference on Beyond Productivity. Minneapolis, 1983.

Merriam-Webster-Dictionnary: Homepage der Merriam-Webster (http://www.m-w.com; letzter Zugriff: 12. September 2002).

Meyer, C.: Betriebswirtschaftliche Kennzahlen und Kennzahlensysteme. Stuttgart, 1994.

Meyer, M. W., Gupta, V.: The Performance Paradox. In: Research in Organisational Behaviour 1994, Vol. 16, S. 309-369.

Microsoft: Active X Data Objects Multidimensional (ADO MD, http://msdn.microsoft.com/library/default.asp?url=/library/enus/ado270/htm/dasdkadooverview.asp; letzter Zugriff: 1. September 2002).

Mintzberg, H.: The Rise and Fall of Strategic Planning. New York, 1994.

Mountfield, A., Schalch, O.: Konzeption von Balanced Scorecard und Umsetzung in ein Management-Informationssystem mit dem SAP Business Information Warehouse. In: Controlling 1998, Vol. 10(5), S. 316-322.

Muchna, C.: Strategische Marketing-Früherkennung auf Investitionsgütermärkten. Wiesbaden, 1988.

Muehlen, M. z., Rosemann, M.: Workflow-based Process Monitoring and Controlling Technical and Organizational Issues. In: Proceedings of the Hawaii International Conference on System Sciences. Hawaii, 2000.

Müller, G.: Strategische Frühaufklärung. München, 1981.

Mulligan, P., Hatten, K., Miller, J.: From issue-based planning to hoshin: different styles for different situations. In: Long Range Planning 1996, Vol. 4(29), S. 473-484. 
Murata, T.: Petri Nets: Properties, Analysis and Applications. In: Proceedings of the IEEE 1989, Vol. 77(4), S. 541-580.

Neely, A.: Measuring Business Performance. London, 1998.

Neely, A.: The performance measurement revolution: why now and what next? In: International Journal of Operations \& Production Management 1999, Vol. 19(2), S. 205-228.

Neely, A.: Performance measurement system design: developing and testing a processbased approach. In: International Journal of Operations \& Production Management 2000, Vol. 20(10), S. 1119-1145.

Neely, A.: The Catalogue of Performance Measures. (http://www.som.cranfield.ac.uk/som/cbp/catperf.htm; letzter Zugriff: 24. August 2002).

Neely, A., Gregory, M., Platts, K.: Performance measurement system design - A literature review and research agenda. In: International Journal of Operations \& Production Management 1995, Vol. 15(4), S. 80-116.

Neely, A., Mills, J., Gregory, M. J., Richards, A. H., Platts, K., Bourne, M.: Getting the measure of your business. London, 1996.

Noack, J., Mehmanesh, H., Mehmaneche, H., Zendler, A.: Architekturen für Network Computing. In: Wirtschaftsinformatik 2000, Vol. 42(1), S. 5-14.

Nonaka, I.: The Knowledge-Creating Company. In: Harvard Business Review 1991, Vol. 69(6), S. 96-104.

Nonaka, I., Takeuchi, H.: The Knowledge-creating Company: How Japanese Companies Create the Dynamics of Innovation. New York, 1995.

Ortega, I., Brügger, P., Endress, G. A., Hubacher, E., Loeffler, D.: Bewertung der Leistungsfähigkeit in ENAPS. St. Gallen, Institut für Technologiemanagement, 1997.

Ortega, I., Brügger, P., Endress, G. A., Hubacher, E., Loeffler, D.: Arbeitspaket 3: Bewertung der Leistungsfähigkeit (Produktivität). St. Gallen, Universität St. Gallen, 1999.

Ortner, E., Söllner, B.: Semantische Datenmodellierung nach der Objekttypenmethode. In: Informatikspektrum 1989, Vol. 1989(12), S. 31-42.

Parasuraman, A., Zeithaml, V., Berry, L.: SERVQUAL: A Multiple-Item Scale for Measuring Consumer Perceptions of Service Quality. In: Journal of Retailing 1988, Vol. 64(1), S. 12-40.

Paulk, M., Weber, C., Chrissis, M., Bush, M.: The Capability Maturity Model - Guidelines for Improving the Software Process. Boston, 1994.

Paulk, M., Weber, C., Garcia, S., Chrissis, M., Bush, M.: Key Practices of the Capability Maturity Model, Pittsburgh, Carnegie Mellon Software Engineering Institute, 1993. 
Pedersen, T. B., Jensen, C. S.: Multidimensional Data Modeling for Compex Data. In: Procedings of the 15th International Conference on Data Engineering. Sydney, 1999, S. 336-345.

PeopleSoft: Homepage der Peoplesoft Inc. (http://www.peoplesoft.com; letzter Zugriff: 25. August 2002).

Peters, T., Waterman, R. H.: In Search of Excellence. New York, 1982.

Petkovic, D.: Microsoft SQL Server 2000. München, 2001.

Poe, V., Klauer, P., Brobst, S.: Building a Data Warehouse for Decision Support. Upper Saddle River, 1997.

Porter, M. E.: Wettbewerbsstrategie (Competitive Strategy). Frankfurt, 1999.

Probst, P., Gomez, P.: Die Praxis des ganzheitlichen Problemlösens. Bern, 1995.

PROMOSYS: A Monitoring System for Information-based Business Processes (http://www2-iiuf.unifr.ch/is/peter/PROMOSYS.htm; letzter Zugriff: 12. September 2002).

Pümpin, C.: Strategische Erfolgspositionen: Methodik der dynamischen strategischen Unternehmensführung. Bern, 1992.

QPR-Software: Homepage der QPR Software Plc. (http:www.qpr.com; letzter Zugriff: 12. September 2002).

Raffée, H., Abel, B.: Wissenschaftstheoretische Grundfragen der Wirtschaftswissenschaften. München, 1979.

Rappaport, A.: Creating Shareholder Value: The New Standard for Business Performance. New York, 1986.

Rappaport, A.: Shareholder Value: Wertsteigerung als Massstab für die Unternehmensführung. Stuttgart, 1995.

Rauh, O.: Gütekriterien für die semantische Datenmodellierung. In: HMD -Praxis der Wirtschaftsinformatik 1991, Vol. 158, S. 91-110.

Rauh, O.: Überlegungen zur Behandlung ableitbarer Daten im Entity-Relationship-Modell (ERM). In: Wirtschaftsinformatik 1992, Vol. 34(3), S. 294-306.

Rautenstrauch, C.: Modellierung und Implementierung von Data-Warehouse-Systemen. Magdeburg, 1997.

Reece, J. S., Cool, W. R.: Measuring investment center performance. In: Harvard Business Review 1978, Vol. 56(3), S. 24ff.

Renggli, R.: Strategische Kontrolle: Theoretische Konzepte und eine empirische Studie in Schweizer Unternehmen. Bern, Lizenziatsarbeit, Institut für Organisation und Personal, Universität Bern, 1996. 
Reuters: Homepage der Reuters (http://www.reuters.com; letzter Zugriff: 12. September 2002).

Roberts, J.: Key performance indicators manual. Melbourne, 1996.

Rockart, J. F.: Chief executives define their own data needs. In: Harvard Business Review 1979, Vol. 57(2), S. 81-92.

Rolstadas, A.: Enterprise performance measurement. In: International Journal of Operations \& Production Management 1998, Vol. 18(9/10), S. 989-999.

Roos, J., Roos, G., Dragonetti, N. C., Edvinsson, L.: Intellectual Capital: Navigating in the New Business Landscape. London, 1997.

Rühli, E.: Unternehmensführung und Unternehmenspolitik. Bern, 1985.

Rummler, G. A., Brache, A. P.: Improving Performance: How to manage the white space on the organization chart: a practical guide for managing organizations, processes, and jobs. San Francisco, 1990.

Rupp, C.: Requirements-Engineering und -Management. München, 2001.

Saldo: Arbeitsplatz - Geld oder Grippe: "Ein Hohn". Saldo, 2002, 30. Januar 2002.

SAP: Homepage der SAP AG (http://www.sap.com; letzter Zugriff: 25. August 2002).

Sapia, C., Blaschka, M., Höfling, G., Dinter, B.: Extending the E/R Model for the Multidimensional Paradigm. In: Proceedings of the International Workshop on Data Warehousing and Data Mining. Singapore, 1998, o.S.

Scheer: Optimieren Sie Ihre Prozess Performance - Process Performance Manager (White Paper). Saarbrücken, IDS Scheer, 2000a.

Scheer: Process Performance Manager (Fact-Sheet). Saarbrücken, IDS Scheer, 2000b.

Scheer, A.-W.: ARIS, Modellierungsmethoden, Metamodell, Anwendungen. Heidelberg, 2001.

Schinzer, H.: Data Warehousing und Data Mining - Marktführende Produkte im Vergleich. München, 1999.

Schmid, U.: Das Anspruchgruppen-Konzept. In: WISU - Das Wirtschaftsstudium 1997, Vol. 26(7), S. 633-635.

Scholz, C.: Planning Procedures in German Companies - Findings \& Consequences. In: Long Range Planning 1984, Vol. 17(6), S. 94-103.

Schreyögg, G., Steinmann, H.: Strategische Kontrolle. In: Zeitschrift für betriebswirtschaftliche Forschung 1985, Vol. 37, S. 391-410.

Seghezzi, H.-D.: Integriertes Qualitätsmanagement: Das St. Galler Konzept. München, 1996. 
Seghezzi, H.-D.: Erfahrungen mit Qualitätsbewertungen und Qualitätswettbewerbern aus der Sicht eines Jurors des European Quality Awards. In: Wunderer, R., Gerig, V., Hauser, R.: Qualitätsorientiertes Personalmanagement. Das Europäische Qualitätsmodell als unternehmerische Herausforderung. München, 1997, S. 107118.

SEI: Capability Maturity Model (SW-CMM) Software (http://www.sei.cmu.edu/cmm/; letzter Zugriff: 25. Juli 2002).

Seidenschwarz, W., Gleich, R.: Controlling und Marketing als Schwesterfunktionen Balanced Scorecard und marktorientiertes Kostenmanagement als verbindende Konzepte. In: Reinecke, S., Tomczak, T., Dittrich, S.: Marketingcontrolling. St. Gallen, 1998, S. 258-272.

Senge, P. M.: The Fifth Discipline. New York, 1990.

Siegwart, H.: Kennzahlen für die Unternehmensführung. Bern, 1998.

Simons, R.: Strategic Orientation and Top Management Attention to Control Systems. In: Strategic Management Journal 1991, Vol. 12(1), S. 49-62.

Simons, R.: Performance Measurement \& Control Systems for Implementing Strategy Text and Cases. Upper Saddle River, 1999.

Simons, R., Davila, A.: How High Is Your Return on Management? In: Harvard Business Review 1998, Vol. 76(1), S. 71-80.

Sinclair, D., Zairi, M.: Effective process management through performance measurement Part III - an integrated model of total quality-based performance measurement. In: Business Process Re-engineering \& Management Journal 1995, Vol. 1(3), S. 50-65.

Sinz, E.: Datenmodellierung betrieblicher Probleme und ihre Unterstützung durch ein wissensbasiertes Entwicklungssystem. Regensburg, 1987.

Skandia: Homepage der Skandia (http://www.skandia.com; letzter Zugriff: 7. September 2002).

Smith, A.: The Wealth of Nations. London, 1776.

Sommerville, I.: Software Engineering. München, 2001.

SPI: Homepage des Strategic Planning Institute (http://pimsonline.com; letzter Zugriff: 12. September 2002).

Steinmann, H., Braun, W.: Zum Prinzip der Wertfreiheit in der Betriebswirtschaftslehre. In: Raffée, H., Abel, B.: Wissenschaftstheoretische Grundfragen der Wirtschaftswissenschaften. München, 1979, S. 191-204.

Steinmann, H., Schreyögg, G.: Zur organisatorischen Umsetzung der strategischen Kontrolle. In: Zeitschrift für betriebswirtschaftliche Forschung 1986, Vol. 38, S. 747-765. 
Steinmann, H., Schreyögg, G.: Management: Grundlagen der Unternehmensführung. Wiesbaden, 1993.

Stern, J. M.: EVA Roundtable. In: Journal of Applied Corporate Finance 1994, Vol. 7(2), S. 46-70.

Stewart, G. B.: The Quest for Value? A Guide for Senior Managers. New York, 1991.

Stewart, T. A.: Intellectual Capital: The New Wealth of Organizations. New York, 1997.

Stroebe, R. W., Stroebe, G. H.: Führungsstile. Heidelberg, 1992.

Stuber, R.: Strategische Investitionsprogrammplanung in divisionalisierten Unternehmen Vorschlag eines heuristischen Verfahrens. Dissertation, Universität Fribourg. Fribourg, 2001.

Sveiby, K.-E.: The Invisible Balance Sheet. Ledarskap. Neben dem Original in schwedisch existiert eine englische Übersetzung, die nie verlegt wurde (verfügbar über: http://www.sveiby.com.au/articles/IntangAss/denosynl.htm; letzter Zugriff: 12. September 2002), 1989.

Sveiby, K.-E.: Homepage der Sveiby Knowledge Associates (http://www.sveiby.com.au; letzter Zugriff: 12. September 2002).

Sveiby-Toolkit: The Sveiby Toolkit (http://www.sveibytoolkit.com; letzter Zugriff: 12. September 2002).

Szyperski, N.: Gegenwärtiger Stand und Tendenzen der Entwicklung betrieblicher Informationssysteme. In: Hansen, H. R., Wahl, M. P.: Problem beim Aufbau betrieblicher Informationssysteme. München, 1973, S. 25 - 48.

Taylor, F. W.: Die Grundsätze wissenschaftlicher Betriebsführung (The Principles of Scientific Management). München, 1919.

Thommen, J.-P.: Betriebswirtschaftslehre - Band 1: Unternehmung und Umwelt, Marketing. Zürich, 1992a.

Thommen, J.-P.: Betriebswirtschaftslehre - Band 2: Materialwirtschaft, Produktion, Finanzierung, Investition. Zürich, 1992b.

Totok, A.: Modellierung von OLAP- und Data-Warehouse-Systemen. Wiesbaden, 2000.

Totok, A., Jaworski, R.: Modellierung von multidimensionalen Datenstrukturen mit ADAPT. Braunschweig, Institut für Wirtschaftswissenschaften, Technische Universität Braunschweig, 1998, Arbeitsbericht Nr. 98/11.

Ulrich, H.: Von der Betriebswirtschaftslehre zur systemorientierten Managementlehre. In: Wunderer, R.: Betriebswirtschaftslehre als Management- und Führungslehre. Stuttgart, 1988, S. 173ff.

UM.IPK.FHG: Zielsystem-Editor. Berlin, Fraunhofer IPK - Unternehmensmanagement, 1996. 
Vester, F.: Der Papiercomputer. In: Management Wissen 1987, Vol. (Oktober), S. 48-57.

Vetter, M.: Konzeptionelle Datenmodellierung. In: Kurbel, K., Strunz, H.: Handbuch Wirtschaftsinformatik. Stuttgart, 1990, S. 383-401.

Villalobos, G.: Web-Application for the Customer Satisfaction Measurement. Diplomarbeit, Departement für Informatik. Universität Fribourg, Fribourg, 2000.

Watson, G.: The Benchmarking Workbook. Cambridge /MA, 1992.

Watty, K., Terzioglu, B.: Performance Measures Employed by Australian Subsidiaries of US Services Multinational Companies: An Empirical Survey. Melbourne, School of Accounting and Law, 1999.

Weber, J.: Einführung in das Controlling. Stuttgart, 1998.

Weber, J., Schäffer, U.: Auf dem Weg zu einem aktiven Kennzahlenmanagement. In: Die Unternehmung 1999, Vol. 53(5), S. 333-350.

Weitz, W.: Basisarchitekturen Web-basierter Informationssysteme. In: Wirtschaftsinformatik 2002, Vol. 44(3), S. 207-216.

Wettstein, T., Küng, P.: Gesamtheitliches Performance Measurement - Vorgehensmodell und informationstechnische Ausgestaltung. Fribourg, Department of Informatics. 2001. Nachfolgend in identischer Form mit umgekehrter Autorenreihenfolge erschienen in: Britzelmaier, B., Geberl, S., Weinmann, S.:

Informationsmanagement - Herausforderungen und Perspektiven. Stuttgart, 2001, S. 229-240.

Wettstein, T., Küng, P.: A Maturity Model for Performance Measurement Systems. In: Prebbia, C. A., Pascola, P.: Management Information Systems. Southampton, 2002, S. 113-122.

Wettstein, T., Küng, P., Meier, A.: Performance Measurement als Ausweg aus dem Information Overload: Ein zielorientierter Ansatz. In: HMD -Praxis der Wirtschaftsinformatik 2001, Vol. 222, S. 49-58.

Wild, J.: Budgetierung. In: Marketing-Enzyklopädie. München, 1974, S. 325-340.

Wild, J.: Grundlagen der Unternehmensplanung. Opladen, 1982.

Winchell, W.: Inspection and Measurement in Manufacturing. Dearborn, 1996.

Wordsmyth: Homepage des Wordsmyth Thesaurus (http://www.wordsmyth.net; letzter Zugriff: 25. Juli 2002).

Workflow-Management-Coalition: Homepage der Workflow Management Coalition (WfMC; http://www.wfmc.org; letzter Zugriff: 12. September 2002).

Wunderer, R.: Führung und Zusammenarbeit. Stuttgart, 1997.

Wunderer, R., Grunwald, W.: Führungslehre: Grundlagen der Führung. Berlin, 1980. 
Zehnder, C. A.: Informatik-Projektentwicklung - Eine Einführung für Informatikstudenten und Praktiker. Zürich, 1991. 


\section{Teil \\ Anhänge}




\title{
Anhang A: Erhebung zum Stand des Performance Measurement und deren IT-Systeme in der Praxis
}

\author{
UNIVERSITÉ DE FRIBOURG SUISSE \\ UNIVERSITÄT FREIBURG SCHWEIZ
}

\author{
DEPARTEMENT FÜR INFORMATIK

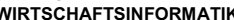 \\ PROF. DR. ANDREAS MEIER \\ Rue Faucigny 2 \\ CH-1700 Fribourg
}

\author{
Actelion Ltd. \\ Konrad P. Wirz \\ Gewerbestrasse 16 \\ 4123 Allschwil
}

Fribourg, Ende November 2001

\begin{abstract}
ERHEBUNG ZUM STAND DES PERFORMANCE MEASUREMENTS UND DEREN IT-SYSTEME IN DER PRAXIS
\end{abstract}

Sehr geehrter Herr Wirz

Ihm Rahmen eines Forschungsprojektes zum Thema Performance Measurement an der Wirtschaftswissenschaftlichen Fakultät der Universität Freiburg, führen wir eine Umfrage bezüglich dem „Stand des Performance Measurements und deren IT-Systeme“ durch.

Performance-Measurement-Systeme unterstützen die Überwachung von finanziellen und nicht-finanziellen Steuerungsgrössen eines Unternehmens (z.B. ROI, EVA, Umsätze, Mitarbeiterzufriedenheit). Mit Ihrer Hilfe möchten wir abklären, wie weit die Entwicklung dieser Systeme in der Praxis vorangeschritten ist und welche Eigenschaften moderne Informationssysteme für das Performance Measurement erfüllen müssen.

Ihre Meinung ist wichtig und ermöglicht uns, in der Theorie formulierte Ansätze auf ihre Verwendbarkeit in der Praxis zu überprüfen. Von Ihrer Teilnahme an der Umfrage können Sie selbst profitieren. Wenn Sie den beigelegten Fragebogen ausfüllen, senden wir Ihnen gerne die Resultate der Befragung zu. Sie verschaffen sich damit einen Überblick der Möglichkeiten zur informationstechnischen Ausgestaltung von Performance-Measurement-Systemen.

Bei Fragen können Sie den zuständigen Assistenten via E-Mail: thomas.wettstein@unifr.ch oder Telefon: 0263008336 kontaktieren.

Wir bitten Sie darum, den beigelegten Fragebogen an die mit der Leistungsmessung beauftragte Stelle, den CFO oder den Leiter Controlling, weiterzuleiten.

Für die Rücksendung des Fragebogens bis Mitte Dezember 2001 danken wir Ihnen im voraus bestens.

Mit freundlichen Grüssen

Prof. Dr. Andreas Meier 


\title{
Erhebung zum Stand des Performance Measurements und deren IT-Systeme in der Praxis
}

\section{BEGRIFFE}

Performance-Measurement-System (PMS) - Ein PMS ist ein Instrument, um das in der Vergangenheit Geleistete zu kontrollieren und quantitativ auszuwerten. Es wird genutzt, um die Geschäftsziele transparent zu machen, das Erreichte quantitativ zu erfassen und zu kommunizieren, den Blickwinkel zu erweitern und die gegenseitigen Beeinflussungen besser zu verstehen. Die betrachteten Gegenstandsbereiche sind die verschiedenen Einheiten einer Organisation. Die Empfänger der Performance-Informationen sind die Stakeholder (Management, Mitarbeiter, evtl. Kunden und Lieferanten) der entsprechenden Organisationseinheiten.

IT-System für das PMS - Darunter verstehen wir ein rechnergestütztes Informationssystem, welches das Sammeln, Verwalten, Analysieren und Kommunizieren der performancerelevanten Daten unterstützt.

Weiterführende Informationen zum Thema Performance Measurement finden Sie auf unserer Projektwebseite www.measure.ch

\section{ADMINISTRATIVES}

Aufwand - Für die Beantwortung der Fragen benötigen Sie etwa 20 Minuten.

Anonymität - Das Departement für Informatik der Universität Fribourg führt seit 1961 regelmässig empirische Untersuchungen durch. Wir garantieren Ihnen Anonymität. Für die Zuordnung zu Branchen- und Grössenklassen, sowie für allfällige Rückfragen benötigen wir Ihre Anschrift. Bei der Auswertung werden diese jedoch entfernt. Aufgrund der Studienresultate können weder auf Sie, noch auf Ihr Unternehmen Rückschlüsse gezogen werden.

Wenn Sie Fragen haben - Wir stehen Ihnen gerne zur Verfügung. Kontaktieren Sie uns unter 02630083 36. Unter dieser Nummer ist auch ein Anrufbeantworter installiert, der Ihre Fragen jederzeit gerne entgegen nimmt. Zusätzliche Kontaktmöglichkeiten sind Fax 0263009726 und E-Mail: christian.beyeler@unifr.ch und thomas.wettstein@unifr.ch.

Rücksendung - Wir bitten Sie, uns den Fragebogen im beiliegenden Antwortumschlag innert vierzehn Tagen zurückzusenden.

Für Ihre wertvolle Mitarbeit danken wir Ihnen bestens.

\author{
Abbildung A-2: Seite 1 des Fragebogens
}


Teil A: Stand der Performance-Measurement-Systeme

1. Ihr Unternehmen

Firma

Name / Vorname

Funktion / Abteilung

Telefon-Nummer

E-Mail

Abbildung A-3: Seite 2 des Fragebogens 
2. Wir setzen in unserem Unternehmen die folgenden Führungsinstrumente ein (Mehrfachnennungen möglich):

$\square \quad$ Value Based Management (z.B. EVA)

$\square \quad$ Balanced Scorecard (nach Kaplan/Norton)

$\square$ Activity Based Management

$\square \quad$ Management by Objectives

$\square$ "Business Excellence Model" - EFQM (European Foundation for Quality Management)

TQM

$\square \quad$ Intellectual Capital

andere:

3. Ist Ihr Unternehmen ISO zertifiziert?

(Mehrfachnennungen möglich):

$\square$ ISO 9000

ISO 14000

\section{Messen der Performance auf der strategischen Ebene}

4.1 Wir überprüfen regelmässig die Umsetzung der Unternehmensstrategie

$\square \quad$ Nein

$\square$ Ja, dabei werden Indikatoren in den folgenden Bereichen verwendet:

\begin{tabular}{|c|c|c|c|}
\hline & Nein & $\mathrm{Ja}$ & $\begin{array}{l}\text { Erhebungs- } \\
\text { häufigkeit }^{1}\end{array}$ \\
\hline Finanzielle Kennzahlen (z.B. ROI, EVA) & $\square$ & $\square$ & …................ \\
\hline Kunden (z.B. Kundenzufriedenheit, Beschwerden) & $\square$ & $\square$ & $\ldots \ldots .$. \\
\hline Mitarbeiter (z.B. Fluktuation, Krankheitstage, Ausbildung) & $\square$ & $\square$ & $\ldots \ldots$ \\
\hline Lieferanten (Lieferpünktlichkeit, Qualität) & $\square$ & $\square$ & $\ldots \ldots . .$. \\
\hline Innovation (z.B. Anz. Forschungsprojekte, Time to Market) & $\square$ & $\square$ & .................... \\
\hline $\begin{array}{l}\text { Gesellschaft (z.B. Papierverbrauch, Anz. positive Nachrichten } \\
\text { in den Medien) }\end{array}$ & $\square$ & $\square$ & $\ldots \ldots$ \\
\hline Andere: & $\square$ & $\square$ & $\ldots \ldots$ \\
\hline
\end{tabular}

${ }^{1}$ In welchen Abständen erheben Sie die Daten (z. B. monatlich, jährlich).

\section{Abbildung A-4: Seite 3 des Fragebogens}


4.2 Mit einem systematischen Controlling überwachen wir unsere strategischen Projekte

$\square \mathrm{Ja}$

$\square \quad$ Nein

4.3 Wir überwachen die vorhandenen Risiken systematisch

\begin{tabular}{|l|c|c|}
\cline { 2 - 3 } \multicolumn{1}{c|}{} & Nein & Ja \\
\hline Strategisches Risikomanagement & $\square$ & $\square$ \\
\hline Operatives Risikomanagement & $\square$ & $\square$ \\
\hline
\end{tabular}

4.4

Wir beobachten die Unternehmensumwelt systematisch

\begin{tabular}{|c|c|c|c|}
\hline & \\
\hline & Nein & $\mathrm{Ja}$ & $\begin{array}{l}\text { Erhebungs- } \\
\text { häufigkeit }^{1}\end{array}$ \\
\hline Benchmarking unserer Mitbewerber & $\square$ & $\square$ & \\
\hline Frühwarnsystem & $\square$ & $\square$ & \\
\hline $\begin{array}{l}\text { Das Frühwarnsystem umfasst die Rahmenbedingungen der } \\
\text { Strategie. }\end{array}$ & $\square$ & $\square$ & \\
\hline
\end{tabular}

\section{Performance-Messung auf der operativen Ebene}

Wir überprüfen regelmässig die Erfüllung der Zielsetzungen auf der operativen Ebene:

$\square \quad$ Nein

$\square$ Ja, wir überprüfen folgende Bereiche:

\begin{tabular}{|c|c|c|c|c|}
\hline & \multirow{2}{*}{$\begin{array}{l}\text { Bereich existiert } \\
\text { in unserem } \\
\text { Unternehmen } \\
\text { nicht }\end{array}$} & \multicolumn{3}{|c|}{ Überwachung der Zielerreichung } \\
\hline & & Nein & $\mathrm{Ja}$ & $\begin{array}{l}\text { Erhebungs- } \\
\text { häufigkeit }\end{array}$ \\
\hline Einkauf & $\square$ & $\square$ & $\square$ & $\ldots \ldots$ \\
\hline Transport & $\square$ & $\square$ & $\square$ & 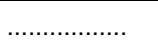 \\
\hline Lager & $\square$ & $\square$ & $\square$ & $x^{\prime} \ldots \ldots \ldots \ldots \ldots \ldots$ \\
\hline Produktion & $\square$ & $\square$ & $\square$ & ...... \\
\hline Marketing \& Verkauf & $\square$ & $\square$ & $\square$ & 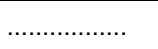 \\
\hline Forschung \& Entwicklung & $\square$ & $\square$ & $\square$ & \\
\hline Rechnungswesen & $\square$ & $\square$ & $\square$ & $x^{\prime} \ldots \ldots \ldots \ldots \ldots \ldots$ \\
\hline IT & $\square$ & $\square$ & $\square$ & $\ldots$. \\
\hline \multicolumn{2}{|c|}{ Andere: } & $\square$ & $\square$ & 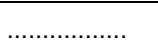 \\
\hline
\end{tabular}

Abbildung A-5: Seite 4 des Fragebogens 


\section{Kommunikation der Performance-Daten}

Performance-Daten werden den folgenden Interessensgruppen zur Verfügung gestellt: (Mehrfachnennungen möglich):
Höheres Kader
Mittleres Kader
$\square$ Mitarbeiter
$\square$ Investoren/Shareholder
$\square$ Kunden
$\square$ Lieferanten
$\square$ Öffentliche Stellen
$\square$ andere:

\section{Wie beurteilen Sie die Verwendung der Performance-Resultate?}

\begin{tabular}{|l|c|c|c|c|}
\cline { 2 - 5 } \multicolumn{1}{l|}{ Performance-Resultate unterstützen ... } & $\begin{array}{c}\text { trifft } \\
\text { nicht zu }\end{array}$ & $\begin{array}{c}\text { trifft kaum } \\
\text { zu }\end{array}$ & $\begin{array}{c}\text { trifft ziemlich } \\
\text { gut zu }\end{array}$ & $\begin{array}{c}\text { trifft } \\
\text { völlig zu }\end{array}$ \\
\hline $\begin{array}{l}\text { das obere Management bei Planungs- und } \\
\text { Führungsaufgaben auf der strategischen Ebene. }\end{array}$ & $\square$ & $\square$ & $\square$ & $\square$ \\
\hline $\begin{array}{l}\text { das mittlere Management bei Planungs- und } \\
\text { Führungsaufgaben auf der taktischen Ebene }\end{array}$ & $\square$ & $\square$ & $\square$ & $\square$ \\
\hline $\begin{array}{l}\text { Teamleiterlnnen und Mitarbeiterlnnen bei } \\
\text { kurzfristigen Entscheidungen. }\end{array}$ & $\square$ & $\square$ & $\square$ & $\square$ \\
\hline
\end{tabular}

\section{Qualität der Performance-Indikatoren?}

Untenstehend finden Sie eine Reihe von Anforderungen, die allgemein an Performance-Indikatoren gestellt werden. Wie beurteilen Sie die in Ihrer Organisation verwendeten Indikatoren bezüglich dieser Kriterien:

\begin{tabular}{|l|c|c|c|c|}
\cline { 2 - 4 } \multicolumn{1}{l|}{} & $\begin{array}{c}\text { trifft } \\
\text { nicht zu }\end{array}$ & $\begin{array}{c}\text { trifft kaum } \\
\text { zu }\end{array}$ & $\begin{array}{c}\text { trifft ziemlich } \\
\text { gut zu }\end{array}$ & $\begin{array}{c}\text { trifft } \\
\text { völlig zu }\end{array}$ \\
\hline $\begin{array}{l}\text { Die verwendeten Indikatoren widerspiegeln die } \\
\text { innen zu Grunde liegenden Ziele. }\end{array}$ & $\square$ & $\square$ & $\square$ & $\square$ \\
\hline $\begin{array}{l}\text { Die verwendeten Indikatoren sind ausgewogen, } \\
\text { d.h. alle Aspekte (Finanzen, Kunden, Mitarbeiter, } \\
\text { Umwelt, etc.) werden berücksichtigt. }\end{array}$ & $\square$ & $\square$ & $\square$ & $\square$ \\
\hline Die Indikatoren sind präzise definiert. & $\square$ & $\square$ & $\square$ & $\square$ \\
\hline $\begin{array}{l}\text { Es besteht Konsens über die Bedeutung } \\
\text { der gewählten Indikatoren. }\end{array}$ & $\square$ & $\square$ & $\square$ & $\square$ \\
\hline
\end{tabular}

\section{Abbildung A-6: Seite 5 des Fragebogens}




\title{
Teil B: Stand der IT-Systeme für das Performance-Measurement
}

\section{Informationstechnologie}

\subsection{Einsatz von Informationstechnologie zur Unterstützung des Performance- Measurements}

Mittels geeigneter Software können Performance-Daten einerseits automatisch gesammelt, gespeichert und analysiert werden; andererseits kann die Kommunikation der Resultate vereinfacht und automatisiert werden. Wir bezeichnen ein System, welches diese Aufgaben erfüllt als „IT-System für das Performance Measurement".

Wird die Performance-Messung in Ihrem Unternehmung durch ein rechnergestütztes Informationssystem für das Performance-Measurement unterstützt?
Nein

Ja

9.2 Welche der folgenden Standardsoftware oder Softwarekomponenten zur Unterstützung der Performance-Messung verwenden Sie?

\author{
Software-Pakete die ein breites Spektrum von Performance-Measurement-Funktionalitäten \\ abdecken (z.B. Cognos, CorVu, Gentia, Hyperion, QPR, SAS).
}

Welche Software setzen Sie ein?

Software-Pakete, die vor allem die visuelle Kommunikation der Performance-Werte unterstützen (z.B. Ergometrics, inSight, Promeasys, Simpel).

Welche Software setzen Sie ein?

Software-Pakete, die vor allem die Analyse der Daten unterstützen (z.B. PS-Explore, SPSS, StatWare, StatSoft, Statistica, Mathlab).

Welche Software setzen Sie ein?

Software-Pakete, welche die Datenakquisition, das Datenmanagement und den ETL-Prozess unterstützen (z.B. Cognos Accelerator, IBM DataPropagator, Oracle Warehouse Builder, PowerMart (Informatica), SAS/Warehouse Adminstrator).

Welche Software setzen Sie ein?

Performance-Measurement-Tools die mit ERP-Systemen gekoppelt sind (z.B. IDS Scheer Process Performance Manager, People Soft - Business Analyst, SAP - BW/SEM).

Welche Software setzen Sie ein?

\section{Abbildung A-7: Seite 6 des Fragebogens}


9.3 Wir haben eine eigene Software-Lösung für das Performance-Measurement entwickelt, unter Berücksichtigung folgender Komponenten:

Relationale Datenbank (z.B. Oracle, IBM DB/2, Microsoft SQL Server, Sybase, Informix).

Welche Software setzen Sie ein?

Multidimensionale Datenbank, resp. Data Warehouse. (z.B. Hyperion ESSBase, Microsoft Analysis Manager).

Welche Software setzen Sie ein?

Entwicklungsumgebung

Welche Software setzen Sie ein?

9.4 Folgende operative Informationssysteme liefern Daten für unser PerformanceMeasurement-System (PMS)

\begin{tabular}{|l|c|c|c|l|}
\cline { 2 - 6 } \multicolumn{1}{c|}{} & $\begin{array}{c}\operatorname{Im} \\
\text { Einsatz }\end{array}$ & \multicolumn{2}{|c|}{ Datenübertragung erfolgt } & $\begin{array}{l}\text { Wenn ja, welche } \\
\text { Daten? }\end{array}$ \\
\cline { 2 - 6 } & & manuell & automatisch
\end{tabular}

\section{Abbildung A-8: Seite 7 des Fragebogens}


Teil C: Anforderungen an IT-Systeme für das Performance Measurement

\section{Anforderungen}

\begin{tabular}{|c|c|c|c|c|c|c|c|c|c|c|}
\hline & \multicolumn{5}{|c|}{ Anforderung an ein PMS } & \multicolumn{5}{|c|}{ Das leistet unser PMS } \\
\hline & \multicolumn{3}{|c|}{ unwichtig } & \multicolumn{2}{|c|}{ sehr wichtig } & \multicolumn{3}{|c|}{$\begin{array}{l}\text { nicht } \\
\text { erfüllt }\end{array}$} & \multicolumn{2}{|c|}{$\begin{array}{r}\begin{array}{r}\text { vollständig } \\
\text { erfüllt }\end{array} \\
\end{array}$} \\
\hline \multicolumn{11}{|l|}{ Berücksichtigte Performance-Indikatoren } \\
\hline $\begin{array}{l}\text { Sowohl finanzielle, als auch nicht-finanzielle } \\
\text { Indikatoren werden gemessen. }\end{array}$ & $\square$ & $\square$ & $\square$ & $\square$ & $\square$ & $\square$ & $\square$ & $\square$ & $\square$ & $\square$ \\
\hline $\begin{array}{l}\text { Die Indikatoren reflektieren die Ziele der } \\
\text { Organisation und der Stakeholder. }\end{array}$ & $\square$ & $\square$ & $\square$ & $\square$ & $\square$ & $\square$ & $\square$ & $\square$ & $\square$ & $\square$ \\
\hline $\begin{array}{l}\text { Die Performance wird auf verschiedenen } \\
\text { organisatorischen Ebenen gemessen. }\end{array}$ & $\square$ & $\square$ & $\square$ & $\square$ & $\square$ & $\square$ & $\square$ & $\square$ & $\square$ & $\square$ \\
\hline $\begin{array}{l}\text { Für jede organisatorische Einheit und für } \\
\text { jeden Kerngeschäftsprozess existiert eine } \\
\text { Reihe definierter Performance-Indikatoren. }\end{array}$ & $\square$ & $\square$ & $\square$ & $\square$ & $\square$ & $\square$ & $\square$ & $\square$ & $\square$ & $\square$ \\
\hline \multicolumn{11}{|l|}{ Sammlung der Daten } \\
\hline $\begin{array}{l}\text { Das System ermöglicht das automatische } \\
\text { Importieren und Konvertieren von Daten aus } \\
\text { anderen Informationssystemen. }\end{array}$ & $\square$ & $\square$ & $\square$ & $\square$ & $\square$ & $\square$ & $\square$ & $\square$ & $\square$ & $\square$ \\
\hline $\begin{array}{l}\text { Das System erlaubt die manuelle Erfassung } \\
\text { von Daten. }\end{array}$ & $\square$ & $\square$ & $\square$ & $\square$ & $\square$ & $\square$ & $\square$ & $\square$ & $\square$ & $\square$ \\
\hline $\begin{array}{l}\text { Die Einbindung unternehmensexterner } \\
\text { Informationsquellen ist möglich. }\end{array}$ & $\square$ & $\square$ & $\square$ & $\square$ & $\square$ & $\square$ & $\square$ & $\square$ & $\square$ & $\square$ \\
\hline \multicolumn{11}{|l|}{ Speicherung der Performance-Daten } \\
\hline $\begin{array}{l}\text { Sämtliche Performance-Daten werden in } \\
\text { einer zentralen Datenbank gespeichert. }\end{array}$ & $\square$ & $\square$ & $\square$ & $\square$ & $\square$ & $\square$ & $\square$ & $\square$ & $\square$ & $\square$ \\
\hline $\begin{array}{l}\text { Die Daten werden in einem integrierten IT- } \\
\text { System verwaltet. }\end{array}$ & $\square$ & $\square$ & $\square$ & $\square$ & $\square$ & $\square$ & $\square$ & $\square$ & $\square$ & $\square$ \\
\hline $\begin{array}{l}\text { Die Performance-Daten sind über einen } \\
\text { langen Zeitraum hinweg verfügbar. }\end{array}$ & $\square$ & $\square$ & $\square$ & $\square$ & $\square$ & $\square$ & $\square$ & $\square$ & $\square$ & $\square$ \\
\hline
\end{tabular}

\section{Abbildung A-9: Seite 8 des Fragebogens}




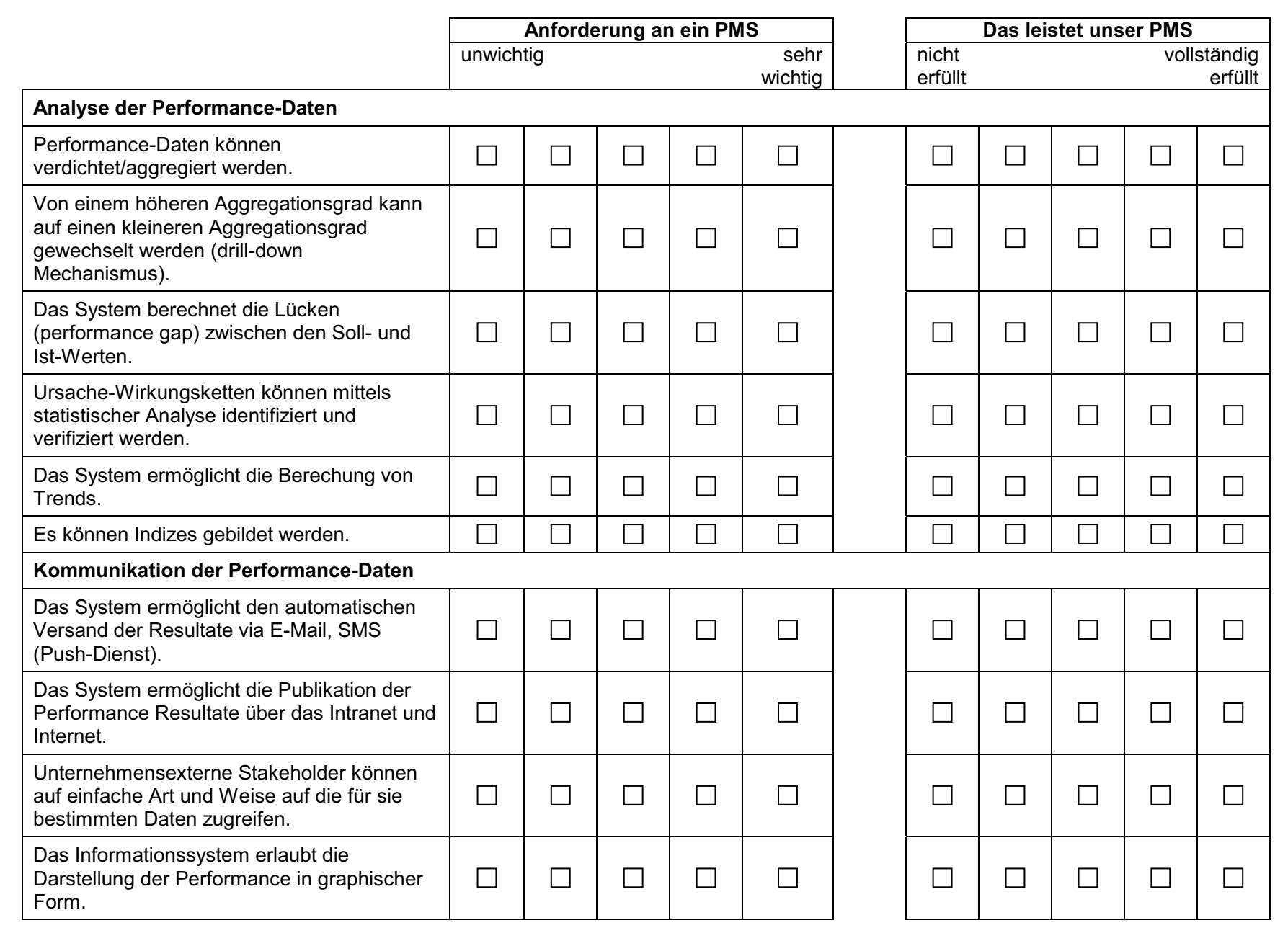

Abbildung A-10: Seite 9 des Fragebogens 


\section{Teil D: Anmerkungen und Bestellung des Studienberichtes}

\section{Anmerkungen}

Hier steht Ihnen noch Raum zur Verfügung für generelle Hinweise, Anliegen, Schilderungen zu Ihrer Situation (die dieser Fragebogen nicht ausreichend berücksichtigt).

\section{Bestellung}

Da Sie diesen Fragebogen ausgefüllt haben, können Sie die Auswertung dieser Studie kostenlos beziehen:

Studienbericht „Stand des Performance-Measurements und deren IT-Systeme in der Praxis 2002“ (Auslieferung ca. April 2002)

Kurzbericht des Studienberichts (4 Seiten, Auslieferung ca. April 2002)

$\rightarrow \quad$ Erste Resultate werden Sie kurz nach Abschluss der Studie auf unserer Projektwebseite (www.measure.ch) abrufen können.

Anschrift (falls Sie keine Anschrift angeben, versenden wir den Bericht elektronisch an die unter Punkt 1 angegebene E-Mail-Adresse.

Firma

Name / Vorname

Funktion / Abteilung

Adresse

PLZ/Ort

\section{Wir danken Ihnen für Ihre wertvolle Mitarbeit!}

\section{Abbildung A-11: Seite 10 des Fragebogens}




\section{Erhebung zum Stand des}

Performance Measurements

und deren IT-Systeme in der Praxis

Den vorliegenden Fragebogen können Sie auch im Internet ausfüllen. Diesen finden Sie unter der folgenden Adresse:

\section{http://www.measure.ch/erhebung/}

Sie können die Adresse entweder direkt eintippen oder auf der http://www.measure.ch Homepage den entsprechenen Link „Erhebung zum Stand des Performance Measurements und deren IT-Systeme in der Praxis" wählen.

Wenn Sie den Fragebogen lieber konventionell bearbeiten möchten, benutzen Sie die beigelegte Papierversion. Ein vorfrankierter und adressierter Umschlag für die Rücksendung liegt bei.

Untenstehend finden Sie die notwendigen Zugangsinformationen:

Actelion Ltd., 4123 Allschwil

\begin{tabular}{|c|c|}
\hline Login: & KoWi \\
\hline
\end{tabular}

Passwort:

K412

Für Fragen stehen wir Ihnen jederzeit gerne zur Verfügung: per Email: christian.beyeler@unifr.ch, thomas.wettstein@unifr.ch oder telefonisch: 0263008336.

\section{Besten Dank!}

\section{Abbildung A-12: Begleitblatt mit Anleitung und Login für Web-Fragebogen}




\section{Erhebung zum Stand des \\ Performance Measurements und deren IT-Systeme in der Praxis}

\section{BEGRIFFE}

Performance-Measurement-System (PMS) - Ein PMS ist ein Instrument, um das in der Vergangenheit Geleistete zu kontrollieren und quantitativ auszuwerten. Es wird genutzt, um die Geschäftsziele transparent zu machen, das Erreichte quantitativ zu erfassen und zu kommunizieren, den Blickwinkel zu erweitern und die gegenseitigen Beeinflussungen besser zu verstehen. Die betrachteten Gegenstandsbereiche sind die verschiedenen Einheiten einer Organisation. Die Empfänger der Performance-Informationen sind die Stakeholder (Management, Mitarbeiter, evtl. Kunden und Lieferanten) der entsprechenden Organisationseinheiten.

IT-System für das PMS - Darunter verstehen wir ein rechnergestütztes Informationssystem, welches das Sammeln, Verwalten, Analysieren und Kommunizieren der performancerelevanten Daten unterstützt.

Weiterführende Informationen zum Thema Performance Measurement finden Sie auf unserer Projektwebseite www.measure.ch.

\section{ADMINISTRATIVES}

Aufwand - Für die Beantwortung der Fragen benötigen Sie etwa 20 Minuten.

Anonymität - Das Departement für Informatik der Universität Fribourg führt seit 1961 regelmässig empirische Untersuchungen durch. Wir garantieren Ihnen Anonymität. Für die Zuordnung zu Branchen- und Grössenklassen, sowie für allfällige Rückfragen benötigen wir Ihre Anschrift. Bei der Auswertung werden diese jedoch entfernt. Aufgrund der Studienresultate können weder auf Sie, noch auf Ihr Unternehmen Rückschlüsse gezogen werden.

Wenn Sie Fragen haben - Wir stehen Ihnen gerne zur Verfügung. Kontaktieren Sie uns unter 02630083 36. Unter dieser Nummer ist auch ein Anrufbeantworter installiert, der Ihre Fragen jederzeit gerne entgegen nimmt. Zusätzliche Kontaktmöglichkeiten sind Fax 0263009726 und E-Mail: christian.beyeler@unifr.ch und thomas.wettstein@unifr.ch

Dauer der Erhebung - Der Fragebogen kann vom 3. bis zum 24. Dezember 2001 erfasst werden. Danach wird diese Seite durch eine allgemeine Information und zu gegebener Zeit mit ersten Resultaten der Erhebung ersetzt.

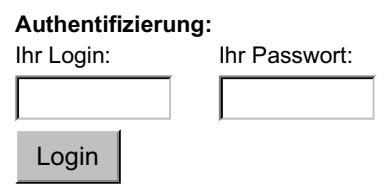

Für Ihre wertvolle Mitarbeit danken wir Ihnen bestens! 


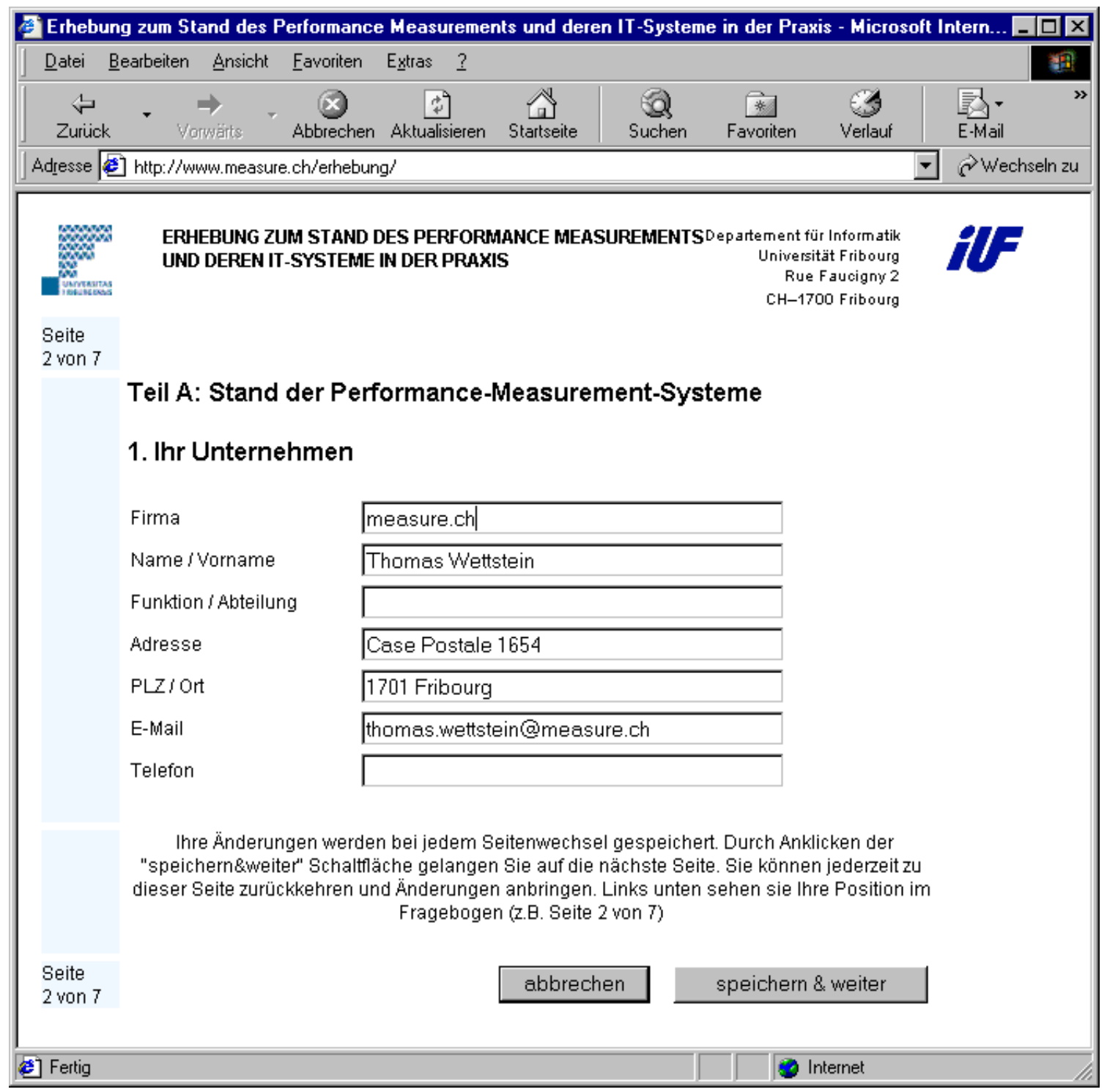

\section{Abbildung A-14: Seite 2 des Webfragebogens}


Anhang B: Ergebnisse der Erhebung zum Stand des Performance Measurement und deren IT-Systeme in der Praxis - Bericht für teilnehmende Unternehmen 
Thomas Wettstein, Christian Beyeler

\section{Ergebnisse der Erhebung zum Stand des Performance Measurements und deren IT-Systeme in der Praxis - Bericht für teilnehmende Unternehmen}

Inhaltsverzeichnis:

1. Zielsetzung und Umfang der Erhebung..........................................

1.1. Ziel der Untersuchung ........................................................

1.2. Methodik und Umfang der Erhebung.........................................

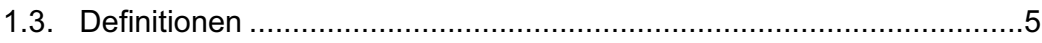

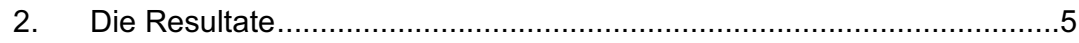

2.1. Stand der Performance-Measurement-Systeme .............................

2.2. Stand der IT-Systeme für das Performance Measurement ..................9

2.3. Anforderungen an Performance-Measurement-Systeme .................11

3. Zusammenfassung der Umfrageergebnisse ....................................14

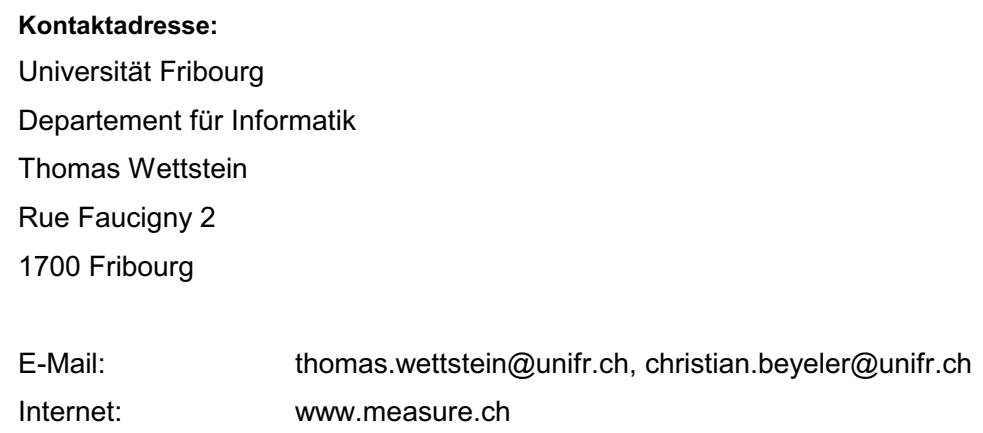

April 2002

\section{Abbildung B-1: Seite 1 des Berichtes}




\section{Zielsetzung und Umfang der Erhebung}

\subsection{Ziel der Untersuchung}

Die Untersuchung soll ein Bild über den Entwicklungsstand von Performance-MeasurementSystemen unter spezieller Berücksichtigung der Ausgestaltung der zugehörigen IT-Systeme bei Schweizer Unternehmen, die an der Börse kotiert sind, liefern. Folgende Fragestellungen waren von besonderem Interesse ${ }^{1}$ :

- Welches ist der Entwicklungsstand von Performance-Measurement-Systemen (PMS)?

- Inwiefern werden PMS durch Informationstechnologie unterstützt?

- Welches sind die Anforderungen an ein modernes IT-System für das Performance Measurement?

\subsection{Methodik und Umfang der Erhebung}

Die Datenerhebung erfolgte durch eine schriftliche Befragung von börsenkotierten Schweizer Unternehmen aus dem deutschsprachigen Raum. Basis bildete die Kotierungsliste des Swiss Performance Index $\left(\mathrm{SPI}^{2}\right)$, jedoch unter Ausschluss der im Swiss Market Index $\left(\mathrm{SMI}^{3}\right)$ enthaltenen Unternehmen. Der Versand der Fragebogen erfolgte Anfang Dezember 2001. Es wurden insgesamt 160 Fragebogen versandt. Empfänger des Fragebogens war der Investor Relations (IR) Manager der zuhanden der schweizerischen Börse (SWX) bekannt gegeben werden muss. Die Stichprobe entspricht der Grundgesamtheit der börsenkotierten Unternehmen, die in der Deutschschweiz domizilieren und nicht dem SMI angehören.

Neben dem Papierfragebogen wurde eine Internet-Version des Fragebogens bereitgestellt, welche im Rahmen der technischen Möglichkeiten identisch aufgebaut wurde. Der elektronische Fragebogen wurde auf der Webseite (www.measure.ch) aufgeschaltet. Die angeschriebenen Unternehmen erhielten zusammen mit dem schriftlichen Fragebogen ein Beiblatt mit den notwendigen Erläuterungen und den Authentifizierungsinformationen. Somit

\footnotetext{
1 Die erste Fragestellung ist Gegenstand einer Diplomarbeit von Christian Beyeler, welche voraussichtlich im Verlaufe dieses Jahres an der wirtschaftswissenschaftlichen Fakultät der Universität Fribourg abgeschlossen wird. Die zweite und dritte Fragestellung stammt von Thomas Wettstein und wird im Rahmen seiner Dissertation zur Verifikation der Anforderungen an ein PMS verwendet. Seine Dissertation wird voraussichtlich im Verlaufe dieses Jahres an der wirtschaftswissenschaftlichen Fakultät der Universität Fribourg abgeschlossen.

${ }^{2}$ Der SPI ist ein dividendenkorrigierter Index, der alle kotierten Aktien inländischer Gesellschaften (inkl. Fürstentum Liechtenstein) des Haupt- und Nebensegmentes der Schweizer Börse enthält (ohne Investmentgesellschaften).

${ }^{3}$ Der SMI ist ein kapitalgewichteter, nicht dividendenkorrigierter Index. Er umfasst bis zu 30 liquide Titel hochkapitalisierter Unternehmungen des Schweizer Aktienmarktes (sog. Blue Chips), die ca. 80\% der Gesamtkapitalisierung repräsentieren.
}

\section{Abbildung B-2: Seite 2 des Berichtes}


wurde sichergestellt, dass nur die ausgewählte Stichprobe an der Untersuchung teilnehmen konnte.

Nach vier Wochen, d.h. Anfang Januar 2002, wurden sämtliche Unternehmen, welche bis dahin noch nicht reagiert hatten, per Telephon nochmals zur Teilnahme angefragt. Der Rücklauf betrug bis Ende Dezember 19\%, bis Mitte Januar erreichte der Rücklauf 24\% (vgl. Tabelle 1).

Die Fragebogen wurden am häufigsten durch den Chief Financial Officer (CFO) und den Leiter Corporate / Group Controlling eingereicht.

Bemerkenswert hoch ist der Anteil der Online-Teilnehmer; mehr als die Hälfte aller TeilnehmerInnen haben die Möglichkeit, den Fragebogen über das Internet einzureichen, genutzt.

Insgesamt wurden die Fragebogen sorgfältig ausgefüllt. Nur zwei Unternehmen haben lediglich die Adressangaben gemacht, um die Studienergebnisse zu erhalten, ohne selbst zu partizipieren.

\begin{tabular}{|l|l|l|l|l|l|}
\hline Universum & Teilnahme & Rücklauf & Ungültig & Bereinigter Rücklauf & Rücklaufquote \\
\hline $\begin{array}{l}160 \\
\text { Unternehmen }\end{array}$ & Internet & 23 & 2 & 21 & \\
\cline { 2 - 7 } & Post & 18 & & 18 & \\
\cline { 2 - 6 } & Total & 41 & & 39 & $24,4 \%$ \\
\hline
\end{tabular}

Tabelle 1: Rücklauf

Für die Analyse der Teilnahme nach Branchenzugehörigkeit ${ }^{4}$ wurde die Gliederung nach SPISektoren an der Schweizer Börse gewählt. Diese seit August 2000 neue Strukturierung lehnt sich an die Gliederung des DJ-STOXX an (vgl. SPI-Reglement vom 28.1.2002). Für die Auswertung wurde lediglich die erste Gliederungsstufe - der Economic Sector berücksichtigt. Mit Ausnahme von Unternehmen der Sektoren Energie und Telekommunikation sind unter den antwortenden Unternehmen sämtliche Sektoren vertreten. Innerhalb der Branchen variiert die Beteiligung beträchtlich. Überdurchschnittlich hoch ist die Beteiligung in den typischen Industriesektoren: „Basic Materials“, „Industrial“ und „Utilities“, unter dem Durchschnitt ist die Beteiligung in den Sektoren „Financial“ und „Consumer, Cyclical“ (vgl. Abbildung 1).

\footnotetext{
4 Bei Tätigkeit eines Unternehmens in mehreren Branchen erfolgt die Zuordnung zur Branche nach dem Geschäftsfeld mit dem grössten Umsatzanteil
}

\section{Abbildung B-3: Seite 3 des Berichtes}


Aus Abbildung 2 geht hervor, dass die Sektoren „Industrial“ und „Financial“ rund die Hälfte der Antworten stellen.

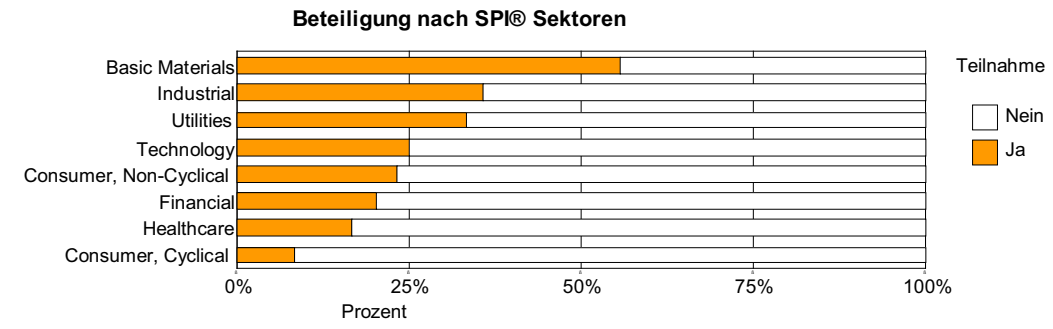

Abbildung 1: Beteiligung nach SPI-Sektoren

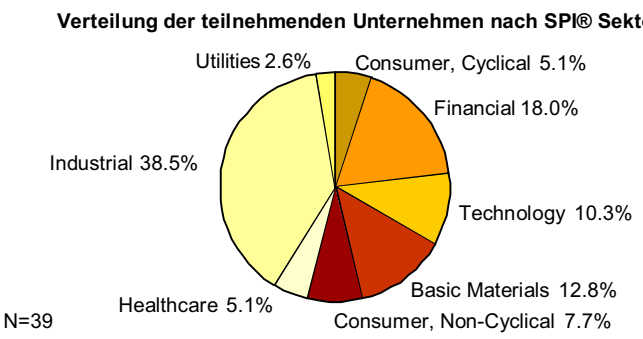

Abbildung 2: Verteilung der teilnehmenden Unternehmen nach SPI-Sektoren

\section{Abbildung B-4: Seite 4 des Berichtes}




\subsection{Definitionen}

Zur Erinnerung sollen die beiden zentralen Begriffe der Untersuchung hier kurz definiert werden ${ }^{5}$ :

Performance-Measurement-System (PMS) - Ein PMS ist ein Instrument, um das in der Vergangenheit Geleistete zu erfassen. Es wird genutzt, um die Geschäftsziele transparent zu machen, das Erreichte quantitativ zu erfassen und zu kommunizieren, den Blickwinkel zu erweitern und die gegenseitigen Beeinflussungen besser zu verstehen. Die betrachteten Gegenstandsbereiche sind die verschiedenen Einheiten einer Organisation. Die Empfänger der Performance-Informationen sind die Stakeholder (Management, Mitarbeiter, evtl. Kunden und Lieferanten) der entsprechenden Organisationseinheiten.

IT-System für das PMS - Darunter wird ein rechnergestütztes Informationssystem verstanden, welches das Sammeln, Verwalten, Analysieren und Kommunizieren der performancerelevanten Daten unterstützt.

\section{Die Resultate}

\subsection{Stand der Performance-Measurement-Systeme}

In einer ersten Frage wurde ermittelt, welche in der Theorie vorgeschlagenen PerformanceMeasurement-Konzepte in der Praxis eingesetzt werden (vgl. Abbildung 3). Management by Objectives (MbO) wird von 82 Prozent der Unternehmen eingesetzt. Ansätze des Value Based Management (VBM) und Balanced Scorecard (BSC) werden je von rund 45\% der Unternehmen benutzt. Eine 1999 durchgeführte Studie $^{6}$ fand Werte von rund $30 \%$ für den VBM und BSC-Ansatz.

Erstaunlich ist, dass der EFQM-Ansatz nur von vier Unternehmen genannt wurde. Dies obwohl hierzulande die lokale Partnerorganisation ESPRIX (www.esprix.ch), erhebliche Anstrengungen zur Verbreitung unternimmt. Kaum verbreitet (1 Nennung) ist auch der Intellectual-Capital-Ansatz. Eine wesentliche Zielsetzung des IC-Ansatzes besteht darin, das intellektuelle Kapital einer Unternehmung optimal zu bewirtschaften. Es kann als Erklärungsansatz der Differenz zwischen Buch- und Marktwert einer Unternehmung herangezogen werden. Bei der gewählten Stichprobe, welche ausschliesslich börsenkotierte Unternehmen enthält, und dem zunehmenden Trade-off von Buch- und Marktwert, hätte man einen grösseren Anteil erwarten können.

${ }^{5}$ vgl. Wettstein, T.;Küng, P.;Meier, A. (2001). "Performance Measurement als Ausweg aus dem Information Overload: Ein zielorientierter Ansatz." HMD -Praxis der Wirtschaftsinformatik 222: 49-58. ${ }^{6}$ vgl. Brunner, J.;Roth, P. (1999). "Performance-Management und Balanced Scorecard in der Praxis." io management 1999(7/8): 50-55.

\section{Abbildung B-5: Seite 5 des Berichtes}




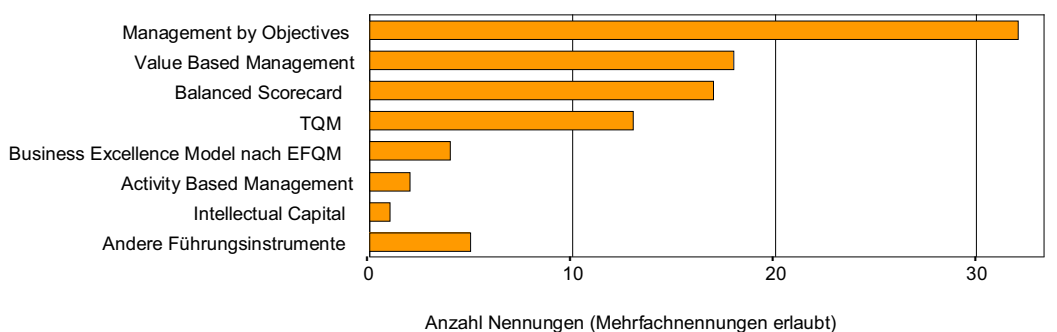

Abbildung 3: Verbreitung von Performance-Measurement-Konzepten

In Abbildung 4 ist die Verbreitung verschiedener Dimensionen von Performance-Indikatoren dargestellt. Finanzielle Performance-Indikatoren werden in sämtlichen Unternehmen zur Überprüfung der Erreichung strategischer Ziele verwendet. Von den nicht-finanziellen Indikatoren werden kundenspezifische Indikatoren (z.B. die Erhebung der Kundenzufriedenheit oder der Beschwerden) am häufigsten (85\% der Unternehmen) erhoben. Indikatoren der Dimension Mitarbeiter (z.B. Mitarbeiterzufriedenheit, Fluktuationsrate) werden in $75 \%$ der Unternehmen berücksichtigt. Indikatoren der Dimension Lieferanten (z.B. Lieferpünktlichkeit, Qualität) werden in 56\% der Unternehmen erhoben. Indikatoren der Dimension Innovation (z.B. Anzahl Forschungsprojekte, Time to Market) werden lediglich in der Hälfte der Unternehmen berücksichtigt. Gesellschaftliche Indikatoren (z.B. Papierverbrauch, Anzahl positive Nachrichten in den Medien) werden in rund $50 \%$ der Unternehmen systematisch erfasst.

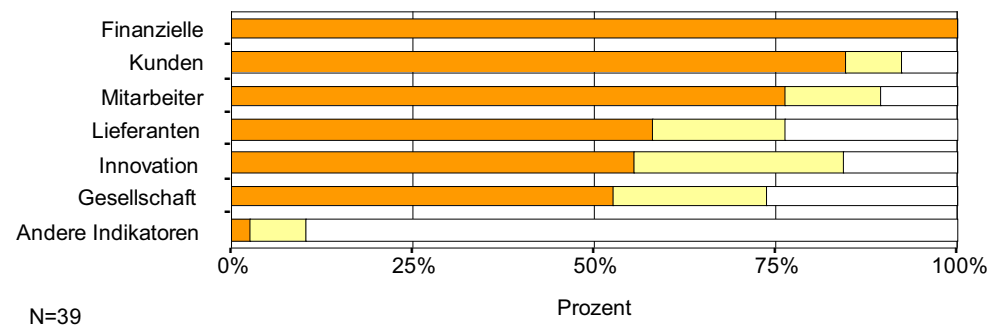

Ja $\square$ Nein $\square$ keine Angabe

Abbildung 4: Verbreitung von verschiedenen Indikatorentypen

Die in der Theorie geforderte Berücksichtigung von Performance-Indikatoren verschiedener Dimensionen (grösser gleich vier Dimensionen) wird von etwas mehr als der Hälfte der Unternehmen wahrgenommen. 15\% der Unternehmen verwenden ausschliesslich finanzielle

\section{Abbildung B-6: Seite 6 des Berichtes}


Indikatoren als Führungsgrössen. Dies erscheint problematisch, da die finanziellen Indikatoren das Ende einer Wirkungskette bilden und somit lediglich eine Auseinandersetzung mit bereits Abgeschlossenem ermöglichen. Aktive, zeitgerechte Steuerung kann mit einer rein finanziellen Führung nach heutigem Verständnis kaum mehr realisiert werden. Positiv zu vermerken ist, dass der Anteil nicht-finanzieller Grössen im Vergleich zu früheren Studien zugenommen hat.

Die Unternehmen wurden weiter zur Erhebungshäufigkeit der Indikatoren befragt. In Abbildung 5 werden die verschiedenen Erhebungsfrequenzen vergleichend dargestellt. Über sämtliche Dimensionen hinweg betrachtet, werden Performance-Indikatoren am häufigsten monatlich erhoben, am zweithäufigsten werden sie jährlich erfasst. Der Anteil der Unternehmen, die Indikatoren in kurzen Abständen (laufend bis zweiwöchentlich) erheben ist, eher klein.

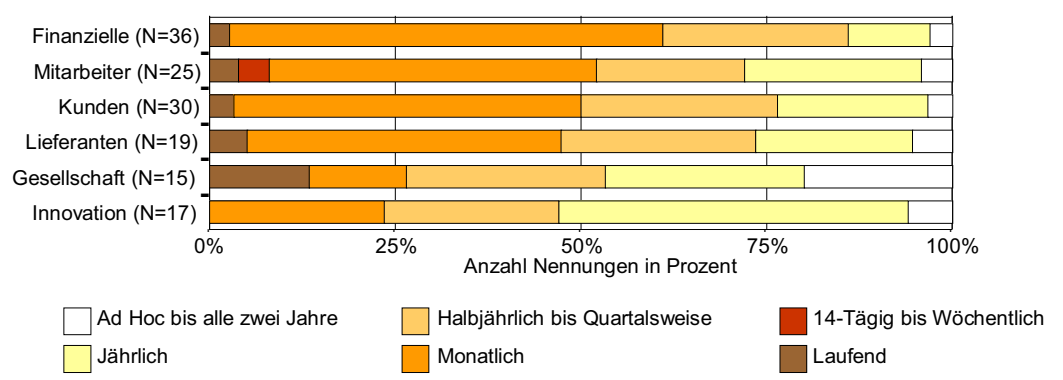

Abbildung 5: Erhebungsfrequenz der Indikatoren auf der strategischen Ebene

Neben der Stakeholder-orientierten Sicht auf die Performance eines Unternehmens, die in der Regel längerfristig ausgelegt ist, werden von den Unternehmen auch strategische Massnahmen in Form von Projekten durchgeführt. Diese sind, entsprechend den Merkmalen eines Projektes durch Einmaligkeit, zeitliche Limitierung und hohe Komplexität gekennzeichnet. Die Frage, ob die Unternehmen ein systematisches Controlling ihrer strategischen Projekte betreiben, haben $80 \%$ bejaht. In diesem Bereich scheint noch Handlungsbedarf zu bestehen, investieren die Unternehmen in dieser Form doch erhebliche Mittel in die Weiterentwicklung ihrer Organisationen.

Das Management von Risiken ist bei einem breiten Begriffsverständnis als Element des Performance Measurements aufzufassen. Die Resultate der Umfrage zeigen, dass Risikomanagement bei operativen Tätigkeiten weit verbreitet ist ( $92 \%$ der Unternehmen), auf der strategischen Ebene jedoch nur in $70 \%$ der Unternehmen erfolgt. Unternehmen, die kein

\section{Abbildung B-7: Seite 7 des Berichtes}


Risikomanagement auf der strategischen Ebene betreiben, sind sich häufig nicht über ihre Risikofähigkeit im Verhältnis zur definierten Strategie im klaren.

Ein Element des Risikomanagements kann ein Frühwarnsystem sein. 73 Prozent der Unternehmen setzen dieses Instrument ein. Frühwarnsysteme haben die Aufgabe, Veränderungen in der Umwelt so frühzeitig aufzuzeigen, dass geeignete Massnahmen ergriffen werden können, bevor der Unternehmung ein Schaden erwächst oder eine Chance entgeht $^{7}$. Damit das Frühwarnsystem richtig greift, muss dieses auf die Strategie des Unternehmens abgestimmt sein, d.h. das Frühwarnsystem muss die Prämissen der Strategie abdecken. $76 \%$ der Unternehmen mit Frühwarnsystem berücksichtigen diesen Aspekt. Mehr als ein Drittel der Unternehmen überprüfen die Strategie laufend.
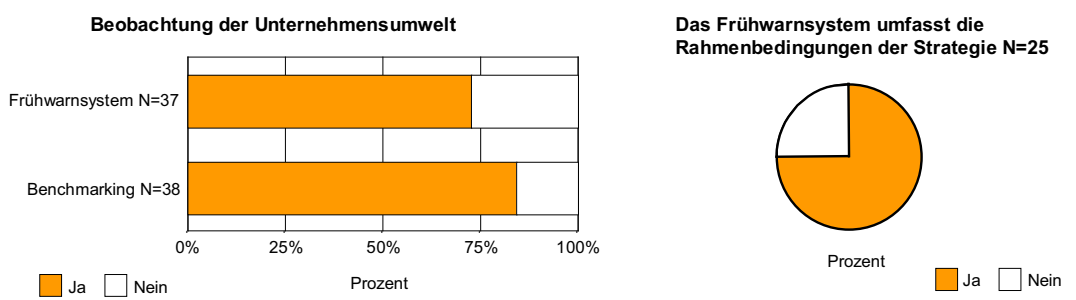

Abbildung 6: Beobachtung der Unternehmensumwelt

Benchmarking kann definiert werden als ein Leistungsvergleich zwischen Institutionen mit gleichem oder ähnlichem Aufgabenbereich mit dem Ziel, in einem qualitativen Prozess von den Besten zu lernen, um die eigene Leistungsfähigkeit zu erhöhen ${ }^{8}$. Es wird in $84 \%$ der Unternehmen eingesetzt, wobei Benchmarking-Daten am häufigsten jährlich erhoben werden (35\% der Unternehmen). Bemerkenswert hoch ist der Anteil an Unternehmen die Daten für das Benchmarking laufend erheben (17\%). Interessant wäre in diesem Zusammenhang das Begriffsverständnis der Unternehmen zu erfahren, die ein kontinuierliches Benchmarking durchführen. Die vorliegende Untersuchung liefert hierzu jedoch keinen Erklärungsansatz.

Als potenzielle Empfänger verschiedener Performance-Daten können sämtliche relevanten Interessensgruppen des Unternehmens in Betracht gezogen werden (z.B. Mitarbeiter, Kunden, Lieferanten, Gesellschaft, Shareholder). In vielen Unternehmen sind PerformanceDaten ausschliesslich dem höheren Kader (39 Nennungen) sowie dem mittleren Kader (37 Nennungen) vorbehalten (vgl. Abbildung 7). Die Kommunikation der Daten an andere Interessengruppen ist weit weniger verbreitet, Investoren/Shareholder (25 Nennungen),

${ }_{8}^{7}$ vgl. Gomez, P. (1983). Frühwarnung in der Unternehmung. Bern.

${ }^{8}$ vgl. Camp, R. C. (1994). Benchmarking. Milwaukee.

\section{Abbildung B-8: Seite 8 des Berichtes}


Mitarbeiter (22). Kontinuierliche Steigerung der Leistungsfähigkeit wird jedoch nicht in erster Linie durch das obere Management, sondern durch das Engagement aller Mitarbeiter eines Unternehmens erreicht. Entsprechende Daten werden jedoch nur in etwa der Hälfte (57\%) der Unternehmen den Mitarbeitern kommuniziert. Weiter erfolgt die Kommunikation der Resultate an die Kunden in 12, den Lieferanten in 10 und den öffentlichen Stellen in 9 Unternehmen. Dass die performance-relevanten Daten primär dem höheren Kader vorbehalten sind, bestätigt auch eine qualitative Studie, die am Departement für Informatik im Jahre 2000 durchgeführt wurde ${ }^{9}$.

\section{Empfänger der performance-relevanten Daten}

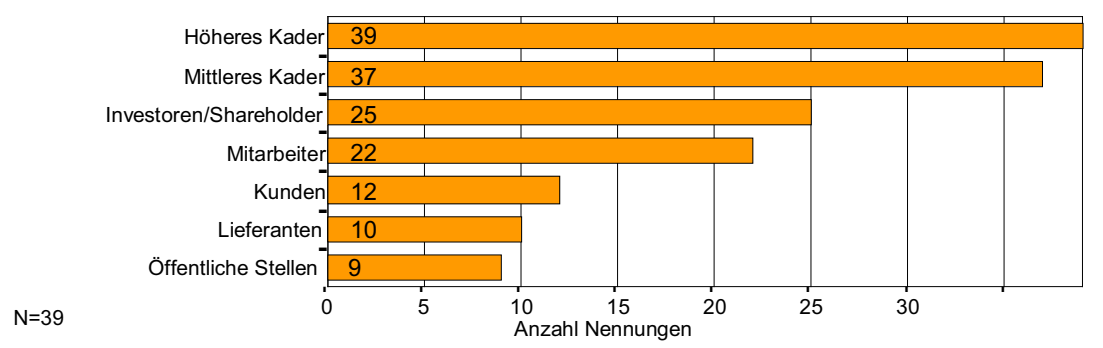

Abbildung 7: Empfänger der performance-relevanten Daten

\subsection{Stand der IT-Systeme für das Performance Measurement}

In 74\% der Unternehmen wird Performance Measurement durch Informationstechnologie unterstützt. Die für das Performance Measurement verwendeten Informationssysteme bestehen meist aus mehreren Softwarekomponenten. Bei der Fragestellung wurde zudem zwischen der Verwendung von Standardsoftware und der Eigenentwicklungen unterschieden. Standardlösungen sind mehr verbreitet $(70 \%)$ als Lösungen, die selbst entwickelt wurden $(30 \%)$.

\footnotetext{
${ }^{9}$ vgl. Küng, P. (2001). "Performance-Measurement-Systeme im Dienstleistungssektor - Das Denken in Wirkungsketten ist noch wenig verbreitet." io management 70(1/2): 56-63.
}

\section{Abbildung B-9: Seite 9 des Berichtes}



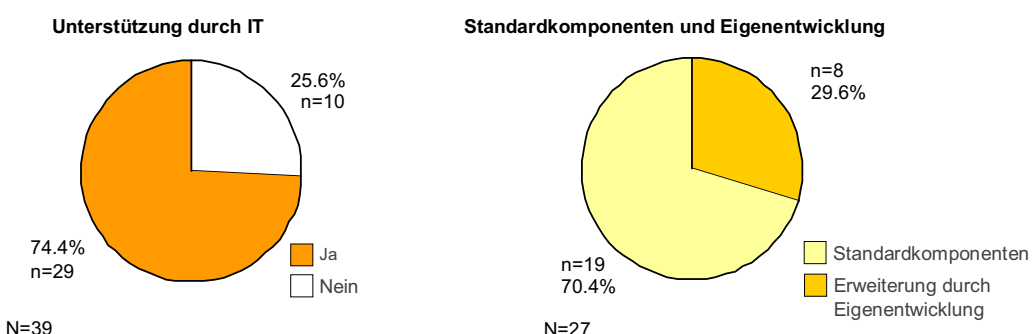

Abbildung 8: Unterstützung durch Informationstechnologie

Am meisten werden Produkte von Cognos und SAP (je 7 Nennungen), Frango und Insight (je 4 Nennungen) und Oracle (2 Nennungen) eingesetzt. Vier Unternehmungen setzen Excel ein. Die meisten der eingesetzten Eigenlösungen basieren auf relationalen Datenbanken. In einer deutschen Studie ${ }^{10}$ finden sich 34 (37\%), die auf SAP R/3, teilweise mit Datenexporten auf Microsoft Excel setzen. 6 Unternehmen nutzen dort Hyperion als IT-System für das Performance Measurement.

Die verschiedenen Produkte wurden in der Umfrage nach fünf Kategorien gegliedert. Danach werden mit 18 Nennungen IT-Systeme für das PMS, die ein breites Spektrum unterstützen und mit 14 Nennungen Tools, die mit ERP-Systemen gekoppelt sind am meisten eingesetzt. Weniger zum Einsatz kommen Produkte, die die Datenanalyse (8 Nennungen), die Datenakquisition, das Datenmanagement und den ETL-Prozess (8 Nennungen) unterstützen (vgl. Abbildung 9).

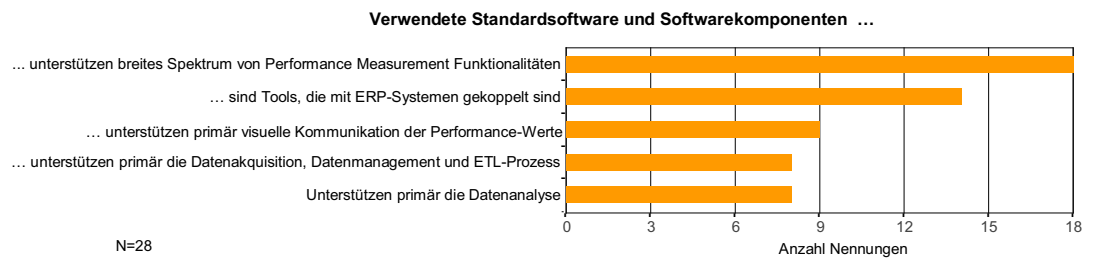

Abbildung 9: Verwendete Standardsoftware und Softwarekomponenten

Das Informationssystem für das Performance Measurement ist den operativen, transaktionsorientierten Informationssystemen übergeordnet, und zwar in dem Sinne, dass es Daten aus operativen Systemen bezieht. Operative Informationssysteme haben somit eine

${ }^{10}$ vgl. Günther, T.;Grüning, M. (2000). Performance Measurement Systems in Germany - A Descriptive Evaluation Report. Dresden, Dresden University of Technology, Department of Economics: 1-27.

Abbildung B-10: Seite 10 des Berichtes 
zentrale Rolle in der Bereitstellung von performance-relevanten Daten. Die Automatisierung der Bereitstellung von Daten erhöht Akzeptanz und Effizienz des PMS. Einerseits erübrigt sich damit die manuelle Datenerfassung, anderseits werden auf diese Weise Daten den Entscheidungsträger zeitnaher zur Verfügung gestellt. Welche operativen Systeme als Datenlieferanten genutzt werden und inwiefern die Bereitstellung der Daten automatisch erfolgt, veranschaulicht Abbildung 10. Buchungs- und Abrechnungssysteme werden in allen Unternehmen (mit Ausnahme eines Unternehmens) als Datenlieferanten für das PMS genannt. Die automatische Bereitstellung von performance-relevanten Daten durch diese Systeme erfolgt in etwas mehr als der Hälfte der Fälle. Mitarbeiterinformationssysteme werden am zweithäufigsten eingesetzt, relevante Daten müssen jedoch in mehr als der Hälfte der Fälle manuell aus den Systemen extrahiert werden. Marketing- und Verkaufsinformationssysteme stellen die relevante Daten in mehr als $60 \%$ der Fälle automatisch bereit. Noch besser sieht die Situation bei Produktionsinformationssystemen aus (75\%). CRM-Software wird in wenigen Unternehmen (8 Nennungen) eingesetzt, die Bereitstellung von Performance-Daten erfolgt jedoch mit einer Ausnahme automatisch. Die Verwendung von externen Informationssystemen ist wenig verbreitet (4 Nennungen), eine automatische Bereitstellung von Daten aus diesen Systemen erfolgt nicht.

Integration operativer Informationssysteme als Datenlieferant für das PMS

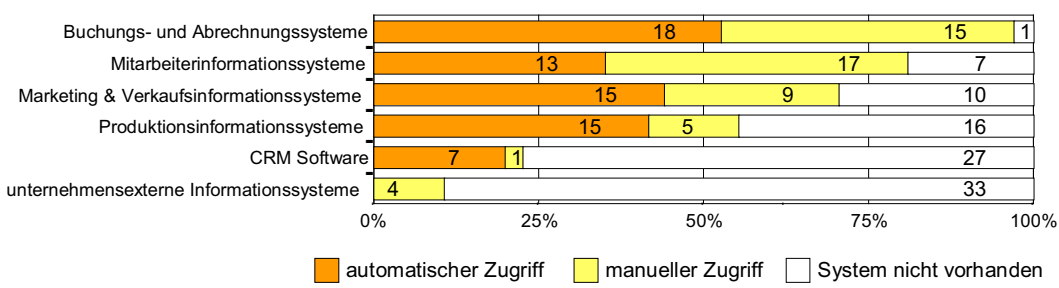

Abbildung 10: Integration operativer Informationssysteme als Datenlieferant für das PMS

\subsection{Anforderungen an Performance-Measurement-Systeme}

In einem dritten Teil wurden Anforderungen, die aus der Literatur zu PerformanceMeasurement-Systemen und anderen empirischen Studien zusammengetragen wurden, bezüglich ihrer Relevanz überprüft. Hierzu wurde einerseits der aktuelle Leistungsgrad der Performance-Measurement-Systeme bezüglich der einzelnen Anforderungen überprüft, andererseits die Wichtigkeit der Anforderung erhoben. Ergebnis ist eine priorisierte Anforderungsliste, ein Überblick über die aktuelle Leistungsfähigkeit von PMS und ein Hinweis darauf, in welchen Bereichen der Handlungsbedarf am grössten ist. Dieser lässt sich

\section{Abbildung B-11: Seite 11 des Berichtes}


aus einer grossen Differenz zwischen aktuellem Abdeckungsgrad und hoher Wichtigkeit ableiten. (vgl. Abbildung 11)

Die Skalierung für das aktuelle Leistungsvermögen einzelner Anforderungen reicht von nicht erfüllt (0) bis zu vollständig erfüllt (4). Die Skalierung für die Wichtigkeit einzelner Anforderungen erstreckt sich von unwichtig (0) bis zu sehr wichtig (4).

Bezüglich der aktuellen Leistung von Performance-Measurement-Systemen fällt auf, dass insbesondere informationssystemtechnische Anforderungen schlecht erfüllt werden. Dies betrifft die automatisierten Schnittstellen zu den operativen Informationssystemen, das Speichern in einer zentralen Datenbank und die Verwaltung der performance-relevanten Daten in einem integrierten Informationssystem. Der damit verbundene Erfassungsaufwand schwächt die Akzeptanz und den Nutzen eines PMS. Zudem scheinen statistische Funktionen, wie das Bilden von Indizes, das Berechnen von Trends und die Validierung von Ursache-Wirkungsketten nur sporadisch eingesetzt zu werden. Gerade der letzte Punkt erscheint von grosser Bedeutung zu sein, werden doch von verschiedenen PerformanceMeasurement-Ansätzen Wirkungszusammenhänge als zentrales Steuerungsinstrument betrachtet. Können die ex-ante aufgebauten Wirkungsnetze jedoch ex-post nicht mit statistischen Daten verifiziert werden, so können bezüglich der Wirksamkeit einzelner Massnahmen nur Vermutungen angestellt werden. Als dritte Schwachstelle ist die Kommunikation der performance-relevanten Daten anzuführen. Der Einsatz von Intranet und Internet scheint noch nicht einen sehr hohen Verbreitungsgrad erreicht zu haben. Gleiches gilt für moderne Kommunikationsarten, wie Push- und Pull-Dienste und die Erweiterung des Benutzerkreises um unternehmensexterne Stakeholder.

Von den 11 wichtigsten Anforderungen, die einen Wert von 3.0 und höher erreicht haben, sind bei deren drei wesentliche Leistungslücken zu den aktuell vorhandenen Systemen festzustellen.

Einerseits die Forderung nach einer zentralen Verwaltung der Performance-Daten in einer Datenbank. Inwiefern die Daten teilweise nicht in digitaler Form, oder aber in anderen Informationssystemen vorhanden sind, geht aus der Frage nicht klar hervor. Der Wunsch nach einer integralen Lösung ist jedoch deutlich.

Im Bereich der automatischen Datenextraktion und -konversion von den operativen Informationssystemen in das PMS besteht eine wesentliche Leistungslücke. Der Aufwand entsprechende Schnittstellen zu realisieren ist erheblich, insbesondere dann, wenn ältere operative Informationssysteme ohne geeignete Schnittstellen einbezogen werden müssen. Es handelt sich jedoch nicht nur um ein Problem des Aufwandes und der Technik, sondern auch der Datenqualität, können doch Daten nur dann automatisch übernommen werden, wenn diese automatisch validiert werden können. Spezialisierte Software, sogenannte ETL-Tools (Extraction-Transformation-Loading), können hier einen Beitrag zur Reduktion des

Abbildung B-12: Seite 12 des Berichtes 


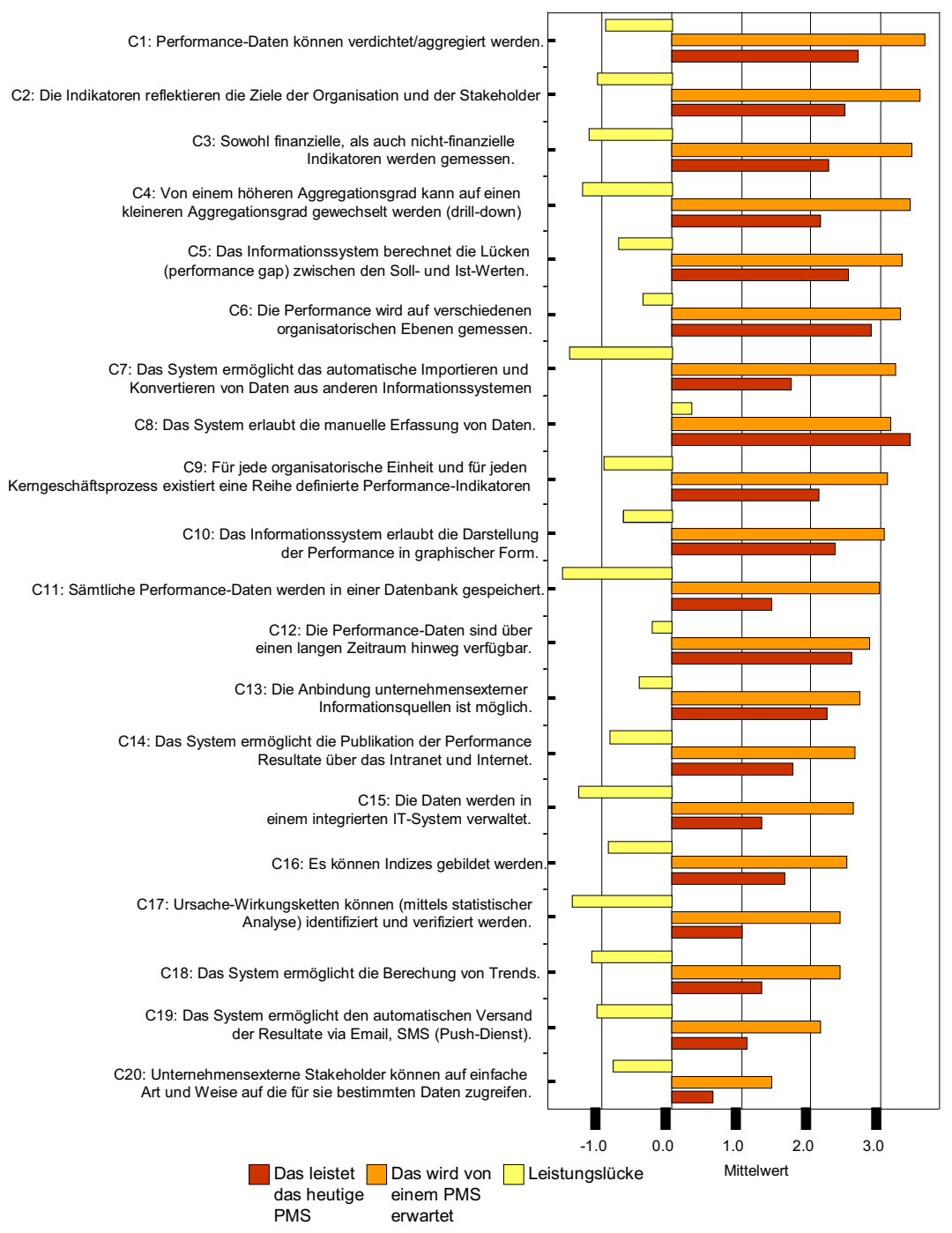

Abbildung 11: Leistungsfähigkeit und Wichtigkeit einzelner Anforderungen an ein PMS

\section{Abbildung B-13: Seite 13 des Berichtes}


Aufwandes leisten. Abzuwarten bleibt zudem, inwiefern neu verfügbar gewordene Techniken wie XML (eXtended Markup Language), dazu beitragen können, dass sich der Austausch von Informationen zwischen Systemen vereinfacht. Öffnen sich sowohl IT-Systeme für das PMS, als auch die operativen Systeme für entsprechende Techniken, so scheint der Ansatz vielversprechend. Zusammenfassend bleibt festzustellen, dass Fortschritte im Bereich der automatischen Datengewinnung entscheidend sind für den Erfolg von PM-Systemen.

Eine wesentliche Leistungslücke weist auch die als wichtig beurteilte Anforderung auf, in den Performance-Daten zu navigieren. Dies ermöglicht ein Performance-Measurement-System, welches auf einem multidimensionalen Datenmodell basiert und dem Benutzer zur Navigation einen entsprechenden OLAP-Client (On-Line Analytical Processing) zur Verfügung stellt.

Die befragten Unternehmen haben die konzeptionellen Aspekte eines PMS den Implementierungsaspekten übergeordnet. Dies schliessen wir aus der hohen Bedeutung von Anforderungen, wie die Zielabdeckung der Indikatoren, die Ausgewogenheit der Indikatoren (finanziell- und nicht-finanziell) und der Berücksichtigung verschiedener organisatorischer Ebenen. Die informationstechnische Umsetzung scheint aber trotzdem eine Voraussetzung für den erfolgreichen Einsatz im betrieblichen Alltag zu sein, wenn man die Wichtigkeit der genannten Anforderungen betrachtet.

Eine Anomalie findet sich bei der Anforderung, dass das System die Erfassung von manuellen Daten erlaubt. Der Leistungswert übersteigt hier die Bedeutung. Dies lässt sich dahingehend interpretieren, dass dies der gängige Weg zur Aufbereitung von performancerelevanten Daten ist und die Unternehmen dies als ungeeignet erachten. Damit wird die Bedeutung des automatischen Sammeln von Daten noch unterstrichen.

Die Bedeutung der Anforderung, dass unternehmensexterne Stakeholder auf einfache Art und Weise auf die für sie bestimmten Daten zugreifen können, wird als niedrig eingestuft. Beobachtet man die Aktivitäten bei der interorganisationellen Optimierung der Wertschöpfungskette, so werden diese Daten bald an Bedeutung gewinnen. Eine offene Informationspolitik gegenüber Shareholdern und Gesellschaft erreicht zudem eine immer höhere Relevanz. Auch hier könnte ein PMS mit der erwähnten Funktion beitragen.

\section{Zusammenfassung der Umfrageergebnisse}

Performance Measurement ist ein wichtiges Thema. Sämtliche antwortenden Unternehmen beschäftigen sich mit dieser Thematik. Die fertigenden Unternehmen haben häufiger an der Umfrage teilgenommen als Dienstleistungsbetriebe. Der in diesen Branchen erhöhte Kostenund Qualitätsdruck, die erhöhte Mobilität der Güter durch die Globalisierung und die teilweise schwierigere Differenzierung als in Dienstleistungsbranchen könnte ein Indiz hierfür sein. In Branchen wie „Healthcare“ oder "Consumer" stehen entsprechende Veränderungen noch in

\section{Abbildung B-14: Seite 14 des Berichtes}


den Anfängen, werden aber vermehrt auch durch staatliche Interventionen gefördert. Es ist daher davon auszugehen, dass die Bedeutung in den entsprechenden Branchen in Zukunft noch zunehmen wird.

Im Vergleich mit älteren Studien scheint der Siegeszug der Ansätze des Value Based Management und der Balanced Scorecard anzuhalten. Neben dem im Personalbereich weit verbreiteten Management by Objectives, welches in die obigen Ansätze integriert werden kann, zeichnet sich ab, dass diese zu den dominierenden Konzepten für das Performance Measurement werden.

Untersucht man die Art der verwendeten Performance-Indikatoren und deren Erhebungshäufigkeit, so liegt die Schlussfolgerung nahe, dass die Orientierung an der kurzfristigen Performance gegenüber der langfristigen dominiert. Diese teilweise auch von den Finanzmärkten provozierte Entwicklung erscheint langfristig problematisch. Eine offene Informationspolitik, gerade auch über nicht-finanzielle Aspekte, könnte das Verständnis der entsprechenden Kreise erhöhen und somit zu einer längerfristigen Orientierung führen. In diesen Bereich gehört auch die Aufnahme von weiteren Empfängern der performancerelevanten Daten. Der Einbezug von Mitarbeitern, Kunden und Lieferanten, aber auch der Öffentlichkeit, um entsprechende Informationen kann sich vorteilhaft auswirken. Ein leistungsfähiges Performance-Measurement-System kann hier einen wesentlichen Beitrag leisten.

Erstaunt hat auch, dass nur $80 \%$ der Unternehmen ihre strategischen Projekte systematisch überwachen. Die Erweiterung der Projekt-Management-Guidelines um ein entsprechendes Multi-Projekt-Management auf der strategischen Ebene und deren konsequente Institutionalisierung ist dringend angezeigt. Elemente wie die Priorisierung, die Risikoüberwachung (Abhängigkeiten von Keyplayern, Externen), die Projektfortschrittskontrolle und die Bereitstellung eines entsprechenden MethodenWerkzeugkastens könnten hier zweckdienlich sein.

Der Einsatz von Informationstechnologie wird allgemein als wichtig erachtet. Drei Viertel der Unternehmen unterstützen ihre PM-Systeme mit Informationstechnologie. Dabei dominiert der Einsatz von Standardkomponenten gegenüber Eigenentwicklungen. Cognos, Frango und Insight sind die meistgenannten offenen Standardtools, die zum Einsatz kommen. SAP Business Warehouse (BW), resp. SAP Strategic Enterprise Management (SEM) ist das Tools, welches in Verbindung mit einer Enterprise Ressource Planning (ERP) - Lösung genannt wird.

Die Integration in die bestehende Informationssystem-Architektur ist im allgemeinen mangelhaft. Die automatisierte Datenübernahmen aus den operativen Systemen in das PMS sind noch zuwenig verbreitet. Es stellt sich daher die Frage, von wem die PMS-Projekte getrieben werden. Kommt der Anstoss primär von der Business-Seite, so besteht die Gefahr,

\section{Abbildung B-15: Seite 15 des Berichtes}


dass „schnellen Lösungen“ gegenüber gut integrierten den Vorzug gegeben wird. Dies führt zwar zu Quick-Wins, die jedoch meist nicht ausbaubar sind, sich nicht an die sich entwickelnde Informationssystem-Landschaft anpassen können und mit grossen laufenden administrativen Aufwendungen, d.h. der manuellen Datenerhebung und -aufbereitung, verbunden sind. Den tiefen Investitionskosten stehen hohe Betriebskosten gegenüber. Neben dem Kostenaspekt ist auch der Zeitaspekt nicht zu unterschätzen. Sind zahlreiche manuelle Eingriffe für die Aufbereitung der performance-relevanten Daten notwendig, so führt dies automatisch zu einer Verzögerung, bis die Daten verfügbar werden, was sich wiederum nachteilig auf den Informationswert und die Motivation der Informationsempfänger auswirkt.

Wird das Leistungsvermögen aktueller PM-Systeme und die Anforderungen, die an entsprechende Systeme gestellt werden, analysiert, so erscheint ein ganzheitlicher Ansatz notwendig. Dabei sollte das IT-System ausgehend von einer organisatorischen Konzeption hergeleitet werden. ${ }^{11}$ Auch wenn, was wir unterstützen, im kleinen begonnen wird, so scheint doch ein umfassendes Datenmodell in einer frühen Projektphase ein Schlüsselfaktor darzustellen. Nur so können die geforderten Analysefunktionen realisiert werden. Dabei wird von den Unternehmen ein freies Bewegen in den performance-relevanten Daten gefordert, was mit einem multidimensionalen Ansatz der Datenrepräsentation erfüllt werden kann.

Die Bedeutung der Kommunikation der performance-relevanten Daten ist noch bescheiden. Dies lässt sich dadurch erklären, dass der Kreis der Daten-Empfänger noch beschränkt ist. Wird dieser erweitert, so wird die Kommunikation an Bedeutung gewinnen.

\section{Projekt-Webseite: www.measure.ch}

Weiterführende Informationen zur Thematik des IT-gestützten Performance Measurements finden sich auf der oben bezeichneten Projektwebseite.

Wir möchten den teilnehmenden Unternehmen an dieser Stelle ganz herzlich danken und hoffen mit dem Feedback auch etwas zurückgeben zu können. Besten Dank! Für allfällige Fragen stehen wir Ihnen gerne zur Verfügung.

${ }^{11}$ vgl. erste Forschungsergebnisse in: Wettstein, T.;Küng, P. (2001). Gesamtheitliches Performance Measurement - Vorgehensmodell und informationstechnische Ausgestaltung. Fribourg, Departement of Informatics.; Wettstein, T.;Küng, P.;Meier, A. (2001). "Performance Measurement als Ausweg aus dem Information Overload: Ein zielorientierter Ansatz." HMD -Praxis der Wirtschaftsinformatik 222: 49-58.; Küng, P.;Wettstein, T. (2001). Performance Measurement Systems - there is a long road ahead. International Workshop on Performance Measurement, Glasgow/UK.

\section{Abbildung B-16: Seite 16 des Berichtes}




\section{Anhang C: Übersicht über kommerzielle IT-Systeme für das PM}

Die nachfolgende Zusammenstellung nennt Produkte, die von den entsprechenden Herstellern als IT-Systeme für das Performance Measurement angeboten werden. Neben dem Hersteller, der Produktebezeichnung, allfälligen Modulangaben und Bemerkungen ist jeweils die Webseite für weiterführende Informationen angegeben. Um die Übersichtlichkeit zu verbessern, wurden die Produkte in fünf Kategorien eingeteilt. Die Zuordnung erfolgte i.d.R. auf Grund der verfügbaren Dokumentation. Bei den mit einer Brille ( $\sigma \sim$ ) bezeichneten Produkten hat der Verfasser weiter gehende Untersuchungen vorgenommen, sei es anhand einer Testinstallation, einem Besuch beim Hersteller oder praktischen Erfahrungen im Rahmen der beruflichen Tätigkeit. Die vorgenommene Einteilung ist nicht immer eindeutig. Einzelne Produkte könnten in mehr als einer Kategorie aufgeführt werden. Des Weiteren erhebt die Liste keinen Anspruch auf Vollständigkeit. Es handelt sich um eine Auswahl des Verfassers, welche durch Fachgespräche, Internetrecherchen und Dokumentenstudium während der Bearbeitungszeit der vorliegenden Dissertation entstanden ist. Eine letzte Aktualisierung der Daten fand im September 2002 statt.

\begin{tabular}{|l|l|l|}
\hline \multicolumn{2}{|l|}{ Firma } & \multicolumn{2}{|l|}{ Produkt le (Module, Bemerkung) } & Webseite \\
\hline \multicolumn{2}{|l|}{ Produkte, die ein breites Spektrum an PM-Funktionalitäten abdecken. } \\
\hline Active Strategy & Active Strategy Enterprise & www.activestrategy.com \\
\hline Brio Technology & $\begin{array}{l}\text { Brio Performance Suite (Brio Intelligence, } \\
\text { Brio Portal, Brio Reports, Brio Metrics } \\
\text { Builder) }\end{array}$ & www.brio.com \\
\hline Business Objects & $\begin{array}{l}\text { Business Objects (Analytic Applications, } \\
\text { Plattform, Portal and Broadcasting, } \\
\text { Administration, Query, Reporting \& } \\
\text { Analysis, Security, Metadata, Database) }\end{array}$ & www.businessobjects.com \\
\hline Cognos & $\begin{array}{l}\text { Cognos Series 7, Cognos Finance, } \\
\text { Analytic Applications }\end{array}$ & www.cognos.com \\
\hline CorVu & $\begin{array}{l}\text { RapidScorecard, CorManage, } \\
\text { CorBusiness, CorPortfolio, CorServer }\end{array}$ & www.corvu.com \\
\hline Crystal Decisions & $\begin{array}{l}\text { Gr Crystal Report, Crystal Enterprise, } \\
\text { Crystal Analysis }\end{array}$ & www.crystaldecisions.com \\
\hline Hyperion & $\begin{array}{l}\text { Gr Business Performance Management } \\
\text { Suite (Performance Scorecarding, } \\
\text { Modeling \& Optimization, Planning, } \\
\text { Budgeting \& Forecasting, Consolidation } \\
\text { \& Reporting) }\end{array}$ & www.hyperion.com \\
\hline
\end{tabular}




\begin{tabular}{|c|c|c|}
\hline Firma & Produkt/e (Module, Bemerkung) & Webseite \\
\hline $\begin{array}{l}\text { Open Ratings } \\
\text { (ehemals Gentia) }\end{array}$ & $\begin{array}{l}\text { EPImpact (Enterprise Performance), } \\
\text { BPImpact (Buyers Performance), } \\
\text { SPImpact (Strategic Performance) }\end{array}$ & www.openratings.com \\
\hline Outlooksoft & $\begin{array}{l}\text { OutlookSoft EAP (Financial } \\
\text { Management, Performance } \\
\text { Measurement, Operational Management) }\end{array}$ & www.outlooksoft.com \\
\hline PROCOS & $\begin{array}{l}\text { STRAT\&GO (bestehend aus Modulen } \\
\text { zur Balanced Scorecard (SBS), } \\
\text { Umsetzung auf Mitarbeiterstufe (MpP), } \\
\text { Analyse (SAM), Budgetierung (CB) und } \\
\text { Kundenzufriedenheit (CSA)) }\end{array}$ & www.procos.com \\
\hline QPR & os QPR Management Software+ & www.qpr.com \\
\hline SAS & $\begin{array}{l}\text { Über } 50 \text { Module, die je nach } \\
\text { Anwendungsbereich und Branche } \\
\text { kombiniert werden. }\end{array}$ & www.sas.com \\
\hline \multicolumn{3}{|c|}{ Produkte, die vor allem die visuelle Kommunikation von Performance-Werten unterstützen } \\
\hline Cartesis & $\begin{array}{l}\text { Strategic Financial Management (SFM), } \\
\text { Cartesis Carat, Cartesis Magnitude } \\
\text { (primär integrierte strategische Planung, } \\
\text { Budgetierung, Berichtswesen, } \\
\text { Konsoliderung und } \\
\text { Erfolgsgrössenmessung) }\end{array}$ & www.cartesis.com \\
\hline Ergometrics & $\begin{array}{l}\text { of Ergometrics (Primär zur Präsentation } \\
\text { von Performance-Daten) }\end{array}$ & www.ergometrics.com \\
\hline ICVisions & $\begin{array}{l}\text { of Dolphin (Primär zur Präsentation von } \\
\text { Performance-Daten nach dem } \\
\text { Intellectual-Capital-Ansatz) }\end{array}$ & www.icvisions.com \\
\hline Inphase & $\begin{array}{l}\text { PerformancePlus (Schwerpunkt } \\
\text { Präsentation) }\end{array}$ & www.inphase.com \\
\hline PBViews & $\begin{array}{l}\text { PBView Pro (Primär zur Präsentation von } \\
\text { Balanced Scorecards) }\end{array}$ & www.Pbviews.com \\
\hline PMExpress & $\begin{array}{l}\text { PMExpress (einfache Applikation, primär } \\
\text { zur Präsentation von Balanced } \\
\text { Scorecards) }\end{array}$ & www.pm-express.com \\
\hline Promeasys & $\begin{array}{l}\text { Gr Promeasys (Pmbuilder, Pmstore, } \\
\text { Pmdesign, Pmview) }\end{array}$ & www.promeasys.com \\
\hline Targit & $\begin{array}{l}\text { Targit Analysis Suite (Analyse und } \\
\text { Reporting-Tool; primär Darstellung) }\end{array}$ & www.targit.com \\
\hline Temtec & $\begin{array}{l}\text { Executive Viewer (primär Präsentation; } \\
\text { Spezialität von Temtec ist eine Lösung } \\
\text { namens Portable Cubes für mobile PM- } \\
\text { Lösungen) }\end{array}$ & www.temtec.com \\
\hline Visual InSight & $\begin{array}{l}\text { Visual InSight (verschiedene Produkte } \\
\text { zur Präsentation und Analyse von Daten, } \\
\text { Schwerpunkt Präsentation und E- } \\
\text { Business Performance Management } \\
\text { (eBPM), d.h. PM für Electronic-Business- } \\
\text { Lösungen) }\end{array}$ & www.visualinsights.com \\
\hline
\end{tabular}




\begin{tabular}{|c|c|c|}
\hline Firma & Produkt/e (Module, Bemerkung) & Webseite \\
\hline \multicolumn{3}{|c|}{ Produkte, die vor allem die Analyse der Daten unterstützen. } \\
\hline Accrue & $\begin{array}{l}\text { Accrue G2, AccrueINSIGHT, PilotSuite, } \\
\text { PilotHITLIST (Schwerpunkt E-Business } \\
\text { Performance Management (eBPM), d.h. } \\
\text { PMS für Electronic-Business-Lösungen) }\end{array}$ & www.accrue.com \\
\hline Mathlab & $\begin{array}{l}\text { Mathlab (Tool für mathematische } \\
\text { Analysen, Visualisierung, statistische } \\
\text { Funktionen, neben dem Hauptprodukt } \\
\text { sind Komponenten zur Datenakquisition } \\
\text { und Programmierung verfügbar) }\end{array}$ & www.mathworks.com \\
\hline SPSS & $\begin{array}{l}\text { Go Rund um das Analyseprogramm } \\
\text { SPSS bietet der Hersteller eine Reihe } \\
\text { von Modulen an: SmartViewerWeb, } \\
\text { AnswerTree, Clementine, DataEntry, } \\
\text { DecisionTime, etc. (Schwerpunkt: } \\
\text { statistische Auswertungen) }\end{array}$ & www.spss.com \\
\hline StatSoft & $\begin{array}{l}\text { Statistica (Base-Modul für statistische } \\
\text { Auswertungen, Zusatzmodule für } \\
\text { verschiedene Bereiche: Quality Control } \\
\text { Charts, Process Analysis, Data Miner, } \\
\text { SPC-System, etc.) }\end{array}$ & www.statsoftinc.com \\
\hline StatWare & Statit e-QC (Prozess-Analyse-Tool) & www.statware.com \\
\hline VGSPS & $\begin{array}{l}\text { PS-Explore (Explore, Report, Database, } \\
\text { StatSys und Toolsys. Schwerpunkt: } \\
\text { statistische Auswertungen) }\end{array}$ & www.ps-explore.de \\
\hline \multicolumn{3}{|c|}{$\begin{array}{l}\text { Produkte, die vor allem die Datenakquisition, das Datenmanagement und den ETL-Prozess } \\
\text { unterstützen. }\end{array}$} \\
\hline IBM & $\begin{array}{l}\text { Data Propagator (spezialisierte } \\
\text { Software für den Austausch von Daten } \\
\text { zwischen verschiedenen Datenbanken) }\end{array}$ & $\begin{array}{l}\text { www- } \\
\text { 4.ibm.com/software/data/dp } \\
\text { ropr/ }\end{array}$ \\
\hline & $\begin{array}{l}\text { ar DB2 OLAP Server (Data Warehouse- } \\
\text { Lösung mit ETL-Komponente, } \\
\text { OLAPMiner, Security-Tool) }\end{array}$ & $\begin{array}{l}\text { www- } \\
\text { 3.ibm.com/software/data/db } \\
\text { 2/db2olap/ }\end{array}$ \\
\hline Informatica & $\begin{array}{l}\text { Primär Produkte für die Datenakquisition } \\
\text { (PowerCenter, PowerMart, } \\
\text { PowerConnect, zusätzlich Produkte für } \\
\text { die Analyse und die Präsentation) }\end{array}$ & www.informatica.com \\
\hline Microsoft & $\begin{array}{l}\text { Go SQL Server } 2000 \text { mit Zusatzmodulen } \\
\text { für ETL (DTS), OLAP, Analyse }\end{array}$ & www.microsoft.com/sql \\
\hline Oracle & $\begin{array}{l}\text { Database 9i, Data Warehousing, OLAP, } \\
\text { DataMining, ETL-Komponente }\end{array}$ & $\begin{array}{l}\text { www.oracle.com/ip/deploy/ } \\
\text { database/oracle9i }\end{array}$ \\
\hline
\end{tabular}




\begin{tabular}{|c|c|c|}
\hline Firma & Produkt/e (Module, Bemerkung) & Webseite \\
\hline \multicolumn{3}{|c|}{ Performance-Measurement-Produkte, die mit ERP-Systemen gekoppelt sind. } \\
\hline IDS Scheer & $\begin{array}{l}\text { Go Process Performance Manager } \\
\text { (Analyse von Prozessen, primäre } \\
\text { Datenquelle SAP R/3) }\end{array}$ & www.ids-scheer.de/ppm \\
\hline People Soft & $\begin{array}{l}\text { Enterprise Performance Management } \\
\text { der ERP-Lösung PeopleSoft }\end{array}$ & $\begin{array}{l}\text { http://www.peoplesoft.com/ } \\
\text { corp/en/products/line/epm }\end{array}$ \\
\hline SAP & $\begin{array}{l}\text { MySAP Business Intelligence } \\
\text { bestehend aus einer Reihe von Modulen: } \\
\text { Data Warehousing, Reporting und } \\
\text { Analyse, Portalintegration, Performance } \\
\text { Management, etc. (primäre Datenquelle } \\
\text { SAP R/3) }\end{array}$ & http://www.sap.com \\
\hline
\end{tabular}

Tabelle C-1: Übersicht über kommerzielle IT-Systeme für das PM 


\section{Anhang D: Weitere PMS-Ansätze}

In der Literatur wird eine Reihe weiterer Ansätze diskutiert. Die Ähnlichkeit mit den vier diskutierten Ansätzen (vgl. Kapitel drei) lässt es jedoch nicht sinnvoll erscheinen, diese im Detail zu besprechen. Insbesondere Ansätze aus der Unternehmensberatungspraxis existieren in einer fast unübersichtlichen Vielfalt (vgl. bspw. die Darstellung bei Klingebiel (2000), der die Ansätze von Arthur Andersen, Booz, Allen \& Hamilton, Coopers \& Lybrand, Ernst \& Young, Gemini Consulting, KMPG, Arthur D. Little und Price Waterhouse bespricht). Bei Gleich (2001) finden sich Informationen zu einer Reihe von weiteren Ansätzen.

In der folgenden Tabelle werden in alphabetischer Reihenfolge einige Ansätze genannt und kurz charakterisiert:

\begin{tabular}{|c|c|c|}
\hline $\begin{array}{l}\text { Bezeichnung } \\
\text { des Ansatzes }\end{array}$ & Charakterisierung & Quellen \\
\hline $\begin{array}{l}\text { IPMS } \\
\text { (Integrated } \\
\text { Performance } \\
\text { Measurement } \\
\text { System) }\end{array}$ & $\begin{array}{l}\text { Die Autoren schlagen vor, die Performance auf den } \\
\text { folgenden vier Ebenen zu messen: (1) Unternehmen, } \\
\text { (2) Business Units, (3) Geschäftsprozesse, (4) } \\
\text { Aktivitäten. Das Modell berücksichtigt auf jeder Ebene } \\
\text { die folgenden Elemente: } \\
\text { - Bedürfnisse der Shareholder } \\
\text { - externe Überwachung } \\
\text { (environmental monitoring) } \\
\text { - Ziele (in Abhängigkeit des Marktes) } \\
\text { - Kennzahlen } \\
\end{array}$ & Bitici et al. (1998) \\
\hline PRISM & $\begin{array}{l}\text { Konzentration auf die Prozesse für die Erstellung eines } \\
\text { PMS. Neely und seine Mitarbeiter schlagen einen } \\
\text { Prozess vor, der im Wesentlichen drei Phasen } \\
\text { umfasst: (1) Entwicklung der Unternehmensziele, (2) } \\
\text { Umsetzung dieser Ziele in geeignete Indikatoren und } \\
\text { (3) die Implementierung der Kennzahlen. Im } \\
\text { Wesentlichen offerieren die Autoren Methoden und } \\
\text { Werkzeuge, um obige Punkte zu erreichen, d.h. ein } \\
\text { passendes, ausgewogenes Set von Indikatoren zu } \\
\text { erhalten, die mit den Zielen des Unternehmens } \\
\text { einhergehen. }\end{array}$ & Neely et al. (1996) \\
\hline ProMES & $\begin{array}{l}\text { ProMES steht für Productivity Measurement and } \\
\text { Enhancement System. Dieser für Dienstleistungs- } \\
\text { unternehmen konzipierte Ansatz stammt von der } \\
\text { Universität Eindhoven. Im Zentrum des Vorschlages } \\
\text { steht ein Closed-Loop-System, welches die } \\
\text { Zielsetzung der Organisation berücksichtigt, diese } \\
\text { operationalisiert, die Ergebnisse misst und sie als } \\
\text { Feed-back an die Betroffenen zurückgibt. Wie für } \\
\text { Ansätze aus personalintensiven Branchen zu erwarten, } \\
\text { liegt der Fokus stark auf den Mitarbeitern. Der Ansatz } \\
\text { umfasst ebenfalls ein Belohnungssystem. }\end{array}$ & Kleingeld (1994) \\
\hline
\end{tabular}




\begin{tabular}{|c|c|c|}
\hline $\begin{array}{l}\text { Tableau de } \\
\text { Bord }\end{array}$ & $\begin{array}{l}\text { Dieses Instrument besteht aus einer Reihe von } \\
\text { Messgrössen, welche ursprünglich wesentliche } \\
\text { Erfolgsfaktoren des Einflussbereiches eines } \\
\text { Produktionsleiters (Ingenieurs) abdeckten. Das Prinzip } \\
\text { wird heute von einem breiteren Kreis von } \\
\text { Führungspersonen v.a. in Frankreich angewandt. } \\
\text { Die Grundidee besteht darin, dass ein Manager durch } \\
\text { das Bewirtschaften der Erfolgsfaktoren Einfluss auf } \\
\text { den finanziellen Erfolg nehmen kann. Häufig finden die } \\
\text { Budgets, die aus den operativen Plänen erstellt } \\
\text { werden, die strategischen Ziele und die } \\
\text { Umwelteinflüsse Eingang in das System. Die nicht- } \\
\text { finanziellen Performance-Indikatoren werden dabei als } \\
\text { so genannte „physikalische Kennzahlen” bezeichnet } \\
\text { (vgl. Lebas (1994)). Das Tableau de Bord wird im } \\
\text { Gegensatz zur traditionellen Kostenrechnung als } \\
\text { dynamisches System begriffen (vgl. Lebas (1994)). Die } \\
\text { zukünftigen Potenziale sind als wichtiger Teil des } \\
\text { "Armaturenbrettes” zu begreifen. Epstein und Manzoni } \\
\text { (1997) schlagen ebenfalls den Einbezug von } \\
\text { (externen) Benchmarking-Informationen vor. Die } \\
\text { Anwender erstellen je nach Bedürnnis ein kurz- } \\
\text { und/oder ein langfristig orientiertes Tableau. Die } \\
\text { Zusammenstellung der Indikatoren erfolgt individuell. }\end{array}$ & $\begin{array}{l}\text { Lebas (1994) } \\
\text { Lebas (1995) } \\
\text { Epstein und Manzoni } \\
\text { (1997) } \\
\text { vgl. auch } \\
\text { Hoffmann (1999) }\end{array}$ \\
\hline
\end{tabular}

Tabelle D-1: Charakterisierung weiterer Performance-Measurement-Ansätze 